\title{
LEGIBILITY
}

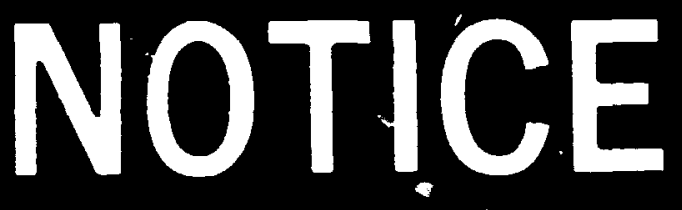

$\Rightarrow$

A major purpose of the Technical Information Center is to provide the broadest dissemination possible of information contained in DOE's Research and Development Reports to business, industry, the academic community, and federal, state and local governments.

Although a small portion of this report is not reproducible, it is being made available to expedite the availability of information on the research discussed herein. 
ORNL-657

Distribution-UC-404

Contract No. DE-ACO5-840R21400

ORNL--6571

DE90 000979

\section{SOLID STATE DIVISION PROGRESS REPORT \\ for Period Ending March 31, 1989}

F. W. Young, Jr., Director

J. B. Roberto, Associate Director

L. A. Boatner, Section Head

J. F. Cooke, Section Head

R. M. Moon, Section Head

D. M. Zehner, Section Head

Edited by:

P. H. Green

D. M. Watson

Date Published - August 1989

OAK RIDGE NATIONAL LABORATORY

Oak Ridge, Tennessee 37831

operated by

MARTIN MARIETTA ENERGY SYSTEMS, INC.

for the

DEPARTMENT OF ENERGY 
Reports preoiousiy issued in this series are as follows:

ORNL-1095

ORNL-1128

ORNL-1214

ORNL-1261

ORNL-1301

ORNL-1359

ORNL-1429

ORNL-1506

ORNL-1606

ORNL-1677

ORNL-1762

ORNL-1851

ORNL-1852

ORNL-1944

ORNL-1945

ORNL-2051

ORNL-2052

ORNL-2188

ORNL-2189

ORNL-2413

ORNL-2414

ORNL-2614

ORNL-2829

ORNL-3017

ORNL-3213

ORNL-3364

ORNL-3480

ORNL-3676

ORNL-3841

ORNL-3935

ORNL-4098

ORNI. -4250

ORNL-4408

ORNL -1526

ORNL-4669

ORNL-4779

ORNL-4861

ORNL-4952

ORNL-5028

ORNL-5135

ORNL-5328

ORNL-5486

ORNL-5640

ORNL-5850

ORNL-5975

ORNL-6128

ORNL -6306

ORNL-6453
Period Endirg April 30, 1951

Period Ending July 31, 1951

Period Ending October 31, 1951

Period Ending Janury 31, 1952

Period Ending May 10, 1952

Periud Ending August 10, 1952

Feriud Ending November 10, 1952

Period Ending February 10, 1953

Period Ending August 30, 1953

Period Ending February 28, 1954

Period Ending August 31, 1954

Period Ending February 28, 1955

Period Ending February 28, 1955

Period Ending August 31, 1955

Period Ending August 31, 1955

Period Ending February 29, 1956

Period Ending February 29, 1956

Period Ending August 31, 1956

Period Enaing August 31, 1956

Pesiod Ending August 31, 1957

Period Ending August 31, 1957

Period Ending August 31, 1958

Period Ending August 31, 1959

Period Ending August 31, 1960

Period Ending August 31, 1961

Period Fnding August 31, 1962

Period linding May 31, 1963

Period Ending May 31, 1964

Period Ending May 31, 1965

Period Ending December 31, 1965

Period Ending December 31, 1966

Period Ending December 31, 1967

Period Ending December 31, 1968

Period Ending December 31, 1969

Period Ending December 31, 1970

Period Ending December 31, 1971

Period Ending December 31, 1972

Period Ending December 31, 1973

Period Ending December 31, 1974

Pariod Ending December 31, 1975

Period Ending April 30, 1977

Teriod Ending September 30, 1978

Period Ending February 29, 1980

Period Ending September 30, 1981

Period Ending March 31, 1983

Period Ending September 30, 1984

Period Ending March 31, 1986

Period Ending Septemher 30, 1987 


\section{Contents}

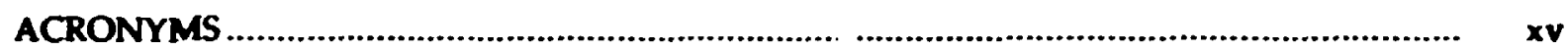

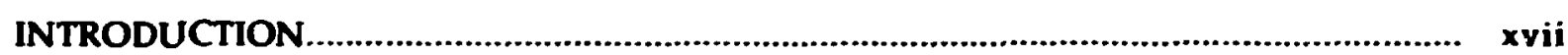

\section{Theoretical Solid State Physics}

\section{ELECTRONIC AND MAGNETIC PROPERTIES}

Pseudoexcitons in Mixed-Valence Mctals -S. H. Liu....................................................... 2

Spin-Fluctuation Resonance in Mixed-Valence Materials-S. H. Liu....................................... 3

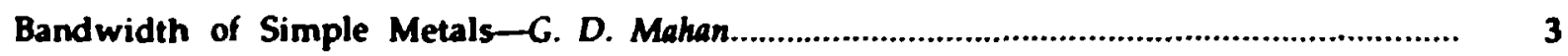

Minority Carrier Band Tails in Semiconductors_C. D. Mahan................................................ \&

Theory of Spin-Modulated Structures in Rare-Earth Metals-S. H. Liu and J. F. Cooke............. 5

Magnetic Excitations in Transition-Metal Systems_J. F. Cooke and J. A. Blaclonan................... 6

Inelastic Electron Scattering and Electron-Hole Excitations in Iron and NickelJ. A. Blackmen and J. F. Cooke ............................................................................... T

Collective Enhancement of Three-Bndy Recombinations in Highly lonized Classical Plasmas-M. Rasolt and F. Perrot................................................................................ 8

Energetics and Lattice Relaxation of Light Impurities in Aluminum-F. Perrol and Mark Rasolt

Divergences in the Force Balance Theory of Resistivity-R. S. Fishman and C. D. Mahan.

The Doublet Splitting of the Real Squashing Modes in Superfluid ${ }^{3} \mathrm{He}-\mathrm{B}-$ R. S. Fishman and I. A. Sauls. 


\section{SUPERCONDUCTIVITY}

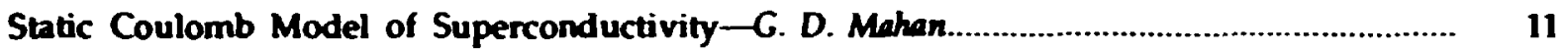

Theory of High- $T_{c}$ Superconductors Within a Realistic Anderson Lattice Model-

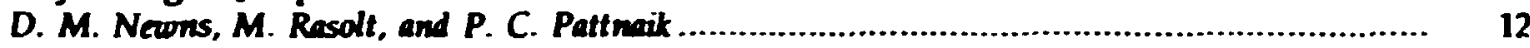

A Spin Polaron Model of High- $T_{c}$ Superconductivity-R. F. Wood, Mark Mostoller, and I. F. Cooke

Antiferromagnetic Band Calculations for the $\mathrm{CuO}_{2}$ Planes in High- $\mathrm{T}_{c}$ Superconductors-

R. F. Wood, Mark Mastoller, and I I. Cooke.

Positron Annihilation in High-T, Copper Oxides-S. H. Liu ................................................. 14

Lattice Vibrations in $\mathrm{La}_{2} \mathrm{CuO}_{4}-$ Mark Mostoller .............................................................

A New Crossover Behavior of Type II Superconductors in Strong Magnetic Fields-

M. Resolt and Z. Tesanovic.

An Expansion in I/z for Granular Superconductors-R. S. Fishman.

\section{SURFACES AND INTERFACES}

Search for "Near Perfect" Agreement in LEED Analysis_-H. L. Davis and I. R. Noonan ............. 18

Surface Structure and Low-Energy Electron Diffraction (LEED) "Bric-A-Brac": A Case

Study of $\mathrm{Ag}(110)-H$. L. Devis and I. R. Noonan.

Rippled Reconstruction of the $\mathrm{HFC}(100)-(1 \times 1)$ Surface-H. L. Davis, J. R. Noonan, and $K$. Müllet

Application of Onsager's Reciprocity Relations to Interface Motion During Phase Transformations-Theodore Kaplan, M. J. Aziz, and L. J. Gray

\section{ION-SOLID INTERACTIONS}

The Showing-Down Time of Energetic Atoms in Solids-M. T. Robinson................................... 22

Channeling Measurement of Solute Vibration Amplitude in an Alloy-I. H. Berrett.................. 23

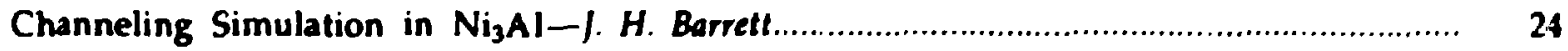

Structurt of the Boror.-Hydrogen Complex in Silicon-A. D. Marwick, C. S. Oehrlein, J. H. Barretl, and N. M. Johnson. 


\section{NEUTRON DIFFRACTION}

Atom-Pair Correlations in Fe-13.5\% V-J. W. Cable, H. R. Child, and Y. Natai....................... 39

Diffuse Neutron Scattering from a Disordered FCC Pd 3 Mn Crystal-H. R. Child,

E. Gurewitz, and J. W. Cable....................................................................................

Thermal Neutron Scattering Lengths and Capture by Even Cakium Isotopes-

S. Raman, S. Kahane, R. M. Moon, I. A. Fernandez-Baca, I. L. Zerestky, I. E. Lymn,

and J. W. Richardson, Ir.

\section{INELASTIC NEUTRON SCATTERING}

A Neutron Scattering Study of the Impulse Approximation in Single-Crystal

Beryllium-D. F. McMorrow, R. A. Cowley, R. M. Nickiow, P. W. Mitchell,

A. D. Taylor, and M. Mostoller.

Neutron Scattering Studies of the Martensitic Transformation in Sodium Metal-

R. Berliner, H. G. Smith, J. R. D. Copley, and J. Trivisonno

Lattice Vibrations of Copper in $\mathrm{YBa}_{2} \mathrm{Cu}_{3} \mathrm{O},-\mathrm{H}$. A. Mook, I. A. Haroey, and

N. W. Hill

Magnetic Dynamics of $\mathrm{La}_{2} \mathrm{CuO}_{4}$ and $\mathrm{La}_{2-x} \mathrm{Ba}_{x} \mathrm{CuO}_{4}-\mathrm{G}$. Aeppli, S. M. Hayden,

H. A. Mook, Z. Fisk, S.-W. Cheong, D. Rytz, J. P. Remeika, G. P. Espinosa, and

A. S. Cooper.

Magnetıc Excitations in Thulium-J. A. Femande-Baca, R. M. Nicklnw, and I. I. Rhyne............ 45

Spin Dynamics of Gd at High Temperatures-J. W. Cable and R. M. Nicklow............................ 46

Spin Dynamics of Gd in an Applied Magnetic Field-J. W. Cable and R. M. Nicklow................. 47

Polarized Neutron Studies of Forbidden Magnon Scattering in Gadolinium-

I. W. Cable and R. M. Nicklow.

Spin Dynamics of Amorphous $\mathrm{Fe}_{00} \mathrm{Ni}_{1} \mathrm{Zr}_{10}-$ J. A. Femandez-Baca, M. Hennion, B. Hennion,

I. J. Rhyne, and G. E. Fish.

\section{INSTRUMENTATION}

Discrete Thin-Film Multilayer Design for Neutron Mirrors-John B. Hayter and

H. A. Mook.

A Three-Pinhole Collimating System for the ORNL 10-m Small-Angle X-Ray

Scattering Camera-C. D. Wignall, I. S. Lin, and S. Spooner.... 
Damage Energy as a Predictor of Frenkel Pair Production Under Channeling Conditions-

O. S. Oen

\section{Neutron Scattering}

\section{SMALL-ANGLE SCATTERING}

Instrumental Resolution Effects in Small-Angle Scattering-G. D. Wignall, D. K. Christen, and $V$. Ramakrishnan.

Interartions in Mixtures of Poly(Ethylene Oxide) with Poly(Methyl Methacrylate)-

G. D. Wignall, T. P. Russell, and H. Ito

Characterization of Carbon-Filled Polymers by Small-Angle Scattering TechniquesG. D. Wignall, N. R. Farrar, and S. Morris.

A Small-Angle Neutron Scattering (SANS) Study of the Dimensions of Polystyrene Formed by Freeze Drying from Dilute Solution-Hugh R. Brown and G. D. Wignall

On the Double-Melting Behavior of Poly(Ether Ether Ketone)-Youngchul Lee, Roger S. Poiter, and J. S. Lin..

Ordered Macromolecular Structures in Ferrofluid Mixtures-John B. Hayter, Roger Pynn, Stuart Charles, Ame T. Slgeltorp, Jill Trewhelle, Gerald Stubbs, and Peter Timmins.

Hydrodynamic Interactions in Concentrated Colloidal Dispersions-lohn B. Hayter, Iff Penfold, and Ian K. Snook.

A Direct Observation of Counterion Condensation Around Cylindrical MicellesC. F. Wu, S. H. Chen, L. B. Shih, and J. S. Lin.

Real-Time Study of Reversion in Mn-33 at.\% Cu-Stephen Spooner, B. D. Caulin, and Y. Morii.

Phase Separation in a Ni-12.5 at.\% A Alloy at $575 \mathrm{~K}-$ J. E. Epperson, J. S. Lin, and P. D. Labarbe.

Hard-Sphere Analysis of the Precipitate Structure in Al-Li AlloysStephen Spoomer, R. Triolo, and E. Coponetti....

Hydride Precipitation in Cathodically Charged Aluminum and NickelStephen Spoomer, E Sirois, and H. K. Birnbaum.

Influence of Severe Deformation on Precipitation in Al-Li Aloys-S. Spoomer, M. D. Kraitchmen, J. Hoyt, D. defontaine, and P. Tortorelli.

The Study of Fractal Seometry on Submicron Length Scales by Small-Angle Scattering-Po-Zen Wong and I. S Lin. 


\section{Physical Properties of Materials}

\section{SUPERCONDUCTORS}

Magnetic Studies of Single-Crystal Superconducting $\mathrm{Er}_{1} \mathrm{Ba}_{2} \mathrm{Cu}_{3} \mathrm{O}_{2}-J$. R. Thompson,

S. T. Sekula, H. A. Deeds, D. K. Christen, B. C. Sales, and L. A. Boatner.

Oxygen Stoichiometry and Superconductivity in $\mathrm{HoBa}_{2} \mathrm{Cu}_{3} \mathrm{O}_{7-x}-$ B. C. Sales, Y. C. Kim,

J. R. Thompson, D. K. Christen, L. A. Boatner, and S. T. Sekula.

Magnetic Properties of Oxygen-Reduced Rare-Earth-Based $\left(\mathrm{RE}_{1} \mathrm{Ba}_{2} \mathrm{Cu}_{3} \mathrm{O}_{(7-x)}\right.$

Materials with RE $=\mathrm{Cd}, \mathrm{Dy}$, and Ho-J. R. Thompson, B. C. Sales, Y. C. Kim,

S. T. Sekula, L. A. Boatner, J. Brymestad, and D. K. Christen.

Anisotropies in the Magnetic Penetration Depth and Critical Current Density of $\mathrm{RBa}_{2} \mathrm{Cu}_{3} \mathrm{O}_{-x}(\mathrm{R}=\mathrm{Y}$ or Ho), Measured on Magnetically Aligned Powder Composites-D. K. Christen, Y. C. Kim, S. T. Sekula, J. R. Thompson, I. Brymestad, B. C. Sales, L. A. Boatner, and J. D. Budai

Superconductivity, Critical Current Density, and Magnetic Properties of Fe-Substituted $\mathrm{Y}_{1} \mathrm{Ba}_{2}\left(\mathrm{Cu}_{1-x} \mathrm{Fe}_{\mathrm{x}}\right)_{3} \mathrm{O}_{2}-\mathrm{S}$. T. Sekula, J. Brynestad, I. Budai,

D. K. Christen, J. R. Thompson, and Y. C. Kim.

Characterization and Superconducting Properties of Phases in the Bi-Sr-Cu-O

System-B. C. Chakoumakos, P. S. Ebey, B. C. Sales, and E. Sonder...

Variation of Superlattice Structure of the $\mathrm{Bi}_{2} \mathrm{Sr}_{2-\mathrm{x}} \mathrm{CuO}_{6}$ Superconductor with

Composition and Thermal History-J. D. Budai, B. C. Chakoumakos, B. C. Sales,

and Edward Sonder.

The Effects of Oxygen and Strontium Vacancies on the Superconductivity of

Single Crystals of $\mathrm{Bi}_{2} \mathrm{Sr}_{2-x} \mathrm{CuO}_{6-y}-$ Edward Sonder, B. C. Chakoumakos, and

B. C. Sales.

Superconductivity, Intergrain, and Intragrain Critical Current Densities of

$\mathrm{T}_{2} \mathrm{Ca}_{2} \mathrm{Ba}_{2} \mathrm{Cu}_{3} \mathrm{O}_{10+8}$ and $\mathrm{Tl}_{2} \mathrm{Ca}_{1} \mathrm{Ba}_{2} \mathrm{Cu}_{2} \mathrm{O}_{8+8}$ Materials-1. $R$. Thompson,

J. Brynestad, D. M. Kroeger, Y. C. Kim, S. T. Sekula, D. K. Christen, and

E. D. Specht.

Geometrically Large Flux-Line Pins-K:. R. Kerchner

\section{VARISTORS AND CARBIDES}

Origin of the Pulse-Response Characteristics of Zinc Oxide Varistors-F. A. Modine,

R. B. Wheeler, Youngjae Shim, and James F. Cordaro 
Zinc Oride Varistor Time Response-F. A. Modine, R. W. Major, S.-1. Choi,

L. B. Bergman, and M. N. Siloer

New Varistor Material-F. A. Modine and H. M. Hyatt

(1xl) Rippled Relaxation of (100) Transition-Metal Carbide Surfaces-C. R. Gruzalski,

D. M. Zehner, I. R. Noonan, H. L. Davis, R. A. DiDio, and K. Müller.

Low-Temperature Electrical Resistivity of Transition-Metal Carbides-C. Y. Allison,

C. B. Finch, M. D. Foegelle, and F. A. Modine.

Single-Crystal Growth of Group IVB and VB Carbides by the Floating-Zone Method-C. B. Finch, Y. K. Chang, and M. M. Abraham

\section{RADIATION EFFECTS}

Structural Inequivalence of the lon-Damage-Produced Amorphous State and the Glass State in Lead Pyrophosphate-B. C. Sales, J. O. Ramey, L. A. Boatner, and J. C. McCallum.

The Effects of Gamma Irradiation on the Superconducting Properties of $\mathrm{GdBa}_{2} \mathrm{Cu}_{3} \mathrm{O}_{-\pi}-$

E. Sonder, L. A. Boatner, B. C. Chakoumakos, and B. C. Sales

Microhardness in Natural Zircon as a Function of $\alpha$-Decay Dose Compared with

Synthetic Zircon Damaged by $\mathrm{Pb}$ lon Implantation-B. C. Chakoumakos,

I. C. McCallum, L. A. Boatner, W. C. Olioe, G. R. Lumplin, and R. C. Ewing

Enhancement of Color Center Luminescence in Neutron-Irradiated $\mathbf{A}_{2} \mathrm{O}_{3}-$

G. I. Pogatshnik, Y. Chen, and B. D. Euans

Stored Energy Recovery of Imadiated Copper-R. T. Richard, R. L. Chaplin,

R. R. Coltman, Ir., H. R. Kerchner, and C. E. Klabunde.

\section{The Synthesis and Characterization of Materials}

\section{THIN-FILM SUPERCONDUCTORS}

Fabrication of Epitaxial YBaCuO Superconducting 'Thin Films by Means of Thermal Coevaporation-R. Feenstra and D. B. Poker

Epitaxial Superconducting Thin Films of $\mathrm{YBa}_{2} \mathrm{Cu}_{3} \mathrm{O}_{7-x}$ on $\mathrm{KTaO}_{3}$ Single Crystals-

R. Feenstra, L. A. Boalmer, J. D. Budai, D. K. Christen, M. F. Chisholm, and

$M$. D. Galloway 
Transport Properties of $\mathrm{Y}_{1} \mathrm{Ba}_{2} \mathrm{Cu}_{3} \mathrm{O}_{7}$ Thin Films-D. K. Christen, C. E. Klabunde,

R. Feenstra, I. R. Thompson, H. R. Kerchner, and S. T. Sekula

Resistive and Structural Pruperties of YBaCuO Films Produced by Means of Thermal Coevaporation-R. Feenstra, J. D. Budai, M. F. Chisholm, M. D. Galloway, and

D. K. Christen

Magnetic AC Response of Superconducting Thin Films-H. $R$. Kerchner, R. Feenstra, J. O. Thomson, I. R. Thompson, D. K. Christen, and S. T. Sekula.

Pulsed-Laser Deposition of Thin Superconducting Films of $\mathrm{HoBa}_{2} \mathrm{Cu}_{3} \mathrm{O}_{7-x}$ and $\mathrm{YBa}_{2} \mathrm{Cu}_{3} \mathrm{O}_{7-x}-$ D. B. Geohegan, D. N. Mashburn, R. J. Culbertson, S. J. Pennycook, I. D. Budai, R. E. Valiga, B. C. Sales, D. H. Lowndes, L. A. Boatner, E. Sonder, D. Eres, D. K. Christen, and W. H. Christie.

Fundamental Studies and Development of Plasma-Assisted Pulsed-Laser

Deposition of Oxide Superconductor Thin Films-D. N. Meshbum, D. H. Lowndes, and

D. B. Geohegan

Transient Optical Absorption Spectroscopy in Laser-Generated Ablation Plumes of High-T, Oxide Superconductors-D. B. Geohegan and D. N. Mashburm.

\section{SOLID ELECTROLYTES AND CERAMIC FILMS}

Fabrication of Thin-Film Lithium Microbatteries-J. B. Bates, N. I. Dudney, Y. T. Chu, and P. Mazumdar

Properties of Electrolyte and Electrode Films Prepared by RF and DC Magnetron Sputtering-J. B. Bate, N. J. Dudney, Y. T. Chu, and P. Mazumdar.

Anomalous Dielectric Constant of $\mathrm{H}_{2} \mathrm{O}$-Saturated Vycor-I. B. Bates and S. K. Anderson.

Enhanced lonic Conductivity in AgI-Alumina Composites-N. J. Dudney

Composite Electrolytes-N. I. Dudney

Ion Exchange Reaction of Silver and Sodium $\beta^{*}$-Alumina in Molten Mercury Salts-

N. J. Dudney and I. B. Bates.

Impedance of a Fractal Electrolyte-Electrode Interface-1. C. Wang

Hopping Model for the Non-Debye Dielectric Response in lonic Crystals-I. C. Wang and J. B. Bales.

The Constant-Phase-Angle Impedance of the Finite Sierpinski Electrode-Y. T. Chu 


\section{OPTICAL AND MAGNETIC CHARACTERIZATION OF CERAMICS}

ihe Characterization of Textured Ceramics by Electron Paramagnetic Resonance

Spectroscopy: Concepts and Theory-L. A. Boatner, I. L Boldú O., and

M. M. Abraham

Optical Study and Analysis of $\mathrm{Pu}^{4+}$ in Single Crystals of $\mathrm{ZrSiO}_{4}-$ I. S. Poirot,

W. K. Kot, N. M. Edelstein, M. M. Abraham, C. B. Finch, and L. A. Boatner

$F^{*}$ and $F$ Luminescence in Thermochemically Reduced MgO Crystals-Y. Chen and

V. M. Orere

Time-Resolved Luminescence and Absorption Spectroscopy of Defects in MgO-

G. P. Williams, Ir., G. H. Rosenblatt, M. I. Ferry, R. T. Williams, and Y. Chen

Optical and Transmission Electron Microscopy Characterization of Metal Precipitates

in Doped Thermochemically Reduced Magnesium Oxjde $-C$. Ballesteros,

$R$. Gonzalez, and $Y$. Chen

Superhyperfine Structure of the $\mathrm{H}^{2}$ - Ion in $\mathrm{MgO}-\mathrm{V}$. M. Orera and $\mathrm{Y}$. Chen

Optical Transitions in Neutron-Irradiated $\mathrm{MgAl}_{2} \mathrm{O}_{4}$ Spinel Crystals-L. S. Cain,

G. J. Pogatshnik, and Y. Chen.

95

Determination of the Optical Dielectric Function of Thin Absorbing Films-Y. T. Chu.

96

\section{Ion Beam and Laser Processing}

\section{ION BEAM PROCESSING}

Ion Beam Deposition of Epitaxial GaAs Using Alternating Low-Energy Beams-

T. E. Haynes, R. A. Zuhr, and S. I. Pennycook.

Heteroepitaxial Growth of Ge Films on GaAs(001) by Ion Beam Deposition-

T. E. Haynes, R. A. Zuhr, S. I. Pennycook, B. C. Larson, and M. D. Galloway.

Metallic Silicides Formed by Ion Beam Deposition-R. A. Zuhr, T. E. Haymes,

O. W. Holland, and S. I. Pennycook.

Ion Beam Deposition of $\beta$-SiC Layers onto $\alpha-S i C$ Substrates-S. P. Withrow,

K. L. More, R. A. Zwhr, and T. E. Haynes.

Development of an Automated Magnet Controller for Altemating lon Beam

Deposition of Compound Layers-I. L. Moore, T. E. Haynes, and R. A. Zuhr. 
Formation of High-Quality Silicon-on-Insulator Structures by High-Dose Oxygen Implantation in Silicon-M. K. El-Ghor, S. J. Panrycook, F. M. Namevar, and N. K. Karam.

Structural Characterization of MeV Damage and Annealing of (100) Si-M. K. El-Ghor,

O. W. Holls: d, C. W. White, and S. I. Pennycook

Noble Metal Ion Implantation of $\mathrm{Ti}$ and Surgical Ti-6Al-4V Alloy-J. M. Williams,

R. A. Buchanan, and In-Seop Lee.

Solid-Phase Epitaxy in $\mathrm{LiNbO}_{3}-D$. B. Poker and D. K. Thomes

108

Effects of Implanted Transition-Metal lons on the Refractive Index of Fused Silica-

R. A. Weeks, H. C. Mogul. R. F. Haglund, G. W. Whichard, and R. A. Zuhr.

109

ECR Microwave Plasma Processing-S. M. Gorbatbin, J. B. Roberto, L A. Berry, and

C. C. Tsai.

\section{ION IMPLANTATION AND ANALYSIS}

MeV Ion Damage in Silicon-O. W. Holland, M. K. El-Ghor, and C. W. White

Interaction of MeV Ions with Preexisting Damage in Si: A New lon Beam

Annealing Mechanism-O. W. Holland.

112

New Mechanisms for Diffusion of lon-Implanted Boron in Si at High Concentration-

O. W. Holland

Oxidation of Si Implanted with Nondopant, Metallic lons- - . W. Holland,

C. W. White, and S. I. Pennycook.

Implantation into Diamonds-S. M. Gorbatbin, R. A. Zuhr, J. Roth, and H. Naramoto.

Nucleation of Damage in Ge by MeV Ion Irradiation-T. P. Sjoreen, O. W. Holland,

M. K. El-Chor, and C. W. White...

High-Dose Oxygen Implantation and Regrowth of Thin YBaCuO Films on $\mathrm{SrTiO}_{3}-$

R. Feenstra, S. I. Pennycook, and C. W. White

The Annealing Behavior of Ion-Implanted Single Crystals of the Type $\mathrm{YBa}_{2} \mathrm{Cu}_{3} \mathrm{O}_{x}-$

I. C. McCallum, C. W. White, and L. A. Boatner.

Annealing Environment Effects in Solid-Phase Epitaxial Regrowth of

Fe-Implanted $\mathrm{Al}_{2} \mathrm{O}_{3}-$ I. C. McCallum, C.W. While, P. S. Sklad, and C. J. McHargue

Time-Resolved Reflectivity Measurements in Ion-Implanted Crystalline Ceramic

Oxides-J. C. McCallum, J. Rankin. C. W. White, and L. A. Boatner.

SIMS Depth Profiling of Multilayer Samples of Isotopically Purified Silicon-

K. Wittmaack, D. B. Poker, and U. Littmark 
High-Resolution X-Ray Studies of the Chemical Environment of Ions Implanted in Insulator Matrices-T. M. Rossed, I. P. Young, C. R. Vare, R. A. Zuhr, and

On the Thermodynamic Driving Force During lon Mixing of the Co-Si Systenr-

W. Xin, S. S. Lau, and D. B. Poker

\section{LASER PROCESSING OF THIN FILM'S}

Photon-Controlled Fabrication of Amorphous Superlattice Structures Using ArF (193 nm) Excimer Laser Photolysis-D. H. Lowndes, D. B. Geohegan, D. Eres, S. J. Pennycook, D. N. Mashburn, and G. E. Jellison, Jr.

Laser Photochemical Deposition of Amorphous Semiconductors from Disilane and Digermane: Spectroscopic Studies and Comparisons with Thermal Chemical Vapor Deposition-Djula Eres and D. H. Lowndes.

First Principles for Low-Temperature Laser-Photon-Controlled Epitaxial Film Growth-D. H. Lowndes, D. Eres, and D. B. Geohegan.

High-Speed Heteroepitaxial Film Gro ; th Using a Pulsed Supersonic Free JetDjula Eres, D. H. Lowndes, and J. Z. Tischler.

Growth of Epitax.: Je Films on (100) GaAs by Pyrolysis of Digermane-Djula Eres, D. H. Lowndes, I. Z. Tischler, J. W. Sharp, S. J. Pennycook, and D. B. Geohegan

Laboratory for Laser-Controlled and Low-Temperature Thin-Film GrowtiD. H. Lowndes, D. Eres, D. B. Geohegan, and D. N. Mashburn

Facility for Measuring Vacuum-Ultraviolet Cross Sections of Novel Molecules Used for Low-Temperature Photochemical Vapor Deposition-D. B. Geohegan,

D. H. Lowndes, and K. Sharp.

In Situ Monitoring of Crystalline and Amorphous Film Growth Using Optical Reflectivity-J. W. Sharp, D. H. Loindes, and D. Eres.

Fabrication of Broadband Cermanium Thermophotovoltaic Cells by Pulsed-Laser Annealing-I. W. McCamy, D. H. Lowndes, and G. E. Jellison, Ir.

Hole Capture at Acceptors in p-Type Germanium-L. S. Darken, P. Sangsinkeow, and G. E. Jellison, Jr.

A New Two-Channel Polarization Modulation Ellipsometer-C. E. Jellison, Ir., and F. A. Modine.

Implementation of Constant Phototube Current in the Presence of Large Variations in Light Intensity-G. E. Jellison, Ir., and F. A. Modine. 


\section{Structure of Solids and Surfaces}

\section{SURFACE PHYSICS}

Atomic Termination and Relaxations of the NiAl(100) Surface- I. R. Nooman and

H. L. Davis.

Reexamination of the Al(111) Surface-J. R. Nooman and H. L. Davis

The First Interlayer Spacing of Ta(100) Determined by Photoelectron

Diffraction-R. A. Bartymsto, D. M. Zehner, D. Heskett, K. Garrison, G. Watson,

W. N. Mei, S. Y. Tong, and X. Pan.

Absolute X-Ray Reflectivity Study of the Au(001) Surface-D. Gibbs, D. M. Zehner,

B. M. Ocko, and S. J. G. Mochrie

A Spot Phofile Analysis Low-Energy Electron Diffraction (LEED) Study of a Stepped

NiAl(111) Surface-John F. Viendelken.

A Spot Profile Analysis Low-Energy Electron Diffraction (LEED) Study of a Stepped

NiAl(110) Surface-John F. Wendelken.

Molecular Oxygen on Cu(110): The Search Extended to $15 \mathrm{~K}$-Arthur P. Baddorf and John F. Wendelken.

Initial Stages in Oxidation of $\mathrm{Cu}(110)$-Anthur P. Baddor' and John F. Wendelken

Surface Ccre-Level Spectroscopy from HfC(100) and HfC(111)-D. M. Zehner,

G. R. Gruzalski, G. W. Ownby, and D. Heskett.

Surface States on NiAl(110)-S. C. Lui, D. M. Zehner, M. H. Keng, E. J. Mele, and

E. W. Plummer .

Electron Spectroscopy of Multicharged lon-Surface Interactions-D. M. Zehmer,

F. W. Meyer, C. C. Havener, K. J. Reed, and K. J. Snowden.

\section{ELECTRON MICROSCOPY}

Chemically Sensitive Structure Imaging with a Scanning Transmission Electron Microscope-S. J. Pennycook and L. A. Boalner

2-Contrast Imaging of $\mathrm{Si}_{1-x} \mathrm{Ge}_{x} / \mathrm{Si}$ Interfaces-S. I. Pennycook, M. F. Chisholm, and O. W. Holland..

Reconstruction of the $\mathrm{COSi}_{2} / \mathrm{Si}(100)$ Interface-S. I. Pennycook and S. M. Yalisove.

Interdiffusion in Strained-Layer Superlattices-S. J. Pennycook, J. M. Baribeau, and

D. C. Houghton. 
Critical Currents and Grain Boundary Dislocations in $\mathrm{YBa}_{2} \mathrm{Cu}_{3} \mathrm{O}_{7-8}$ Superconductors-

M. F. Chisholm and S. J. Pennycook.

Oriented Growth of $\mathrm{YBa}_{2} \mathrm{Cu}_{3} \mathrm{O}_{2}$ on $\mathrm{MgO}_{8} \mathrm{O}$ by Ion Sputtering - M. F. Chisholm.

I. P. Doyle, and R. A. Roy.

Anisotropic Recrystallization of lon-Implanted $\mathrm{YBa}_{2} \mathrm{Cu}_{3} \mathrm{O}_{7-x}-\mathrm{S}$. J. Pennycook,

R. Feenstre, and M. F. Chisholm.

Formation of Defect-Free Shallow Junctions in Ast-Implanted Si-M. K. El-Ghor,

S. J. Pennycook, and R. A. Zuhr

Dopant Segregation During Solid-Phase-Epitaxial Growth-S. J. Pennycook

Delacalization Corrections for Electron Channeling Analysis-S. J. Pennycook.

\section{X-RAY DIFFRACTION}

Nuclear Bragg Diffration from Epitaxially Grown ${ }^{57} \mathrm{Fe}_{2} \mathrm{O}_{3}-$ J. Z. Tisctler and

B. C. Larson

X-Ray Study of In-Plane Epitaxy of $\mathrm{YBa}_{2} \mathrm{Cu}_{3} \mathrm{O}_{2}$ Thin Films-J. D. Budai, R. Feenstru, ani L. A. Boatner.

Time-Resolved X-Ray Study of Ge and Si at Nanosecond Resolution During

Pulsed-Laser Melting-J. Z. Tischler, B. C. Larson, and D. M. Mills

X-Ray Measurements of Phason Distortion in Ion-Implanted A-Mn-J. D. Budai

Asymptotic Diffuse Scattering from Co Precipitates in $\mathrm{Cu}-$ B. C. Larson and

M. D. Calloway.

Vacancy and Interstitial Loops in Light-Ion-Irradiated Copper-H. Maete,

B. C. Lasson, T. P. Sjoreen, D. K. Thomas, O. S. Oen, and J. D. Lewis.

Development of Microstructures in Fe-15Ni-15Cr Single-Crystal Electron Beam

Welds-M. Rappaz, S. A. David, J. M. Vitek, and L. A. Boatmet

The Crystal Structure of $\mathrm{Ceo}_{0.9} \mathrm{U}_{0.1} \mathrm{PO}_{4}-D$. F. Mullica, E. L. jappenfield, G. A. Wilson,

and L. A. Boatner.

PUBLICATIONS AND PAPERS

SEMINARS

SCIENTIFIC ACTIVITIES, AWARDS, AND HONORS.

PERSONNEL CHANGES.

ORGANIZATION CHART 


\section{Acronyms}

AF - antiferromagnetic

ALH - Anderson iattice hamiltonian

ASRO - atomic short-range order

BCA - binary collision approximation

CLBEs - core-level binding energies

CPA - constar.t phase angle

CVD - chemi:al vapor deposition

DOS - density of states

DSC - differential scanning calorimetry

ECR - electron cyclotron resonance

EPR - electron paramagnetic resonance

FLL - flux-line lattice

HIXSE - heary-ion-induced $x$-ray satellite emission

IBD - ion beam deposition

IFT - indirect Fourier transform

LEED - low-energy electron diffraction

LPVD - laser photochemical vapor deposition

MC - Monte Carlo

MF - mean field

MO - metalorganic

MO - molecular orbital

MPM - mechanical properties microprobe
OCV - open circuit voltage

PLD - plasma-assisted laser-ablation deposition

PMT - photomultiplier tube

RBS - Rutherford backscattering spectroscopy

RPA - random phase approximation

RSG - reentrant spin glasses

rsq-real squashing

SANS - small-angle neutron scattering

SAXS - small-angle $x$-ray scattering

SEM - scanning electron microscopy

SF]-CBE - supersonic free jet chemical beam epitaxy

SIMS - secondary ion mass spectrometry

SPE - solid-phase epitaxy

STEM - scanning transmission electron microscope

SW - spin wave

TEM - transmission electron microscopy

TRR - time-resolved optical reflectivity

TVP - thermophotovoltaic

XPS - $x$ ray photoemission spectroscopy

XTEM - cross-sectional transmission

elec'.ron microscopy 


\section{INTRODUCTION}

This report covers the progress of the Solid State Division from October 1, 1987, to March 31, 1969. During this period, a new director was appointed for ORNL, the management and onganization of the division have changed, and our research reactors have remained shut down.

Major improvements have been made to the Surface Modification and Characterization Collaborative Research Center. The tandem accelerator has been placed in operation; a new 500-kV LINAC implantation accelerator has been installed, and acceptance tests have been completed. The user program for this facility continues to serve a broad spectrum of scientists from universities, industries, and other national laboratories. Scientists from the division continue their involvement in participating research :-ams at the National Synchrotron Light Source at Brookhaven National Laboratory: one with scientists from the University of Pennsylvania operates a surface research facility at the VUV ring, and the other, involving several universities and ORNL, operates a diffraction facility at the $x$-ray ring.

The division has continued a strong emphasis in processing science. Utilizing support from a number of sources, we now have research programs involving laser photochemical vapor deposition for the preparation of thin films and multilayer structures of semiconductors, the preparation of thin films of the high- $T_{c}$ superconductors by laser ablation and by physical vapor deposition, electron cyclotron resonance microwave plasma processing of thin films, the preparation of thin films by ion beam deposition, and the synthesis of ceramic nanocomposite and macromolecular thin films.

Research on the high-T $T_{c}$ superconductors is emphasized at ORNL and throughout the DOE laboratories. This research program is coordinated through collaborative arrangements, specifically for ORNL with similar programs at Los Alamos National Laboratory and Lawrence Berkel 3y Laboratory, and through Division of Materials Sciences coordination meetings. The formation of high$T_{C}$ superconductivity pilot centers, one at ORNL, has served to promote collaborations between our research programs and a number of industrial firms. Of course, information about superconductivity is very rapidly dispersed by the numerous meetings, joumals, and other pertinent literature. The research on superconductivity in the division has been collected in a subsection in this report.

As a consequence of the lack of operating reactors, the program supporting the Low-Temperature Neutron Irradiation Facility was terminated, and the support from the National Science Foundation 
for the National Center for Small-Angle Scattering Research was ended. To compensate for the lack of neutrons, our scientists have taken advantage of user facilities a' nuclear reactors and accelerator neutron sources both in this country and in Europe. We have also used this period to revitalize the neutrolı scattering farilities at the High Flux Isotope Reactor. While the Advanced Neutron Source project is not specifically a part of the divisicn, the long-range $\mathrm{pl}$. . for the division is inimately related to this facility. During this period we have been concerned with design of neutron scattering instruments that will be incorporated into this premier of neutron scattering user facilities. We anxiously await HFIR restart so as to reestablish our neutron scattering user programs and to continue the upgrading and developing of neutron instrumentation.

This report has been arranged into chapters titled Theoretical Solid State Physics, Neutron Scattering, Physical Properties of Materials, the Synthesis and Characterization of Materials, Ion Beam and Laser Processing, and Structure of Solids and Surfaces; and each of these chapters is further subdivided into sections in an attempt to group the research into logical entities. An introduction provides an overview of the goals, objectives, and directions of the research covered in each chapter. However, because of the cooperative nature of the division's various programs, there is inevitable overlap and the reader may find related programs in more than one section. 


\section{Theoretical Solid State Physics}

The Solid State Theory Program reflects the broad range of experimental research programs in the division. In addition to work carried out in collaboration with experimental and other theory programs at ORNL and elsewhere, research is initiated on problems dictated by interests in new directions that are important for maintaining a strong and productive effort. A comprehensive review of current activities, which are somewhat arbitrarily organized into four sections, is provided in this chapter.

A significant fraction of the research described in this chapte- is associated with highly correlated electronic systems, including work on mixed-valence metals, high- $T_{c}$ superconductors, and itinerant magnetic metals. The spinfluctuation resonance proposed for mixed-valence materials has been shown to have negligible $f$ character and, therefore, to be inconsistent with experiment. An explanation of phonon mode splitting at temperatures above the spin-fluctuation temperature has also been proposed. Research on high- $T_{c}$ superconductivity encompasses investigations of a number of proposed pairing mechanisms, electronic band structure calculations, lattice dynamics, a positron annihilation study, and the prediction of a new state of type II superconductivity. Itinerant electron theory has revealed the reason for incorrect predictions of spin-wave behavior from local density theory.

Research on single-particle properties of materials includes investigations of bandwidths of simple metals determined from photoemission and of electron-hole excitation spectra from inelastic electron scattering experiments. Low-energy electron diffraction (LEED) theory has been used to study reconstruction of binary metallic systems. In addition, a reexamination of the nonstructural parameters of the theory has led to "near perfect" agreement between calculated and experimental LEED profiles. Long-standing programs on particle-solid interactions have expanded into new areas. For example, numerical simulation of channeling and computer simulation of damage produced by energetic particles have been modified in ways which significantly add to the versatility of these widely used techniques. 


\section{ELECTRONIC AND MAGNETIC PROPERTIES}

\author{
PSEUDOEXCTTONS IN \\ MIXED-VALENCE METALS 1
}

\section{S. H. Lin}

Recent neutron scattering studies of the phonon dispersion relations of a-Ce and $\mathrm{CePd}_{3}$ have revealed a mode splitting in the (100) LA branch at temperatures around the spin fluctuation temperature of the materials. Since the LA phonon is a pressure wave and in mixedvalence materials the position of the $f$ level is sensitive to hydrostatic pressure, it is expected that the mode splitting has its physical origin in the interaction between acoustic phonons and $f$ electrons. At low temperatures when the $f$ electrons are in band-like states, the interaction is known to produce a mode softening, as is seen experimentally. It is shown in this work that at higher temperatures where the $f$ electrons are in incoherent local levels, the pressure wave can promote an f electron into the conduction band and create a localized $f$ hole. The hole is very effectively screened by the band electrons. The entity formed by this hole and the screening charge is named a psuedoexciton becouse it resembles an exciton in semiconductors except that it leads only a transient existence. Nevertheless, it can mix with LA phonuns to produce the observed mode splitting. The theory predicts that at temperatures much higher than the spin fluctuation temperature the pseudoexciton lifetime becomes so short and the linewidth becomes so large that the mode splitting disappears. The evolution of line shape as a function of phonon energy and temperature is depicted in Fig. 1.1. In this figure

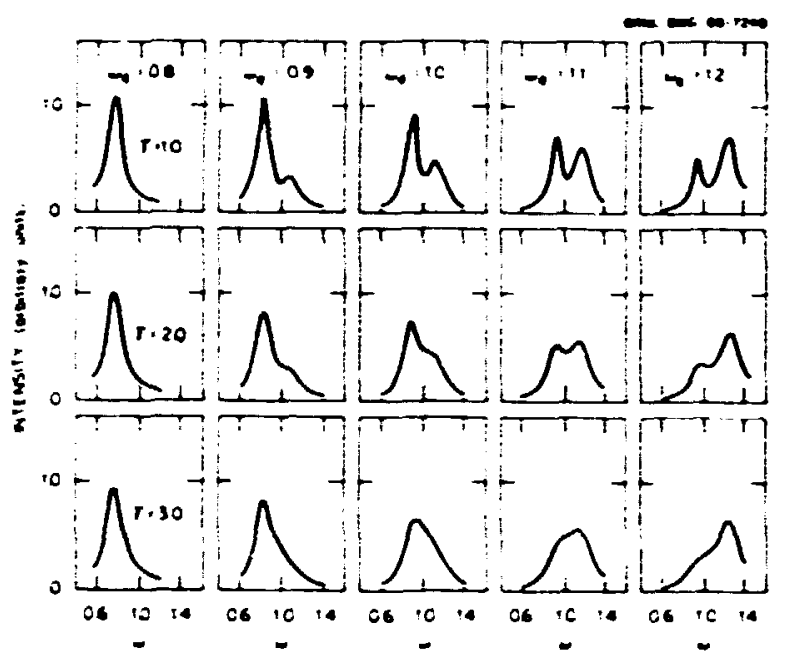

Fig. 1.1. Line shapes of LA phonons in the energy and temperature range where mode splitting occurs. All energies as well as temperatures are in units of cos which is a measure of the spin-fluctuation temperature.

both phonon energy and temperature are measured in units of the spin fluctuation temperalure $T_{f}$. Line splitting occurs when the phonon energy matches the energy of the flevel, which is measured by the spin fluctuation temperature. The line shape at $T=3 T_{f}$ resembles very closely what is seen in the high-temperature Yphase Ce.

1. Summary of paper: Phys. Reo. B 39, 1403 (1989). 


\section{SPIN-FLUCTUATION RESONANCE IN MDXED-VALENCE MATERIALS ${ }^{1}$}

\section{S. H. Lin}

In the Anderson model for mixed-valence systems, it is now well established that singlesite spin fluctuation produces a resonance very close to the Fermi level. Inclusion of this resonance level in the thermodynamic and transport calculations accounts for an improssive array of observed properties of dilute alloys of Ce. This has encouraged the speculation that mixed-valence metals, which are dense lattices of Ce or some other lanthanide or actinide, may be treated as a lattice of spin-fluctuation resonances. The basic idea is that an electron in this resonance level may propagate from site to site to create a narrow band, and such a band is needed to explain the anomalous thermodynamic and transport properties of these materials. This paper demonstrates for the first time that the wave function of the resonance level has predominantly the spatial dependence of the conduction band electrons at the Ferm level and has a negligible amount of $f$ character. It further shows that this result has interesting implications.

When a magnetic field is applied to this narrow band of electrons, a lattice of magnetic moments is induced and the spatial dependence of the moment follows that of the band electrons. Such experiments have been carried out on real materials by measuring the neutron magnetic form factor, which is the Fourier transform of the spatial distribution of the induced moments. The results of these mea- surements on mixed-valence and heavy-electron materials have shown unanimously that the induced moments have the spatial distribution of the f shell electrons, not band electrons. Therefore, there is unambiguous disagreement between the spin-fluctuation resonance model and the experimental findings. This suggests that, contrary to general expectations, the marrow band of electrons in mixed-valence and heavy-electron metals is not produced by spinfluctuation resonance but by itinerant $f$ electrons.

In some Ce-based materials, for example $a-C e, C e S n_{3}$, and CePd, the magnetic susceptibility rises very sharply at temperatures below $40 \mathrm{~K}$, well below the spin-fluctuation temperature of these materials. $\mathrm{N}$ - tron magnetic form factor measurements have revealed that the rise is associated with the emergence of a new magnetic moment contribution which has the spatial dependence of Ce $5 d$ electrons. We suggest that this is the signature of the spinfluctuation resonance effect hecause it has both the expected form factor and temperature dependence.

1. Summary of paper: Physical Review B (in press).

\section{BANDWIDTH OF SMMPLE METALS}

$$
\text { C. D. Mahan' }
$$

W. Plummer's group at the University of Pennsylvania has used photoemission to measure the width of the occupied energy bands in 
simple metals such as sodium, magnesium, and aluminum. ${ }^{2}$ He concludes that the bandwidths are significantly reduced and that the reduction is due to electron-electron inteactions. We disagree with both of these assertions. We do not think that electron-electron interaction causes a significant reduction in the occupied bandwidth.

Many years ago Hedin calculated the change in the bandwidth using random phase approximation (RPA). Recently numerous groups have tried to improve this calculation by including various improvements in the dielectric function. We show that one must also include vertex corrections, which make a significant contribution to the predicted bandwidths. ${ }^{3}$ We show that improving the screening and the vertex functions must be done rogether. Then one finds that the predicted bandwidths are almost iden:ical to those of Hedin. Our calculations, which use the most up-to-date dielectric functions and vertex corrections, predict the changes in the bandwidth which are nearly identical to RPA, which omits all of these corrections.

We also assert that Plummer's group misinterpreted the data. Our extensive calculations of photoemission in sodium show that surface interference will shift the observed peak positions to the values where they are observed experimentally. In this case the bands are not narrowed, but only appear so because the experiment is sensitive to the surface. His photoemitted electrons come from the first one or two atomic layers in the surface. In aluminum, Plummer's analysis neglects the band structure effects. Published energy bands by Callaway and Segail show a significant reduction due to one electron effects. 5 This is the reason Plummer observes a narrowing of these bands, rather than due to electron-electron interactions.

1. ORNL/UT Distinguished Scientist.

2. I. W. Lyo and E. W. Plummer, Phys. Reo. Lett. 60, 1558 (1988).

3. G. D. Mahan and B. E. Sernelius, Physical Review Letters (in press).

4. K.W. K. Shung and G. D. Mahan, Phys. Reo. B 38, 3856 (1988).

5. F. Szmulowiz and B. Segall, Phys. Reo. B 24, 892 (1981); S. P. Singhal and J. Callaway, Phys. Reo. B 16, 1744 (1977).

\section{MINORITY CARRIER BAND TAIL IN SEMICONDUCTORS}

$$
\text { G. D. Mahan' }
$$

Semiconductors without impurities have energy gaps between the conduction and valence bands. Semiconductors with impurities have electron states with energies in the gap region. When there is a high density of impurities, the conduction and valence band states are known to have "tails." These band tails are electron states in the energy gap. Their density decreases exponentially according to the encrgy difference from the nominal band edge of the undoped semiconductor.

Numerous theories have been constructed to describe the band tails of the majority carrier: clectrons in n-type semiconductors or holes in ptype semiconductors. The low-energy states are due to clustering of the impurities. Usually the 
impurity locations are considered to be random, and clustering is due to fluctuations in a random distribution.

We constructed the first model of band tail states of the minority carrier: electrons in $p$ type semiconductors or holes in n-type semiconductors. Although these band tails states have been observed for years, no prior theory has ever been presented. Our model also assumes the impurities are randomly located in space. Due to fluctuations, there are regions which have no impurities. Since the charge of the minority carrier has the same sign as the impurity, the minority carrier prefers these regions which lack any impurities. We constructed a variational wave function of the minority carrier in the many-particle system. This wave function has an eigenvalue for the minority carrier that is less than the band edge. The wave function was constructed to prefer the regions in space which lacked the impurities.

1. ORNL/UT Distinguished Scientist.

THEORY OF SPIN-MODULATED STRUCTURES NN RARE-EARTH METALS

\section{S. H. Liu and J. F. Cooke}

Over twenty-five years ago, neutron scattering experiments carried out at ORNL revealed that a c-axis modulated spin order occurred in thulium below $56 \mathrm{~K}$. As the temperature was lowered, it was also found that this structure "squared up" and eventually evolved into a ferromagnetic antidomain spin structure (i.e., a spin structure with four planes of moments pointing up followed by three planes with moments pointing down). A relatively simple theoretical explanation for the squaring up of this c-axis modulated spin structure has been found. In addition, an expression for the spinwave dispersion curve has been derived for the four-up three-down spin structure.

The theory is based on a Heisenberg spin Hamiltonian which includes isotropic exchange interactions and relevant crystal field terms. Self-consistent calculations of the thermally averaged spin operator were carried out using the molecular field approximation. Results of the calculation are shown in Fig. 1.2.

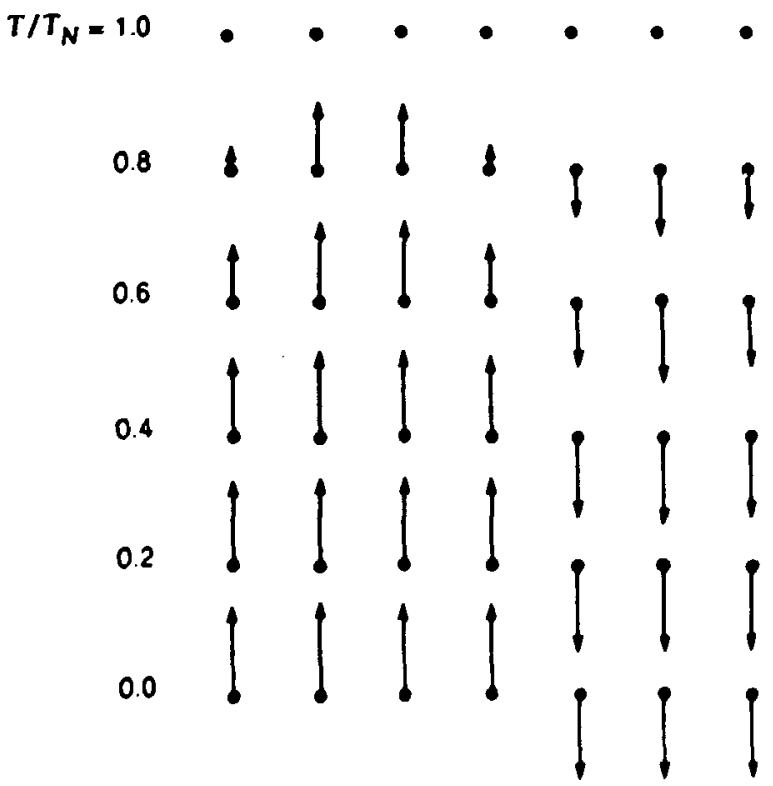

Fig. 1.2. The squaring up of the $c$-axis modulated spin structure in thulium as a function of temperature. $T_{N}$ is the transition temperature. 
These results demonstrate, for the first time, that the complicated squaring up of the spins simply results from the molecular field.

The spin-wave dispersion s arve was found to consist of fourteen branches. This results from a combination of the hcp crystal structure and the seven-layer repeat distance for the spin structure. Along the c-axis, pairs of branches are dcgenerate at the Brillouin zone boundary. In the extended zone scheme, this yields seven branches separated by energy gaps which result from the spin structure. Good fits to the recently measured dispersion curve along the $c$ axis in thulium have been obtained for a wide range of exchange and crystal field parameters. Unfortunately, not enought experimental information is available to determine these parameters uniquely, but it does appear that thulium is a system where the crystal field dominates the exchange interaction.

\section{MAGNETIC EXCTTATIONS IN TRANSITION-METAL SYSTEMS ${ }^{1}$}

\section{F. Cooke and I. A. Blackman'}

Magnetic excitations in transition-metal systems are linked in a fundamental way to the electronic band structure. This complexity leads to unusual properties suc , as the existence of spin waves over part of the Brillouin zone and "optic" modes. The prediction and subsequent observation of these as well as other effects indicate that itinerant electron theory provides an excellent description of the lowtemperature properties of Iransition-metal ferromagnets. The description, however, is not exact. The prediction that the lower branch of the spin-wave dispersion curve persists out to the Brillouin zone boundary along (100) in nickel has not been confirmed experimentally. In addition, calculations based on local density theory do not yield the "optic" mudes predicted by our theoretical approach and observed experimentally. In order to investigate these problems, our formalism has been generalized to include the most general form of the coulomb matrix element consistent with symmetry. This increases the number of independent parameters from two to ten with two parameters fixed by knowledge of the spin-splitting energy.

Calculations of the transverse part of the inelastic neutron scattering cross section for arbitrary values of the eight independent parameters have revealed a wider variety of spin-wave behavior than was obtained previously. Some of the results obtained along (100) for nickel are shown in Fig. 1.3. They range from a single branch to multiple branches which exist over limited parts of the zone. These results are representative of a number of proposed band structures. It might be surmised that local density theory incorrectly generates exchange matrix elements which are consistent with the single branch case. Results obtained thus far do not remove the problem along (100) in nickel.

1. Summary of paper: I. Phys. (France) 49, C8-81 (1988).

2. University of Reading, Reading, United Kingdom. 


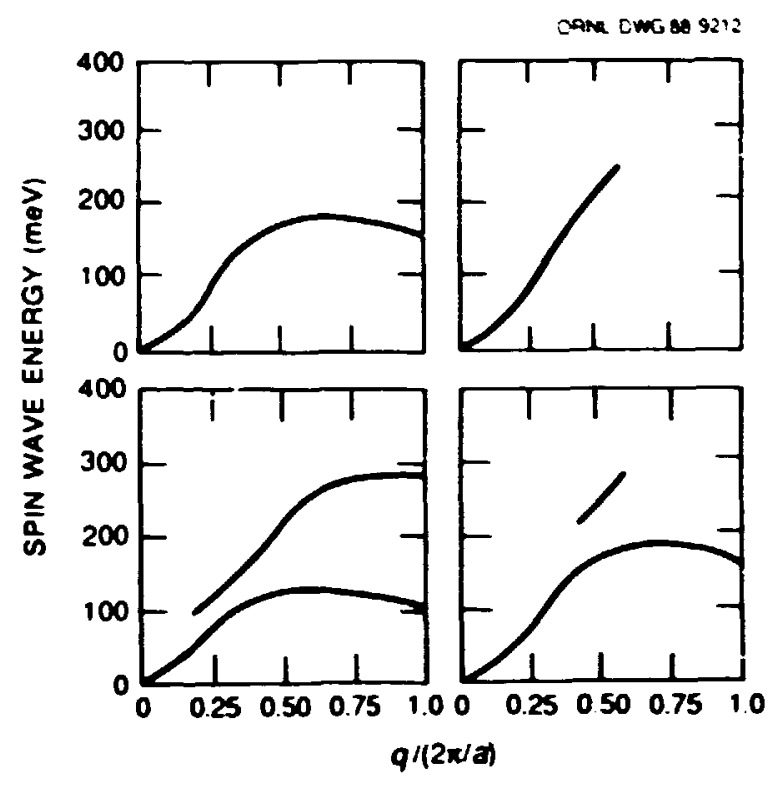

Fig. 1.3. Spin-wave energy for nickel along (100) for various choices of Coulomb matrix element parameters.

\section{INELASTIC ELECTRON SCATTERING AND ELECTRON-HOLE EXCTTATIONS IN IRON AND NICKEL ${ }^{1}$}

\section{J. A. Blackman ${ }^{2}$ and J. F. Cooke}

Spin-resolved electron-energy-loss spectroscopy has been used to study electron-hole excitations in the transition-metal ferromagnets iron and nickel. Electrons with polarization antiparallel to the magnetic moment displayed greater inelastic scattering than those with parallel polarization. Furthermore, this asymmetry in the polarizationdependent scattering was found to be a maximum for energy losses near the spinsplitting energy, indicaling that spin-flip excitations played an important role. Two mechanisms have been proposed to explain the experimental results which, at an elementary level, differ on whether the scattering is dominated by spin-flip (Stoner excitations) or nonspin-flip processes.

In order to investigate the origin of the features observed in the asymmetric part of the scattering, calculations based on realistic energy bands of the energy and wave-vector dependence of the relevant correlation function have been carried out for ferromagnetic iron and nickel. A representative result for iron for $q=$ $(1 / 2,1 / 2,0)$, in units of $2 \pi /($ lattice parameter), is shown in Fig. 1.4. The asymmetry changes sign between 1.0 and $1.5 \mathrm{eV}$ and is peaked between 2.5 and $3.0 \mathrm{eV}$. This peak is fairly close to the spin-splitting energy, but a numerical analysis reveals that there is no connection between the two. Rather, this peak is roughly associated with the center of the bandwidth for nonspin-flip excitations. This describes the bel avior over a wide range of $q$ values in the

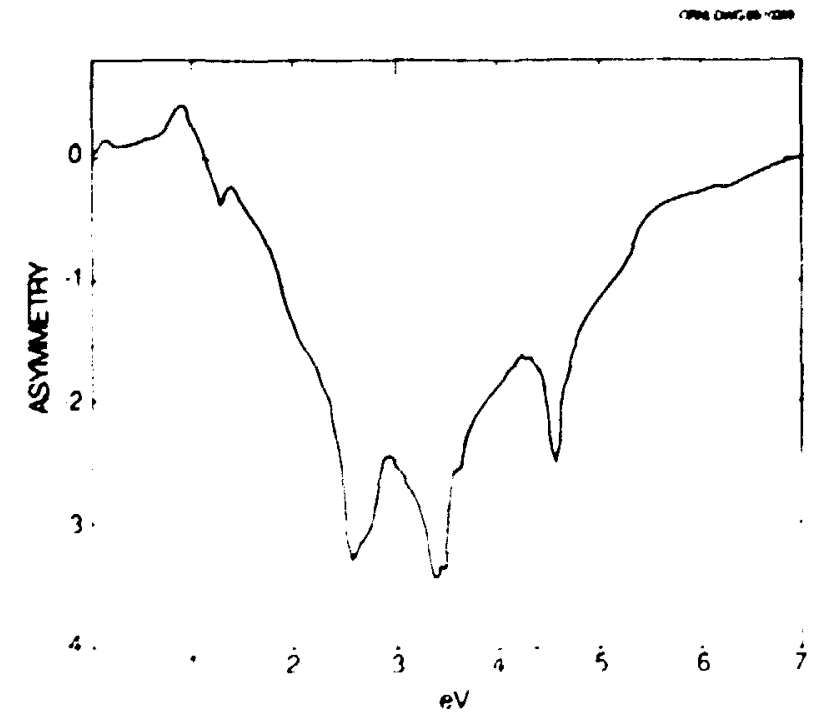

Fig. 1.4. Asymmetry in the polarization dependent scaltering for iron at $q=(1 / 2,1 / 2,0)$, in units of $2 \pi /$ (lattice parameter). Asymmetry is in arbitrary units. 
(100) direction. Similar results were found for nickel. Apart from this feature, comparison with experimental results does not provide strong evidence for either of the proposed mechanisms.

1. Summary of paper: J. Phys. (France) 49, C8-57 (1988).

2. University of Reading, Reading, United Kingdom.

\section{COLLECTIVE ENHANCEMENT OF THREE- BODY RECOMBINATIONS IN HIGHLY IONIZED CLASSICAL PLASMAS'}

M. Rasolt and F. Perrot

An ionized classical plasma will recombine to its lowest free energy state (i.e., the ionized electrons will return to the core states), conserving energy through an emission of a photon or through a three-body (3b) collision (or higher). The cross sections of these two channels are fundamental to the temporal behavior of the plasma. The crucial $3 \mathrm{~b}$ cross sections, so far, have been attributed strictly to the single particle-like behavior of the plasma. It has been shown that in some regions in density $p$ and temperature $T$ of the plasma, the collective behavior (i.e., plasma frequency $\omega_{p}$ ) plays a crucial role in the $3 \mathrm{~b}$ recombination cross section. When $\omega_{p}$ matches the transition energy of the ionized electron to the core states, the $3 \mathrm{~b}$ cross section is strongly enhanced by the collective plasma resonance. This region of $p$ and $T$ is well within experimental interest (for example, in an aluminum plasma at $T=100 \mathrm{eV}$, this occurs at roughly electron densities of bulk Al) and will thercfore profoundly change the experimental interpretation of such highly ionized plasmas.

The density and temperature dependence of this enhanced $3 b$ recombination is shown in Fig. 1.5. The curve labeled $P 1$ is the contribution of the plasma enhancement of the $3 \mathrm{~b}$ recombination of the ionized electron into the Al $3 p$ core state. Unlike the single particlelike $3 \mathrm{~b}$ recombination, the collective resonantenhanced recombination peaks at some finite

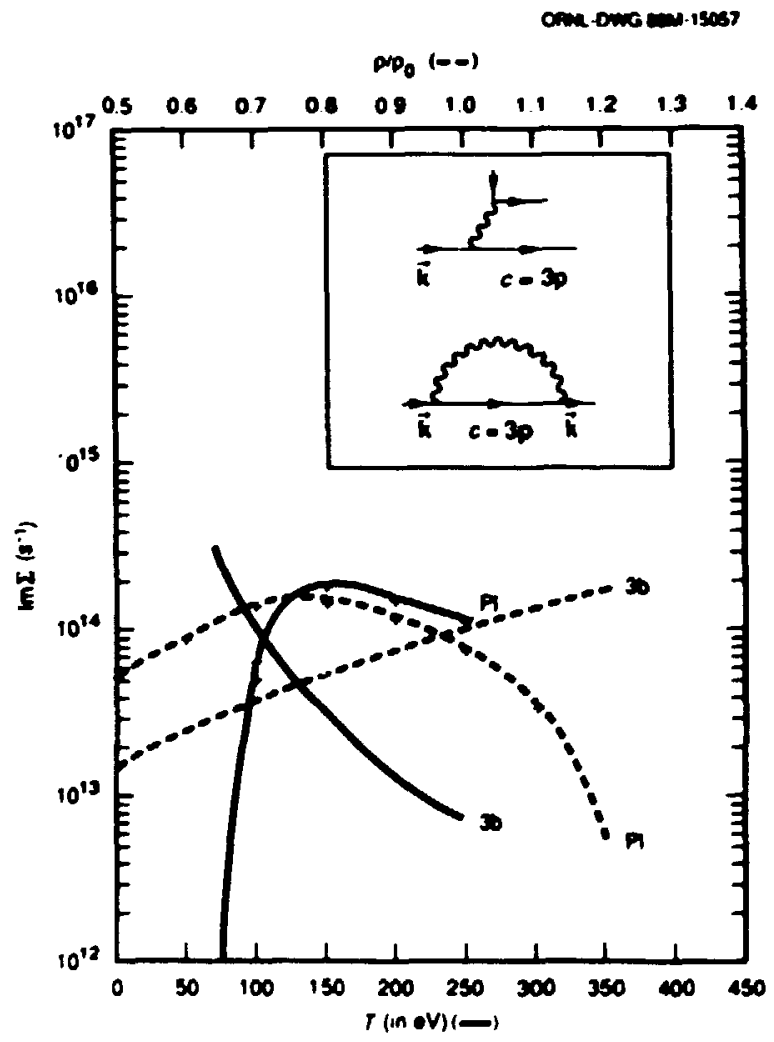

Fig. 1.5. The inverse $1 / \tau_{R}$ of the $3 b$ recombination time as a function of temperature $T$ (full curve) anc of relasive density $\rho / \rho_{0}$ (dashed curve) in aluminum. Comparison is made between the single-partisle approximation (3b) and the plasma-enhanced form (PI). 
temperature at fixed $\left(\rho / \rho_{0}=1\right)$ or finite density (at fixed $T=100 \mathrm{eV}$ ). In that window of $p-T$ plane (where resonance conditions are satisfied), the recombination is enhanced by an order of magnitude. In this region, the temporal evolution of the plasma will be much different than in other regions of the $\rho-T$ plane, and this must have important experimental implications. For example, the optical properties of the plasma, which depend on the corelevel occupation will change drastically.

1. Summary of paper: Physical Reciew Letters (in press).

2. Commissariat a l'Energie Atomique, Villenzuve-Saint-Georges, France.

\section{ENERGETICS AND LATTICE RELAXATION OF LIGHT IMPIRTIIES IN ALUMUNUM'}

\section{F. Petrot ${ }^{2}$ and Mark Rasolt}

Previous calculaiions on the energetics of a single $\mathrm{H}$ impurity in an $\mathrm{Al}$ host ${ }^{3,4}$ have been extended to :relude $\mathrm{He}, \mathrm{Li}, \mathrm{Be}, \mathrm{B}, \mathrm{C}, \mathrm{N}, \mathrm{C}, \mathrm{F}$, and $\mathrm{Ne}$ impurities in $\mathrm{Al}$. Mr: motivation is $\mathrm{ts}$ in restigate the possibility of strong corre'ations bet:veen impurity lattice coupling (i.e., heat of solution, diffusion barrier, and lattice relaxations) and the shell structure of the impurities and to sce if the calculated charge densities around the impurities can succeed in the prediction of such fine details of groundstate properties of impurity-host coupling (even in simple metal hosts likn Al).

As an example, the results for the lattice relaxations when impurities ranging from $\mathrm{H}$ to Ne are placed in the octahedral site of an aluminum lattice are shown in Fig. 1.6. The relaxation displacements of the ions around the impurities at the octahedral site can be seen to vary rather smoothly with the impurity atomic number and show some structure. It is also found that these results cannot simply be correlated with the size of the impurity in its atomic state, but involve the details of the density displaced in the host aluminum lattice.

1. Summary of paper. Phys. Reo. B 38, 2147 (1988).

2. Cormissariat a l'Energie Atomique, Villeneuve-Sain:-Georges, France.

3. L. M. Kohn, F. Perrot, and M. Rasolt, Phys. Reo. B 21, 5594 (1980).

4. F. Perrot and M. Rasolt, Phys. Reo. B 23, 6534 (1381).

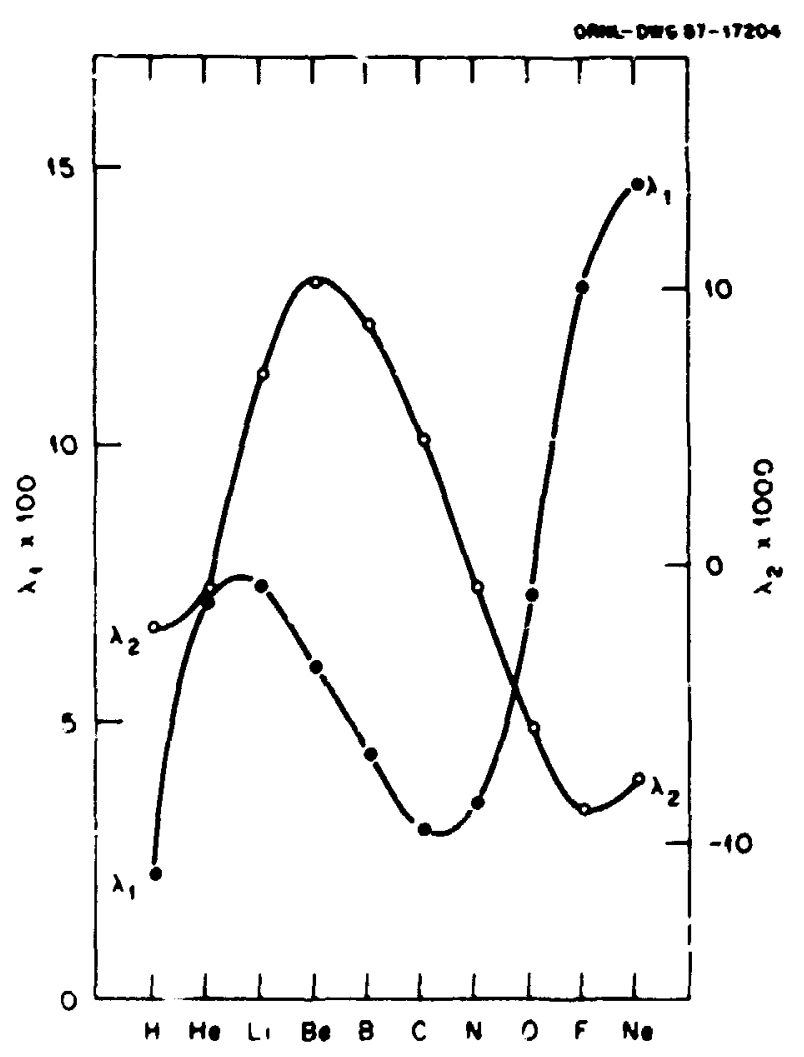

Fig. 1.6. Lattice relaxation, first $\left(\lambda_{1}\right)$ and second $\left(\lambda_{2}\right)$ shells for impurities ranfing from $\mathrm{H}$ to $\mathrm{Ne}$ at the octahedral site. 
DTVERGENCES IN THE FORCE-BALANCE THEORY OF RESISTIVITY

\section{R. S. Fishman ${ }^{2}$ and G. D. Mahan}

The force-balance theory is a method for calculating the electrical resistivity and other transport properties of metals by separating the electron coordinates and momenta into center-of-mass and relative variables. The frictional force of phonons and impurities is then balanced by the force of the electric field. The advantage of this approach is that the resistivity can be expanded perturbatively in powers of the electron-phonon and the impurity interactions.

Unfortunately, the result of this theory for the impurity resistivity $p_{i}-\langle 1 / \tau\rangle$ disagrees with the result of the Boltzman equation $p_{i} \sim 1 /<>$ at nonzero temperatures. To explain this discrepancy, some workers have claimed that the Boltzman equation is valid only under adiabatic conditions, when the electrons are heated by the electric field, while the forcebalance theory is valid under the isothermal conditions realized experimentally.

We show that the expansion of the impurity resistivity $\rho_{i}$ in powers of the impurity interaction $|\phi(q)|^{2}$ is divergent. The divergent terms must be re-summed by taking the limit of weak impurity interaction $\left(|\phi|^{2} \rightarrow 0\right)$ only after the long tin.e limit $(t \rightarrow \infty)$, so that $t$ is always larger than the electron relaxation time $\tau \sim 1 /||^{2}$. A tor resumming two sets of divergent terms, we find that the force-balance method yiclds the same result as the Boltzman equation. Since this calculation was proformed in the presence of a heat bath of phonons, the "isothermal" and "adiabatic" resistivities are equal to first-order in the electric field.

Though the force-balance method is formally exact, an infinite number of terms must be summed to calculate the resistivity. This eliminates the advantage of this method as an exact formalism. However, the lowest order nisults of the force-balance method may still prove useful as approximate guides for more rigorous studies.

1. Summary of papers: Phys. Reo. B 39, 2990 (1988); Phys. Reo. B 39, 2994 (1988).

2. Guest scientist from the ORNL/UT Distinguished Scientist Program.

3. ORNL/UT Distinguished Scientist.

\section{THE DOUBLET SPLTTING OF THE REAL SOUASHING MODES IN SUPERFLUID ${ }^{3} \mathrm{He}-\mathrm{B}^{1}$}

\section{R. S. Fishman ${ }^{2}$ and J. A. Sauls ${ }^{3}$}

The superfluid phases of ${ }^{3} \mathrm{He}$ support a rich spectrum of collective excitations which have been studied extensively using nuclear magnetic resonance and ultrasound. These collective modes correspond to excitations of the $L=1$, $S=1$ order parameter $\Delta R\left(\theta_{L} \hat{n}\right)_{\alpha i}$, where $R_{\alpha i}$ is the matrix for a rotation about the vector $\hat{n}$ by the angle $\theta_{L}$. The set of collective modes which correspond to a $/=2$, real excitation of the order parameter, are called the real squashing (rsq) modes. In a magnetic field $H$, these modes split into $2 J+1=5$ substates. Recently, Shivaram et al.4 observed a sixth rsq mode, which forms a doublet with the central substate, as shown in 
Fig. 1.7. Our work presents a quantitative theory of the doublet splitting in terms of the textural bending of the order parameter in the experimental cell.

Previous theoretical work on the highfrequency $(\omega-\Delta)$ collective modes assumed that the equilibrium superfluid is spatially uniform. We use a gradient expansion to calculate the shifts in the rsq mode frequencies due to bending of the $\hat{\mathbf{n}}$ vector when the scale of the inhomogeneity is much longer than the coherence length $h o f / \pi \Delta \sim 500 \mathrm{~A}$. We find that the sixth rsq mode is excited near the walls of the experimental cell while the other five modes are excited in the bulk. The doublet frequency $\omega_{d}$ depends on various Fermi-liquid

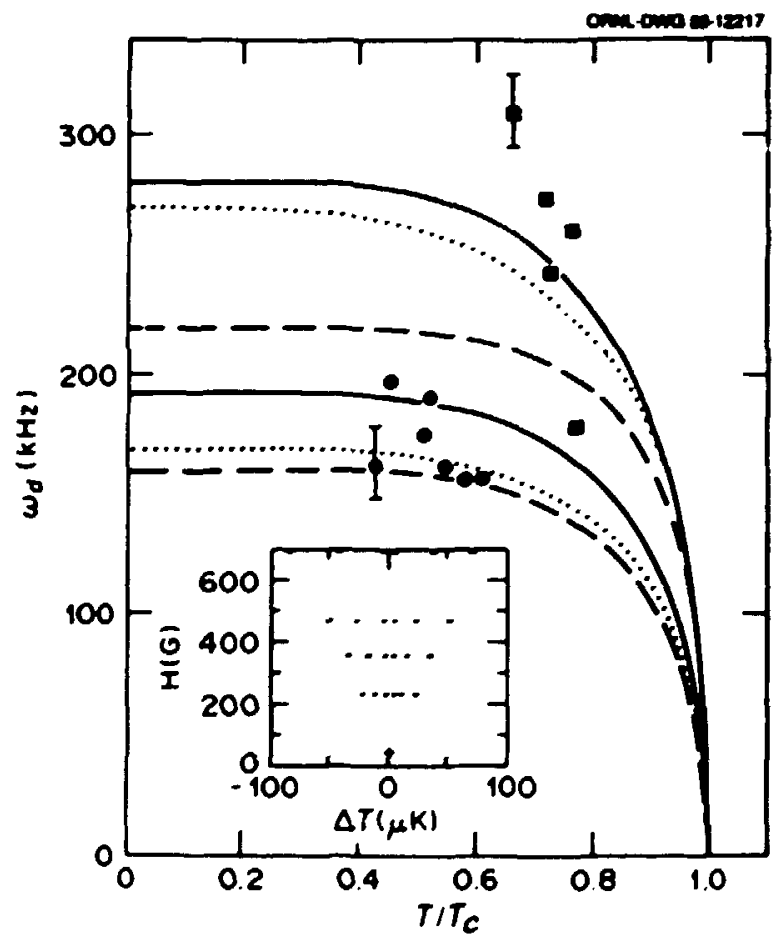

Fig.1.7. The experimental linset, squares ( 1 bar), and circles ( 5 bar) $)$ and theoretical values of the doublet spiitting. parameters. As shown in Fig. 1.7, agreement between the experimental splittings for $1 \mathrm{bar}$ (squares) and 5 bar (circles) and the theoretical predictions is very good.

The smooth disappearance of the doublet and the nonlinear evolution of the rsq mode frequencies at low magnetic fields are explained in terms of a crossover to the dispersion-dominated regime when $(q \circ \rho)^{2} / \Delta>\gamma H$.

1. Summary of paper: Phys. Reo. Lett. 61, 2871 (1988).

2. Guest scientist from the ORNL/UT Distinguished Scientist Program.

3. Northwestern University, Evanston, III.

4. B. S. Shivaram et al., Phys. Reo. Lett. 49, 1646 (1982).

\section{SUPERCONDUCTIVITY}

\section{STATIC COULOMB MODEL OF SUPERCONDUCTTVITY}

G. D. Mahan'

A model of high-temperature superconductivity that utilizes only the static Coulomb interaction between electrons is constructed. Since the copper-oxide planes are inhomugeneous, the fourier transform of the potential $V(q)$ has regions of wave vector $q$ where the interaction is attractive. A Bardeen-CooperSchrieffer-type pairing wave function which utilizes these a!tractive regions is constructed. High-temperature superconductivity is predicted. This model explains the occurrence of 
high-temperature superconductivity as due to the structure of the atoms in the plane.

A conventional three-band model is used for the sigma bonded states in the plane. The top band is the conduction band. The electronelectron interaction is calculated using these tight-binding wave functions. This potential has attractive regions for wave vectors near to the value $Q=\pi( \pm 1, \pm 1) / a$, which is one-half the diagonal vector across the Brillouin zone. This wave vector also spans the Fermi surface. For two electrons on the Fermi surface, the Coulomb interaction is attractive when the electrons scatter through large wave vectors, and it is repulsive for small-angle scattering.

The most important aspect of the calculation is the nature of dielectric screening by the electrons. The random phase approximation equations for the tight-binding model are solved approximately for the electrons in the conduction band. The dielectric function reduces the small-angle scattering, while enhancing the large-angle scattering. Superconductivity is predicted for some regions of parameter space. These regions have been calculated in detail.

1. ORNL/UI Distinguished Scientist.

\section{THEORY OF HIGH-T SUPERCONDUCTORS WITHIN A REALISTIC ANDERSON LATTICE MODEL 1}

D. M. Newns, ${ }^{2}$ M. Rasolt, and P. C. Pattnaik ${ }^{2}$

It is proposed that the origin for superconductivity in a strongly correlated electron band is an indirect one via the Hubbard U. For a half-filled Hubbard band, such a system is believed to be a strongly fluctuating quantum Heisenberg antiferromagnet. The limit of a small number of holes introduced into such a half-filled band has been largely treated within the resonating-valence-bond approach. We concentrated primarily on the opposite limit of a large number of holes $-a$ limit which we find is better treated within an itinerant picture (as is consistent with recent measurements of the Fermi surface) and by generalization of the Hubbard model to the Anderson lattice Hamiltonian (ALH).

A detailed description of $\mathrm{La}_{2-x} \mathrm{Sr}_{x} \mathrm{CuO}_{4}$ using the ALH both in the normal and superconducting states has been completed. An accurate parameterization of the band structure of $\mathrm{La}_{2-x} \mathrm{Sr}_{x} \mathrm{CuO}_{4}$ has been used to generate the ALH, which was investigated using a $1 / N$ expansion in the normal and superconducting states. In mean field (leading $N$ ), Fermi fluid is found above $T_{c}$ with a mass enhancement of $m^{*} / m-5$, a superconducting long-range-order parameter below $T_{c}$, and a narrow but observable critical region. The correct behavior for the trend of thermodynamic and transport properties both above and below $T_{c}$, as a function of the hole concentration $x_{h}$, is predicted with one adjustable parameter.

1. Summary of paper: Phys. Rev. B 38, 7033 (1988).

2. IBM Research Division, Thomas J. Watson Research Center, Yorktown Heights, N. Y. 


\section{A SPIN POLARON MODEL OF HIGH-T, SURERCONDUCTIVTY'}

\section{R. F. Wood, Merk Mostoller, and J. F. Cooke}

The seemingly close relationship between antiferromagnetism (AF) and superconductivity in the new high- $T_{c}$ oxide superconductors has led many authors to study the role magnetic excitations may play in the pairing mechanism. The fact that very small concentrations of oxygen vacancies in $\mathrm{La}_{2} \mathrm{CuO}_{4}$ can apparently induce three-dimensional (3D) AF ordering is striking. The observation of this effect suggested that there is a strong tendency toward AF ordering in the stoichiometric material. Neutron scattering experiments by Shirani et al. ${ }^{2}$ and inelastic light scattering experiments by Lyons et al. ${ }^{3}$ have established that such long-range 2D AF fluctuations are present to very high temperatures.

In the spin-polaron model described here, the viewpoint is taken that the 2D AF correlations can indeed provide a pairing mechanism for superconductivity. We emphasize immediately, however, that these correlations can be relatively short-range, even though our starting point will be a certain type of AF band calculation. Our premise is that it is better to include the AF fluctuations approximately from the beginning rather than to ignore them altogether, as in conventional ba.ıd calculations. Stoichinmetric $\mathrm{La}_{2} \mathrm{CuO}_{4}$ is taken as the prototypical material. It is : sumed to be a strongly correlated system with long-range AF fluctuations of the $\mathrm{Cu}$ spins in the $\mathrm{CuO}_{2}$ plane. The results of parameterized AF band calcula- tions show how strong electron correlation modeled by an on-site Hubbard term opens a gap in the bands obtained by a more conventional band calculation. The material becomes a Mott-Hubbard $(\mathrm{M}-\mathrm{H})$ insulator with the $\mathrm{Cu}$ $3 d^{9}$ configuration playing a predominant role. Doping with $\mathrm{Sr}$ introduces holes on the $\mathbf{O}$ sublattice and gives rise to the observed hole-type conductivity. The holes induce spin deviations on the $\mathrm{Cu}$ sites to form the spin polarons. These are small spin polarons (the spin deviations are well localized about the hole), with an effective mass of $\mathbf{- 5}$. Pairing comes about because the localized spin deviations associated with two polarons can repair or heal one another; the pairing is most likely singlet.

Consideration of the gap equation and the band structure results indicate that the singlet pairing can be very complex but is probably slike and highly anisotropic. The proximity of the Fermi level to the top of the $\mathrm{M}-\mathrm{H}$ valence band and the anisotropy of the pairing interaction greatly complicate the solution of the gap equation. However, approximate solutions readily explain how the gap and $T_{c}$ vary as the whole concentration in the $\mathrm{CuO}_{2}$ plane increases from zero.

1. Summary of paper to be published.

2. G. Shirane et al., Phys. Rev. Lett. 59, 1613 (1987).

3. K. B. Lyons et al., Phys. Reo. B 37, 2553 (1988); Phys. Rev. Lett. 60. 732 (1988).

4. R. F. Wood, Mark Mostoller, and I. F. Cooke, Bull. Am. Phys. Soc. 33, 260 (1988); Bull. Am. Phys. Soc. 34, 642 (1989). 


\section{ANTIFERROMAGNETIC BAND \\ CALCULATIONS FOR THE CuO ${ }_{2}$ PLANES \\ IN HIGH-T SUPERCONDUCTORS}

\section{R. F. Wood, Mark Mostoller, and J. F. Cooke}

Previously constructed Slater-Koster-type tight-binding calculations of $\mathrm{CuO}_{2}$ planes in the new high- $T_{c}$ superconductors were extended to study the antiferromagnetic (AF) structure of these planes. The approach follows that introduced originally by Slater to study AF chromium, but with a Hubbard $U$ term included to keep down-spin electrons off up-spin Cu sites. The calculations show how a Mott-Hubbard (M-H) gap opens in the density of states (DOS) obtained from conventional band calculations. The presence of the $\mathrm{M}-\mathrm{H}$ gap converts stoichiometric $\mathrm{La}_{2} \mathrm{CuO}_{4}$ from a metal to an $\mathrm{M}-\mathrm{H}$ insulator or semiconductor.

Calculations were also carried out for several sets of parameters that shift the $02 p \pi$ bands relative to the $\mathrm{O} 2 p \sigma$ and $\mathrm{Cu} d\left(x^{2}-y^{2}\right)$. The results suggest that in some materials, holes may be introduced into the $02 p \pi$ bands initially but that spin polarons do not form because of the weak exchange interaction with the Cu $d$ electrons. Later, as holes begin to populate the $\mathrm{O} 2 \mathrm{po}-\mathrm{Cu} d\left(x^{2}-y^{2}\right)$ bands, the spin polarons form and give rise to superconductivity. This picture agrees with experimental data for $\mathrm{La}_{2-x} \mathrm{Sr}_{2} \mathrm{CuO}_{4}$ that show superconductivity does not occur until $x$ exceeds $\sim 0.05$. In addition, the DOS and the magnetic moment per $\mathrm{Cu}$ atom can be brought into good agreement with experiment by a reasonable choice of parameters in the band calculations.
In collaboration with scientists at Erlangen University in the Federal Republic of Germany, these AF band calculations have recently been put on a first-principles basis using an unrestricted Hartree-Foch approach with the inclusion of correlation effects. Quantities such as exchange, Coulomb, and overlap integrals that bear on the present parameterized calculations have been evaluated. These results are currently being used in an effort to understand better the effects of introducing holes into filled $\mathrm{M}-\mathrm{H}$ bands, in particular, to study in greater detail the difference in behavior between the 2po and $2 p \pi$ orbitals on the oxygen sites and to investigate the way in which increasing concentrations of holes disrupt the long-range AF correlations.

\section{POSITRON ANNIHILATION IN HIGH-T ${ }_{c}$ COPPER OXIDES}

\section{S. H. Lin}

In collaboraticn with $W$. F. Huang at the University of Louisville, we have been studying the positron annihilation in high- $T_{c}$ vttrium-barium-copper oxides. Single-phase powder samples of the 123 material with oxygen content neal 6.85 were prepanvi by $M . K$. Wu at the University of Alatama at Huntsville and now at Columbia L'niversity. The Doppler-broadened positron annihilation spectra were measured first at room temperature and then with the sample rapidly quenched in liquid nitrogen. The difference spectrum between the two measurements was 
calculated and analyzed, and this proves to yield more information than the commonly used shape parameter analysis. In every case studied, the spectrum at room temperature has "shoulders" on both sides of the central peak. At nitrogen temperature the shoulders are less discernable. The difference spectrum, shown in Figs. 1.8 and 1.9 for two different samples, is consistent with the opening of an energy gap at the lower temperature. A careful comparison between theory and experiment shows that the gap is not associated with superconductivity but with a charge density wave in the $\mathrm{Cu}-\mathrm{O}$ chain. The solid curves in the figures are theoretical results based on this assumption. The two samples differ slightly in $O$ content. They have essentially the same $T_{c}$, but it is clear from the difference spectra that they have very different Fermi momenta and gap parameters. At present we do not know whether the charge-density-wave transition

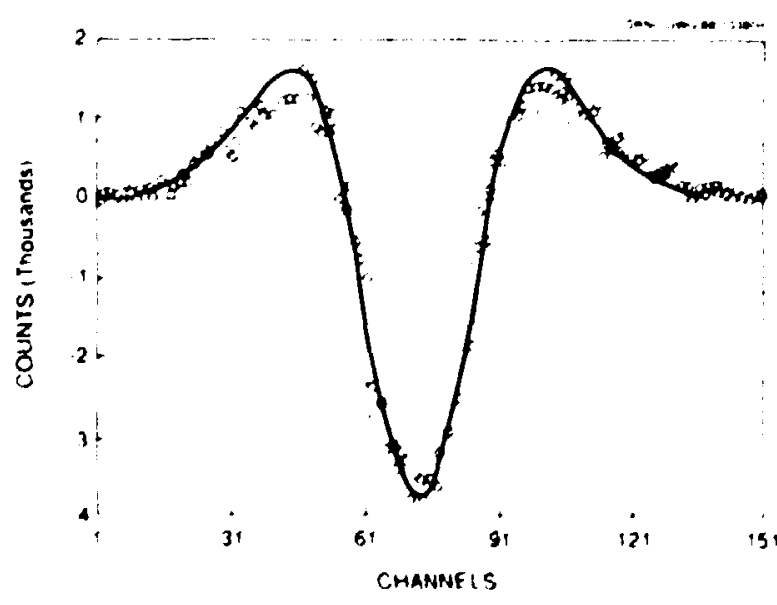

Fig. 1.8. The difference between the Doppler broadened spectra of normal and superconducting states of $\mathrm{YBa}_{2} \mathrm{Cu}_{3} \mathrm{O}_{3} \cdot x(x=$ 0.15 ). Each channel corresponds to a Doppler momentum of $1.37 \times 10^{4} \mathrm{mc}$.

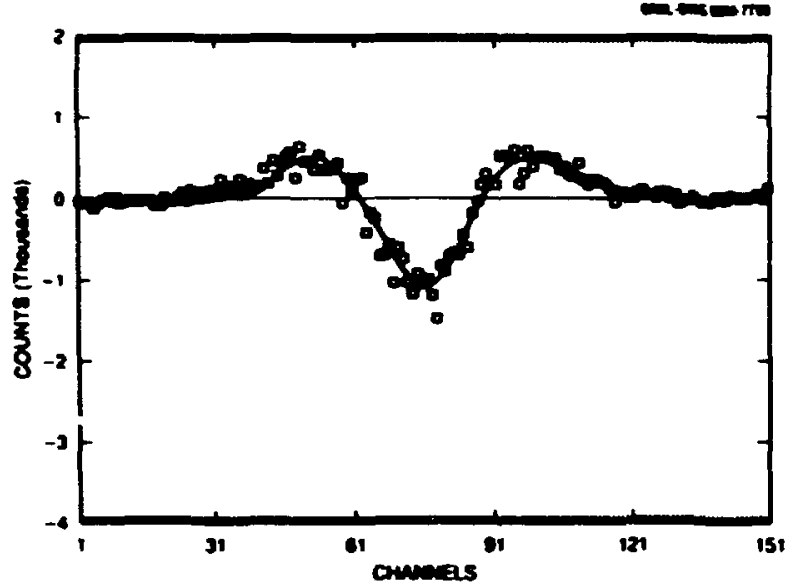

Fig. 1.9. Same as Fig. 1.8 for a sample containing slightly less but unspecified amount of oxygen.

occurs concurrently with superconductivity. A similar measurement over a series of temperatures will be carried out to check this point.

\section{LATTICE VIBRATIONS IN La $\mathrm{CuO}_{4}$ Mark Mostoller}

The pairing mechanism in the new high- $T_{c}$ materials remains in question, but it seems unlikely that conventional electron-phonon coupling is the source of the high superconducting transition temperatures. Nevertheless, their phonon spectra are of interest. Recently, optical and neutron scattering data from single crystals of $\mathrm{La}_{2} \mathrm{CuO}_{4}$ have been reported. These data are still somewhat scant and not in total agreement, but they are sufficient to allow exploratory studies of the interatomic forces.

The available data at the zone center and along the [110] direction have been fitted with a simple shell model with all ions polarizable. 
The calculated results exhibit the soft mode at the $X$ point that drives the tetragonal to orthorhombic structural phase transition and appear to favor the optical measurements of the IBM group' over those of Eklund et al.,' which are in disagreement with respect to the $A_{2 u}$ modes at $q=0$. Figure 1.10 shows the

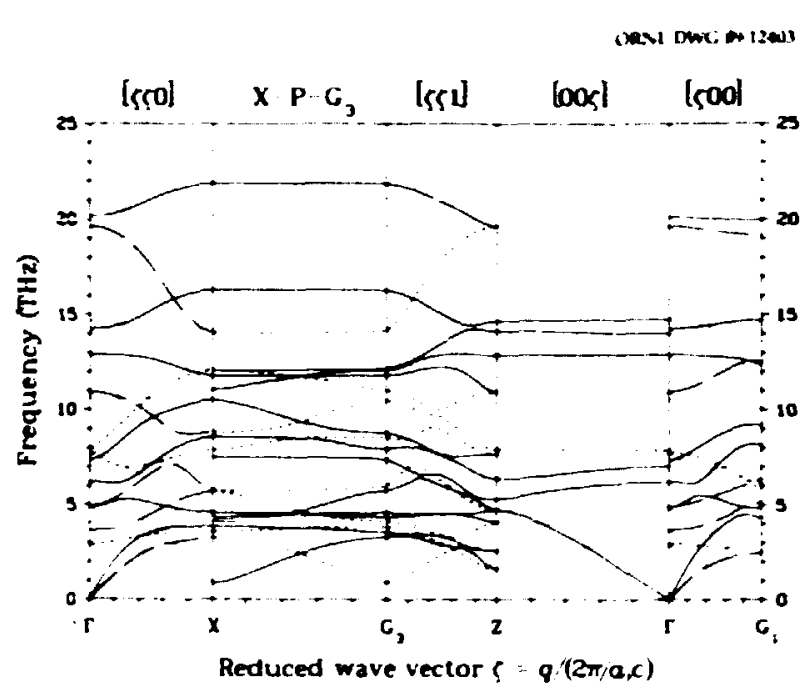

Fig. 1.10. Calculated phonon dispersion curves for $\mathrm{La}_{2} \mathrm{CuO}_{4}$.

calculated frequencies along high-symmetry directions in the Brillouin zone for a set of shell model parameters that gives a reasonable fit to the data of Collins et al. ${ }^{1}$ and Boni et al. ${ }^{3}$

1. R. T. Collins et al., Phys. Rev. B 39, 2251 (1989).

2. P. C. Eklund et al., J. Opt. Soc. B (1989).

3. P. Boni et al., Phys. Rev. B 38, 185 (1988).

\section{A NEW CROSSOVER BEHA VIOR OF TYPE II SUPERCONDUCTORS IN STRONG MAGNETC FIEDS ${ }^{1}$}

\section{Rasolt and Z. Tesanovic ${ }^{2}$}

The fluctuations of vortex lines in type II superconductors have been a subject of considerable attention recently, particularly in reference to high-temperature superconductivity. Such fluctuations can melt the flux lattice and destroy the long-range order. The melting transition has been observed experimentally. In the context of these new results about the role of fluctuations, we have reexamined our understanding of the flux lattice at the mean-field level.

In a classic paper Abrikosov showed that in type II superconductors the Meissner effect is incomplete and part of the external magnetic field flux penetrates into the superconductor, resulting in a lattice of quantized vortex lines. This theory and its more microscopic extensions lead to a critical temperature which decreases monotonically to zero as a function of the external field $H_{c 2}$ (the $H_{c 2}$ line in Fig. 1.11). $T_{c}$ is identically zero when $H_{c 2}=\phi_{0} / 2 \pi \xi_{0}^{2}$, where $\phi_{0}$ is the elementary flux and $\xi_{0}$ is the coherence length at zero temperature. This well-known result has been shown to be incorrect. The Abrikosov-Gorkov curve in Fig. 1.11 is the consequence of a commonly employed semiclassical approximation in which the Landau level structure is neglected. Detailed analytical and exact numerical calculations with full Landau level structures reveal a nonmonotonic $T_{c}(H)$ as the flux lattice crosses over to the quantum limit, and remarkably, in external 


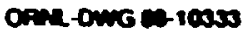

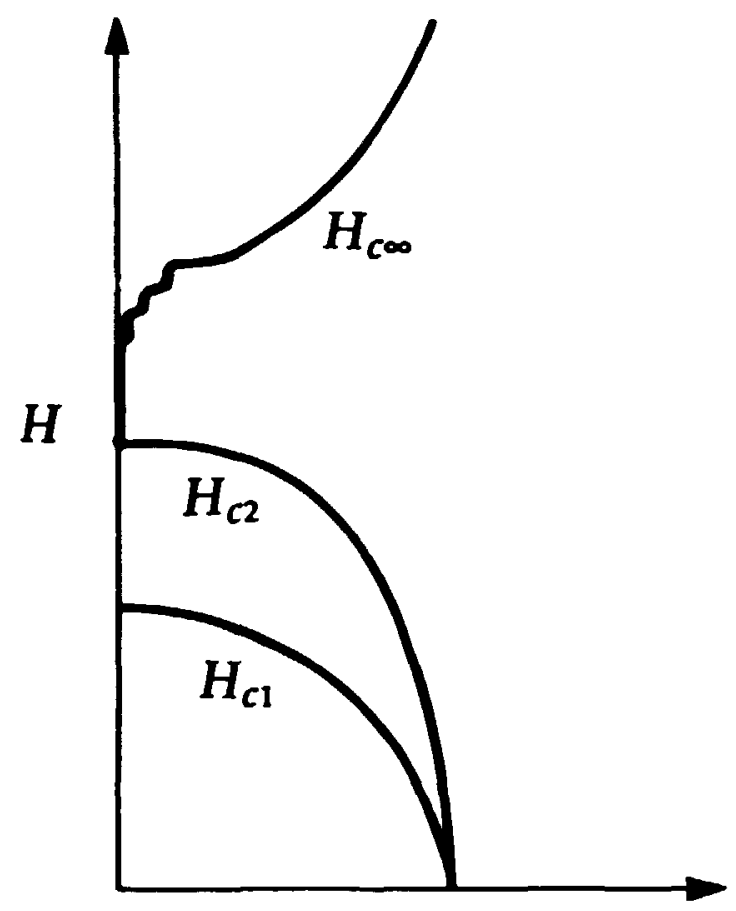

Fig. 1.11. Phase diagram of a type II superconductor in the $H-T$ plane. The $H_{c 2}$ line terminating at $T=0$ is the semiclassical prediction of the Abrikosov theory. Our full quantum mechanical treatment does not permit $H_{c 2}$ to go identically to zero. Rather, for very bw temperatures $T^{*}$ (i.e., $T^{*}<\left(T_{c} / E_{F}\right) T_{c}$ l we predict a crossover to a new superconducting state given by $H_{c o s}$. The step-like behavior in the crossover region reflects the depopulation of higher Landau levels.

fields larger than $H_{c 2}(0)$, superconductivity is enhanced by the increasing field. This entirely new state of type II superconductors is characterized by breakdown to the London relation between the supercurrent and the vector potential. Furthermore, the critical temperature of a BCS superconductor remains finite in an arbitrapily strong ficld. We also considered circumstances under which such remarkable deviations from the standard AbrikosovGorkov theory could be experimentally observed along with the Zeeman spin splitting and disorder on the new superconducting state.

1. Summary of paper to be published.

2. Harvard University, Cambridge, Mass.

\section{AN DXPANSION IN 1/z FOR GRANULAR SUPERCONDUCTORS ${ }^{1}$}

\section{R. S. Fishman ${ }^{2}$}

Granular superconductors can be conveniently modeled as arrays of superconducting grains coupled by Josephson junctions. The Hamiltonian for such an array is a function of the phase $\phi_{i}$ of the order parameter at each grain. The tunneling of Cooper pairs between grains favors the alignment of all the superconducting phases \%. Upon the onset of global phase coherence, when the order parameter $\left\langle\cos \phi_{i}>\right.$ becomes nonzero, the resistivity of the granular superconductor vanishes. It is well-known that the finite self-capacitance of the grains induces quantum fluctuations of the phases is which suppress the order parameter and the transition temperature of the array. When the selfcapacitance or dimensionless grain diameter $\alpha$ is lower than a critical value $\alpha_{c}$, these quantum fluctuations destroy the superconducting order.

The most common method of studying granular superconductors is mean-field (MF) theory, which neglects the coupling of phase fuctuations on neighboring grains. By performing an expansion in $1 / z$, where $z$ is the number of neapest neighbors for each grain, the lowest order 
corrections to the MF order parameter and transition temperature have been calculated. These lowest order corrections incorporate the coupling of phase fluctuations over an infinite number of grains. As expected, fluctuations suppress the phase-coherence of the granular superconductor. The $1 / 2$ correction to the MF order parameter is negative and diverges at the MF transition temperature. Associated with this divergence is a shift in the transition temperature away from the MF value. The $1 / 2$ correction to the transition temperature is also negative and diverges at the MF value of the critical grain diameter $\alpha_{c}$ signaling a shift in $a_{c}$ away from the MF value of 2 .

Using this technique, which allows a systematic study of the effect of phase fluctuations, the short-range-order parameter $\left\langle\cos \left(\phi_{1}-\phi_{2}\right)\right\rangle$, where grains 1 and 2 are nearest neighbors, and the specific heat have been calculated. Both show signs of an enhanced phase coherence at a temperature inversely related to the grain diameter. At this temperature, a Cooper pair is thermally excited between grains. This quasi-reentrance is related to the observed dip in the normal state resistivity of granular films.

1. Summary of papers: Phys. Rev. B 39, 7228 (1989); to be published.

2. Cuest scientist from the ORNL/UT Distinguished Scientist Program.

\section{SURFACES AND INTERFACES}

\section{SEARCH FOR "NEAR PERFECT" AGREEMENT IN LEED ANALYSIS}

\author{
H. L. Davis and I. R. Noonen
}

Existing low-energy electron diffraction (LEED) 1-V data sets for the $\mathrm{Cu}(100), \mathrm{Al}(110)$, and NiAl(110) surfaces have been reanalyzed in order to investigate the differences that existed in the original analyses between calculated and experimental I-V profiles. Although these differences were modest (the original analyses had very small $\boldsymbol{R}$-factors), it was anticipated that reanalyses, using a more extensive search of LEED model parameters, would provide improved agreement. Also, and more importantly, since the accuracy and reliability of LEED analysis are related to the quality of agreement (between calculated and experimental I-V's), it was hoped that reanalyses would provide some clues on how the accuracy of LEED could be improved by examination of those I-V features, which are enhanced by better model parameters. The $\mathrm{Cu}(100), \mathrm{Al}(110)$, and $\mathrm{NiAl}(110)$ surfaces were selected specifically for the investigation because each of their original analyses ended with excellent agreement between calculated and experimental I-V's and any improved agreement obtained in the reanalyses would provide an indication that our new procedures are correct and contain some valid physics. Be that as it may, results of the reanalyses for each of the three surfaces did provide a significant reduction in $R$-factor, 
and almost "near perfect" agreement was obtained between calculated and experimental I-V's. For example, the Zamazzi-Jona R-factor for Al(110) has been reduced to 0.023 from the value of 0.034 , which was obtained in the original analysis. For $\mathrm{Cu}(100)$ it has been reduced to 0.026 from 0.038 , and for $\mathrm{NiAl}(110)$ it has been reduced to 0.010 from 0.053 . Such improved agreement was achieved by extensive modeling of the LEED bric-a-brac (i.e., nonstructural parameters of the theoretical model), especially by considering different thermal vibrations in successive atomic layers and by including an energy dependence in the complex optical potential. There should be no doubt that these new results provide insights useful for improving the accuracy obtainable in LEED analysis, and for suitable surfaces, distances between atoms soon should be able to be obtained with error limits being of the order of $0.01 \AA$ or smaller.

\section{SURFACE STRUCTURE AND LOW-ENERGY ELECTRON DIFFRACTION (LEED) 'BRIC-A- BRAC": A CASE STUDY OF Ag(110)}

\section{H. L. Davis and I. R. Noonan}

Significant advances in the reliability of LEED analysis for surface structure determination have been made over the past 15 years. This has been partially due to the fact that the theoretical model was sufficiently accurate so that structural results obtained in LEED analyses usually had been found to be relatively insensitive to the various nonstructural param- eters of the theoretical model that were used to produce calculated I-V profiles to compare with experimental profiles. The nonstructural parameters of the theoretical model are the scattering phase shifts, the functional forms of the real and imaginary components of the optical potential, the vibrations of the various atomic sites in the surface region, etc.-things that we denote as the Tric-a-brac" of LEED.

The many past successes of LEED have lulled one into ignoring possible effects of the bric-abrac on the structural conclusion obtained in an analysis. As an example, in 1981 a LEED anal ysis for Ag(110), which had an acceptable $R$ factor of 0.10, indicated a surface relaxation of $\Delta d_{12}=-5.7 \%$ and $\Delta d_{23}=+2.2 \%$. $\quad\left(\Delta d_{i j}=\right.$ the change in the spacing between layers $i$ and $j$ relative to the bulk interlayer spacing.) A recent mediumenergy ion scattering study, however, indicates that $\Delta d_{12}=-9.5 \%$ and $\Delta d_{23}=+6.0 \%$. To investigate this significant disagreement, an extensive reanalys:. of the LEED data for $A g(110)$ has been performed. Surprisingly, it was determined that, in the new analysis, the structural conclusion for $A g(110)$ is quite sensitive to the choice of vibrational model and other LEED bric-a-brac. The final results of the LEED reanalysis are also in much better agreement with the ion scattering results, since the surface relaxations obtained, with an $R$-factor of 0.06 , are $\Delta d_{12}=-8.0 \%$ and $\Delta d_{23}=+3.7 \%$. However, whether the new LEED results or the ion results more nearly reflect the actual surface must await future work. 
The most interesting result of the Ag(110) LEED reanalysis is that it serves as an excellent illustration of the necessity of making an extensive consideration of the bric-a-brac when other surfaces are analyzed, even when initial results have a very acceptable $\boldsymbol{R}$-factor. The Ag(110) reanalysis also has opened an exciting possibility concerning the sensitivity of LEED analyses. Today it is thought that atomic positions in suitable surfaces can be determined to within $0.02 \AA$ by a LEED analysis, but could a more detailed examination of the bric-a-brac enable the sensitivity to be improved to $0.01 \AA$ or even lower? This possibility is now being actively pursued.

1. E. Holub-Krappe et al., Surf. Sci. 188, 335 (1987).

\section{RIPPLED RECONSTRUCTION OF THE HFC(100)-(1X1) SURFACE}

\section{H. L. Devis, J. R. Noonan, and K. Müller'}

Transition-metal carbides have significant technological interest because of their resistance to corrosion and abrasion. Such interest naturally leads to research which is concemed with the metal carbide surfaces. Past research ${ }^{2}$ has demonstrated that the atoms at the $\mathrm{TaC}(100)$ surface are relaxed away from, their truncated bulk positions, because of electronic density redistribution at the surface, in a manner to induce a rippled reconstruction in the atomic structure. Since samples of HfC were readily available, it was of interest to deter- mine whether such a reconstruction also existed in the $\mathrm{HCC}(100)$ surface. At the same time, an HfC investigation would also expand the available, quantitative, experimental information concerning the surface structure of binary metallic systems. Such information is of considerable value in testing numerical simulations and/or theories of pair potentials for binary systems, as has been demonstrated by the wide theoretical interest shown in previous ORNL work on $\mathrm{NiAl}(110)$.

A HfC(100) surface was prepared by $\mathrm{Ar}^{+}$ion sputtering followed by flashing to $1800 \mathrm{C}$ for approximately $10 \mathrm{~s}$. These processes produced a clean, stoichiometric, well-ordered $\mathrm{H} f(100)$ surface. With the primary electron beam at normal incidence, equivalent-beam-averaged I-V profile data for the eight lowest indexed diffraction beams were collected. These experimental I-V profiles were then compared with profiles calculated for various structural models of the surface. In order to obtain excellent agreement between the calculated and experimental profiles, the various nonstructural parameters of the low-energy electron diffraction (LEED) t'leoretical model were also varied. The best agreement was obtained for a rippled reconstruction model in which the $C$ atoms of the topmost $(100)$ plane are $0.11 \mathrm{~A}$ above the $\mathrm{Hf}$ atoms of the plane, while in a bulk plane the $H f$ and $C$ atoms are coplanar. Also, the $C$ atoms of the second (100) plane of the surface are $0.03 \AA$ above its $H f$ atoms. Since Hf and Ta are adjacent in the periodic table, it was surprising that the $0.11-\AA$ rippling in the 
topmost (100) HKC plane was found to be about one-half that determined for $\mathrm{TaC}$ (i.e., $0.20 \AA$ ).

An important result from our $\mathrm{HfC}(100)$ investigation, and one that is expected to have significant impact for future work on binary systems, is that a forward-scattering-thenbackscattering effect has produced, in the LEED analysis, a sensitivity to $C$ atom position that is almost equal to the sensitivity to Hf position. Such sensitivity to $\mathrm{C}$ position is contrary to what is expected from simple kinematic scattering considerations. It is anticipated that this effect will be exploited more fully in future work on binary systems.

1. Guest scientist from Universitat Erlangen-Nümberg, Federal Republic of Germany.

2. J. R. Noonan, H. L. Davis, and G. R. Gruzalski, I. Vac. Sci. and Technol. A 5, 787 (1987).

\section{APPLICATION OF ONSAGER'S RECIPROCTIY RELATIONS TO INTERFACE MOTION DURING PHASE TRANSFORMATIONS ${ }^{1}$}

Theodore Kaplan, M. J. Aziz, ${ }^{2}$ and L. I. Gray ${ }^{3}$

Experiments on splat quenching of molten metallic alloys and pulsed-laser melting of doped semiconductors have demonstrated the breakdown of local equilibrium at the crystalmelt interface and a coupling between the motions of the two atomic species across the interface during rapid interface motion. This work has inspired a number of kinctic models for the deviation from local equilibrium at the interface. Theories have also been developed for interface motion during other phase transformations, a common goal being the prediction of the interface velocity and the composition of the growing phase in terms of temperature and composition of the parent phase at the interface. Due to obvious experimental difficulties involved in measuring the relevant quantities at a rapidly moving interface, only partial tests of some of these models have been possible to date. Onsager's reciprocity relations, deduced by the application of time-reversal symmetry to microscopic fluctuations, can provide some help in evaluating theories for coupled irreversible processes such as alloy solidification. By setting a criterion that the theories have to match, some theories can be eliminated without the need for experimental tests.

We have shown how to choose properly the conjugate fluxes and forces for Onsager's reciprocity relations to hold at the moving interface in the alloy solidification problem. These relations have been applied to two variations of the continuous growth theory for solidification. 5 One theory identified as withsolute drag was shown to violate Onsager's relations and is therefore incorrect. The theory without-solute drag was shown to be consistent with Onsager's relations and therefore can still be considered as a potentially valid theory of solidification. During this study it became clear that particular care must be taken in determining Onsager coefficients from a system of highly nonlinear equations. In particular, 
the analysis must consider the domain in which physical solutions of the equations can exist.

1. Sunumary ef paper. I. Chem. Phys. 90, 1133 (1989).

2. Oak Ridge Associated Universities faculty research participant from Harvard University, Cambridge, Mass.

3. Engineering Physics and Mathematics Division, ORNL.

4. L. Onsager, Phys. Reo. 37, 405 (1931); 38, 2265 (1931).

5. M. J. Aziz and T. Kaplan, Acta Metall. 36, 2335 (1988).

\section{ION-SOLID INTERACTIONS}

\section{THE SLOWING-DOWN TIME OF ENERGETIC ATOMS N SOLIDS ${ }^{1}$}

\section{M.T. Robinson}

Computer simulation models for the slowing-down of energetic atoms in matter are often based on the binary collision approximation (BCA). Such models typically ignore the temporal aspects of the problem. A method is described whereby calculations of the times at which energetic particles reach their collision points may be included in such BCA simulations without altering the essential "event-driven" character of the model. This method was implemented in an enhanced version of the BCA simulation code MARLOWE?

The enhanced BCA model was illustrated by a series of calculations concerning the slowing down of $10-\mathrm{eV}$ to $10-\mathrm{keV} \mathrm{Cu}$ atoms re- coiling isotropically from lattice sites in both crystalline and amorphous $\mathrm{Cu}$ targets and of similar atoms injected from an ion beam into polycrystaliine $\mathrm{Cu}$ targets. The ranges of the projectiles were recorded as well as the time required to slow down. In addition to distributions of the ranges and slowing-down times, correlations between the two were obtained.

A typical set of results is shown in Fig. 1.12. The two panels on the lef? show distrilutions of the slowing-down time and the penetration (the projectile range projected onto the target normal) for 10-keV atoms in polycrystalline targets. The right-hand pinel shows the correlation between these two quantities. As expected, there is a general correlation in the sense that particles which travel farther take longer to do so, but because there are many routes in buth energy and space which particles can traverse in reaching their terminal points, the time distribution at a single penetration is broadened. More interestingly, the joint distribution shows a penetrating tail due to channeling: in fact, there is evidence of about three different channels, each with its own relationship between range and slowing-down time.

1. Summary of paper to be published.

2. M. T. Robinson and I. M. Torrens, Phys. Rev. B 9, 5008 (1974). 

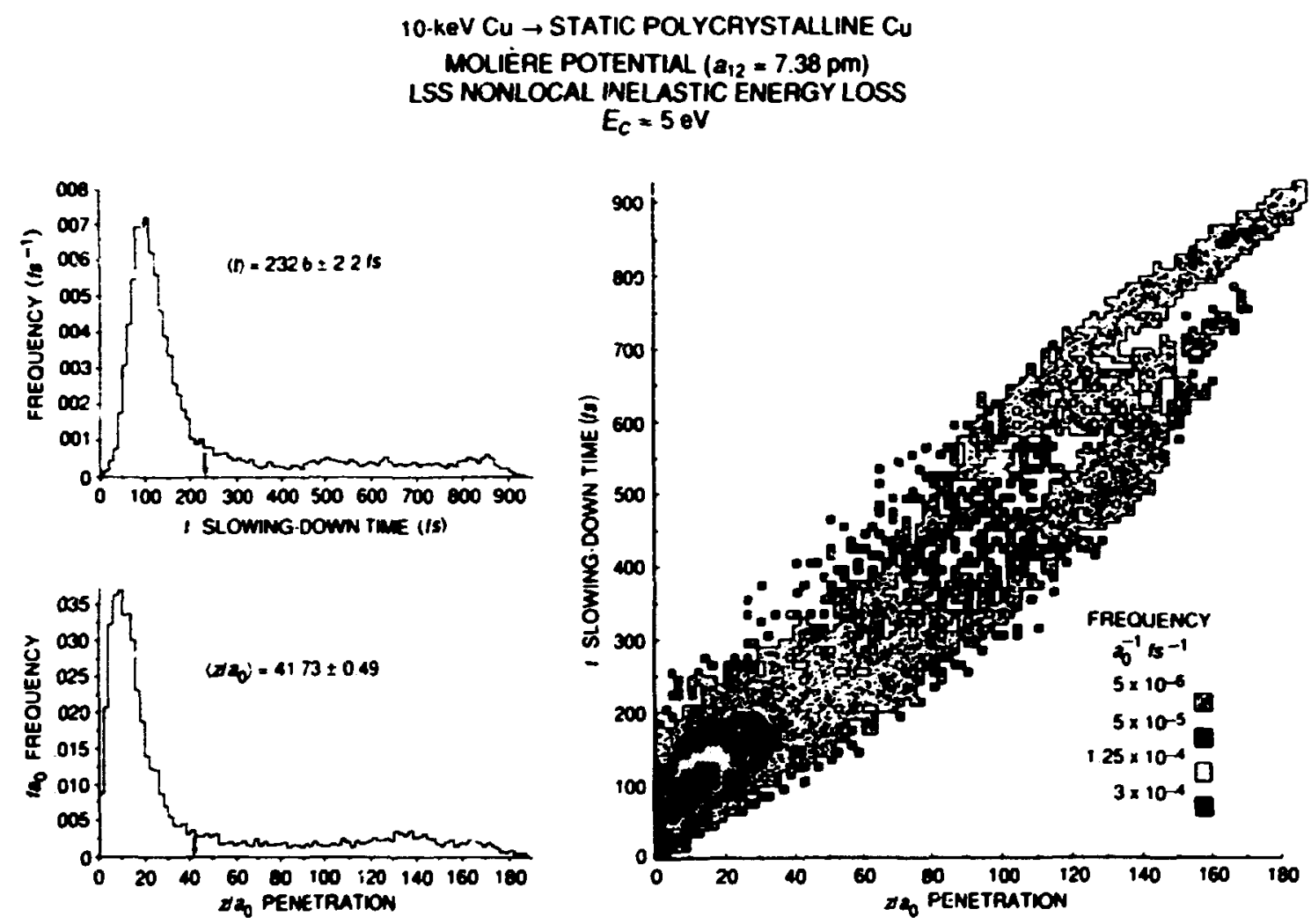

Fig. 1.12. Penetration and slowing-down time distributions for 10-keV Cu atoms at normal incidence upon polycrystalline $\mathrm{Cu}$. The arrows mark the mean values in the two singlevariable distributions. The + marks the maximum in the joint distribution. Four frequency ranges are shown by the shading.

\section{CHANNELING MEASUREMENT OF SOLUTE VIBRATION AMPLITUDE IN AN ALLOY ${ }^{1}$}

\section{J. H. Burrett}

The measurements ${ }^{2}$ on a $\mathrm{Cu}(2$ at. \% Au) alloy shown in Fig. 1.13(a) have long been regarded as the classical example of channeling behavior of a solute or impurity atom on a substitutional site in a host crystal. One of their features, however, suggests that the measurements contain additional information; this feature is that all the Au points lie below the corresponding $\mathrm{Cu}$ points out to angles of almost $2^{\circ}$. This feature means that the Au channeling

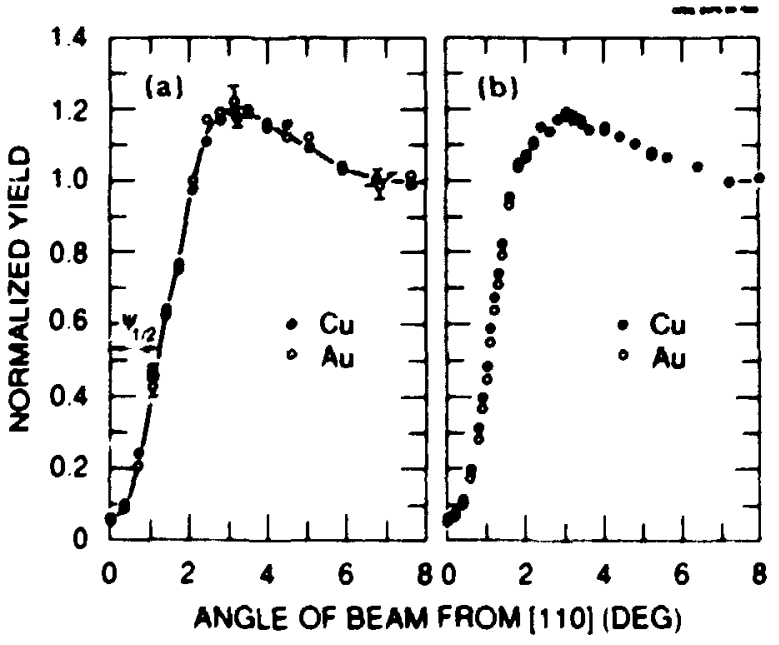

Fig. 1.13. Backscattering of $1.2-\mathrm{MeV}$ He ions incident near the $[110]$ direction in $\mathrm{Cu}(2$ at. $\% A u$ ): (a) measurements (from Ref. 2), (b) computer simulations. 
dip has a slightly larger half angle $\Psi_{1 / 2}$ than does the $\mathrm{Cu}$ dip and a larger half angle suggrsts a smaller thermal vibration amplitude. The effect of the vibration amplitude of the Au atoms was explored by doing computer simulations of the channeling with $2 \%$ of the atoms being $A u$ distributed randomly on the lattice sites and the Au atoms being allowed to have a vibration amplitude smaller than that of $\mathrm{Cu}$. Simulation results are shown in Fig. 1.13(b) for vibration amplitudes of $8.4 \mathrm{pm}$ for $\mathrm{Cu}$ and of $7.0 \mathrm{pm}$ for Au; the relationship of the Au and Cu points looks very much as it does in part (a). The Au points are lower than the Cu ones in the vicinity of a yield of 0.5 by about $0.030 \pm 0.015$ in part (a) and by about 0.035 in part (b). From these two lowerings, the Au amplitude appears to be $7.2 \pm 0.6 \mathrm{pm}$ or $86 \pm 7 \%$ as large as that of Cu. For future measurements made specifically for application of this analysis method, the accuracy should be better.

1. Summary of paper: Phys. Rev. B 38, 5069 (1988).

2. R. B. Alexander and J. M. Poate, Radiat. Eff. 12, 211 (1972).

\section{CHANNELING SIMULATION IN Ni $\mathrm{NI}^{1}$}

\section{H. Barrett}

Measurements made by ion channeling and Rutherford scattering ${ }^{2}$ on an ordered alloy of $\mathrm{Ni}_{3} \mathrm{Al}$ containing $1 \% \mathrm{Hf}$ have indicated that the $\mathrm{Hf}$ is mostly on the Ni sites. An atom probe experiment ${ }^{3}$ on a similar specimen, however, has lead to the conclusion that Hf is mostly on the Al sites. Computer simulations of the channeling and Rutherford scattering have been done to reexamine the interpretation of ion measurements in the case of an alloy. The simulations accumulated separate results for the $\mathrm{Ni}$ and the $\mathrm{Al}$ sites and allowed for the possibility that the $\mathrm{Hf}$ atoms might have a displacement amplitude different from the value of $8.0 \mathrm{pm}$ used for the host atoms.

In the simulation results, a change in the displacement amplitude of the $\mathrm{Hf}$ atoms has an effect very similar to a change in the fraction of Hf atoms on each of the host sites. This similarity in the effects produced by these two factors in the calculations causes difficulty in interpreting the measurements. If the $\mathrm{Hf}$ atoms are taken to have the same displacement amplitude as the host atoms, then about $60 \%$ of them would be on the Ni sites, close to the value inferred in Ref. 2. If one assumes that $76 \%$ of the $\mathrm{Hf}$ atoms are on the $\mathrm{Al}$ sites as in Ref. 3, then the displacement amplitude of the $\mathrm{Hf}$ atoms would be $4.9 \mathrm{pm}$ or $60 \%$ as large as that of the host atoms. Each of these two combinations gives equally good agreement with the neasurements. At present the displacement amplitude for the $\mathrm{Hf}$ atoms is unknown so that it is not possible to choose between these or various other possible interpretations.

1. Summary of paper: Nucl. Instrum. and Methods Phys. Res. Sect. B 30, 546 (1988).

2. H. Bohn et al., p. 127 in HighTemperature Ordered Alloys, ed. by $\mathrm{O}$. Izumi et al., Norti-Holland, New York, 1987.

3. M. K. Miller and J. A. Horion, Ref. 2, p. 117. 


\section{STRUCTURE OF THE BORON-HYDROGEN COMPLEX IN SILCON1}

\author{
A. D. Marwick, ${ }^{2}$ G. S. Ochrlein, ${ }^{2}$ \\ J. H. Barrett, and N. M. Johnson 3
}

Channeling measurements using nuclear re actions have been applied ${ }^{4}$ to the question of where $\mathrm{B}$ and $\mathrm{H}$ atoms are located in $\mathrm{B}-\mathrm{H}$ complexes in Si. It wis determined that $B$ atoms are displaced from lattice sites by $0.22 \pm 0.04 \mathrm{~A}$ and that $\mathrm{H}$ atoms occupy preferentially bondcentered sites. Further precision has now been sought in these determinations by means of computer simulations of ion channeling in $\mathrm{Si}$. During simulations for the same directions of ion beam incidence as used in the experiments, ion fluxes were collected at several depths and stored. Subsequently, the stored flux distributions were used with many possible B or $\mathrm{H}$ locations to determine which location gave best agreement with experiment. In the case of $B$, the displacement from the lattice sites was now determined to be $0.28 \pm 0.03 A$. In the specirnen uspd for the $H$ measurements, the $H$ and $B$ atoms were present in equal concentrations at all depths except near the surface, where there was an excess of $\mathrm{H}$ comprising $\mathbf{2 8 \%}$ of the total. Because other measurements have indicated such an excess to have random lattice locations, we made such an assignment here. Of the remaining $H$, the best fit to the measurements occurred for an assignment of $83 \%$ to a bondcentered site (for which the displacement was found to be between 1.2 and $1.5 \AA$ ) and $17 \%$ to a back-bonded site.

1. Summary of paper: Mat. Res. Soc. Symp. Proc. 104, 259 (1988).
2. IBM T. J. Watson Research Center, Yorktown Heights, N. Y.

3. Xerox Palo Alto Research Center, Palo Alto, Calif.

4. A. D. Marwick, G. S. Oehrlein, and N. M. Johnson, Phys. Reo. B 36, 4539 (1987).

\section{DAMAGE ENERGY AS A PREDICTOR OF FRENTEL PAIR PRODUCTION UNDER CHANNELING CONDITIONS}

$$
\text { O. S. Oen }
$$

A fundamental prediction of radiation damage theory in semiconductors and metals is that the number of Frenkel pairs produced during the slowing down of an energetic ion is directly proportional to the amount of energy ending up in nuclear motion. Because of this relationship, this energy is called the damage energy. An ingredient of this prediction is the stochastic nature of the nuclear and electronic energy loses incurred by the ion and its progeny as they slow down to rest in a structureless solid. The question arises as to whecher the above prediction is valid in a crystalline solid, especially under channeling conditions where correlated collisions occur and where the division of energy losces between nuclear and electronic may be greatly altered from that of a random solid. Channeling acts as a switch to "tuin off" the more violent nuclear collisions. To test the hypothesis that the damage energy is a good predictor of the Frenkel pair production in crystalline solids when channeling is present, calculations were done using the computer simulation code MARLOWE to calculate simultaneously the number of Frenkel pairs (determined by interstitial-vacancy separation distance) and the energy ending up in nuclear 
motion. Light and heavy ions slowing down in silicon were studied. The ions chosen were B, $\mathrm{Si}, \mathrm{As}$, and $\mathrm{Bi}$ with initial energies ranging from 1 to $30 \mathrm{keV}$. The single-crystalline silicon was bombarded along [110], [100], and in a random direction (12 deg from [110]). The ions and the knocked-on atoms that were produced were followed collision by collision until their energy dropped below $15 \mathrm{eV}$. A Molière interatomic potential was used to describe the nuclear collisions, and the electronic losses were treated using the Lindhard-Scharff-Schiott formalism. Thermal vibrations of the target atoms were included. At the higher ion energies, channeling is found to reduce tive amount of energy going into nuclear motion by a factor of 6 or more. For random direction irradiations, it was found that the number of Frenkel pairs produced was directly proportional to the deposited damage energy. When channeling occurred, the damage energy overpredicted slightly (15 to 30\%) the number of Frenkel paiss produced. It is believed that this overprediction arises from the excess nuclear collisional losses below the displacement energy $(15 \mathrm{eV})$ of the silicon atoms. When it is considered that the damage energy changes by a factor of 6 , a prediction that is off by even $30 \%$ is a fair approximation. It is concluded that the damage energy is a useful although not precise predictor of the number of Frenkel pairs. Its usefulness resides in that it is much easier to calculate than the Frenkel pair production. 


\section{Neutron Scattering}

The High Flux Isotope Reactor (HFIR) was restarted in April 1989 and ran for a few weeks at low power before being shut down again for review of operating procedures. At present, it is hoped that there will be a restart in August and full power operation in October.

Members of the Neutron Scattering Section have continued to be scientific nomads, traveling to laboratories in France, England, Canada, and within the United States to perform experiments. At ORNL, some substantial upgrades to the triple-axis spectrometers were completed, finishing touches on a new high-resolution powder diffractometer were added, and a move was made into our new office building across the street from HFIR. The loss of the Oak Ridge Research Reactor has left the Ames Laboratory without a neutron source. This problem is being solved jointly by installing a new triple-axis spectrometer at the HFIR that will be utilized by both the ORNL and Ames neutron scattering programs.

The only facility that has remained in operation is the small-angle $x$-ray scattering (SAXS) instrument. The SAXS user program has continued with 44 external users making 69 visits during this reporting period. An improvement in the collimation system for the SAXS instrument has greatly reduced the instrumental background and allowed a significant reduction in the minimum scattering vector. A new PC-based data analysis scheme has been developed and will be installed soon on both the SAXS and small-angle neutron scattering instruments

There is a growing effort aimed at designing the beam tubes and guides for the Advanced Neutron Source. While this facility is still ten years in the future, decisions on the number and shape of beam delivery systems must be made soon.

This chapter is organized into subsections on small-angle scattering, neutron diffraction, inelastic neutron scattering, and instrumentation. The small-angle work includes topics in polymer science, colloids, and metallurgy. 
Inelastic topics include work on beryllium, lithium, rare-earth metals, high- $T_{c}$ superconductors, and amorphous magnets.

\section{SMALL-ANGLE SCATTERING}

\section{INSTRUMENTAL RESOLUTION EFFECTS IN SMALL-ANGLE SCATTERING ${ }^{1}$}
G. D. Wignall, D. K. Christen, and V. Ramakrishnan ${ }^{2}$

Experimentally measured scattering data differ from theoretical curves because of departures from point geometry in a real instrument. In a small-angle neutron scattering (SANS) instrument, there are essentially three contributions to the smearing of an ideal curve: (1) the finite divergence of the beam, (2) the finite resolution of the detector, and (3) the polychromatic nature of the beam. Where the scattering is azimuthally symmetric about the incident beam, indirect Fourier transform (IFT) methods riay be used not only to smear an ideal scattering curve but also to desmear an observed pattern. For experiments where the assumption of azimuthal symmetry cannot be made, alternative procedures based on Monte Carlo (MC) techniques have been developed to simulate the smearing of a given theoretical scattering function. This procedure permits the evaluation of smearing effects in anisotropic systems. Both IFT and MC procedures have been used with a range of applications from data taken on the 30-m SANS facility at the High Flux Isotope Reactor. It was shown that, for exper:ments with scattering dimensions $<200 \AA$, smearing effects are small and that dimensions up to $-1200 A$ may be accurately resolved after proper evaluation of resolution effects. These findings are illustrated in Table 2.1, which compares polymer core-shell latex diameters measured via SANS and other techniques. These procedures have also been extended to

Table 2 4. Polymer latex diameters from different techniques.

\begin{tabular}{lccc}
\hline Sample & \multicolumn{3}{c}{ Quter diamite $(\mathcal{A})$} \\
\cline { 2 - 4 } & SANS & Light scattering & Electron microscopy \\
\hline $\mathrm{Cl}$ (core) & 992 & 980 & - \\
$\mathrm{C} 2$ (core) & 1218 & 1230 & - \\
A (core) & 1010 & 900 & 1120 \\
B (core + shell) & 1200 & 1070 & 1300 \\
\hline
\end{tabular}


simulate resolution effects on the ORNL $10-\mathrm{m}$ SAXS facility. As with the 30-m SANS instrument, resolution effects are negligible for $R_{g}<100 \AA$ and begin to be observable for $R_{g}>$ 200 A. For an irradiated Al-8 calibration standard, ${ }^{3}$ the observed $R_{8}=218 \AA$ via $S A X S$ is slightly lower than the value (227 A) measured independently. Simulation of an ideal Guinier scattering law with $R_{g}=227$ A gives a smeared $R_{g}=218 \pm 2 \AA$, which equals the observed result within the experimental error.

1. Summary of paper. J. Appl. Crystallogr. 21, 438 (1988).

2. Brookhaven National Laboratory, Upton, N. Y.

3. T. P. Russell et al., J. Appl. Crystallogr. 21, 629 (1988).

4. R. W. Hendricks, J. Schelten, and W. Schmatz, Phil. Mag. 30, 819 (1974).

\section{INTERACTIONS IN MIXTURES OF POLY(ETHMENE OXIDE) WITH POLY(METHYL METHACRYATE)'}

\section{G. D. Wignall, T. P. Russell, ${ }^{2}$ and H. Ito}

Small-angle neutron scattering (SANS) measurements have been performed on mixtures of amorphous poly(ethylene oxide), PEO, with protio- and deuteriopoly(methyl methacrylate), PMMAH and PMMAD, respectively, to evaluate the Flory-Huggins interaction parameter $X_{A B}$. It has been found that $X_{A B}$ varies from $-5 \times 10^{-3}$ to $-1 \times 10^{-3}$ as the total PMMA corcentration increases from 0.3 to 0.7 , and this concentration dependence is in good agreement with independent measurements ${ }^{3}$ undertaken at $\emptyset=0.9$ (Fig. 2.1). The temperature dependence of the scattering combined with other results indicates that entropic, rather than enthalpic, contributionis dominate $X_{A B}$. The magnitude of $X_{A B}$ is found to be on the same order of magnitude as that expected between PMMAH and PA IMAD, particularly at high total concentrations of PMMA. Studies of PMMA at higher scattering vectors show that, down to length scales of $25 \AA$, no changes are observed in the conformation of PMMA in the mixtures in comparison with that of PMMA in the bulk. On crystallizing from the amorphous state at $50^{\circ} \mathrm{C}$, small-angle $x$-ray scattering (SAXS) and SANS data indicate that PMMA is incorporated into the amorphous phase between the crystalline lamellae. In addition, the thickness of the crystalline lamellae does not change with temperature, which is in keeping with a small interaction parameter

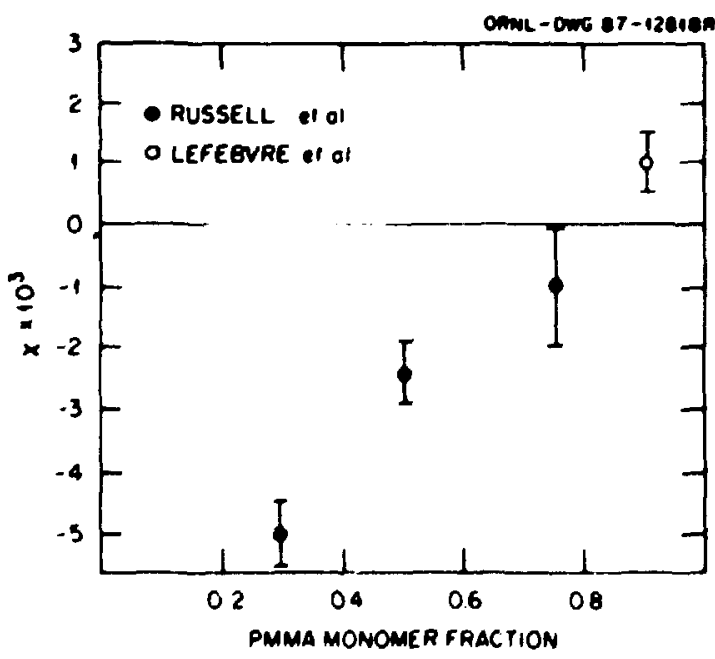

Fig. 2.1. Interaction parameter between PEO and PMMA as a function of the monomer fraction $(H+D)$ of PMMA. 
between the PEO and PMMA. The diffusephase boundary between the crystalline and amorphous phase is $-15 \AA$ greater for the SAXS measursments than that measured by SANS. These results suggest the existence of a region on the crystal surface in which the crystalline order dissipates and from which the noncrystallizible PMMA is excluded.

1. Summary of papers: Macromol. 20, 2213 (1987); 21, 1703 (1988).

2. IBM Almaden Research Center, San Jose, Calif.

3. J. M. R. Lefebvre, R. S. Porter, and G. D. Wignall, Polym. Eng. Sci. 27(6), 433 (1987).

\section{CHARACTERIZATION OF CARBON-FILLED POLYMERS BY SMALL-ANGLE SCATTERING TECHNIQUES}

\section{G. D. Wignall, N. R. Farrar, ${ }^{2}$ and S. Morris ${ }^{3}$}

Carbon black is used extensively to modify the mechanical ind electrical properties of polymers and elastomers. The size and distribution of the individual particles and agglomerates have an important effect on properties. Agglomeration of the particles into larger aggregates is known to occur in highly loaded polymers and can significantly affect the strength and electrical resistivity. Carbonblack polymer composites are not amenable to investigation by optical methods because they are opaque and only microtomed sections may be examined by electron microscopy. A combination of small-angle neutron scattering (SANS) and small-angle $x$-ray scaltering (SAXS) has been used for the first time to provide information not available previously. In a collaboration with Raychem Corporation, highresolution methods were used to evaluate particle sizes and aggregation in the size range 100-800 $A$. The SANS data give an excellent fit to the Debye-Bueche two-correlation function model 4.5 as indicated in Fig. 2.2 for a concentration of 34.8 vol\% carbon black in polyethylene. The absolute magnitude of the scattering cross section is also consistent with the dimensions of the model parameters and the neutron contrast factors, thus providing an independent check on the validity of the model. Comparison of SAXS and SANS data indicates the presence of a third phase, most probably voids at the surface of the carbon particles.

1. Summary of paper: Journal of Materials Science (in press).

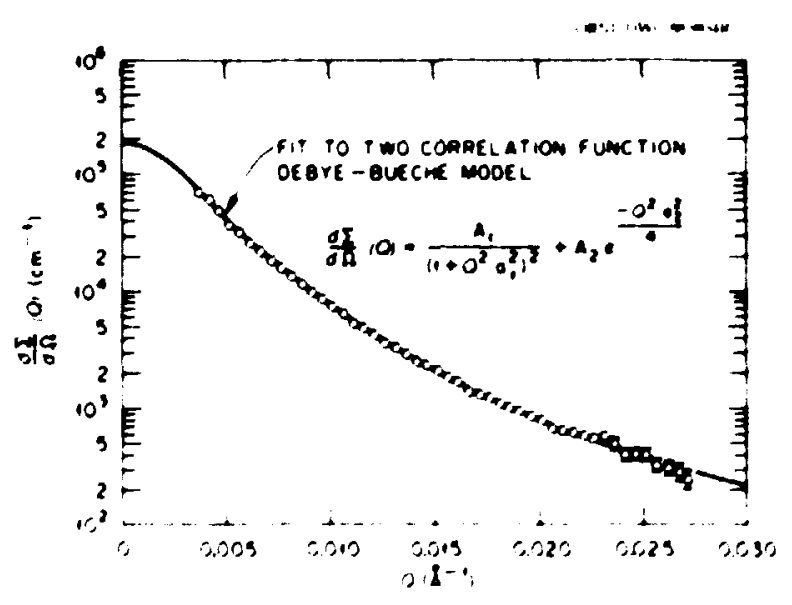

Fig. 2.2. Coherent SANS cross section from 34.8 vol\% carbon black in polyethylene. 
2. Hewlett Packard Laboratories, Palo Alto, Calif.

3. Raychem Corporation, Menlo Park, Calif.

4. P. Debye and A. Bueche, I. Appl. Phys. 20, 519 (1949).

5. P. Debye, $H . R$. Anderson, and $H$. Brumberger, J. Appl. Phys. 28, 679 (1957).

\section{A SMALLANGLE NEUTRON SCATTERING (SANS) STUDY OF THE DIMENSIONS OF POLYSTYRENE FORMED BY FREEZE DRYNG FROM DIUUTE SOLUTIONI}

\section{Hugh R. Brown ${ }^{2}$ and G. D. Wignall}

Polymer molecules in the bulk state or in concentrated solution are highly interpenetrating and hence show considerable entanglement. In dilute solutions below the overlap concentration $c^{*}$, there is little or no interpenetration. It seems reasonable therefore that a noninterpenetrating polymer solid may be made from a dilute solution by rapid drying or quenching techniques. It has been suggested in the literature ${ }^{3}$ that a polymer solid consisting of nonoverlapping collapsed coils can be made by freeze drying, and from fluorescence techniques, evidence has been presented that the resulting material consists of noninterpenetrating molecules. Measurements were undertaken to verify the latter assertion using the SANS technique, which permits a direct measurement of the size of a polymer coil, so that the degree of collapse of the coils is very clear. The data on polystyrene showed that the freeze-dried material was not in the form of collapsed coils but had the normal dimensions characteristic of the bulk polymer. This observation is illustrated in Table 2.2, which gives the results of fitting the data to a throretical scattering function of a Gaussian coil. Values of the differential scattering cross section at $\mathbf{Q}=\mathbf{0}$, derived from the fits, were in the range $50-57 \mathrm{~cm}^{-1}$, to be compared with a value of $53.6 \pm 4 \mathrm{~cm}^{-1}$ calculated from the polymerization indices of the labeled (deuterated) and normal chains. Values of the

Table 2.2. Results from fitting data to a Gaussian coil scattering function.

\begin{tabular}{cccc}
$\begin{array}{c}\text { Initial solution } \\
\text { concentration } \\
(\mathrm{g} / \mathrm{dL})\end{array}$ & $\begin{array}{c}\text { Sample-detector } \\
\text { distance }(\mathrm{m})\end{array}$ & $\begin{array}{c}\text { Cross section } \\
(\mathrm{Q}=0)\end{array}$ & $R_{\mathrm{g}}(\AA)$ \\
\hline 2.50 & 2.66 & $56.3,57.1$ & $59.6,58.2$ \\
& 7.51 & $50.6,53.6$ & $60.8,60.8$ \\
3.33 & 2.66 & $52.0,53.6$ & $57.8,58.8$ \\
& 7.51 & $58.8,52.7$ & $59.7,60.9$ \\
& & $50.2,51.4$ & $60.2,60.8$ \\
\hline
\end{tabular}


chain radius of gyration $\left(R_{8}\right)$ were in the range $58-61 \AA$, compared with a value of $60 \pm 2 \AA$ characteristic of the bulk polymer. The corresponding $R_{8}$ for collapsed coils is $-27 \AA$. Further SANS measurements are planned to explore the apparent contradiction with the data from other techniques. The work is being undertaken in collaboration with IBM to study effects of entanglements on the mechanical properties of the polymer.

1. Summary of paper to be published.

2. IBM Almaden Research Center, San Jose, Calif.

3. J. Jachowicz and H. Morawetz, Macromol. 15,828 (1982).

\section{ON THE DOUBLE-MELTING BEHAVIOR OF POLY(ETHER ETHER KETONE)!}

Youngchul Lee, ${ }^{2}$ Roger S. Porter, ${ }^{3}$ and J. S. Lin

The double-melting behavior of poly(ether ether ketone) (PEEK) has been investigated using differential scanning calorimetry (DSC) and wide- and small-angle $x$-ray scattering The peak temperatures of two melting endotherms have been found to depend on the DSC heating rate. At a high heating rate, two endotherms coalesce into a single endotherm which has been considered to represent complete molting without reorganization. The peak temperature of the coalesced endotherm has been found to be approximately the average of the two former peak temperatures at lower heating rates. The first peak is belicved to be caused by the melting of crystals originally present, whereas the second peak is caused by the melting of crystals which are formed by recrystallization of the material during the course of the DSC measurement. This behavior has also been observed for poly(ethylene terephthalate). In the semicrystalline state the microstructure consists of alternate layers of amorphous and crystalline material with a well-defined periodicity. This period has been measured as a function of temperature using SAXS techniques. The total fraction of material in the crystalline layers has been measured by wide-angle $x$-ray diffraction as a function of temperature. These experiments also show that there is only one crystal structure, regardless of the multiplicity of melting endotherms. The $x$-ray data have been used with the DSC data in the ThompsonGibbs equation to estimate the thermodynamic melting point $\left(384^{\circ} \mathrm{C}\right)$ and the surface free energy $\left(39 \mathrm{erg} / \mathrm{cm}^{2}\right)$ of crystalline PEEK.

1. Jummary of paper: Macromol. 22, 1756 (1989).

2. The University of Tennessee, Knoxville, Tenn.

3. University of Massachusetts, Amherst, Mass.

4. P. J. Holdsworth and A. Tumer-Jones, Polymer 12, 195 (1971).

\section{ORDERED MACROMOLECULAR STRUCTURES IN FERROFLUID MIXTURES'}

\footnotetext{
John B. Hayter, Roger Pynn, ${ }^{2}$ Stuart Charles, ${ }^{3}$ Arne T. Skjellorp, Jill Trewhella, Gerald Stubbs, ${ }^{5}$ and Peler Timmins
}

We have observed ordering of dilute dispersions of spherical and cylindrical macromolecules by suspending them in magnetized 
ferrofluids. The order results from structural correlations between macromolecular and ferrofluid particles rather than from macroscopic magnetostatic effects. We have aligned elongated macromolecules by this technique and have obtained anisotropic neutron diffraction patterns, which reflect the internal structure of the macromolecules. The method provides a new way of orienting suspended macromolecular assemblies which are not amenable to conventional high-field or shear alignment techniques. An example of the efficacy of ferrodispersion as an alignment tool is depicted in Fig. 2.3, which shows blanksubtracted small-angle neutron scattering (SANS) intensity patterns of the rod-like tobacco mosaic virus (TMV) suspended at $6 \%$ volume fraction in water-based ferrofluid in a horizontal field $H=0.5 \mathrm{~T}$. (In these patterns, white corresponds to minimum, black to maximum intensity.) At low q [Fig. 2.3(a)], the intensity observed perpendicular to the field corresponds to scattering from the cross section of the aligned rods. At higher $q$ [Fig. 2.3(b)], the intensity peaks which appear parallel to the field are caused by Bragg scattering from the helical pitch of the aligned virus. The method may have interesting applications for submicron engineering in general, especially when combined with field gradients to manipulate the microstructure.

1. Summary of paper: Phys. Rev. Lett. 62, 1667 (1989).

2. LANSCE, Los Alamos National Laboratory, Los Alamos, N. Mex.
3. University College of North Wales, Bangor, United Kingdom.

4. Institute for Energy Technology, Kjeller, Norway.

5. Vanderbilt University, Nashville, Tenn.

6. Institut Laue-Langevin, Grenoble, France.

ommenos on
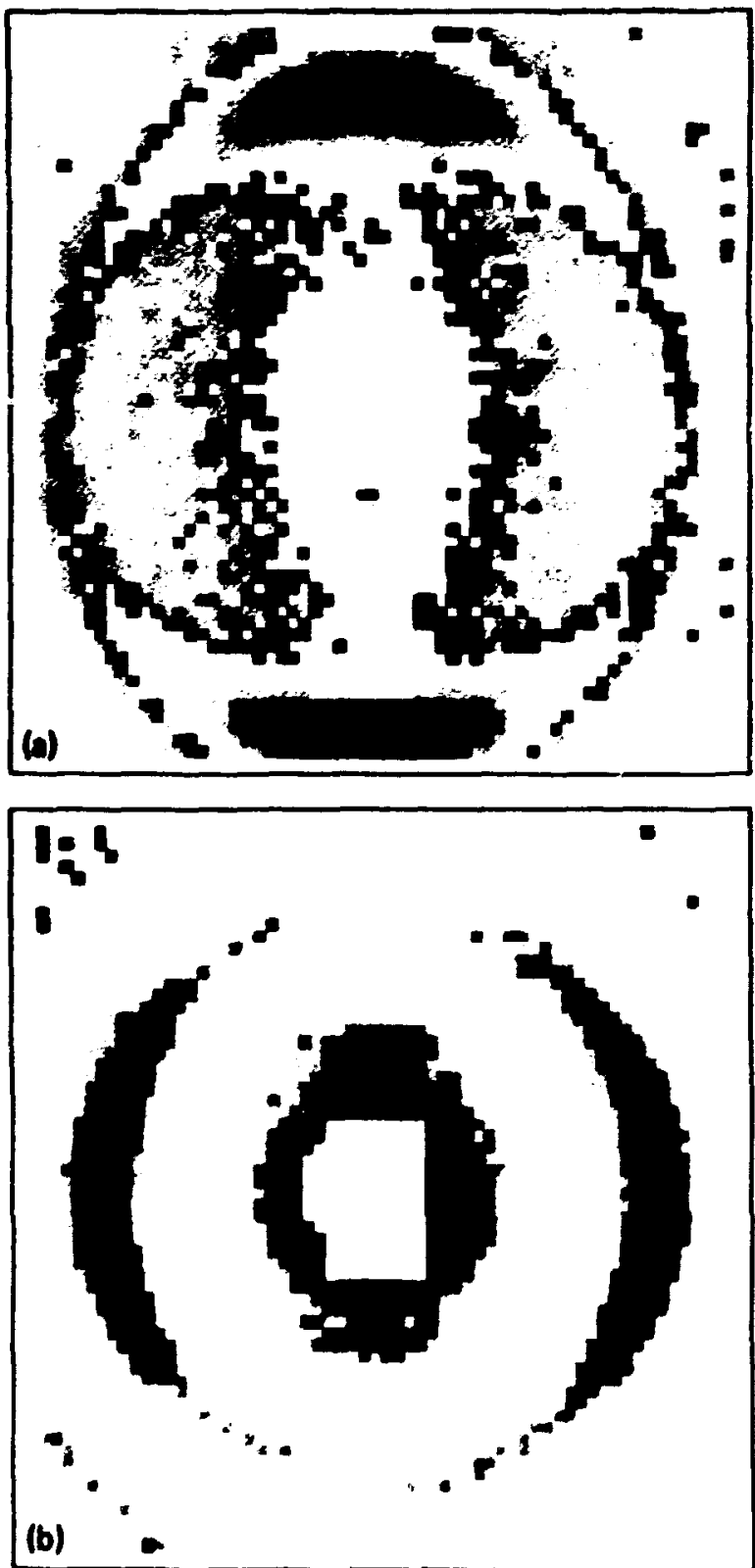

Fig. 2.3. SANS from ferrodispersed TMV. The vertical and horizontal dimensions each correspond to momentum transfer 29 max, where (a) $q_{\max }=0.20 \mathrm{~nm}^{-1}$ and (b) $q_{\max }=3.55 \mathrm{~nm}^{-1}$. 


\section{HYDRODYNAMTC INTERACTIONS IN CONCENTRATED COLLOIDAL DISPERSIONS ${ }^{1}$}

John B. Hayter, leff Penfold, and lan K. Snook ${ }^{3}$

The time-dependent field autocorrelation function $F(q, t)$, measured by dynamic scattering techniques such as photon correlation or neutron spin-echo spectroscopy, is very difficult to interpret because there are virtually no suitable techniques of nonequilibrium statistical mechanics which are both accurate and computationally tractable. In particular, one needs the indirect, solvent-mediated hydrodynamic interactions between the particles. These interactions may be represented by a mobility tensor $\mu$, which is long-ranged, has a manybody character, and is only known in the form of a series expansion. We have developed a practical analytical technique by introducing an effective volume-fraction dependent twobody hydrodynamic tensor,

$\mu_{i j}^{e f f}=\mu_{0}\left(B_{i j}(r)\left(1-\delta_{i j}\right)+1+\sum_{k} A_{i k}(r) \delta_{i j}\right)$.

where the two-body functions $A$ and $B$ may be evaluated from integrals over the pair correlation function. Two methods have been crolved, one via an effective screened Navier-Stokes equation and the other using a volume-fraction dependent effective viscosity. Both approaches give a good description of existing light-scattering and ncutron spin-echo data for either electrostatically or sterically stabilized suspensions.

1. Summary of paper to be published.
2. Rutherford Appleton Laboratory, Chilton, Didcot, England.

3. Royal Melboume Institute of Technology, Melbourne, Australia.

4. C. W. J. Beenakker and P. Mazur, Physica 126A, 349 (1984).

\section{A DIRECT OBSERVATION OF COUNTERION CONDENSATION AROUND CYLINDRICAL MICELLES ${ }^{1}$}

C. F. Wu, S. H. Chen, ${ }^{2}$ L. B. Shih, ${ }^{3}$ and J. S. Lin Small-angle $x$-ray scattering (SAXS) and small-angle neutron scattering (SANS) techniques have been used to determine the size and shape of cylindrical micelles formed by a combshaped copolymer, poly(1-octadecene-comaleic anhydride), abbreviated as PODMA, in aqueous solution. Upon full neutralization by the addition of alkaline hydroxide, PODMA becomes a polyelectrolyte carrying two negative charges per repeating unit. SANS measurements on PODMA in $D_{2} 0$ solutions indicate cylindrical scattering bodies with a radius of 20 $\AA$ and a length of $110 \AA$. SAXS measurements :cere made for a series of $1 \%$ PODMA solutions fully neutralized by $\mathrm{CsOH}$. The linear charge density at the micellar surface was varied by adding an ionic surfactant, sodium dodecyl sulfate. The dimensionless linear charge density parameter, $\xi \equiv \ell_{B} / b$, where $\ell_{B}$ is the Bjetrum length $\left(\ell_{B}=7.15 \AA\right.$ for water solvent at $\left.295 \mathrm{~K}\right)$ and $b$ is the linear spacing between unit charges on the micellar surface, varies from 32.96 to 67.21. Since the electron densities of the micellar core and the aquenus solvent are nearly equal, the SAXS intensity distribution function is a Bessel transform of the Cs counterion distribution around the cylindrical micelles. 
The results obtained from. SAXS intensity distribution analyses are in agreement with the prediction of Manning's condensation theory.

1. Summary of paper. I. Appl. Crystallogr. 21, 853 (1988).

2. Massachusetts Institute of Technology, Cambridge, Mass.

3. S. C. Johnson \& Son, Inc., Raciue, Wis.

4. G. S. Manning Acc. Chem. Re. 12443 (1979).

\section{REAL-TIME STUDY OF REVERSION IN Mn-33 AT.7 Cu}

\section{Stephen Spooner, B. D. Gaulin,' and Y. Moriz'}

In the Cahn-Hilliard formulation for the kinetics of spinodal decomposition, the scattering intensity change as a function of time is $I(Q, t)=I_{0}(Q) e^{R(Q) t},\left(R(Q)=-\left(M / N_{0}\right)(P+A+\right.$ $\left.B Q^{2}\right) Q^{2}$, where $M=$ atomic mobility, $N_{0}=$ atomic density, $A=$ a positive parameter associated with lattice strain, $B=a$ positive parameter associated with compositon gradients, $f=$ the second derivative of the Helmholz free energy, and $Q=$ the scattering vectorl. An effective interdiffusion coefficient is defined as $\vec{D}=M f / N_{0}$. Above the miscibility gap, $f$ is always positive, thereby ensuring monotonic decay of deviations from the equilibrium state, while below the miscibility gap and within the so-called spinodal, $r$ is negative and leads to positive values of $R(Q)$ for $Q$ less than a critical value. A Mn-33 at.\% $\mathrm{Cu}$ alloy was quenched from 800 to $500^{\circ} \mathrm{C}$, annealed for $30 \mathrm{~min}$ to develop an easily measured scattering curve, and "up-quenched" to $575^{\circ} \mathrm{C}$ (several degrees above the miscibility gap). There the scattering curves were recorded at approximately 30 -s intervals for a period of more than $2 \mathrm{~min}$. The results of this reversion treatment are shown on the left of Fig. 2.4. Kinetic analysis of the curves revealed that $R(Q)$ was simply proportional to $Q^{2}$, indicating that the gradient term is negligibly small. Using this result, the set of scattering curves at the right of Fig. 2.4 were calculated. With the assumption that $A / F \times 1, \tilde{D}$ was found to be $40 \times$ $10^{16} \mathrm{~cm}^{2} / \mathrm{s}$, a value almost 2 orders of magnitude smaller than self-diffusion in copper, which suggests some critical slowing near the spinodal.

1. Present address: Physics Department, McMaster University, Hamilton, Ont., Canada.

2. Guest scientist from the Japan Atomic Energy Research Institute, Tokai, Japan.

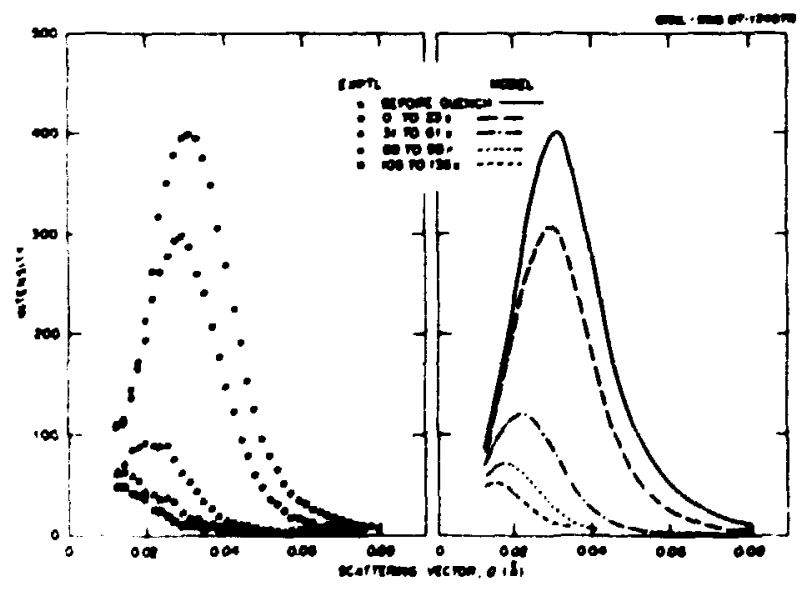

Fig. 2.4. Calculated scattering curves showing that the time dependences arising from "upquenching" are in good agreement with the experimental data. 


\section{PHASE SEPARATION IN A Ni-12.5 AT.\% AI ALLOY AT $575 \mathrm{~K}$}

I. E. Epperson, I. S. Lin, and P. D. Labarbe?

The phase separation in a supersaturated $\mathrm{Ni}-12.5$ at.\% $\mathrm{Al}$ alloy has been investigated by high-angle diffuse scattering, small-angle scattering, transmission electron microscopy, and electrical resistivity methods. High-angle $x$-ray diffuse scattering measured at room temperature on a bulk single crystal homogenized and quenched from $1323 \mathrm{~K}$ showed welldeveloped short-range atomic ordering. Subse quent electrical resistivity measurements on samples heid at various temperatures for a fixed time show a marked peak at about $675 \mathrm{~K}$. Small-angle scattering measurements on samples annealed at $823 \mathrm{~K}$ clearly indicate that, at this temperature, phase separation occurs by a nucleation and growth process. It has been argued that a cluster coagulation model well represents the processes occurring at $823 \mathrm{~K}$. It is, however, not required that the same process be operational at lower temperatures, particularly below the break in the resistivity isochrone. Measurements for annealing times up to $840 \mathrm{~h}$ at $575 \mathrm{~K}$ reveal weak small-angle scaltering that has some of the characteristics of a continuous transformation. However, much longer transformation times at the lower temperature will need to be investigated before the possibility that this is only a kinetic effect associated with slow nucleation of the second phase can be ruled out. III.
2. Laboratoire de Science des Materiaux Vitreus, Montpellier, France.

\section{HARD-SPHERE ANALYSIS OF THE PRECIPITATE STRUCTURE DN Al-Li ALLOYS}

Stephen Spooner, R. Triolo, 'and E. Caponetti ${ }^{1}$

Analyzing small-angle scattering from polydisperse scattering centers at volume fractions greater than $10 \%$ reyuires the modeling of the center size distribution and the center-tocenter structure. A complete solution appears to be formally impossible since the interaction between scattering centers is dependent on center size and the solution of this many-body problem is beyond the practical reach of any known computational scheme. Small-angle scattering curves measured from an alloy undergoing precipitation eventually obey dynamical scaling when the precipitate and matrix reach steady-state compositions (except for interface curvature perturbations which drive the coarsening process) and the precipitate size distribution function, as well as the interprecipitate structure, expands with a single length scale. The scattering curves change by a simple affine shrinkage of the scattering vector siale as structural coarsening progresses. Therefore, it is of general interest to describe the shape of the scaltering curves in the scaling regime. A sludy of aging in a Al-7.8 at.\% Li alloy was undertaken to test an approximation to such a description. The model used assumes scattering from a set of cllipsoidal precipitates with a distribution described by the Perkus-Yevick hard-sphere model of a liquid. The principal parameters of 
the hard-sphe structure function model are the "sphere" vulume fraction and the average inter-"sphere" separation. This liquid-like structure function is rombined with the precipitate form factor whose parameters are the ellipsoidal eccentricity and the mean precipitate size. A good fit is achieved for the data at larger scattering angles, and it appears that decoupling the inter-precipitate structure from the size distribution is an effective approximation. However, the model breaks down at small angles where the calculated scattering cross section corresponds to an unreasonably low system compressibility.

1. Consultant fom the University of Palermo, Palermo, Italy.

\section{HMDRIDE PRECIPITATION IN CATHODICALLY CHARGED ALUMINUM AND NICKEL}

\section{Stephen Spooner, E. Sirois, and H.K. Birnbaum ${ }^{1}$}

Formation of thin hydride plates in polycrystalline and single-crystal samples of aluminum and nickel has been confirmed in smallangie $x$-ray scattering (SAXS) experiments on the $10-\mathrm{m}$ machine. Hydrogen was cathudically charged into thin sheets of each metal at room temperature and then examined in highresolution geometry on the SAXS machine. The grair. size of polycrystalline materials was large enough so that only a small number of grains was sampled in the beam. The result was that both polycrystal and single-crystal samples exhibited highly anisotropic scattering patterns, exemplified in Fig. 2.5. The single-crystal SAXS experiments combined with Laue back-reflection scattering analysis indicate that (211) and (111) are the habit planes for the hydride plates. The SAXS diagrams indicate that the plates may be several micrometers in diameter and several hundred angstroms in thickness. In pure materials, it is difficult to suppress formation of these plates although extended annealing at high temperatures is largely successful in eliminating the anisotropic scattering. It is surprising that transmission electron microscopy (TEM) observation of these plates is so rare. The conditions for TEM sample preparation and observation in the electron beam may be responsible. The presence of these hydrides can significantly alter mechanical properties.

1. University of Illinois, Urbana, 111.

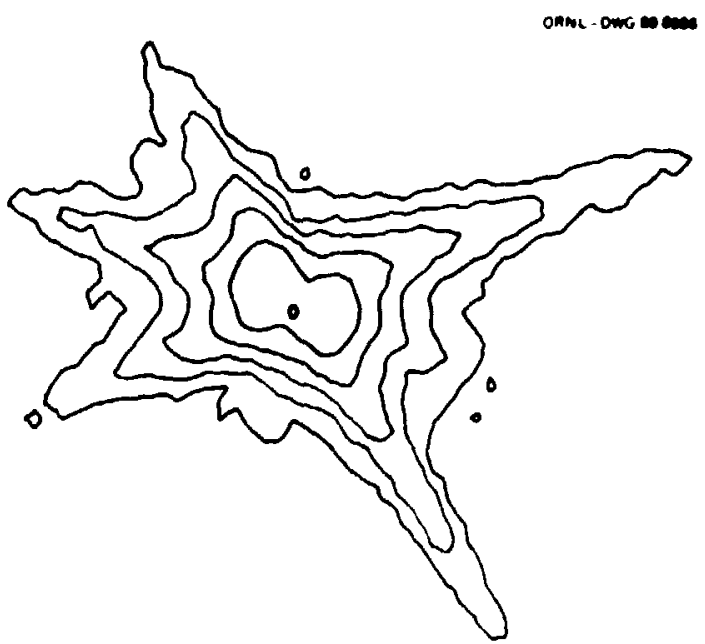

Fig. 2.5. Anisotropic SAXS from a largezrained polycrystalline aluminum sheet which has been cathodically cha, ged with hydrogen. 


\section{INFLUENCE OF SEVERE DEFORMATION ON PRECIPITATION IN AI-Li ALLOYS}

S. Spooner, M. D. Kraitchman, I. Hoyt, D. deFontaine,' and P. Torturelli ${ }^{3}$

An Al-9.8 at.\% Li alloy was made from high-purity components that were melted in an alumina crucible under a purified argon atmosphere. The effect of cold deformation on precipitation was investigated by aging two samples at $110^{\circ} \mathrm{C}$. One sample was reannealed after rolling at $520^{\circ} \mathrm{C}$, auenched, and preparerl for in situ aging. A 3-mm disk was mounted in a small furnace in the 10-m SAXS machine. Scattering from the sample in its starting condition was recorded, and the furnace was heated to $110^{\circ} \mathrm{C}$. Scattering data sets were accumulated during this aging treatment in 1-h intervals up to $11 \mathrm{~h}$. The second sample was rolled to approximately $120 \mu \mathrm{m}$ (a total deformation of $95 \%$ ), and a $3-\mathrm{mm}$ disk was punched from the thinned sheet and treated as in the previous experiment. Fig. 2.6 presents the scattering curves in a plot of $Q^{2} l(Q)$ vs $Q$. The difference in integrated intensity between the initial and 11-h aged state is shown as a shaded area, which is proportional to the increase in the volume fraction of precipitates. The peak in the curve is a rough measure of the inverse mean precipitate size. The results of aging after annealing are shown in (A), and the results of aging after deformation are shown in (B). The volume fraction of precipitate is smaller, and the mean size of the precipitate is smaller in the alloy aged after cold deformation. At all times precipitation is less advanced in the sample which begins aging in the deformed state. Recent evidence has shown that a long-range-ordered state appears in quenched $\mathrm{Al}-\mathrm{Li}$ alloys before precipitation begins. It is suggested that either deformation destroys the ordered state or introduces sufficient dislocation density, either of which Ifects can suppress precipitate formation. Calif.

1. University of California, Berkeley,

2. Washington State University, Pullman, Wash.

3. Metals and Ceramics Division, ORNL.

4. B. J. Shaiu, H. T. Li, and Haydn Chen, in Proceedings of the Aluminum-Lithium Conference, March 1989, Charlottesville, Va. (in press).

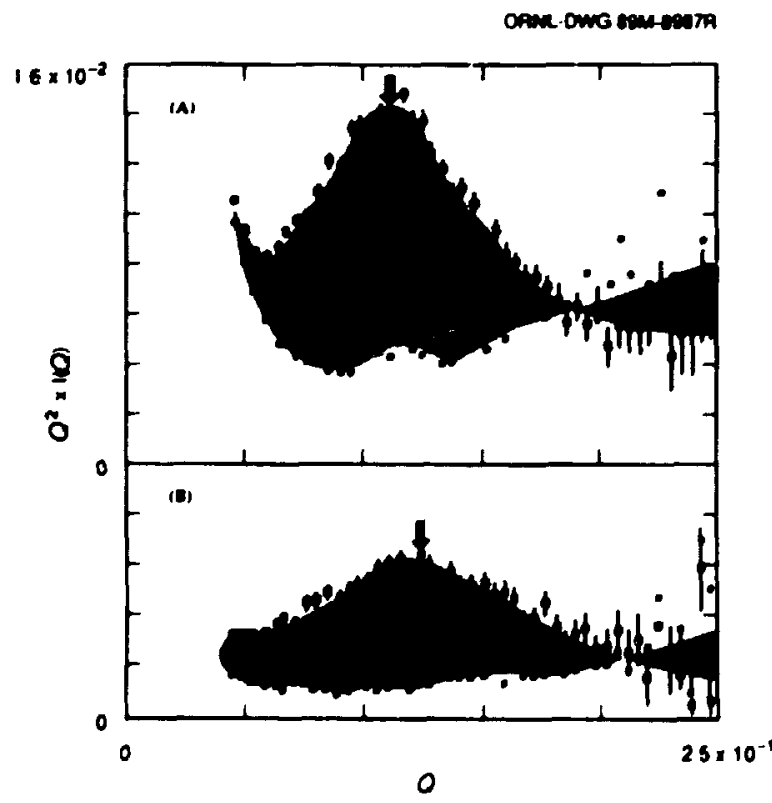

Fig. 2.6. $Q I(Q)$ versus $Q$ for the initial and final state after 11 -h aging at $110^{\circ} \mathrm{C}$ in anncaled (A) and rolled (B) matcrial, showing that the volume fraction and average precipilate size are smaller in the rolled material. 
THE STUDY OF FRACTAL GEOMETRY ON SUBMICRON LENGTH SCALES BY SMALL-ANGLE SCATTERING ${ }^{\prime}$

$$
\text { Po-Zen Wong and J. S. Lin }
$$

Fractal is a term introduced by Mandelbrot to describe the geometry of objects that look the same upon change of length scales. Such objects are ubiquitous in nature. Recent studies have shown that internal surfaces of porous geological materials, such as sedimentary rocks and lignite coals, can be described by fractals down to atomic length scales. Two major types of fractals are self-similar fractals and selfaffine fractals. Self-similar fractals are isotropic; they look the same when the length scales along all spatial directions are changed by the same factor. Self-affine fractals are anisotropic; they look the same only if the length scales along different directions are changed by different factors.

Small-angle $x$-ray and neutron scattering techniques were used to determine the fractal dimensions $D$ of sandstones and shales. All the data exhibit slopes between -3 and -4 on a $\log$ log plot (scattering cross section vs scattering vector), which implies that pore surfaces in sandstones are fractals with $D$ between 2 and 3, consistent with studies by the microscopy technique and the adsorption method.

Small-angle scattering experiments can be used to distinguish volume (mass) fractals from surface (boundary) fractals. Mass fractals exhibit $I(q) \sim 1 / q D$ with $D$ being less than 3, while surface fractals have $I(q)-1 / q(6-D)$ where the exponent $(6-D)$ is greater than 3 .

1. Summary of paper: Math. Geol. 20, 655 (1988).

2. University of Massachusetts, Amherst, Mass.

\section{NEUTRON DIFFRACTION}

\section{ATOM-PAIR CORRELATIONS IN Fe-13.5\% VI}

I. W. Cable, H. R. Child, and Y. Nakai ${ }^{2}$

In this paper, we report a neutron study of the stomic short-range order (ASRO) in an Ferich, Fe-V alloy. The nuclear and magnetic diffuse scattering intensities for this alloy are comparable and must be separated fo: the measurement of either. The magnetic scattering was extinguished by the application of a saturating magnetic field parallel to the scattering vector, and the remaining nuclear diffuse intensity was then measured along the [001], [110], and [111] directions. The observed cross sections exhibit considerable structure with the most intense diffuse peaks occurring at (100) and (111) for this bcc lattice. This observation indicates an anticorrelation for first-neighbor atom pairs and a tendency toward the $\beta-\mathrm{CuZn}$ type of ordering. The data are well described by either Warren-Cowley 
ASRO parameters or Krivoglaz-Clapp-Moss pair potentials with only a slight dependence of the fitted parameters on the number of parameters included in the fit. However, at least eight neighboring-shell parameters are required to reproduce the sharpness of the diffuse peak at (111) and the double peak observed near $(1 / 2,1 / 2,1 / 2)$.

To place the fitted pair-potential parameters on an energy scale, an effective temperature must be assigned to the quenchedin, high-temperature state of the alloy. For this sample, which was quenched from about $1270 \mathrm{~K}$, we estimate an effective temperature of about $900 \mathrm{~K}$ or $K_{B} T-80 \mathrm{meV}$. The first three observed pair potentials then have energies corresponding to 34,22 , and $-4 \mathrm{meV}$, to be compared with the calculated ${ }^{3}$ values of 46,10 , and $2 \mathrm{meV}$. We consider this to be reasonable agreement, especially in view of the fact that the experimental results correspond to the high-temperature limit while the calculations are carried out in the $T=0$ limit.

1. Summary of paper: Physica B 156, 50 (1989).

2. Guest scientist from Osaka University, Osaka, Japan.

3. M. Hennion, I. Phys. F 13, 2351 (1983).

\section{DIFFUSE NEUTRON SCATTERING FROM A

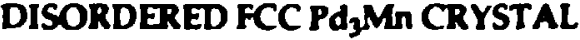

H. R. Child, E. Curewitz, and I. W. Cable

$\mathrm{Pd}_{3} \mathrm{Mn}$ crystallizes in the fcc structure in which the Pd and $\mathrm{Mn}$ atoms occupy at random the vertices and the face centers of the cube. The disordered and ordered atomic arrange ments show differences in the long range mag netic order. ${ }^{2,3}$ Neutron diffuse scattering from a disordered single crystal was measured at temperatures from 296 to $9 \mathrm{~K}$. At $296 \mathrm{~K}$ we observed weak Bragg peaks at the superlattice positions (001) and (110), indicating some degree of atomic long-range order. In addition, diffuse peaks were centered at (110) $\pm\left(\begin{array}{lll}0 & 0 & 0.12\end{array}\right)$ and (110) $\pm\left(\begin{array}{ll}0 & 0\end{array} 0.12\right)$, indicating complicated shortrange atomic order. Both of these features grew stronger as the temperature was lowered to $9 \mathrm{~K}$. In addition, magnetic Bragg peaks appeared below $106 \mathrm{~K}$, located at the center of the diffuse peaks. The width of these peaks indicates ordered regions of about 8 unit cells. A successful analysis of these data has not yet been achieved.

1. Guest scientist from the Atomic Energy Commission, Nuclear Research Center-Negev, Beer-Sheva, Israel.

2. J. W. Cable et al., Phys. Rev. 128, 2118 (1962).

3. B. D. Rainford, J. Magn. and Magn. Mater. 14, 197 (1979).

\section{THERMAL NEUTRON SCATTERING LENGTHS AND CAPTURE BY EVEN CALCIUM ISOTOPES}

S. Raman, ${ }^{2}$ S. Kahane, ${ }^{3}$ R. M. Moon,

I. A. Fermandez-Baca, I. L. Zarestky, 4

I. E. Lynn, and I. W. Richardson, Ir. ${ }^{6}$

Neutron diffraction patterns have been measured for isotopically enriched powder samples of calcite at the Oak Ridge Research 
Reactor and at the Intense Pulsed Neutror. Source at Argonne National Laboratory. Greatly enhanced precision over previous work has been achieved for the scattering lengths of ${ }^{40} \mathrm{Ca}$ and ${ }^{44} \mathrm{Ca}$, while the results for ${ }^{42} \mathrm{Ca},{ }^{43} \mathrm{Ca}$, and ${ }^{48} \mathrm{Ca}$ represent new data. These results, given in Table 2.3, have been used in an analysis of primary electric-dipole gamma rays from

Table 2.3. Nuclear coherent scattering lengths for $\mathrm{Ca}$ isotopes.

\begin{tabular}{cc}
\hline Isotope & $b(\mathrm{fm})$ \\
\hline${ }^{40} \mathrm{Ca}$ & $4.73 \pm 0.05$ \\
${ }^{42} \mathrm{Ca}$ & $3.36 \pm 0.10$ \\
${ }^{43 \mathrm{Ca}}$ & $-1.56 \pm 0.09$ \\
${ }^{4} \mathrm{Ca}$ & $1.42 \pm 0.06$ \\
${ }^{48 \mathrm{Ca}}$ & $0.36 ; 0.09$ \\
\hline
\end{tabular}

thermal neutron capture by the even isotopes. In three cases $\left({ }^{40} \mathrm{Ca},{ }^{42} \mathrm{Ca}\right.$, and $\left.{ }^{48} \mathrm{Ca}\right)$, the estimates from a spherical optical model formulation of the direct-capture mechanism are in agreement with experimental cross sections. For ${ }^{44} \mathrm{Ca}$, the theory overestimates the maasured cross sections by about $60 \%$; this discrepancy can be explained by considering modifications to the th-ory due to collective vibrations of the ${ }^{4} \mathrm{Ca}$ case.

1. Summary of paper: Physical Review C (in press).
2. Physics Division, ORNL.

3. Nuclear Research Centre-Negev, BeerSheva, Israel.

4. Guest scientist from Ames Laboratory, Ames, lowa.

5. Atomic Energy Research Establishment, Harwell, England. III.

6. Argonne National Laboratory, Argonne,

\section{INELASTIC NEUTRON SCATTERING}

\section{A NEUTRON SCATTERING STUDY OF THE IMPULSE APPROXIMATION IN SINGLE-CRYSTAL BERYULIUM}
D. F. McMorrow, ${ }^{1}$ R. A. Cowley, ${ }^{1}$
R. M. Nicklow, P. W. Mitchell,2
A. D. Taylor, ${ }^{3}$ and M. Mostoller

The inelastic scattering of highenergy neutrons from a single crystal of beryllium has been measured in order to test the validity of the impulse approximation. Separate measurements were performed, all with the sample at room temperature with the wave-vector transfer along the (100), (110), and (001) crystallographic directions using the time-offlight spectrometer HET at ISIS, the spallation neutron source at the Rutherford Appleton Laboratory, England. For wave-vector transfers $\geq 35 A^{-1}$, corresponding to an incident neutron energy of $-1 \mathrm{eV}$, we find that the scattering is centered precisely at the recoil energy given by the impulse approximation (Fig. 2.7). Also, in this regime the energy width is independent of the orientation of the wave-vector transfer with respect to the crystal axes and has a value consistent with that expected from a harmonic 


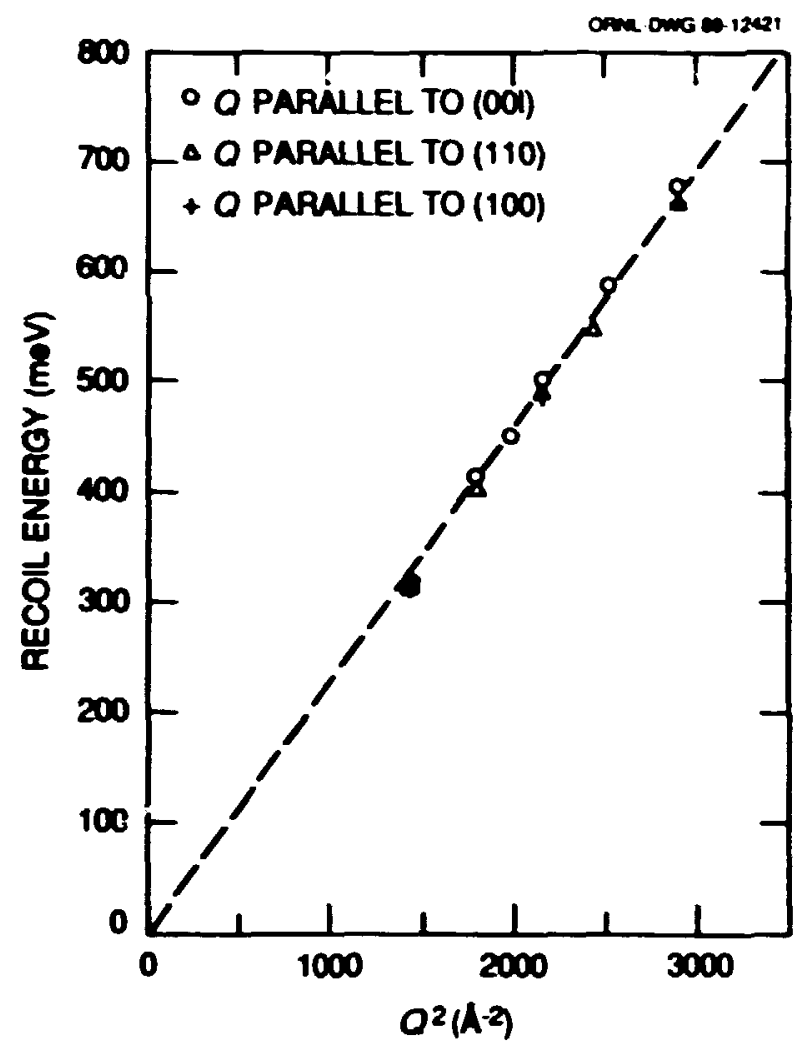

Fig. 2.7. The fitted peak position of the neutron recoil scattering from Be plotted against the square of the wave-vector transfer. The straight line represents the locus of the center of the recoil scattering calculated from the impulse approximation. Errors in the fitted peak positions are comparable to the size of the symbols.

model for the lattice vibrations of beryllium. We find that the experimental criterion for the validity of the impulse approximation is ir. agreement with a numerical calculation of th: asymptotic behavior of the exact incoherent neutron dynamic structure factor, based on the phonon density of states.

1. University of Edinburgh, Edinburgh, Scotland.
2. Schuster Laboratory, University of Manchester, Manchester, England.

3. Rutherford Appleton Laboratory, Chilton, Didcot, England.

\section{NEUTRON SCATTERING STUDIES OF THE MARTENSITC TRANSFORMATION IN SODIUM METAL}

\author{
R. Berliner,' H. G. Smith, \\ I. R. D. Copley. ${ }^{2}$ and I. Trioisonno ${ }^{3}$
}

Neutron powder diffraction experiments on polycrystalline sodium metal have shown that it undergoes a martensitic phase transformation at about $35 \mathrm{~K}$ from the bcc structure to a new hexagonal form. The structure of the new phase appears to be the same as the structure adopted by lithium metal at low temperatures (i.e., the 9R samarium-type structure).

We have measured the phonon dispersion curve for the $\Sigma$ branch as a function of temperature and the structure of the transformed phase at $30 \mathrm{~K}$ with a single crystal, using the triple-axis spectrometer BT9 at the National Institute of Standards and Technology. No sub stantial softening of the phonon branch was observed. The crystallography of the transformed phase is somewhat different than the one observed earlier in lithium metal. Studies of the (10 2) reflections showed that the new phase contains both a $9 R$ (samarium-type) structure and a substantial fraction of an hcp phase. Preliminary investigations of both phases show the same kind of shifting and broadening that was observed in lithium. Careful monitoring of the diffraction pattem as 
the crystal warmed showed that there was a substantial temperature range, starting at $55 \mathrm{~K}$, over which the $9 R$ phase transformed to bec, leaving the hcp phase intact. Upon further warming the hcp phase also reverted to bcc, and the system remained a single crystal in its original orientation up to a temperature in the range $250-300 \mathrm{~K}$, where it broke into several pieces, probably caused by a too rapid warming rate in this latter region.

1. University of Missouri, Coiumbia, Mo.

2. National Institute of Standards and Technology, Gaithersburg, Md.

3. John Carroll University, Cleveland, Tenn.

\section{LATTICE VIBRATIONS OF COPPER IN $\mathrm{YB}_{2} \mathrm{Cu}_{3} \mathrm{O}_{7}$}

H. A. Mook, I. A. Harocy, and N. W. Hill'

It is generally thought that the standard electron-phonon interaction is too small to account for superconductivity at $90 \mathrm{~K}$ unless phonons are at much higher energies than are expected. Neutron measurements of the phonon densities of states suggest that phonons extend to energies near $100 \mathrm{meV}$. These measurements, however, cannot determine which atom is contributing to a particular energy. One would assume that the high-energy modes in the density-of-states measurement correspond to the oxygen modes; however, that had not been established. In fact, lkeda et al. ${ }^{2}$ purformed a high-energy scattering experiment using neutron energies above $1 \mathrm{cV}$, which they claimed signified anomalously high $\mathrm{Cu}$ vibration frequencies in $\mathrm{YBa}_{2} \mathrm{Cu}_{3} \mathrm{O}$. They relied on a model to separate the frequencies of the $\mathrm{Cu}$ and $O$ atoms that resulted in an effective temperature of $750 \mathrm{~K}$ for the $\mathrm{Cu}$ in the $\mathrm{YBa}_{2} \mathrm{Cu}_{3} \mathrm{O}_{7}$ compound compared with $319 \mathrm{~K}$ for $\mathrm{Cu}$ metal. It was then suggested that this very high effective temperature for $\mathrm{Cu}$ in the compound may result in high superconducting transition temperatures.

If one has a neutron source with sufficient resolution and intensity, the lattice frequencies of individual atoms can be studied by observing the width of a narrow resonance absorption line. Cu has a resonance absorption line at about $230 \mathrm{eV}$ that has a natural width of $0.300 \mathrm{eV}$. Most of the observed width of this line thus results from Doppler broadening or lattice vibrations of the $\mathrm{Cu}$ atoms. Figure $\mathbf{2 . 8}$

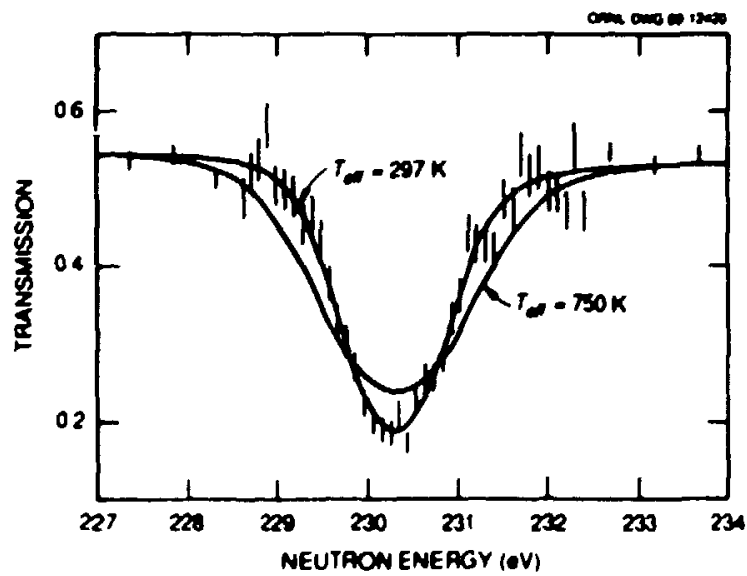

Fig. 2.8. Neutron transmission of $\mathrm{YBa}_{2} \mathrm{Cu}_{3} \mathrm{O}$, as function of neutron energy near the $230-\mathrm{eV}$ resonance of copper. The linewidth depends on the thermal vibration spectra of the Cu atoms. The solid lines are calculated for two different effective temperatures for the Cu atoms. 
shows a measurement of this resonance mode or. $\mathrm{YBa}_{2} \mathrm{Cu}_{3} \mathrm{O}_{7}$ at the the ORNL ORELA neutron source. The sample was in the form of a powder and had excellent superconducting properties. Magnetic measurements showed bulk superconductivity with a sharp transition at $92 \mathrm{~K}$. The energy resolution of the neutron measurement was $0.13 \mathrm{eV}$. The solid line in Fig. 2.8 that gives a good least-squares fit in the dald results in an effective temperature for the $\mathrm{Cu}$ of $297 \pm 20 \mathrm{~K}$. This observation is very similar to that for Cu metal, showing unambiguously that the $\mathrm{Cu}$ in $\mathrm{YBa}_{2} \mathrm{Cu}_{3} \mathrm{O}_{7}$ has a vibration spectra no more energetic than that for $\mathrm{Cu}$ metal. Also shown is the calculated curve for an effective temperature of $750 \mathrm{~K}$. This curve clearly does not agree with the data; therefore, one must look elsewhere for the mechanism for hightemperature superconductivity in $\mathrm{YBa}_{2} \mathrm{Cu}_{3} \mathrm{O}_{3}$.

1. Engineering Physics and Mathematics Division, ORNL.

2. Instrumentation and Controls Division, ORNL.

3. S. Ikeda et al., Phys. Lett. A 134, 191 (1988).

\section{MAGNETIC DYNAMACS OF} $\mathrm{La}_{2} \mathrm{CuO}_{4}$ AND $\mathrm{La}_{2-x} \mathrm{Ba} \mathrm{a}_{x} \mathrm{CuO}$

G. Aeppli, ${ }^{1}$ S. M. Hayden, ${ }^{2}$ H. A. Mook, Z. Fisk, ${ }^{3}$ S. -W. Cheong, ${ }^{3} D$. Rytz, I. P. Remeika,' G. P. Espinosa,' and A. S. Cooper'

$\mathrm{La}_{2-x} \mathrm{Ba}_{x} \mathrm{CuO}_{4}$ has been extensiv ely studied by a number of techniques including neutron scattering; however, because of restrictions on the incident beam energies and resolutions, the magnetic excitations have not been characterized properly. By using the hot source tripleaxis spectro.neter at the Institut LaueLangevin, we have been able to perform neutron scattering experiments that resolve the antiferromagnetic spin-wave excitations that are very closely spaced in $q$. The steepness of the branches requires a small resolution function (shown in the insert of Fig. 2.9 by the shaded ellipse) to make it possible to separate the branches in a constant energy scan. The data in Fig. 2.9 show a constant energy scan at $100 \mathrm{meV}$ through the dispersion surface. The solid line is a least-squares fit to the data that takes into account the spectrometer resolution and results in a spin-wave velocity of $0.78 \pm 0.04 \mathrm{eV}-\mathrm{A}$ for $293 \mathrm{~K}$. At $5 \mathrm{~K}$ the velocity is larger $(0.85 \pm 0.03$

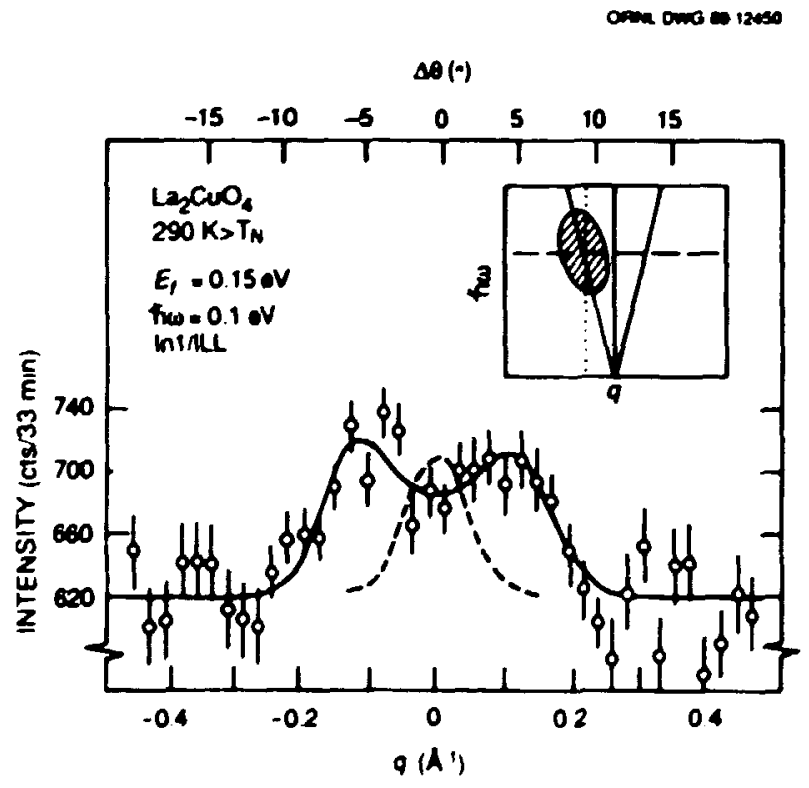

Fig. 2.9 Constant energy scan at $100 \mathrm{meV}$ showing neutron intensity scattered by $\mathrm{La}_{2} \mathrm{CuO}_{4}$. Two antiferro:nagnetic spin waves are ubserved, closely sfaced in $\mathbf{q}$. 
eV-A), corresponding to a Heisenberg nearneighbor exchange of $0.16 \mathrm{cV}$. Analysis of the spin-wave intensities at a number of energy transfers showed that the measurements are well explained by conventional spin-wave theory, and it is not necessary to introduce novel quantum effects to understand the magnetic dynamics.

Measurements on a sample of $\mathrm{La}_{1.95} \mathrm{Ba}_{20.05} \mathrm{CuO}_{4}$ were also made. This sample has sufficient $\mathrm{Ba}$ that the material is a reentrant spin glass with a maximum in the magnetic order parameter found near $30 \mathrm{~K}$. Constant energy scans for this material were much wider than for the material without $\mathrm{Ba}$, showing either considerable spin-wave lifetime effects or a greatlv reduced exchange constant or both. Two-axis measurements showed a magnetic correlation length of about $50 \AA$ i,or the Ba-doped material so that the spin-wave broadening is apparent at wave vectors large compared with the inverse correlation length.

1. AT\&T Bell Laboratories, Murray Hill, N. J.

2. Institut Laue-Langevin, Grenoble, France.

3. Los Alamos National Laboratory, Los Alamos, N. Mex.

4. Hughes Research Laboratory, Malibu, Calif.

\section{MAGNETIC EXCTTATIONS IN THULIUM}

\section{A. Fernandez-Baca, R. M. Nicklow, and I. I. Rhyne'}

The study of magnetic excitations in the heavy rare-earth elements is of considerable interest because it contributes to understanding the magnetic interactions that give rise to the complicated magnetic structures found in these elements. We have performed inelastic neutron scattering measurements on a single crystal of Im at wave vectors $Q=(1,1, \zeta)$ and $(0,0,2+\zeta)(\zeta=$ $0, \ldots, 1)$. Most of the measurements have been made at $T=5 \mathrm{~K}$, where $T \mathrm{~m}$ exhibits a sevenlayer ferrimagnetic-antiphase-domain structure $^{2}$ (four moments up, parallel to the c-axis, followed by three moments down). At this temperature, the excitation spectra IFig. 2.10(a)] consist of three peaks. The lower energy excitations have been identified as originating from magneto-vibrational scattering from the TA phonon Isce dispersion relation in Fig. 2.10(b)), while the higher energy excitation is truly magnetic and exhibits only a weak dispersion (between 8.3 and $9.6 \mathrm{meV}$ ). These excitations persist at $T=50 \mathrm{~K}$, a temper. ature at which the system exhibits a $c$-axis sinusoidally modulated structure, ${ }^{2}$ with significant softening and broadening of the magnetic mode. The magncto-vibrational scattering vanishes above the Néel temperature $\left(T_{N}=58 \mathrm{~K}\right)$ while the magnetic mode persists at least up to $T=70 \mathrm{~K}$. 

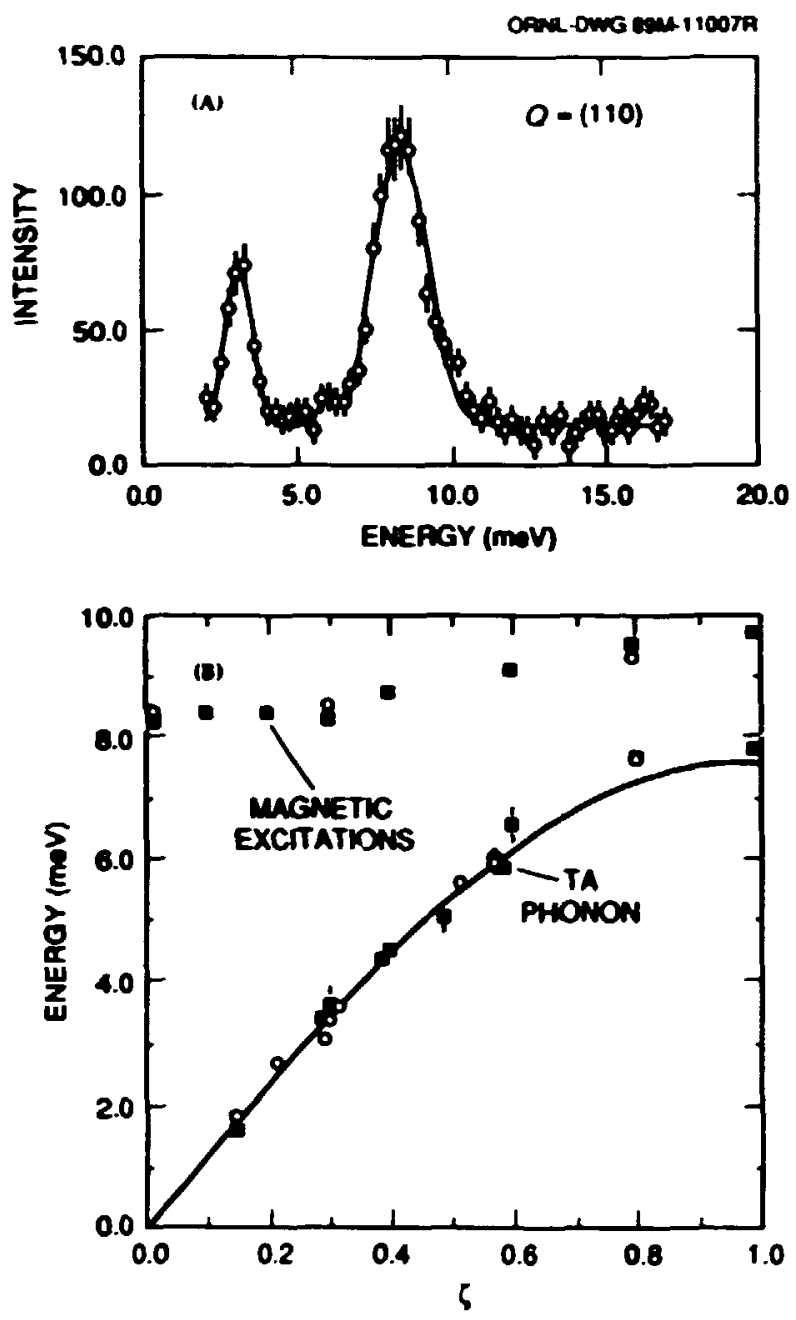

Fig. 2.10. (A) Constant-Q $\operatorname{scan} \mid Q=(1,1,0)]$ for $\mathrm{Tm}$ at $T=5 \mathrm{~K}$. The solid line is the fit to three Gaussians. The lower energy excitations have been identified as magneto-vibrational scattering, while the higher energy peak is a truly magnetic excitation. (B) Dispersion relation for $\mathrm{Tm}$ along the $(1,1, \zeta)$ (open circles) and $(0,0,2+\zeta)$ (full squares) directions. The solid line is the dispersion relation of the TA phonon.

These results suggest that the Hamiltonian in this system is dominated by a strong crystal field term and that the exchange interaction is relatively weak and plays the rolc of a small perturbation. We found no evidence of the excitation at $15 \mathrm{meV}$ reported by McEwen and Steigenberger. ${ }^{3}$

1. National Institute of Standards and Technology, Gaithersburg, Md.

2. W. C. Koehler et al., Phys. Reo. 126, 1672 (1962).

3. K. A. McEwen and U. Steigenberger, Journal de Physique (in press).

\section{SPIN DYNAMICS OF GA AT HIGH TEMPERATURES ${ }^{1}$}

\section{W. Cable and R. M. Nicklow}

The magnetic excitation spectra of Gd were measured by neutron inelastic scattering over the entire Brillouin zone in the $<110\rangle$ direction at temperatures from 250 to $850 \mathrm{~K}\left(T_{C}=293 \mathrm{~K}\right)$. The data were fitted to a damped harmonic oscillator (DHO) form for the spectral weight function (Fig. 2.11) and were placed on an absolute cross-section basis. Wave-vector dependent susceptibilities were obtained by integration over energy. They clearly show the presence of static spin correistions even at $T=$ $850 \mathrm{~K}$. At $T \geq T_{c}$, we observe a crossover from spin diffusive motion at small $q$ to spin-wave behavior at large $q$. The wave vector $q_{c}$ at which this crossover occurs is determined by the ratio of the damping parameter to the second moment $\left\langle\omega^{2}\right\rangle$ of the frequency distribution. Surprisingly, $\left\langle\omega^{2}\right\rangle$ remains inoependent of temperature above $T_{c}$, and the temperature dependence of $q_{c}$ is therefore determined by a gradual change in the damping. This results in a very weak $q_{c}$-vs- $T$ 


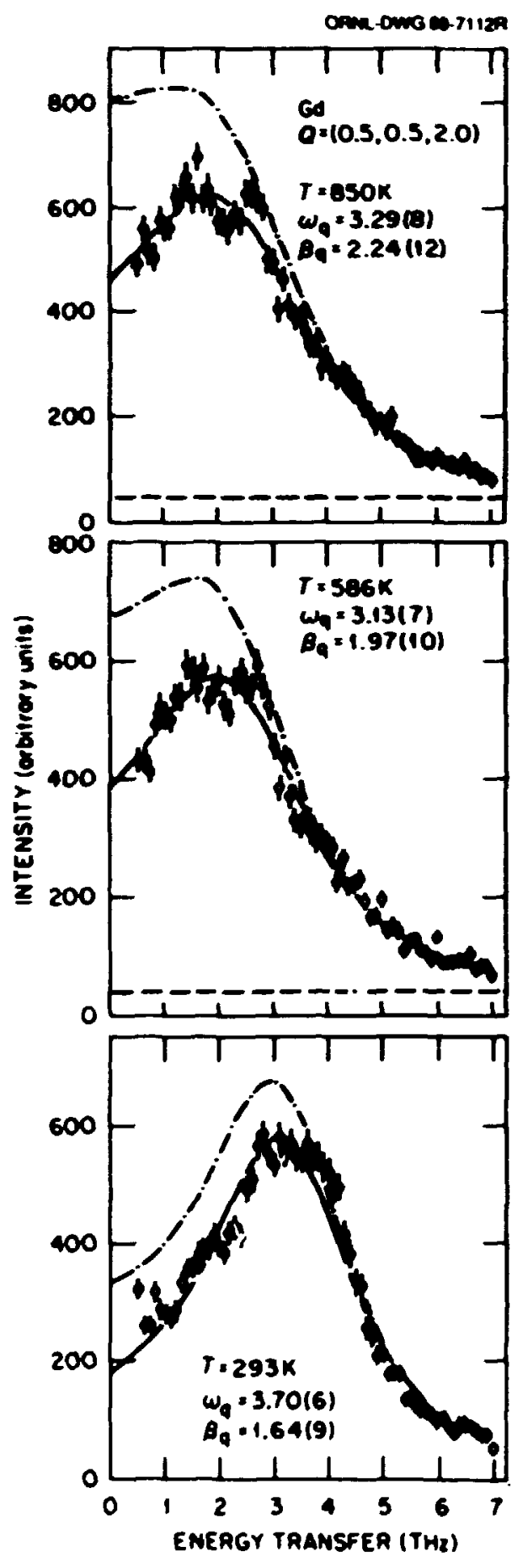

Fig. 2.11. Magnetic excitation spectra of $\mathrm{Cd}$ at $T \geq T_{c}$ and $Q=(0.5, C .5,2.0)$. The solid curves are fitted to a DHO function, and the dashed lines indicate the fitted background levels (this is zero at $T=293 \mathrm{~K}$ ). The dash-dot curves represent the fitted DHO curves after correction for $g(E)$, the higher order wavelength contamination in the monitor response. dependence that is not consistent with a magnetic short-range-order interpretation for the existence of spin waves above $T_{C}$.

\section{Summary of paper: Physical Review B (in press).}

\section{SPIN DYNAMICS OF Gd IN AN APPUIED MAGNETC FIELD}

\section{J. W. Cable and R. M. Nicklow}

In this paper, we report a neutron study of the effect of an applied magnetic field on the spin dynamics of the Heisenberg ferromagnet, Gd. In such a system the spin at a given lattice site experiences an effective molecular field comprised of the near-neighbor exchange field plus the applied field. At temperatures near $T_{c}$, even small applied fields will dramatically alter both the order parameter $\left\langle S^{z}\right\rangle$ and the spin-pair correlations $\left\langle S_{i}^{\alpha} S_{j}^{\alpha}\right\rangle-\left\langle S_{j}^{\alpha}\right\rangle^{2}$. In the present experiment, we investigate the field dependence of the inelastic magnetic scattering near $T_{C}\left(T_{C}=293 \mathrm{~K}\right)$.

Typical results are given in Fig. 2.12, which shows energy spectra for the two spin states at $Q=\langle 0,0,2-\zeta\rangle$ for $\zeta=0.2$ and 0.3 under various conditions of $H$ and $T$. These spectra show an increase in the spin-wave energies with both $\zeta$ and the magnetization s. At these elevated temperatures, the spin-wave energies are proportional to the magnetization, as in linearspin-wave theory, only at small $\zeta$. At larger wave vectors, these energies shift upward because of local correlations of the spin 


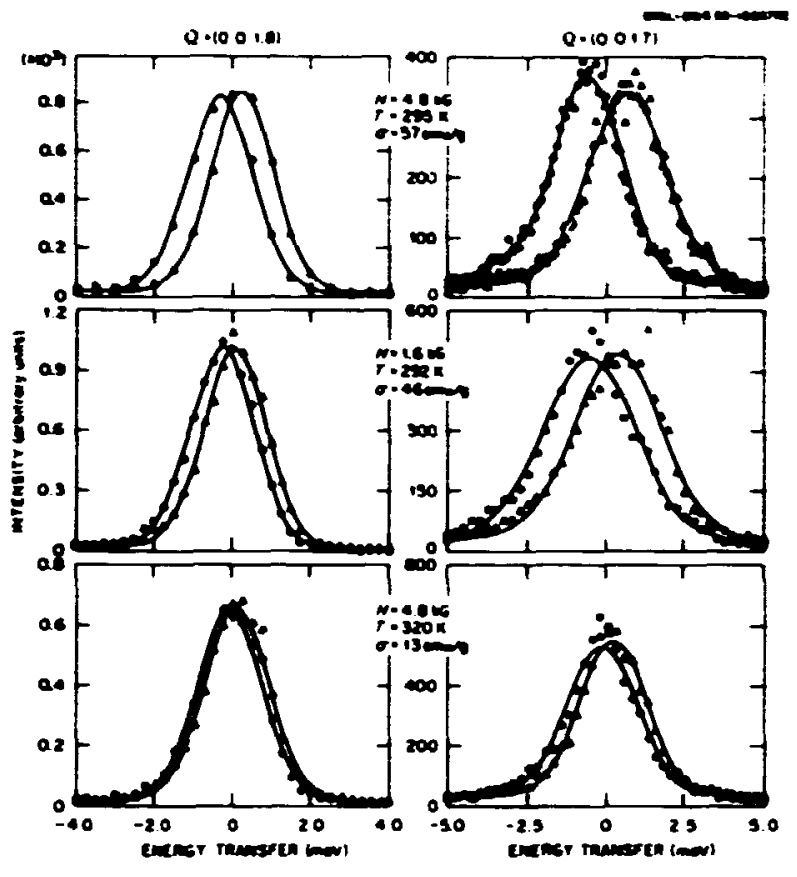

Fig. 2.12. Polarized-neutron, magnetic excitation spectra of $\mathrm{Gd}$ in forbidden magnon geometry and $H \| Q \mathbf{Q}$. The open triangles correspond to flipper-off data and spin-wavecreation spectra, while the open circles represent flipper-on data and spin-waveannihilation spectra.

fluctuations away from long-range order and are no longer directly proportional to the magnetization. The observed behavior is consistent with the correlation theory treatment by Lindgard. ${ }^{2}$ Comparison of the spin-wave spectra obtained in an applied field with previous zero field data shows that the spectra are the same for $H$ and $T$ conditions that produce the same magnetizations, whether induced or spontancous.

1. Summary of paper to be published.

2. P. A. Lindgard, Phys. Rev. B. 30, 2729 (1984).

\section{POLARIZED NEUTRON STUDIES OF FORBIDDEN MAGNON SCATTERNG IN GADOLINIUM'}

\section{W. Cable and R. M. Nicklow}

For a single domain ferromagnet at low temperatures, magnon creation is forbidden for incident neutrons polarized paralled to both $\mathbf{Q}$ and the magnetization. Measuremen: of the evolution of this forbidden scattering at elevated temperatures (as the spin system develops regions of magnetic short-range order misoriented relative to the net magnetization) provides information about how the spin system disorders.

Triple-axis, constant-E scans along $\mathbf{Q}=0,0, \xi$ at temperatures near and above $T_{C}$ for the Heisenberg ferromagnet $\mathrm{Cd}\left(T_{c}=293 \mathrm{~K}\right)$ show that forbidden scattering does not occur for $T \leq$ $T_{c}$ but develops progressively at $T>T_{C}$. However, peaks in constant-E scans may arise even from diffusive spin fluctuations so that constant- $\mathbf{Q}$ measurements are required to determine the presence of underdamped spin waves. We therefore carried out a series of constant-Q measurements near $T_{C}$. No forbidden magnon peaks were observed at finite energy transfer out to $Q=(0,0,1.4)$ in the temperature range from 270 to $300 \mathrm{~K}$.

The existence of forbidden magnons is presumably associated with the presence of magnetically short-range-ordered regions that are misoriented relative to the sample magnetization and that exist over large enough correlation lengths and time scales for the magnons to propagate. In that context, the present results 
may be reconciled with the earlier observations of forbidden magnons in itinerant electron systems since magnetic short-range order is expected to be much less pronounced in Heisenberg systems such as $\mathrm{Gd}$.

1. Summary of paper to be published.

\section{SFIN DYNAMICS OF AMORPHOUS Fegeni $\mathrm{Ni}_{\mathbf{1}} \mathbf{T}_{10}$}

\section{A. Fernandez-Baca, M. Hennion,i}

B. Hennion, I. J. Rhyme, ${ }^{2}$ and G. E. Fish ${ }^{3}$

Reentrant spin glasses (RSG) are magnetic systems that behave like ferromagnets below a transition temperature but at lower temperatures exhibit spin freezing that results from magnetic "frustration." Some theories predict that the ferromagnetism is completely destroyed in this low-temperature phase, while others have suggested that there should be a coexistence of ferromagnetism and spinglass-like order. We have performed a highresolution inelastic scattering experiment in order to determine if spin-wave (SW) propagating excitations exist at low temperatures in the amorphous RSG $\mathrm{Fe}_{g g} \mathrm{Ni}_{1} \mathrm{Zr}_{10}$ and, if so, what are the temperature and wave vector dependence of these excitations. We have observed (Fig. 2.13) long-wavelength SW excitations at all temperatures below $T_{c}=245 \mathrm{~K}$ under study $(23 \mathrm{~K}<T<150 \mathrm{~K})$. As in a conventional ferromagnet these excitations follow a quadratic dispersion relation. Unlike a

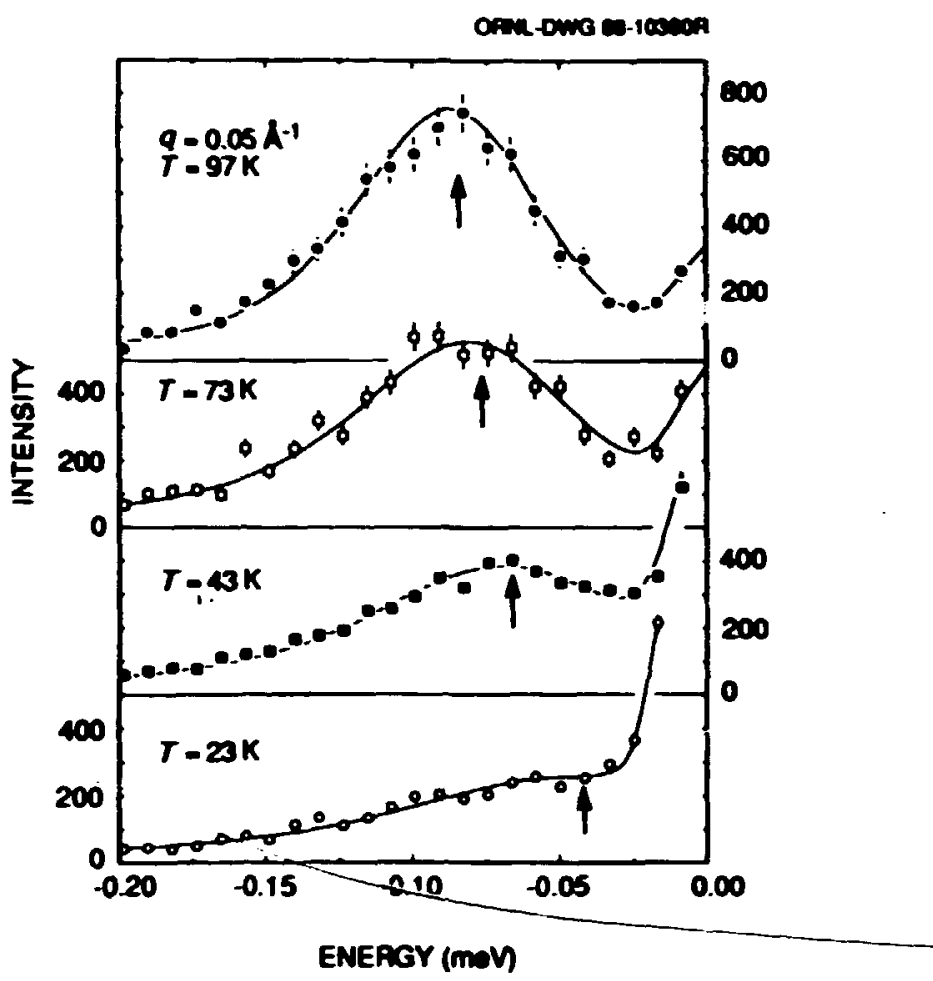

Fig. 2.13. Constant- $q$ scans $\left(q=0.05 \mathrm{~A}^{-1}\right)$ for $\mathrm{Fe}_{89} \mathrm{Ni}_{1} \mathrm{Zr}_{10}$ at $\mathrm{T}=23,43,73$, and $97 \mathrm{~K}$. The solid lines are the result of least-squares fits that assume Lorentzian line shapes and include the effects of the instrumental resolution. The arrows indicate the energy of the excitations obtained from our analysis.

conventional ferromagnet, the SW excitations soften below $T \simeq 100 \mathrm{~K}$, at the same time that they become broader. Below $100 \mathrm{~K}$ there is also a drastic increase in the intensity of the elastic component of the scattering that indicates the development of the spin-glass-like phase. These results are consistent with a picture in which ferromagnetism does not vanish completely in the spin-glass-like phase, but 
the effects of magnetic frustration are to soften and broaden the SW propagating excitations.

1. Laboratoire Leon Brillouin, CEN Saclay, France.

2. National Institute of Standards and Technology, Gaithersburg, Md.

3. Allied Signal, Inc. Morristown, N. J.

4. Experiment carried out at the ORPHEE reactor at the Laboratoire Leon Brillouin.

\section{INSTRUMENTATION}

\section{DISCRETE THIN-FILM MULTILAYER DESIGN FOR NEUTRON MIRRORS'}

John B. Hayter and H. A. Mook

Many modem developments in neutron scattering are based on optical techniques which rely on reflection or refraction of the neutron wave. Since the neutron refractive index is very close to unity $\left(1-n \approx 10^{-5}\right)$, only small angular deviations are possible, and applications have been limited by the small acceptance angles. In recent years, various multilayer optical designs, termed supermirrors, have been proposed to extend the angular range reflected by the mirror coating.

To date, designs for supermirrors ${ }^{2,3}$ have been based on continuum approaches in which refraction between layers has not been included in an optimal way. We have been able to show that a better design may be obtained using a discrete difference equation technique. The key to the new design is a method of computing the impact of a single layer on an existing multi- layer structure, so that the supermirror may be built up layer-by-layer with each new layer beginning to reflect at the angle where the previous structure just ceases to reflect. This new approach leads to a design which maintains essenually perfect reflectivity up to larger cutoff angles than are obtained with any of the continuum methods. Further, an optimal technique for trading reflectivity against angular range emerges naturally from the physics of the design, so that experiments where large angles are critical may be accommodated. This design is the first to show that perfect reflectivity in the supermirror range is at least theoretically possible, overcoming the previous barrier to the development of high-efficiency transmission polarizers, which would revolutionize certain classes of experiments, such as small-angle scattering.

1. Summary of paper to be published.

2. F. Mezei and P. A. Dagleish, Commun. Phys. 2, 41 (1977).

3. J. Schelten and K. Mika, Nucl. Instrum. and Methods 160, 287 (1979).

\section{A THREE-PDNHOLE COLLDMATING SYSTEM FOR THE ORNL 10-m SMALL-ANGLE $X$-RAY SCATTERING CAMERA ${ }^{1}$ \\ G. D. Wignall, I. S. Lin, and S. Spooner}

A series of experiments have been undertaken on the ORNL 10-m small-angle $x$-ray scattering (SAXS) camera to provide quantitative data on the level of background (parasitic) scattering generated by different types of beveled collimating slits. The addition of a 


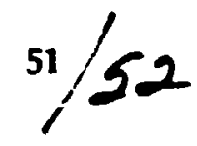

third (guard) slit, positioned close to the sample, resulted in a reduction of over an order of magnitude in the parasitic background generated by the best two-slit combination. The improvement in the parasitic background is indicated in Fig. 2.14, which compares the twodimensional contour plots from the original (two-slit) and the final (three-slit) collimation systems. This slit system made it possible to reduce the size of the beam stop, permitting useful data to be collected down to a value of the scattcring vector $Q=4 \pi \lambda^{-1} \sin \theta=3 \times 10^{-3}$ $A^{-1}$, where $\lambda$ is the wavelength and $2 \theta$ is the angle of scatter. This experimental configuration permits the resolution of distances $D$ $2 \pi / Q$ up to $2000 A$. The current instrumental signal-to-noise ratio for weakly scattering systems compares favorably with that available at synchrotron SAXS facilities.

1. Summary of paper to be published.
CONTOURS AT $0.2,0.4,0.8 \ldots 102.4$ COUNTSICHANNEL

(a)

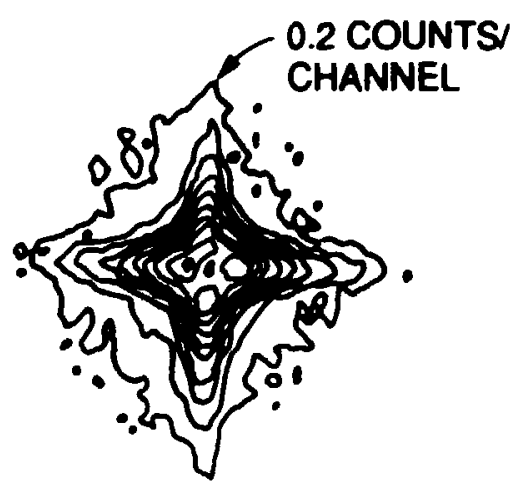

CONTOURS AT 0.2, 0.4, 0.8 AND 1.6 COUNTSICHANNEL

(b)

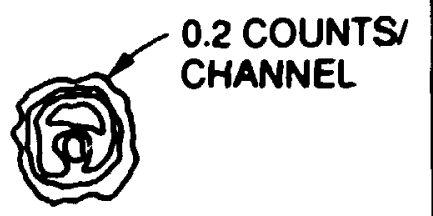

Fig. 2.14. Two dimensional parasitic scattering contours from (a) original slit system, and (b) 3-slit collimation system. 


\section{Physical Properties of Materials}

Research efforts in the field of high-temperature superconductivity have assumed an increasingly important role in the activities of the ORNL Solid State Division during the period covered by this report. During this time, new superconducting materials based on oxides of thallium and bismuth have been discovered, and the SSD is actively pursuing research on these new materials as well as yttrium-barium-copper oxide superconductors. While examples of these wide-range research activities exist throughout this report, the present chapter st-mmarizes the results of investigations of the magnetic and transport properties of bulk superconductors. Investigations of critical currents in bulk superconducting materials and of the effects of giant flux creep are reported

This chapter also describes research activities in the areas of varistor physics and physical and electronic properties of transition-metal carbides. The pulseresponse characteristics of varistors have been determined, and new varistor materials have been investigated. Techniques for the growth of single crystals of transition-metal carbides have been developed in the division, and these crystals have been used in surface physics investigations as well as in fundamental studies of electrical conductivity.

Research in the general area of radiation effects in bulk materials no longer receives special emphasis in the SSD, but the existing expertise and capabilities in this field are still applied to particularly interesting research opportunities. This activity is reported in the final section of this chapter. The work dealing with investigations of the structural inequivalence of the amorphous state produced by ion damage and the glass state is particularly noteworthy. 


\section{SUPERCONDUCTORS}

\section{MAGNETC STUDIES OF SINGE-CRYSTAL SUPERCONDUCTING $\mathrm{Er}_{1} \mathrm{Ba}_{2} \mathrm{Cu}_{3} \mathrm{O}_{2}$}

I. R. Thompson, ' S, T. Sekula, H. A. Deeds, ${ }^{2}$ D. K. Christen, B. C. Sales, and L. A. Boatner

Single crystals of $\mathrm{Er}_{1} \mathrm{Ba}_{2} \mathrm{Cu}_{3} \mathrm{O}_{2}$ were prepared for an investigation of the suferconducting magnetization $M(H, T)$ and the critical current density $J_{C}(H, T)$ along principal crystal directions. In addition, the normal state magnetic susceptibility $\chi(T)$ was determined along the principal crystal axes in order to correlate the results with the observed orientation of crystallites in magnetically aligned composites. Magnetic measurements were made on a single crystal $0.8 \times 0.8 \times 0.24 \mathrm{~mm}^{3}$ in a SQUID based magnetometer for $H \| c$ and $H \| a b$ in the temperature interval from 6 to $287 \mathrm{~K}$ and in magnetic fields up to $53 \mathrm{kOe}$. The onset of the superconducting magnetic transition occurred at $92 \mathrm{~K}$ with the midpoint at $90.5 \mathrm{~K}$.

The superconducting magnetization for the case $\boldsymbol{H} \mathbf{I}$ ab is smaller by an order of magnitude than for $H \|_{c}$ because of the weaker superconducting current sustainable in a direction perpendicular to the $\mathrm{Cu}-\mathrm{O}$ planes. From the hysteresis in the magnetization process, values of $I_{c}(H, T)$ were deduced, using the Bean critical state model. For both orientations, $J_{c}$ decreases exponentially with temperature as $J_{C}(T)=J_{c}(0)$ $\exp \left(-T / T_{0}\right)$, where the values of the slope $T_{0}$ can be used to estimate the effective well depth $U_{0}$ for flux pinning in this material. This analysis yields values for $I_{c}(0)$ of $4.0 \times 10^{6}$ and $2.7 \times$
$10^{5} \mathrm{~A} / \mathrm{cm}^{2}$ and values of $U_{0}=41$ and $54 \mathrm{meV}$ for the cases $H \| c$ and $H \|_{a b}$, respectively.

The normal-state magnetic susceptibility $x$ follows a Curie-Weiss behavior for both orientations, with $\chi(a b)>\chi(c)$ over the temperature range from $T_{c}$ to $287 \mathrm{~K}$.

1. Adjunct research and development participant from The University of Tennessee, Knoxville, Tenn.

2. Graduate student from The University of Tennessee, Knoxville, Tenn.

\section{OXYGEN STOICHIOMETRY AND SUPERCONDUCTIVITY IN $\mathrm{HOBa}_{2} \mathrm{Cu}_{3} \mathrm{O}_{7-x}$}

B. C. Sales, Y. C. Kim, ${ }^{2}$ I. R. Thompson, D. K. Christen, L. A. Boatner, and S. T. Sekula

The effects o! varying oxygen content on the superconducting transition temperature $T_{c}$, resistivity, and crystal structure of $\mathrm{HoBa}_{2} \mathrm{Cu}_{3} \mathrm{O}_{7-x}(0<x<1)$ have been investigated. A plateau in the $T_{c}$ vs $x$ curve is observed (Fig. 3.1) for values of $x$ between 0.3 and 0.4 with the value of $T_{c}$ (10\% onset measured magnetically) remaining relatively constant at about $55 \mathrm{~K}$. The width of the superconducting transition, as measured both resistively and magnetically, is substantially narrower for $x=$ $0.35 \pm 0.05$ than for slightly larger or smaller values of $x$. High-resolution powder $x$-ray diffraction analysis was used to measure the lattice constants of both the orthorhombic and tetragonal phascs as $x$ was varied. With increasing $x$ the temperature coefficient of the resistivity changed from positive (metallic) for $x \leq 0.2$ to negative (semiconducting) for $x \geq 0.3$. 


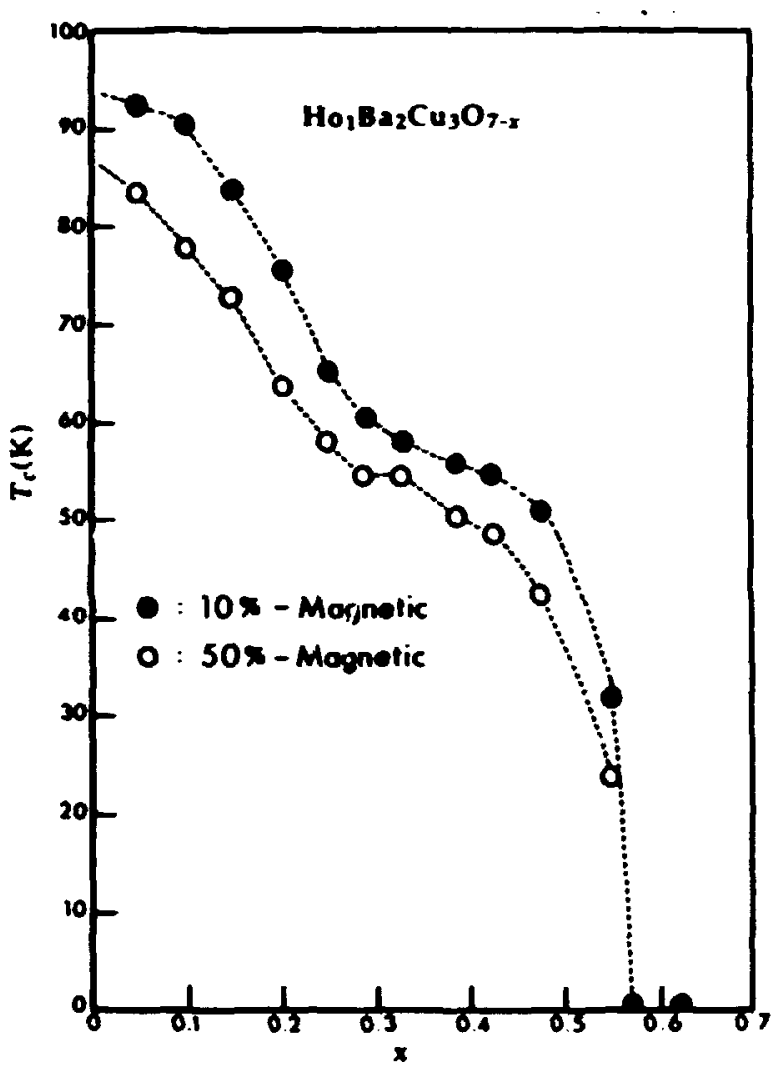

Fig. 3.1. The superconducting transition temperature vs oxygen content $x$ for $\mathrm{Ho}_{1} \mathrm{Ba}_{2} \mathrm{Cu}_{3} \mathrm{O}_{7-x}$.

These results are very similar to those previously reported for $\mathrm{YBa}_{2} \mathrm{Cu}_{3} \mathrm{O}_{7-x}$ by several authors. The present results demonstrate that the complete substitution of magnetic Ho for $y$ has no significant effect on variation of $T_{c}$ with $x$ and suggest that there is an ordering of the oxygen vacancies in the $a-b$ plane and the existence of an ordered compound with a $T_{c}$ of $55 \mathrm{~K}$.

1. Summary of paper: p. 591 in HighTemperature Superconductors, ed. by Merwyn B. Brodsky et al., Materials Research Society, Pittsburgh, Pa., 1988.

2. Graduate student from The University of Tennessee, Knoxville, Tenn.
MAGNETC PROPERTIES OF OXYGENREDUCED, RARE-EARTH-BASED

(RE) $\mathrm{Ba}_{2} \mathrm{Cu}_{3} \mathrm{O}\left(\mathrm{C}_{2-\pi}\right)$ MATERIALS WTTH $R E=G d, D y, A N D$ Hol

I. R. Thompson, ${ }^{2}$ B. C. Sales, Y. C. Kim, 3 S. T. Setula, L. A. Boatrer, J. Brymestad, and D. K. Christen

Bulk magnetic properties of tetragonal, oxygen-depleted, polycrystalline samples of sintered ( $\mathrm{RE})_{1} \mathrm{Ba}_{2} \mathrm{Cu}_{3} \mathrm{O}_{7-x}$ with $x=0.95$ have been investigated. Materials containing the rare-earth (RE) ions Gd, Dy, and Ho were studied for comparison with their oxygenated superconducting counterparts. 5,6 For each system, the magnetic susceptibility $\boldsymbol{X}$ was described by a Curie-Weiss dependence with effective moments that were very nearly equal to the free-ion values, a behavior very similar to that observed for the corresponcing superconducting, rare-earth-based materials. However, the field dependence of the magnetization $M(H, T)$ diffred substantially from the free-ion behavior-an effect that demonstrates a significant influence of the crystal electric field on the magnetic properties of these compounds accompanied by some contribution from RE-RE interactions.

1. Summary of Paper: Phys. Reo. B 37, 9395 (1988).

2. Adjunct research and development participant from The University of Tennessee, Knoxville, Tenn.

3. Graduate student from The University of Tennescee, Knoxville, Tenn.

4. Chemistry Division, ORNL.

5. J. R. Thompson et al., Phys. Reo. B 36, 836 (1987).

6. J. R. Thompson et al., Phys. Rev. B 36, 718 (1987). 
ANISOTROPIES IN THE MAGNETIC PENETRATION DEPTH AND CRITICAL CURRENT DENSITY OF $\mathrm{RBa}_{2} \mathrm{Cu}_{3} \mathrm{O}_{7-x}$ $(R=$ Y OR Ho), MEASURED ON MAGNETICALIY ALIGNED POWDER COMPOSITES'

D. K. Christen, Y. C. Kim, ${ }^{2}$ S. T. Sekula, J. R. Thompson, ${ }^{3}$ J. Brymestad, B. C. Sales, L. A. Boatner, and J.D. Budai

The highly anisotropic properties of the high- $T_{c}$ oxide superconductors may have important ramifications for many potential applications. In particular, the very large magnetic penetration depth $\lambda$ corresponding to $c$-axis shielding currents raises questions regarding applications involving flux screening in devices of dimensions comparable to $\lambda$. For power applications, a knowledge of anisotropic effects on the critical current density $J_{c}$ is required to assess the need for texturing of bulk materials.

Crystallite powder particles of $\mathrm{YBa}_{2} \mathrm{Cu}_{3} \mathrm{O}_{2}$ (Y123) and $\mathrm{HoBa}_{2} \mathrm{Cu}_{3} \mathrm{O}_{2}$ (Ho123) were sized, suspended in an epoxy matrix, and magnetically aligned to yield c-axis composites of about $10 \%$ superconducting volume iraction. The samples were characterized magnetically as a function of field and temperature for orientations of the $c$-axis either parallel or perpendicular to the applied magnetic field. By treating the particles as spheres and properly accounting for the large magnetic moment of $10.6 \mu_{B}$ carried by the $\mathrm{Ho}^{3+}$ ions, anisotropy ratios of $\lambda_{c} / \lambda_{a b}=4.6$ and 2.9 were derived for Y123 and Ho123, respectively. From the magnetization hysteresis $\Delta M$, the derived critical current densities show an approximate order- of-magnitude anisotropy and an exponential decay with temperature, $I_{c}(T)=J_{c}(0)$ $\exp \left(-T / T_{0}\right)$, that is also observed in single crystals and is likely a nuanifestation of large flux creep effects. 5

1. Summary of paper. IEEE Trans. Magn. 25, 2324 (1989).

2. Graduate student from The University of Tennessee, Knoxville, Tenn.

3. Adjunct research and development participant from The University of Tennessee, Knoxville, Tenn.

4. Chemistry Division, ORNL.

5. J. R. Thompson et al., "Magnetic Studies of Single-Crystal Superconducting $\mathrm{Er}_{1} \mathrm{Ba}_{2} \mathrm{Cu}_{3} \mathrm{O}_{2}{ }^{n}$ this report.

\section{SUPERCONDUCTTVITY, CRITCAL CURRENT DENSITY, AND MAGNETIC PROPERTIES OF FE-SUBSTITUTED

$$
\mathrm{Y}_{1} \mathrm{Ba}_{2}\left(\mathrm{Cu}_{1-\mathrm{x}} \mathrm{Fe}_{\mathbf{x}}\right)_{3} \mathrm{O}_{2}{ }^{1}
$$

S. T. Sekula, J. Brymestad, ${ }^{2}$ J. Budai, D. K. Christen, I. R. Thompson, and Y. C. Kim

A series of Fe-substituted $\mathrm{Y}_{1} \mathrm{Ba}_{2}\left(\mathrm{Cu}_{1-x} \mathrm{Fe}_{x}\right) \mathrm{O}_{2}$ materials with $x=0$ to $10 \%$ was prepared by standard methods. Investigations of the superconducting properties were carried out with a vibrating sample magnetometer over a temperature interval 4.2 to $90 \mathrm{~K}$ in magnetic fields up to $8.0 \mathrm{~T}$. Optical metallographic examination of these materials indicated the disappearance of twinning for $x>3 \%$. This result is consistent with $x$-ray diffraction work which showed that the orthorhombic distortion $(2(b-a) /(b+a)]$, where $a$ and $b$ are unit cell dimensions, abruptly decreases for $x>3 \%$. However, the superconducting Iransition 
temperature $T_{c}$ was found to decrease smoothly at a rate of about $6.7 \mathrm{~K} /(\mathrm{at} . \% \mathrm{Fe})$ over the range $3 \%<x<10 \%$ with no apparent correlation with the structural alteration. From the isothermal magnetization data, the hysteresis in the magnetization $M(T, H)$ was determined, and using the Bean model, values of the intragranular critical current density $I C T, H)$ were deduced. Further analysis of these data showed that at a fixed value of magnetic field, $J_{c}$ decreased exponentially with the reduced temperature $T / T_{c}$ and with $F e$ concentration. This latter result is illustrated in Fig. 3.2.

Since the Fe substitutions occur primarily in the $\mathrm{Cu}-\mathrm{O}$ chains, thereby driving the crystal

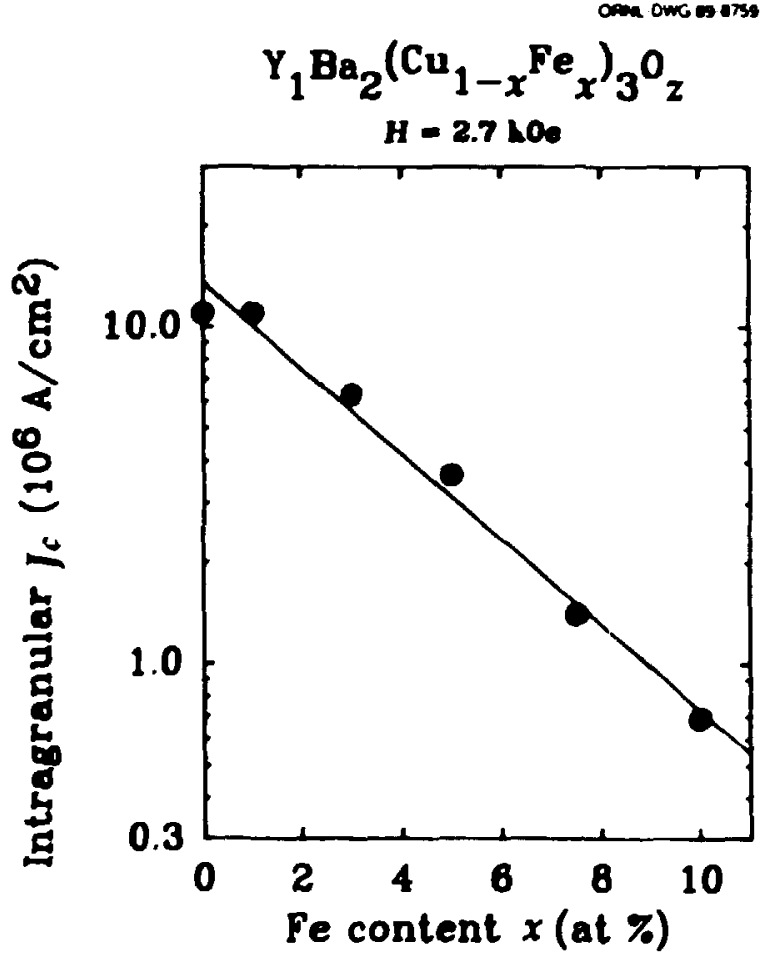

Fig. 3.2 Critical current density $I_{c}$ in a field of $2.7 \mathrm{kOe}$ (extrapolated to $T=0$ ) vs Fe content $x$. structure toward tetragonality, it is concluded that neither $\mathrm{Cu}-\mathrm{O}$ chains nor associated twinning is essential underpinnings of superconductivity in these compounds.

1. Summary of paper: IEEE Truns. Magn. 25, 2266 (1989).

2. Chemistry Division, ORNL.

3. Adjunct research and development participant from The University of Tennessee, Knoxville, Tenn.

4. Graduate student from The University of Tennessee, Knoxville, Tenn.

5. Z. Hiroi et al., Jpn. I. Appl. Phys. 27, L580 (1988).

\section{CHARACTEREATION AND SUPERCONDUCTING PROPERTIES OF PHASES IN THE BI-SR-Cu-O SYSTEM 1}

B. C. Chakoumakos, P. S. Ebay,

B. C. Sales, and E. Sonder

Phase formation in the system Bi-Sr-Cu-O has been examined as a function of composition, temperature, ambient atmosphere, cooling his tory, and annealing time. Ceramic processing and melt crystallization techniques were used. For the ceramic materials (using $\mathrm{Bi}_{2} \mathrm{O}_{3}, \mathrm{SrCO}_{3}$. and $\mathrm{CuO}$ ) processed at $700^{\circ} \mathrm{C}$ in air, the $\mathrm{Bi}_{2} \mathrm{Sr}_{2} \mathrm{CuO}_{6}$ composition (221) crystallizes to a mixture of $\mathrm{CuO}, \mathrm{SrCO}_{3}$, and the rhombohedral $\mathrm{Bi}_{2} \mathrm{O}_{3} \times 5 \mathrm{SHO}$ solid solution. At $800-830^{\circ} \mathrm{C}$ in air for short durations ( $5 \mathrm{~min}$ to $2 \mathrm{~h}$ ) the reacted products consist principally of the ideal 221 phase with minor amounts of $\mathrm{CuO}$. For longer reaction times $(2-400 \mathrm{~h})$ the reacted products consist of the ideal 221-type structure with $c=$ $24.64 A$ and $a=3.804 A, a$ "collapsed" 221 
structure with $c=23.6 \AA$, and CuO. With increasing reaction time the collapsed 221 phase gradually grows at the expense of the ideal 221 phase. The collapsed 221 phase is not an oxycarbonate and appears to be a distinct ternary compound near the 221 composition, with a layered structure having a $1-\AA$ smaller stacking repeat. The ideal 221 phase is a solid solution with variable $\mathrm{Sr}$ content. With decreasing $S r$ in the starting mixture [ 2 to 1.25 atoms per formula unit (afu)] we observe the following: (1) the formation of the collapsed 221 structure is inhibited; (2) for the ideal 221 phase the c-cell dimension decreases significantly $(0.2 \AA)$ and the a-cell dimension increases slightly $(0.02 \quad A)$; 13$)$ the lowtemperature resistivity behavior changes from superconducting with a $T_{c}$ onset of $7 \mathrm{~K}$ for $\mathrm{Sr}>$ 1.5 afu to semiconducting for $\mathrm{Sr}<1.5$ afu; and (4) the positions of the superlattice peaks around the $(002)$ reflections become more incommensurate with respect to the parent structure. Rapid quenching ( $<5$ s) from temperatures near the melting point $\left(900^{\circ} \mathrm{C}\right)$ can raise the superconducting $T_{c}$ onset to $10 \mathrm{~K}$. Independent of the cell variation with $\mathrm{Sr}$ content, quenching causes the $c$-cell dimension to expand by $0.03 A$ on average while the $a$-cell dimension remains invariant. A preliminary phase compatibility diagram for $800^{\circ} \mathrm{C}$ in an oxygen atmosphere has been made for the system $\mathrm{Bi}_{2} \mathrm{O}_{3}$-SrO-CuO.

1. Summary of paper to be published.
2. Great Lakes Collcges Association and the Associated Colleges of the Midwest Science Semester student research participant from St. Olaf College, Northfield, Minn.

\section{VARIATION OF GUPERLATTICE STRUCTURE OF THE Bi $\mathrm{Sr}_{2-x} \mathrm{CuO}_{6}$ SUPERCONDUCTOR WITH COMPOSITION AND THERMAL HISTORY! \\ J. D. Budai, B. C. Chakoumakos, \\ B. C. Sales, and Edward Sonder}

Nominal $\mathrm{Bi}_{2} \mathrm{Sr}_{2-x} \mathrm{Cu}_{1.5} \mathrm{O}_{6}(x=0-0.25)$ ceramic compositions reacted in oxygen at $830^{\circ} \mathrm{C}$ yield the ideal $\mathrm{Bi}_{2} \mathrm{Sr}_{2} \mathrm{CuO}_{6}$ phase as the major product. Based on a tetragonal cell choice, with decreasing $\mathrm{Sr}$ content in the starting mixture, the $c$-cell dimension decreases significantly $(0.2$ $A$ overall) and the a-cell dimension increases slightly ( $0.02 \AA$ overall). Ind-ypendent of the cell variation with $\mathrm{Sr}$ content, quenching causes the c-cell dimension to expand by $0.03 \AA$ on average while the a-cell dimension is unchanged. A small number of oxygen vacancies are quenched in from high temperature and presumably originate in the $\mathrm{Bi}_{2} \mathrm{O}_{2}$ layer. All single crystals (up to $1 \times 10 \mathrm{~mm}$ ) grown from CuO-rich melts with the same range of $\mathrm{Sr}$ deficiency display prominent satellite peaks around their $(002)$ reflections. Energy dispersive analytical $x$-ray spectra of single-crystal cleavages confirm decreasing $\mathrm{Sr}$ content of the crystals. The satellite peaks shift systematically with changing Sr content. The superstructure has been determined to be commensurate or incommensurate depending on Sr content. 
With decreasing Sr content, the ceramic samples exhibit a metal-to-insulator transition as measured resistively to $4.2 \mathrm{~K}$. The onset $T_{c}$ in the superconducting samples is $7 \mathrm{~K}$. As grown from the melt, crystals have semiconducting resistivity at low temperatures; but with an additional high-temperature anneal in oxygen, metallic resistivity is restored with the superconducting onset at $7 \mathrm{~K}$. As grown from the melt, the crystals are deficient in oxygen. The posibion of the superlattice reflections are invariant with oxygen content, implying that the supercsnductivity is not related to the superlattice modulations. Using single-crystal $x$-ray diffraction methods, correlations between the superlattice modulations and the $\mathrm{Sr}$ content are quantified.

1. Summary of paper: Materials Research Society Symposium Proceedings (in press).

\section{THE EFFECTS OF OXYGEN AND STRONTIUM VACANCIES ON THE SUPERCONDUCTIVITY OF SINGLE CRYSTALS OF $\mathrm{Bi}_{2} \mathrm{Sr}_{2-x} \mathrm{CuO}_{-y} 1$}

Edward Sonder, B. C. Chakoumakos, and B. C. Sales

The compound $\mathrm{Bi}_{2} \mathrm{Sr}_{2} \mathrm{CuO}_{6}$ was the first phase discovered ${ }^{2}$ and one of the simplest members in a large family of superconducting compounds ( $\mathrm{BiO}_{2} \mathrm{Sr}_{2} \mathrm{Ca}_{n-1} \mathrm{Cu}_{n} \mathrm{O}_{2 n+2}(n=1,2,3)$, and $\left(\mathrm{TIO}_{2} \mathrm{Ba}_{2} \mathrm{Ca}_{n-1} \mathrm{Cu}_{n} \mathrm{O}_{2+2 n}(n=1,2,3)\right.$. Single crystals of $\mathrm{Bi}_{2} \mathrm{Sr}_{2-2} \mathrm{CuO}_{6-2}$ were grown by slowly cooling melts of $\mathrm{Bi}_{2} \mathrm{O}_{3}, \mathrm{SrCO}_{3}$, and $\mathrm{CuO}$ from $950^{\circ} \mathrm{C}$ in either air or flowing oxygen. The melts were close to stoichiometric with the exception of an excess of $\mathrm{Cu}$ to ensure that the resulting solid phases were only $\mathrm{Bi}_{2} \mathrm{Sr}_{2-x} \mathrm{CuO}_{6-2}$ (hereafter denoted by 2201) and CuO. Previous work ${ }^{3}$ had shown that the ideal superconducting phase is deficient in Sr and is a solid solution that can tolerate a range of Sr deficiency. Thus, crystals with composition $\mathrm{Bi}_{2} \mathrm{Sr}_{2-2} \mathrm{CuO}_{6}$ $(x=0.1-0.5)$ were grown by varying the starting concentration of $\mathrm{Sr}$ in the melt. The as-grown crystals consisted of large micaceous sheets (typically $5 \times 5 \times 0.01 \mathrm{~mm}$ ) that were oxygen deficient. These oxygen-deficient crystals were not superconducting above $4.2 \mathrm{~K}$ and showed semiconducting behavior that became more pronounced with smaller Sr content. When heated in flowing oxygen at temperatures ranging from 600 to $830 \mathrm{C}$, the as-grown crystals increased in weight by $0.5-1 \%$, which correspionds to a change of 0.25-0.5 oxygen per formula unit. For crystals with near stoichiometric St content, this oxygen treatment resulted in bulk superconductivity as determined by resistivity and ac susceptibility measurements with a maximum $T_{c}$ of $9 \mathrm{~K}$. Results of electrical measurements are shown in Fig. 3.3. This figure also shows that the resistivity (measured in the $a-b$ plane) of the superconducting crystals is almost perfectly linear in temperature frum $15-300 \mathrm{~K}$.

1. Summary of paper to be published.

2. C. Michel et. al., Z. Physik B 68, 417 (1987).

3. B. C. Chakoumakous et al., Journal of Materials Research (in press). 


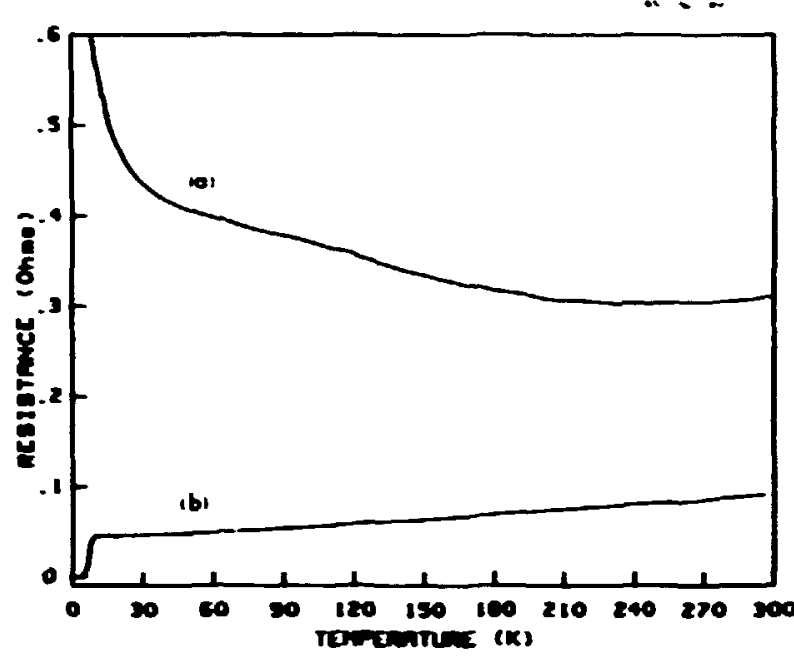

Fig. 3.3. Resistivity vs temperature for (a) an as-grown crystal of $\mathrm{Bi}_{2} \mathrm{Sr}_{1.85} \mathrm{Cu}_{1} \mathrm{O}_{6-y}$ and (b) for the same crystal after heating in oxygen at $800 \mathrm{C}$.

\section{SUPERCONDUCTIVITY, INTERGRAN, AND INIRAGRAN CRTICAL CURRENT DENSTIES OF $\mathrm{M}_{2} \mathrm{C}_{2} \mathrm{Ba}_{2} \mathrm{Cu}_{3} \mathrm{O}_{10+6}$ AND $\mathrm{Tl}_{2} \mathrm{Ca}_{1} \mathrm{Ba}_{2} \mathrm{Cu}_{2} \mathrm{O}_{2+8}$ MATERIALS 1}

\section{J. R. Thompson, 2 J. Brynested, 3 D. M. Kroeger," Y. C. Kim, S. T. Sekula, \\ D. K. Christen, and E. D. Specht}

Bulk-sintered as well as powdered samples of the high-temperature superconducting compounds $\mathrm{T}_{2} \mathrm{Ca}_{2} \mathrm{Ba}_{2} \mathrm{Cu}_{3} \mathrm{O}_{10+8}(\mathrm{~T}-2223)$ and $\mathrm{Tl}_{2} \mathrm{Ca}_{1} \mathrm{Ba}_{2} \mathrm{Cu}_{2} \mathrm{O}_{8+8}$ (T1-2122) have been synthesized. Stoichiometric mixtures of pure oxides of $\mathrm{Tl}, \mathrm{Ba}, \mathrm{Ca}$, and $\mathrm{Cu}$ were used to achieve final materials with phase purities of approximately 90\%. Preparation of materials was monitored by $x$-ray diffraction, netallographic, and electron microprobe analyses. Electronic and superconductive properties were investigated by measurements of the electrical resistivity and the critical current dcrisity /c using transport methods and by extensive magnetization measuraments in the temperature range from $4.2 \mathrm{~K}$ to $T_{c}$ in applied fields up to 80 kOe.

The primary results and conclusions of this work are that (1) the intragrain /c values were large, much larger than the transport values, which in low magnetic fields exhibited features typical of weakly soupled Josephson junction arrays; (2) both sintered and powdered materials exhibited large flux creep; and (3) the $J_{c}$ decreased exponentially with temperature. From analysis of the temperature dependence of $J_{c}$ in $2.7 \mathrm{kOe}$, we estimate for $U_{0}$ the effective well depth for flux pinning, the values of 50 and $40 \mathrm{meV}$ for $71-2 \mathrm{m23}$ and $\pi-2122$, respectively. Qualitatively, these features are similar to those found in the corresponding $\mathrm{Y}_{1} \mathrm{Ba}_{2} \mathrm{Cu}_{3} \mathrm{O}_{2}$ series of compounds.

1. Surnmary of paper: Phys. Reo. B 39, 6652 (1989).

2. Adjunct research and development participant from The University of Tennessee, Knoxville, Tenn.

3. Chemistry Division, ORNL.

4. Metals and Ceramics Division, ORNL.

5. Graduate student from The University of Tennessee, Knoxville, Tenn.

\section{GEOMETRICALLY LARGE FLUX-LNE PINS 1}

\section{H. R. Kerchner}

The extensive theoretical work on the statistical tummation of flux-line point pins cannot be applied, in principle, to the numerous experimental studies of pinning by large crystalline 
defects [i.e., with dimensions larger than the

flux-line-lattice (FLL) parameter]. However, qualitative similarities exist between the theory of point pins and many observations of the critical current density in coarsely grained polycrystalline, coid-worked, and two-phase superconducting materials. The theory recently developed for understanding pinning by grain boundaries $^{2}$ and ellipsoidal precipitates ${ }^{3}$ assumes contiguity of a FLL plane with the crystalline defect surface. Contiguous pinning by ellipsoidal precipitates accounts for the observed anisotropy of the critical current density in a Nb crystal containing a gaseous impurity' (contradicting the viewpoint expressed in Ref. 4). The required alignment ${ }^{2}$ of a flat defect surface with a FLL layer, as illustrated in Fig. 3.4, is impossible over the vast majority of grain boundary and dislocation cell wall area. How then can one understand the often observed sonnection between grain size and critical current density? A clue to the answer arises in a detailed technical analysis of the magnetic ac response and TEM observations which provides strong evidence that the dislocation-cell vertices are effective point pins in a cold-worked $\mathbf{N b Z r}$ sample. Similar analysis of $\mathbf{N b}_{3} \mathbf{S n}$ and NbTi multifilamentary cable will be needed to map out clearly the proper inlerpretation of critical current measurements on commonly used superconducting wire.

1. Summary of paper: Cryogenics 29, 251 (1989).

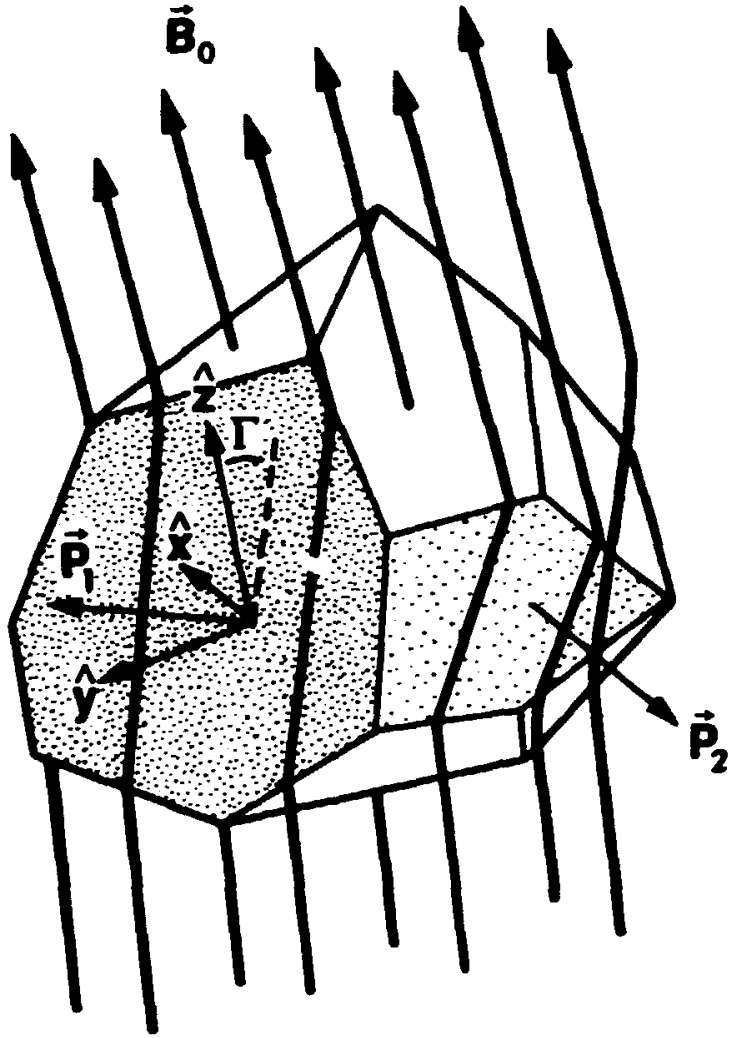

Fig. 3.4. Schematic illustration of a single grain in polycrystalline material. For clarity, only two FLL planes are shown; one is contiguous with the planar grain face supplying a pinning force $P_{1}$, and a FL from each FLL plane shown together form the diagonal FLL plane contiguous with the grain face supplying $\mathbf{P}_{\mathbf{2}}$.

2. E. V. Thuneberg, p. 18 in Proceedings of the International Symposium on Flux Pinning and Electromagnetic Properties of Superconductors, ed. by F. Matsushita, K. Yamafuji, and F. Irie, Matuskuma, Fukuoka, Japan, 1985; H. R. Kerchner et al., ibid., p. 38.

3. A. I. Larkin and Yu. N. Ovchinnikov, Zh. Eksp. Teor. Fiz. 80, 2334 (1981) [Soo. Phys. JETP 53, 1221 (1981)].

4. G. Antesberger and $\mathrm{H}$. Ulimaier, Phys. Rev. Lett. 35, 59 (1975). 


\section{VARISTORS AND CARBIDES}

\section{ORIGIN OF THE PULSE-RESPONSE CHARACTERISTICS OF ZINC OXIDE VARISTORS 1}

\section{F. A. Modine, R. B. Wheeler, \\ Youngjee Shim, ${ }^{3}$ and lames F. Cordaro}

Pulse-response characteristics were measured for simple varistor materials in the $\mathrm{ZnO}$ $\mathrm{Bi}_{2} \mathrm{O}_{3}-\mathrm{MnO}_{2}$ system. It was found that smail additions of $\mathrm{Li}_{2} \mathrm{O}$ or $\ln _{2} \mathrm{O}_{3}$ can enhance or eliminate the materials inductive behavior, which is responsible for voltage overshoot and current undershoot. Figure 35 shows the currizt response of three samples to identical pulses from a low-impedance source. The indium-

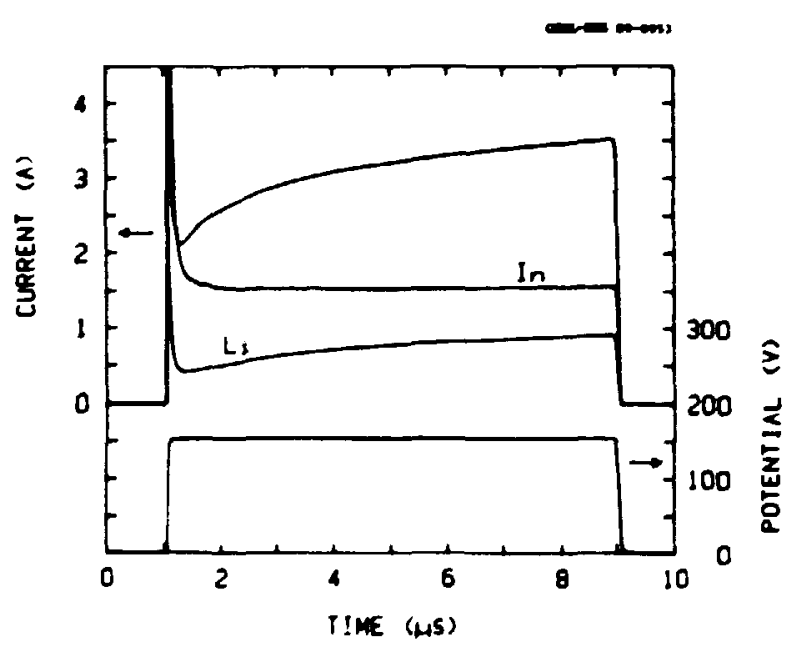

Fig. 3.5. Current responses of the undoped, $\ln _{2} \mathrm{O}_{3}$-doped, and $\mathrm{Li}_{2} \mathrm{O}$-doped samples to an identical step voltage. The displacement current spike on the leading edge of the pulse is off scale for the $\operatorname{In}_{2} \mathrm{O}_{3}$-doped sample. A negative-going-current spike on the trailing edge of each pulse has been clipped at zero. doped sample contains $-60 \mathrm{ppm}$ in, and the lithium-doped sample contains -6 ppm Li by atomic fraction. The undoped sample shows a current undershoot of about $40 \%$. The addition of $\mathrm{Li}_{2} \mathrm{O}$ increases the undershoot to more than $60 \%$. The $\mathrm{Li}_{2} \mathrm{O}$ also reduces the steady-state current and the displacement current that are revealed by a sharp spike on the leading edge of the current response. The addition of indium suppresses the current undershoot to the point where it is no longer visible.

The room-temperature equivalence of the emission time of electrons from deep traps and the rise time of the varistor current has led several investigators to associate the inductive behavior with deep electron traps within the depletion regions on either side of the junction. Iowever, this association is not supported by the temperature dependence of the pulseresponse characteristics. The current rises more quickly at low temperatures, whereas the emission is much slower.

A phenomenological description of the inductive response of a varistor is obtained by associating a time lag with voltage-induced changes in resistance and/or the capacitance of a varistor. Changes in the resistance and capacitance are readily attributed to changes in the height and width of the Schottky barriers that accompany charge motion in the barrier region.

1. Summary of paper to be published.

2. Present address: Los Alamos Nationa! Laboratory, Los Alamos, N. Mex.

3. Alfred University, Alfred, N. Y. 


\section{ZINC OXIDE VARISTOR TMME RESPONSEI}

\section{F. A. Modine, R. W. Major, ${ }^{2}$ S.-I. Choi, ${ }^{3}$}

L. B. Bergman, 3 and M. N. Siloer ${ }^{3}$

The application of a voltage to a zinc oxide varistor produces transient currents that persist on a time scale extending from $10^{-8}$ to $10^{5} \mathrm{~s}$ at the least. The transient currents are characterized by a power-law time dependence (i.e., $I=I_{0} / t^{m}$, where $m$ is a little less than unity) and a weak temperature dependence. Figure 3.6 shows the discharge currents that follow removal of an applied voltage. The inset of Fig. 3.6 is an Arrhenius plot of the temperature dependence of the current measured at fixed times after removing the applied voltage. The results imply two processes with activation energies of about 160 and $10 \mathrm{meV}$. The higher energy is close to the $170 \mathrm{meV}$ observed in deeplevel transient spectroscopy and can be inter-

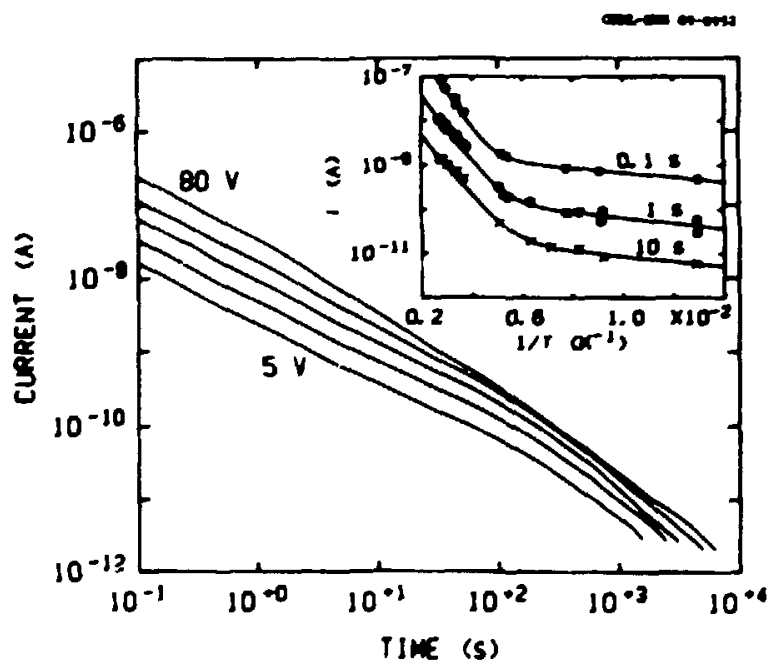

Fig. 3.6. Discharge current transients indured in a medium-voltage varistor at $295 \mathrm{~K}$ by removal of voltages that differ by factors of 2 . Inset is an Arrhenius plot of the current measured at $0.1,1$, and $10 \mathrm{~s}$. preted as thermal emission from a deep level. However, $10 \mathrm{meV}$ is too low an energy to be interpreted in terms of thermal emission because the current persists too long. The time dependence is confirmed by an ac admittance that increases as a noninteger power of the frequency [i.e., $Y=K(j \omega)^{m}$ ], with Fourier theory providing the connection between the time and frequency measurements. Also, the transient currents are accompanied by changes in the ac admittance measured at fixed frequencies that reveal similar time and temperature dependencies (e.g., $I \sim V d C / d t$, where $C$ is the capacitance). The transient currents are not well understood, but they are typical of the non-Debye electrical response that is found in many materials. An explanation of the transients follows from a distribution of relaxation times that varies as $\tau^{-1}$. This distribution is often an ad hoc assumption, but a similar distribution can be derived if the current is associated with tunneling from deep traps in the depletion region.

1. Summary of paper to be published.

2. Oak Ridge Associated Universities faculty research participant from the University of Richmond, Richmond, Va.

3. University of North Carolina, Chapel Hill, N. C.

\section{NEW VARISTOR MATERIAL'}

F. A. Modine and H. M. Hyatt ${ }^{2}$

A new composite varistor material containing a metal, an insulator, and a semiconductor was evaluated. Although other constituents 
can be substituted, the materials that were investigated contained varying proportions of nickel metal, silicon dioxide, and silicon carbide in a silicon rubber binder. The material has rubber-like flexibility and is easily formed into complex shapes. A higher leakage resistivity $\left(-10^{12} \mathrm{ohm} \mathrm{cm}\right)$ and greater nonlinearity $(-10)$ than for commercial silicon carbide varistors will permit the material to be used as a gapless surge suppressor. The breakdown voltage $(1 \mathrm{kV} / \mathrm{cm}-10 \mathrm{kV} / \mathrm{cm})$ and other properties of the material vary with composition. High-current capability $\left(>200 \mathrm{~A} / \mathrm{cm}^{2}\right)$ and good energy absorption $\left(>40 \mathrm{~J} / \mathrm{cm}^{3}\right)$ are found. The material exhibits a low-temperalure coefficient $\left(\sim \times 10^{-3} / \mathrm{K}\right)$ and a low-dielectric constant $(\sim 10)$ with no observed loss peak. These dielectric properties are suited to highfrequency applications, such as antenna protection. The material has a response time ( $<1 \mathrm{~ns})$ that should provide some protection against fast pulses generated by nuclear and directedbeam weapons. At this stage, the physics of the material is mostly conjecture, but the electrical conduction is almost certainly controlled by grain-boundary barriers. However, the conduction is not thermally activated, and it probably has a percolative character. The material will not replace zinc oxide varistors at this time, but it will find specialized applications in which its flexibility, formability, and frequency response are advantageous.

1. Summary of paper: J. Appl. Phys. 64, 4229 (1988); ORNL/TM-10886 (August 1988).

2. Applied Pulse Corp., Ventura, Calif.
(1X) RIPPLED RELAXATION OF (100) TRANSITION-METAL CARBIDE SURFACES'

G. R. Gruzalski, D. M. Zehner, J. R. Noonan,

H. L. Davis, R. A. DiDio,' and K. Müller ${ }^{3}$

An investigation of $\mathrm{TaC}(100)$ and $\mathrm{HFC}(100)$ was performed using low-energy electron diffraction (LEED) and angle-resolved ultraviolet photoemission spectroscopy with synchrotron radiation. Detailed LEED I-V analyses show that, on both surfaces, carbon atoms displace outward and metal atoms inward, giving rise to $(1 \times 1)$ rippled relaxations, with the magnitude of the ripple being larger on $\mathrm{TaC}(100)$. Photoemission spectra of metal if levels from these surfaces show no surface core-level shifts.

Using $x$-ray photoelectron spectroscopy and Auget electron spectroscopy, changes in (bulk) core-level binding energies, valence-band structures, and local carbon environments were determined for $\mathrm{TaC}_{x}(0.5<x<1.0)$ and $\mathrm{HFC}_{x}$ $(0.6<x<1.0)$. From these bulk studies it was inferred that, as $x$ decreases, added negative charge accumulates in the vicinity of metal atoms and is depleted from remaining carbon atoms.

One explanation for the rippling is that carbon atoms in the outermost layer of both $\mathrm{TaC}(100)$ and $\mathrm{HfC}(100)$ move outward to allow their compressed s,p electrons to expand to a state of lower kinetic energy and that metal atoms move inward owing to added charge that accumulates in their vicinity. We propose that the larger first-layr.r ripple on $\mathrm{TaC}(100)$ is a result of a larger accumulation of charge near 
the metal atoms on that surface, consistent with results from our bulk studies.

1. Sumrary of paper. Journal of Vacuum Science and Technology $A$ (in press).

2. Present address: La Salle University, Philadelphia, Pa.

3. Universität Erlangen-Nürnberg, Federal Republic of Germany.

\section{LOW-TEMPERATURE ELECTRICAL RESISTIVITY OF TRANSTIION-METAL CARBIDES ${ }^{\prime}$}

C. Y. Allison, ${ }^{2}$ C. B. Finch,

M. D. Foegelle, ${ }^{3}$ and F. A. Modine

The van der Pauw technique was used to make electrical resistivity measurements from 4 to $350 \mathrm{~K}$ on single crystals of four transitionmetal carbides: vanadium, zirconium, niobium, and tantalum carbide. The crystals were chosen to include (1) superconducting and nonsuperconducting carbides, (2) carbides from both groups IV and V, (3) a carbide with a nearly stoichiometric composition, and (4) carbides with randomly distributed and with ordered carbon vacancies. An analysis of the data revealed that the Bloch-Gruneisen theory of electrical resistivity gives a good fit to the zirconium carbide and the vanadium carbide measurements. In contrast, the resistivities of the two superconducting crystals, tantalum carbide and niobium carbide, show excellent agreement with the Wilson mode!. The appropriate model appears to be correlated with the superconducting properties of the crystals, but not with the carbon content. The Bloch-Gruneisen equation is

$\rho(T)=p_{0}+\varphi_{1} T(T / \theta) / J_{5}(\theta / T)$,

where $\rho_{0} \rho_{1}$, and $\theta$ are parameters and $J_{5}(\theta / T)$ is the transport integral defined by

$\ln (\theta / T)=2^{n-1} \int_{0}^{\theta / 2 \pi} x^{n / \sinh ^{2} x d x}$

Wilson's modification of Eq. (1) is

$p(T)=p_{0}+2 p_{1} T(T / \theta)^{2} / 3(\theta / T)$.

Since Eqs. (1) and (3) vary as $T^{5}$ and $T^{3}$, respectively, at low temperatures, we also fitted the resistivities up to a temperature $T_{0}$ with the simpler expression

$p(T)=p_{0}+A T^{n}$,

where $A$ and $n$ are constants. The results of the analyses are given in Table 3.1 .

1. Summary of paper: Solid State Commun. 68, 387 (1988).

2. Guest srientist from Oklahoma State University, Stillwater, Okla.

3. Oak Ridge Associated Universities student research participant from Oklahoma State University, Stillwater, Okla. 
Table 3.1. Parameters describing the electrical resistivity of transition-metal carbides. Values for $\mathrm{ZrC}_{0.53}$ and $\mathrm{VC}_{0.3}$ were obsained by fitting the data with Eq. (1), and values for $\mathrm{Nb}_{0.95}$ and $\mathrm{Ta} \mathrm{C}_{a .9}$ were obtained by fitting with Eq. (3). The exponents $n$ were oblained by fiuting the resistivity dat for temperatures less than $T_{0}$ to Eq. (4).

\begin{tabular}{lcccccc}
\hline & $\begin{array}{c}\rho_{0} \\
(\mu \Omega-\mathrm{cm})\end{array}$ & $\begin{array}{c}\theta \\
(\mathrm{K})\end{array}$ & $\begin{array}{c}\rho_{1} \\
(\mu \Omega-\mathrm{cm} / \mathrm{K})\end{array}$ & $n$ & $\begin{array}{c}\sigma_{0} \\
(\mathrm{~K})\end{array}$ \\
\hline $\mathrm{ZrC}_{0.93}$ & 180.79 & 570 & 0.490 & $4.8-4.0$ & $35-50$ \\
$\mathrm{VC}_{0.83}$ & 2.46 & 587 & 0.123 & $4.4-3.8$ & 4070 \\
$\mathrm{NbC} C_{0.95}$ & 70.45 & 431 & 0.0373 & $3.0 \pm 0.1$ & $40-0$ \\
$\mathrm{TaC} C_{0.99}$ & 12.53 & 272 & 0.0495 & $3.0 \pm 0.1$ & $25-40$ \\
\hline
\end{tabular}

\section{SINGLE-CRYSTAL GROWTH OF GROUP IVB AND VD CARBIDES BY THE FLOATING-ZONE METHOD}

\section{B. Finch, Y. K. Chang, and M. M. Abraham}

The preparation of Group IVB and VB car. bide single crystals has been an important topic of research for over two decades. Large highquality single-crystal samples are often required in investigate the physical properties of these high-temperature cubic materials that are of considerable fundamental interest. In addition, some carbide single crystals have practical applications as substrates, as wearresistant components, and in high-temperature electronir devices. Their preparation extends c ystal-growth procedures to extreme temperatures and growth co:ıditions, entailir.g process temperatures of up to nearly $4000^{\circ} \mathrm{C}$ and gas prescures of up to $20 \mathrm{~atm}$

The floating-zone method (melt-growth) has been comnonly employed to prepare large single-crystal samples of the transition-metal carbides, although arc, flux, and recrystallization procedures have also been used occasionally. In the floating-zone method, a molten zone, usually inductively heated, is passed slowly through a vertical polycrystalline carbide feed rod. With proper control, the solid crystallizing behind the zone is a single crystal. The molten zone is supported laterally by ifs surface tension and vertically by the solid rods. The technique employs $m$ crucible and can produce high-purity homogeneous single crystals of dimensions often exceeding $1 \mathrm{~cm}$. The difficulty in obtaining good single crystals of these compounds prompted the presently described crystal growth program, the goal of which was to make a wide range of research specimens available for in-depth physical property studies.

We have systematically investigated the technique and prepared large single crystals of $\mathrm{TiC}_{0.95}, \mathrm{ZCC}_{0.93}, \mathrm{ZrC}_{0.98}, \mathrm{VC}_{0.80}, \mathrm{NbC}_{0.95}$, and 
$\mathrm{TaC}_{0.89}$. Optimal growth conditions were in the $0.5-2.0 \mathrm{~cm} / \mathrm{h}$ range under 8-12 atm helium. Good crystal-growth results were achieved with hot-pressed starting rods of 90-95\% density, using a "double pancake" induction coil and a $200-k H z / 100-k W$ if power supply. Growth rates were confirmed to be $0.5-2.5 \mathrm{~cm} / \mathrm{h}$ under 8-12 atm helium.

1. Present address: 809 Chateaugay Road, Knoxville, Tenn.

\section{RADIATION EFFECTS}

\section{STRUCTURAL INEQUIVALENCE OF THE ION-DAMAGE-PRODUCED AMORPHOUS STATE AND THE GLASS STATE IN LEAD PYROPHOSPHATE'}

B. C. Sales, J. O. Remey, L. A. Boatner, and J. C. McCallum ${ }^{2}$

The detailed structural changes that occur during the ion-beam-induced amorphization of a solid have been experimentally determined for the first time using single-crystal surfaces of lead pyrophosphate that were implanted with varying doses $\left(10^{11}\right.$ to $5 \times 10^{15}$ ions $\left./ \mathrm{cm}^{2}\right)$ of $\mathrm{Pb}^{+3}$ ions at $540 \mathrm{keV}$ per ion. The structural information for either the amorphous, the partially disordered, or the glass state was obtained using the modern technique of highperformance liquid chromatography. For all Pb-implant doses the phosphate anion distribution (structure) was found to be significant!y different than the structure of leac pyrophus- phate glass prepared via thermal quenching of the molten liquid. This finding indicates that for this material the ion-damage process should not be viewed as a mechanism that is equivalent to melting the material followed by a thermal quench of the molten liquid. A comparison of the chromatogram of a lead pyrophosphate glass and that from a 100-nmthick amorphous layer formed by ion implantation $\left(10^{15} \mathrm{~Pb}^{+3}\right.$ ions $\left./ \mathrm{cm}^{2}\right)$ is shown in Fig. 3.7. Gross structural differences are obvious for the two materials. Relative to the glass, the percentage of pyrophosphate groups $\left(\mathrm{P}_{2}\right)$ is much lower for the ion-damaged layer, which

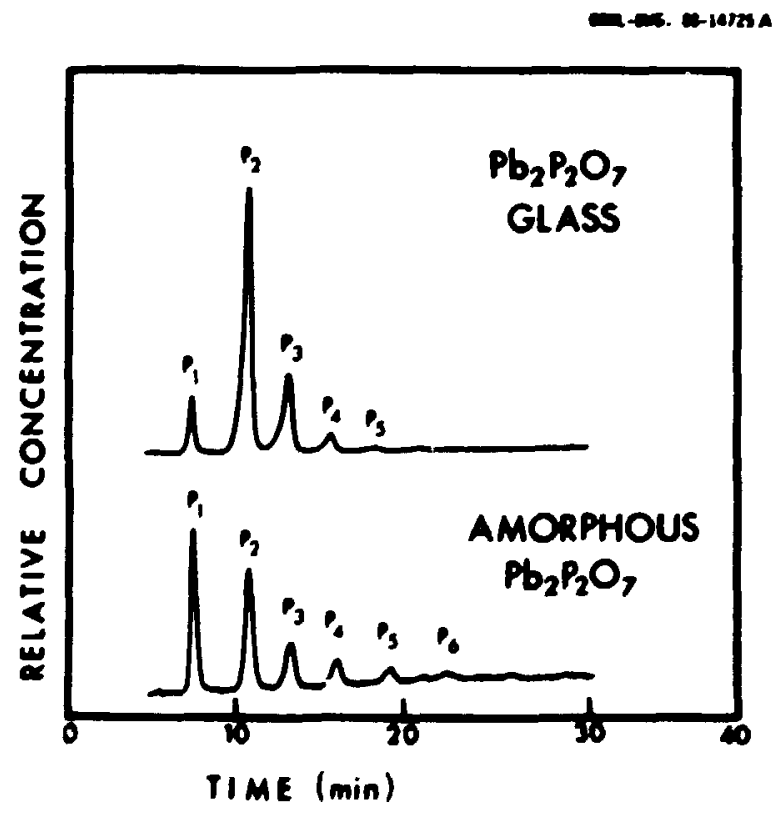

Fig 3.7. Liquid chromatograms from a lead pyrophosphate glase produced by quenching the melt (rop) and from a 100-nm-thick iondamage-produced amorphous layer (bottom) on the surface of a lead pyrophosphate single crystal implanted with $10^{15} \mathrm{~Pb}^{3}$ ions $/ \mathrm{cm}^{2}$ at $540 \mathrm{keV} / \mathrm{ion}$. 
indicates that this layer is actually "more amorphous" or more disordered than the thermally quenched glass. In addition, the glass has a much larger percentage of $P_{3}$ anions, whereas the amorphous-implanted layer has significantly higher concentrations of $P_{1}, P_{4}$, and $P_{5}$ anions. Variations in the local chemical composition (i.e., the local lead to phosphorus ratio) caused by the ion implantation process are believed to be responsible for the formation of long phosphate chains in the ion-damaged layer. This hypothesis is being checked using numerical simulations of the ion damage process. $^{3}$

1. Summary of paper: Phys. Rev. Lett.62, 1138 (1989).

2 Oak Ridge Associated Universities postgraduate resuarch participant.

3. Work in progress.

\section{THE EFFECTS OF GAMMA RRADIATION ON THE SUPERCONDUCTING PROPERTIES OF $\mathrm{CdBa}_{2} \mathrm{Cu}_{3} \mathrm{O}_{7}{ }^{1}$}

\section{E. Sonder, L. A. Bnatiner,}

B. C. Chakoumatos, and B. C. Sales

The effects of gamma irradiation on the superconducting properties of $\mathrm{CdBa}_{2} \mathrm{Cu}_{3} \mathrm{O}_{7 \cdot x}$ have been investigated. No significant changes in the superconducting transition temperature $T_{c}$ or the normal-state resistivity could be ascribed to the gamma irradiation, irrespective of the ambient gas, for gamma dosess up to $1.8 \times$ $10^{10} \mathrm{rad}$ (Fig. 3.8). This result contradicts two previous studies reported in the literature. 2,3

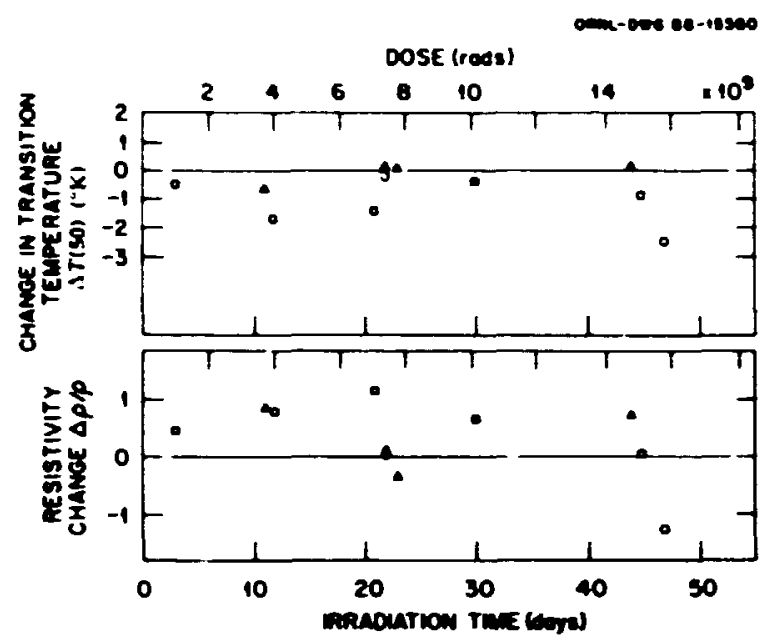

Fig. 3.8. Differences in the measured transition temperature (a) and the normal-state resistivity (b) for irradiated samples (open symbols) and control samples (filled symbols). The different symbols indicate different ambients: $(\Delta)$ irradiated in vacuum, ( $(0) 0.9 \mathrm{~atm} \mathrm{He}$, and (o) 0.9 atm oxygen.

Reasonable explanations for these discrepancies were developed based on the observed inherent sample-to-somple variations of $T_{c}$ or the normal-state resistivity, the extreme sensitivity of these materials to inadvertent he.ating at temperatures as low as $200^{\circ} \mathrm{C}$, and the tendency of these ceramics to develop microcracks with thermal cycling.

1. Summary of paper to be published.

2. E. Adem et al., I. Mater. Res. 3, 807 (1988).

3. W. G. Maisch et al., IEEE Trans. Nucl. Sci. NS34, 1781 (1987). 
MICROHARDNESS IN NATURAL ZIRCON AS A FUNCTION OF Q-DECAY DOSE COMPARED WITH SYNTHETC ZIRCON DAMAGED BY FO ION IMPLANTATION

\author{
B. C. Chatoumatos, J. C. McCallum, 2 \\ L. A. Boatner. W. C. Oliver. \\ G. R. Lumplin, and R. C. Ewing
}

A mechanical properties microprobe (MPM) has been used to measure the hardness and elastic modulus as a function of a-decay dose in a natural single crystal of zircon $\mathrm{ZZSiO}_{4}$ from Sri Lanka) that exhibited zonation (1 to 100 mm thick) in its $U$ and Th concentration. The probed zones have $U$ and Th concentrations such that the $\alpha$-decay dose varies between $0.2 \times 10^{16}$ and $0.8 \times 10^{16} \alpha$-decays $/ \mathrm{mg}(0.15$ to $0.60 \mathrm{dpa})$, a dose range that covers the crystalline-tometamict transition. A traverse of 75 indent pairs across zones spanning the entire dose range reveals hardness values that correlate $\left(r^{2}=0.99\right)$ inversely with increasing $\alpha$-decay dose. For comparison, pure synthetic single crystals of zircon I(100) growth surfaces] were implanted with $540 \mathrm{keV}{ }^{208} \mathrm{~Pb}^{3+}$ ions with fluences of $3.3 \times 10^{11}$ to $5 \times 10^{15}$ ions $/ \mathrm{cm}^{2}$, a dose range which completely spans the crystallineto-metamict transition as determined by Rutherford backscattering analysis. The implanted crystals, similarly examined by MPM, exhibit the same radiation-induced softening through the crystalline-to-metamict transition. Below the transition, radiation-induced hardening, which may be caused by the accumulation of interstitials, occurs.

1. Summary of paper to be published.
2. Oak Ridge Associated Universities postgraduate research participant.

3. Metals and Ceramics Division, ORNL

4. Australia Nuclear Science and Technology Organization, New South Wales, Australia.

5. University of New Mexico, Albuquerque, N. Mex.

\section{ENHANCEMENT OF COLOR CENTER LUMINESCENCE IN NEUTRON- IRRADIATED $\mathrm{Al}_{2} \mathrm{O}_{3}{ }^{1}$}

\section{G. J. Pogatshnit, 2 Y. Chen, and B. D. Eanns}

Neutron-irradiated sapphire is somewhat unique among the oxide crystals in that it can support a number of singlesite and aggregate Ft, e color centers. In addition to the $F$ and $F^{+}$ defects, there are others consisting of dusters of two or more F-type centers. The spectroscopic properties of these aggregate defects depend on the size of the cluster, their valence state, and their orientation in the crystal matrix. A number of these centers are efficient emitters whose luminescence covers the spectral range from the near uv to beyond $1 \mu \mathrm{m}$ and which are potentially useful as hosts for solid state tunable lasers.

Neutron irradiation produced broad absorption throughout the visible region. In the ultraviolet, the absorption was dominated by the presence of the $F$ and $F^{+}$centers. Annealing at $773 \mathrm{~K}$ produced distinct absorption bands associated with various charge states of anion vacancy clusters. These bands were highly anisotropic and covered much of the visible spectrum. The absorption bands near 300 and $355 \mathrm{~nm}$ have been tentatively identified as $\boldsymbol{F}_{\mathbf{2}}$ type centers with differing charge states. The 
$F_{2}$ center associated with the $300-n m$ absorption band is thought to be charge neutral with respect to the lattice. The 355-nm band results from an $F_{2}$ center which has been singly ionized and is designated an $\mathrm{F}_{2}{ }^{+}$center. ${ }^{5}$ Recent results from photobleaching experiments were consistent with this assignment.

Optical excitation of the $\mathrm{F}_{2}{ }^{+}$center produced a broad band emission which peaked at $385 \mathrm{~nm}$. The emission intensity of this $\mathrm{F}_{2}{ }^{+}$center may be enhanced in several ways. We observed that the emission was highly quenched at room temperature so that cooling the sample to $77 \mathrm{~K}$ produced an order of magnitude increase in the luminescence. A productive method to increase $\mathrm{F}_{2}{ }^{+}$-center concentration was by photoionization of $F_{2}$ centers. The excited state of the $F_{2}$ center lies near the conduction band; $300-\mathrm{nm}$ illununation ionized these centers at room temperature. In our samples, we have been able to convert about $90 \%$ of the $F_{2}$ centers to $F_{2}{ }^{+}$. The reduction of the absorption near $\mathrm{F}_{2}{ }^{+}$center emission wavelengths enhanced the $385-\mathrm{nm}$ luminescence.

1. Summary of paper: I. Lumin. 40/41, 315 (1988).

2. Guest scientist from North Carolina State University, Raleigh, N. C.

3. Boeing Electronics Co., Seattle, Wash.

4. G. P. Pells, A. E. Hughes, and L. S.

Welch, I. Phys. C 13, 1805 (i25;0).

5. V. I. Baryshnikov and E. F. Martynovich, Fiz. Tverd. Tela. 28, 1258 (1986); Sov. Phys.Solid Slate 28, 710 (1986).

\section{STORED ENERGY RFCŌVERY OF IRRADLATED COPPERI}

\author{
R. T. Richard, ${ }^{2}$ R. L. Chaplin, 2 \\ R. R. Coltman, Jr., ${ }^{3} H$. R. Kerchner, \\ and $C$. E. Klabunde
}

The stored energy released in stage I recovery of reactor neutron-irradiated copper was measured by differential thermal calorimetry. This study was implemented using the ORNL Low-Temperature Neutron Irradiation Facility (LTNIF). Irradiations up to a maximum of $3.5 \times$ $10^{18} \mathrm{n} / \mathrm{cm}^{2}$ ( $\left.\mathrm{E}>0.1 \mathrm{MeV}\right)$ were carried out at 4 K. A stored energy dependence upon fluence and a tendency toward saturatijin were observed. Theoretical reaction rate processes were fitted to the experimental rates of stored energy release, and the parameters associated with the theory were compared with results from previous measurements. Good agreement was found in the case of several parameters, but major differences from previous 40-50-K annealing results led to the conclusion that the point defect model may not describe materials experiencing this dose of neutron damage.

Computer studies of warmup rates predict a catastrophic stored energy release for damage comparable to the highest fluence carricd out in the LTNIF. Figure 3.9 shows the expected warming of copper with applied heat and with the applied heat turned off when the sample reaches $34 \mathrm{~K}$. The primary motivations for this study were the identification of difficult-tocontrol temperature ranges for postirradiation 


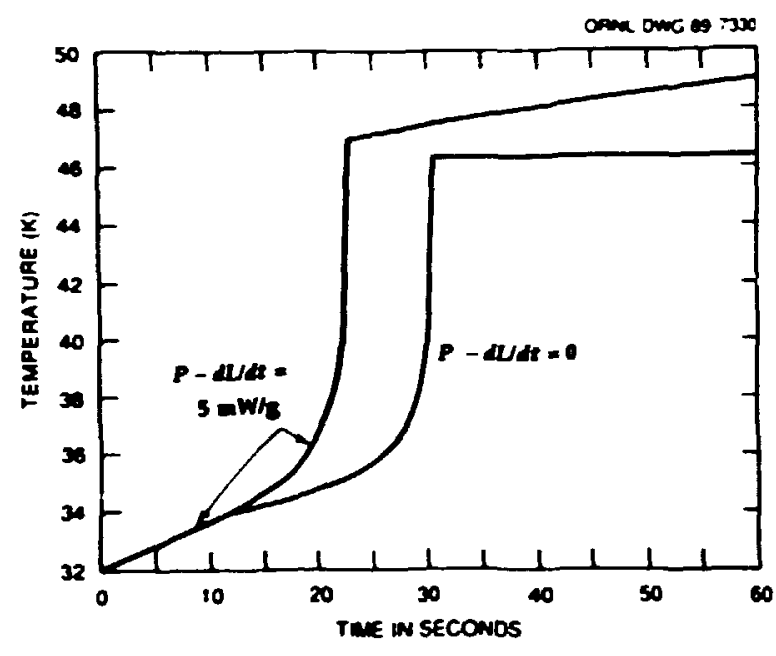

Fig. 3.9. The response of copper when the applied heat less the heat lost, $P-d L / d t$, is reduced to zero close to the onset of a stored energy release (34 K). A fraction of $1 \times 10^{-4}$ displaced atoms and an activation energy of $\mathbf{0 . 1 0}$ eV for defect migration were assumed.
LTNIF studies and the identification of likely temperature bursts which designers of future fusion reactors must anticipate.

1. Summary of papers to be published.

2. Clemson University, Gemson, S. C.

3. Consultant representing self. 


\section{The Synthesis and Characterization of Materials}

Activities in the areas of the synthesis and characterization of materials with new or unique properties represent one of the cornerstones on which the advancement of solid state physics and materials science is based. Efforts of this type have traditionally played a significant role in the overall Solid State Division research program and are strongly linked to either significant energy-related technological problems or to important fundamental scientific investigations.

The first section of this chapter describes the division's research program in the area of thin-film high-temperature superconductors. Two different filn. deposition methods are being employed in this work to produce epitaxial thin films on a variety of substrate materials: a coevaporation method in which yttrium, copper, and barium fluoride are co-deposited and then reacted in wet oxygen to form the Y123 phase and a pulsed-laser ablation method. One highlight of the effort in this area has been the discovery of a new substrate material, $\mathrm{KTaO}_{3}$, for the epitaxial growth of thin films of the $\mathrm{Y} 123$ family of superconductors. This perovskite-structure oxide can be grown either in a highly insulating or semiconducting form or as a ferroelectric material.

The second section of the chapter reports ongoing research efforts in the increasingly important field of solid dielectrics and in the relatively new field of thin-film ceramics and composites. These efforts include a recently initiated program directed toward the realization of a new class of thin-film microbatteries. The final section describes the research activities in the area of materials characterization by optical and magnetic methods. In particular, a new technique for the study of texturing or preferred orientation effects in insulating ceramics has been developed, based on the application of paramagnetic resonance methods. This technique has been applied to the study of texturing effects in sintered $\mathrm{MgO}$ ceramics formed using faceted precursor powders. 


\section{THIN-FILM SUPERCONDUCTORS}

\section{FABRICATION OF EPITAXIAL YBaCuO SUPERCONDUCTING THIN FILMS BY MEANS OF THERMAL COEVAPORATTON}

\section{R. Feenstra and D. B. Poker}

Because of the highly anisotropic crystal structure of $\mathrm{YBa}_{2} \mathrm{Cu}_{3} \mathrm{O}_{7-x}$ (Y123), epitaxial films with different types of orientation are of great scientific interest and potentially of significant future practical value. Thin films offer the additional advantage of having a geometry which is very suitable for characterization and modification by means of ion beams.

The epitaxial Y123 films $(150-400 \mathrm{~nm})$ were produced by means of a coevaporation of $Y, C u$, and $\mathrm{BaF}_{2}$, followed by an ex situ anneal in wet oxygen. This method has emerged as one of the most reproducible established to date. ${ }^{1}$ The films were deposited at a substrate temperature of $450^{\circ} \mathrm{C}$ in a background pressure of oxygen stabilized at $5 \times 10^{-6}$ Torr. The $\mathrm{BaF}_{2}$ was heated resistively, and e-beam guns were used for $Y$ and $\mathrm{Cu}$. A homogeneous composition throughout the films was achieved by separate feedback control of the evaporation rates, which were measured with vibrating quartz thickness monitors located directly above each shielded evaporation source.

As deposited, the films were amorphous, transparent, and insulating. The superconducting Y123 compound was formed by a 30-min anneal at $850^{\circ} \mathrm{C}$ in flowing oxygen bubbled through distilled water, followed by a soak in dry oxygen at $550^{\circ} \mathrm{C}$ to enhance oxygen saturation of the films. Rutherford backscattering (RBS) spectroscopy was used to monitor various stages of the fabrication process. Compositional aralyses by means oi RBS with $5-\mathrm{MeV}$ $\mathrm{He}^{2+}$ ions provided the calibration if evaporation rates needed to obtain the proper Y:Bd:Cu ratios. The molecular deposition of $\mathrm{BaF}_{2}$, responsible for the enhanced stability of as-deposited films, was confirmed by the $\mathrm{F}: \mathrm{Ba}=2: 1$ ratio in films on polished carbon substrates. The substitution of oxygen for fluorine upon annealing in the presence of water vapor was studied for 150 -nm-thick films on $\mathrm{MgO}$ and $\mathrm{He}^{+}$energies below $3.0 \mathrm{MeV}$. For films on (001) $\mathrm{SrTiO}_{3}$, the epitaxial ordering of the films was confirmed by ion channeling with the incident $2-\mathrm{MeV} \mathrm{He}{ }^{+}$beam aligned with the (001) direction of the substrate. Typically, minimum backscattering yields of $40-60 \%$ were obtained.

Different epitaxial orientations $(a \perp, c \perp)$ could be obtained by varying the annealing procedure. In particular, significantly larger c $1: a \perp$ ratios were observed in films that were preannealed at $750^{\circ} \mathrm{C}$ in dry oxygen (before the anneal at $850^{\circ} \mathrm{C}$ in wet oxygen). The cause for this enhancement of $c \perp$ growth, favorable for the achievernent of high critical currents, is not clear and is currently under investigation.

1. P. M. Mankiewich ct al., Appl. Phys. Lett. 51, 1753 (1987). 
EPTTAXAL SUPERCONDUCTING

THIN FILMS OF $\mathrm{YB}_{2} \mathrm{Cu}_{3} \mathrm{O}_{7} \mathrm{ON}$

KTaO $\mathrm{O}_{3}$ SINGLE CRYSTALS

R. Feenstre, L. A. Boatner, J. D. Budai, D. K. Christen, M. F. Chisholm, and $M$. D. Galloway

Until recently, the best superconducting $\mathrm{YBa}_{2} \mathrm{Cu}_{3} \mathrm{O}_{7-x}$ (Y123) films were obtained on (001) $\mathrm{SrTiO}_{3}$, which combines a relativeiy low chemical affinity for Y123 with an excellent lattice match between the substrate and film. With other substrates such as $\mathrm{Al}_{2} \mathrm{O}_{3}$, cubic $\mathrm{Z} \mathrm{rO}_{2}, \mathrm{Si} / \mathrm{SiO}_{2}$, and $\mathrm{MgO}$, the superconducting properties are adversely affected by a severe interdiffusion across the film-substrate interface or by the formation of a reaction layer during the fabrication process. Alternate "clean" substrate materials that are capable of sustaining high-temperature anneals are obviously desirable for both fundamental studies and possible device applications.

In this work, single-crystalline $\mathrm{KTaO}_{3}$ (a cubic perovskite, like $\mathrm{SrTiO}_{3}$ ) was shown to be a promising altemate substrate for the epitaxial growth of Y123 thin films. Doped and updoped single crystals of $\mathrm{KTaO}_{3}$ were grown by a direct solidification process. From these crystals, (001) growth surfaces and cleaved or polished surfaces were used as substrates. The Y123 films were grown by coevaporation of $Y$, $\mathrm{BaF}_{2}$, and $\mathrm{Cu}$ followed by a postdeposition anneal at $850^{\circ} \mathrm{C}$ in wet oxygen. Films with a composition close to the 1:2:3 cation ratio exhibited sharp superconducting transitions at $90 \mathrm{~K}$ (Fig. 4.1). The microstructure of Y123 films

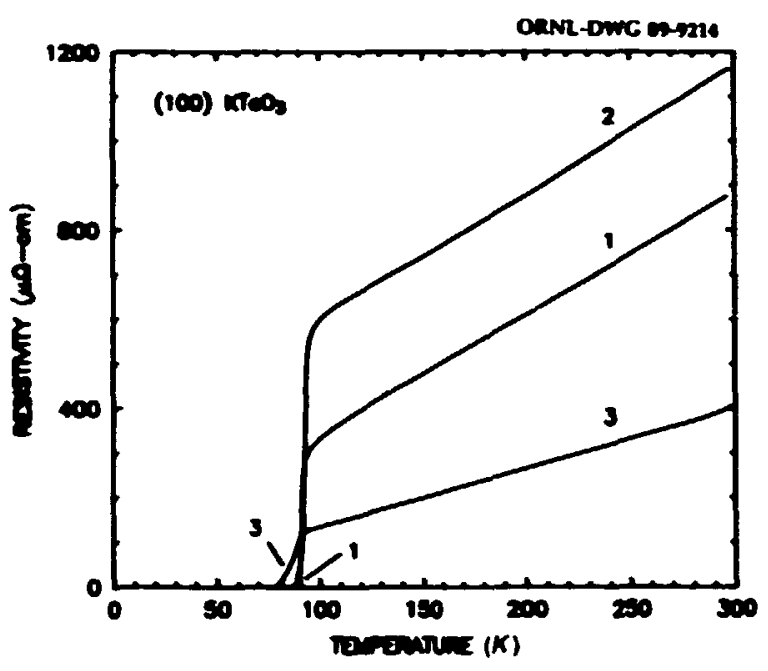

Fig. 4.1. Resistivity vs temperature for stoichiometric Y123 films deposited on the (001) growth surface (curve 1) and a (001) cleaved surface (curve 2) of a $\mathrm{KTaO}_{3}$ single crystal. Film 1 contained more $c \perp$ domains than $a \perp$ domains, whereas film 2 contained more a $\perp$ domains. Curve 3 is for a Cu-rich finin $(i / \mathrm{Cu}=$ $0.27 ; \mathrm{Ba} / \mathrm{Cu}=0.50$ ), containing the $\mathrm{Y} 248$ phase with $T_{c}=80 \mathrm{~K}$ on (001) $\mathrm{KTaO}_{3}$ (growth surface).

on (001) $\mathrm{KTaO}_{3}$ was found to be quite similar to that of films on (001) $\mathrm{SrTiO}_{3}$ prepared under the same conditions. The films were epitaxial in three dimensions, and no reaction layer was observed at the film-substrate interface. Critical current densities in excess of 0.3 $\mathrm{MA} / \mathrm{cm}^{2}$ at $77 \mathrm{~K}$ and $1 \mathrm{MA} / \mathrm{cm}^{2}$ at $4 \mathrm{~K}$ were measured in zero magnetic field.

1. Summary of paper: Appl Phys. Lett. 54, 1063 (1989). 
TRANSPORT PROPERTIES OF $\mathrm{Y}_{1} \mathrm{Ba}_{2} \mathrm{Cu}_{3} \mathrm{O}_{7}$ THIN FILMS

D. K. Christen, C. E. Klabunde, R. Feenstra, J. R. Thompson,'

H. R. Kerchner, and S. T. Sekula

Thin films of $\mathrm{Y}_{1} \mathrm{Ba}_{2} \mathrm{Cu}_{3} \mathrm{O}_{7}$ were produced by coevaporation of $\mathrm{Cu}, \mathrm{Y}$, and $\mathrm{BaF}_{2}$ onto substrates of single-crystal $\mathrm{SrTiO}_{3}$ and $\mathrm{KTaO}_{3}$ followed by annealing at $850 \mathrm{C}$ in wet oxygen. Depending on the processing conditions, films deposited on (100) substrate surfaces showed preferential epitaxy corresponding to $c, a$, or mixed $c$ and $a$ axes parallel to the surface normal. Depositions on (110) substrates produced films composed of finely divided domains having [110]- and [103]-type orientations. Transport critical current densities $/ c$ were strongly correlated with the film morphology; in particular, the (110) films had $I_{c}$ values about four orders-of-magnitude smaller than those of the best c-oriented films, which showed dissipation-free conduction that approached estimated material limits with $I_{c}>1 \mathrm{MA} / \mathrm{cm}^{2}$ in zero magnetic field at $85 \mathrm{~K}$. These results are illustrated in Fig. 4.2, where the high-temperature data for three of the best films are plotted in comparison with estimated intrinsic upper limits to /c. The curves represent the theoretical depairing limit, given by

$$
I_{c}=\frac{1.73 c H_{c}(0)}{3 \sqrt{3} \pi \lambda(0)}(1-8)^{3 / 2}
$$

and the limit imposed by a current-induced sclf-field $H_{c l}$, given by

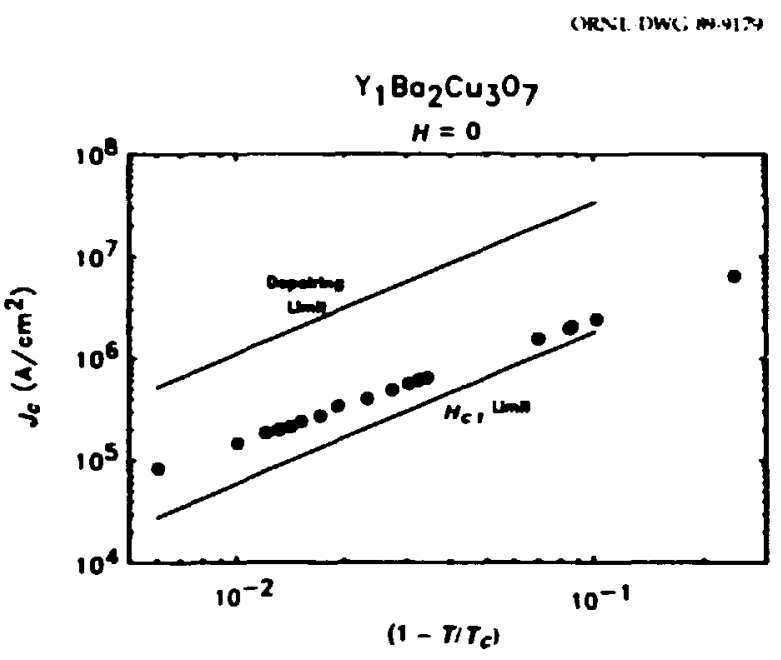

Fig. 4.2. The transport critical current density $I_{c}$ as a function of reduced temperature near the transition temperature $T_{c}$. The solid lines are intrinsic upper bounds on $l_{c}$ imposed by two different theoretical limits.

$$
I_{c}=\frac{c H_{c \mid}(0)}{2 \pi \lambda(0)}(1-t)^{3 / 2}
$$

where $t=T / T_{c}$. For these estimates, published superconducting parameters obtained from measurements on high-quality single crystals were used. ${ }^{2}$ Current-voltage dependencies in applied field indicate substantial flux creep effects and can be compared to flux creep parameters determined sy dc magnetization relaxation and ac loss measurements.

1. Adjunct research and development participant from The University of Tennessea, Knoxville, Tenn.

2. A. Umezawa et al., Phys. Rev. B 38, 2843 (1988). 
RESISTTVE AND STRUCTURAL PROPERTIES OF YBaCuO FILMS PRODUCED BY MEANS OF THERMAL COEVAPORATIONI

R. Feenstra, J. D. Budai, M. F. Chisholm, M. D. Galloway, and D. K. Christen

The microstructure of $\mathrm{YBa}_{2} \mathrm{Cu}_{3} \mathrm{O}_{7 \cdot x}\left(\mathrm{Y}_{123)}\right.$ films (thickness $200-400 \mathrm{~nm}$ ) on $\mathrm{SrTiO}_{3}$ singlecrystal substrates was studied by $x$-ray diffraction, scanning electron microscopy (SEM), transmission electron microscopy (TEM), and ion beam analyses (Rutherford backscattering-ion channeling). The films, produced by a coevaporation of $\mathrm{Y}, \mathrm{BaF}_{2}$, and $\mathrm{Cu}$, followed by a postdeposition anneal at $850^{\circ} \mathrm{C}$ in wet oxygen, were granular with grain dimensions between 0.2 and $10 \mu \mathrm{m}$. The grains were in (3D) epitaxial registry with the substrate with either the $c$-axis or the $a$-axis of the Y123 unit cell perpendicular to (001) $\mathrm{SrTiO}_{3}$ substrates. Generally both orientations existed within the same film. TEM cross seciions showed that the plate-like $c \perp$ domains were predominantly situated at the film-substrate interface, with the $a \perp$ platelets growing out of the $c \perp$ layer, thus forming a connected rectangular network of grains at the surface of films containing a sufficiently large fraction of a $\perp$ domains. Secondary phases were commonly observed at the surface of the films and also as granular inclusions, even if the $Y: B a: C u$ ratio was within $5 \%$ of the 123 stoichiometry. As a result, the films were rough with thickness variations of 50-100 nm.

The superconducting and normal state resistive properties of the films depended critically on the microstructure and orientation of the film, as expected. In Fig. 4.3, this dependence

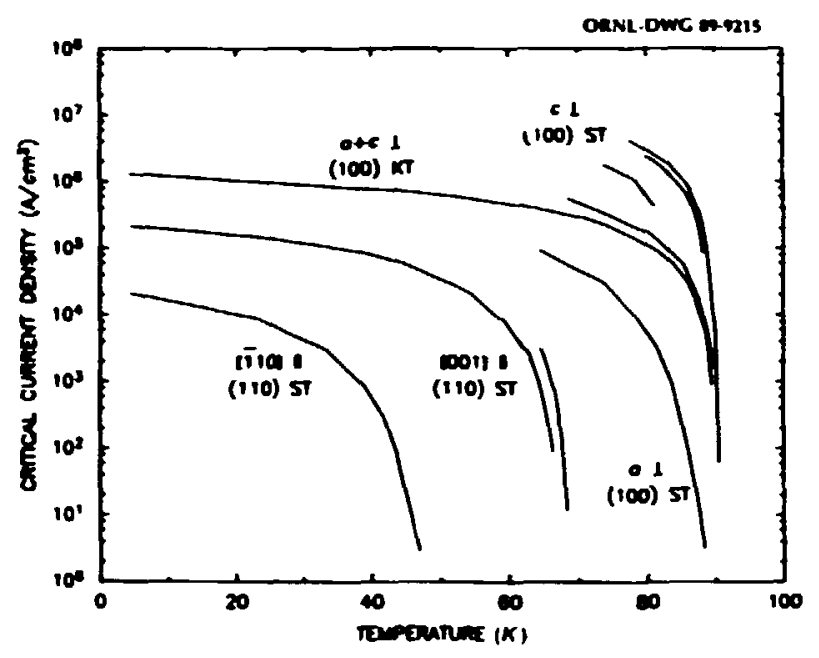

Fig. 43. Critical current density as a function of temperature for epitaxial Y123 films on (001) and (110) $\mathrm{SrTiO}_{3}$ and (001) $\mathrm{KTaO}_{3}$ substrates. The dominant epitaxial orientations are indicated in the figure. For the films on (110) $\mathrm{SrTiO}_{3}$, the critical currents were measured along two in-plane lattice directions.

is illustrated for the critical current density $J_{c}$. In films that were mainly $c \perp$, critical currents as high as $4 \mathrm{MA} / \mathrm{cm}^{2}$ at $77 \mathrm{~K}$ were measured repeatedly in several samples. In films with a high $a \perp: C \perp$ ratio, depending on temperature $J_{c}$ was about 100-400 times smaller. In Y123 fil.ns on (110) $\mathrm{SrTiO}_{3}$ consisting of many small grains with the conductive $\mathrm{Cu}-\mathrm{O}$ planes either perpendicular to the substrate $([110] \perp)$ or at a $45^{\circ}$ angle with the substrate $([103] \perp), I_{c}$ was 3 to 4 orders of magnitude smaller than in the $c 1$ films.

1. Summary of paper: Proceedings of the Conference on the Science and Technology of Thin-Film Superconductors, Colorado Springs, Colo., November 14-18, 1988 (in press). 


\section{MAGNETIC AC RESPONSE OF SUPERCONDUCTING THIN FILMS ${ }^{1}$}

H. R. Kerchner, R. Feenstra, I. O. Thomson, ${ }^{2}$ I. R. Thompson, ${ }^{3}$

D. K. Christen, and S. T. Setula

The ac magnetic susceptibility of $\mathrm{YBa}_{2} \mathrm{Cu}_{3} \mathrm{O}_{x}$ films was studied in perpendicular $\mathrm{dc}$ and collinear ac magnetic fields. Films with different epitaxial orientations on single-crystal $\mathrm{SrTiO}_{3}$ were produced by coevaporation of $\mathrm{Y}$, $\mathrm{Cu}$, and $\mathrm{BaF}_{2}$ followed by an anneal at $850^{\circ} \mathrm{C}$. The apparent critical current density of these films as deduced from the ac susceptibility is in reasonable agreement with that determined from measurements of dc magnetic hysteresis and four-terminal current transport. The voltage waveform from pickup coils of the mutual inductance bridge clearly shows slow decay of the film's transport supercurrent. As a result of these complex dynamic characteristics, the deduced critical current density is frequency dependent.

Figure 4.4 shows the applied ac-fieldamplitude dependence of a film's ac moment at a temperature of $20 \mathrm{~K}$. At high amplitudes the moment is independent of $H_{e c}$ and gives the circulating transport current indicated on the ordinate scale. The frequency dependence is apparently nonlinear as expected from the very curved current-voltage characteristics described elsewhere."

1. Summary of paper to be published.

2. Consultant from The University of Tennessee, Knoxville, Tenn.
3. Adjunct research and development participant from The University of Tennessee, Knoxville, Tenn.

4. D. K. Christen et al., "Transport Dissipation in $\mathrm{Y}_{1} \mathrm{Ba}_{2} \mathrm{Cu}_{3} \mathrm{O}_{7}$ Thin Films," this report.

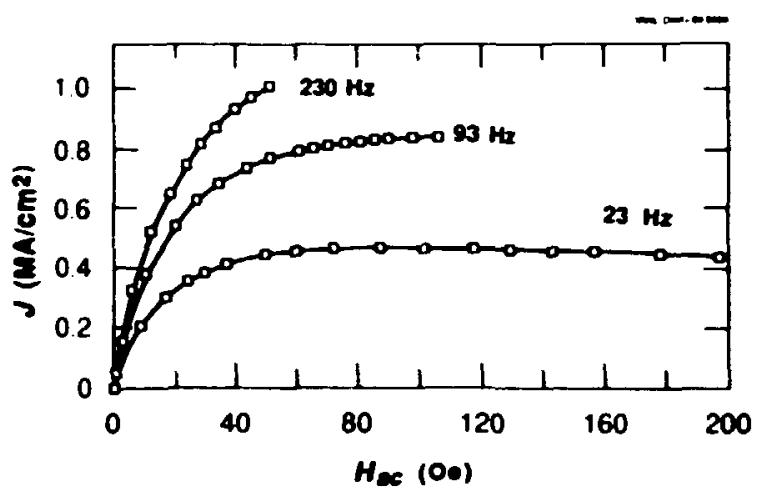

Fig. 4.4. Circulating current induced in an $\mathrm{YBa}_{2} \mathrm{Cu}_{3} \mathrm{O}_{x}$ film in an applied dc field of $1.2 \mathrm{~T}$ as a function of the applied ac field amplitude at three frequencies.

\section{PULSED-LASER DEPOSITION OF THIN SUPERCONDUCTING FILMS OF $\mathrm{HoBa}_{2} \mathrm{Cu}_{3} \mathrm{O}_{7-x} \wedge \mathrm{ND} \mathrm{YBa}_{2} \mathrm{Cu}_{3} \mathrm{O}_{7-x} 1$}
D. B. Geohegan, D. N. Mashburn,

R. I. Culbertson,2 S. I. Pennycook, I. D. Budai,

R. E. Valiga, ${ }^{3}$ B. C. Sales, D. H. Lowndes,

L. A. Boatner, E. Sonder, D. Eres,

D. K. Christen, and W. H. Christic ${ }^{3}$

Thin superconducting films of high- $T_{c}$ $\mathrm{HoBa}_{2} \mathrm{Cu}_{3} \mathrm{O}_{7-2}$ and $\mathrm{YBa}_{2} \mathrm{Cu}_{3} \mathrm{O}_{7-2}$ were deposited by pulsed $248-\mathrm{nm}$ laser ablation of stoichiometric target pellets. These films were subsequently studied by secondary un mass spectroscopy, Rutherford backscattering, energy dispersive $x$-ray analysis, transmission electron microscopy (TEM), and $x$-ray diffraction 
(XRD). The stoichiometry of as-deposited films was investigated for varying laser energy densities. Two regimes were observed. Below $0.5 \mathrm{~J} / \mathrm{cm}^{-2}$, stoichiometric 123 films were unattainable because of a deficiency of $\mathrm{Y}$ or $\mathrm{Ho}$ in the films, as one would expect based upon evaporation alone. Between 0.8 and $3.5 \mathrm{~J} / \mathrm{cm}^{-2}$, however, a laser-generated plasma formed, and the stoichiometry of the films matched that of the pellet to within 10\%. This is the laser ablation regime.

Four-point probe resistance vs temperature profiles of the annealed films deposited on (100) $\mathrm{SrTiO}_{3}$ (Fig. 4.5) gave superconducting transition temperatures nearly equal to those of the bulk material $\left(T_{c}=93 \mathrm{~K}\right)$, but with the transitions they broadened to $10 \mathrm{~K}$ (vs $\sim 2 \mathrm{~K}$ for the pellets). Microstructural analysis with TEM revealed epitaxial alignment with the

OAMK.DWC LAM.82238

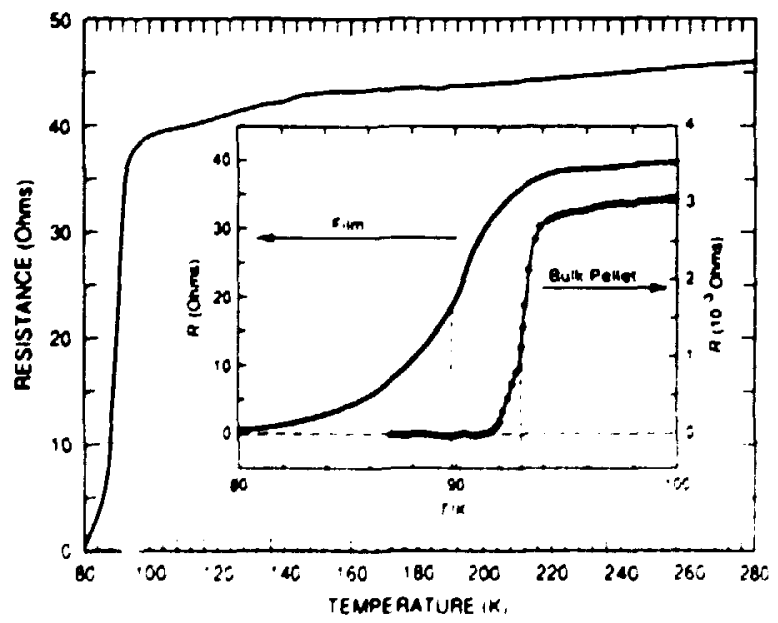

Fig. 4.5. Four-point probe resistance vs temperature of a $1-\mu \mathrm{m}$-thick $\mathrm{HoBa}_{2} \mathrm{Cu}_{3} \mathrm{O}_{7}$ film deposited on $\mathrm{SrTiO}_{3}$ in vacuum at $40{ }^{\circ} \mathrm{C}$. The inset compares the superconducting transition region for this film and a bulk pellet. substrate (with either the a- or c-axis of the superconductor unit cell perpendicular to the substrate $(a \perp$ or $c \perp)$ and a clean, sharp interface between substrate and film. $X$-ray diffraction studies showed that both orientations were aligned within $0.4-0.9^{\circ}$ of surface normal and that the majority of the films were oriented $a \perp$. In addition, lattice constants measured by XRD ( $a \perp$ : $a=3.84 \pm 0.005 \AA$ ) deviated significantly from fully oxygenated bulk material ( $a=3.82 A$ ), indicating strains imposed by epitaxy with the substrate.

1. Summary of paper. I. Mater. Res. 3, 1169 (1988).

2. Present address: U.S. Army Materials Technology Laboratory, Watertown, Mass. 3. Analytical Chemistry Division, ORNL.

\section{FUNDAMENTAL STUDIES AND DEVELOPMENT OF PLASMA-ASSISTED PULSED-LASER DEPOSITION OF OXIDE SUPERCONDUCTOR THIN FIUMS}

\section{N. Mashbum, D. H. Lowndes, and D. B. Geohegan}

Research in the Solid State Division, at The State University of New York-Buffalo, and at Bell Communications Research Laboratories has established plasma-assisted laser deposition (PLD) as a promising process for reduced temperature $\left(-600^{\circ} \mathrm{C}\right)$ oxide superconductor thin-film deposition on the laboratory scale. Detailed understanding of the physical mechanisms at work during reduced temperature deposition is no longer just a matter of scientific curiosity but has become essential knowledge 
for process development and possible wider utilization of this technology. We are currently carrying out a coordinated basic research and process development program, with the goal of developing a practical method for rapid, continuous deposition of oxide superconductor thin films with high current-carrying capacity on technologically important substrate materials. Pulsed-laser ablation is being used together with a glow discharge to achieve rapid in situ oxidation at reduced substrate temperatures in orde to overcome problems associated with the inreractions between superconducting films and substrates at elevated $\left(-850-900^{\circ} \mathrm{C}\right)$ temperatures. Other essential parts of the work include the deposition of crystallographically oriented c-axisnormal oxide superconductor films on thin buffer layers and sequential deposition of superconductor, insulator, and normal metal films from multiple laser-ablation targets in a single chamber. In these ways we hope to obtain high-critical current densities in practical oxide superconductor structures.

Early in 1989, a second PLD facility was constructed, based on a large turbo-pumped stainless steel cylinder equipped with 12 access ports in the target-substrate-laser beam plane. It can be pumped to the low $10^{-7}$ Torr range or throttled for continuous operation at wellcontrolled pressures in the 1-1000 mTorr $\mathrm{O}_{2}$ pressure range. The larger chamber volume offers considerable freedom for a varicty of experimental setups. It is equipped with a substrate heater (to $800^{\circ} \mathrm{C}$ ) that can be used in vacuum, atmospheric pressure oxygen, or glow discharges in whirh electrical isolation is required. The chamber is equipped with UVgrade fused silica windows, laser beam-focusing optics and a laser-energy monitor, electrically isolated rotation of the ablation target, highvoltage feedthroughs, and a flow-controlled oxygen injector. This facility has been installed in the time-resolved laser spectroscopy laboratory in order to take advantage of existing transient digitizers, a streak camera, visible and VUV spectrometers, a mass spectrometer, excimer and $\mathrm{Ar}^{+}$lasers, and a computer-based data-acquisition system. We plan to add to this facility a reflection highenergy electron diffraction capability and a remote measuring microscope.

\section{TRANSIENT OPTICAL ABSORPTION SPECTROSCOPY IN LASER-GENERATED ABLATION PLUMES OF HIGH-T $\boldsymbol{T}_{c}$ OXIDE SUPERCONDUCTORS}

\section{B. Geohegan and D. N. Mashbum}

Recent experiments suggest that laser ablation has several unique features for deposition of high- $T_{c}$ oxide superconductor thin films. Epitaxial films can be prepared with the correct stoichiometry and oxygenated in situ at lower temperatures than with other techniques. The high translational energies (1-40 eV) of the neutrals, ions, and electrons in the excited laser-generated ablation plume are believed to assist these processes. Previous spectroscopic techniques aimed at understanding 
the complex transport in the ablation process have centered on time-of-flight measurements of fluorescence to determine the species in the plume and their velocities. The fluorescence arises from electron collisional excitation of the neutrals and recombination with the ions in the plume as the laser-produced plasma expands under the constraints of space-charge neutrality. In contrast, our current research centers on monitoring the time dependence of the ground state neutrals of $\mathrm{Y}, \mathrm{Ba}$, and $\mathrm{Cu}$ by transient optical absorption. A pulsed-Xe lamp provides an intense broadband source to probe the ablated vapor at various delay times after the arrival of a high-intensity ArF (193-nm) laser pulse. A $1-m$ spectrometer with $0.07-\AA$ resolution enables us to observe atomic absorption lines that are typically broadened to $\sim 0.3 A$ in the plume. Ground state ions (e.g., $\mathrm{Y}^{+}, \mathrm{Ba}^{+}$) and molecules (e.g., YO) also are observable in absorption.

The time dependences of the absorption by ground state Y, Ba, and $\mathrm{Cu}$ (Fig. 4.6) reveal an additional, long-lived component to the ablation transport process, with neutrals existing for times much longer than the fluorescence from the laser plume (see inset). The dissimilar time dependences of $\mathrm{Y}, \mathrm{Ba}$, and $\mathrm{Cu}$ neutrals measured with this technique profoundly alter our understanding of pulsed-laser deposition since they reveal a component of the process that results in layered deposition as the $\mathrm{Y}, \mathrm{Ba}$, and $\mathrm{Cu}$ neutrals arrive at the substrate at different times.

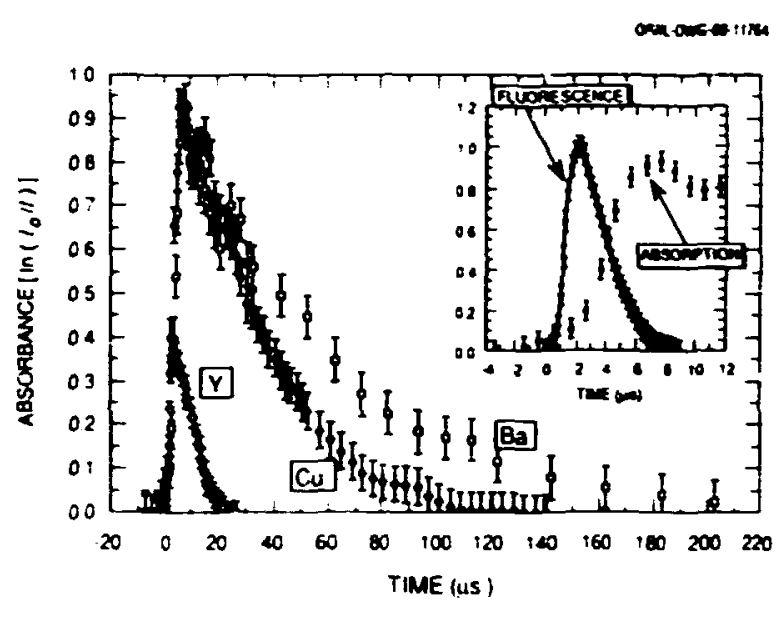

Fig. 4.6. The time dependences of absorption by $\mathrm{Y}, \mathrm{Ba}$, and $\mathrm{Cu}$ neutrals in the laser-generated ablation plume, $2.0 \mathrm{~cm}$ from a $\mathrm{YBa}_{2} \mathrm{Cu}_{3} \mathrm{O}_{7}$ pellet irradiated by Ari: laser pulses (193 nn, 25 ns, 1 $\mathrm{J} / \mathrm{cm}^{2}$ at $t=0$ ). The time dependences contrast with those obtained by time-of-flight nuorescence, which indicate nearly equal velocities for the three components. The inset compares the absorption and emission time dependences for the barium $6 p^{1} P_{1}-6 s^{1} S_{0}$ resonance line at $553.5 \mathrm{~nm}$ for $t<12 \mathrm{~ms}$.

\section{SOLID ELECTROLYTES AND CERAMIC FILMS}

\section{FABRICATION OF THIN-FTLM UTHTUM MICROBATTERIES}

\author{
J. B. Bates, N. I. Dudnay, \\ Y. T. Chu, and P. Mazumdar
}

Thin-film rechargeable lithium microbatteries could have important advantages over commercially available cells as a backup power source for integrated circuits. Being all solid state, their operating temperature range is much larger, and they can potentially be 
fabricated directly onto a chip using standard deposition techniques.

A schematic diagram of one of the prototype celis with a $\mathrm{V}_{2} \mathrm{O}_{5}$ cathode is shown in Fig. 4.J. The open circuit voltage (OCV) of this cell is determined by the free energy of reaction be tween lithium and $\mathrm{V}_{2} \mathrm{O}_{5}: x \mathrm{Li}+\mathrm{V}_{2} \mathrm{O}_{5}=\mathrm{ii}_{\mathrm{x}} \cdot{ }_{2} \mathrm{O}_{5}$ $(x \leq 1)$. The substrate was a microscope slide with a sputtered gold current collector. A l-im $\times 1-\mathrm{cm} \times 2-\mu \mathrm{m}$-thick $\mathrm{V}_{2} \mathrm{O}_{5}$ cathode film deposited on the gold current collector was covered by a $1.5-\mathrm{cm} \times 1.5-\mathrm{cm} \times 2-\mu \mathrm{m}$-thick electrolyte film. ${ }^{3}$ A $0.8-\mathrm{cm} \times 0.8-\mathrm{cm} \times 1.5-\mu \mathrm{m}$-thick $\mathrm{Li}$ anode was deposited on the electrolyte by electron beam evaporation and covered by a $1-\mathrm{cm} \times 2-\mathrm{cm} \times 1.0-\mu \mathrm{m}$-thick e-beam-evaporated Ni film to protect the anode and serve as a cur-

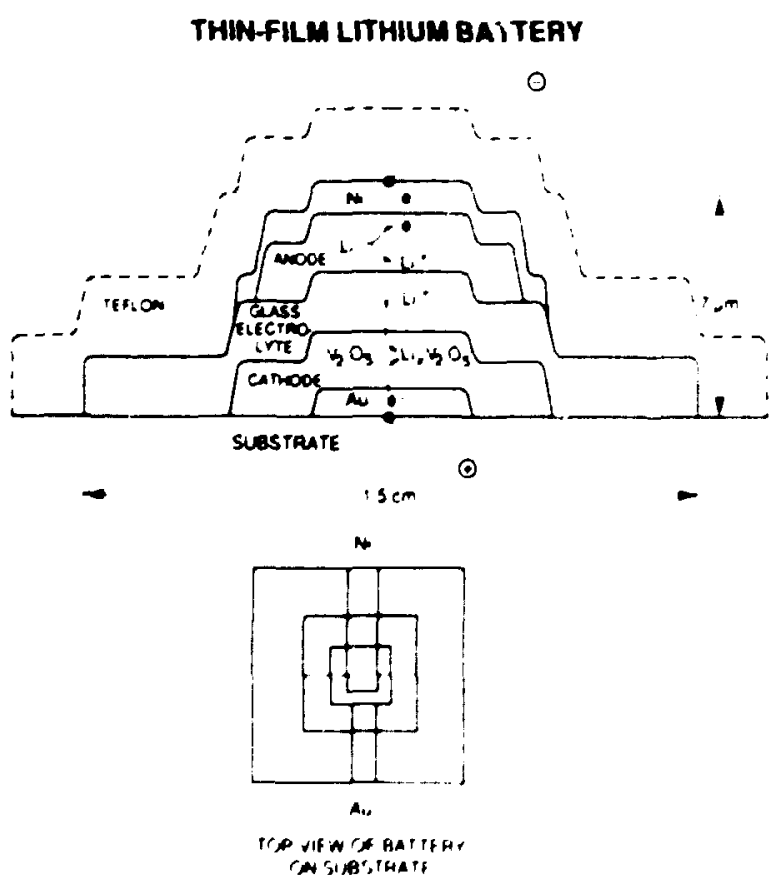

Fig. 4.7. Schematic diagram of the lithium microbattery. rent collector. The initial OCV of this cell was $3.5 \mathrm{~V}$, which decreased to $3.2 \mathrm{~V}$ after standing for a week. Discharge experiments were carried out at currents from $2 \mathrm{nA}$ to $1.5 \mu \mathrm{A}$. The voltage remained steady at $3.2 \mathrm{~V}$ after one hour of discharge at $1.5 \mu \mathrm{A}$. After discharge to $0.1 \mathrm{~V}$, the cell could be recharged to just $1 \mathrm{~V}$. Changes in the color and texture of the anode side of the cell and an increase in the internal resistance from 33 to $710 \mathrm{k} \Omega$ after several weeks of testing suggest that the battery failed due to a slow reaction of the lithium anode with the electrolyte.

1. Present address: Schaffner Technologies, Inc., Knoxville, Tenn.

2. Guest scientist representing self.

3. J. B. Bates et al., "Properties or Electro!yte and Electrode Films Prepared by RF and DC Magnetron Sputtering," this report.

\section{PROPERTIES OF ELECYROLYTE AND ELECTRODE FILMS PREPARED 3 Y RF AND DC MAGNETRON SPUTTERING'}

\author{
J. B. Bates, N. J. Dudney, \\ Y. T. Chu, and P. Mazumdar ${ }^{3}$
}

Thin films of lithium-ion-conducting glass electrolytes and crystalline $\mathrm{TiS}_{2}$ and $\mathrm{V}_{2} \mathrm{O}_{5}$ cathodes were deposited in a high-vacuum chamber by reactive $\mathrm{dc}$ and if magnetron sputtering using small sources designed for $2.54-\mathrm{cm}$ targets. The stainless steel bell jar, which forms the top part of the chamber, opens into a glove box so that films can be protected from exposure to oxygen and water vapor.

Targets for the deposition of lithium in conducting glass films were prepared by cold 
pressing and sintering mixed powders of $\mathrm{Li}_{4} \mathrm{SiO}_{4}, \mathrm{Li}_{3} \mathrm{PU}_{4}$, and $\mathrm{Li}_{2} \mathrm{CO}_{3}$, the latter added to make up for the possible loss of lithium during sputtering. Targets were also prepared from sectors of the pure compounds. Films were deposited on heated $\left(-100^{\circ} \mathrm{C}\right)$ alumina and glass substrates by if (13.56 $\mathrm{MHz})$ magnetron sputtering in a $3 / 2 \mathrm{Ar} / \mathrm{O}_{2}$ gzs mixture at a sotal pressure of $20 \mathrm{mTorr}$. In order to achieve a composition in the glass films near $\mathrm{Li}_{4} \mathrm{SiO}_{4}: \mathrm{Li}_{3} \mathrm{PO}_{4}$ it was necessary to use targets with $\mathrm{P} / \mathrm{Si}$ ratios from 4/3 to 2/1. In addition to composition and type of target, the compositions of the films depended on the if power delivered to the target, which was typically between 40 and $70 \mathrm{~W}$. Conductivities of the films ranged from $-2 \times 10^{-7} \mathrm{~S} / \mathrm{cm}$ to $-3 \times 10^{-10} \mathrm{~S} / \mathrm{cm}$, depending on the target and deposition conditions.

Crystallirie films of $\mathrm{V}_{2} \mathrm{O}_{5}$ were prepared by reactive dc magnetron sputtering of vanadium in mixtures of 15 to $50 \% \mathrm{O}_{2}$ in $\mathrm{Ar}$ at a total pressure of $\mathbf{2 0} \mathrm{mTorr}$. Films were deposited on

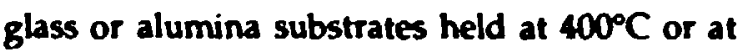
ambient temperatures which increased from 25 to about $50^{\circ} \mathrm{C}$ during deposition. The $\mathrm{V}_{2} \mathrm{O}_{5}$ phase was confirmed by $x$-ray diffraction and Raman scattering. Films deposited on substrates at $400^{\circ} \mathrm{C}$ consisted of loosely packed platelets measuring about $0.5-\mu \mathrm{m}$ thick by ahout $2 \mu \mathrm{m}$ along the edge. Films grown on substrates at ambient temperatures consisted of fibers, $\sim 70 \mathrm{~nm}$ or smaller in diam, that were organized into large clusters about $5 \mu \mathrm{m}$ in diam.
Both kinds of films were highly oriented with the $c$-axis lying in the plane of the substrate.

1. Summary of paper: Meterials Research Saciety Symposium Proceedings (in press).

2. Present address: Schaffner Technologies, Inc., Knoxville, Tenn.

3. Guest scientist representing self.

\section{ANOMALOUS DIEEECTRIC CONSTANT OF $\mathrm{H}_{2}$ O-SATURATED VYCOR'}

\section{J. B. Bates and S. K. Anderson ${ }^{2}$}

Vycor is a high-silica, porous glass with a 28\% void space due to pores that range from 40 to $70 A$ in diam. Our interest in $\mathrm{H}_{2} \mathrm{O}$-saturated vycor stems from renewed efforts to understand anomalous dielectric constants of inhomogeneous materials, especially rocks containing brine for which the dielectric constant can exceed ${ }^{3} 10^{6}$.

Disc-shaped samples of vycor (Coming 1930) measuring $25.4 \mathrm{~mm}$ in diam by $1.2-$ to $3-\mathrm{mm}$ thick were cleaned by treatment in hydrogen peroxide, nitric acid, and distilled water. Wire leads were attached with silver paint to 1-cm-diam sputtered gold contacts on the samples and covered with waterproof epoxy. Prior to each series of measurements, a sample was suspended from the leads in distilled, deionized water $\left(\sigma=4 \times 10^{-6} \mathrm{~S} / \mathrm{cm}\right)$ in a sealed container for several days. Before a measurement, the sample was allowed to stand for several 
hours suspended in the saturated vapor above the liquid.

The results of impedance measurements on the $\mathrm{H}_{2} \mathrm{O}$-saturated vycor are illustrated in Fig. 4.8(a). The solid lines through the data
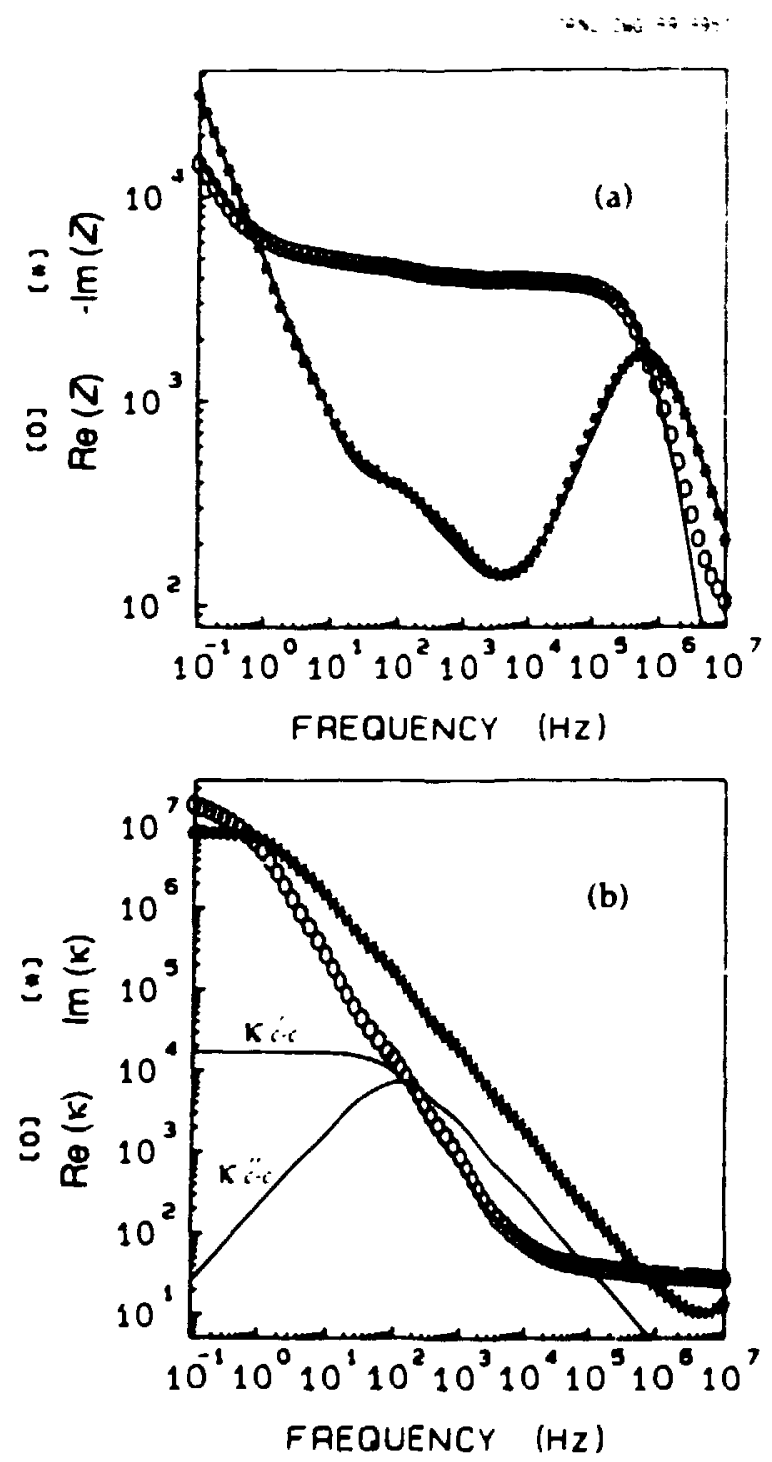

Fig. 4.8. Impedance (a) and permittivity (b) of $\mathrm{H}_{2} \mathrm{O}$-saturated vycor. The solid lines through the data points are the respective calculated values of real and imaginary parts of $Z$ and $K$. The solid lines in (b) are the graphs of $\operatorname{Re}(K)=K_{c \cdot c} \cdot$ and $\operatorname{Im}(K)=K_{\vec{c}-c}$ for the Cole-Cole function representing the anomalous response points are the result of a least-squares fit of a model which includes a Cole-Cole function to represent the anomalous feature in $\operatorname{lm}(Z)$ centered near $200 \mathrm{~Hz}$. In Fig. 4.8(b), the solid line is the graph of the Cole-Cole function showing the contribution of the anomalous response to the total permittivily of the sample which includes the bult resistance and the contact impedance. It can be seen from these results that the static dielectric constant of the sample is about $2 \times 10^{4}$. Attempts to fit extensions of the Maxwell-Garnet or Maxivell-Wagner models to this large dielectric constant resulted in unreasonable aspect ratios for the pores.

1. Summary of paper to be published.

2. Great Lakes College Association science participant from St. Olaf College, Northfield, Minn.

3. P. N. Sen, Appl. Phys. Lett. 39, 667 (1981).

\section{ENHANCED IONIC CONDUCTIVITY IN AgI-ALUMINA COMPOSITES}

\section{N. I. Dudney}

A number of studies demonstrating that the ionic conductivity of AgI below the $\beta-\alpha$ phase transition can be enhanced up to 3 orders of magnitude by the addition of submicron alumina particles have been reported in the literature. This phenomenon has generally becn attributed to the formation of space charge layers with increased carrier concentrations surrounding the alumina particles. However, the enhanced conductivily and thermoclectric power reported for the composites are very 
similar to those reported for the metastable rAgl phase. Studies were initiated to determine if this high-conductivity metastable phase is present in the composites.

Composites were formed by solidifying molten AgI in a low-density alumina fiber paper. Several composites also contained a small volume fraction of alumina particles. The AgI phase was determined by $x$-ray diffraction. As prepared, only the stable $\beta A g I$ phase was detected. However, when the samples were cold pressed, the matrix completely transformed to the rAgl phase. This was accompanied by a large increase in the conductivity. Upon annealing at temperatures near the $\beta-a$ transition, the conductivity decreased and much of the AgI transformed back to the stable phase. Due to coincidence of many of the diffraction lines for the beta and gamma phases, $x$-ray diffraction is not a good technique for evaluating the relative phase composition in samples where there is a significant concentration of the $\beta A g^{\prime}$ phase. Other techniques for determining the phase composition are being evaluated.

\section{COMPOSTTE ELECTROLYTES ${ }^{1}$}

\section{N. J. Dudncy}

The ionic conductivity of over 20 different electrolytic crystalline materials has been increased dramatically, by 10-1000 times, through the addition of fine particles of an insoluble second phase. Typically composites containing 10 to 40 vol \% of submicron alumina particles have the highest conductivities. The mechanism(s) responsible for this enhancement in the ionic conductivity is still a matter of some debate. This review emphasizes the variety of processes that may be contributing to the increased ion transport.

Most studies of composite electrolytes in the literature have focused on evidence for enhanced conduction along the matrix-particle interface. This high conductivity may be caused by enhanced defect mobilities or the formation of sface-charge layers at the interface. However, there is also evidence that the enhanced conductivity is not restricted to the particle interface. The dispersed phase may in fact chemically dope the matrix through partial solution of the very fine particles, react or introduce impurities forming high conductivity phases, alter the grain size or dislocation density of the matrix phase, or stabilize a different crystal phase of the matrix material. Contrary to the impressions given by earlier work on composite electrolytes, there is no reason to expect one mechanism to dominate the conductivity in all the composite electrolyte systems that have been reported.

Recommendations for future research stress the need for more complete characterization of the composites, greater use of well-defined model systems such as single interfaces, and use of advanced processing techniques to prepare composites with well-aligned and regular microstructures.

1. Summary of paper: Ann. Rev. Maler. Sci. 19, 103 (1989). 


\section{ION EXCHANGE REACTION OF SILVER AND SODIUM $\beta "$ "-ALUMINA IN MOLTEN MERCURY SALTS ${ }^{1}$}

\section{N. I. Dudney and I. B. Bales}

Sodium $\beta^{\prime \prime}$-alumina is a crystalline fast $\mathrm{Na}^{+}$ ion conductor. The sodium in the structure can be readily exchanged for a variety of monovalent, divalent, and trivalent cations ty soaking the material in appropriate molten salts. Attempts were made to prepare $\mathrm{Hg} \boldsymbol{\beta}^{\prime \prime}$-alumina for potential use as an electrolyte for a mercury heat engine. Both single-crystal and polycrystalline samples of $\mathrm{Na}$ and $\mathrm{Ag} \boldsymbol{\beta}^{\prime \prime-a l u m i n a s}$ were treated in several molten mercuric salts. The composition of the partially exchanged crystals were analyzed by quantitative analysis of the scanning electron microscopy-energy dispersion $x$-ray spectra.

The results of a number of exchange treatments indicate that the exchange reaction is severely limited by unfavorable thermodynamics. The equilibrium distribution coefficients were evaluated for leactions of $\mathrm{Na}$ and $\mathrm{Ag} \beta^{\prime \prime}$-aluminas in both $\mathrm{Hgl}_{2}$ and $\mathrm{HgCl}_{2}$ sealed under vacuum or backfilled with a small oxygen pressure. The largest exchanges, of about $38 \%$, were achieved for $\mathrm{Ag} \boldsymbol{B}^{\prime \prime}$-alumina crystals treated for five days in a large excess of $\mathrm{HgI}_{2}$.

Calculations were made to predict the conditions that would be necessary to achieve near complete exchange for $\mathrm{Hg}^{2+}$ ions by these molten salt treatments. The composition dependence of the calion activity coefficients in the partially exchanged crystal was assumed to follow a regular solution model. For the range of interation terms considered, it was apparent that multiple exchange treatments with a large excess of a very pure mercury salt would be necessary to produce a viable mercury $\beta$ "-alumina solid electrolyte.

1. Summary of paper: Solid State lonics (in press).

\section{IMPEDANCE OF A FRACTAL ELECTROLYTE-ELECTRODE INTERFACE'}

$$
\text { I. C. Wang }
$$

The interfacial impedance between an electrolyte and a blocking electrode of ten shows a fractional-power dependence on angular frequency $\omega: Z(\omega)=A(j \omega)^{-n}$, where $A$ is independent of $\omega, j=\sqrt{-1}$, and $1>n>0.5$. This is also called a constant-phase-angle behavior. According to Nyikos and Pajkossy (N-P), 2,3 this behavior can be explained if the interface is a fractal. In this work, a critical analysis of the N-P theory was made. It was demonstrated that the above claim by N-P is invalid because some of their equations are mathematically inconsistent and some scaling assumptions made by them are incorrect.

$N-P$ assumed that when the whole system is magnified conventionally by a factor $r$ (Fig. 4.9), the interfacial admitlance is increased by a factor $r^{2}$. As can be seen from the figure, this is true only for the flat part of the electrode: $C_{0}^{\prime}=r^{2} C_{0}$. For the long pore, because the admittance is proportional to $(C / R)^{1 / 2}$, the interfacial admittance is increased by a factor $r^{3 / 2}$. This demonstrates that for a general interface with pores of various sizes and 
ORNL-DWG 89-8899

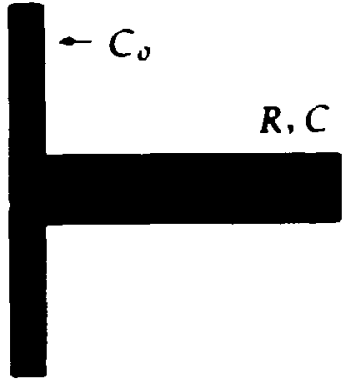

(a)

$$
\begin{aligned}
& R^{\prime}=R / r^{2} \\
& C^{\prime}=r C
\end{aligned}
$$

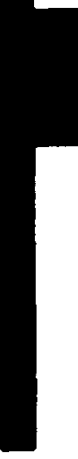

(b)

Fig. 4.9. Schematic diagram showing the conventional magnification (from a to b) of an interface formed by an electrolyte (shaded area) on the left and an electrode on the right. The electrode consists of a flat surface that has a double-layer capacitance $C_{o}$ and a long cylindrical pore that has $a$ iesistance and capacitance per unit length, $R$ and $C$, respectively.

shapes, the convcntional scaling assumption used by N-P is invalid.

Under fractal magnification, one enlarges a fractal interface and introduces new stages at the same time. Several scaling assumptions conceining the interfacial admittance were tested with numerical calculations for interfaces having an electrolyte-metal boundary in the shape of a generalized Koch curve. It was demonstrated that some of them are either valid over only limited frequency ranges or not valid at all.

1. Summary of papers: Electrochimica Acte 33, 707 (1988); Electrochimice Acta (in press).

2. L. Nyikos and T. Pakossy, Electrochimice Acta 30, 1533 (1985).

3. T. Pajkossy and L. Nyikos, Electrochimice Acta 33, 713 (1988).

\section{HOPPING MODEL FOR THE NON-DEBYE DIELECTRIC RESPONSE IN IONIC CRYSTALS'}

\section{C. Wang and I. B. Bates}

The bulk dielectric response of many materials shows a loss peak, the existence of which can be qualitatively understood from a version of the Debye model: charged particles associated with fixed centers, such as $\mathrm{O}^{2-}$ vacancies in stabilized zirconia, are each assumed to hop back and forth in a double-well potential. According to diffusion theories, the transition rate has the thermally activated form with a single activation energy which is equal to the difference between the minimum potential energy of the crystal when a jumping particle is at the saddle point and the potential energy when it is at the bottom of a well. However, many materials show a non-Debye 
dielectric response characterized by a much broader loss peak and a constant-phase-angle (CPA) behavior at frequencies above the loss peak.

To understand this phenomenon, an attempt has been made to nemove the "average" nature of previous diffusion theories. It is assumed that the probability function for the potential energy of the whole crystal is in the form of Boltzmann's factor both when the jumping particle is at the saddle point and when it is near the bottom of the well before the jump. This results in a distribution of activation energies $G(E)$, which decays exponentially on both sides of some given value $E_{o}$ - It is demonstrated that (a) the existence of a loss peak is a result of the decay of $G(E)$ for $E>E_{0}$. (b) the CPA behavior above the loss peak is associated with the decay of $G(E)$ for $E<E_{0}$, and (c) $C(E)$ can produce all the main features of the empirical Havriliak-Negami (H-N) function ${ }^{2}$ (Fig - 4.i0). An interesting property of this $G(E)$ is that it broadens with increasing temperature, consistent with many experimental observations.

1. Summary of paper: Materials Research Society Symposium Proceedings (in press).

2. S. Havriliak and S. Negami, Polymer 8, 161 (1967).

\section{THE CONSTANT-PHASE-ANGLE IMPEDANCE OF THE FINITE SIERPINSKI ELECTRODE ${ }^{I}$}

$$
\text { Y.T. Chu }{ }^{2}
$$

The ac impedance of rough or porous bloxkıng electrodes at low frequencies is represented by

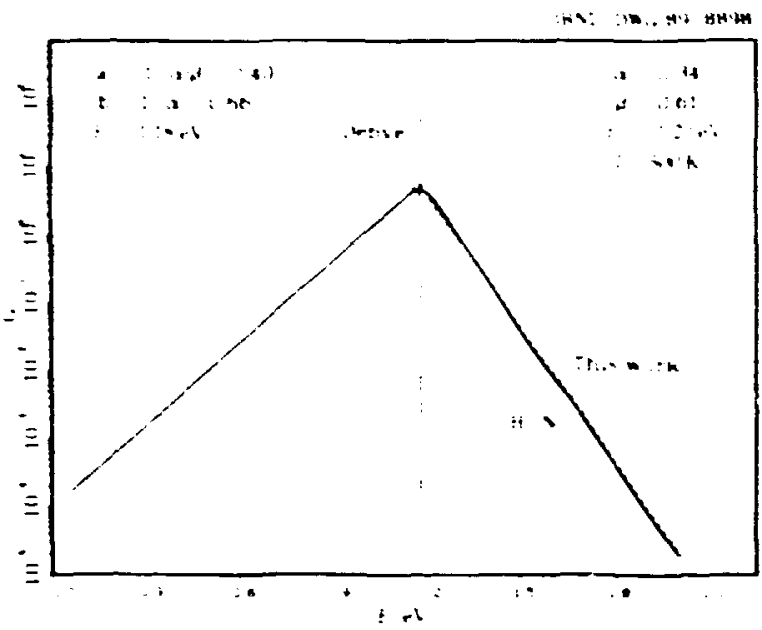

Fig. 4.10. Comparison of $G(E)$ 's of the H-N model, Debye model, and model of this work (dashed line). The value of $E_{0}$ for the present model was estimated from the conductivity data of stabilized zirconia.

the so-called constant-phase-angle (CPA) impedance:

$$
Z(\omega)=A(j \omega)^{-n}
$$

where $j=\sqrt{-1}, \omega$ is the angular frequency, and $A$ and $n$ are constants. Recently, Sapoval ${ }^{3}$ reported the results of numerical calculations on the impedance of the finite Sierpinski electrode (Fig. 4.11), from which he concluded that the frequency exponent $n$ of the CPA impedance is related to the fractal dimension $D$ of the electrode by the relation $n=3-D$ provided that $D<5 / 2$.

The cross section of the working electrode is the Sierpinski carpet of fractal dimension $D_{p}=$ $\log (N) / \log (\alpha)$, where $N$ is the rati, of the total number of holes at stage $n$ to those at stage $n-1$ and $\alpha$ is the reduction ratio of the side lengths of the holes at conseculive stages. Because of the shape of the pores in the electrode 
(Fig. 4.11), the fractal dimension of the Sierpinski electrode is $D=D_{p}+1$. If the ionic resistance between working and counter electrodes is taken into account, it can be shown that at sufficiently low frequency the impedance of the Sierpinski electrode can be represented by a parallel combination of series RC elements representing the impedance of a single pore, in which $R$ and $C$ are related to the reduction ratio $\alpha$. From this equivalent circuit, it can be shown analytically that the relation $n=3-D$ holds without the restriction $D<5 / 2$.

1. Summary of paper: Solid State lonics 26, 299 (1988).

2. Present address: Schaffner Technologies, Inc., Knoxville, Tenn.

OANL DWG 87-19342

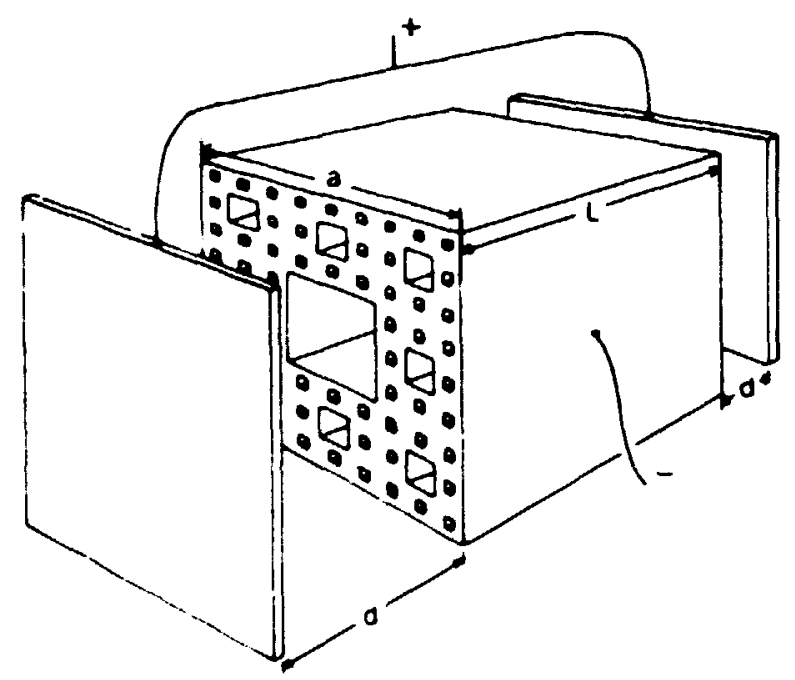

Fig. 4.11. Sierpinski working electrode and counter electrode.

\section{MODEL FOR LTHIUM INTERCALATION INTO TIS,}

J. C. Wang

Lithium intercalation into the layered electrode material $\mathrm{TiS}_{2}$ is being studied with potential energy calculations. It is assumed that the presence of a $\mathrm{L}^{+}$ion in the van der Waals gap between two conducting $\mathrm{TSS}_{2}$ layers induces electronic charges in the layers. Potentials included in the calculations are (1) Coulomb interaction between the $\mathrm{Li}^{+}$ion and the induced charges, (2) Coulomb interaction between induced charges in neighboring layers, (3) shortrange repulsive interaction between the $\mathrm{Li}^{+}$ion and sulphur atoms, and (4) short-range repulsive and van der Waals interaction between sulphur atoms in neighboring layers.

As shown in Fig. 4.12, the expansion of the caxis with increasing $\mathrm{Lj}^{+}$concentration ( $x$ in $\mathrm{Li}_{x} \mathrm{TiS}_{x}$ ) is almost linear for $x<0.3$, but levels off as $x$ approaches $1 .^{1-3}$ In order to explain this result, the distorted region caused by an intercalated $\mathrm{Li}^{+}$ion should have a limited size, about $3 A$ in radius perpendicular to the $c$ axis. This implies that only the three sulphur atoms above and three below a $\mathrm{Lj}^{+}$ion are puckered by the intercalation.

Another interesting property of $\mathrm{Li}_{x} \mathrm{TiS}_{x}$ is the decrease of the cell voltage of a $\mathrm{Li}_{-} \mathrm{TiS}$ cell with increasing $x$. A dominating factor is the interaction of a $\mathrm{Li}^{+}$ion with other $\mathrm{Lj}^{+}$ions and with the electronic charges ind:sed by these ions in the neighboring $\mathrm{TiS}_{2}$ layers. This interaction can be calculated with the method of 


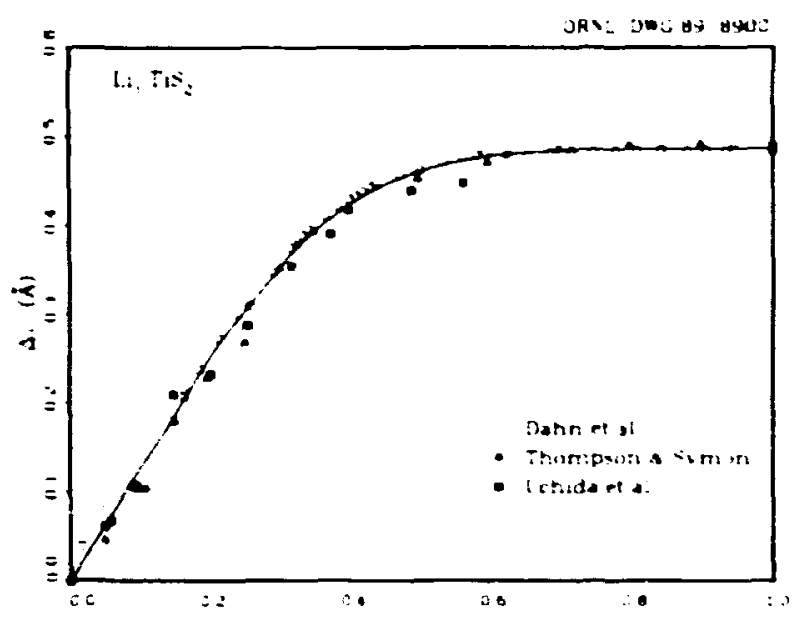

Fig. 4.12. Expansion of the $\mathrm{c}$-axis vs $\mathrm{Li}^{+}$ion concentration. The solid curve is a fit to published experimental data using the present model.

image and Coulomb lattice summation. The result is quite similar to that of a double-layer capacitance problem with the discrete charge oi the $\mathrm{Li}^{+}$ion taken into account explicitly.

1. A. H. Thompson and C. R. Symon, Solid State lonics 3/4, 175 (1981).

2. J. R. Dahn et al., Can. J. Phys. 58, 207 (1980).

3. T. Uchida et al., Maler. Res. Soc. Bull. 22, 935 (1987).

4. T. Jacobsen et al., Electrochimica Acta 27, 1007 (1982).

\section{OPTICAL \& MAGNETIC CHARACTERIZATION OF CERAMICS}

\section{THE CHARACTERIZATION OF TEXTURED CERAMICS BY ELECTRON PARAMAGNETIC RESONANCE SPECTROSCOPY: CONCEPTS AND THEORY}

\author{
L. A. Boatner, J. L. Boldú O.,' \\ and M. M. Abraham
}

Various physical alterations of polycrystalline systems can result in the formation of materials in which the microstructure consists of crystalline grains that exhibit a nonrandom or preferred orientation. Such preferred orientations are frequently referred to as "texture" by ceramists and materials scientists.

We have shown that electron paramagnetic resonance (EPR) spectroscopy is a powerful and versatile technique for the detection and characterization of preferred orientation effects in insulating ceramics. Although the technique is applicable to both cubic and noncubic ceramics, in the initial work the conceptual and theoretical basis for the analysis of textured cubic materials was established. The practical utility of the EPR technique has been illustrated for the specific case of a paramagnetic impurity $\left(\mathrm{Mn}^{2+}\right)$ in the cubic ceramic $\mathrm{MgO}$. As shown in Fig. 4.13, EPR methods establish that texturing occurs in a cold-pressed, sintered $\mathrm{MgO}$ ceramic and that the preferred orientation corresponds to $\langle 111\rangle$ axes lying nearly parallel to the direction of the cold pressing force. The usc of the EPR technique in characterizing preferred orientation effects in ceramic materials 


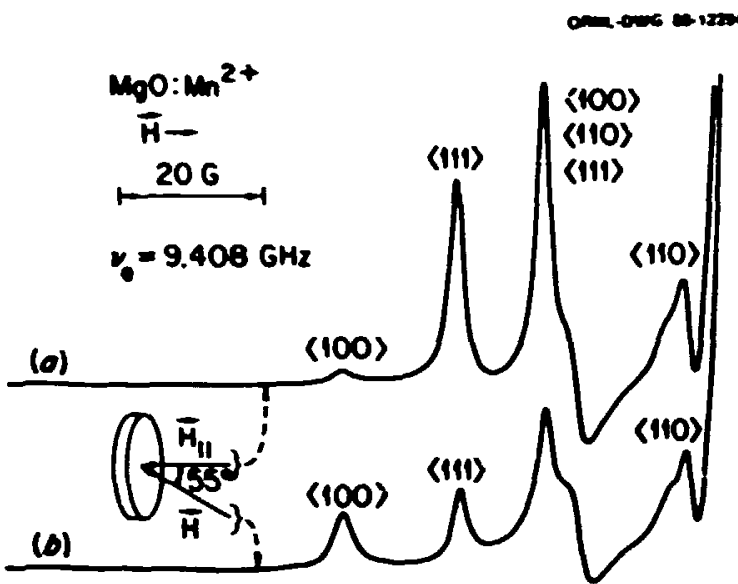

Mgo Sintered of $1450^{\circ} \mathrm{C}$.

Fig. 4.13. EPR spectra of $\mathrm{Mn}^{2+}$ in a sintered $\mathrm{MgO}$ ceramic in a first-derivative presentation. (a) The applied magnetic field (H is oriented parallel to the force used to cold press the green body). The intensity at the <111> extreme field value is large relative to that at the $\langle 100\rangle$ field value; (b) $\mathrm{H}$ is rotated $\sim 55^{\circ}$ away from the axis corresponding to the cold-pressing force. Changes that occur in the relative intensities of the <111 $>$ and $<100\rangle$ shoulders as $\mathrm{H}$ is rotated between the two orientations show that texturing has developed during sintering with an alignment of $\langle 111\rangle$ axes parallel to the cold-pressing force.

offers several advantages over more traditional diffraction-based and optical characterization methods.

1. Guest scientist from the National Autonomous University of México, D.F., México.

\section{OPTICAL STUDY AND ANALYSIS OF Püt IN SINGLE CRYSTALS OF ZRSIO,}

I. S. Poirot,' W. K. Kot,' N. M. Edelstein,' M. M. Abraham, C. B. Finch, and L. A. Boatner

Optical spectroscopic investigations of the narrow absorption and fuorescence spectra of trivalent lanthanide and actinide ions in various host single crystals have been carried out for over 30 years. The parametric theory used to interpret such spectra has been quite successful for these ionic systerns, and in particular, it has proven to be very useful when applied to the lanthanides. The same theory applied to tetravalent actinide ions such as $\mathrm{U}^{4+}$, however, results in fits to the experimental spectra that are much worse than those found for the trivalent ions. At the present time, these findings are based on only a relatively small number of experimental studies of tetravalent ions with atomic number $Z$ greater than 92 cased, in part, by the problems associated with the radioactivity of these elements. Since the heavier actinide ions become more lanthanide-like in their electronic properties because of the contraction of the 5 orbitals, one would expect the conventional parametric theory to be more successful when applied to the higher $Z$ tetravalent actinides. Accordingly, the present work represents one portion of a systematic study of the higher $\mathbf{Z}$ tetravalent actinide ions diluted as impurities in various host single crystals.

The optical spectrum of $\mathrm{ZrSiO}_{4}: \mathrm{U}^{4+}$ has been extensively investigated, and an optical and electron paramagnetic resonance study of tetravalent neptunium in single-crystal $\mathrm{ZSSO}_{4}$ has recently been published.? The current work represents an investigation of the optical spectrum of $\mathrm{Pu}^{4+}$ doped into single crystals of $\mathrm{ZrSiO}_{4}$ and a parametric analysis of these data. In carrying out this analysis, it has been assumed that the crystal-field parameters obtained for $\mathrm{ZrSiO}_{4}: \mathrm{Np}^{4+}$ represent reasonable 
initial values for the $\mathrm{ZrSiO}_{4}: \mathrm{Pu}^{4+}$ case, since $\mathrm{Np}$ and $\mathrm{Pu}$ are neighboring elements in the periodic table.

Single crystals of $\mathrm{ZrSiO}_{4}$ (zircon) are tetragonal with the tetravalent metal ion located at a $D_{2} d$ symmetry site. The $\mathrm{Zr}^{d+}$ ion is surrounded by eight oxygen atums in a dodecahedral array with the $S_{4}$ axis parallel to the optic axis of the crystal. The electronic structure of tetravalent plutonium incorporated as a dilute impurity in single crystals of the tetragonal host $\mathrm{ZrSiO}_{4}$ was studied using polarized optical spectroscopy. No attempt was made to measure the Zeeman splittings, since the calculated 8 values are generally small for the doubly degenerate $r_{5}$ states and the line widths are relatively broad. Fifty-three crystal-field levels were assigned and used to obtain a fit to a parametric Hamiltonian to within an rms deviation of $33 \mathrm{~cm}^{-1}$. The resulting crystalfield parameters were similar to those obtained for the $\mathrm{ZrSiO}_{4}: \mathrm{U}^{4+}$ and $\mathrm{ZrSiO}_{4}: \mathrm{Np}$ systems. The parametric fit improves with increasing atomic number across the actinide series, suggesting that the tetravalent actinides are more "lanthanide-like" as the atomic nımber increases.

1. Lawrence Berkeley Laboratory, Berkeley, Calif.

2. I. Poirot et al., Phys. Rev. B 37, 3255 (1988).

\section{F+ AND F LUMINESCENCE IN THERMOCHEMICALLY REDUCED MgO CRYSTALS \\ Y. Chen and V. M. Orera ${ }^{2}$}

Thermochemically reduced $\mathrm{MgO}$ crystals ( $\mathrm{MgO}: \mathrm{Mg}$ ) contain $F^{+}$and $F$ centers (anion vacancies with one and two electrons, respertively) and $\mathrm{H}^{-}$ions that occupy $\mathrm{O}^{2-}$ ion sites. Both the $\mathrm{F}^{+}$and $\mathrm{F}$ centers absorb at $4.95 \mathrm{eV}$ (250 $\mathrm{nm}$ ). Excitation at this wavelength yields either the $F$ or $F^{+}$luminescence at 2.4 and $3.1 \mathrm{cV}$, respectively or both. Absorption of 4.95-eV light excites electrons from the ${ }^{1} A_{1 / g}$ ground state of the $F$ center into its ${ }^{1} T_{1_{u}}$ excited state. Since the excited state is close to the conduction band (within $0.01 \mathrm{eV}$ ), the electrons are thermally ionized and are trapped at impurity sites, leaving behind $F^{+}$centers. After optical excitation at low temperatures, $F$ center thermoluminescence peaks are observed below $100 \mathrm{~K}$ and at 260 and $500 \mathrm{~K}$ These three peaks correspond to three different electron traps.

The trap responsible for the $500 \mathrm{~K}$ peak was shown by Kappers and Hensley ${ }^{3}$ to be due to $\mathrm{Fe}^{2+}$. The process is $\mathrm{F}+\mathrm{Fe}^{2+} \rightarrow \mathrm{F}^{+}+\mathrm{Fe}^{+}$. The peak observed below $100 \mathrm{~K}$ is associated with electrons trapped at $\mathrm{H}^{-}$ions (nr $\mathrm{H}^{-}$complexes). Eiectron paramagnetic resor ance measurements and theoretical calculations showed that stable, $S=1 / 2$, substitutional $H^{2-}$ ions were formed when a substitutional $\mathrm{H}^{\circ}$ ion trapped an elertron. Irradiation with blue $(400 \mathrm{~nm})$ light was most effective in forming these $\mathrm{H}^{2-}$ ions. 
Optical excitation into the 4.95-eV absorption band near $295 \mathrm{~K}$ creates a long-lived $F$ phosphorescence. The lifetime of this emission depends on the hydrogen content and can extend up to $10^{3} \mathrm{~s}$. We have identified a complex containing an $\mathrm{H}^{-}$ion which has trapped an electron during opical excitation of the $F$ center. At $77 \mathrm{~K}$, a $\mathrm{MgO}: \mathrm{Mg}$ crystal exhibits infrared absorption bands at 1056, 1034, and $1027 \mathrm{~cm}^{-1}$. These bands are caused by different $\mathrm{H}^{-}$species. Optical pumping into the $F$ band (235-270 nm light) at $77 \mathrm{~K}$ decreased the $1027-\mathrm{cm}^{-1}$ local mode and created a sew band at $137 \mathrm{~cm}^{-1}$. During the excitation, an electron is released into the conduction band and is captured by one of the $\mathrm{H}^{-}$complexes, referred to as $\left[\mathrm{H}_{\mathrm{x}}\right]^{+}$, and forms the $\left[H_{x}\right]^{\circ}$ complex, where the subscript $x$ refers to the perturbin; wecies. We attribute the 1027 and $1037 \mathrm{~cm}^{-1}$ bands as due to the local modes of $\left[\mathrm{H}_{x}^{-}\right]^{+}$and $\left[\mathrm{H}_{x}^{-}\right]^{\circ}$ ions, respectively. The process is described by

$$
\begin{aligned}
& F+h v(4.95 \mathrm{eV}) \rightarrow F^{*} \rightarrow F^{+}+e^{-}, \\
& e^{-}+\left[\mathrm{H}_{x}\right]^{+} \rightarrow\left[\mathrm{H}_{x}^{-}\right]^{0} .
\end{aligned}
$$

This complex is not the same defect identified previously as $\mathrm{H}^{2-}$ ions ${ }^{3}$ by EPR at low temperatures.

1. Summary of paper: I. Lumin. 40/41, 325 (1988).

2. Guest scientist from the University of Zaragoza, Zarazoza, Spain.

3. L. A. Kappers and E. B. Hensley, Phys. Reo. B 6. 2474 (1972).

\section{TIME-RESOLVED LUMINESCENCE AND ABSORPTION SPECTROSCOPY OF DEFETS IN MgO'}

G. P. Williams, Jr., ${ }^{2}$ G. H. Rosenblatt, 2

M. I. Ferry, ${ }^{2}$. T. Williars, ${ }^{2}$ and $Y$. Chen

Lattice defects in a number of oxide crystals including MgO exhibit broad luminescence bands in the visible and near-ultraviolet spectrum. There has been interest in the possibility of developing color-center lasers in oxide crystals, both because of the potential shortwavelength tining range and the robust nature of the refractory oxide crystals and their defests.

Time-resolved luminescence irom $F, F^{+}$, and $F$-aggregate mnters following excimer laser excitation was investigated in thermochemically reduced $\mathrm{MgO}$. In $\mathrm{MgO}$, two electrons trapped at the oxygen vacancy ( $F$ center) absorb near $250 \mathrm{~nm}$ and emit in a broad band at $530 \mathrm{~nm}$. The oxygen vacancy with one electron $\left(F^{+}\right.$center) absorbs in a nearly coincident band near $250 \mathrm{~nm}$ but emits near $390 \mathrm{~nm}$. Hence both centers are efficiently excited by KrF radiation at $248 \mathrm{~nm}$. The $F$ luminescence has a long lifetime correlated to the concentration of $\mathrm{H}^{-}$ions (protons occupying $F$ centers), extending to many minutes in crystals with significant $\mathrm{H}^{-}$concentration. ${ }^{3}$

The 390-nm $\left(F^{+}\right)$and 530-nm $(F)$ luminescence bands in low-hydrogen $\mathrm{MgO}_{\mathbf{g}} \mathrm{O}$ above $100 \mathrm{~K}$ decay at effectively the same rate over a period from $10 \mu \mathrm{s}$ to $10 \mathrm{~s} .4$ We believe this requires a charge transfer model in which the same photoionized charges (electrons) from both centers retum to their respective recombination sites from a common collection of traps including $\mathrm{H}^{-}$centers. 
This implies that the $F^{+}$excited states lie near the conduction band edge (unless donor-acceptor pair tunneling occurs) and that the long-lived $F^{+}$luminescent transition is of the form

$$
F^{2+}+e^{-} \stackrel{h v}{\rightarrow} F^{+}
$$

Luminescence from every thermochemically reduced $\mathrm{MgO}$ crystal, regardless of hydrogen content or nature of the late spectrum, appears as $F^{+}$in the beginning.

1. Summary of paper: !. Lumin. 40/41, 339 (1988).

2. Wake Forest University, Winston-Salem, N. C.

3. Y. Chen and R. Gonzalez, Opt. Lett. 10, 276 (1985).

4. G. H. Rosenblatt et al., Physical Review $B$ (in press).

\section{OPTICAL AND TRANSMISSION ELECTRON MICROSCOPY CHARACTERIZATION OF METAL PRECIPITATES IN DOPED THERMOCHEMICALLY REDUCED MAGNESIUM OXIDE'}

\section{Ballesteros, ${ }^{2}$ R. Gonzalez, ${ }^{2}$ and $Y$. Chen}

Recently, a method based on thermochemical reduction has been developed to produce metal precipitates in oxides. MgO crystals doped with $\mathrm{Co}, \mathrm{Cu}$, and $\mathrm{V}$ were thermochemically reduced at $-2000 \mathrm{~K}$ in -4 atm of $\mathrm{Mg}$ vapor. Changes in coloration were observed in all of these crystals because of broad extinction bands in the visible region. Analytical transmission electron microscopy techniques indicated the presence of metallic precipitates consisting of
Co in the Co-doped sample, Cu with some precipitates of $\mathrm{Fe}$ in the Cu-doped sample, and a $\mathrm{V}$-Fe-Cr alloy in the V-doped sample. iNicrodiffraction patterns indicated that the Co precipitates possessed the fcc $\left(a_{0}=3.54 \AA\right)$ crystal structure, and V-Fe-C.r alloy precipitates showed a bcc $\left(a_{0}=2.89 \AA\right)$ crystal structure.

As an illustration, in the as-grown $\mathrm{MgO}$ :Co crystal, two bands due to Co impurities appeared at 2.4 and $4.3 \mathrm{eV}$. This initially pink crystal turned dark and almost opaque after reduction. A broad and intense extinction was then observed at $3.7 \mathrm{eV}$. Transmission electron microscopy demonstrated that there were regions containing a high concentration of pre cipitates about $100 \mathrm{~nm}$ in diameter. Often the precipitates appeared to follow irregular lines that were associated with dislocations (Fig. 4.14). Using microdiffraction techniques,

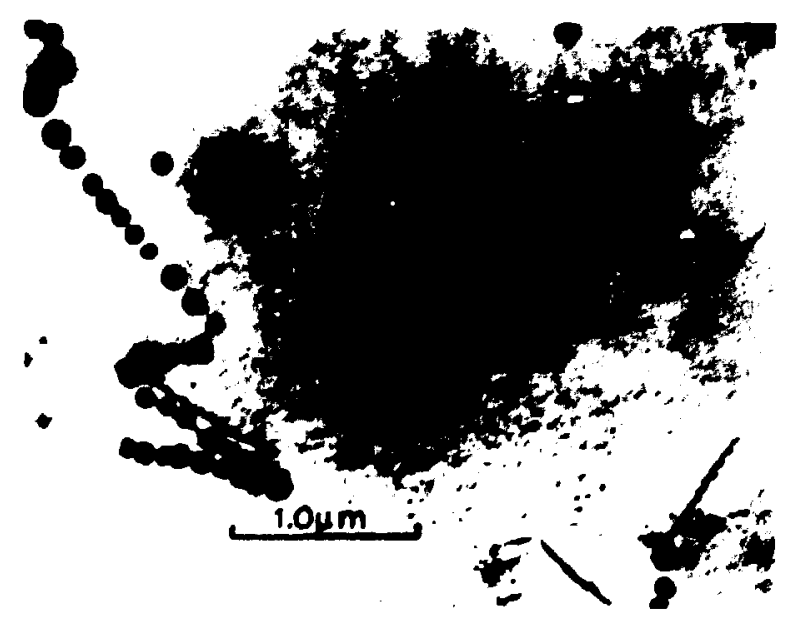

Fig. 4.14. Electron micrograph of aligned cobalt precipitates in a $\mathrm{MgO}: \mathrm{Co}$ specim:n. 
the structure of the precipitates and the crystallographic relationship between matrix and precipitates were found:

$<001>_{p}||<001>_{m}$ and $<010>_{p}||<010>_{m}$

1. Summary of paper: Phys. Reo. B 37, 8008 (1988).

2. Guest scientist from the University of Madrid Complutense, Madrid, Spain.

\section{SUPEKHYPERFINE STRUCTURE OF THE $\mathrm{H}^{2-}$ ION DN MgO'}

V. M. Grera ${ }^{2}$ and $Y$. Chen

An electron paramagnetic resonance (EPR) signal consisting of two isotropic lines with equal intensity separated by $52.7 \times 10^{-4} \mathrm{~cm}^{-1}$ and centered at about $g \sim 2$ has been observed from defects in thermochemically reduced MgO. ${ }^{3}$ A comparison with deuterated samples unequivocally linked these defects with the presence of hydrogen.

The low-field EPR line as measured with the external magnetic field $H \|$ [111] is shown in Fig. 4.15. An intense central line is flanked by six equally spaced lines of equal intensity. The spectra are anisotropic and split when the magnetic field departs from the [111] direction, showing the characteristic behavior of a defect with axial symmetry along the [100] direction. The ratio between the intensity of the central line and the sum of that of the six lines is $\mathbf{1 . 6}$ for any magnetic field orientation. These observations lead us to attribute the signals to the superhyperfine interaction with neighboring

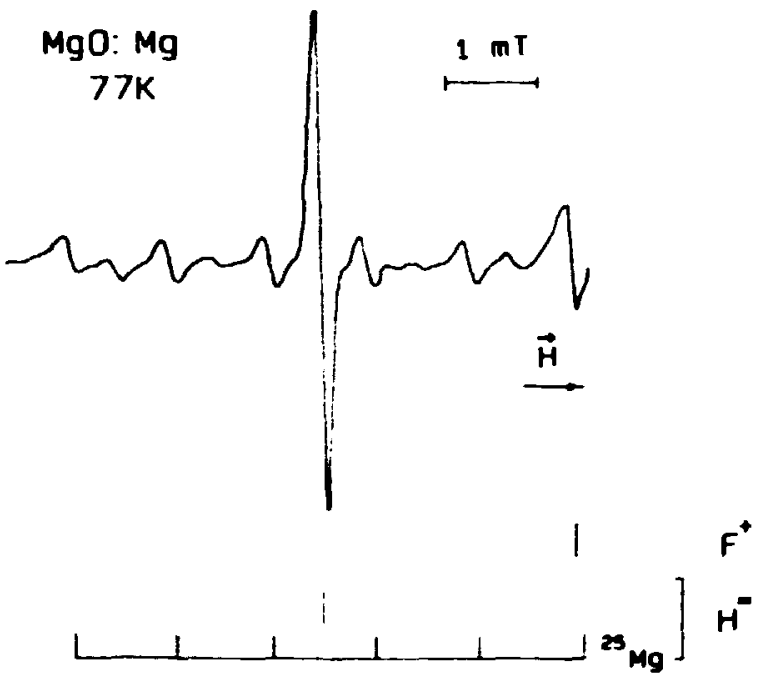

Fig. 4.15. Low-field hyperfine group of the $\mathrm{H}^{2-}$ ion measured with $\mathrm{H} \|[111]$ at $77 \mathrm{~K}$ showing the ${ }^{25} \mathrm{Mg}$ superhyperfine lines.

${ }^{25} \mathrm{Mg}$ isotopes $(U=5 / 2)$. The analysis of this superhyperfine structure associated with the EPR spectrum confirms the structure of the defect as $\mathrm{H}^{2-}$, a proton with three electrons occupying an oxygen vacancy site.

1. Summary of paper: Phys. Reo. B 36, 5576 (1987).

2. Guest scientist from the University of Zaragoza, Zaragoza, Spain.

3. J. Tombrello et al., Phys. Reo. B 30, 7374 (1984).

\section{OPTICAL TRANSITIONS IN NEUTRON- [RRADLATED M\&Al $\mathrm{O}_{4}$ SPINEL CRYSTALS ${ }^{1}$}

L. S. Cain, ${ }^{2}$ G. I. Pogatshnik, and Y. Chen

Neutron-irradiated single crystals of $\mathrm{MgAl}_{2} \mathrm{O}_{4}$ spinel containing about $10 \mathrm{ppm} \mathrm{Cr}^{3+}$ exhibited a prominent absorption band at 
$5.3 \mathrm{eV}(235 \mathrm{~nm})$ that increased with neutren dose and was attributed to $F$ centers (anion vacancies each with two electrons).

The emission spectrum due to $\mathrm{Cr}^{3+}$ consisted of a series of sharp lines near $700 \mathrm{~nm}$ and a sideband that peaks near $720 \mathrm{~nm}$. The absorption spectrum showed an absorption band at $540 \mathrm{nrn}$ for the ${ }^{4} A_{2} \rightarrow{ }^{4} T_{2}$ transition and a band near $390 \mathrm{~nm}$ for the ${ }^{4} A_{2} \rightarrow{ }^{4} T_{1}$ transition. The excitation spectra for $\mathrm{Cr}^{3+}$ in the spinel are shown in Fig. $4.16 \mathrm{fcr}$ an as-received sample and for the neutron-i, spectra were obtained with the emission spectrometer tuned to $720 \mathrm{~nm}$. The as-received sample showed two excitation peaks at 540 and $390 \mathrm{~nm}$. With increasing neutron dose, the excitation peaks became much more intense and shifted to higher energies. In the sample irradiated with $1 \times 10^{17} \mathrm{n} / \mathrm{cm}^{2}$ (curve $\mathrm{c}$ ) the shift in

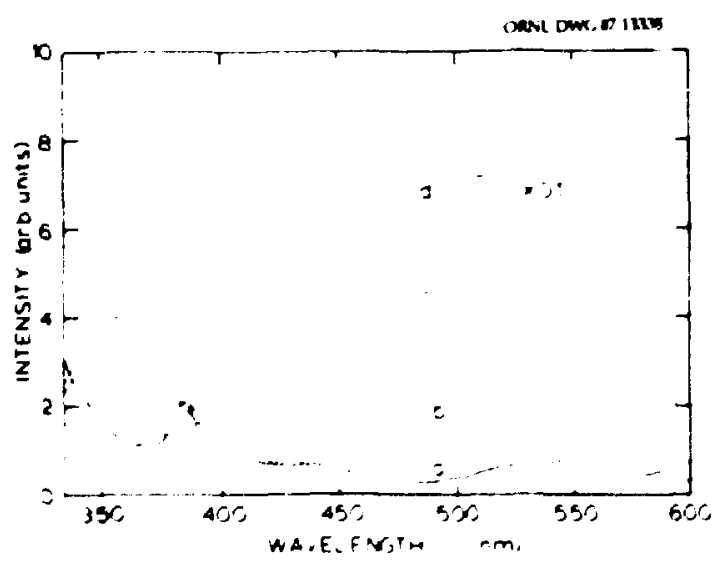

Fig. 4.16. Excitation spectra of the $\mathrm{MgAl}_{2} \mathrm{O}_{4}$ spinel for the $\mathrm{Cr}^{3+}$ emission at $720 \mathrm{~nm}$ for crystals with differing exposures to neutron irradiation. The excitation spectrum for the asreceived sample (curve a) and crystals irradiated with $2 \times 10^{16}$ (curve b), $1 \times 10^{17}$ (curve c), and $3 \times 10^{17} \mathrm{n} / \mathrm{cm}^{2}$ (curved). the excitation bands was clear. The new excitation peaks were at 360 and $515 \mathrm{~nm}$.

A model which explains the observed feasures of the $\mathrm{Cr}^{3+}$ emission and excitat $3 \mathrm{~s}$ spectra is that of $\mathrm{Cr}^{3+}$ implrity ions perturbed by the presence of a nearby $F$ center. According to the Tanabe-Sugano diagram for $\mathrm{Cr}^{3+}$, the optical transitions from the ${ }^{4} A_{2}$ ground state to the ${ }^{4} T_{2}$ and ${ }^{4} T_{1}$ excited states can be modified by an effective increase in the crystal field. The increased crystal field strength would shift the positions of the optical transitions to higher energies. In addition to the shift in energy, the perturbation induced by the $F$ center can increase the oscillator strength by adding odd components to the crystal field or by exchange coupling between the $F$ center and the $\mathrm{Cr}^{3+}$ impurity.

1. Summary of paper: Phys. Reo. B 37, 2645 (1988).

2. Oak Ridge Associated Universities faculty research jarticipant from Davidson College, Davidsx $n, N$. C.

3. Guest scirntist from North Carolina State University, Raleigh, N. C.

\section{DETERMINATION OF THE OPTICAL DIELECTRIC FUNCTION OF THIN ABSORBINC FILMS ${ }^{1}$}

$$
\text { Y.T. Chu }{ }^{2}
$$

Detcrmining the optical dielectric function of a thin film overlayed on a thick absorbing substrate is often difficult because the measurements of transmittance may be impossible and the shapes of reflectivity speetra are 
affected strongly by the substrate. It was found that these difficulties can be overcome with a simple technique which is based on determining the ratio $\left(R / R_{5}\right)$ of the sample (substrate + film) reflectivity to the reflectivity of the substrate.

This method was applied to a determination of the optical dielectric function of a $1600-A$ thick film of amorphous alumina $\left(a-\mathrm{Al}_{2} \mathrm{O}_{3}\right)$ on a single-crystal $\mathrm{Al}_{2} \mathrm{O}_{3}$ substrate. Because the two higher frequency modes of $a-\mathrm{Al}_{2} \mathrm{O}_{3}$ have relatively small damping factors, they can be readily identified in the reflection spectrum. ${ }^{3}$ Problems occurred in confirming the presence of modes suspected to occur in the $300-550 \mathrm{~cm}^{-1}$ region of the reflection spectrum because of their large damping factors. However, the reflection ratio plotted in Fig. 4.17 confirms the presence of two modes in this region. From an analysis based on Fresnel's equation for reflectivity, the ratio $R / R_{5}$ should be less than unity in the absence of these modes. The fact that $R / R_{s}>1$ and the good agreement between the

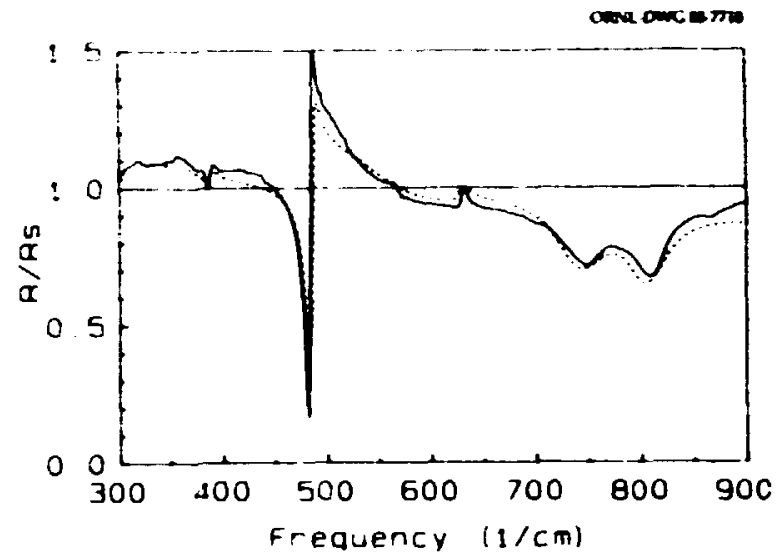

Fig. 4.17. The measured (solid line) and calculated (dashed line) ratio $R / R$, of the reflectivity of a $1600-A$-thick amorphous $\mathrm{Al}_{2} \mathrm{O}_{3}$ film on single $\mathrm{Al}_{2} \mathrm{O}_{3}$ to the reflectivity of the $\mathrm{Al}_{2} \mathrm{O}_{3}$ substrate.

measured and calculated reflectivity ratios confirm the presence of two modes in the 300$550 \mathrm{~cm}^{-1}$ region.

1. Summary of paper to be published.

2. Present address: Schaffner Technologies, Inc., Knoxville, Tenn.

3. Y. T. Chu et al., I. Appl. Phys. 64, 3727 


\section{Ion Beam and Laser Processing}

Materials processing research using ion beams and lasers continues to provide new opportunities for both basic research and new materials development. In this chapter, progress in ion beam processing, ion beam analysis and ion implantation, and laser-assisted processing of materials is reported. This research is centered at the Surface Modification and Characterization Collaborative Research Center and at the newly developed laser photochemical vapor deposition facility.

Research on ion beam deposition-the direct deposition of mass-analyzed ions at tens of $\mathrm{eV}$-has been extended to the fabrication of epitaxial GaAs films by alternating deposition of the two cunstituent species. These are the first epitaxial compound films to be grown using ion beams. New results are also reported on heteroepitaxial growth of Ge on CaAs, the formation of lowresistance metal silicides, and low-temperature preparation of beta-SiC. Other processing research using ion beams has included the formation of silicon-oninsulator structures, the effects of Ir implantation on the electrochemical behavior of biosensors, and the solid phase epitaxy of ion-implanted waveguide materials.

Substantial progress has also been made in the fundamental understanding of ion implantation phenomena. Research on high-energy implantation of semiconductors points to important differences in damage and annealing mechanisms between $\mathrm{MeV}$ and conventional implant energies. Facilities for ion implantation were substantially upgraded this year by installation of a high-current $500-\mathrm{keV}$ linear accelerator/implanter-the first of its kind in a research laboratory.

Research on laser processing of thin films has centered on fabrication of superlattice structures using laser photolysis and preparation of high- $T_{\text {c }}$ superconducting films using laser ablation (Chapter 4). This research has included in-situ spectroscopic studies as well as materials characterization. 
An exciting new epitaxial growth technique based on directing a pulsed supersonic jet of precursor molecules onto a heated substrate has been developed and has demonstrated very high growth rates of up to $10 \mu / \mathrm{min}$.

\section{ION BEAM PROCESSING}

\section{ION BEAM DEPOSITION OF EPITAXIAL GaAs USING ALTERNATING LOW-ENERGY BEAMS ${ }^{1}$}

\section{T. E. Haynes, R. A. Zuhr, and S. I. Pennycoot}

We have recently shown that isotopically pure GaAs films can be grown epitaxially on $\mathrm{Si}$ and Ge substrates by low-energy ion beam deposition (IBD). These films were the first epitaxial compound layers to be successfully grown using only ionized, energetic species for deposition. Compound growth was achieved by alternately depositing thin layers of the two constituent species $\left({ }^{69} \mathrm{Ga}^{+}\right.$and $\left.{ }^{75} \mathrm{As}^{+}\right)$onto a heated substrate. Ga and As ion species were obtained simultaneously from the ion source of the Extrion ion implanter, extracted at $35 \mathrm{kV}$, mass-analyzed, and decelerated to the final deposition energy at the substrate. Deposition was alternated between $\mathrm{Ga}^{+}$and $\mathrm{As}^{+}$under computer control using a new automated analyzer magnet controller. ${ }^{2}$

GaAs films similar to that illustrated in Fig. 5.1 have been grown at temperatures from 520 to $250^{\circ} \mathrm{C}$ by alternate deposition of approximately monolayer amounts of $A s$ and $\mathrm{Ga}$ on Ce(D01) substrates. Ion channeling minimuin yields for these samples were consistently $=6 \%$. Cross-section transmission clectron

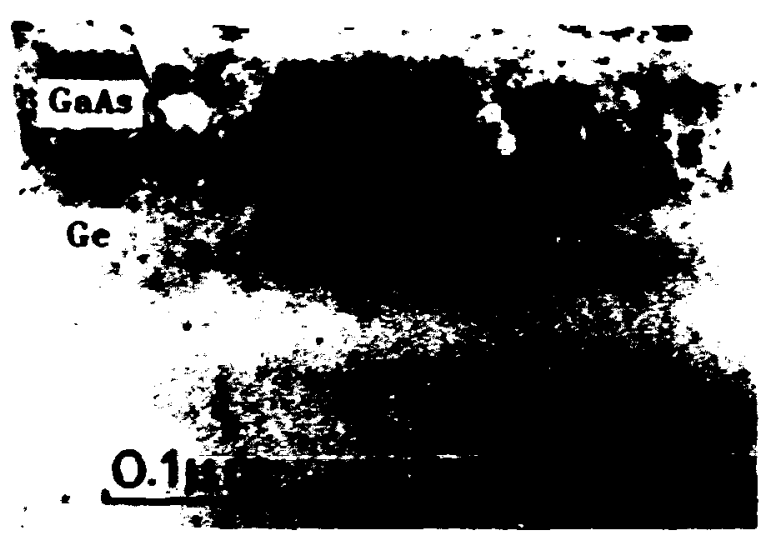

Fig. 5.1. Cross-section TEM micrograph of a 90-nm-thick ${ }^{69} \mathrm{Ga}{ }^{75}$ As layer deposited by IBD on $\mathrm{Ge}(100)$ at $400^{\circ} \mathrm{C}$.

microscopy (TEM) typically shows a few threading dislocations and disiocation loops along with some precipitates in the films. GaAs layers were also grown epitaxially on Si(001) by IBD, but these were highly defective as a result of the large lattice mismatch $(\Delta a / a=4 \%)$, with ion channeling minimum yields around $50 \%$ and a high density of microtwins and stacking faults.

1. Summary of papers: Appl. Phys. Lett. 54, 1439 (1989); Materials Research Society Sym. posium Proceedings (in press).

2. J. L. Moore, T. E. Haynes, and R. A. Zuhr, "Development of an Automated Magnet Controller for Alternating Ion Beam Deposition of Compround layers," this report. 


\section{HETEROEPTT AXIAL GROWTH OF GE FIMMS ON GaAs(00) BY ION BEAM DEPOSITION}

\section{T. E. Haynes, R. A. Zuhr, S. I. Pennycook, B. C. Larson, and M. D. Galloway}

Epitaxial ${ }^{76} \mathrm{Ge}$ thin films have been grown on $\mathrm{CaAs}(001)$ substrates at temperatures from 150 to $400^{\circ} \mathrm{C}$ using the ion beam deposition (IBD) technique with $40-\mathrm{eV}{ }^{76} \mathrm{Ge}$ ions. These layrss were characterized by ion channeling, cruss-section transmission electron microscopy (TEM), and $x$-ray diffraction rocking curves. Deposition of Ge on GaAs at temperatures $2250^{\circ} \mathrm{C}$ produced single-crystal epitaxial films (Fig. 5.2). Small tislocation loops and a few precipitates were typically found in all IBDgrown Ge layers. Ion channeling minimum yields as low as $3 \%$ were obtained, and the dechanneling rate was shown to increase slightly as the deposition temperature was lowered, indicating an increased density of extended defects.

In double-crystal $x$-ray diffraction rocking curves, several orders of satellite peaks were visible, indicating the spatial uniformity of the lattice parameter and the flatness of the layer. However, the (004) reflection from the IBD layer was found to be substantially shifted from the position expected for a coherently strained Ge overlayer. This shift is consistent with nearly complete relaxation of the misfit strain, but we note that threading dislocations, which are usually associated with misfit strain relaxation, were not seen in TEM cross sections. Furthermore, relaxation is not expected for such small misfits in thin films, and we are presently investigating other possi-
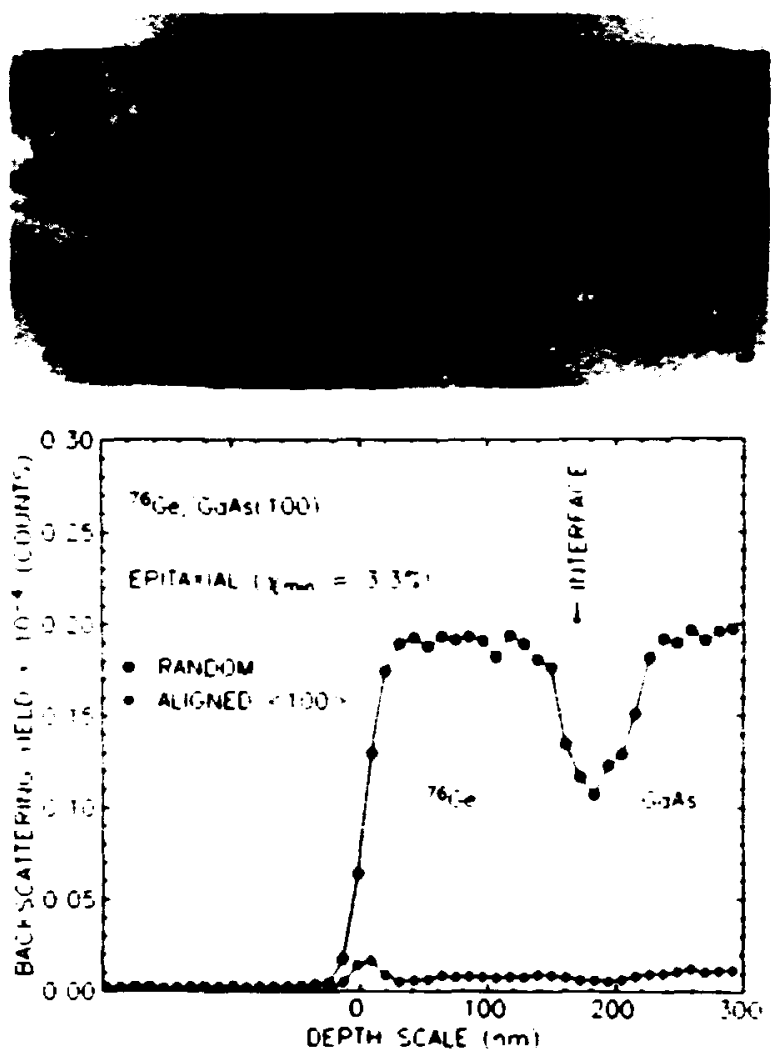

Fig. 5.2. A 180 -nm-thick ${ }^{76} \mathrm{Ge}$ layer deposited by IBD on $\mathrm{CaAs}(100)$ at $400^{\circ} \mathrm{C}$ : (top) cross-section TEM micrograph; (bottom) ion channeling and random spectra.

ble explanations for the measured shift, including isotope effects.

1. T. E. Haynes et al., I. Vac. Sci. and Technol. A 7, 1372 (1989).

\section{METALUC SILCTDES FORMED BYION BEAM DEPOSITION}

R. A. Zuhr, T. E. Haynes,

O. W. Holland, and S. I. Pennycook

Low-energy ion beam deposition (IBD) of the metal ion has been shown to be a viable technique for the formation of silicides on singlecrystal Si at low temperatures. Silicides of $\mathrm{Co}$, 
Fe, Ni, Ti, and $\mathrm{W}$ have been formed at temperatures near $500^{\circ} \mathrm{C}$. This is a temperature at which diffusion or loss of previously implanted dopants should be minimized. For the silicides in which $\mathrm{Si}$ is the dominant diffusing element (Fe, Ti, W), a low-resistance stoichiometric disilicide phase has formed in all cases. For silicides in which the metal is the dominant diffuser $(\mathrm{Co}, \mathrm{Ni})$, the stoichiometry has varied. Co and Ni silicides are also known to form epitaxial films, and this has been achieved using IBD with the best results for Co on Si(111) at $550^{\circ} \mathrm{C}$. However, even where epitaxial growth has occurred (Fig. 5.3), the stoichiometry of these silicides is not constant and the resistivities are relatively high.

The results support a model in which both thermal and athermal processes are significant and the effectiveness of silicide growth is dependent upon the diffusion characteristics of the particular silicide system. Silicides in which $\mathrm{Si}$ is the dominant diffusing atom (Fe, Mo, Ti, Ta, W) can be grown most effectively by the IBD technique. As a result, low-resistance films of the disilicide phase, notably $\mathrm{TiSi}_{2}$, that exhibit bulk-like resistivities without high-temperature annealing can be produced at temperatures below $550^{\circ} \mathrm{C}$ for Si diffusing systems. The characteristics of these films are at least comparable with those of films produced thermally at significantly higher temperatures. The formation of high-quality lowresistance silicide films by IBD at low $1 \mathrm{~cm}$. peratures can lower the thermal budget during semiconductor processing and could find appli.
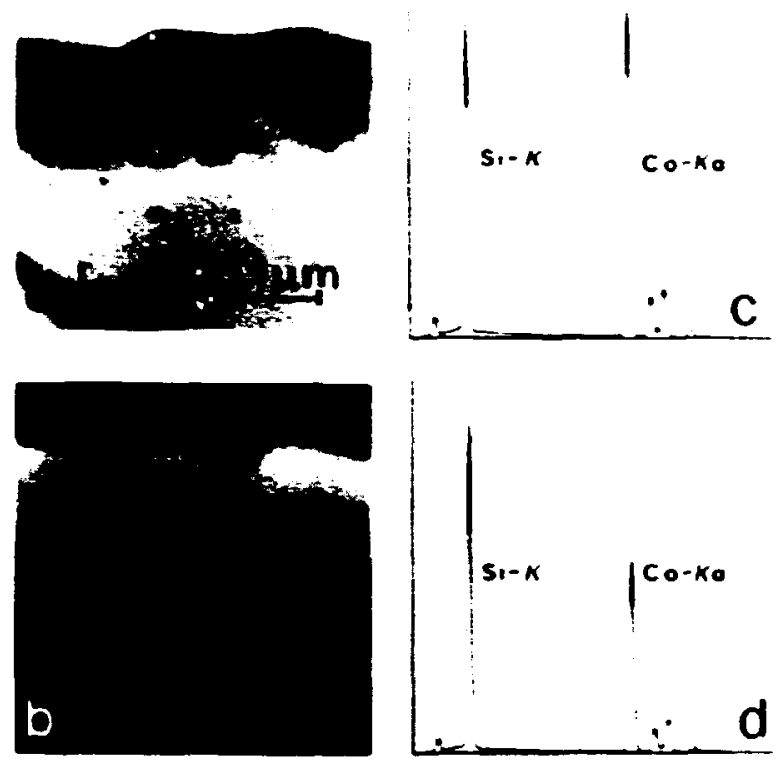

Fig. 5.3. Cross-section transmission electron micrograph (a), Z-contrast image (b), and electron-induced $x$-ray spectra from the upper (c) and lower (d) layers of a Co-Si film grown at $40 \mathrm{eV}$ and $500^{\circ} \mathrm{C}$ by IBD on a $\mathrm{Si}(100)$ substrate. The stoichiometry of the film crianges from $\mathrm{CoSi}_{2}$ (c) to $\mathrm{CoSi}$ (d) as film thickness increases because of reduced availability of $\mathrm{Si}$ from the substrate.

cation in several areas of integrated circuit fabrication.

\section{ION BEAM DEPOSITION OF $\beta$-SiC LAYERS ONTO $\alpha$-SiC SUBSTRATES}

\section{S. P. Withrow, K. L. More,' \\ R. A. Zuhr, and T. E. Haynes}

In recent years, the use of $\mathrm{SiC}$ for applica. tions in high-temperature, high-frequency, and high-power electronic devices has emerged as a promising technology. Successful growth of thin films of $\beta$-SiC using chemical vapor depor)sition ${ }^{2}$ and molecular beam epitaxy $y^{3}$ has been 
reported. However, both of these techniques require growth temperatures well above $1000^{\circ} \mathrm{C}$. Very recently the heteroepitaxial growth of B-SiC on Si substrates held below $800^{\circ} \mathrm{C}$ using a reactive ion beam deposition (IBD) technique has been reported.4

In this work, thin films of $\beta$-SiC have been grown epitaxially on $6 \mathrm{H}$ a-SiC substrates by direct IBD of altemating layers of ${ }^{13} \mathrm{C}^{+}$and $30 \mathrm{Si}^{+}$. The substrate was chosen because of the lattice match between the (0001) 6H a-SiC and the (111) B-SiC surfaces. The carbon and silicon ions were ohtained from an ion implanter by decelerating mass-analyzed ion beams to $40 \mathrm{eV}$. The substrate was held at $-700^{\circ} \mathrm{C}$ during growth. Rutherford backscattering (RBS) and high-resolution transmission electron microscopy (TEM) techniques were used to determine the composition and structure of the resulting $\mathrm{B}-\mathrm{SiC}$ layer and the nature of the interface.

Figure 5.4 shows a high-resolution TEM image of the resulting overlayer and substrate. The film is crystalline, with a variety of

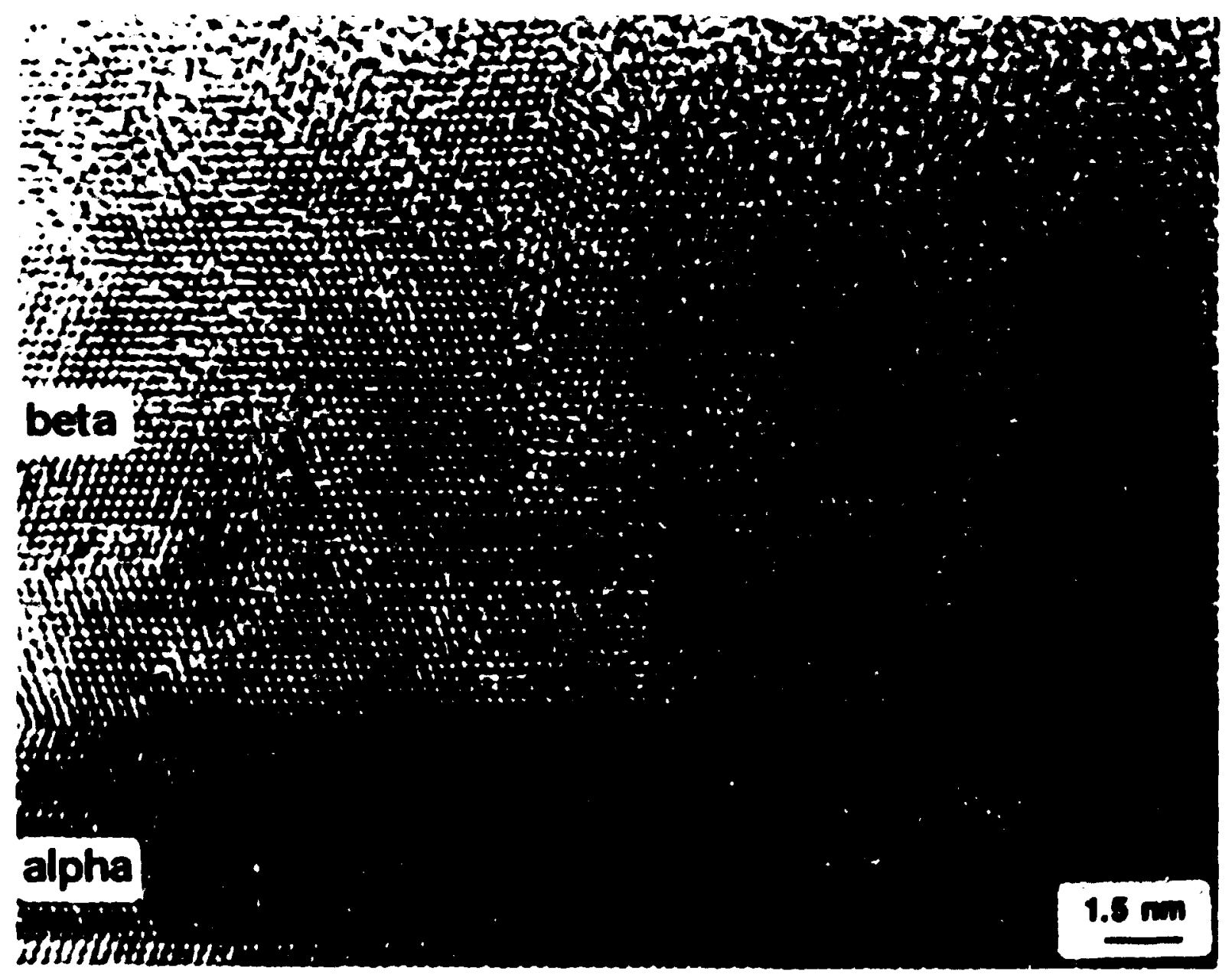

Fig. 5.4. Lattice image of IBD-deposited B-SiC on an $\alpha-S i C$ substrate. 
defects present. The most common defects, identified by arrows in the figure, are small areas or boundaries which appear amorphous in nature, but the remainder of the B-SiC overlayer is epitaxial. RBS results indicated that the film had an excess of about $30 \%$ carbon. In addition, no well-defined channeling axis was found in the overlayer. This result, taken together with the TEM images, suggests that the crystal is highly strained. Both TEM and RBS indicated that the overlayer thickness is not uniform. This work represents the lowest reported temperature for formation of $\beta$-SiC.

1. Metals and Ceramics Division, ORNL.

2. H. P. Liaw and R. F. Davis, J. Electrochem. Soc. 132, 642 (1985).

3. Shigeo Kaneda et al., J. Cryst. Growth 81, 536 (1987).

4. Hiroshi Yamada, J. Appl. Phys. 65, 2084 (1989).

\section{DEVELOPMENT OF AN AUTOMATED MAGNET CONTROLLER FOR ALTERNATING ION BEAM DEPOSITION OF COMPOUND LA YERS}

\section{L. Moore, T. E. Haynes, and R. A. Zuhr}

Previous sludies of ion beam deposition (IBD) at ORNL have focused on the deposition of the elemental semiconductors $\mathrm{Si}$ and Ge. These studies demonstrated that isotopically pure, epitaxial Si and Ge films could be grown successfully at modest temperatures by I8D. In order to extend the application of IBD to a wider varicty of materials systems, a program was initiated to apply IBD to alloy and com- pound layers. The chief experimental barrier to depositing compound layers by IBD had been to find a suitable method for combining (wo or more elements from low-energy (decelerated) ion beams onto a single target. Several methods were considered, including the use of molecular ion species and the use of multiple independent ion sources operating simultaneously. However, we have recently discovered thai some compound layers can be grown by simply switching rapidly between two elemental ion beams during deposition. 1

This research required the development of an automatic controller which was capable of switching the analyzer magnet quickly and repetitively between ion species after predefinable deposited fluences. An open-loop controller was developed based on a microcomputer linked by fiber optic cables to the beam current integrator (measured fluence) and the magnet current adjustment. The following criteria were considered in the design of the controller: reproducibility of settings, flexibility (programmable in BASIC), simplicity of operation ("user friendly"), and upward compatibility to meet future needs. The present controller has performed satisfactorily in work on GaAs and SiC deposition, and upgrades to extend the performance over a wider range of masses are planned.

1. T. E. Haynes et al., Appl. Phys. Lett. 54, 1439 (1989). 


\section{FORMATION OF HIGH-QUALTY SIUCON-ON-INSULATOR STRUCTURES BY HICH-DOSE OXYGEN MMPLANTATION IN SILCON ${ }^{1}$}

M. K. El-Ghor, ${ }^{2}$ S. J. Pennycook, F. M. Nameoar, ${ }^{3}$ and N. K. Karam ${ }^{3}$

There is increasing interest in the production of device quality silicon-on-insulator material for possible microelectronic applications. One of the most promising techniques is buried oxide structures formed by high doses of oxygen implanted in silicon (SIMOX). The two types of defects present in the Si film of the annealed structure are precipitates and threading dislocations. So far, three SIMOX methods have been reported that produce a dislocation density $<10^{5} \mathrm{~cm}^{-2}$. We present a new method that also produces a low dislocation density."

SIMOX structures were produced by implanting n-type (100) Si wafers with $16 \mathrm{O}^{+}$ $\left(200 \mathrm{keV}, 1.8 \times 10^{16} / \mathrm{cm}^{2}, 12 \mu \mathrm{A} / \mathrm{cm}^{2}\right)$ while the surface temperature was kept at $610^{\circ} \mathrm{C}$. Figure $5.5(a)$ shows the microstructure of the asimplanted sample, which contains a buried oxide layer $-2700 A$ thick. The top silicon layer consists of a region -1300 \& wide below the surface, which contains a high density of randomly arranged cavities ranging in size from 30 to $200 \AA$ in diameter. The region below the cavities extending to the oxide interface is heavily damaged, containing a high density of small precipitates with stacking faults and extended dislocations.

After annealing at $1300^{\circ} \mathrm{C}$ for $6 \mathrm{~h}$ in dry $\mathrm{N}_{2}$, the structure in Fig. 5.5(b) was obtained. The buried oxide layer increased in thickness to
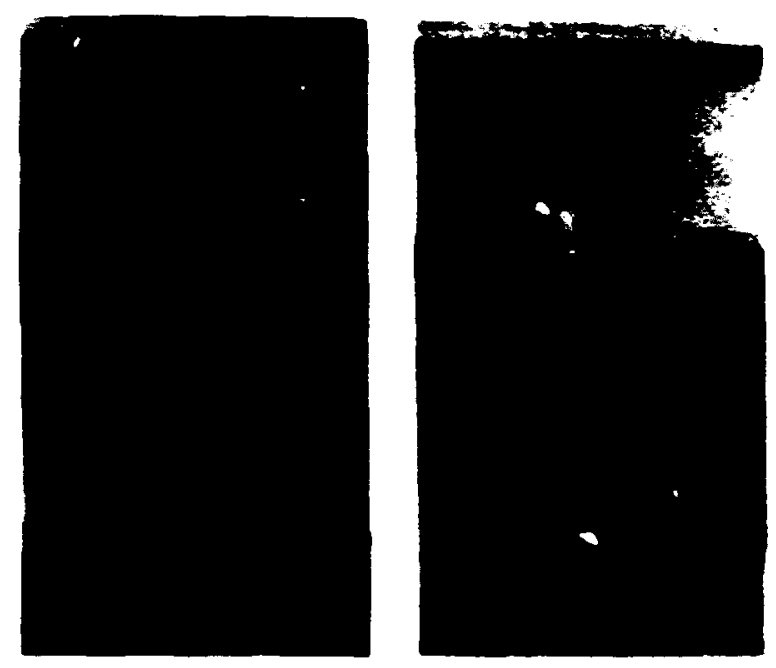

Fig. 55. Bright field cross-sectional TEM micrographs of Si(100i samples implanted at $610^{\circ} \mathrm{C}$ with ${ }^{16} \mathrm{O}^{+}\left(200 \mathrm{keV}, 12 \mu \mathrm{A} / \mathrm{cm}^{2}\right)$ at a dose of $1.8 \times 10^{18} \mathrm{O}^{+} / \mathrm{cm}^{2}$. (a) as-implanted and (b) after annealing at $1300^{\circ} \mathrm{C}$ for $6 \mathrm{~h}$ in $\mathrm{N}_{2}$. Sample surface is denoted by $S$.

4000 A primarily by the motion of the upper interface. The most important result of this work is the absence of precipitates and threading dislocations in the top Si layer. This is confirmed by plan view samples, which showed a dislocation density $<10^{5} \mathrm{~cm}^{-2}$. The high quality of the top Si layer was further substantiated by Rutherford backscattering/channeling results.

1. Summary of paper to be published.

2. Oak Ridge Associated Universities postgraduate research participant.

3. Spire Corporation, Bedford, Mass.

4. M. K. El-Ghor et al., Mat. Res. Soc. Symp. Proc. 74, 591 (1987). 
STRUCTURAI CHARACTERIZATION

OF MEV DAMAGE AND

ANNEALNG OF (100) Si

M. K. El-Ghor, 2 O. W. Hoiland,

C. W. White, and S. J. Penmycook

Implantation using $\mathrm{MeV}$ ion beams is of considerable current interest since it produces a relatively damage-free near-surface region, which is essential in very large scale integrated circuit applications. In the present work, transmission electron microscopy (TEM) has been used to investigate the microstructure of buried amorphous layers produced by Si selfimplantation at $R T$ and $L N_{2}$. The buried layers were produced using $1.25-\mathrm{MeV}{ }^{28} \mathrm{Si}$ ions at a current density of $0.3 \mu \mathrm{A} / \mathrm{cm}^{2}$. Doses of $1.0 \times$ $10^{16} \mathrm{~cm}^{-2}$ and $5.0 \times 10^{16} \mathrm{~cm}^{-2}$ were used at RT and $\mathrm{LN}_{2}$, respectively. Postimplantation annealing was conducted at temperatures of $400-800^{\circ} \mathrm{C}$ at 30 min in dry $\mathrm{N}_{2}$.

In the as-implanted condition, the amorphous/crystalline interfaces formed at RT were rough and were surrounded by distocation loops and large single-crystalline islands. At $\mathrm{LN}_{2}$, the interface was observed to be very diffuse [Fig. 5.6(a)]. The diffuse nature of the $a / c$ interface at $\mathbf{L N}_{\mathbf{2}}$ resulted in a wide transition region, which contains a mixture of minute single-crystalline $\mathrm{Si}$ islands and amorphous zones. Substantial reordering is observed in this mixed-phase region after annealing at $\mathbf{4 0 0}$ and $500^{\circ} \mathrm{C}$ [Figs. 5.6(b) and 5.6(c)]. Further annealing to $550^{\circ} \mathrm{C}$ [Fig. $5.6(\mathrm{~d})$ ] shows the evolution of inairpin dislocations from the advancing upper interface where the recrystallization front intersects dislocation

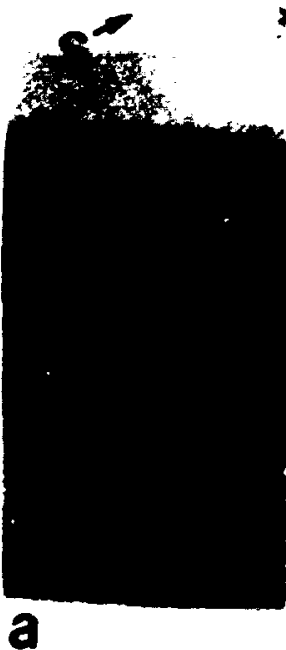

AS IMPLANTED

\subsection{MOV Si -IMPLANTED SI (100) AT LN}
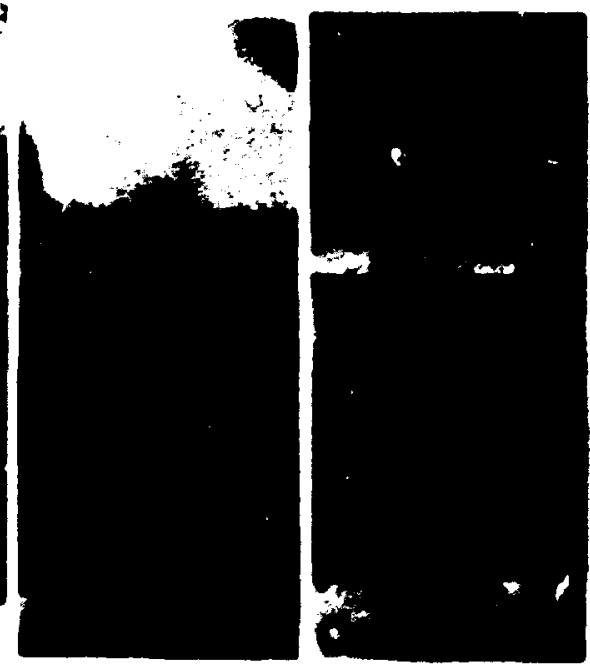

$500^{\circ} \mathrm{C}$

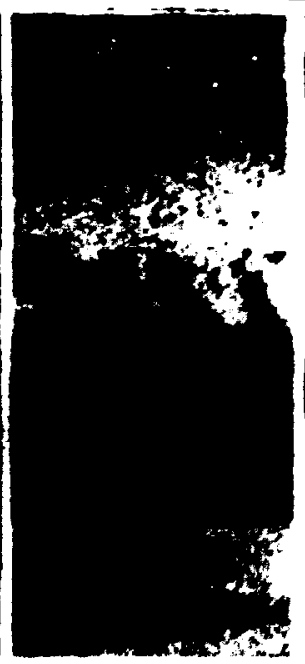

600

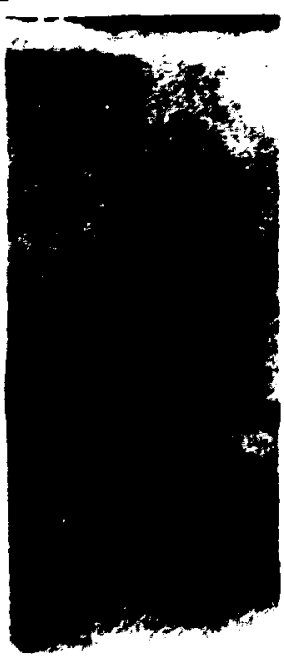

$800^{\circ} \mathrm{C}$

Fig. 5.6. Bright field cross-sectional TEM micrographs of Si(100) samples at an energy of $1.25 \mathrm{MeV}$ implanted at $\mathrm{LN}_{2}$. (a) as-implanted; after annealing for $30 \mathrm{~min}$ at (b) $400^{\circ} \mathrm{C}$, (c) $500^{\circ} \mathrm{C}$, (d) $550^{\circ} \mathrm{C}$, and (e) $800^{\circ} \mathrm{C}$. Sample surface is denoled by $\mathrm{S}$. 
loops above a critical size. Fig. 5.6(e) shows an $800^{\circ} \mathrm{C}$ anneal where complete recrystallization sccurs and both fronts meet at a central line in which hairpin dislocations are incorporated to reduce their surface energy. Rod-like defects lying on (111) planes and along $<110\rangle$ directions appeared at that temperature.

1. Summary of paper to be published.

2. Oak Ridge Associated Universities postgraduate research participant.

\section{NOBLE METAL ION MMPLANTATION OF TI AND SURGICAL Ti-GAH-AV ALLOY1}

\author{
J. M. Williams, R. A. Buchaman, \\ and In-Seop Lee?
}

The effects of ion implantation of Ir on electrocherrical behavior of $T i$ and surgical Ti-6Al-4V alloy have been investigated. For possible applications in the field of surgical materials, the surface properties of interest include not only corrosion resistance in neutral saline media but also the properties of iridium oxide that pertain to possible use of the oxide as a biosensor and neural electrode. These latter properties are illustrated by the cyclic voltammagram (Fig. 5.7), which comprises part of the results of the present study. One of several goals is to maximize the "charge injection" properties represented, in effect, by the areas of the loops such as those shown in Fig. 5.7.

Experimental techniques have included ion implantation, standard electrochemical tech-

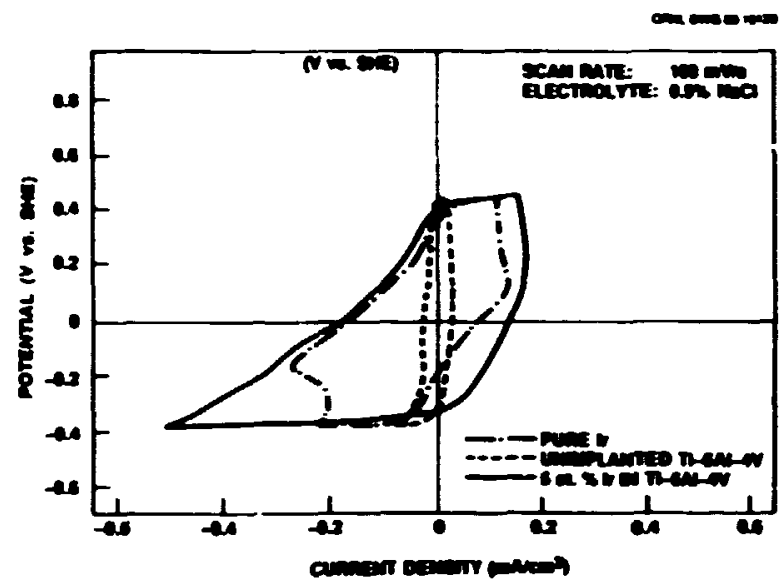

Fig. 5.7. Cyclic voltammetry curves (potential vs current density) for the sample materials indicated. The implanted alloy sample was also acid treated.

niques, Rutherford backscattering spectroscopy, scanning electron microscopy, electron spectroscopy for chemical analysis, and profilometry. Some implanted samples were subjixcted to a corrosion pretreatment in $1 \mathrm{~N} \mathrm{H}_{2} \mathrm{SO}_{4}$ solution. For alloy samples, this selectively removed the alloy components and left the ionimplanted Ir essentially as a coating on the surface. At least $80 \%$ of the implanted Ir appears to have been retained. The resulting cyclic voltammagram in saline is shown in Fig. 5.7, which also has curves for pure Ir and unimplanted alloy control samples.

The area of the loop for the implanted alloy (Fig. 5.7) can be accounted for if $66 \%$ of the retained Ir is involved in the reversible transition from $\mathrm{InO}_{2}$ to $\mathrm{Ir}_{2} \mathrm{O}_{3}$ during the cycle. This seemingly close correlation between the charge injection and the amount of Ir available supports the view that the reversible transition, 
rather than double-layer capacitance, is respcnsible for the charge injection.

1. Summary of papers: Nucl. Instrum. and Methods Phys. Res. Sect. B 37/38, 676 (1989); p. 53 in Proceedings of the Conference on lon Implantation and Plasma-Assistea Processes for Industrial Applications, ASM International, Metals Park, Ohio (1989); Sens. and Actuators 2, 399 (1982); Materials Res.arch Society Symposium Proceedings (in press); Joumal of Biomedical Malirials Research (in press).

2. The University of Tennessee, Knoxville, Tenn.

\section{SOLID-PHASE EPITAXY IN LiNbO ${ }_{3}^{1}$}

\section{B. Poker and D. K. Thomas}

Ion implaritation of $\mathrm{Ti}$ into $\mathrm{LiNbO}_{3}$ has been shown to be an effective means of introducing dopant for the purpose of producing an optical waveguide. The process of ion implantation resu!ts in more precise control of the dopant concentration profile, shallower waveguides, and higher dopant conrentrations than can be achieved with conventional thermal diffusir: techniques. Implantation at low temperatures produces an amorphous implanted region, which must be regrown by solid-phase epitaxy (SPE) to produce the waveguide. SPE of $\mathrm{LiNbO}_{3}$ following low doses of $\mathrm{Ti}\left(<3 \times 10^{16}\right.$ $\mathrm{Ti} / \mathrm{cm}^{2}$ ) was shown to proceed at $250 \AA / \mathrm{s}$ at $400^{\circ} \mathrm{C}$, with an activation energy of $2.0 \mathrm{cV}$. However, regrowth at higher doses was slower due to segregation of Ti at the interface. Samples implanted to doses high enough to produce waveguides, $-2.5 \times 10^{17} \mathrm{Ti} / \mathrm{cm}^{2}$, must be regrown at $1000^{\circ} \mathrm{C}$ for several hours.

Two other known waveguide dopants, Ag and $\mathrm{Pb}$, were investigated as implanted dopants, since they exhibit a lower tendency to oxidize and should interfere less with the SPE. Even high doses of $\mathrm{Ag}$ show no interference with the SPE, as illustrated in Fig. 5.8. However, at the temperatures required for complete annealing of the residual radiation damage $\left(-800^{\circ} \mathrm{C}\right)$, the $\mathrm{Ag}$ dopant diffuses rapidly, destroying the concentration profile. $\mathrm{Pb}$ exhibits behavior intermediate between $\mathrm{Ti}$ and Ag but deas not occupy substitutional sites, even after annealing at $1000^{\circ} \mathrm{C}$.

1. Summary of papers: I. Mater. Res. 4,412 (1989); Nucl. Instrum. and Methods Phys. Res. Sect. B 39, 716 (1989).

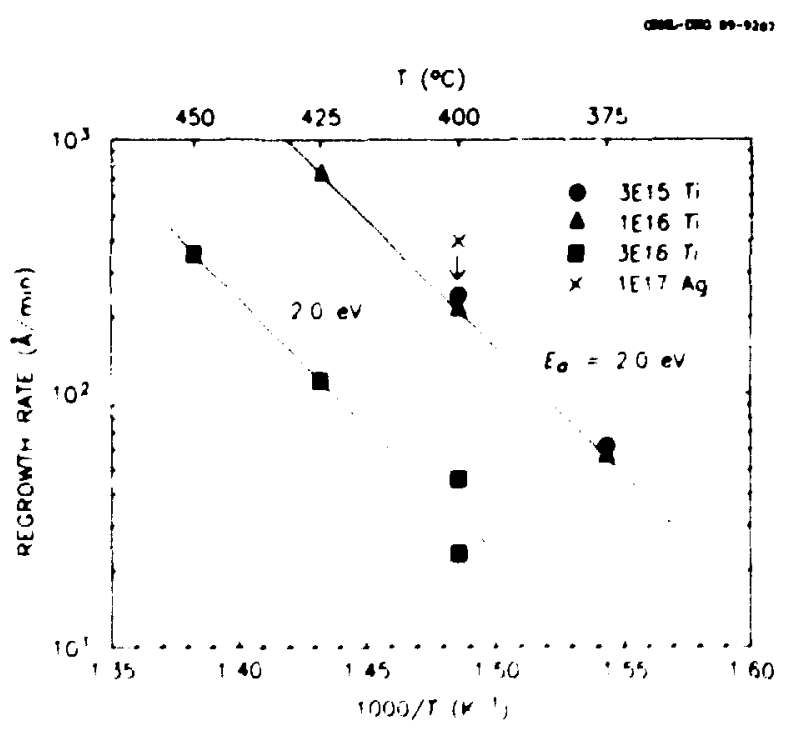

Fig. 5.8. Arrhenius plot of regrowth rate vs inverse temperature for SPE of $\mathrm{Ti}$ - and $\mathrm{Ag}$ implanted $\mathrm{LiNbO}_{3}$. The straight lines are least-squares fits to the data with an activation energy of $2.0 \mathrm{eV}$. 


\section{EFFECTS OF IMPLANTED TRANSITION- METAL IONS ON THE REFRACTTVE INDEX OF FUSED SILICA}

\section{R. A. Weeks, ${ }^{1}$ H. C. Mogul,' R. F. Haglund, G. W. Whichard, 1 and R. A. Zuhr}

We have made ellipsometric measurements of changes in the refractive index of highpurity silica glass produced by implantation of $\mathrm{Ti}^{+}, \mathrm{Cr}^{+}, \mathrm{Mn}^{+}, \mathrm{Fe}^{+}$, and $\mathrm{Cu}^{+}$ions at $160 \mathrm{keV}$, at doses ranging from $0.1 \times 10^{16}$ ions $/ \mathrm{cm}^{2}$ to $6 \times 10^{16}$ ions $/ \mathrm{cm}^{2}$. Distributions of the implanted ions were determined by conventional ion backscattering techniques, and a single-layer model of the implanted ions was found to be consistent with both the backscattering and eilipsometric data. We interpret the implantation-induced changes in the refractive index (Fig. 5.9) as arising from (1) changes in the density caused by radiation damage and (2) alterations in the polarizabilities of the substrate with its

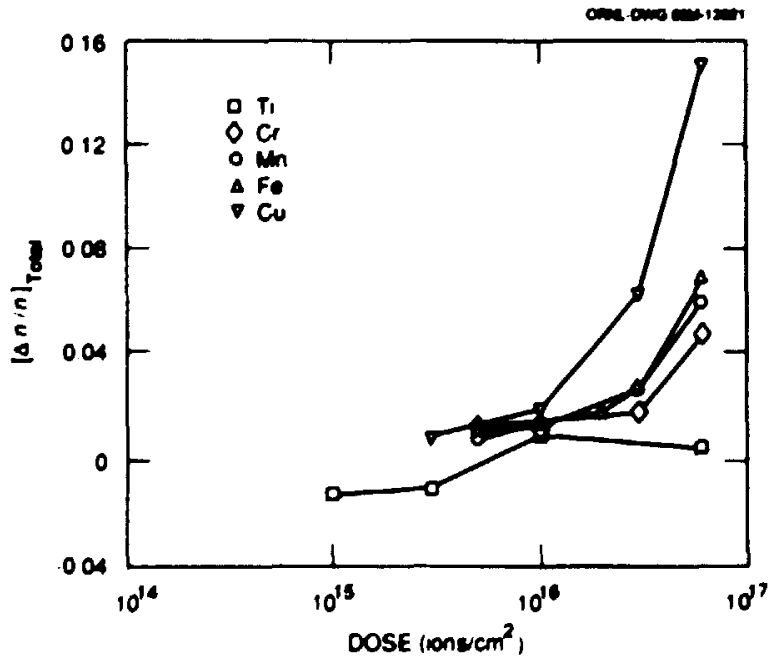

Fig. 5.9. Measured ellipsometric values of $(\Delta r / n)_{\text {Total }}$ for Spectrosile implanted with the indicated transition-metal ions from the first series, as a function of total ion dose. implanted ions. The increased density comes from the compaction of the substrate caused by radiation damage by some $2.5 \%$ for the dose range covered here and from the filling of the glass "free volume" by the implanted ions. The differences between the total changes in refractive index and the changes caused solely by the density variations are attributed to changes in the polarizatility of the ions in the implanted region as calculated from the Lorentz-Lorenz relation. The scaling of these variations with implanted ion species appears to be correlated with the electronic structure of the implanted ions. At the highest doses, and especially for the $\mathrm{Cu}^{+}$and $\mathrm{Ti}^{+}$ions, more dramatic changes in refractive index may signal breakdown of the single-layer model, shifts in the electronic (or chemical) character of the binding of the implanted ions, or both.

1. Vanderbilt University, Nasiiville, Tenn.

\section{ECR MICROWAVE PLASMA PROCESSING \\ S. M. Gorbatkin, J. B. Roberto, \\ L. A. Berry, and C. C. Tsail}

Electron cyclotron resonance (ECR) microwave plasmas offer significant advantages for thin-film processing in comparison with conventional plasmas. ECR plasmas can be operated at lower gas pressures for the same plasma density, yield high current densities at low ion energy (a few 10's of eV), and allow more independent control over operating 
parameters such as pressure, ion density, and ion energy.

Two ECR systems have been assembled and operated over the past year. A UHV deposition system has been constructed in the Solid State Division based on a commercial two-coil mirror ECR microwave ion source. This system is currently being operated with $A r$ and $N_{2}$ to study the effect of operating conditions (pressure, magnetic field profile, power, etc.) on plasma properties. ${ }^{2}$ Gas-handling equipment is being installed, and initial materials studies will focus on BN thin-film systems.

A second ECR system based on a permanent magnet line cusp plasma confinement chamber coupled to a compact single-coil ECR source has been assembled in the Fusion Energy Division. ${ }^{3}$ The resulting plasma source (shown in Fig. 5.10) is capable of producing large-area $(20-\mathrm{cm}$ diam), highly uniform $( \pm 5 \%)$ ion beams with current densities above $5 \mathrm{~mA} / \mathrm{cm}^{2}$. This source is a prototype for a commercial cusp field ECR

OAMU OWG 3001C FEOA

\section{ECR PLASMATAON}

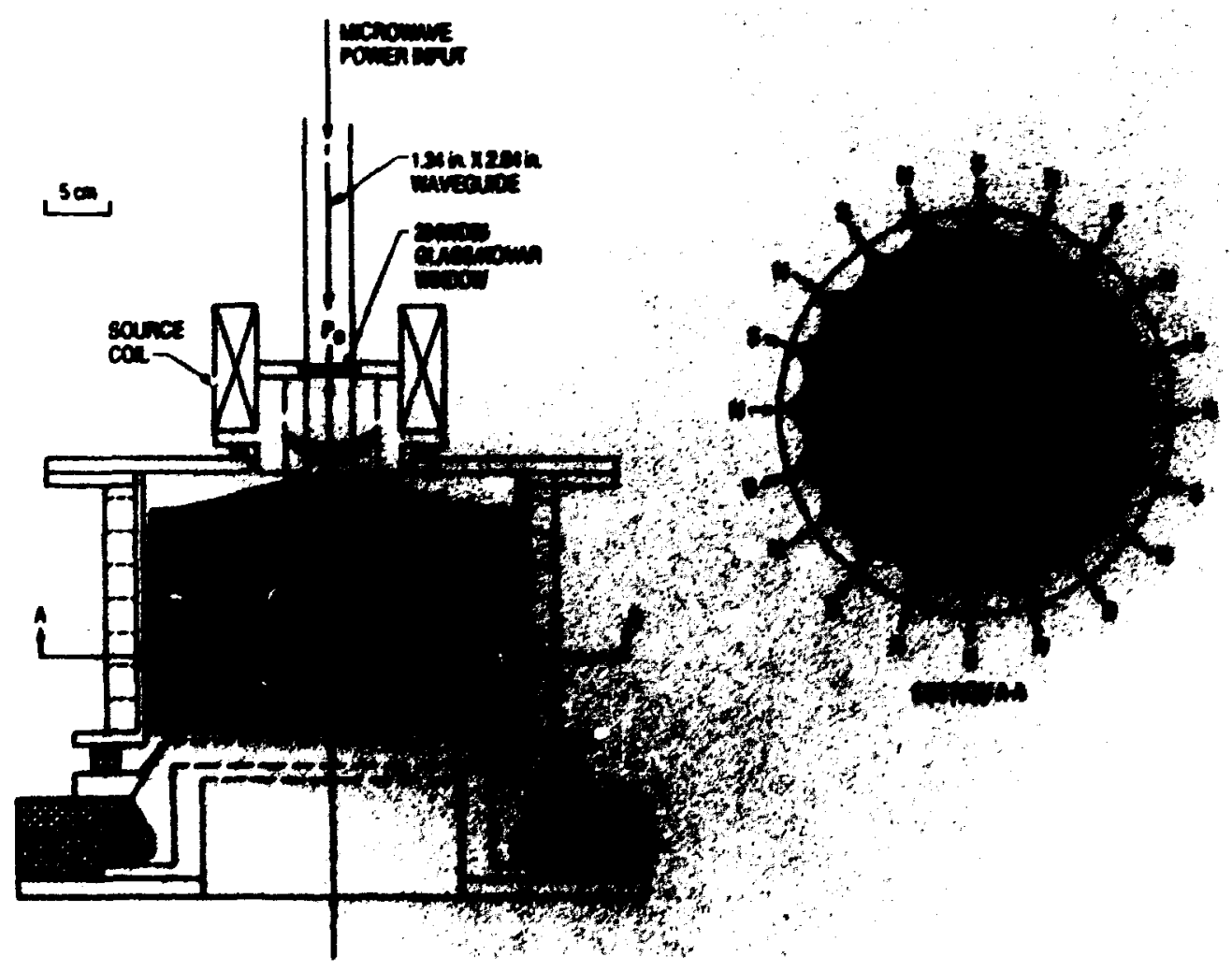

Fig. 5.10. The ORNL ECR multicusp plasmatron. 
system and has been used to etch photoresist on 5-in.-diam silicon wafers with good uniformity. The source is currently being optimized to operate over a wider range of operating conditions at higher current density and uniformity.

1. Fusion Energy Division, ORNL.

2. Summary of paper to be published.

3. L. A. Berry et al., Proceedings of SRC Topical Research Conference on Plasma Etching, MIT, Cambridge, Massachusetts, February 1-2, 1989 (in press).

\section{ION IMPLANTATION AND ANALYSIS}

\section{MEV ION DAMAGE IN SIUICON'}

\author{
O. W. Holland, M. K. El-Ghor, \\ and C. W. White
}

The large penetration depth of $\mathrm{MeV}$ ions offers the possibility for studying ion-induced damage growth mechanisms over an extended range of nuclear and electronic stopping. Thus in $\mathrm{Si}$ the ion path consists of segments in which different damage nucleation and growth mechanisms dominate. Evidence for this can be seen in Fig. 5.11, which compares Rutherford backscattering specira from $\mathrm{Si}$ single crystals implanted with $1.25 \mathrm{MeV} \mathrm{Si}$ ions at two different doses. The aligned scattering yield from both samples is seen to be essentially the same over the top $\sim 1.0$ micron, indicating a saturation of damage growth in this region at some low level. However, in the region centered on the end of range (EOR) of the ions

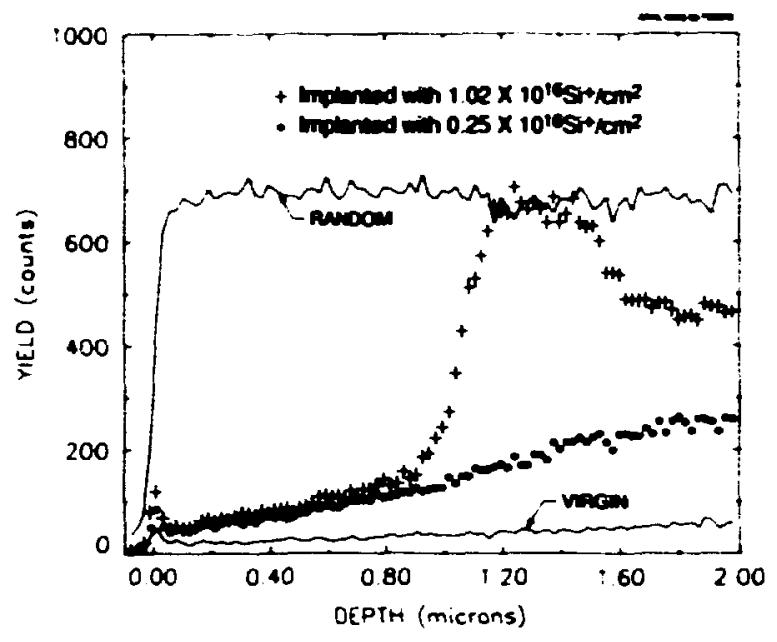

Fig. 5.11. <110> aligned spectra from $\mathrm{Si}(100)$ samples implanted with $1.25-\mathrm{MeV} \mathrm{Si}{ }^{+}$ ions.

(-1.3 micron), the scattering yield is seen to increase with ion dose up to the random level. Interestingly, no defects were observed by transmission electron microscopy in the saturated region. Isothermal heating of the implanted samples showed that the damage in this region annealed at a very low temperature $\left(200^{\circ} \mathrm{C}\right.$ for $\left.1 \mathrm{~h}\right)$. This annealing behavior is the same as for the divacancy in $\mathrm{Si}$ and strongly suggests that the divacancy is the predominate defect in this region.

The defect interactions were modeled using chemical rate equations in which damage nucleation and growth were assumed to occur homogeneously within the irradiated volume as a result of interaction between the Frenkel pairs produced by the ions and various defect sinks. It was shown that damage saturation ahead of the EOR of the ions is easily accounted for by the model if no extended 
defects (i.e., dislocations) are present within the reaction volume. The model also predicts a dose-rate dependence on the saturation level, which has been confirmed.

1. Summary of paper. Appl. Phys. Lett. 53, 1282 (1988).

2. Oak Ridge Associated Universities postgraduate research participant.

\section{INTERACTION OF MEV IONS WITH PREEXISTING DAMAGE IN Si: A NEW ION BEAM ANNEALING MECHANISM ${ }^{1}$}

\section{O. W. Hollend}

The interaction of $\mathrm{MeV}$ ions with preexisting damage in Si was investigated. Predamaging of Si, prior to the high-energy ion irradiation, was accomplished by $100-\mathrm{keV} \mathrm{Si}^{+}$ion implantation. A variety of analytical techniques, such as Rutherford backscattering spectroscopy and cross-sectional transmission electron microscopy (XTEM) in conjunction with isothermal annealing techniques, were used to determine the microstructure of the damage. Ion-channeling spectra (along the $\langle 110\rangle$ axis) are shown in Fig. 5.12 from samples which had been implanted with $100-\mathrm{keV}$ ions to a fluence of (a) $2 \times 10^{14}$ and (b) $3 \times 10^{14} \mathrm{Si}^{+} / \mathrm{cm}^{-2}$. Also shown in Fig. 5.12 are aligned spectra from similarly implanted samples which were subsequently irradiated with two different flu. ences $\left(2 \times 10^{15}\right.$ and $\left.1 \times 1 n^{16} \mathrm{~cm}^{-2}\right)$ of $1.25 \mathrm{MeV}$ $\mathrm{Si}^{+}$ions. A damage peak cintered at $\sim 0.13 \mu \mathrm{m}$ as a result of the $100-\mathrm{keV}$ implantation is seen in both predamaged samples.

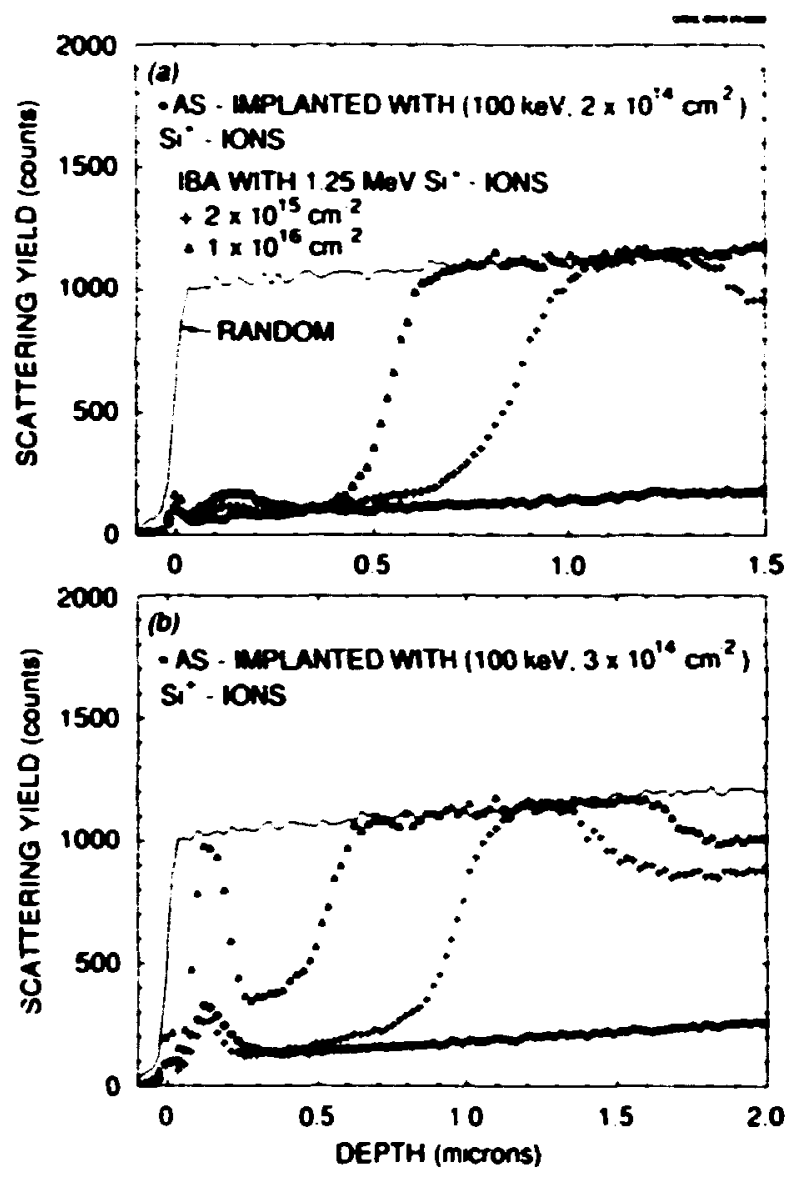

Fig. 5.12. Comparison of aligned spectra showing the effect of different fuences of 1.25$\mathrm{MeV} \mathrm{Si}^{+}$ions on samples predamaged with 100 $\mathrm{keV} \mathrm{Si}^{+}$ions implanted at (a) $2 \times 10^{14}$ and (b) $3 \times 10^{14} \mathrm{~cm}^{-2}$.

The MeV ion irradiation is seen [Fig. 5.12(a)] to reduce the scattering yield in the predamaged region of the sample produced by the lower fluence, $100-\mathrm{keV} \mathrm{Si}{ }^{+}$irradiation. However, the interaction of the MeV ions with the damage produced by the higher fluence of 100 $\mathrm{keV}$ ions [Fig. 5.12(b)] results in a very different behavior. The scaltering yield from the predamaged region is seen to be slightly decreased by the $1.25 \mathrm{MeV}, 2 \times 10^{15} \mathrm{~cm}^{-2}$ 
irradiation, but is greatly increased by the higher fluence. In fact, XTEM analysis showed that a buried amorphous layer was formed in this region of the sample. Analysis of the predamaged morphology showed that only simple types of defects such as divacancies were formed in $\mathrm{Si}$ at the lower fluence $\left(2 \times 10^{14}\right.$ $\mathrm{cm}^{-2}$ ) of 100-keV ions, while more complex defects, such as dislocation loops and small amorphous regions, were formed at the higher fluence. A model based on homogeneous nucleation and growth of damage was shown to account qualitatively for the interaction of the $\mathrm{MeV}$ ions with the different damage morphologies.

1. Summary of paper. Appl. Phys. Lett. 54, 320 (1989).

\section{NEW MECHANISMS FOR DIFFUSION OF ION-IMPLANTED BORON IN Si AT HIGH CONCENTRATION'}

\section{O. W. Holland}

Modeling of the diffusion of ion-implanted boron in $\mathrm{Si}$ at high concentrations was shown to be inconsistent with vacancy-controlled, substitutional motion. The depth profile of boron, determined by secondary ion mass spectroscopy, in a $\mathrm{Si}(100)$ single crystal after annealing at $900^{\circ} \mathrm{C}$ for $15 \mathrm{~min}$ is shown in Fig. 5.13. The sample was implanted at $65 \mathrm{keV}$ with $5 \times$ $10^{15} \mathrm{~cm}^{-2}$ of $\mathrm{BF}_{2}{ }^{+}$ions. It is seen that the profile has an inflection at $-0.1 \mu \mathrm{m}$. Ahead of this point, the boron is relatively immobile (at the

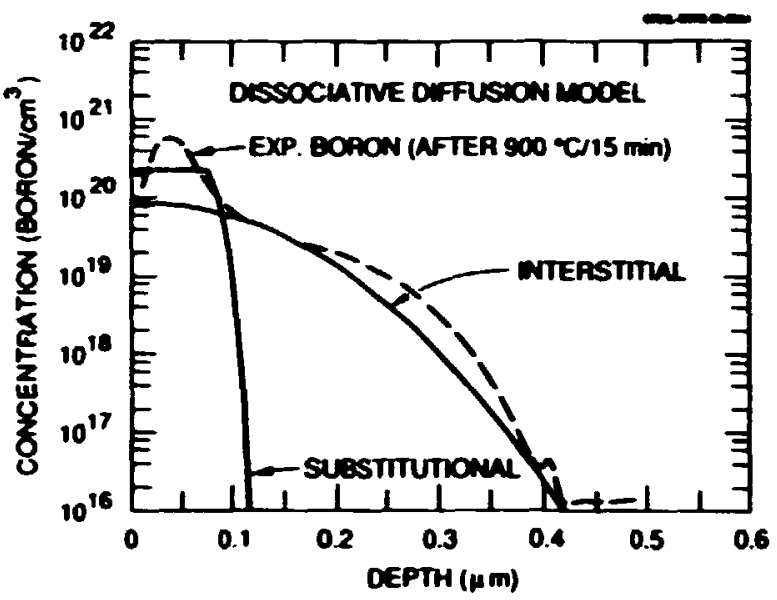

Fig. 5.13. Comparison of the experimental concentration profile of boron in Si diffused at $900^{\circ} \mathrm{C}$ with calculated profiles using the dissociative model.

annealing temperature) and is located near the original (as-implanted) profile. Beyond this point there is a large, diffused tail. Sheet resistance measurements showed that much of the boron in the immobile peak was electrically active.

In the charged-vacancy model, diffusion under extrinsic conditions is greatly enhanced because the concentration of charged vacancies increases with the doping level. Computer simulation using this model predicts the boron profile to be abrupt, having a reasonably uniform concentration over its distribution and terminated by a very steep gradient. The lack of these features in the experimental profile suggests that the diffusion of the boron which produces the profile tail is not substitutional. This led to a two-stream model in which both substitutional and interstitial boron diffuse interdependently in $\mathrm{Si}$. II is envisioned that at 
high boron concentrations, the interstitial component is large and of the same order of magnitude as the substitutional boron. Simulation results of the two-stream diffusion model, which produced both the interstitial and substitutional profiles, are shown in Fig. 5.13 and clearly reproduce the main features of the diffusion profiles of boron implanted in Si.

1. Summary of paper. Appl. Phys. Lett. 54, 798 (1989).

\section{OXIDATION OF SI IMPLANTED WITH NONDCPANT, METALLIC IONS ${ }^{1}$}

O. W. Holland, C. W. White, and S. J. Pennycook

The effect of implantation of low-solubility metallic impurities on the oxidation kinetics of $S i$ in steam was studied. It was found that novel morphological structures could be formed during oxidation of such implanted Si crystals. All impurities studied did not behave in an identical manner during oxidation, but $\mathrm{Pb}$ exhibited many of the interesting features which were observed in other impurity/Si systems. Initially it was found that there was very little substitutional incorporation of the $\mathrm{Pb}$ within the Si lattice, even after annealing at high temperatures in an inert ambient. Transmission electron microscopy showed that after annealing, much of the $\mathrm{Pb}$ was located in randomly aligned precipitates and polycrystalline silicon had formed within the irradiated region of the sample. However, the $\mathrm{Pb}$ precipitates were observed to align themselves with the Si lattice during oxidation.

The degree of alignment (as determined by ion channeling measurements) is shown in Fig- 5.14. The data points represent the measured values, while the solid curve is corrected for the special morphology encountered in these samples. The curve shows that the alignment of the $\mathrm{Pb}$ precipitates during oxidation occurs rapidly at first but then more slowly. The initial improvement in the alignment is the result of the reorientation of the $\mathrm{Pb}$ precipitates, which are rejected from the oxide during growth, with less-defective $\mathrm{Si}$ at the oxide interface. This process continues until all the polycrystalline $\mathrm{Si}$ is consumed. Further improvement is thought to occur as a result of a ripening process in which size of the precipitates increases.

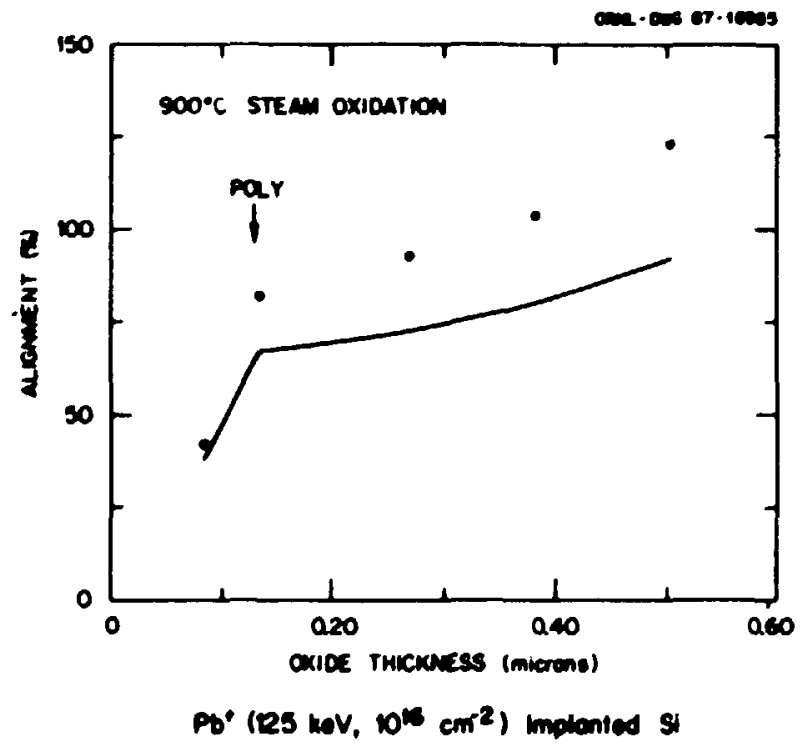

Fig. 5.14. The percent alignment along $<100\rangle$ of $\mathrm{Pb}$ precipitates as a function of oxide thickness in $\mathrm{Pb}^{+}$-implanted Si. 
A similar behavior was observed for other impurities such as $\mathrm{Ag}_{\mathbf{g}}$ which have a lattice structure fcc with symmetry similar to the diamond lattice. Impurities, such as $\mathbf{T}, S_{n}, \mathbf{Y b}$, and Co (which do not have such symmetry), were segregated during oxidation and piled up at the growth interface but were not crystallographically aligned. Yb was unique in that it was completely segregated into the oxide during the growth.

1. Summary of paper. J. Moter. Res. 3,898 (1988).

\section{MMLANTATION INTO DLAMONDS ${ }^{1}$}

S. M. Gorbattin, R. A. Zwhr,

J. Roth, ${ }^{2}$ and H. Naramoto ${ }^{3}$

The unique properties of diamond, such as high-thermal conductivity, extreme hardness, wide band gap, and good thermal and chemical stability, make it attractive for use in hightemperature semiconductor devices, Doping diamond, however, cannot be achieved by thermal diffusion due to the low diffusivity of impurities at high temperatures. Ion implantation is a prime candidate technique for doping, but the issues of annealing radiation damage, avoiding graphitization, and incorporating implanted species into substitutional sites have not been completely resolved.

In this work, Rutherford backscattering spectroscopy was used to study the damage and impurity location in the lattice, as a function of total dose and dose rate, for synthetic diamonds

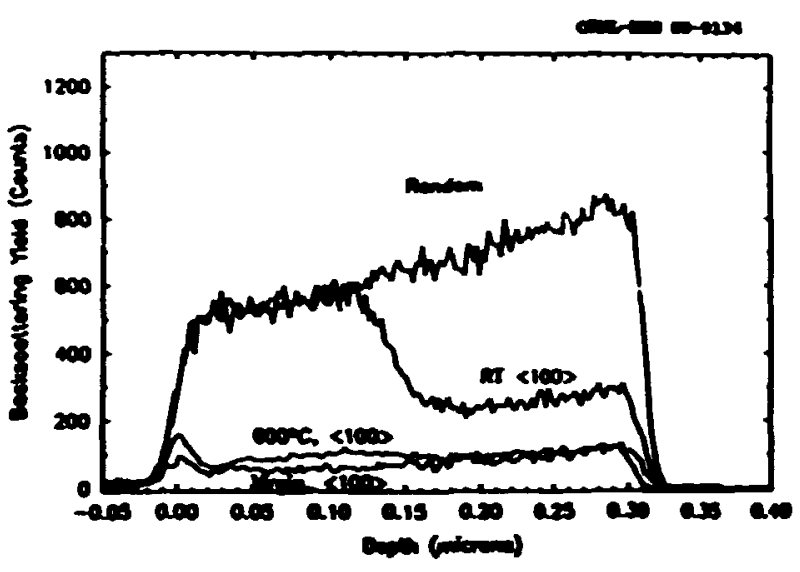

Fig. 5.15. Rutherford backscattering spectrum showing the effect of implantation temperature on lattice damage during implantation of $1 \times 10^{15} \mathrm{~cm}^{-2} 75$ As into diamond at $250 \mathrm{keV}$.

implanted with ${ }^{75} \mathrm{As}$ at $250 \mathrm{keV}$ and $600{ }^{\circ} \mathrm{C}$. Figure 5.15 shows the large reduction in damage found by increasing the temperature from room temperature to $600^{\circ} \mathrm{C}$ during implantation of $1 \times 10^{15} \mathrm{~cm}^{-2} 75$ As into diamond. Postimplantation anneals are normally ineffective for damage reduction, and the annealing behavior cf these diamonds is currently being studied. The fraction of As occupying substitutional sites was as high as $50 \%$ at the lowest implanted doses $\left(5 \times 10^{14} \mathrm{~cm}^{-2}\right)$.

1. Summary of paper to be published.

2. Max-Planck-Institut für Plasmaphysik, Garching, Federal Republic of Cermany.

3. Japan Atomic Energy Research Institute, Tokai, Japan.

4. V. S. Vavilov, Rodiat. Eff. 37, 229 (1978). 


\section{NUCLEATION OF DAMAGE DN GE BY MEV ION IRRADIATION'}

T. P. Sjoreen, O. W. Holland, M. K. El-Ghor, ${ }^{2}$ and C. W. White

Recently it has been shown ${ }^{3}$ that roomtemperature irradiation of $\mathrm{Si}$ by $\mathrm{MeV}$ ions results in a damage morphology which changes dramatically over the range of the ion. Since Ge has lattice properties similar to $\mathrm{Si}_{\text {, }}$ it is of interest to observe whether the heavier mass Ge lattice would have similar damage nucleation and growth mechanisms. Analysis by
Rutherford backscattering of channeled He ions and cross-sectional transmission electron microscopy (XTEM) of (100) Ge irradiated at room temperature with $1.3-\mathrm{MeV} 16 \mathrm{O}$ and 2.5 MeV ${ }^{74} \mathrm{Ge}$ ions shows that damage increases monotonically with dose over the entire range of the ion. This is different than $\mathrm{Si}$ in which saturation of damage is observed ${ }^{3}$ over the first half of the range of the ions.

The damage morphology for self-implanted Ge is shown for two doses in the XTEM micrographs of Fig. 5.16. At the lower dose of $0.5 \times$

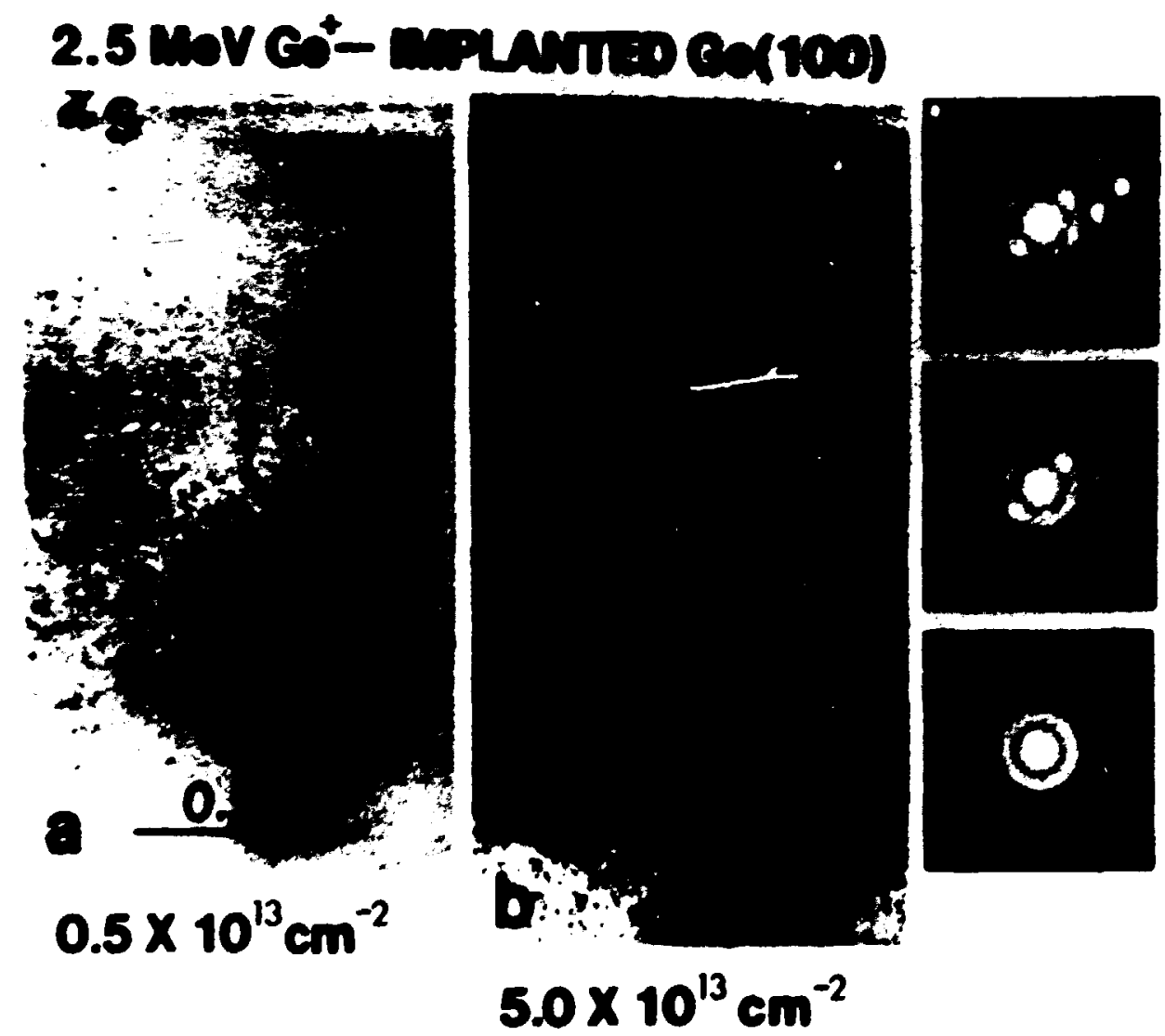

Fig. 5.16. Cross-sectional transmission electron micrographs of Ce single crystals implanted with $2.5-\mathrm{MeV}{ }^{74} \mathrm{Ge}$ for doses of (a) $0.5 \times 10^{13} / \mathrm{cm}^{2}$ and (b) 5.0 $\times 10^{13} / \mathrm{cm}^{2}$. The three insets on the right are microdiffraction images. 
$10^{13} / \mathrm{cm}^{2}$ [Fig. 5.16(a)] the damage is characterized by crystalline clusters (such as dislocation loops) that increase in density with depth reaching a maximum near $1.0 \mu \mathrm{m}$ as expected from linear cascade theory. At the higher dose of $5.0 \times 10^{13} / \mathrm{cm}^{2}$ [Fig. 5.16(b)l, the implanted layer is highly damaged and the microdiffraction images indicate that both amorphous (ring pattern) and crystalline (spot pattern) phases exist with the amorphous fraction increasing with depth. It is clear that no continuous amorphous layer forms at this dose and that no distinct interface ahead of the damaged region can be identified as in $\mathrm{Si}^{3}$ Eventually, at a Ge dose of $2.0 \times 10^{14} / \mathrm{cm}^{2}$, the entire implanted volume is amorphized.

These results are consistent with a heterogeneous damage nucleation mechanism for $\mathrm{Ge}$ in which individual ion cascades produce extended crystalline defects. As the dose is increased, these cascades overlap to produce amorphous zones that completely fill the irradiated volume at a sufficiently high dose.

1. Summary of papers: Materials Research Society Symposium Proceedings (in press); to be published.

2. Oak Ridge Associated Universities postgraduate research participant.

3. O. W. Holland, M. K. El-Chor, and C. W. White, this report; Appl. Phys. Lett. 53, 1282 (1988).

\section{HGH-DOSE OXYGEN MMPLANTATION AND REGROWTH OF THIN VBCuO FIMS ON SrTIO3}

R. Feenstre, S. J. Pennycook, and C. W. White

Epitaxial films (thickness $200-400 \mathrm{~nm}$ ) of $\mathrm{YBa}_{2} \mathrm{Cu}_{3} \mathrm{O}_{7-\mathbf{x}}$ (Y123) on (001) $\mathrm{SrTiO}_{3}$ were implanted with oxygen ions in the dose range $1 \times 10^{14}$ to $3 \times 10^{16}$ jons $/ \mathrm{cm}^{2}$. The films ( $\mathrm{c} \perp$ or mixed $a+c \perp$ ) were produced by a coevapuration of $\mathrm{Y}, \mathrm{BaF}_{2}$ and $\mathrm{Cu}$, followed by a postdeposition anneal at $850^{\circ} \mathrm{C}$ in wet oxygen. At implantation doses of $1 \times 10^{15}$ ions $/ \mathrm{cm}^{2}$ and higher, the implanted regions became amorphous and insulating but remained homogeneous. At low implantation energies (40-60 keV), the implantation zones were limited to nearsurface regions (typically $100 \mathrm{~nm}$ ) of the films. In such samples, a buried layer of the superconductor remained for which the resistive properties below $300 \mathrm{~K}$ were measured using a standard four-probe technique with current and voltage contacts on either side of the implantation zone. The boundary between the amorphized and buried crystalline layers showed sharp interfaces in TEM cross sections of the films. The resistance measurements, however, suggested the existence of an additional, highly defective interface layer, presumably associated with the tail of the implantation profile.

The regrowth of the implanted regions upon reannealing was monitored by resistance measurements and Rutherford backscattering 
(RBS). For all films, a substantial regrowth of the amorphous region was observed after reannealing at $850^{\circ} \mathrm{C}$ in flewing oxygen, leading to a restoration of metallic conductivity in the normal state as well as superconducting properties at or below $90 \mathrm{~K}$. As an example, in Fig. 5.17 the epitaxial regrowth of a $230-\mathrm{nm}$ thick, predominantly $c \perp$ film, implanted at 77 $K$ with $1 \times 10^{16}$ oxygen ions at $60 \mathrm{keV}$, is shown after reannealing at $850^{\circ} \mathrm{C}$. For this film, the normal state resistance after negrowth was only slightly higher than before implantation, and a superconducting transition was observed with zero resistance at $90 \mathrm{~K}$. At lower annealing temperatures, the "regrowth" appeared to be limited to an annealing of lattice defects underneath the amorphous region only.

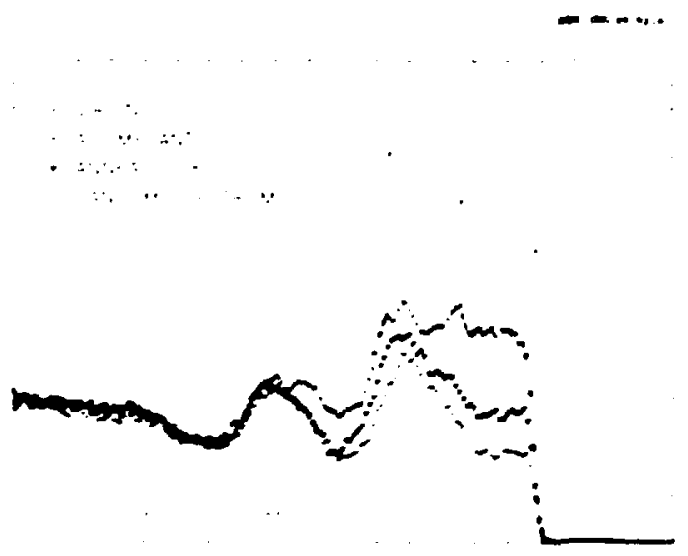

Fig. 5.17. RBS and ion channcling spectra of a $230 \mathrm{~nm}$ c $\perp$ Y123 film on (001) $\mathrm{SrTiO}_{3}$ after various stages of the implantation and regrowth sequence. The spectra were measured with 2-MeV He+ ions at near-normal incidence and 16$)^{\circ}$ scattering angle.

\section{THE ANNEALING BEHAVIOR OF ION- IMPLANTED SINGLE CRYSTALS OF THE TYPE $\mathrm{YBa}_{2} \mathrm{Cu}_{3} \mathrm{O}_{2}{ }^{1}$}

\author{
J. C. McCallum, ${ }^{2}$ C. W. White, \\ and L. A. Boatner
}

Ion implantation followed by thermal annealing offer: an attractive means of altering the stoichiometry in the surface region of single crystals of the high- $T_{c}$ superconducting oxides of the type $\mathrm{YBa}_{2} \mathrm{Cu}_{3} \mathrm{O}_{2}$ in a controlled and reproducible manner. However, recent measurements have shown that the structural disorder induced by the implantation of ions into these materials can lead to significant decomposition of the implanted region during subsequent thermal processing. ${ }^{3}$ We have used Rutherford backscattering spectrometry and ion channeling to examine the effects of ion implantation and thermal annealing on the near-surface composition of these materials. A number of different ion species, ion doses, and annealing environments have been used in the study. Our results indicate that the decomposition of the implanted region of the crystals oxcurs for ion doses above the threshold required to produced a fully amorphous surface layer when thermal anneals are carried out at temperatures greater than $500^{\circ} \mathrm{C}$ in a variety of atmospheres $\left(\mathrm{O}_{2}, \mathrm{O}_{2}+\mathrm{H}_{2} \mathrm{O}\right.$, Ar). However, the implanted region of the crystals can be substan. tially stabilized against decomposition during thermal annealing by including a disk of the polycrystalline $\mathrm{YBa}_{2} \mathrm{Cu}_{3} \mathrm{O}_{x}$ ceramic in the annealing environment."' With this arrangement, it has been possible to obtain substantial 
damage removal during high-temperature anneals $\left(\mathrm{O}_{2}, 850^{\circ} \mathrm{C}, 2 \mathrm{~h}\right.$ followed by $500^{\circ} \mathrm{C}$, $1 \mathrm{~h}) .^{\prime}$ At this stage, it is not clear whether the damage recovery occurs by solid-phase epitaxy or by growth of oriented grains. This study provides important insight into the possible application of ion implantation to processing of high-T, materials of this type.

1. Summary of papers: Mater. Lett. 6, 374 (1988); Nuclear Instruments and Methods in Physics Research, Section B (in press).

2. Oak Ridge Associated Universities postgraduate research participant.

3. N. G. Stoffet et al., Mat. Res. Soc. Symp. Proc. 99, 507 (1988).

\section{ANNEALING ENVIRONMENT EFFECTS IN SOLID-PHASE EPTAXIAL REGROWTH OF FE-IMPLANTED $\mathrm{Al}_{2} \mathrm{O}_{3} 1$}

I. C. McCallum, 2 C. W. White, P. S. Stlad, ${ }^{3}$ and C. J. McHargue ${ }^{3}$

Samples of $c$-axis $\mathrm{Al}_{2} \mathrm{O}_{3}$ were implanted with Fe $\left(160 \mathrm{keV}, 4 \times 10^{16} / \mathrm{cm}^{2}\right)$ at liquid nitrogen temperature and then thermally annealed at temperatures of 900 and $1100^{\circ} \mathrm{C}$ in a variety of ambients $\left(\mathrm{O}_{2}, A r, A r+\mathrm{H}_{2}\right)$. Rutherford backscattering spectrometry, ion channeling. and transmission electron microsiopy of the crystals revealed differences in the annealing characteristics of the implanted layers that depended on the annealing envirorument used. Notably, the reducing atmosphere $\left(\mathrm{Ar}+\mathrm{H}_{2}\right)$ produced fe distributions which differed markedly from those obtained in the other two atmospheres $\left(\mathrm{O}_{2}\right.$, Ar). These results indicate the importance of the anneaiing atmosphere in determining the characteristics of epitaxially regrown layers in ion-implanted and thermally annealed $\mathrm{Al}_{2} \mathrm{O}_{3}$.

1. Summary of paper to be published.

2. Oak Ridge Associated Universities postgraduate research participant.

3. Metals and Ceramics Division, ORNL

\section{TME-RESOLVED RERECTIVTY MEASUREMIENTS IN ION-MMLANTED CRYSTALINE CERAMIC OXIDES'}

\author{
J. C. MoCallum, 2 I. Rankin, 3 \\ C. W. White, and L. A. Boatner
}

Time-resolved optical reflectivity (TRR) is a simple and elegant technique for dynamically monitoring the interface motion that occurs during crystallization of thin films and amorphous layers on crystalline substrates. This in situ technique has enabled measurementst of the solid-phase epitaxial regrowth rate of amorphous silicon layers produced by ion implantation to be extended by over five orders of magnitude to rates in excess of $10^{6} \mathrm{~A} / \mathrm{s}$. TRR is also well suited to measurements of crystallization kinetics in ion-implanted ceramic oxides. Figure 5.18 shows the reflectivity trace for a thermal anneal $\left(355^{\circ} \mathrm{C}\right.$ in $\left.\mathrm{N}_{2}\right)$ of a sample of single-crystal $\mathrm{SrTiO}_{3}$ which had previously been implanted with $\mathrm{Pb}\left(2.0 \mathrm{MeV}, 1 \times 10^{15} / \mathrm{cm}^{2}\right)$ to amorphize the surface region of the crystal 10 a depth of $\sim 6000$ A. Each full cycle in the trace corresponds to movement of the growth interface thrnugh a depth interval of 1733 A. 


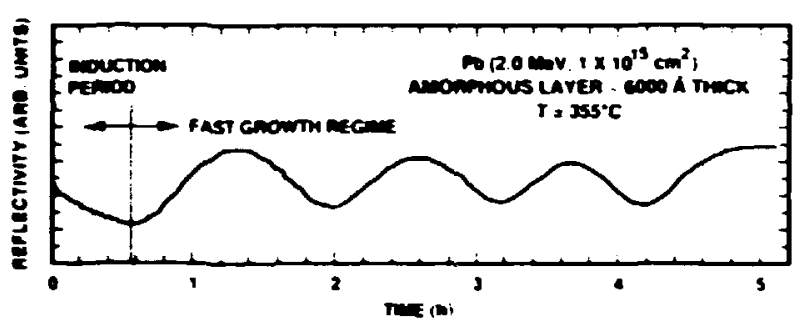

Fig. 5.18. TRR trace of an $\mathrm{SrTiO}_{3}$ single crystal implanted with $\mathrm{Pb}(2.0 \mathrm{MeV}, 1 \times$ $\left.10^{15} / \mathrm{cm}^{2}\right)$ at liquid nitrogen temperature and then thermally annealed $\left(355^{\circ} \mathrm{C}\right.$ in $\left.\mathrm{N}_{2}\right)$. The amorphous layer produced by implantation was -6000 A thick.

We have used TRR to monitor directly epitaxial regrowth during thermal annealing of amorphous layers produced by ion implantation in the crystalline perovskites $\mathrm{SrTiO}_{3}$ and $\mathrm{CaTiO}_{3}$. In particular, the effect of ambient water vapor on the epitaxial regrowth rate of amorphous layers in these materials has been examined. 5 This study provides important insight into the role of water in regrowth of materials of this nature and clearly illustrates the utility of TRR in measurements of crystallization rates in ceramic oxides.

1. Summary of paper to be published.

2. Oak Ridge Associated Universities postgraduate rescarch participant.

3. Oak Ridge Associated Universities Laboratory graduate participant from the Massachusetts Institute of Technology, Cambridge, Mass.

4. G. L. Olson and J. A. Roth, Mater. Sci. Rep. 3, 1 (1988).

5. J. Rankin et al., p. 207 in Selected Topics in Electronic Materials, ed. by B. R. Appleton, D. K. Biegelsen, and J. A. Knapp, Materials Research Society, Pittsburgh, Pa., 1988.

\section{SLMS DEPTH PROFILNG OF MULTLAYER SAMPLES OF ISOTOPICALLY PURIFIED SILICON'}

\section{K. Wittmaack, 2 D. B. Poker, and U. Littmark ${ }^{3}$}

Profile broadening due to ion beam mixing is one of the determining factors of the depth resolution of secondary ion mass spectrometry (SIMS). Ir. order to study the properties of this effect, with the objective of reducing its influence on resolution, multilayer films of isotopically purified silicon $\left({ }^{28} \mathrm{Si},{ }^{29} \mathrm{Si}\right.$, and $\left.{ }^{30} \mathrm{Si}\right)$ have been prepared using the ion beam deposition technique. In this process, the beam from an ion implantation accelerator is focused and decelerated onto a sample, impinging on the surface with energies on the order of $100 \mathrm{eV}$. Such low energies reduce the amount of sputtering and mixing that occurs and allow a uniform thin film to be deposited on the surface. The low impact energies also ensure that the interfaces remain very sharp, typically less than $1 \mathrm{~nm}$ in width. The accelerator provides excellent mass resolution, so that isotopically pure layers can be sequentially deposited.

The thickness of individual layers was varied between 1 and $50 \mathrm{~nm}$, and impurities of $C$ and $F$ were occasionally introduced intentionally between layers. The composition of the samples was investigated by SIMS using different primary ion beams (Ne, Ar, Xe, Cs, and $\mathrm{O}_{2}$ ) at energies between 1 and $10 \mathrm{keV} /$ atom. The impurity content (i.e., the fraction of unintentionally deposited $\mathrm{Si}$ isotopes) was found to range from $10^{-3}$ to $10^{-2}$. The beam-induced broadening of the interfaces between adjacent 
layers increased with increasing primary ion energy $E$ as $E^{n}$, where $n \sim 0.5$. In contrast to the oretical predictions based upon a model of cascade mixing, the interface broadening observed with ion beams of inert gases or Cs did not depend upon the primary ion mass at a fixed energy. Much improved depth resolution was obtained with $O$ atoms. This effect is attributed to the form jon of $\mathrm{SiO}_{2}$, in which case the $O$ subsystem suppresses the relocation of Si atoms.

1. Summary of paper to be published.

2. Gesellschaft für Strahlert- und Umweltforschung mbH, München, Federal Republic of Germany.

3. Kernforschungsanlage, Jülich, Federal Republic of Germany.

\section{HIGH-RESOLUTION X-RAY STUDIES OF THE CHEMICAL ENVIRONMENT OF IONS IMPLANTED IN INSULATOR MATRICES}

T. M. Rosseel, ' I. P. Young, ' C. R. Vane, R. A. Zuhr, and R. S. Peterson ${ }^{3}$

A program has been initiated to study the chemical environment of ions implanted in insulator matrices, e.g., $\mathrm{SiO}_{2}$ (quartz glass), as a function of the initial charge state, concentration, encrgy (depth), deposition rate, and substrate matrix using heavy-ion-induced $x$-ray satellite emiszion (HIXSE) spectroscopy. HIXSE is an x-ray fluorescence technique which uses fast $(\simeq 1 \mathrm{MeV} / \mathrm{amu})$, heavy ions for excitation and yields intense, chemically sensitive satellite lines. If combines the advantages of po :tive ion-induced x-ray emis- sion (i.e., being able to detect an element in almost any matrix) with the chemical information obtainable from electron spectroscopy methods. Unlike surface analysis techniques, HIXSE probes the near-surface region ( $\simeq 5-1000 \mathrm{~nm})$, making it ideally suited for the relatively nondestructive examination of the local chemistry of implanted ions and thin-film interfaces.

A series of high-resolution sulfur $\mathbf{K}_{a} \times$-ray satellite spectra has been produced by $39 \mathrm{MeV}$ C ion irradiation of quartz glass previously implanted with $70-k e V$ sulfur ions at $\mathbf{2 . 5}$ $\mu \mathrm{A} / \mathrm{cm}^{2}$ to doses ranging from 1 to $10 \times 10^{16} / \mathrm{cm}^{2}$. Comparison of the normalized and corrected HDXE spectra as a function of decreasing sulfur dose clearly reveals a shift in the relative intensity of the $K L^{n}$ satellite lines from higher to lower order lines. These spectral variations were parameterized with a term called the average $L$-shell vacancy fraction. A plot of this parameter vs the valence electron distribution, a term which describes the local chemical environment of the target, yiel Is a systematic correlation with the local sulfur density. These results suggest a rossible method for nondestructively probing implant profiles which is relatively free from restrictions on the $\mathrm{Z}$ of the implant or matrix.

1. Analytical Chemistry Division, ORNL.

2. Physics Division, ORNL.

3. The University of Tennessee at Chattanooga, Chattanooga, Tenn. 


\section{ON THE THERMODYNAMIC DRIVING FORCE DURING ION MDXNG OF THE CO-Si SYSTEM'}

W. Xia, ${ }^{2}$ S. S. Lau, ${ }^{2}$ and D. B. Poker

The relative importance of the thermodynamic driving force as compared with kinetics during thermal annealing and ion mi ing in the thermally activated regime has not been clarified. To probe the role of the thermodynamic driving force in reactions between metals and silicon, the Co-Si system was chosen for investigation. in general, three silicide phases are formed during thermal annealing of Co thin films deposited on Si substrates [i.e., $\mathrm{CO}_{2} \mathrm{Si}$ (the first phase to form, with a heat of formation, $\Delta H_{f}=-9 \mathrm{kcal} / \mathrm{g}$ atom $), \operatorname{CoSi}\left(\Delta H_{f}=\right.$ $-12 \mathrm{kcal} / \mathrm{g}$ atom) and $\mathrm{CoSi}_{2}$ (the last phase to form, with $\Delta H_{f}=-8.2 \mathrm{kcal} / \mathrm{g}$ atom)]. Previous experiments have shown that annealing a sample of $\mathrm{Si} / \mathrm{CoSi} / \mathrm{Co}$ converts $\mathrm{Co}$ into $\mathrm{CO}_{2} \mathrm{Si}$ instead of a continuous growth of CoSi. This reaction is apparently unrelated to the magnitude of the thermodynamic driving force, since $\Delta H_{f}$ of CoSi is significantly larger than those of $\mathrm{CO}_{2} \mathrm{Si}$ and $\mathrm{CoSi}_{2}$, but the reaction is kinetically restricted instead. Under ton mixing conditions the kinetic restriction is relaxed due to the enhanced atomic mobilities during ion irradiation. Hence the ion-induced reaction should be driven by tharmudynamics.

In thermal annealing, $\mathrm{CO}_{2} \mathrm{Si}$ is the first phase to form, and $\mathrm{Co}_{\mathrm{o}}$ is the dominant moving species. In ion mixing, both $\mathrm{CoSi}$ and $\mathrm{CO}_{2} \mathrm{Si}$ are observed to form. At low temperatures the formation of CoSi dominates. As the substrate temperature is increased, the formation of
$\mathrm{CO}_{2} \mathrm{Si}$ becomes more significant. $\mathrm{Co}$ and $\mathrm{Si}$ are found to be the dominant moving species in the ion-beam-assisted formation of $\mathrm{CO}_{2} \mathrm{Si}$ and $\mathrm{COSi}$, respectively. By introducing the concept of an effective heat of formation based upon the availability of reactant atoms at the interface, the initial phase formation in both ion mixing and thermal annealing can be explained in terms of the thermodynamic driving force.

\section{LASER PROCESSING OF THIN FILMS}

\section{PHOTON-CONTROLLED FABRICATION OF AMORPHOUS SUPERLATTICE STRUCTURES USING ArF (193 nm) EXCIMER LASER PHOTOLYSIS'}

\author{
D. H. Lowndes, D. B. Geohegan, D. Eres, \\ S. I. Peinizycook, D. N. Mashbum, \\ and $G$. E. Jellison, Jr.
}

We recently reported' the first experiments in which superlattice structures have been fatricated entirely under photolytic control by using ArF (193 nm) excimer laser photolysis of appropriate parent gas molecules to deposit multilayered amorphous $\mathrm{Si}_{2} \mathrm{Si}_{3} \mathrm{~N}_{4}$ and $\mathrm{Si} / \mathrm{Ce}$ structures. Amorphous $\mathrm{Si}$ was deposited by photolysis of disilane $\left(\mathrm{Si}_{2} \mathrm{H}_{6}\right), \mathrm{Si}_{3} \mathrm{~N}_{4}$ by simultanc uus photolysis of disilane-ammonia mixtures, and amorphous Ge by photolysis of germane $\left(\mathrm{CeH}_{4}\right)$. We found that complete 
"photonic" control of deposition could be obtained at low substrate temperatures, in that deposition ceased when the laser was turned off. For example, at $350^{\circ} \mathrm{C}$ the ratio of photolytic-to-pyrolytic deposition of a-Si from disilane was $-10^{3}$, so that "background" thermal deposition was negligible. Thus, quite smali (-monolayer) thickness steps can be introduced simply by switching the laser on and off, and abrupt compositional changes can be made by changing gas composition with the laser off.

Figure 5.19 shows transmission electron microscope cross-section views of a photolytically grown nine-layer a-Si/a-Ge structure and of a 32-layer $a-\mathrm{Si}_{2} / \mathrm{Si}_{3} \mathrm{~N}_{4}$ structure. The layer thicknesses were controlled by keeping the product of the laser power and deposition time a constant for corresponding layers. For the a-Si/a-Ge structure, the average thickness of the a-Si layers is $10.7( \pm 0.4) \mathrm{nm}$, while for the first, third, and fourth a-Ge layers the average thickness is $5.4( \pm 0.2) \mathrm{nm}$. The second $a-G e$ layer was deposited with the power-time product increased by approximately $24 \%$ and resulted in a layer $30 \%$ thicker $(7.0 \pm 0.3 \mathrm{~nm})$ than the average of the others. For the a$\mathrm{Si} / \mathrm{Si}_{3} \mathrm{~N}_{4}$ structure, the average $a-\mathrm{Si}$ and $\mathrm{Si}_{3} \mathrm{~N}_{4}$ layer thicknesses were $13.3( \pm 0.4) \mathrm{nm}$ and 26.6 (士2) $\mathrm{nm}$, respectively.

1. Summary of paper: Appl. Phys. Lett. 52, 1868 (1988).
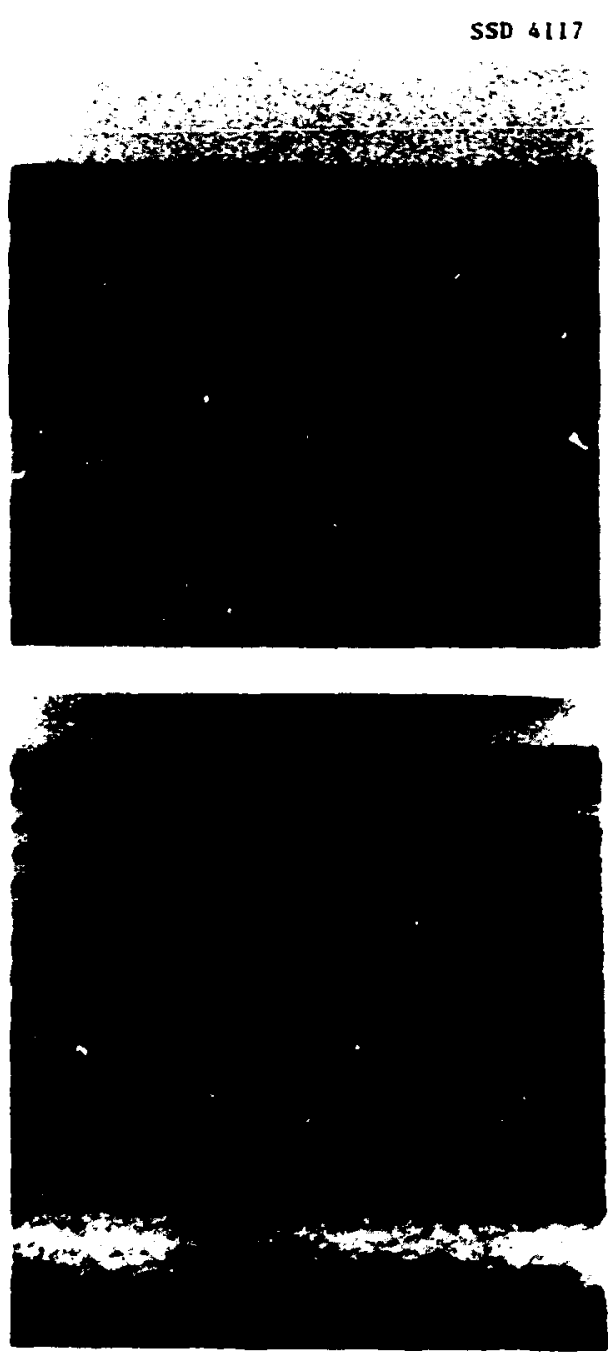

Fig. 5.19. (Top) Nine-layer a-Si(light)/aGe(dark) superlattice deposited at $250^{\circ} \mathrm{C}$ and (Bottom) 32-layer a-Si/Sig $\mathrm{N}_{4}$ superlattice deposited at $350^{\circ} \mathrm{C}$.

\section{LASER PHOTOCHEMICAL DEPOSITION OF AMORPHOUS SEMICONDUCTORS FROM DISILANE AND DIGERMNE: SPECTROSCOFIC STUDIES AND COMPARISONS WITH THERMAL CHEMICAL VAPOR DEPOSTION'}

\section{Djule Eres and D. H. Lowndes}

Because of their relatively large absorption cross sections near $193 \mathrm{~nm}$, efficient photofragmentation of disilane and digermane can be 
achieved by an ArF pulsed excimer laser. Spectroscopic investigation of the fuorescence emitted by the photofragments following ArF laser irradiation of disilane $10 \%$ in helium carrier gas) in a flowing gas reactor environment was carried out. Molecular species such as $\mathrm{SiH}$ and $\mathrm{SiH}_{2}$ were positively identified as fragmentation products. The picture that emerges from these investigaticns is that while energy deposition by absorption of photons leads to fragmentation of parent molecules, no specific molecular species could be directly associated (from fragments that emit in the visible) with film deposition. The primary photodissociation products undergo secondary gas phase reactions with other photofragments and/or parent molecuies creating highly excited gas phase species. The role of the substrate is to facilitate bond formation into an amorphous network by deactivating these species. The chemical reaction taking the central role in the surface rearrangement reactions is believed to be $\mathrm{H}_{2}$ desorption. Preliminary results indicate that the same reaction pathways govem amorphous Ge deposition by photochemical vapor decomposition of digermane. Based on the above results, we conclude that, in common with thermal chemical vapor deposition, the rate limiting step in laserinduced photochemical vapor decomposition is a thermally activated surface reaction.

1. Summary of papers: Appl. Surf. Sci.36, 70 (1989); Fourth Intermational Laser Science Conference (October 2-6, 1988, Allanta, Ga.) (in press); American Instilute of Physics Conference Proceedings (in press).

\section{FIRST PRINCIPLES FOR LOW- TEMPERATURE LASER-PHOTON- CONIROLLED EPITAXIAL FILM GROWTH'}
D. H. Lowndes,
D. Eres, and
D. B. Geohegan

The use of thermal energy (substrate temperature $T_{s}$ ) to drive film growth reactions in conventional pyrolytic chemical vapor deposition degrades some thin films and multilayered structures through thermally activated processes such as the generation of vacancies and vacancy-dopant atom complexes, through thermal diffusion of impurities, or by vacancyenhanced diffusion that can produce precipitates and grain boundaries. For several classes of materials (e.g., the II-VI cumpounds), such processes make it difficult to form well-defined interfaces, to dope reproducibly, or to produce abrupt compositional changes at conventional pyrolysis temperatures.

Laser photochemical vapor deposition (LPVD) is an altemative process in which UV excimer laser photons are used to photodissociate parent molecules, resulting in primarily photonic control of film growth at temperatures too low for pyrolysis. However, recent work shows that the possibility of achieving low- $T_{s}$ LPVD epitaxial growth depends sensitively on the gas-phase photodissociation reaction products. For example, if $T_{s}$ is reduced sufficiently to allow photolysis to dominate pyrolysis, it is often found that only amorphous or polycrystalline films can be obtainrd with the laser beam parallel to the substrate. This occurs if gas-phase photolysis doc: nit directly produce the atomic species needed but only an intermediate radical that must undergo further 
surface reactions. In such cases, additional surfacn energy input is needed to stimulate the surface processes of bond-breaking and desorption that result in low- $T_{s}$ epitaxy. On the other hand, if the atomic species is produced by gas-phase photolysis, then $T_{s}$ generally can be reduced. This appears to be the situation for growth of II-VI compounds by photolysis of metalorganic (MO) alkyls in the parallel-laser-beam geometry.

Several practical considerations also enter into the design of LPVD experiments. The molecular photodissociation cross section must be large enough (typically $>3 \times 10^{-18} \mathrm{~cm}^{2}$ ) at the laser wavelength for rapid growth; when using MO alkyls, secondary photolysis reactions of carbon-bearing ligands must be avoided to prevent carbon contamination of films; normally incident pulsed-laser radiation may cause periodic twin growth within an epitaxial film because of interference between the incident laser beam and scattered surface waves; and chamber windows often need to be flushed continuously with an inert or etchant gas to avoid window film deposition.

With such fundamental and practical considerations in mind, our research into efficient low- $T$, photon-controlled epitaxial growth is currently exploring the use of new molecules (obtained through collaborations with industrial synthetic chemistry research grrups) and combining pulsed gas beams with pulsed excimer lasers.

1. Summary of paper: International laser Science Conference-IV, October 1-2, 1988, Atlanta, Ga. (in press).

\section{HIGH-SPEED HETEROEPTTAXIAL FIMM GROWTH USING A PULSED SUPERSONIC FREE JET ${ }^{1}$}

Djule Eres, D. H. Lowndes, and J. Z. Tischler

Epitaxial films have been grown for the first time from pulsed supersonic jets of parent molecules seeded in helium gas. Very high Ge epitaxial growth rates (up to $-0.25 \mu \mathrm{m} / \mathrm{s}$ ), not attainable by conventional vapor-phase filmgrowth methods, were obtained together with excellent crystal quality.

A fast acting (short rise and fall time) pulsed molecular beam valve was used to produce a high-pressure jet containing digermane $\left(\mathrm{Ce}_{2} \mathrm{H}_{6}\right)$ molecules directed toward a heated GaAs substrate in a high-vacuum environment $\left(10^{-6}\right.$ Torr). The main characteristics of this method of parent-molecule delivery are the high intensity and increased kinetic energy of the Ge-bearing molecules and the high directionality (line-of-sight delivery) of the gas jet, all of which contribute to a dramatic increase in growth rates compared with conventional chemical vapor deposition (CVD). Crowth rates of $1000-4000 \mathrm{~nm} / \mathrm{min}$ were achieved at substrate temperatures of $450-625^{\circ} \mathrm{C}$ (Fig. 5.20), using a $4.9 \%$ digermane $/ 95.1 \%$ helium gas mixture. X-ray double-crystal diffractometry showed that the Ge films were of high crystalline quality and were completely commensurate with the $(100)$ CaAs substrate. The films were very smooth and planar, being free of surface fealures or faceting down to the $-0.1-\mu \mathrm{m}$ length scale and highly uniform in thickness, in comparison with slowly grown conventional-CVD Ge films. ${ }^{2}$ The very high 


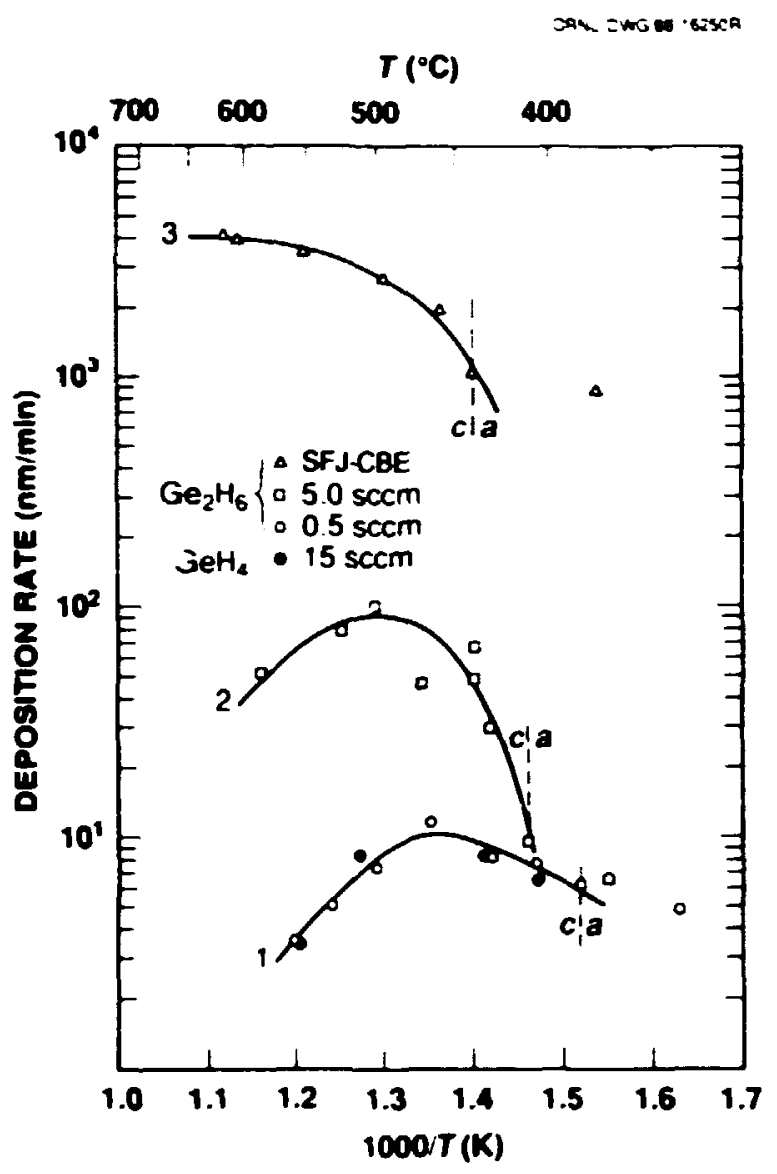

Fig. 5.20. Logarithm of deposition rate vs reciprocal substrate temperature. The total pressure during deposition was $40 \mathrm{mT}$ Trr and 1 mTorr for 5.0 and $0.5 \mathrm{sccm}$ digermane $(5 \%$ in helium), respectively, and $100 \mathrm{mTorr}$ for 15 scem diger mane (10\% in helium). Growth data using germane are shown for comparison. The a-c transition temperature for a particular incidence rate is given by the dashed vertical line.

growth rates effectively "freeze" the Ge-GaAs interface, resulting in minimal interfacial diffusion and reduced ir.corporation of impuritics from the surrounding growth environment. In contrast, slower film growth $(-10 \mathrm{~nm} / \mathrm{min})$ at $510^{\circ} \mathrm{C}$ produced Ce-GaAs structures in which both the Ge and GaAs (400) diffraction peaks were significantly broadened. This broadening is believed to be caused by nonuniform strain due to differential thermal expansion.

Epitaxial Ge film growth is believed to be initiated by dissociative chemisorption of the impinging digermane molecules: therefore, the name supersonic free jet chemical beam epitaxy (SFJ-CBE). SFJ-CBE differs significantly from conventional pyrolytic CVD, since it is governed by molecular scattering events involving thermal decomposition products and undecomposed digermane molecules, rather than by equilibrium thermodynamic and reactor design (gas-flow) considerations. The sole reliance of SFJ-CBE on surface reactions and a moderatevacuum environment results in certain practical advantages. For example, it completely avoids contamination of films by microparticles that are formed in homogeneous vapor-phase reactions under equilibrium conditions, and the high directionality of a supersonic jet confines growth to the heated substrate region so that frequent cleaning of the growth chamber is not required.

1. Summary of paper: Applied Physics Letfers (in press).

2. Djula Eres et al., "Growth of Epitaxial Ge Films on (100) GaAs by Pyrolysis of Digermane," this report. 


\section{GROWTH OF EPTIAXAL Ge FILMS ON (100)} GaAs BY PYROLYSIS OF DIGERMANE'

Diula Eres, D. H. Lowndes, I. Z. Tischler, J. W. Sharp. ${ }^{2}$ S. I. Pennycoot, and D. B. Geohegan

The first studies of ersitaxial Ge thin-film growth from digermane $\left(\mathrm{Ce}_{2} \mathrm{H}_{6}\right)$ were carried out by thermal chemical vapor decomposition (CVD) at substrate temperatures in the 380 $600 \mathrm{C}$ range. Low-temperature growth rates up to three orders of magnitude greater than obtained from germane ( $\mathrm{GeH}_{4}$ ) were observed as a consequence of the lower thermal stability of digermane. Digermane (5\%) mixed in a He carrier gas flowed over a heated GaAs (100) substrate at total reactor pressures of a few Torr. From the growth curves, which present the growth rate as a function of reciprocal substrate temperature, two different growth regimes were identified (Fig. 5.20). At low substrate temperatures $\left(380-450^{\circ} \mathrm{C}\right)$, growth is governed by dissociative chemisorption, while at higher substrate temperatures (above $500^{\circ} \mathrm{C}$ and depending on digermane partial pressure), desorption of $\mathrm{Ge}$ atoms from the growing film surface becomes rate limiting, causing a maximum and reversal of slope of the gro $v$ th curves.

Investigation of the crystalline quality of the Ge epitaxial layers by double-crystal $x$-ray diffractometry revealcd a high degree of crystalline perfection over the entire $380-600^{\circ} \mathrm{C}$ substrate temperature range. $X$-ray diffractometry also showed that the Ge films were completely commensurate with the CaAs (100) substrate, meaning that the as-grown $\mathrm{Ce}$ films have the lattice constant of GaAs in the plane of the interface and are tetragonally distorted perpendicular to the interface (the direction of growth) in order to accommodate the elastic strain. Cross-section transmission electron microscopy revealed a high-quality interface with no dislocations or extended defects of any kind. However, slowly grown Ge films were found to have rougher surfaces than films grown rapidly.

1. Summary of paper: Applied Physics Letters (in press).

2. Graduate student from The University of Tennessee, Knoxville, Tenn.

\section{LABORATORY FOR LASER-CONTROLLED AND LOW-TEMPERATURE THIN-FILM GROWTH \\ D. H. Lowndes, D. Eres, D. B. Geohegan, and D. N. Mashburn}

During the past three years the Solid State Division, with support from the ORNL Director's Research and Development Fund, has developed a new program and laboratory facilities for research into low-temperature vapor-phase growth of thin films and artificially structured materials. i . program presently is focused on epitaxial film growth, with the goal of developing a fundamental molecular-level understanding of photoncontrolled (or other low-temperature) chemical reactions, both in the gas phase and on surfaces, in order to identify the reaction paths that can lead to low-temperature epitaxy.

Three different approaches to lowtemperature and/or rapid epitaxial growth are 
being studied: (1) laser photochemical vapor deposition (LPVD), (2) supersonic free jet chemical beam epitaxy (SF)-CBE), and (3) plasma-assisted laser-ablation deposition (PLD). The program also includes research to identify and to synthesize (through collaborations with industrial synthetic chemistry research groups and specialty gas suppliers) new molecules that are specifically designed for efficient photon-controlled or pyrolytic growth at low substrate temperatures. The program is concentrating on low-temperature and/or rapid epitaxial growth for three technologically important families of materials: (1) $\mathrm{Si}, \mathrm{Ge}$, and their alloys; (2) II-VI and III-V compound semiconductors; and (3) high- $T_{c}$ oxide superconductors (using PLD).

Because LPVD and SFJ-CBE use toxic and/or pyrophoric gases, they are carried out within continuously vented enclosures. Two film-growth chambers and gas-handling manifolds for vapor-phase $\mathrm{Si}$-Ge growth are located within one enclosure. A second enclosure houses a laser photolytic MOCVD system (for II-VI compound growth) and a pulsed gas (molecular) beam chamber. Two pulsed excimer lasers and an excimer-pumped dye laser provide photon energies from 3.5 to $7.9 \mathrm{eV}$. Support facilities include a turbo-pumped VUV spectrometer, optical multichannel analyzer, and gas-mixing station for absorption/emission studies of new film-growth molecules. The VUV spectrometer also is used, together with a quadrupole mass analyzer, to detect intermediate products of photo- and thermal-dissociation reactions.
PLD of oxide superconductor films is carried out in a large turbo-pumped chamber to which a second UV spectrometer has been interfaced for absorption and emission studies of the laser ablation "plume."

All the film-deposition and gas siorage f-cilities are designed so that no toxic gas lines run outside of the vented enclosures. A twostage system consisting of a gas bumer and gas scrubber is used to dispose of effluent gases. An automatic safety interlock system monitors some 35 different conditions in the laboratory and can warn the operator of a system malfunction or of the onset of potentially unsafe operating conditions.

\section{FACILITY FOR MEASURING VACUUM- ULTRA VIOLET CROSS SECTIONS OF NOVEL MOLECULES USED FOR LOW. TEMPERATURE PHOTOCHEMICAL VAPOR DEPOSITION}

\section{B. Geohegan, D. H. Lowndes, and K. Sharp'}

The advent of high-powered UV excimer lasers has made it possible to drive thin-film growth reactions photolytically. Consequently, new low-temperature methods for semiconductor deposition focus on synthesizing molecules that have large absorption cross sections in the UV and VUV. Molecules can be synthesized for either deposition or etching, or for a combination of the two; for example, by varying the number of halogen substitutions in the hydrides of Cie and Si. Both the absorption spectrum and absolute cross sections are needed throughout the UV and VUV in order to assess 
which orbitals of the molecules are absorbing, whether dissociation takes place, and how effective attempts to drive the reaction with a particular laser line will be.

A facility has been constructed to measure the absorption of molecules for wavelengths greater than $105 \mathrm{~nm}$. The broadband continuum emission of either a hollow-cathode deuterium lamp or a pulsed, high-pressure Xe-arc lamp is dispersed by a $0.3-m$ crossed Czerny-Turner vacuum monochromator with $\mathrm{MgF}_{2}$ coated optics. The resolution of the system is $0.2 \mathrm{~nm}$ at $121.5 \mathrm{~nm}$. The light is then passed through an absorption cell with $1-m m$-thick $\mathbf{M g F}_{2}$ windows, where it is then converted into the visible by a sodium-salicylate-coated window and detected by a photomultiplier. A second converter/photomultiplier detects a fraction of the incident light intensity, and both signals are measured and averaged with a boxcar averaget/computer system. The monochromator and intermediate chambers are evacuated by a turbomolecular pump. An on-line gashandling manifold allows for evacuation and for pressure control of the gas under study. Mich.

1. Dow Coming Corporation, Midland,

\section{IN SITU MONITORNG OF CRYSTALLNE AND AMORPHOUS FILM GROWTH USINC OPTICAL REFLECTIVTTY}

\section{J. W. Sharp.' D. H. Lowndes, and D. Eres}

Optical reflectivity techniques have been developed to obtain in situ information about pyrolytic and laser-photolytic vapor-phase growth of both amorphous and crystalline thin films under varying experimental conditions. A low-power HeNe $(632.8 \mathrm{~nm})$ laser beam is reflected at near-normal incidence from the gas-film and film-substrate interfaces, and the reflected intensity is detected using a largearea Si photodiode.

For amorphous films, this technique makes possible rapid, precise measurements of changes in film thickness and deposition rates. For a film deposited under constant conditions, its mean optical properties can be determined by using the index of refraction $\boldsymbol{n}$ and absorption coefficient $k$ as parameters to fit the decaying interference oscillations that are observed with increasing film thickness. Changes in amorphous film thickness can be monitored in real time with submonolayer sensitivity (averaging the film thickness over the $-0.5-m m$-diam spot size of the HeNe beam), and deposition rates of $<10^{-3} \mathrm{~A} / \mathrm{min}$ can be measured.

Information about the film-growth mode and the amorphous-to-crystalline (a-c) transition temperature (a function of film growth conditions) also can be obtained. Roughness (and/or faceting) of a growing c-Ge surface, due to island growth, is manifested in a nonspecular reflectivity component. The slowly damped successive oscillations characteristic of a perfectly smooth film are lost in the rapid decay of the specularly reflected component [Fig. 5.21, Curve (a)). The rate and extent of reflectivity decay depend on the vertical and lateral scales of surface feature size, so that model calculations can be compared directly with the 


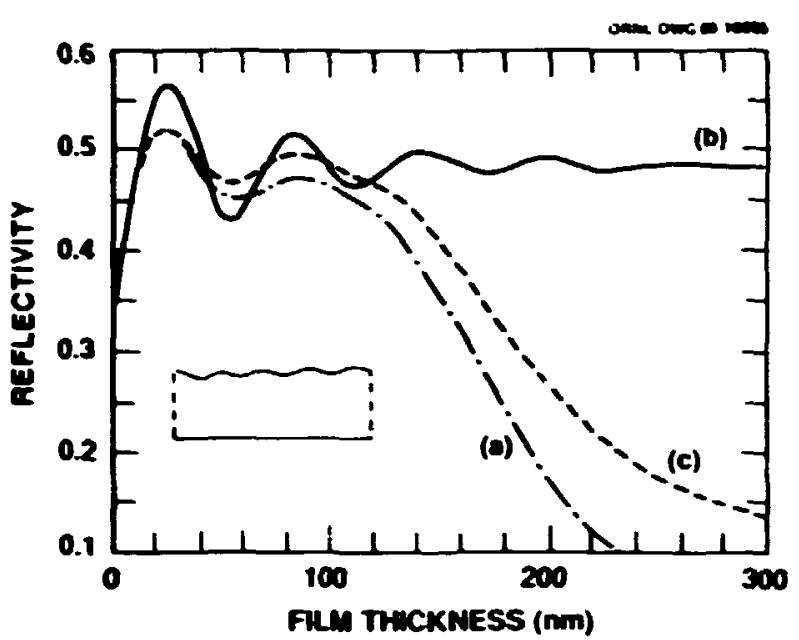

Fig. 5.21. (a) Experimental reflectivity signal for slowly grown c-Ce film on (100) CaAs. Reflectivity curve (b) is a model cakulation for a smooth, specularly reflective $c$-Ce film on GaAs, while (c) shows the specularly reflected component for a Ge film with surface features illustrated qualitatively in the inset. Curve $(c$, can be made to agree with (a) by adjusting the roughness parameters of the surface model.

reflectivity data to determine surface roughness parameters. Using a model that assumes statistical distributions centered on mean values for the surface feature height and lateral separation, we find that we can simulate the experimental reflectivity curves (Fig. 5.21, Curve (c)].

1. Graduate student from The University of Tennessee, Knoxville, Tenn.

\section{FABRICATION OF BROADBAND GERMANIUM THIERMOPHOTOVOLTAIC CELLS BY PULSED-LASER ANNEALING}

\author{
I. W. McCamy', D. H. Lowndes, \\ and G. E. Jellison, Jr.
}

Because its energy band gap corresponds to a photon wavelength $-1.8 \mu \mathrm{m}$, Ge lends itself to direct thermophotovoltaic (TPV) conversion of high-temperature blackbody radiation (e.g., power plant waste heat) to electricity. This application requires cells that can operate at IR illumination levels oi $-10 \mathrm{~W} / \mathrm{cm}^{2}$, about 100 times greater than for nonconcentrating terrestrial photovoltaic cells. In early work on Ge, we found that certain photolithographic and other processing steps developed for $\mathrm{Si}$ solar cells were unsuitable for $\mathrm{Ge}$. By using pulsedlaser annealing tech:wology developed earlier at ORNL, with other processing changes, we have successfully fabricated Ge TPV cells. There are critical requirt-nents and differences in the Ge TPV cell process: (1) additional cleaning steps are needed to prevent surface damage during pulsed-laser annealing; (2) metal contacts that are wider and thicker than for conventional Si solar cells are needed to provide the necessary current-carrying capacity with reduced ohmic losses; (3) mesa isolation is used at the end of the cell fabrication process to eliminate leakage current paths between the emitter and base by isolating the emitter from the rest of the cell; and (4) the Ge surface must be protected during electroplating and mesa fabrication because of differences in the chemical properties of $G e$ and Si and of their oxides. 
Pulsed-Jaser-annealed TPV cells were fabricated using Ga-doped $\left(N_{a}-2 \times 10^{15} / \mathrm{cm}^{3}\right)$ (100) Ge single-crystal wafers. The $n^{+}-p$ junction was formed by low-cost glow discharge ion implantatien of P from a 5\% $\mathrm{PH}_{3} / 95 \%$ Ar mixture followed by $\mathrm{XeCl}(303 \mathrm{~nm})$ pulsed excimer laser annealing using energy densities $\left(E_{\ell}\right)$ of $0.5-1.8 \mathrm{~J} / \mathrm{cm}^{2}$. An unexpected result was the significant broadband spectral response of these cells (internal quantum efficiency $>20 \%$ for 550 $\mathrm{nm}<\lambda<1800 \mathrm{~nm}$ ). The spectral response from 400 to $1500 \mathrm{~nm}$ was found to depend on the $E_{\mathrm{R}}$ used to form the $p-n$ junction, with significant blue response occurring at the lower values of $E_{\mathbf{q}}$. This finding apparently results from the very short photon absorption length in the blue part of the Ge absorption spectrum, combined with the increase of $p-n$ junction depth below the surface with increasing $E_{\mathcal{L}}$ so that blue photons can be absorbed in the base region only for low $E_{1}$. Thus, we have developed a technique by which Ge TPV cells can be fabricated with a "custom designed" spectral response. In order to understand more fully the response of these cells and the effect of processing changes on their absolute quantum efficiency, spectrally selective measurements of I-V characteristics and of cell efficiency at wavelengths of $400,800,1200$, and $1500 \mathrm{~nm}$ are being carried out.

1. Oak Ridge Associated Universities Professional Internship Program graduate student from The University of Tennessee, Knoxville, Tenn.

\section{HOLE CAPTURE AT ACCLPTORS IN P-TMPE GERMANIUM'}

\section{S. Darken,2 P. Sengsinkeow, and G. E. Jellison, Jr.}

Transient capacitance techniques have been used to characterize trapping centers introduced during crystal growth or subsequent processing in high-purity $\left(\left|N_{A}-N_{D}\right| \leq 10^{10} \mathrm{~cm}^{-3}\right)$ germanium. Because of the low free-carrier concentration, the capture cross sections of all observed trapping centers can be mensured using the variable pulse width technique. Using optical as well as thermal emission, measurements of the iemperature dependence of the capture cross sections have been extended down to $-10 \mathrm{~K}$, where freese-out of the hydrogenic centers is the limiting factor. We have measured capture cross sections for substitutional impurities (B, $\mathrm{Zn}$, and $\mathrm{Cu}$ ), impurity complexes $\left(\mathrm{CuH}_{2}\right)$, and vacancy-related complexes $\left(\mathrm{V}_{2} \mathrm{H}\right)$. For all types of centers, the experimental cross section can be given by

$$
\sigma_{\exp } \sim g k T / h N_{0} \sigma_{t h} .
$$

where $g$ is the degeneracy of the ground state, $k$ is Boltzman's constant, $T$ is the temperature, $h$ is Planck's constant, $N_{v}$ is the effective concentration of holes at the valence band edge, and $O_{\text {in }}$ is the thermal velocity of the hole.

The present experimental results are not consistent with the cascade theory ${ }^{3}$ for hole capture by deep levels in germanium; the cascade mechanism predicts capture times that are 
too slow and predicts temperature and charge state dependences which are at variance with our experimental results.

The capture of holes by negatively charged acceptors in germanium exhibits features similiar to those attributed to the capture of carriers in wide-band gap III-V semiconductors. In particular, (1) the data for hole capture in germanium are consistent with capture directly from the band states into the electronic ground state, without passing through any intermediate stationary states; and (2) for some centers, capture is "activated." In this case, the righthand side of Eq. 1 is multiplied by exp $(-E / k T)$.

The actual hole capture mechanism is not yet completely understood. However, the results do rule out the published multiphonon emission theories based on the adiabatic approximation of the wave function and utilizing the Colden Rule to obtain transition probabilities. Such theories require strong coupling (a large Huang-Rhys factor) to obtain large cross sections. Lange unactivated cross sections have been observed for many centers, and when activation is present, the cross section is reduced by the activation factor.

1. Summary of paper to be published.

2. Tennelec, Oak Ridge, Tenn.

3. M. Lax, Phys. Rev. 119, 1502 (1960).

\section{A NEW TWO-CHANNEL POLARIZATION MODULATION EULIPSOMETER}

\section{E. Jellison, Ir., and F. A. Modine}

A new two-channel polarization modulation ellipsometer that is capable of simultancously measuring all three of the ellipsometric parameters, $N=\cos 2 \psi, S=\sin 2 \psi \sin \Delta$, and $C=$ $\sin 2 \Psi \cos \Delta$, has been mnstructed. Light is collimated with an off-axis parabolic mirror and then passes through a polarizer (P) modulator (M) combination, which polarizes the light with an ellipticity oscillating at $50 \mathrm{kHz}$ IAn additional compensator (C) and/or polarizer (P) can be added to the light path for calibration purposes.] The light then reflects off the sample and is analyzed by a Wollaston prism (W), which splits the incoming beam into two beams with mutually orthogonal polarizations. The intensity of each beam is detected with a photomultiplier whose voltage is controlled to obtain a constant dc current.' The output current from each phototube consists of a dc component and $50-\mathrm{kHz}_{\mathrm{z}}$ harmonics. The dc, fundamental, and second harmonic are converted to voltages and then measured by a digital voltmeter (dc) or lock-in amplifiers (fundamental and second harmonic).

If the azimuthal angle of the polarizer is set to $\pm 45^{\circ}$ with respect to the modulator, the azimuthal angle of the modulator is set to $\pm 22.5^{\circ}$ or $\pm 67.5^{\circ}$, and the Wollaston prism analyzer is set to $\pm 45^{\circ}$, then

$R_{i 1}=I_{1}(\omega) / I_{i}(d c)=(-1)^{i} 2 I_{1}(A) S$,

$$
\begin{gathered}
R_{D}=I_{1}(2 \omega) / I_{1}(\mathrm{dc})=2 I_{2}(\mathrm{~A}) \\
\left(y N+(-1)^{i} \times C\right) / \sqrt{2},
\end{gathered}
$$

where $I_{i}$ represents the intensity in the $i^{\text {th }}$ channel, at a frequency of dc, $\omega$, or $2 \omega$. The $l_{1}(A)$ and $l_{2}(A)$ represent integer Bessel functions, whose argument $A$ depends upon the 
driving amplitude of the photoelastic modulator. The quantities $x$ and $y$ are +1 or -1 and depend only on the azimuthal angle of the modulator. Clearly, Eq. 1 can be solved for $S$, $N$, and $C$.

This ellipsometer has several advantages over previous designs, arising from its ability to measure $N, S$, and $C$ independently and simultaneously: (1) there is no value of $\nabla$ or $\Delta$ to which this instrument is insensitive (other instruments are insensitive if $\Delta \sim 0^{\circ}$ or $\Delta-180^{\circ}$ ); (2) there is never an ambiguity as to the sign of $\checkmark$ or $\Delta$; and (3) the quantity $\beta^{2}=N^{2}+S^{2}+C^{2}$ can be calculated, allowing for an on-line consistency check and an indication of sample depolarization effects.

1. G. E. Jellison, Jr., and F. A. Modine, "A Simple Phototube Power Supply for Constant Phototube Current in the Presence of Large Variations in Light Intensity," this report.

\section{RMLEMENTATION OF CONSTANT PHOTOTUBE CURRENT IN THE PRESENCE OF LARGE VARIATIONS IN UGHT INTENSITY1}

\section{G. E. Jellison, Jr., and F. A. Modine}

In polarization modulation ellipsometry,' as well as many other experimental situations where photoelastic modulators are used (such as magnetic dichroism experiments), it is necessary to operate a photomultiplier tube (PMT) at constant dc output while the light intensity on the PMT varies significantly. This is normally done by controlling the voltage of the PMT, which in turn controls the gain of the PMT.

In most applications, a separate feedback control circuit had to be built to control a power supply. In this work we have shown that a commercially available high-voltage power supply (Kepco OPS 1000B) can be adapted to control the PMT current simply by strapping appropriate resistors onto the rear control panel in a manner that reverses the current and voltage control channels that are usually employed. Two input voltages are used to control the PMT output current and limiting bias voltage, respectively; both can be computercontrolled using digital-to-analog converters since their ranges are -10 to $+10 \mathrm{~V}$. The PMT can be operated in either voltage control or current control mode.

The rise time of the circuit is $\sim 10$ ms, meaning that the bias voltage of the PMT can follow $60-\mathrm{Hz}$ fuctuations in the incident light level but cannot follow the $50-$ or $100-\mathrm{kHz}$ modulation arising from the photoelastic modulator. Thus, this power supply automatically controls the dc level without attenuating the highfrequency signals.

1. G. E. Jellison, Jr., and F. A. Modine, "A New Two-Channel Polarization Modulation Ellipsometer," this report. 


\section{Structure of Solids and Surfaces}

Structural properties of solids and surfaces are the major focus of this chapter. The research utilizes a variety of analytical techniques, including surface spectroscopies, electrun microscopy, and electron and $x$-ray diffraction in conjunction with synchrotron radiation.

The continuing emphasis in the division on determining the arrangement of atoms at the surface of a solid is indicated in the first section. Results of a low-energy electron diffraction analysis of the Al(111) surface indicate an expansion in the outermost interlayer spacing with respect to the bulk value. New studies using synchrotron radiation include photoelectron diffraction from $\mathrm{Ta}(100)$ and abso'ute $x$-ray reflectivity measurements of $\mathrm{Au}(100)$. The spot profile analysis technique has been used to determine step heights on NiAl surfaces. Adsorption of molecular oxygen on $\mathrm{Cu}(110)$ at $15 \mathrm{~K}$, as well as the initial stages of oxidation, has been examined with a new cooling stage.

Installation of the new VG HB-501 scanning transmission electron microscope with a specially modified objective pole piece has provided unique research capabilities to the Solid State Division. Chemically sensitive atomic structure imaging has been achieved for the first time, providing a fundamentally new and powerful approach to the determination of the structure and chemistry of materials. Initial efforts have been focused on exploring the limits of this new technique. These studies have provided unique images of defects in a number of technologically important materials, with emphasis on the high-temperature superconductors and multilayered semiconductors.

$X$-ray investigations have included work on novel $x$-ray monochromators. the structure of thin-film superconductors, and studies of lattice defects. Mössbauer diffraction measurements have shown that isotopically enriched ${ }^{57} \mathrm{Fe}_{2} \mathrm{O}_{3}$ crystals can be grown epitaxially and are suitable for obtaining submicrovolt $x$-ray beams from synchrotrons. $X$-ray scattering studies of $\mathrm{YBa}_{2} \mathrm{Cu}_{3} \mathrm{O}_{x}$ thin films grown on single-crystal substrates have revealed unexpected epitaxial relationships between the film and the substrate. 


\section{SURFACE PHYSICS}

\section{ATOMIC TERMINATION AND \\ RELAXATIONS OF THE NiAl(100) SURFACE}

\section{R. Noonan and H. L. Davis}

NiAl forms an ordered intermetallic compound which has the $\mathrm{CsCl}$ structure. It has been determined that (110), (111), and (100) surfaces of NiAl that are ordered and stoichiometric can be prepared. Since bulk (100) planes of NiAl have an $A \cdot B \cdot A \cdot B \cdots A \cdot B \cdot$ stacking sequence, where $A$ represents a plane of $A l$ atoms and $B$ a plane of $\mathrm{Ni}$ atoms, the (100) surface could be terminated with an Al layer, a Ni layer, or a surface with a mixture of Alterminated and $\mathrm{Ni}$-terminated domains, as has been identified for $\mathrm{NiAl}(111)$. The (100) surface has a complication not observed with the other surfaces. The surface initially orders in a $c(\sqrt{2} \times 3 \sqrt{2}) R 45^{\circ}$ structure after sputtering and annealing above $-400^{\circ} \mathrm{C}$. After anneals to temperatures above $-900^{\circ} \mathrm{C}$, the superlattice beams start to disappear, and by $\sim 1000^{\circ} \mathrm{C}$ a (1x1) pattern is observed. However, the observation of structure in the diffuse scattering suggests that remnants of the reconstructed surface are present, but only in very small domains since no superlattice diffraction beams are observed.

Six equivalent-beam-averaged I-V spectra were measured from the $(1 \times 1) \mathrm{NiAl}(10(0))$ surfoce obtained by annealing to $1000^{\circ} \mathrm{C}$. Thesc spectra were compared to spectra calculated for a variety of models to test surface termination, interlayer relaxations, and nonstructural parameters. Since the best agreement achieved for any Ni-terminated surface was $\mathbf{0 . 1 8}$ (Zanazzi-Jona $R$-factor formula) and the best for the Al-terminated surface was $\mathbf{0 . 0 8 4}$, it was concluded that $\mathrm{NiAl}(100)$ terminates in an $\mathrm{Al}$ layer. In addition, the surface exhibits multilayer relaxations of $\Delta d_{12}=\mathbf{- 8 . 5 \%}$ and $\Delta d_{23}=$ $+4.0 \%$. Figure 6.1 illustrates the convergence of the analysis to the optimum structure.

Cawt owe 07.1172a

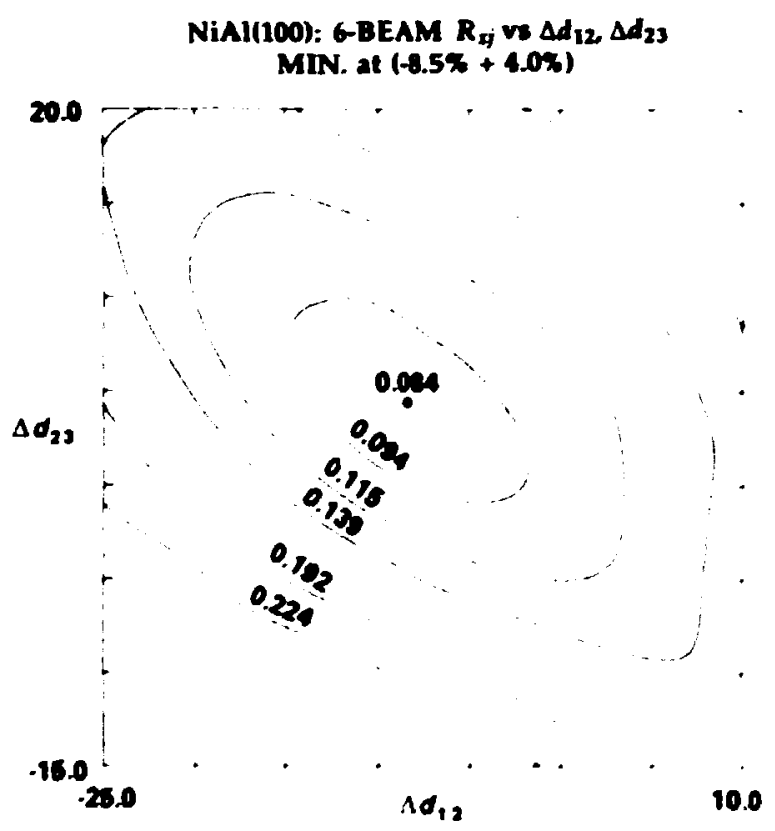

Fig. 6.1. $R$-factor topograph for the Alterminated NiAl(100) surface as a function of $\Delta d_{12}$ and $\Delta d_{23}$, the surface relaxations from bulk values between the first and second atomic layers and sccond and third atonic layers, respectively. 


\section{REEXAMINATION OF THE Al(111) SLRFACE}

\section{R. Noonan ana H. L. Davis}

Although it is well recognized that the creation of a surface can induce changes in the atomic structure of a solid rear the surface, the processes involved are not fully understood. For example, a dilemma has arisen between the measured and theoretically predicted relaxations of the AI(111) surface. The Al(111) surface has been investigated using low-energy electron diffraction (LEED) analysis, and the first interlayer spacing was found to be expanded by $2.2 \% 1$ and $0.9 \% 2$ with respect to the interlayer spacing of the bulk. Theoretical models, however, predict a contraction of $\mathbf{3 . 3}$ and 3.6\%.3,4 Since a verification of an expanded interlayer spacing would have important theoretical consequences, it was decided to reinvestigate the $\mathrm{Al}(111)$ surface.

I-V spectra for the five lowest index LEED beams from a room-temperature surface of Al(111) have been measured at normal incidence. in the analysis of these spectra, a very detailed investigation of LEED nonstructural parameters was considered in addition to the usual structural variations of the model. Figure 6.2 illustrates the agreement between the measured spectra and the spectra calculated for the best theoretical model. The analysis confirms that the surface layer is expanded, by $1.8 \pm$ $0.5 \%$. The minimum value of the total reliability factor for the analysis of the five
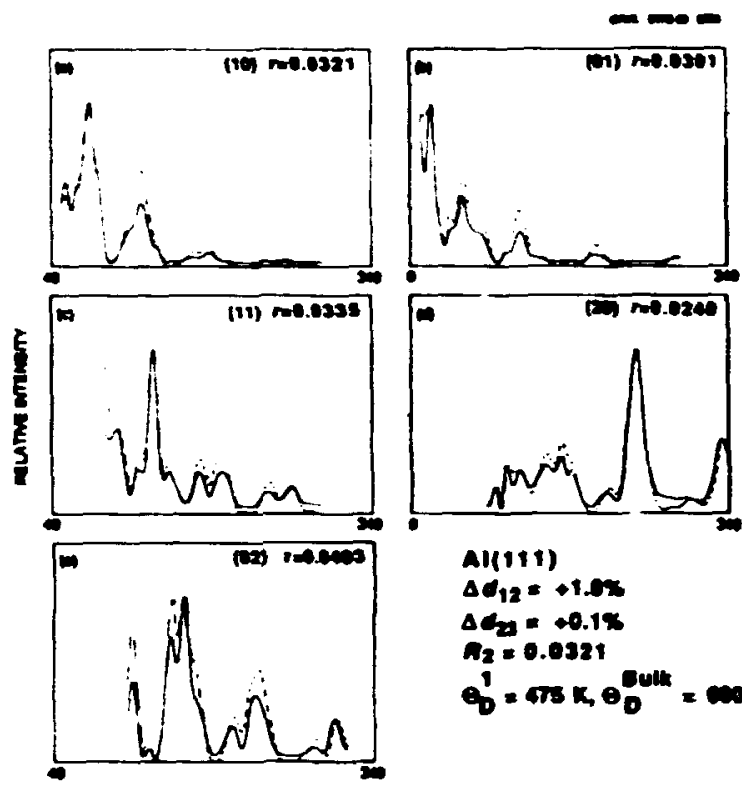

A|(11)

$\Delta C_{12}=+1.015$

$\Delta+4=0.1 \%$

$a_{2}=6.0221$

$e^{\prime}=\sin e_{0}^{\text {Dulk }}=\cos$

acernonedion

Fig. 6.2. Comparison of measured (solid line) and calculated (dashed line) I-V spectra for five LEED beams for Al(111). The model parameters are given in the figure label.

beams was 0.032 for the $R_{2}$ formula. This is the smallest $R_{2}$ value reported for any LEED analysis, which adds credibility to the conclusion that the first interlayer spacing is expanded.

1. F. Jona et al., J. Phys. C 13, L155 (1980).

2. H. B. Nielsen and D. L. Adams, J. Phys. C 15, 615 (1982).

3. John R. Smith and A. Banerjea, Phys. Reo. B 37, 10411 (1988).

4. Ning Ting et al., Surf. Sci. 206, L857 (1988). 


\section{THE FIRST INTERLAYER SPACING OF Ta(100) DETERMINED BY PHOTOELECTRON DIFFRACTION ${ }^{\natural}$}

\section{R. A. Bartynski, ${ }^{2}$ D. M. Zehner, D. Heskett, ${ }^{3}$ K. Garrison, ${ }^{2}$ G. Watson, ${ }^{3}$ W. N. Mei, S. Y. Tong, ${ }^{5}$ and X. Pan ${ }^{3}$}

Using the bulk and surface shifted $4 f$ core levels, a photoelectron diffraction study of $\mathrm{Ta}(100)$ at the National Synchrotron Light Source was performed to determine the first interlayer spacing of this surface. Photoemission intensities were measured as a function of photon energy at normal emission and near the $<111>$ direction of the crystal. To eliminate the necessicy of extensive normalization, the ratios of the photoemission intensities from the bulk and surface-shifted $4 f$ core levels were determined. A multiple scattering photoemission calculation for several first interlayer spacings ranging from the bulk value $\left(d_{12}=1.6550 \AA\right)$ to a $15 \%$ contraction $\left(d_{12}=1.4089\right.$ $A)$ in $5 \%$ increments has been performed.

Shown in Fig. 6.3'a) are the measured surface/bulk intensity ratios (open squares) as a function of photon energy at normal emission. The solid curves are the results of the multiple scattering calculation assuming different first interlayer spacings. Best agreement with experiment is obtained for the $d_{12}=1.4894 A$ curve. From the considerable disagreement with results of calculations for both 5 and 15\% contractions, a contraction of $10 \pm 3 \%$ is determined. The intensity ratios obtained away from normal emission are shown in Fig. 6.3(b).

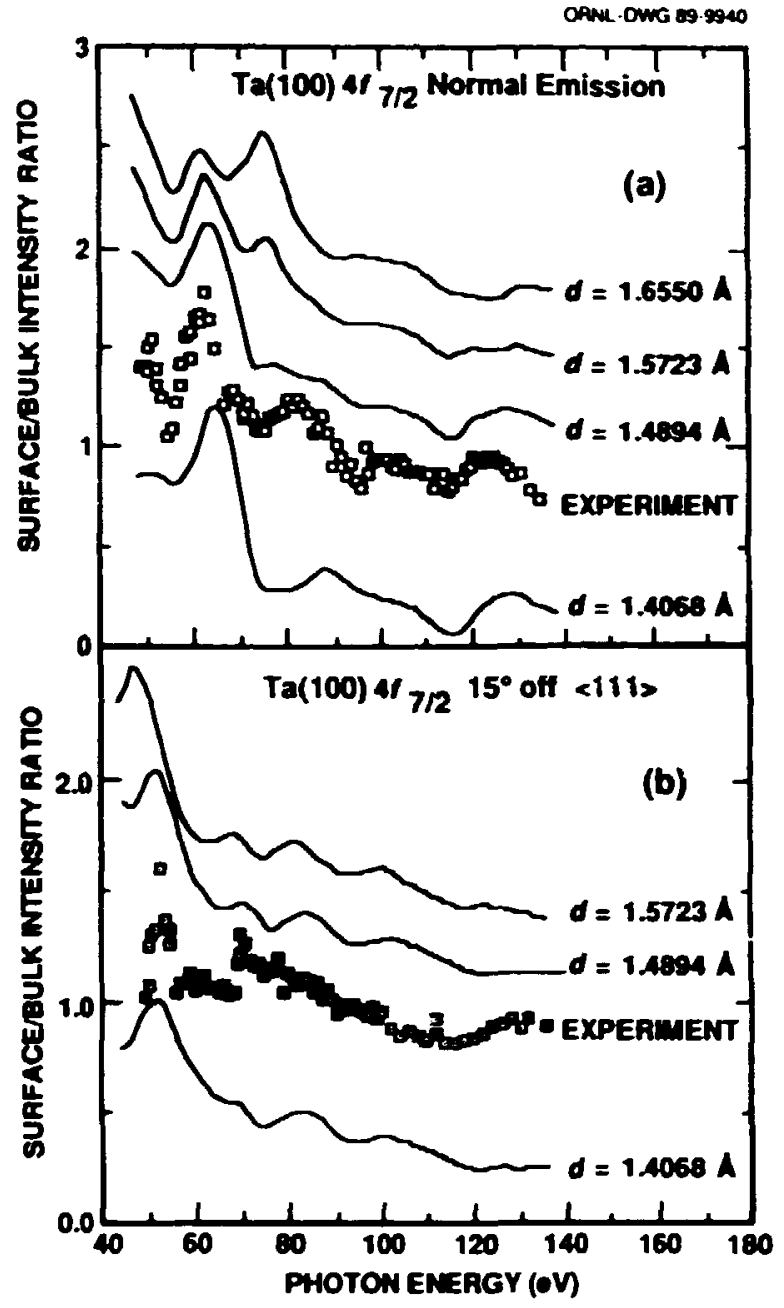

Fig. 6.3. Experimental (open squares) and theoretical (solid lines) intensity ratios of the surface and bulk $4 f$ core levels of $\mathrm{Ta}(100)$; (a) normal emission and (b) near the (111) direction.

Considerably less intensity modulation than for the normal emission results is scen for the offnormal data, and the results of the calculations for this geometry are not as sensitive to changes in the first interlayer spacing. Nevertheless, 
best agreement between theory and experiment is also obtained for a $10 \%$ contraction.

1. Summary of paper. Joumal of Vacuum Science and Technology (in press).

2. Rutgers University, Piscataway, N. J.

3. University of Pennsylvania, Philadelphia, $\mathbf{P a}$.

4. University of Nebraska, Omaha, Nebr. Wis.

5. University of Wisconsin, Milwaukee,

\section{ABSOLUTE X-RAY REFLECTIVITY STUDY OF THE Au(COI) SURFACE}

\section{Gibbs, ${ }^{2}$ D. M. Zehner, B. M. Ocko, and S.J.G. Mockric ${ }^{3}$}

It is well known that the clean $A u(001)$ surface is reconstructed and that this reconstruction can be eliminated by adsorbing small quantities $(\sim 5 \%)$ of foreign atoms on the surface. Although many surface-sensitive techniques have been employed in studying this surface, a complete and satisfactory description of the microscopic details does not yet exist. In this research we have used $x$-ray scattering to study the $A u(001)$ surface from room temperature to $1250 \mathrm{~K}$. Samples were cleaned by conventional sputter-annealing techniques after insertion into a small portable vacuum chamber, and measurements were performed using a rotating anode. The reflected intensity, normalized on an absolute scale, was measured along the surface r.ormal through the (002) and (022) bulk reflections. From these data it has been determined that for clean annealed samples (1) the $A u(001)$ surface is atomically smooth c ver length scales of several thousand angstroms; (2) the asymmetric angular dependence of the reflectivity is consistent with an expanded (19\% of the bulk interlayer spacing), buckled (20\% amplitude), hexagonal (25\% increase in mass) overlayer; and (3) the inplane order is not simply commensurate with the bulk. The expansion in the outermost interlayer spacing is a new result not previously determined by any other technique.

Significant differences in line shapes are observed for deliberately contaminated or sputtered surfaces. In the latter case the best model is one consisting of a rough surface layer (4.5-A ms roughness), in which Au atoms are not positioned in lattice planes and are separated from the undamaged bulk by a relatively smooth inter race (0.5-A rms roughness).

Additional measurements made as a function of sample temperature show that at $1175 \mathrm{~K}$ the surface undergoes a structural phase transition marked by a reduced asymmetry in the specular reflectivity, as would be expected for surface melting of the hexagonal overlayer and/or transformation to the bulk termination. The measured line shape of the $(002)$ is remarkably similar to the symmetric profile of the ideally terminated bulk with a root mean-square amplitude of $-0.25 \AA$ for atoms in the top layer.

1. Summary of paper: Phys. Rev. B 38, 7303 (1988).

2. Brookhaven National Laboratory, Upton, N. Y.

3. Massachusetts Institute of Technology, Cambridge, Mass. 


\section{A SPOT PROFILE ANALYSIS LOW-ENERGY ELECTRON DIFFRACTION (LEED) STUEY OF A STEPPED NiAl(111) SURFACE}

\author{
John F. Wendelken
}

A NiAl(111) surface with no atomic steps would be terminated with either a $\mathrm{Ni}$ or an $\mathrm{Al}$ layer with underlying layers alternating between the two eleinents. However, LEED (I vs $V^{1}$ and low-energy ion scattering studies ${ }^{2}$ indicate a mixed termination for this surface. The two terminations are assumed to exist in random domains whose domain boundaries are steps.

In order to examine the step structure and the question of a preferential surface termination, a highly stepped surface was prepared by cutting and polishing a NiAl surface with a surface normal 1.7 deg from the [111]. Spot profile analysis of selected LEED beams was then used to measure the average step height. Typically, LEED beams from a highly stepped surface with well-ordered steps will exhibit a splitting which is periodic in energy. However, for this surface, only a periodic broadening for the LEED beams was observed, which indicates an almost random distribution of terrace widths. The periodicity of the broadening is inversely related to the step height. Figure 6.4 shows the measured (01) diffraction beamwidth in momentum units in the direction perpendicular to the idealized step edges as a function of electron beam energy. The solid line shows the best calculated fit to the periodicity which was obtained for a step height of $1.63 \AA$, just

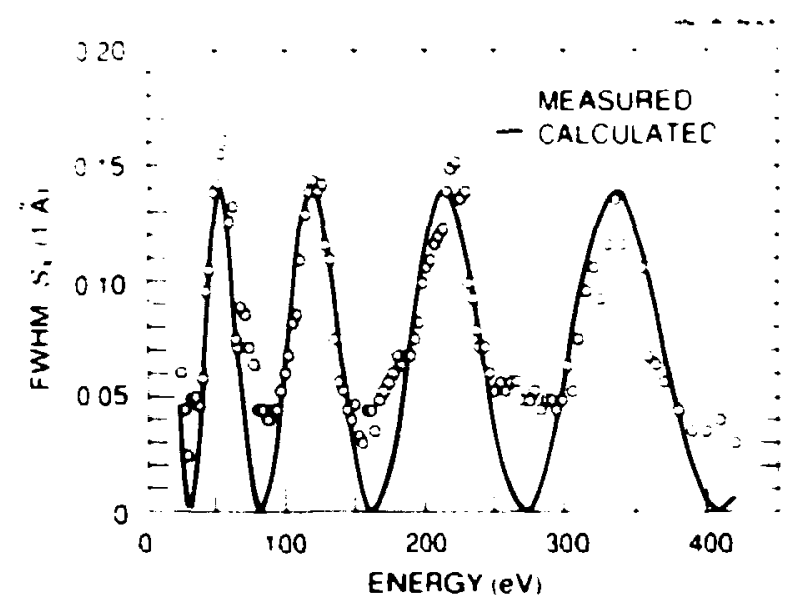

Fig. 6.4. The measured FWHM vs energy of the [01] electron diffraction beam in the direction perpendicular to the step edges of a highly stepped $\mathrm{NiAl}(111)$ surface and the best fit calculation, indicating a double-layer step height of $1.63 \AA$.

slightly less than the bulk double-layer spacing.

The apparent double-layer spacing indicates the possibility of a preferred termination on the highly stepped surface. Low-energy ion scattering studies are under way to confirm this. This is the first examination of a stepped surface in which the terraces could have alternating terminations. The possibility that single steps could, in this case, give the appearance of double steps because of the different phase factors will also be investigated.

1. J. R. Noonan and H. L. Davis, Phys. Rev. Lett. 59, 1714 (1987).

2. H. Nichus, Nucl. Instrum. and Methods Phys. Res. Sect. B 33, 876 (1988). 


\section{A SPOT PROFILE ANALYSIS LOW-ENERGY ELECTRON DIFFRACTION (LEED) STUDY OF A STEPPED NIAI(110) SURFACE}

\section{John F. Wendelken}

For comparison to the results obtained in the study of a stepped NiAl(111) surface, a stepped NiAl(110) surface has been studied with the same spot profile analysis LEED technique. The ideal $\mathrm{NiAl}(110)$ surface contains an equal mixture of $\mathrm{Ni}$ and $\mathrm{Al}$ atoms. Experimentally, this surface has been found to exhibit this bulk stoichiometry, but a rippling that places the $\mathrm{Al}$ atoms approximately $0.2 \AA$ above the $\mathrm{Ni}$ atoms has been noted. 2,3 Since every terrace on a stepped surface should have the same composition, single-layer steps are expected in contrast to the double-layer step height results of the NiAl(111) study.

Angular profiles from a highly stepped NiAl(110) surface for which the surface normal is $3.8 \mathrm{deg}$ from the $[110]$ are shown in Fig. 6.5. The intensities have been normalized, and the baseline of each profile has been offset vertically with increasing energy expressed in terms of the phase jump associated with the energy at a single-layer step. The LEED beam shapes are quite different from those observed with the [111] surface in that the beams show a distinct splitting which is periodic with energy. The splitting is indicative of a strcng step-step interaction and a more or tered step structure than was observed with 'ne $\mathrm{NiAl}(111)$ surface. A single-layer step height is confirmed by the occurrence of splitting when the phase jump calculated for single steps is $(2 n+1) \pi$. From the splitting an average terrace width of $33 \AA$ can

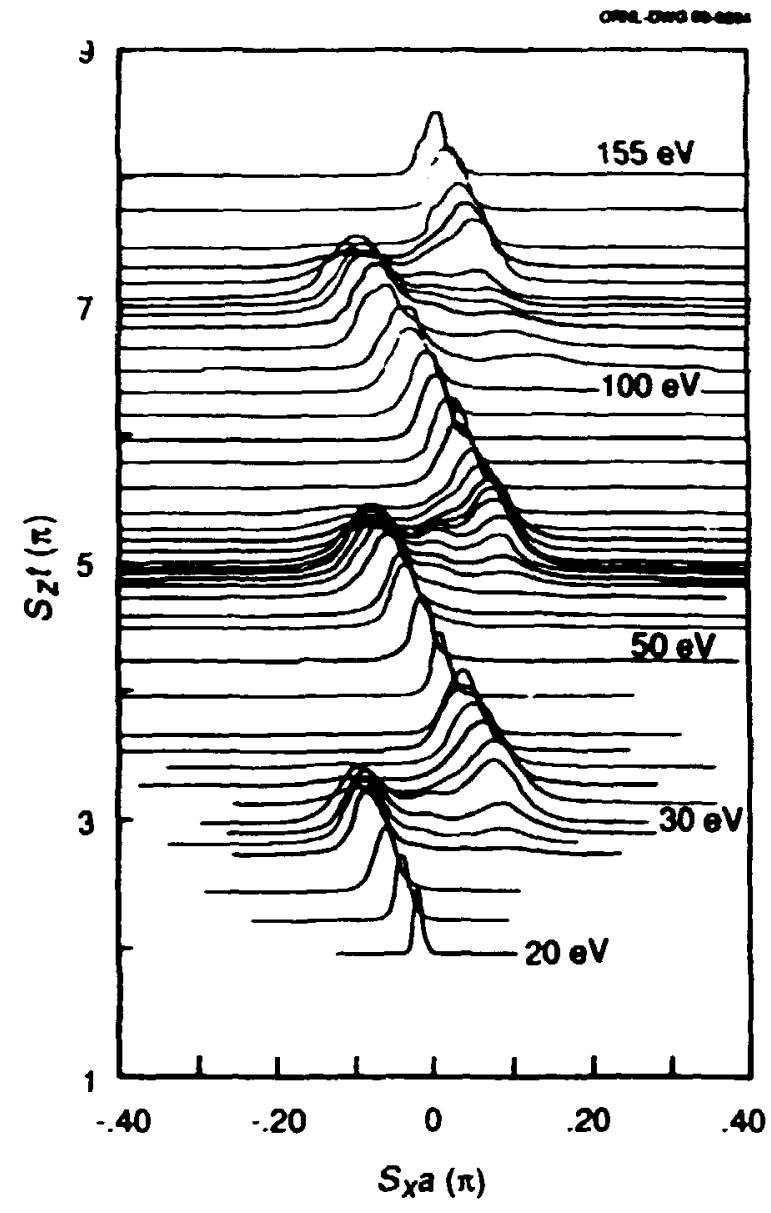

Fig. 6.5. Electron diffraction angular profiles in the direction perpendicular to step edges on a highly stepped NiAl(110) surface. The vertical offset is the energy-dependentphase jump $S_{z} t$ associated with a single-layer step height of $2.04 \mathrm{~A} . S_{x^{a}}$ and $S_{z} t$ are the product of the momentum parallel and perpendicular to the surface with the row spacing and step height, respectively.

be calculated, which is consistent with the single-layer step height and the 3.8-deg surface inclination.

Also observed in the angular profiles at energies where the splitting occurs is a small central peak. This indicates that approximately $5 \%$ of the surface is in regions where the step density is very low, while the rest has the 
step density indicated by the intense split peaks.

1. John F. Wendelken, "A Spot Profile Analysis Low-Energy Electron Diffraction (LEED) Study of a Stepped NiAl(111) Surface," this report.

2. S. M. Yalisove and W. R. Graham, Surf. Sci. 183, 556 (1987).

3. D. R. Mullins and S. H. Overbury, Surf. Sci. 199, 141 (1988).

\section{MOLECULAR OXYGEN ON CU(110): THE SEARCH EX:ENDED TO $15 \mathrm{~K}^{\mathbf{l}}$}

\section{Arthur P. Baddorf and John F. Wendelken}

Oxygen exists as a diatomic molecule in the gas phase, but bonds to metals as an atom. Dissociation occurs as the oxygen molecule interacts with the metal surface. This occurs very rapidly at room temperature, but between 100 and $180 \mathrm{~K}$ a molecular species has been observed on a number of single-crystal metal surfaces. Despite attempts by several research groups, such a precursor to dissociation has not been observed at these temperatures on Cu(110).

We have used electron energy luss spectroscopy and $x$-ray pholoemission spectroscopy to search for molecular oxygen on $\mathrm{Cu}(110)$ at crystal temperatures down to $15 \mathrm{~K}$. We have found that even at $15 \mathrm{~K}$., molecular oxygen dissociates on the clean surface. The atomic oxygen which bonds to the surface is identified by an O-metal vibration of $49 \mathrm{meV}$ (inhomogeneously broadened) and an (Uls core level al 530-eV binding energy. Dissociation continues until a coverage of $-1 / 2$ monolayer of atomic oxygen is formed.
Additional exposure dias result in molecular species on the surface. Aboce $0.5 \mathrm{~L}$. two new oxygen species are observes. One of the species has a 192-meV vibrational energy and a 538.2. eV Ols binding energy. These values are close to those observed for gas phase $\mathrm{O}_{2}$ and correspond to molecular oxygen condensed on the surface with no chemical bonding to the metal. Exposures above $5 \mathrm{~L}$ lead to multiple layers of condensed $\mathrm{O}_{2}$.

The second molecular specie observed has an O-O stretching energy of $130 \mathrm{meV}$, much lower than the gas phase energy. This correlates to a bond order of 1.4 , reduced from the gas phase bond order of 2 . The Ols core level of this molecule shows a chemical shift from that of the gas phase to $537.0 \mathrm{cV}$. This is a chemisorbed molecule and the precursor to dissociation we had been secking.

As the crystal temperature is increased, the molecular species desorb or dissociate. Annealing to $35 \mathrm{~K}$ desorbs the multilayers of condensed $\mathrm{O}_{2}$. Between 50 and $70 \mathrm{~K}$ the chemisorbed $\mathrm{O}_{2}$ dissociates, leaving atomic coverages well above 1/2 monolayer.

These molecular species are sensitive to radiation, and concentrations were altered by $\mathrm{MgK}_{\alpha} \mathrm{X}$ rays $(1253.6 \mathrm{eV})$. Exposures to $X$ rays dissociate the molecular species and also convert condensed $\mathrm{O}_{2}$ into chemisorbed $\mathrm{O}_{2}$. thus promoting oxidation. These experiments show the exceptional reactivity of $\mathrm{Cu}(110)$ with oxygen. The reactivity is more like that seen on W or Cr than on other noble metals, Pd or P't.

1. Summary of paper to be published. 


\section{NITIAL STAGES IN OXIDATION} OF $\mathrm{Cu}(110)^{1}$

\section{Arthur P. Baddorf and John F. Wendelken}

The prevalence of oxygen in the atmosphere makes oxidation common and important for many materials processes. Unfortunately, most surface spectroscopies are not compatible with the high oxygen pressures required to oxidize many metals, making studies of the microscopic details of oxidation difficult or impossible. We have discovered that oxidation of $\mathrm{Cu}(110)$ is possible without large pressures by condensing $\mathrm{O}_{2}$ on the surface of $\mathrm{Cu}(110)$ at $15-25 \mathrm{~K}$ and subsequently bombarding the molecule with electrons or $X$ rays. We have utilized this discovery to study $\mathrm{O} / \mathrm{Cu}(110)$ using high-resolution electron energy loss spectroscopy, x-ray photoemission spectroscopy (XPS), and lowenergy electron diffraction (LEED).

Previous studies have revealed that below $1 / 2$ monolayer, oxygen bonds in a long-bridge site with a vibrational energy of $49 \mathrm{meV} .^{2}$ The oxygen fills alternate rows in the $[100]$ direction, producing a $p(2 \times 1)$ LEED pattern and causing a buckled row reconstruction. Higher exposures (above $10^{5} \mathrm{~L}$ at $300 \mathrm{~K}$ ) produce oxygen coverages up to 5/6 monolayer. ${ }^{3}$ The additional oxygen is forced to occupy a second site (probably four-coordinated) with a vibrational energy of $44 \mathrm{meV}$, and it produces further surface reconstruction associated with a $c(6 \times 2)$ LEED pattern. A small vibrational peak is also observed at $62 \mathrm{meV}$, although this was not identified. ${ }^{2}$
We have discovered that above $5 / 6$ monolayer coverage the copper begins to oxidize in isolated islands. Two new vibrational modes appear with energies of 19 and $81 \mathrm{meV}$. These are identified as the infrared active vibrations at the surface of $\mathrm{Cu}_{2} \mathrm{O} .4$ XPS discloses a $\mathrm{Cu}$ $2 p_{3 / 2}$ shift to lower binding energy with no satellite, confirming the growth of $\mathrm{Cr}_{2} \mathrm{O}$ and not $\mathrm{CuO}$. Because $\mathrm{Cu}_{2} \mathrm{O}(110)$ has an $18 \%$ lattice mismatch with $\mathrm{Cu}(110)$, the growth of $\mathrm{Cu}_{2} \mathrm{O}$ islands is accompanied by diffuse LEED spots, and no pattern is observed above -1 monolayer.

Even higher oxygen coverages are associated with growth of the 62-meV vibration. Apparently excess oxygen is forced into surface sites by the limited mobility of subsurface oxygen. Annealing above $150^{\circ} \mathrm{C}$ removes the oxygen from this surface site. Further annealing continues to remove oxygen from the surface and subsurface region. Annealing above $350^{\circ} \mathrm{C}$ removes evidence of $\mathrm{Cu}_{2} \mathrm{O}$, since by this temperature the oxygen has migrated into the copper bulk.

1. Summary of paper to be published.

2. J. M. Mundenar et al., Surf. Sci. 188, 15 (1987).

3. G. R. Gruzalski, D. M. Zehner, and J. F. Wendelken, Surf. Sci. 147, L623 (1984).

4. L. H. Dubois, Surf. Sci. 119, 399 (1982). 


\section{SURFACE CORE-LEVEL SPECTROSCOPY} FROM HFC(100) AND HfC(111)'

\author{
D. M. Zehner, G. R. Gruzalski, \\ G. W. Ownby, and D. Heskett ${ }^{2}$
}

The electronic environment of $\mathrm{Hf}$ in the outermost layers of $\mathrm{HFC}(100)$ and $\mathrm{HFC}(111)$ has been investigated using angle-resolved ultraviolet photoemission with synchrotron radiation at the National Synchrotron Light Source to measure the $\mathrm{Hf} 4 \mathrm{f}$ core-level binding energies (CLBEs). By carefully choosing the incident photon energy and by varying the collection angle, such spectra can yield the required surface sensitivity to measure CLBEs of surface atoms, if these CLBEs sufficiently differ from those of bulk atoms.

Shown in Fig. 6.6 (solid line) are spectra obtained for two different collection angles from a clean $\mathrm{HfC}(100)$ surface. By measuring the photoemission at a collection angle $\left(\theta_{c}\right)$ of $70^{\circ}$ with respect to the surface normal, the electron mean-free path is reduced by a factor of 3 as compared with that for normal emission, $\theta_{c}=0^{\circ}$. These data show one set of spin-orbit split $4 f$ levels, no evidence of surface core-level shifted peaks, and no change with detection angle. This observation is consistent with the rippling of the outermost layers determined by LEED in which the $C$ atoms are displaced outward and the $\mathrm{Hf}$ atoms inward, creating an environment for the surface $\mathrm{Hf}$ atoms similar to that in the bulk.

In contrast, the results in Fig. 6.6 idashed line) for the (111) surface show additional intensity on the high binding energy side of the $4 f$

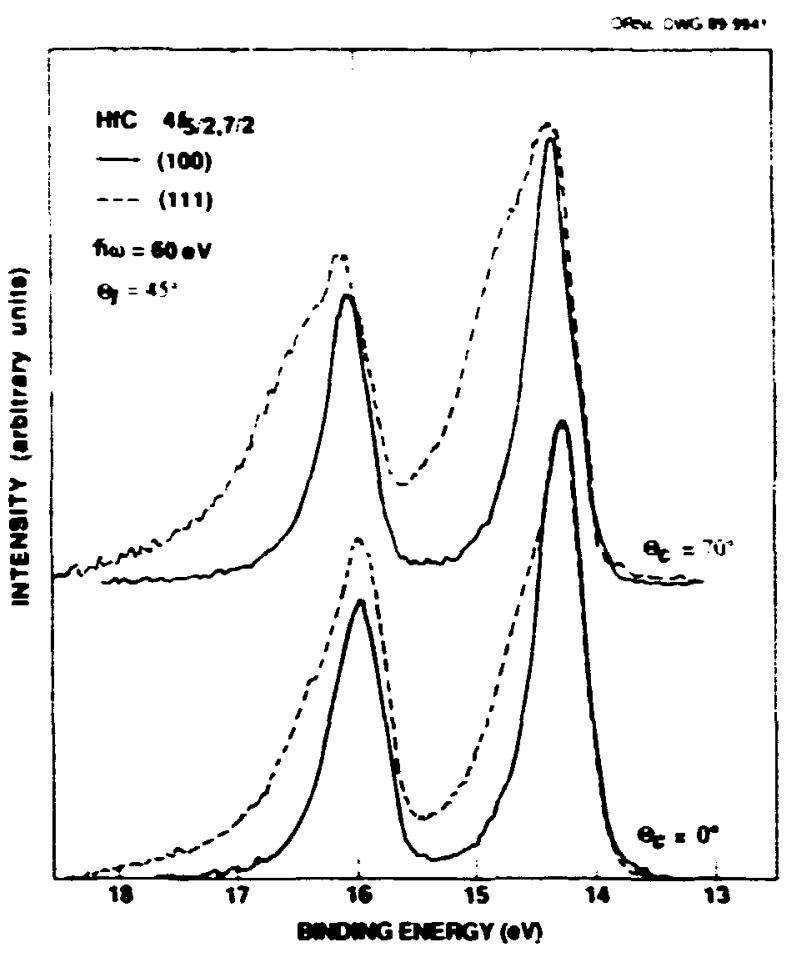

Fig. 6.6. Photoemission spectra of $\mathrm{Hf} 4 f$ region from (a) $\mathrm{HfC}(100$ and (b) $\mathrm{HfC}(111)$.

peaks for $\theta_{c}=0^{\circ}$. This intensity increases when more surface-sensitive detection conditions are used $\left(\theta_{c}=70^{\circ}\right)$. We propose that these data are consistent with at least a portion of this surface being terminated by Hf. Support for this interpretation comes from data for $\mathrm{H}(0001)$ (not shown), where a similar core-level shift is observed.

1. Summary of paper to be published.

2. University of Rhode Island, Kingston, 


\section{SURFACE STATES ON NiAI(110) ${ }^{1}$}

S. C. Lui, ${ }^{2}$ D. M. Zehner, M. H. Kang, ${ }^{3}$ E. I. Mele, ${ }^{3}$ and E. W. Plummer ${ }^{3}$

The (110) surface of NiAl exhibits a rippled relaxation in the top layer with $\mathrm{Al}$ atoms displaced outward and $\mathrm{Ni}$ atoms inward. This structure has been investigated extensively using many different experimental techniques. The calculated surface electronic structure shows that surface and resonance states localized at the $\mathrm{Ni}$ site are partly responsible for the inward relaxation of the $\mathrm{Ni}$ atoms. Thus, measurements of these surfaces states and comparisons with results of calculations could help to elucidate the driving mechanism of the rippled relaxation as well as other physical properties that are different from the bulk. Specifically, since bulk NiAl has a filled d band and is nonmagnetic, a determination of the surface electronic structure would be particularly interesting because a change in the electron configuration of the surfase $\mathrm{Ni}$ atoms could produce a magnetic surface layer.

Surface states on NiAl(110) were investigated experimentally using angle-resolved photoemission and synchrotron radiation at the National Synchrotron Light Source. Two surface states with binding energies of 1.08 and $1.12 \mathrm{eV}$ and one surface resonance state with binding energy of $1.37 \mathrm{eV}$ were observed at normal emission. The dispersions of these surface and resonance states throughout the surface Brillouin zone were also measured. Both types of states exhibit very strong polarization dependence, which indicates that the symme- try of the surface peaks is well defined. Theoretically, the surface was simulated by a fivelayer slab. A first-principles calculation was performed using a self-consistent pseudopotential scheme within the local density approximation. Results of the calculation qualitatively reproduce the energy positions and dispersions of the surface/resonance states. Agreement between theory and experiment is encouraging especially considering the complexities associated with an alloy surface. $O$ particular interest, the first moment of the surface density of states (DOS) is shifted toward the Fermi level by $-0.5 \mathrm{eV}$, suggesting that the surface $\mathrm{Ni}$ and $\mathrm{Al}$ atoms in the alloy have recovered some of their individual bonding behavior. However, the total surface DOS at the Fermi level shows negligible change from that of the bulk, thus indicating the absence of a magnetic surface layer.

1. Summary of paper. Physical Review B (in press).

2. Oak Ridge Associated Universities postgraduate research participant.

3. University of Pennsylvania, Philadelphia, Pa.

4. M. H. Kang and E. J. Mele, Phys. Reo. B 36, 7371 (1987).

\section{ELECTRON SPECTROSCOPY OF MULTICHARGED ION-SURFACE INTERACTIONS ${ }^{1}$}

D. M. Zehner, F. W. Meyer, 2 C. C. Haoener, 2 K. J. Reed, ${ }^{3}$ and ‥ I. Snowden 4

In order to interpret results obtained for the interaction of multicharged ions with surfaces 
of single crystals, the variable screening modet of Eichler and Wille ${ }^{5}$ has been used to calculate adiabatic molecular orbital (MO) energy levels for a number of collision systems. Results from these calculations provide information about the range of internuclear separations at which MO pseudocrossings or rotational couplings may occur. Specifically, they give a more realistic description of the possible pathways along which innershell excitations occur and how sensitive these pathways are to the collision partners making up the quasi-molecule. For example, the calculations have provided a qualitative explanation of the experimental observation that projectile $K$-shell excitation for Li-like ions occurs for $N, O$, and $F$ ions incident on a Cu target, but not for a Au target. The vacancies produced by the collision are seen to decay from more neutral ions, while vacancies brought into the collision can, in addition, decay from higher charge states.

New measurements have been made using a small hemispherical-sector electron-energy analyzer mounted on a rotatable stage. The electron spectrometer also has a deceleration/focusing stage, by which the electron pass energy can be reduced with only a minimal loss of signal. This instrument thus provides the capability for angle- as well as energyresolved measurements. Initial results for both projectile and target Auger emission have been obtained for 70-keV $\mathrm{O}^{++}$projectiles incident on a Au target. Since the observed O KLL prak position is relatively insensitive to the angle of incidence of the projectile (i.e., time spent above the surface), it is inferred that the total ion neutralization rate is large compared with the relevant $K L L$ Auger decay rate. Oscillations in intensity with respect to take-off angle of target emission peaks are attributed to diffraction and thus indicate that penetration of the projectile and subsequent vacancy transfer to target atoms occur for several layers in the selvedge region.

1. Summary of paper: Surface Science (in press).

2. Physics Division, ORNL.

3. Lawrence Livermore National Laboratory, Livermore, Calif.

4. Osnabrück University, Osnabrück, Federal Republic of Germany.

5. J. Eichler and U. Wille, Phys. Reo. A 11, 1973 (1975).

\section{ELECTRON MICROSCOPY}

\section{CHEMICALLY SENSITIVE STRUCTURE MMAGING WITH A SCANNING TRANSMISSION ELECTRON MICROSCOPE ${ }^{1}$}

\section{S. J. Pennycook and L. A. Boatner}

Conventional high-resolution electron microscopy uses the phase-contrast method, in which the diffracted beams emerging from the sample are recombined on the viewing screen of the microscope. ${ }^{2}$ The resultant contrast depends on the relative phases of the diffracted beams, which are sensitive to microscope and sample parameters, so that images must be interpreted by means of simulation and defect models are 
somewhat empirical. By using a high-angle detector in a scanning transmission electron microscope (STEM), these problems may be avoided and high atomic-number or $Z$ contrast may be obtained. The scattering at high angles is highly localized so that each atomic nucleus scatters independently of its neighbors with the $Z^{2}$ cross section of Rutherford scattering. resulting in images with strong chemical sensitivity and an incoherent character.

In this report, chemically sensitive atomic structure Z-contrast imaging has been achieved for the first time using an HB501 STEM with specially designed optics to provide a $2.2-\AA$ diam probe beam. Figure 6.7 shows images from cross sections of $\mathrm{YBa}_{2} \mathrm{Cu}_{3} \mathrm{O}_{7-x}$ and $\mathrm{ErBaC}_{13} \mathrm{O}_{7-x}$ in which the tripled perovskite cell is clearly visible, the bright lines within the cell corresponding to the planes containing the heavy metal ions. The strong $Z$ sensitivity results in the $Y(Z=39)$ plane showing lower intensity than the Ba $(Z=56)$ planes [Fig. 6.7(a)], while in the rare-earth-substituted material the Er $(Z=68)$ plane is brighter than the $B$ planes [Fig. 6.7(b)].

In a conventional phase-contrast image, atoms can appear bright or dark depending on both the objective lens focus and sample thickness, whereas in the $Z$-contrast image atoms are always imaged in their correct location with an intensity dependent on $Z^{2}$. Precise knowledge of thickness and focus is therefore not required for interpreting $Z$-contrast images, even at interfaces. With conventional phase-contrast imaging, images of interfaces cannot be inter-
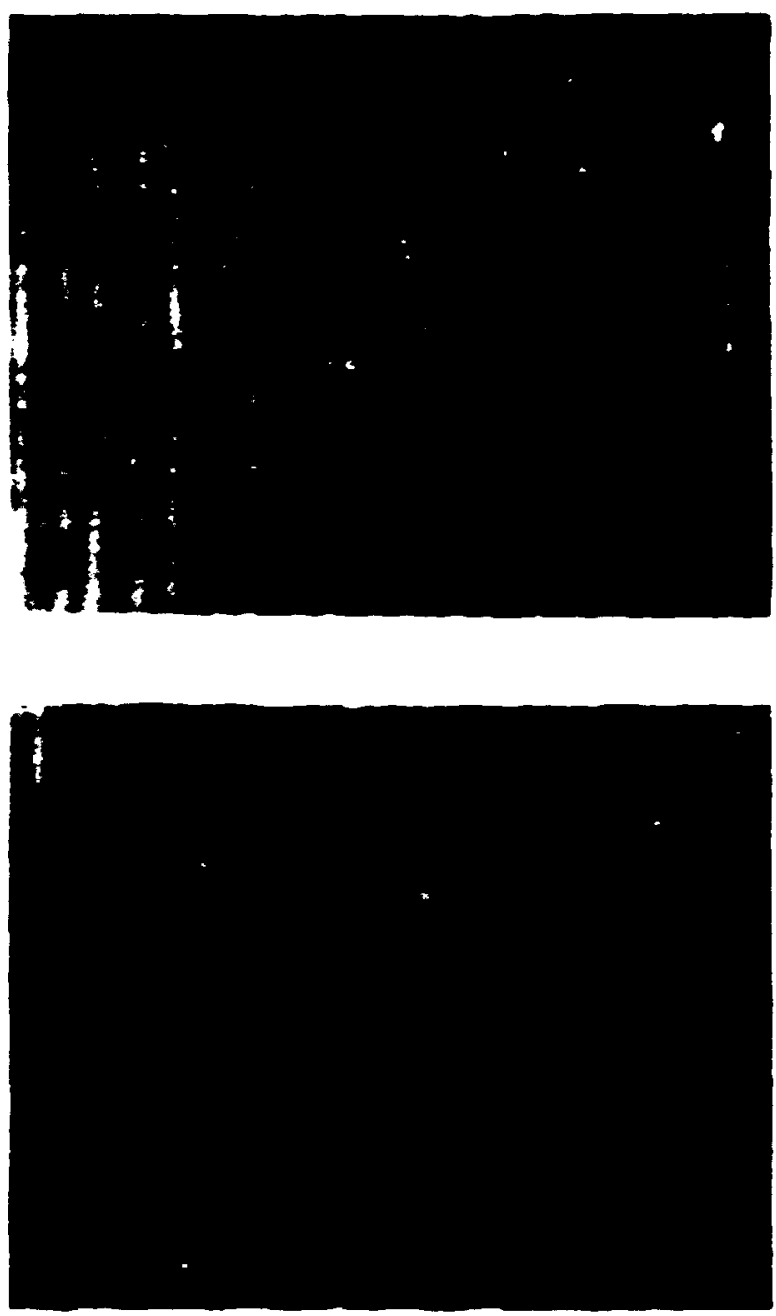

Fig. 6.7. Z-contrast STEM images of (a) $\mathrm{YBa}_{2} \mathrm{Cu}_{3} \mathrm{O}_{7-x}$ and (b) $\mathrm{ErBa}_{2} \mathrm{Cu}_{3} \mathrm{O}_{7-x}$ single crystals viewed parallel to the $c$ planes.

preted from the images of the adjacent perfect crystals but must be simulated from first principles. Z-contrast imaging provides a fundamentally new and powerful approach to the determination of the atomic structure and chemistry of materials.

1. Summary of paper: Nature 336,565 (1988).

2. O. Scherzer, I. Appl. Phys. 20,20 (1949). 


\section{Z-CONTRAST IMAGING OF

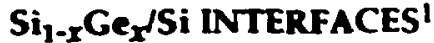

\section{S. I. Pennycook, M. F. Chisholm, and $\mathrm{O}$. W. Holland}

Imaging of an interface between isostructural $\mathrm{Si}_{1-x} \mathrm{Ge}_{x}$ and $\mathrm{Si}$ by conventional phase-contrast techniques requires that particular conditions of sample thickness and objective lens defocus be chosen to maximize interfacial contrast. ${ }^{2}$ These conditions depend on the composition $x$. With Z-contrast imaging the defocus is set fo: the minimum probe size, and the image intensity at all thicknesses is sensitive to composition. Atomic resolution Z-contrast images may be obtained using the Solid State Division's specially modified $\mathrm{HB} 50 \mathrm{l}$ scanning transmission electron microscope.

Figure 6.8 shows images of a thin epitaxial layer of $\mathrm{Si}_{1-x} \mathrm{Ge}_{x}$ on $\mathrm{Si}$, with $x$ estimated at 0.9 , grown by oxidation of Ge-implanted $\mathrm{Si}^{3}$ With this nonequilibrium growth technique, the Ge layer segregates ahead of the $\mathrm{SiO}_{2}$ layer but has insufficient time to diffuse into the $\mathrm{Si}$ and, therefore, forms a thin strained epitaxial layer. The phase-contrast image in Fig. 6.8(a) shows no contrast from the Ge layer, whereas in the Z-contrast image [Fig. 6.8(b)] the interface can be located to within a monolayer.

This image provides a map of the interfa se composition at the atomic scale, since each atomic columr. is resolved with an intensity dependent on the concentration of $\mathrm{Ge}$ within it. The upper $\mathrm{Ge} / \mathrm{SiO}_{2}$ interface also can be studied using the Z-contrast image. In the phasecontrast image of this interface, the characteristic random speckle pattern of the amorphous phase overlaps and obscures the last layer of the Ge film. In the incoherent $Z$-contrast image
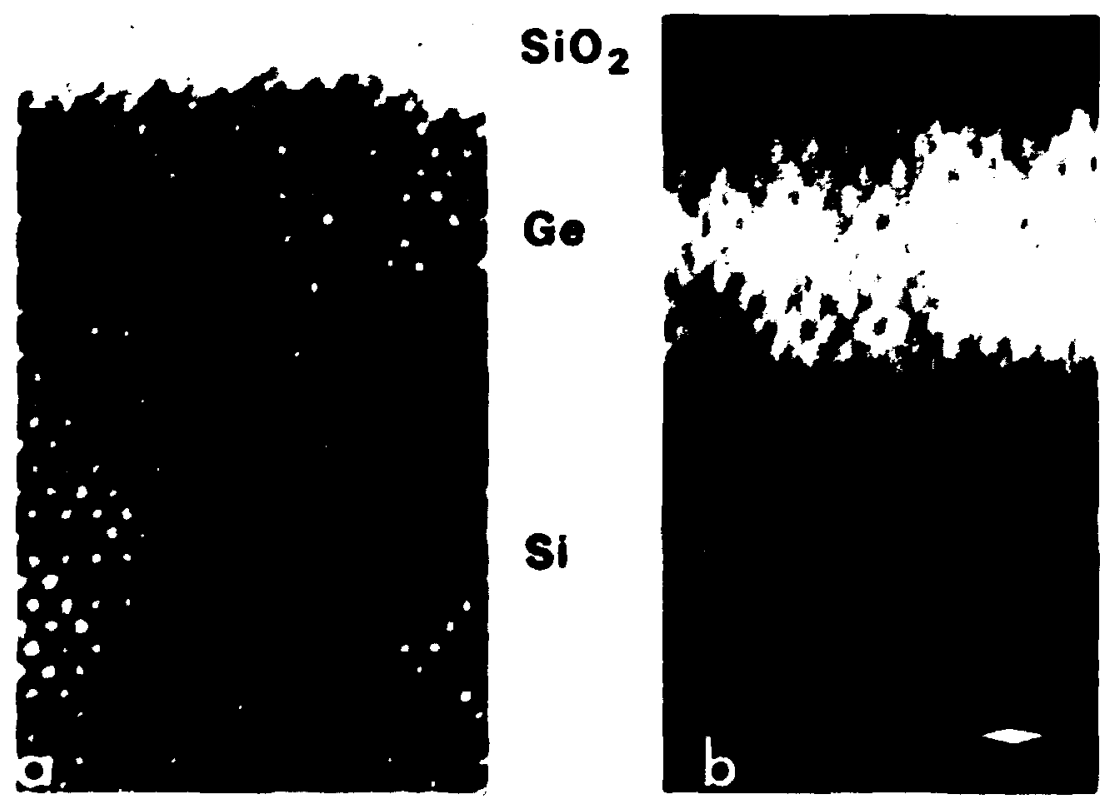

Fig. 6.8. Phase-contrast (a) and $Z$-contrast (b) images of $\mathrm{Si}_{1-x} \mathrm{Gc}_{x}$ epitaxial layer on Si grown by an oxidation process. 
there is no contrast from within an amorphous phase, and the last Ge layer can be studied for interface roughness or atomic steps.

1. Summary of paper to be published.

2. R. Hull, ] '4. Gibson, and J. C. Bean, Appl. Phys. Lett. 46, 179 (1985).

3. O. W. Holland, C. W. White, and D. Fathy, Appl. Phys. Lett. 51, 520 (1987).

\section{RECONSTRUCTION OF THE $\mathrm{COSi}_{2} / \mathrm{Si}(100)$ INTERFACE}

\section{S. J. Pennycook and S. M. Yalisove'}

The $\mathrm{CoSi}_{2} / \mathrm{Si}(100)$ interface has been observed by diffraction studies to undergo a $2 \times 1$ reconstruction. 2 The same reconstruction is seen whether the silicide is formed by reaction with deposited Co, which destroys the original ineerface, or by evaporation, which might in principle preserve the $2 \times 1$ reconstruction of the original Si surface. Figure 6.9 shows a Zcontrast image of a film formed by a template method, followed by homoepitaxial growth to a thickness of $6.5 \mathrm{~nm}$. The Z-contrast image picks out the Co columns in the $\mathrm{CoSi}_{2}$ and clearly shows atomic resolution chemical information at the interface. An interface reconstruction can be seen, but it is in a $4 \times n$ form and chemical in nature. The interface is periodically located either above a complete (200) silicide plane or above the next (partially complete) (200) layer.

Figure 6.9 shows an intersection of two "domains" of the interface reconstruction; the three Co column pairs in the partially complete

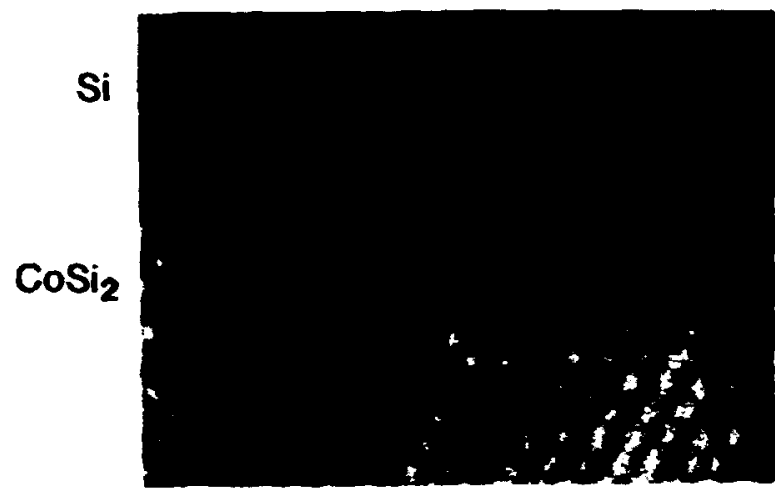

Fig. 6.9. Z-contrast image of a $\mathrm{CoSi}_{2} / \mathrm{Si}(100)$ interface showing interfacial reconstruction.

(200) layer on the left of Fig. 6.9 are out of sequence with the Co column pair on the right and are separated by a faint partially complete Co column pair. Such domains are commonly observed. One possible explanation of why only a $2 \times 1$ reconstruction has been seen before is the extremely small domain size of the $4 \times n$ reconstruction, which would reduce the intensity of the corresponding diffraction spots. The relative shift between the two crystal lattices is consistent with an interface model in which, at least for a flat interface, the Co atoms retain eightfold coordination. ${ }^{3}$

1. AT\&T Bell Laboratories, Murray Hill, N. J.

2. S. M. Yalisove, R. T. Tung, and J. L. Batstone, Mat. Res. Soc. Symp. Proc. 116, 439 (1988).

3. D. Chems, C. J. D. Hetherington, and C. J. Humphreys, Philos. Mag. A 49, 165 (1984). 


\section{INTERDIFFUSION IN STRAINED-LAYER SLPERLATTICES}

\section{S. 1. Pennycook, I. M. Baribeau,' and D.C. Houghton}

Many attempts have been made to grow ultrathin multilayers of the form $\left(\mathrm{Si}_{m} \mathrm{Ge}_{n}\right)_{p}$, where $m$ and $n$ refer to alternating $(400)$ monolayers of Si and Ge repeated p times. Such artificial crystals have the potential for realizing a direct band-gap superconductor with novel electronic properties. ${ }^{2}$ However, the growth of such sharf compositionally modulated materials is hampered by interdiffusion, which is significantly enhanced over tracer diffusion coefficients due to the extremely large strains in these materiais. ${ }^{3}$ Z-contrast scanning transmission electron microscopy (STEM) provides a means for measuring interdiffusion at individual interfaces in these materials, for example, to correlate with the depth from the surface or with atomic steps on the interfaces.

Figure 6.10 shows $Z$-contrast images of a $\left(\mathrm{Si}_{8} \mathrm{Ge}_{2}\right)_{100}$ ultrathin superlattice in $\langle 110\rangle$ projection obtained using the Solid State Division's specially modified high-resolution scanning transmission electron microscope. The dots represent the zigzag chains of atoms seen in this projection so that each vertical row of dots corresponds to two $\{400]$ monolayers. The image is, therefore, sensitive to composition on the monolayer scale, and it is quite clear that significant interdiffusion has occurred in this sample, which was grown by molrcular beam epitaxy at a substrate temperature of $4(X)^{\circ} \mathrm{C}$ and a deposition rate of $0.02 \mathrm{nms}^{-1}$. Cursently we
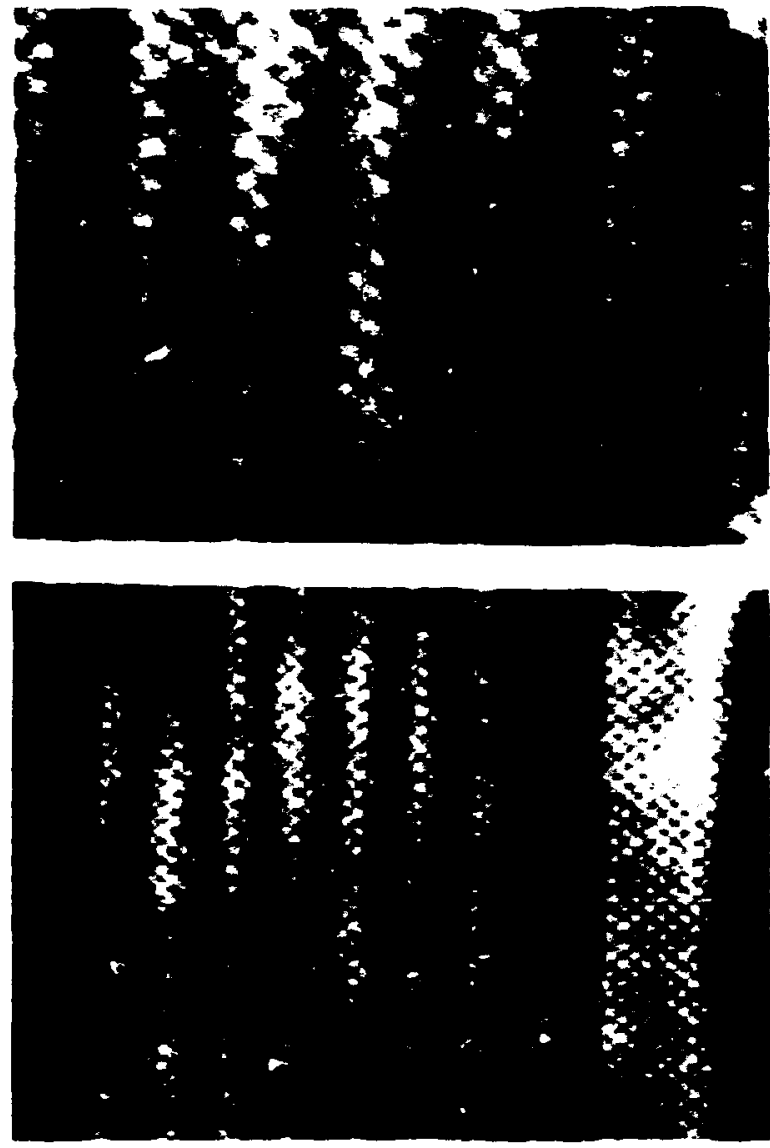

Fig. 6.10. $Z$-contrast STEM image of ultrathin multilayer $\left(\mathrm{Si}_{8} \mathrm{Ge}_{2}\right)_{100}$ showing (a) interdiffusion on the monolayer scale and (b) a stacking mistake due to failure of the Ge shutter to close resulting in a wide alloy layer.

are studying samples grown at $350^{\circ} \mathrm{C}$, in which the interdiffusion should be less; we could then study the thermal relaxation of the sample directly by Z-contrast STEM.

1. National Research Council of Canada, Ottawa, Ontario, Canada

2. D. C. Houghton et al., J. Cryst. Growth 81, 434 (1987).

3. F. Spaepen, Mat. Res. Soc. Symp. Proc. 37, 295 (1985). 


\section{CRIMCAL CURRENTS AND GRAIN BOUNDARY DISLOCATIONS IN $\mathrm{YBa}_{2} \mathrm{Cu}_{3} \mathrm{O}_{7-8}$ SUPERCONDUCTORS}

\section{F. Chisholm and S. J. Pennycook}

A new electron microscopy technique for forming high-resolution images with strong chemical sensitivity has been used to examine grain boundaries in high-temperature oxide superconductors. The new technique forms images using large-angle elastically scattered electrons. Thus, the image is not a reconstruction of the object from diffracted beams, as in conventional high-resolution microscopy, but is instead a map showing at atomic resolution the scattering power of the specimen. The scattering power for high-angle scattering is strongly dependent on atomic number $Z$, giving the images chemical sensitivity. Using this technique, we have shown for the first time the nature of the defects present at individual lowangle tilt grain boundaries. These defects are responsible for the rapid reduction of the critical current density across grain boundaries with increasing tilt angle observed in $\mathrm{YBa}_{2} \mathrm{Cu}_{3} \mathrm{O}_{7}-\delta$ superconductors. 2 Such boundaries are seen to consist of an asymmetric array of dislocations. When the boundary tilt angle exceeds $-7.5^{\circ}$, the defects appear amorphous and chemically identical to the adjacent $\mathrm{YBa}_{2} \mathrm{Cu}_{3} \mathrm{O}_{7-8}$ grains (Fig. 6.11).

This research demonstrates that the achievement of high critical current densities in the oxide superconductors depends strongly on the boundary geometry. Segregation of impurities or the formation of intergranular glassy
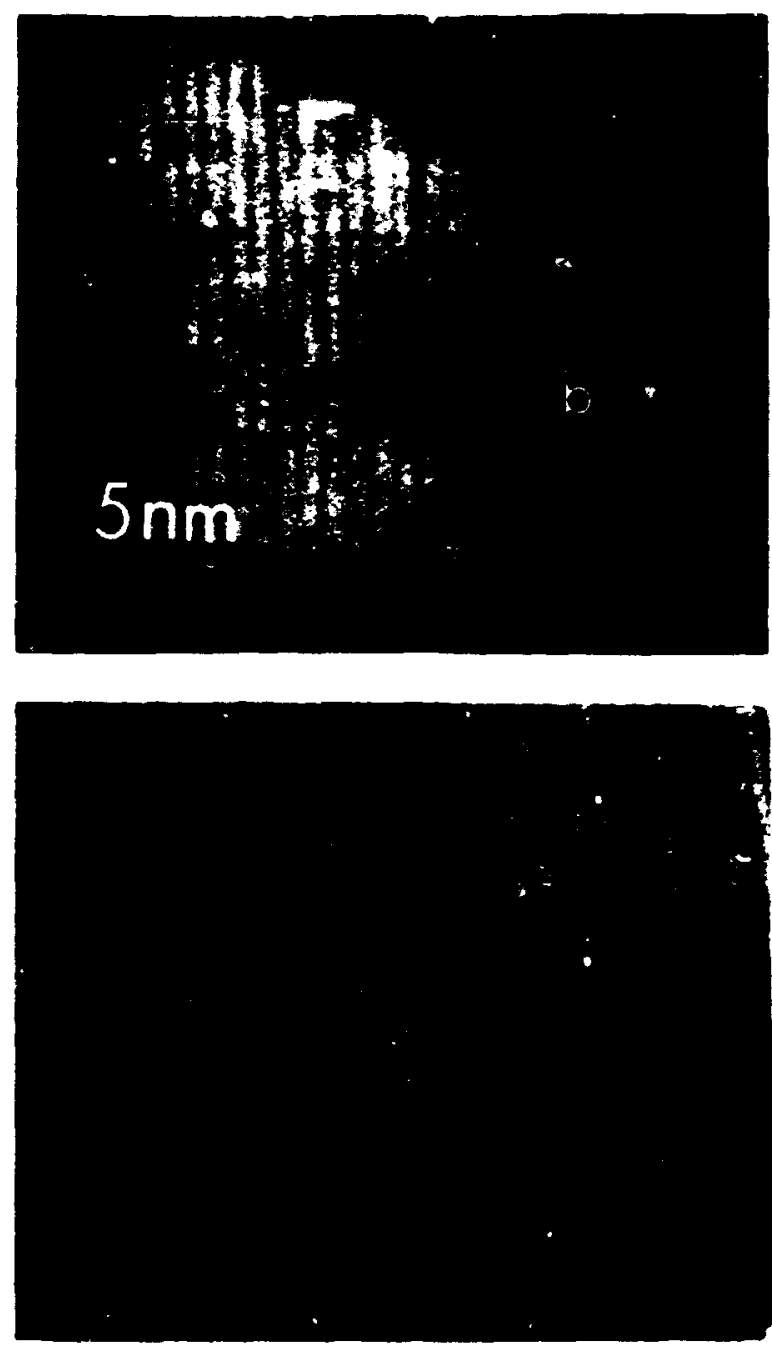

Fig. 6.11. Z-contrast images of a $13^{\circ}$-tilt boundary in $\mathrm{YBa}_{2} \mathrm{Cu}_{3} \mathrm{O}_{7-8}$. (a) The specimen has been tilted so that no channeling effects are produced in the grain on the right side of the micrograph. The uniform contrast seen in this grain and along the grain boundary indicates that the grain-boundary region is chemically indistinguishable from the bslk of the grain. (b) A higher magnification Image of the dislocation cores in this boundary. The specimen has been oriented so that electron channeling effects can be seen in both grains.

phases is not necessary to produce drastic reductions in critical currents. The disturbed region associated with the defects is the result 
of intrinsic structural relaxation of a chemically clean boundary. These zones are obstacles to curront flow. As the tilt angle increases, the number of defects increases linearly, further restricting the superconducting path. This behavior is modeled by the AmbegaukarBaratoff equation for the critical current density of a Josephson junction. ${ }^{3}$

1. Summary of paper to be published.

2. D. Dimos et al., Phys. Rev. Lett. 61, 219 (1988).

3. V. Ambegaokar and A. Baratoff, Phys. Rev. Lett. 10, 486 (1963); Phys. Rev. Lett. 11, 104(E) (1963).

\section{ORIENTED GROWTH OF $\mathrm{YBa}_{2} \mathrm{Cu}_{3} \mathrm{O}_{x}$ ON MgO BY ION SPUTTERING'}

\section{F. Chisholm, J. P. Doyle, and R. A. Roy ${ }^{2}$}

The superconductivity properties of $\mathrm{YBa}_{2} \mathrm{Cu}_{3} \mathrm{O}_{x}$ hase been found to be extremely anisotropic, so that for many applications alignment of the ceramic is desirable. In addition, a key factor for microelectronic applications is their ability to be fabricated into thin films. Epitaxial growth of $\mathrm{YBa}_{2} \mathrm{Cu}_{3} \mathrm{O}_{2}$ has been demonstrated on a number of substrates, all with similar lattice parameters and with structures similar to the perovskite structure of the high-temperature $\mathrm{CuO}$ superconductors. A!l the presently known substrates for epitaxial growth of $\mathrm{YBa}_{2} \mathrm{Cu}_{3} \mathrm{O}_{x}$ offer good geometrical fit and strong chemical interaction.

In this study we found that oriented growth of the superconducting film could te achieved on $\mathrm{MgO}$, a substrate with poor lattice match and little chemical interaction with $\mathrm{YBa}_{2} \mathrm{Cu}_{3} \mathrm{O}_{\mathbf{x}}$, by ion beam sputter deposition. ${ }^{3}$ In addition, it was found that the orientation of the deposit is a function of deposition rate. For deposition rates below $2 \mathrm{~nm} / \mathrm{min}$, the films exhibit a (001) texture as shown in Fig. 6.12(a). When the deposition rate is between 2 and 4 $\mathrm{nm} / \mathrm{min}$, a mixture of (110) and (103) texture is observed as in Fig. 6.12(b). For deposition rates above $5 \mathrm{~nm} / \mathrm{min}$, a (100) texture is seen [Fig. 6.12(c)].

The production of highly oriented superconducting films on $\mathrm{MgO}$ opens up the possibility that other substrates, cheaper and less reactive than those presently being used, can be used to obtain oriented thin films. This would allow one to match the properties of the substrate (e.g., low dielectric constant in applications involving fast pulses) to a specific application and still obtain oriented films.

1. Summary of paper to be published.

2. IBM, T. J. Watson Research Center, Yorktown Heights, N. Y.

3. J. P. Doyle et al., Proceedings of the 35th AVS Symposium and Topical Conference on High-T $T_{C}$ Superconductors, Atlanta, Georgia, 1988.

\section{ANISOTROPIC RECRYSTALLIZATION OF ION-IMPLANTED $\mathrm{YBa}_{2} \mathrm{Cu}_{3} \mathrm{O}_{7-x}$}

\section{S. I. Pennycook, R. Feenstra, and M. F. Chisholm}

lon implantation doping of high- $T_{C}$ superconductors is of considerable interest for 


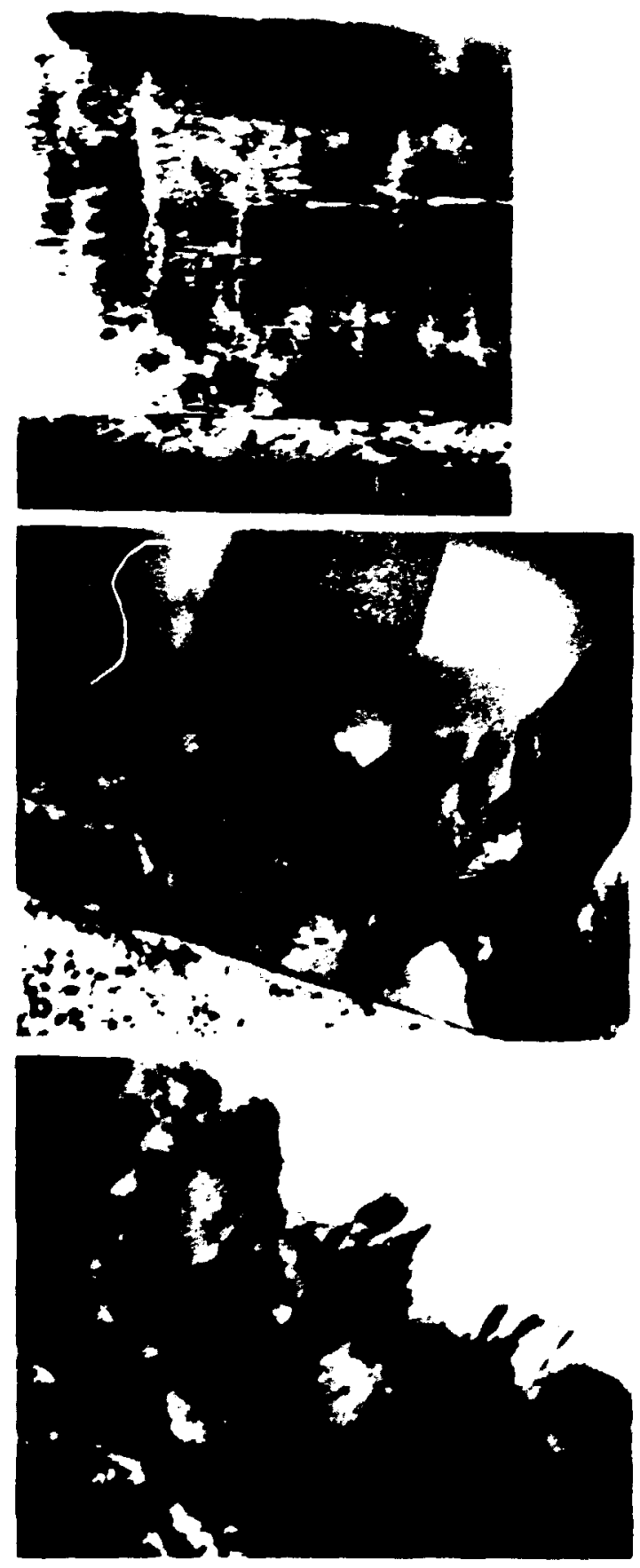

Fig. 6.12. Electron micrographs of thrce film/substrate interfaces viewed in cross section. (a) Film grown at $1.6 \mathrm{~nm} / \mathrm{min}$ showing columnar grains with their $c$ axis normal to the substrate surface. (b) Film grown at $3.8 \mathrm{~nm} / \mathrm{min}$. Grains with a [103] and [110] surface normal are identified. (c) Film grown at $5.4 \mathrm{~nm} / \mathrm{min}$ showing majority of the film is aligned with its aaxis normal to the surface. the potential modification of superconducting properties, particularly for thin-film applications. However, there are three major problems compared with the now standard procedures used for semiconductors: first, the extremely reactive nature of the material; second, the uncertainty in the thermodynamic stability of the phase at the low temperatures at which regrowth is desired; and third, the problem of the kinetics of a complex unit cell, which would suggest very anisotropic regrowth behavior. In the case of $\mathrm{YBa}_{2} \mathrm{Cu}_{3} \mathrm{O}_{7-x}$, growth along the $c$ direction would require ordering on the $12-\AA$ scale ahead of the growth front. Growth along the $a$ or $b$ directions would only entail ordering on a 4- $\AA$ scale ahead of the interface and would be expected to be much faster, ordering along the $c$ direction now being possible through fast interface diffusion.

Our experiments were designed to test this idea using superconducting thin films of mixed $a \perp$ and $c \perp$ orientation deposited on $\mathrm{KTaO}_{3}$, which were implanted with $\mathrm{O}^{+}\left(40 \mathrm{keV}, 1 \times 10^{16}\right.$ $\mathrm{cm}^{-2}$ ). Figure 6.13(a) is a cross-section image of the as-implanted film showing a sharp amorphous/crystalline interface, although the surface of the film has been lost on the left of the figure through ion milling. The a 1 grains tend to stand above the strface of the films as seen in the left side. After annealing in flowing $\mathrm{O}_{2}$ at $800^{\circ} \mathrm{C}$ for $20 \mathrm{~min}$, polycrystalline $\mathrm{YBa}_{2} \mathrm{Cu}_{3} \mathrm{O}_{7-x}$ is seen above the $c \perp$ grain on the left of Fig. 6.13(b). A sn:all region of buried amorphous phase is visible, indicating that the polycrystalline material nucleated at the 

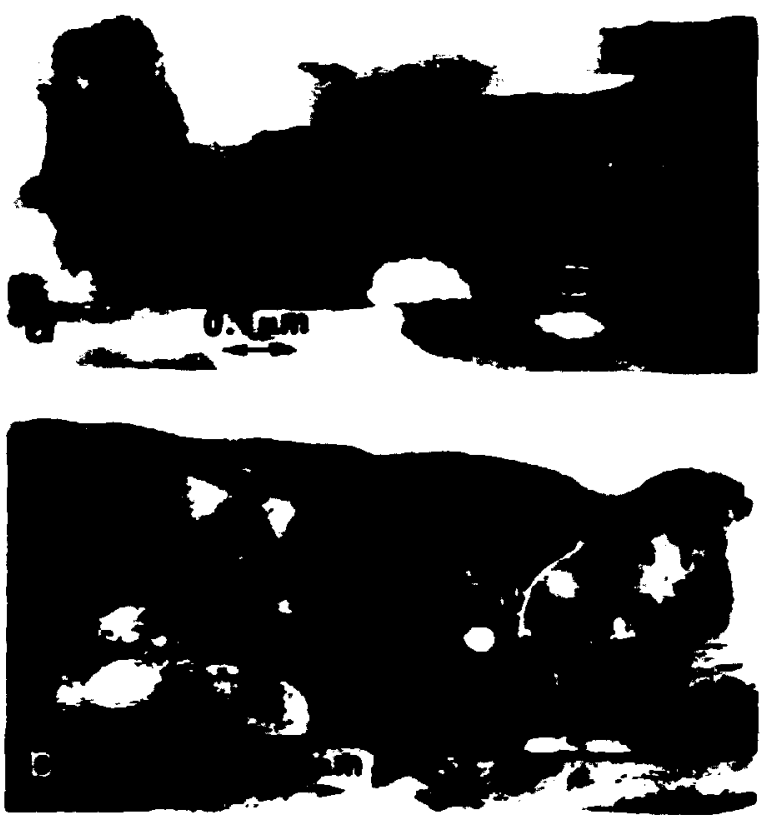

Fig. 6.13. Cross-section TEM images of $\mathrm{YBa}_{2} \mathrm{Cu}_{3} \mathrm{O}_{7 \cdot x}$ thin films on $\mathrm{KTaO}_{3}$ (a) after implantation with $\mathrm{O}^{+}\left(40 \mathrm{keV}, 1 \times 10^{16} \mathrm{~cm}^{-2}\right)$ showing amorphous layer and (b) after recrystallization at $800^{\circ} \mathrm{C}$ for $20 \mathrm{~min}$ showing epitaxial growth of $a \perp$ grains but not $c \perp$ grains.

surface. On further annealing at $850^{\circ} \mathrm{C}$, grain alignment leads to reasonably good ion channeling in the surface layer of the film. The $a \perp$ grain on the right of Fig. 6.13 is crystalline right to the surface. Checking thicker regions of film, where there was no possibility of the surface having been milled away, all $a \perp$ grains had recrystallized to the surface. Therefore, for ion implantation doping at low temperatures, it would seem promising to recrystallize along the $c$-layer planes, either by furnace annealing of $a \perp$ films or, perhaps, by a scanned CW laser annealing of $c \perp$ films.

1. Summary of paper to be published.
FORMATION OF DEFECT-FREE SHALLOW JUNCTIONS IN As ${ }^{+}$-IMPLANTED Si ${ }^{1}$

\section{K. El-Ghor, ${ }^{2}$ S. J. Pennycook, and R. A. Zuhr}

High dose $\mathrm{As}^{+}$implantation is widely used for source and drain formation in metal-oxidesemiconductor devices. In response to the push toward smaller devices requiring shallower junctions, rapid thermal arinealing (RTA) was successfully introduced as a means of removing the structural damage with minimum dopant diffusion. This high-temperature short-time anneal favors the high activation energy process (damage removal by $\mathrm{Si}$ self diffusion) over the process with tive lower activation energy (dopant diffusion). In the present work, we have shown that as the damage is brought closer to the surface a different annealing mechanism becomes important, allowing highquality junctions to be formed at processing temperatures of only $700^{\circ} \mathrm{C}$, significantly lower than any previously reported studies. ${ }^{3}$ The mechanism relies on the close proximity of the surface, which induces glide of the dislocation loops to the free surface under the action of image forces. This mechanism consequently becomes more effective as the implantation energy is reduced.

In this study, $\mathrm{Si}(100)$ samples were implanted at $17.5 \mathrm{keV}$ using molecular $\mathrm{As}_{2}^{+}$and from 2 to $8 \mathrm{keV}$ using the deceleration system used for ion beam deposition. Figure 6.14 compares furnace and rapid thermal annealing of a samp!c implanted at $17.5 \mathrm{keV}$ and a dose of $8.8 \times 10^{15} \mathrm{~cm}^{-2}$. In both the long-time (furnace) and short-time anneals, the end-of-range 

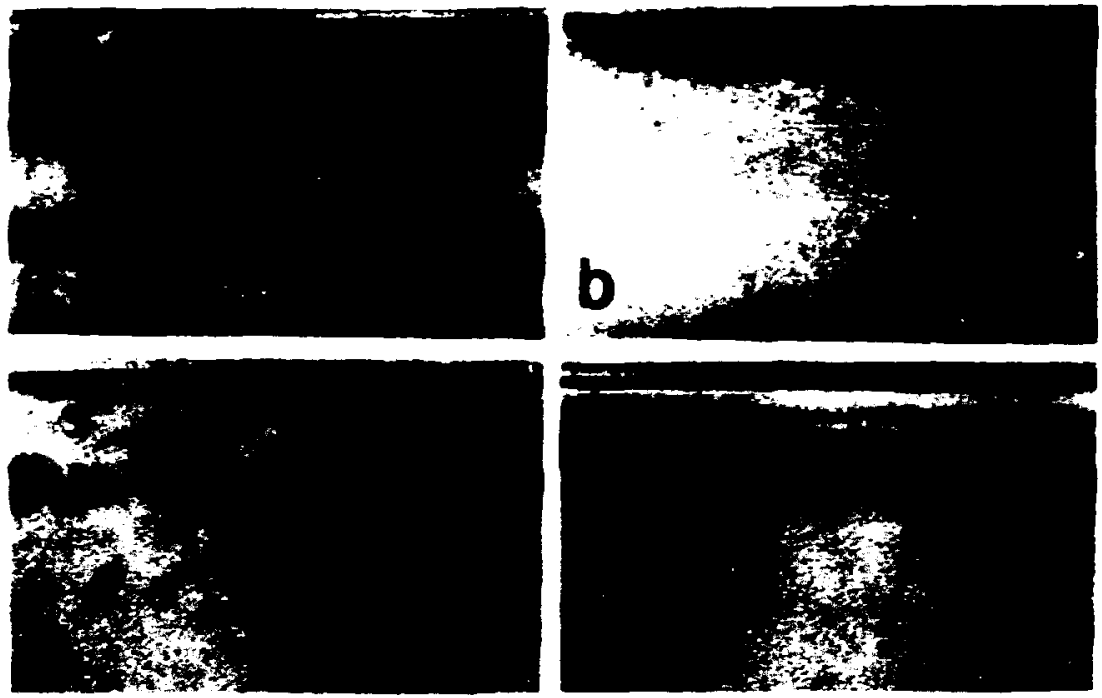

Fig. 6.14. Cross-sectional transmission electron micrographs of $\mathrm{As}^{+}$ (17.5 $\mathrm{keV}, 8.8 \times 10^{15} \mathrm{~cm}^{-2}$ ) implantation into $\mathrm{Si}(100)$ after furnace annealing at (a) $600^{\circ} \mathrm{C} / 30 \mathrm{~min}+800^{\circ} \mathrm{C} / 30 \mathrm{~min}$ and (b) $600^{\circ} \mathrm{C} / 30 \mathrm{~min}+$ $900^{\circ} \mathrm{C} / 30 \mathrm{~min}$ and after RTA at (c) $800^{\circ} \mathrm{C} / 40 \mathrm{~s}$ and (d) $900^{\circ} \mathrm{C} / 40 \mathrm{~s}$.

damage is present after $800^{\circ} \mathrm{C}$ annealing but absent after $900^{\circ} \mathrm{C}$ annealing. This behavior is inconsistent with a process involving diffusion of point defects but is quite characteristic of a process involving glide of dislocations to the surface. For samples implanted at $2 \mathrm{keV}$, complete damage removal was observed after annealing at $700^{\circ} \mathrm{C}$ for only $40 \mathrm{~s}$. The use of image forces allows defect-free shallow junctions to be formed at temperatures and times significantly lower than previously achieved.

1. Summary of paper to be published.

2. Oak Ridge Associated Universities postgraduate research participant.

3. E. Myers et al., Appl. Phys. Lett. 53, 228 (1988).

\section{DOPANT SEGREGATION DURING SOUD-PHASE-EPTTAXAL GROWTH'}

\section{S. J. Pennycook}

Dopant segregation during solid-phaseepitaxial (SPE) growth has been observed for low-melting-point dopants such as $\ln ^{2,3}$ but not for the high-melting-point dopants such as Sb, although in both cases it is expected to result from the higher solubility in amorphous $\mathrm{Si}$ compared with crystalline Si. Z-contrast scanning transmission electron microscopy (STEM) provides a sensitive means to study these effects with high spatial resolution, giving fundamental information on diffusion coefficients and solubilities in amorphous Si. Figure 6.15 shows a $Z$-contrast STEM image from $\mathrm{Sb}$ implanted (80 keV, $\left.1.5 \times 10^{16} \mathrm{~cm}^{-2}\right) \mathrm{Si}(100)$ 


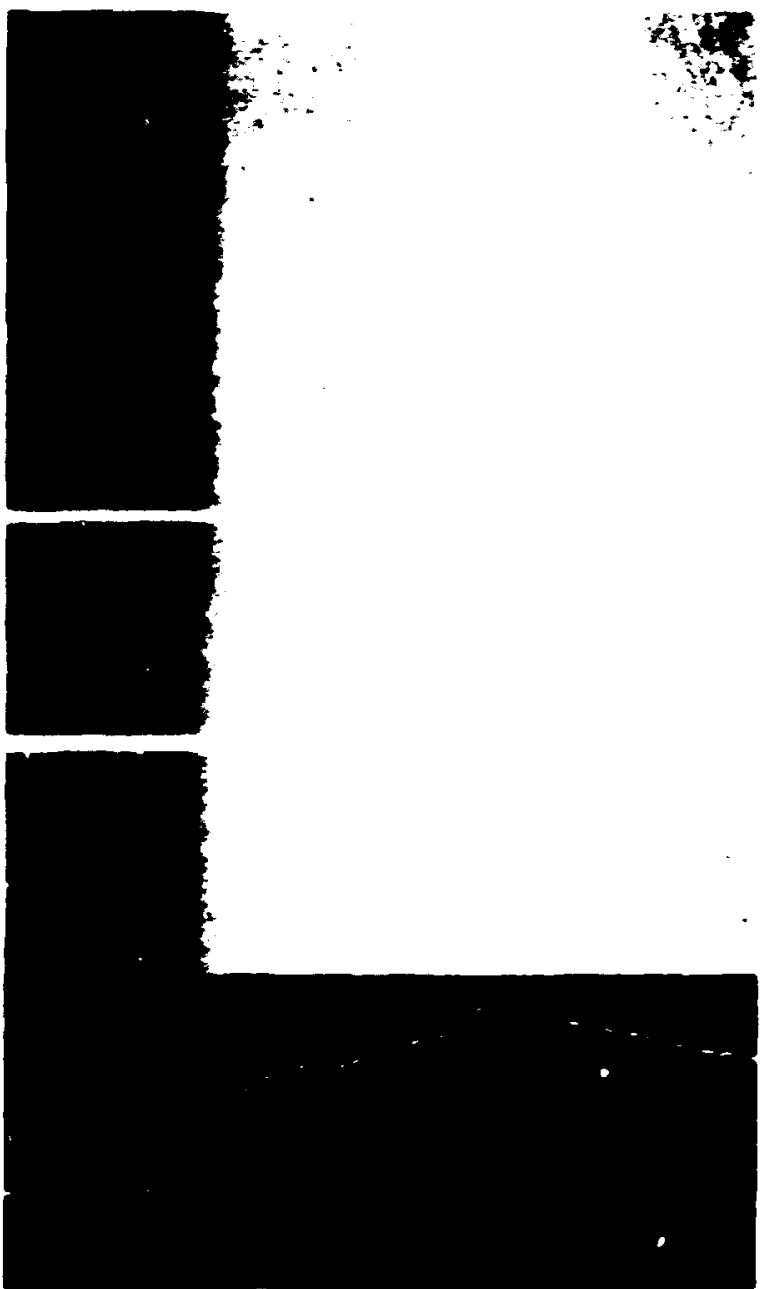

Fig. 6.15. Z-contrast STEM image and intensity profile showing segregation of $\mathrm{Sb}$ at the Si amorphous/crystalline interlace during SPE growth

regrown part way to the surface. A narrow band of high $\mathrm{Sb}$ concentration is seen segregated ahead of the amorphous/crystalline interface. The intensity profile wbtained by vertical averaging within the box area shows the interface to have just passed the peak Sb concentration. This high!y doped reguon is responsible for the nucleation of twins and the brcakdown of planar epilaxial growth. The width of the segregated peak allows the diffusion cocticient of $\mathrm{Sb}$ in amorphous $\mathrm{Si}$ to be estimated as $2.4 \times 10^{-15} \mathrm{~cm}^{2} \mathrm{~s}^{-1}$, almost nine orders of magnitude above tracer crystalline values. However, the same Sb concentration incorporated into crystalline Si by SPE growth would show a transient diffusion confficient only somewhat lower, approximately $10^{-16} \mathrm{~cm}^{2}$ $s^{-1}+$ We believe these effects to have a common origin; the enhanced diffusion in amorphous $\mathrm{Si}$ is caused by a high density of defects. a large fraction of which are incorporated into the crystalline Si during SPE growth, where subsequently they can cause the observed enhanced dopant deactivation.

1. Summary of paper to be published.

2. J. S. Williams and R. G. Elliman, Appl. Phys. Lett. 40, 266 (1982).

3. S. J. Pennycook, R. S. Culbertson, and S. D. Berger, Mat. Res. Soc. Symp. Proc. 100, 411 (1988).

4. S. J. Pennycook, R. J. Culbertson, and J. Narayan, 1. Mater. Res. 1, 476 (1986).

\section{DELOCALIZATION CORRECTIONS FOR ELECTRON CHANNELING ANALYSIS ${ }^{\prime}$}

\section{S. I. Pennycook}

In recent years terhniques have heen developed for the spatially resolved investigation of impurity lattice location using channeling effects in electron diffraction. 24 These methods are based on measuring and comparing the channeling effects seen in the clertron inducid $x$-ray fluorescence. An interesting effect occurs 
for ionization of the weaker bound shells, since the channeling effect can concentrate the flux to the extent that it is not uniform over the range of impact parameters contributing to the excitation. Then the channeling effect becomes dependent on the ionization energy of the shell and is weaker for the less bound shells. Experimentally, this delocalization effect can be measured and incorporated into the formulation in the form of a fractional excitation of the shell (a $c$ factor). ${ }^{3,4}$ This factor is not dependent on the actual channeling effect measured experimentally, which is sensitive to many other experimental parameters.

Figure 6.16 shows how this partial excitation arises. Highly localized interactions such

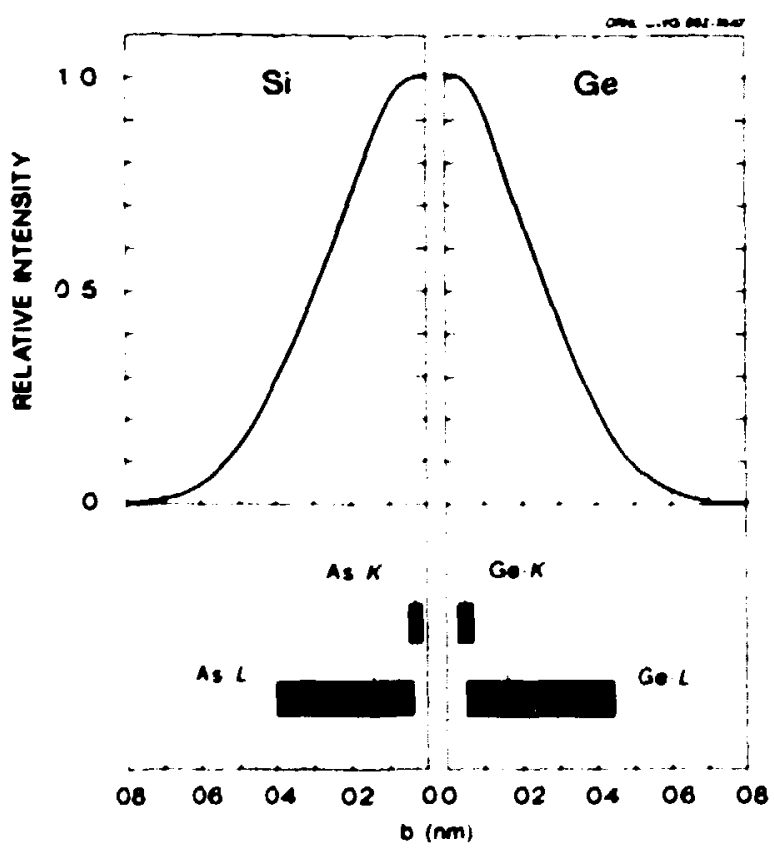

Fig. 6.16. Normalized distribution of electron flux about the atomic strings for $\langle 100\rangle$ axial channeling in $\mathrm{Si}$ and $\mathrm{Ge}$ with $100-\mathrm{keV}$ electrons, together with the average impact parameters (vertical lines) and the range of impact parameters (horizontal bars) contributing to various inner shell excitations. as the As- $K$ and Ce- $K$ shells feel the full fux enhancement close to the atomic strings, whereas less localized interactions such as the $K$-shell excitations are excited by electrons further from the string, resulting in less enhancement. Using a simple impact parameter description of the differential inelastic cross section and numerically integrating over the electron distribution, $c$ factors have been calculated in good agreernent with experimentally determined values. These results show that the effective excitation of the shell is given simply by the flux lying at a radius equal to the average (root mean square) impact parameter. This allows $c$ factors to be simply estimated for any channeling condition and greatly extends the applicability of the electron channeling methods.

1. Summary of paper: Ultramicroscopy 26, 239 (1988).

2. J. C. H. Spence and J. Tafto, J. Microsc. 130, 147 (1983).

3. S. J. Pennycook and J. Narayan, Phys. Reo. Lett. 54, 1543 (1985).

4. S. J. Pennycook, Scanning Microsc. 2, 21 (1988).

\section{X-RAY DIFFRACTION}

\section{NUCLEAR BRAGG DIFFRACTION FROM EPTTAXIALLY GROWN ${ }^{37} \mathrm{Fe}_{2} \mathrm{O}_{3}$}

\section{Z. Tischler and B. C. Larson}

$X$-ray monochromators using pure magnetic superlattice reflections from antiferromagnetic crystals enriched with Mössbauer nuclei offer 
the possibility of producing sub- $\mu \mathrm{eV}$ energy resolution $x$-ray beams from synchrotron sources. In this study, we have characterized $\sim 1-\mu \mathrm{m}$ epitaxial crystalline films of ${ }^{57} \mathrm{Fe}_{2} \mathrm{O}_{3}$ that were grown by chemical vapor transport on natural $\mathrm{Fe}_{2} \mathrm{O}_{3}$ single-crystal substrates as potential Mössbauer monocivomators.

The films were quantitatively characterized by absolute integrated intensity measurements of the allowed (666) reflection and the normally forbidden (777) antiferromagnetic superlattice reflection using collimated 14.4$\mathrm{KeV} \gamma$ rays from a Doppler-shifted ${ }^{5 ?}$ Co Mössbauer source. Since the (777) magnetic superlattice reflection is forbidden for all but nuclear resonant scaltering at the energy of the Mössbauer transition in ${ }^{57} \mathrm{Fe}$, its measurement provides a direct test of the nuclear resonant scattering propertics of the thin epitaxial layer.

Figure 6.17 shows on-resonance and offresonance rocking curves of the (777) reflection. The on-resonance rocking curve (at $+8.5 \mathrm{~mm} / \mathrm{s}$ )

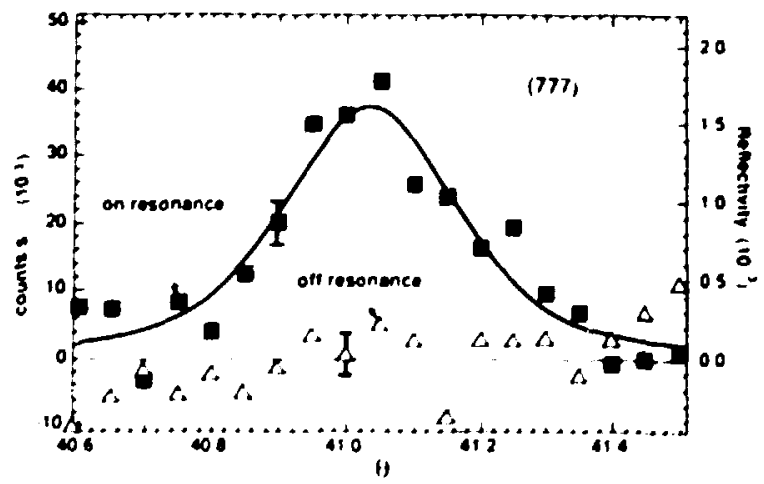

Fig. 6.17. On-resonance and off-resonance rocking curves of the (777) superlattice reflection. Counting time was $5000 \mathrm{~s} /$ point; incident beam was 23.2 counls/s. shou's a well-defined peak from the Mössbauer resonant photons, while the off-resonance rocking curve (at $-10 \mathrm{~mm} / \mathrm{s}$ ) shows no evidence for diffraction. The integrated intensity of this (77) superlattice reflection, as well as measurements of the allowed (666) reflection, was found to be very close to thre calculated values, indicating that thin epitaxial ${ }^{57} \mathrm{Fe}_{2} \mathrm{O}_{3}$ films such as these can be used for nuclear Bragg scattering experiments and that they are suitable for the construction of sub- $\mu \mathrm{eV}$ monochromators.

\section{X-RAY STUDY OF IN-PLANE EPITAXY OF $\mathrm{YBa}_{\mathbf{x}} \mathrm{Cu}_{3} \mathrm{O}_{\mathbf{x}}$ THIN FILMS}

\section{D. Budai, R. Feenstra, and L. A. Boatner}

$X$-ray diffraction measurements have been used to investigate the epitaxy and microstructure of $\mathrm{YBa}_{2} \mathrm{Cu}_{3} \mathrm{O}_{x}(Y 123)$ thin films produced by coevaporation on single-crystal (001) $\mathrm{SrTiO}_{3}$ and $\mathrm{KTaO}_{3}$ substrates. The epitaxial relationship between the film and the substrate is made particularly important by the large anisotropy of the superconducting properties of the cuprate oxides. The films consisted of orthorhombic Y123 domains oriented with either the $a$ axis ( $\perp \perp$ grains) or the $c$ axis ( $\_\perp$ grains) aligned with the substrate surface normal. Small but systematic differences were found between the lattice parameters measured for the $a_{1}$ and the $c 1$ domains within the same sample. These differences are consistent with shifts expected from strains induced by interactions with the slightly larger lattice parameters of the cubic substrates. 
An analysis of the diffraction line shapes revealed that the in-plane axes of the film and the substrate are aligned: that is, the threedimensional epitaxy. In the schematic shown in Fig-6.18, the heavy lines represent a projection of the $a$ and $b$ axes of the cubic substrate onto a reciprocal lattice plane perpendicular to the surface normal. X-ray scans obtained by rotating the sample about its surface normal 14 scans) reveal that, contrary to general belief, the $a$ and $b$ axes of the Y123 grains in the $C \perp$ orientation are not exactly aligned with the cubic substrate axes. Instead, as depicted in Fig. 6.18, it is the $<110\rangle$ in-plane directions of the $c \perp$ Y123 grains and the substrate that are parallel. We speculate that this alignment is associated with the formation of $(110)$ twins as a means of minimizing strains which develop

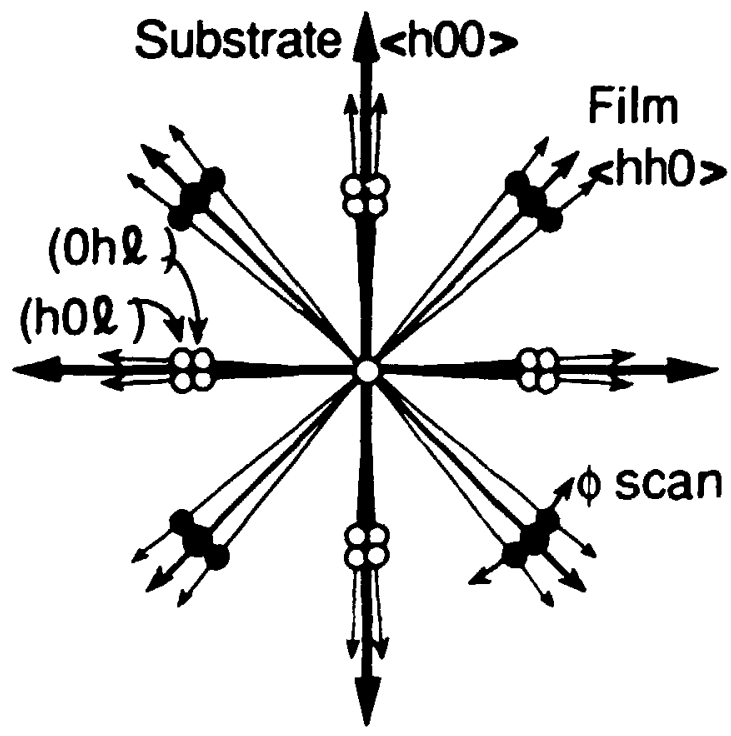

Fig. 6.18. Schematic drawing showing the in-plane epitaxial relationship between the Y123 axes for c: grains (fine lines) and the substrate $<h(0)>$ axes (heavy lines). within $c \perp$ grains while the sample is cooled (during processing) through the tetragonal-toorthorhombic phase transition $\left(650^{\circ} \mathrm{C}\right)$. The absence of $b_{\perp}$ domains implies that the $a_{\perp}$ grains do not contain the conventional (110) a-b twins. The observed differences between Y123 domains with different orientations indicate that the substrate plays a significant role in determining the thin-film microstructure.

1. Summary of paper: Physical Review B (in press).

\section{TMEE-RESOLVED X-RAY STUDY OF Ge AND Si AT NANOSECOND RESOLUTION DURING PULSED-LASER MELTING'}

\section{Z. Tischler, B. C. Larson, and D. M. Mills ${ }^{2}$}

Time-resolved $x$-ray scattering has been used to study interfacial overheating and undercooling in $\mathrm{Ge}$ and $\mathrm{Si}$ through measurements of the liquid-solid interface temperature and lattice temperature distribution during laserinduced melting and recrystallization. Overheating as a function of interface velocity on the (001) surface during laser melting was found to be $-5 \mathrm{~K} / \mathrm{m} / \mathrm{s}$ for $\mathrm{Ge}$ and $<2 \mathrm{~K} / \mathrm{m} / \mathrm{s}$ for $\mathrm{Si}$, while undercooling on the (001) surface during regrowth was found to be $-5 \mathrm{~K} / \mathrm{m} / \mathrm{s}$ for both Ge and Si. On the (111) surface, overheating during melting was found to be small as well; however, undercooling on the (111) surfaces was found to $\mathrm{t}:-20 \mathrm{~K} / \mathrm{m} / \mathrm{s}$ for $C_{C}$ and $\sim 12 \mathrm{~K} / \mathrm{m} / \mathrm{s}$ for Si, which are substantially larger than for the 
(001) surface. This asymmetry in the thermodynamic driving force associated with melting and regrowth indicates that crystallization on the (111) surface is significantly more difficult than melting. Since the interface velocities involved $(<10 \mathrm{~m} / \mathrm{s})$ are not near the collision limited interface velocity, nonlinear behavior is not expected and the asymmetry may indicate a breakdown of planar interface geometry for the (111) surface during melting. The gradients of the lattice temperature distributions were steeper than expected from heat flow calculations, suggesting a departure from linear heat flow and equilibrium thermal conductivity under these extreme conditions. This last aspect is presently under investigation.

1. Summary of papers: Appl. Phys. Lett. 52, 1785 (1988); Mat. Res. Soc. Symp. Proc. 100, 513 (1988)

2. CHESS and Comell University, Ithaca, N. Y. Present address: Argonne National Laboratory, Argonne, III.

\section{X-RAY MEASUREMENTS OF PHASON DISTORTION IN ION-IMPLANTED AI-Mn'}

\section{J. D. Budai}

The nature of structural defects present in icosahedral quasicrystals has been studied using high-resolution $x$-ray diffraction. Since a quasicrystal can be considered a nonperiodic arrangement of more than one unit cell, the resulting $x$-ray scattering should consist of delta-function Bragg peaks located at incommensurate, but well-defined, positions in reciprocal space. A rearrangement of the quasicrys. tal unit cells introduces disorder known as phason strain which can broaden or shift diffraction peaks. We have studied phason strain in quasicrystal samples produced by implanting Mn ions into (001) and (110) Al single crystals at elevated temperatures. An important characteristic of samples formed by this technique is that the icosahedral Al-Mn grains are found to align with respect to the original Al crystal, allowing us to obtain "single quasicrystal" $x$ ray measurements with a four-circle diffractometer.

We find that, for the (G,1) sample, the shifts in peak positions away from exact icosahedral symmetry can be quantitatively described by a $3 \times 3$ matrix representing a simple linear phason strain. Although such a simple matrix representation is not found for the (110) sample, an examination of the peak shifts allows us to reach several conclusions. First, we find that the phason distortions are not directly associated with a quasicoherency of icosahedral density waves with the $(002) \mathrm{Al}$ lattice planes. Second, like most defects, the phason strain depends strongly on the environment in which the quasicrystal grains are formed. In particular, we find that phason strain is more strongly influenced by the fcc matrix than by the orientation of the grains relative to the surface normal. Finally, wr find that the measured peak positions are inconsistent with the rational indexing scheme (i.e., crystalline model) proposed by Pauling. ${ }^{2}$

1. Summary of paper: Mater. Sci. Forum 2224, 369 (1987).

2. L. Pauling, Phys. Rev. Lett. 58, 365 (19:37). 


\section{ASYMPTOTIC DIFFUSE SCATTERNG FROM CO PRECIPITATES IN Cu}

\section{B. C. Larson and M. D. Galloway}

Huang diffuse scattering from the long-range distortion fields of defect clusters can be used ${ }^{1}$ to obtain information on the symmetry of defect clusters through a determination of the dipoleforce tensor. We have made measurements of $x$ ray diffuse scattering from spherical precipitates in aged $\mathrm{Cu}-1 \% \mathrm{Co}$, demonstrating that diffuse scattering in the asymptotic scattering region contains detailed information on the symmetry of defect clusters as well.

Scattering measurements vere made in the $\left(q_{x}, q_{y}, 0\right)$ plane as a function of the distance $q$ from the $(200)$ reciprocal lattice point, using a linear position-sensitive detector and a rotating anode $x$-ray source. The data are shown in the form of a contour plot in Fig. 6.19(a), where the scattering intensity has been scaled by $q^{4}$ in order to emphasize the features relating to the structure and symmetry of the clusters. The scattering is dominated by the large peak at $q$ = $(0.07,0,0) \AA^{-1}$ (resulting from the direct scattering from the precipitates) with subsidiary maxima on the opposite side of the $(200)$ reciprocal lattice point (genetsted by scattering from the distortions outside the precipitates); very low scattering is observed in the regions to the right and left of the (200) point. These data are to be compared with diffuse scattering calculations for spherical precipitates in an isotropic material shown in Fig. 6.19(b). These calculations were made using previously determined $^{2}$ size distributions and internal strain values for the precipitates and show the same general features as the measurements. Asymptotic scattering from dislocation loops is qualitatively differ'ent from Fig. 6.19 in that dilatation-free rotational strains generated by loops give rise to large scattering peaks in the
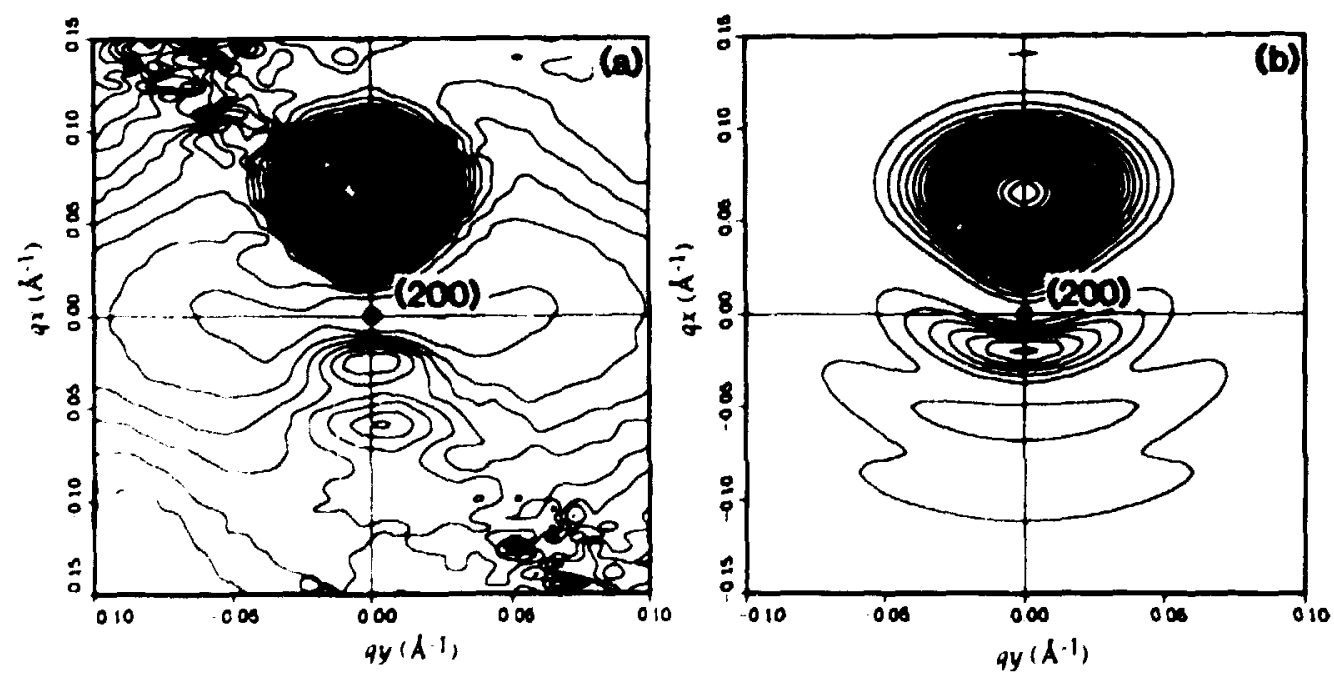

Fig. 6.19. Contour plots of diffuse scattering intensity $\times q^{4}$, (a) measured near (200) on Cu-1\% Co sample and (b) calculated using isotropic elasticity. 
regions to the left and right of the reciprocal lattice point.

1. B. C. Larson and W. Schmatz, Phys. Reo. B 10, 2307 (1974).

2. B. C. Larson et al., Mat. Res. Soc. Symp. Proc. 82, 3 (1987).

\section{VACANCY AND INTERSTTIIAL LOOPS IN UGHT-ION-IRRADIATED COPPER ${ }^{1}$}

\section{H. Maeta, ${ }^{2}$ B. C. Larson, T. P. Sjoreen,} D. K. Thomas, O.S. Oen, and I. D. Lewis ${ }^{3}$

The defect production process associated with high-energy displacement cascades in solids has been the subject of considerable theoretical and experimental interest in relation to irradiation damage, ion implantation, and surface modification of materials. As part of a program to investigate fundamental aspects of defect production in metals, we have used $x$-ray diffuse scattering to study light-ioninduced dislocation loops in copper. Singlecrystal copper samples were irradiated at room temperature with $\mathrm{He}, \mathrm{O}$, and $\mathrm{Si}$ ions using a 1.7MeV tandem accelerator. Multiple irradiations and varying ion energies were used to obtain surface layers with uniform damage energies of $2 \times 10^{12} \mathrm{MeV} / \mu \mathrm{m}$ for each ion spacie. The diffuse scattering measurements were made in the "asyrnptotic" scattering region near the (111) Bragg reflection, and they were analyzed in terms of vacancy and interstitial dislocation loops using numerically calculated scattering cross sections.
The number of defects retained ir the form of dislocation loops was found to be $0.7,1.4$, and $3.1 \%$ of the defects created for the $\mathrm{He}, \mathrm{O}$, and $\mathrm{Si}$ ion irradiations, respectively. Interstitial loops of $20-30-\AA$ radius were produced in each irradiation, and vacancy loops of $-10-\AA$ radius were found in the $\mathbf{O}$ and Si-irradiated samples. Essentially no vacancy loops were detected in the sample irradiated with He ions. Variations in the number of retained defects are apparently related to the effect of ion mass on the initial cascade structure, and these results may be compared to $\mathrm{Ni}$ ion irradiation of coppert for which $-6 \%$ of the defects were retained. The lack of vacancy loop formation during the $\mathrm{He}$ ion irradiation is believed caused by He-vacancy interactions resultirg in vacancy trapping or uncollapsed vacancy clusters.

1. Summary of paper: Characterization of the Structure anu Chemistry of Defects in Materials, ed. by B. C. Larson, M. Rühle, and D. N. Seidman, Materials Research Society, Pittsburgh, Pennsylvania (in press).

2. Guest Scientist from the Japan Atomic Energy Research Establishment, Tokai, Japan.

3. Present address: Paducah Gaseous Diffusion Plant, Paducah, Ky.

4. S. J. Zinkle, I. Nucl. Mater. 137, 73 (1985). 


\section{DEVELOPMENT OF MICROSTRUCTURES \\ IN Fe-15Ni-15Cr SINGLE-CRYSTAL ELECTRON BEAM WELDS ${ }^{1}$}
M. Rappaz,2 S. A. David, 3
J. M. Vitek, ${ }^{3}$ and L. A. Boatner

The grain structure of the fusion zone in a weld has a strong influence on its mechanical properties. Although several investigators have focused on the development of fusion-zone grain structure, a fundamental understanding of the development of this structure in welds is lacking. It is well established that initial solidification within the fusion zone occurs epitaxially at the partially melted grains along the fusion line. In welds, the average growth direction during solidification of the weld pool is approximately perpendicular to the solid-liquid interface and parallel to the heat flow direction. Crystallographic effects can influence grain growth by favoring growth along a preferred crystallographic direction; namely, the easy growth direction. Consequently, the grains that are oriented with their easy growth directions aligned parallel to the heat flow direction grow preferentially with respect to those with other orientations. Hence, the overall microstructure of the fusion zone is controlled by the interplay between the shape of the weld pool and the growth crystallography.

By employing single-crystal specimens rather than normal polycrystalline material, it is possible to delineate clearly the effects of growth crystallography on the microstructure of welds. A detailed analysis of the microstructures produced in an autogenously welded single crystal of $\mathrm{Fe}-15 \mathrm{Ni}-15 \mathrm{Cr}$ was performed in order to investigate the relationship between growth crystallography and solidification behavior. Electron beam welds were made at various speeds on the (001) surface of single crystals, in either the [100] or [110] directions. A geometrical analysis was carried out in order to relate the dendrite growth velocities in the three $<100\rangle$ directions to the weld velocities for the different crystallographic orientations examined. From this analysis, the preferred dendrite trunk directions were determined as a function of the solidification front orientation based upon a minimum velocity or minimum undercooling criterion. A thorough examination of the weld microstructures and a comparison with the geometrical relationships developed in this work permitted a three-dimensional reconstrurtion of the weld pool shape to be performed. In addition, the dendrite spacings were measured, and the variation in spacings as a function of growth velocity was compared with theoretical predictions. It was found that the range of velocities over which dendritic growth is expected agreed with the experimental findings and, furthermore, that the change in dendrite spacing with growth velocity varied as predicted by theory. These results clearly demonstrate the effect of crystallography on the microstructural development during weld pool solidification. The results also show that 
the resultant microstructures and pool shapes can be explained by geometrical analysis in conjunction with existing solidification models.

1. Summary of paper: Metallurgical Transactions (in press).

2. Guest scientist from Ecole Polytechnique Fédérale de Lausanne, Lausanne, Switzerland.

3. Metals and Ceramics Division, ORNL.

\section{THE CRYSTAL STRUCTURE OF Ce..9 $\mathrm{U}_{0.1} \mathrm{PO}_{4}{ }^{1}$}

D. F. Mullica, 2 E. L. Sappenfiela,

G. A. Wilson, ${ }^{2}$ and L. A. Boalner

The geological and commercial importance of natural monazite and the potential application of synthetic analogs of this mineral to nuclear waste disposal have stimulated a number of investigations of its morphological and structural properties. These studies include structural refinements of pure synthetic lanthanide orthophosphates as well as a more recent study of a mixed $1: 1 \mathrm{Gd} / \mathrm{YbPO}_{4}$ system. Investigations of the mixed orthophosphates are possible since solid solutions of these materials can be synthesized over a wide range of rare-earth compositions. The first complete structural analysis of a mixed lanthanide- actinide orthophosphate has been carried out. The crystal structure of cerium-uranium orthophosphate $\left(\mathrm{Ce}_{0.9} \mathrm{U}_{0.1} \mathrm{PO}_{4}\right)$ was determined by means of single-crystal $x$-ray diffraction techniques. The mixed compound crystallizes in the monoclinic space group $P 2_{1} / n$ (No. 14) $[a=6.796(2), b=7.026(2), c=6.478 \AA, \beta=$ 103.52(2); and $Z=41$. The lattice constants were obtained from powder diffraction data, $F(30)=37(0.016,50)$ and $M(20)=36.2$. A final least-squares, full-matrix refinement for $\mathbf{5 4 0}$ unique reflections yielded $R=0.055$ and $R_{w}=$ 0.054. The $C e$ and $U$ atoms share the same general site with refined occupancies of 0.9 and 0.1 , respectively, and are nine-coordinated to oxygen atoms. The geometric polyhedron is best described as a pentagonal interpenetrating tetrahedral polyhedron. Selected bond distances and angles are presented. These results bear on the question of incorporating radioactive cations in the chemically stable lanthanide orthophosphate lattice for the purpose of synthesizing nuclear waste forms.

1. Summary of paper: Lanthanide and Actinide Research (in press).

2. Baylor University, Waco, Tex. 


\section{Publications and Papers}

\section{JOURNAL ARTICLES}

C. Y. Allison, C. B. Finch, M. D. Foegelle, and F. A. Modine, "Low-Temperature Electrical Resistivity of Transition-Metal Carbides," Solid State Commun. 68, 387 (1988).

B. Alzyab, C. H. Perry, C. Zahopoulos, O. A. Pringle, and R. M. Nicklow, "High-Presiure Neutron Scattering Studies of Graphite and Graphite-SbCl 5 (Stage-Two)," Phys. Reo. B 38, 1544 (1988).

J. H. An, A. M. Fernandez, G. D. Wignall, and L. H. Sperling, "Development of Multiphase Morphology in Poly(Cross-Butadiene)-Interpoly(Crcss-Styrene) Interpenetrating Polymer Networks by SmallAngle Neutron Scattering," Polym. Mater. Sci. Eng. 56, 541 (1987).

Paul R. Ashley, William S. C. Chang, Chris J. Buchal, and Darrell K. Thomas, "Guided Wave Modulators in Ti Ion-Implanted $\mathrm{LiNbO}_{3}$ Waveguides," I. Lightwave Technol. 7, 855 (1989).

M. J. Aziz and Theodore Kaplan, "Continuous Growth Model for Interface Motion During Alloy Solidification," Acta Metall. 36, 2335 (1988).

C. Ballesteros, R. Gonzalez, and Y. Chen, "Optical and Transmission Electron Microscopy Characterization of Metal Precipitates in Doped Thermochemically Reduced Magnesium Oxide," Phys. Rev. B 37, 8008 (1988).

C. Ballesteros, R. Gonzalez, S. J. Pennycook, and Y. Chen, "Optical and Analytical Transmission Electron Microssopy Studies of Thermochemically Reduced MgO Crystals," Phys. Reu. B 38, 4231 (1988).

John H. Barrett, "Channeling Simulation in NizAl," Nucl. Instrum. and Methods Phys. Res. Sect. B 30, $546(1988)$

J. H. Barrett, "Channeling Measurement of Solute Vibration Amplitude in an Alloy," Phys. Rer. B 38, 5069 (1988).

F. S. Bates, M. Muthukumar, G. D. Wignall, and L. J. Fetters, "Thermodynamics of Isotopic Polymer Mixturcs: Significance of Local Structural Symmetry," J. Chem. Phys. 89, 535 (1988).

F. S. Bates, S. B. Dierker, and G. D. Wignall, "Phase Behavior of Amorphous Binary Mixtures of Perteuterated and Normal 1,4-Polybutadienes," Macromol. 19, 1938 (1986).

F. S. Bates, L. J. Fetters, and G. D. Wignall, "Thermodynamirs of Isotopic Polymer Mixtures: Po!y(Vinylethylene) and Poly(Ethylethylene)," Macromol. 21, 1086 (1988) 
J. B. Bates, Y. T. Chu, and W. T. Stribling, "Surface Topography and Impedance of Metal-Electrolyte Interfaces," Phys. Rev. Lett. 60, 627 (1988).

J. B. Bates and J. C. Wang, "Dielectric Response of lonic Conductors," Solid State lonics 28-30, 115 (1988).

J. B. Bates and Y. T. Chu, "Surface Topography and Electrical Response of Metal-Electrolyte Interfaces," Solid State Ionics 28-30, 1388 (1988).

D. P. Bellanger, V. Jaccarino, A. R. King, and R. Nicklow, "Neutron Scattering Obங்̀rvations of Extreme Critical Slowing Down in a d=3 Random Field System," Phys. Rev. Lett. 59,930 (1987).

R. B. Besson, S. P. Withrow, and S. J. Pennycook, "Formation of an Al $\mathbf{M n}$ Precipitate in Aluminum Annealed After Implantation with 3.5 Atomic Percent Manganese," Mater. Lett. 6, 93 (1988).

D. Bhattacharyya, K. Tano, G. J. Bunick, E. C. Uberbacher, W. D. Behnke, and S. Mitra, "Rapid, LargeScale Purification and Characterization of 'Ada Protein' ( $\mathrm{O}^{6}$-Methylguanine-DNA Methyltransferase) of E. Coli," Nuc. Acids Res. 16, 6397 (1988).

J. A. Blackman and J. F. Cooke, "Inelastic Electron Scattering and Electron-Hole Excitations in Iron and Nickel," J. Phys. (France) 49, C8-57 (1988).

J. D. Budai, "X-Ray Measurements of Phason Distortion in Ion-Implanted Al-Mn," Muter. Sci. Forum 2224, 369 (1987).

S. J. Burkitt, R. H. Ottewill, J. B. Hayter, and B. T. Ingram, "Small-Angle Neutron Scattering Studies on Micellar Systems. Part 1. Ammonium Octanoate, Ammonium Decanoate, and Ammonium PerfluorOctanoate," Colloid and Polym. Sci. 265, 619 (1987).

S. J. Burkitt, R. H. Ottewill, J. B. Hayter, and B. T. Ingram, "Small-Angle Neutron Scattering Studies on Micellar Systems. Part 2. Mixed Systems of Ammonium Decanoate and Ammonium PerfluoroOctanoate," Colloid and Polym. Sci. 265, 628 (1987).

B. L. Burks, M. A. G. Fernandes, G. R. Satchler, D. J. Horen, F. E. Bertrand, J. L. Blankenship, J. L. C. Ford, Jr., E. E. Gross, D. C. Hensley, R. O. Sayer, D. Shapira, and T. P. Sjoreen, "Optical Model and Coupled-Channel Analyses of the Elastic and Inelastic Scattering of ${ }^{18} \mathrm{O}$ from ${ }^{28} \mathrm{Si}$ and $352 \mathrm{MeV}$," Phys. Rev. C 38, 1680 (1988).

J. W. Cable and R. M. Nicklow, "Polarized Neutron Studies of Forbidden Magnon Scattering in Gadolinium," I. Phys. (France) 49, C8-361 (1988).

J. W. Cable, G. Parette, and Y. Tsunoda, "Spin Correlations in $A u_{0.85} \mathrm{Fe}_{0.15}$," Phys. Rev. B 36, 8467 (1987).

J. W. Cable, R. M. Nicklow, and T. Tsunoda, Polarization-Analysis Study of the Atomic and Spin-Pair Correlations in $\mathrm{Ni}_{0.76} \mathrm{Mn}_{0.24}$," Phys. Rev. B 36, 5311 (1987).

J. W. Cable, "Magnetic Form Factor and Spin-Density Asphericity of NiCu," Phys. Rev, B 36, 8837 (1987).

J. W. Cable, "Magnetic Diffuse Scaltering," Mater. Sci. Forum 27/28, 181 (1988). 
J. W. Cable, H. R. Child, and Y. Nakai, "Atom-Pair Correlations in Fe-13.5\% V," Physica B 156 \& 157, 50 (1989).

L. S. Cain, G. J. Pogatshnik, and Y. Chen, "Optical Transitions in Neutron-Irradiated $\mathrm{MgAl}_{2} \mathrm{O}_{4}$ Spinel," Phys. Reo. B 37, 2645 (1988).

G. S. Canright, "Time-Dependent Screening in the Electron Gas," Phys. Reo. B 38, 1647 (1988).

C. Y. Chen, G. J. Pogatshnik, Y. Chen, and M. R. Kokta, "Optical and Electron Paramagnetic Resonance Studies of Fe Impurities in Yttrium Aluminum Camet Crystals," Phys. Rev. B 38, 3888 (1988).

Y. Chen, V. M. Orera, R. Gonzalez, and C. Ballesteros, "Opportunities and Problems in Wavelength Tunable Lasers Based on Vacancy Defects in Refractory Oxides," Cryst. Lattice Defects and Amorphous Mater. 14, 283 (1987).

Y. Chen and V. M. Orera, "F and $F^{+}$Luminescence in Thermochemically Reduced MgO Crystals," $I$. Lumin. 40-41, 325 (1988).

D. K. Christen, S. T. Sekula, J. T. Ellis, J. D. Lewis, and J. M. Williams, "Formation, Properties, and Jon Irradiation Effects of Hexagonal Structure MoN Thin Films," IEEE Trans. Magn. 23, 1014 (1987).

D. K. Christen, Y. C. Kim, S. T. Sekula, J. R. Thompson, J. Brynestad, B. C. Sales, L. A. Boatner, and J. D. Budai, "Anisotropies in the Magnetic Penetration Depth and Critical Current Density of $\mathrm{R}_{1} \mathrm{Ba}_{2} \mathrm{Cu}_{3} \mathrm{O}_{7-x}(\mathrm{R}=\mathrm{Y}$ or $\mathrm{Ho}$ ) Measured on Magnetically Aligned Powder Composites," IEEE Trans. Magn. 25, 2324 (1989).

Y. T. Chu, J. B. Bates, C. W. White, and G. C. Farlow, "Optical Dielectric Functions for Amorphous $\mathrm{Al}_{2} \mathrm{O}_{3}$ and $\gamma \mathrm{Al}_{2} \mathrm{O}_{3}$," I. Appl. Phys. 64, 3727 (1988).

Y. T. Chu, "The Constant Phase A .gle Impedance of the Finite Sierpinski Electrode," Solid State lonics 26, 299 (1988).

Thomas G. Consler, Edward C. Uberbacher, Gerard J. Bunick, Michael N. Liebman, and James C. Lee, "Domain Interaction in Rabbit Muscle Pyruvate Kinase. II. Small-Angle Neutron Scattering and Computer Simulation," I. Biol. Chem. 263, 2794 (1988).

J. F. Cooke, S. H. Liu, and A. J. Liu, "Paramagnetic Form Factors for Cubic Itinerant Electron Systems," Phys. Reo. B 37, 289 (1988).

J. F. Cooke and J. A. Blackman, "Magnetic Excitations in Transition-Metal Systems," I. Phys. (France) 49. C8-81 (1988).

B. Crist and G. D. Wignall, "Small-Angle Neutron Scattering Studies of Chain Conformation in Hydrogenated Polybutadiene," I. Appl. Crystallogr. 21, 701 (1988).

Phillip G. Cummins, Edwin Staples, John B. Hayter, and Jeffrey Penfold, "A Small-Angle Neutron Scattering Investigation of Rod-Like Micelles Aligned by Shear Flow," J. Chem. Soc. Faraday Trans. 183,2773 (1987).

L. S. Darken, "Photochemical Ionizatior. Spectroscopy in High-Purity Germanium," J. Appl. Phys. 65, 1118 (1989). 
N. J. Dudney and J. B. Bates, "Hydration of Sodium B-and B"-Alumina," J. Am. Ceram. Soc. 70,816 (1987).

N. J. Dudney, "Enhanced lonic Conductivity in Composite Electrolytes," Solid State lonics 28-30, 1065 (1988).

J. A. Edmond, R. F. Davis, S. P. Withrow, and K. L. More, "Ion Implantation in B-SiC: Effect of Channeling Direction and Criticai Energy for Amorphization," I. Mater. Res. 3, 321 (1988).

P. C. EkJund, G. D. Mahan, I. G. Spolar, J. M. Zhang, E. T. Arakawa, and D. M. Hoffman, "Resonant Raman Scattering in Metals at the Interband Absorption Threshold," Phys. Rev. B 37, 691 (1988).

J. E. Epperson, B. A. Loomis, J. Faber, Jr., J. S. Lin, and R. W. Hendricks, "The Decomposition of a Ni-12\% Al Alloy at $550^{\circ} \mathrm{C}$ Investigated by Small-Angle $X-R a y$ Scattering and Transmission Electron Spectroscopy," Metall. Trans. A 18, 2027 (1987).

J. E. Epperson, J. S. Lin, and P. D. Labarbe, "Thase Separztion in a Ni-12.5 at.\% Alloy at 302\%," J. Appl. Crystallogr. 21, 873 (1988).

D. Eres, D. B. Geohegan, D. H. Lowndes, and D. N. Mashburn, "ArF Laser Photochemical Deposition of Amorphous Silicon from Disilane: Spectroscopic Studies and Comparison with Thermal CVD," Appl. Surf. Sri. 36, 70 (1988).

D. Fathy, O. W. Holland, and C. W. White, "Formation of Epitaxial Layers of Ge on Si Substrates by Ge Implantation and Oxidation," Appl. Phys. Lett. 51, 1337 (1987).

R. Feenstra, L. A. Boatner, J. D. Budai, D. K. Christen, M. D. Galloway, and D. B. Poket, "Epitaxial Superconducting Thin Films of $\mathrm{YBa}_{2} \mathrm{Cu}_{3} \mathrm{O}_{7-x}$ on $\mathrm{KTaO}_{3}$ Single Ciystals," Appl. Phys. Lett. 54, 1063 (1989).

J. A. Fermandez-Baca, J. W. Lynn, J. J. Rhyne, and G. E. Fish, "Long Wavelength Spin-Wave Energies and Linewidths of the Amorphous Invar Alloy Fe $e_{100-x} B_{x}$," Phys. Rev. B 36, 8497 (1987)

J. A. Fernandez-Baca, J. W. Lynn, J. J. Rhyne, and G. E. Fish, "Low-Temperature Spin Waves in Amorphous Feg0-x $\mathrm{Ni}_{x} \mathrm{Zr}_{10}, "$ I. Appl. Phys. 63, 3749 (1988).

J. A. Femandez-Baca, J. J. Rhyne, R. W. Erwin, and G. E. Fish, "Neutron Srattering Study of the Magnetic Correlations of Iron-Rich Fe-Zr Glasses," I. Phys. (France) 49, C8-1207 (1988).

L. W. Fisher, S. M. Melpolder, J. M. O'Reilly, V. Ramakrishnan, and G. D. Wignall, "Neutron Scattering from Interfacially Polymerized Core-Shell Latexes," I. Colloid Inlerface Sci. 123, 24 (1988).

R. S. Fishman, "Adiabatic a.nd Isothermal Resistivities," Phys. Rev. B 39, 2994 (1989).

R. S. Fishman, "Perturt ative Results for the Short-Range-Order Parameter, Speciíc Heat, and Resistivity of Cranular Superconductors," Phys. Reo. B 38, 4437 (1988).

R. S. Fishman, "Constructing a Well-Defined Free Energy from the Harmonic Approximation for Josephson Junction Arrays," Phys. Rev. B 38, 11996 (1988). 
R. S. Fishman and G. D. Mahan, "Divergences in the Force-Balance Theory of Resistivity," Phys. Reo. B 39, 2990 (1988).

R. S. Fishman and J. A. Sauls, "Doublet Splitting and the Low Field Evolution of the Real Squashing Modes in Superfluid ${ }^{3} \mathrm{He}$ B," Phys. Reo. Lett. 61, 2871 (1988).

R. 'j. Fishman, "Fluctuations in Granular Superconductors," Phys. Rep. B 39, 7228 (1989).

E. Fogarassy, C. W. White, A. Slaoui, C. Fuchs, P. Siffert, and S. J. Pennycook, "Excimer-Laser-Induced Oxidation of Ion-Implanted Silicon," Appl. Phys. Lett. 53, 1720 (1988).

B. D. Gaulin, M. F. Collins, and T. E. Mason, "Critical Scattering from Paramagnetic CsMnBr3: An XY Antiferromagnet with Chiral Degeneracy," Physice 156/157, 244 (1989).

B. D. Gaulin, M. Hagen, and H. R. Child, "A Critical Scattering Study of the Helical Antiferromagnets Ho and Dy," J. Phys. (France) 49, C8-327 (1988).

D. B. Geohegan and J. G. Eden, " $\mathrm{Xe}_{2} \mathrm{Cl}$ and $\mathrm{K}_{2} \mathrm{~F}$ Excited State (4 ${ }^{2} \Gamma$ ) Absorption Spectra: Measurements of A.bsolute Cross Sections," Chem. Phys. Lett. 139, 519 (1987).

D. B. Geohegan and J. G. Eden, "Absorttion of $\mathrm{Kr}_{2} \mathrm{~F}\left(4^{2} \Gamma\right)$ in the Near Ultraviolet and Visible (335 $\leq \lambda \leq 600 \mathrm{~nm})$ : Comparison with $\mathrm{Kr}_{2}\left(1\left(\frac{1}{2} \mathrm{tu}\right)\right.$ Measurements," I. Chem. Phys. 89,3410 (1988).

D. B. Geohegan, D. N. Mashburn, R. J. Culbertson, S. J. Pennycook, J. D. Budai, R. E. Valiga, B. C. Sales, D. H. Lowndes, L. A. Boatner, E. Sonder, D. Eres, D. K. Christen, and W. H. Christie, Pulsed-Laser

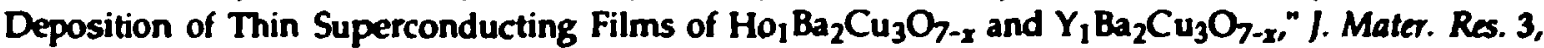
1169 (1988).

Doon Gibts, B. M. Ocko, D. M. Zehner, and S. G. J. Mochrie, "Absolute X-Ray Reflectivity Study of the Au(100) Surface," Phys. Reo. B 38, 7303 (1988)

H. Y. Cöksu, S. J. Pennycook, and L. M. Brown, "Thermoluminescence and Cathodoluminescence Studies of Calcite and MgO: Surface Defects and Heat Treatment," Nucl. Tracks and Radiat. Meas. 14, 365 (1988).

R. Gonzalez, Y. Chen, and M. M. Abraham, "Transmutation-Induced Tritium in $\mathrm{LiNbO}_{3}$ Single Crystals," Phys. Rev. B 37, 6433 (1988).

G. N. Greaves, S. H. Gurman, L. Gladden, C. Spense, P. Cox, B. C. Sales, L. A. Boatner, and R. N. Jenkins, "A Structural Basis for the Corrosion Resistance of Lead-Iron Phosphate Glasses: An X-Ray Absorption Spectroscopy Study," Philos. Mag. B 58, 271 (1988).

C. R. Gruzalski, D. M. Zehner, J. R. Noonan, H. L. Davis, R. A. DiDio, and K. Müller, "(1x1) Rippled Relaxation of (100) Transition-Metal Carbide Surfaces," I. Vac. Sci, and Technol. A 7, 2054 (1989).

M. Hagen, W. G. Stirling, and C. H. Lander, "The Temperature Dependence of the Low-Frequency Magnetic Excitations in USb," F'hys. Rev. B 37, 1846 (1988).

M. Hagen, B. D. Gaulin, and H. R. Child, "A Study of the Critical Scattering in the Helical Antiferromagnets Dy and Ho," Phys. Rev. B 38, 7365 (1988). 
M. Hagen and S. A. Higgins, "A Computer Simulation of the Spin Waves in a Competing Anisotropy Antiferromagnet: $\mathrm{K}_{2} \mathrm{Co}_{2} \mathrm{Fe}_{1-\mathrm{x}} \mathrm{F}_{4}, "$ I. Phys. C 21, 2701 (1988).

H. J. M. Hanley, G. C. Straty, C. J. Glinka, and J. B. Hayter, "Low-Q Neutron Diffraction from Supercooled D-Glycerol," Mol. Phys. 62, 1165 (1987).

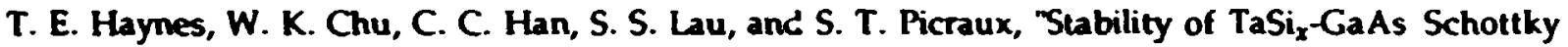
Barriers in Rapid Thermal Processing," Appl. Phys. Lett. 53, 2200 (1988).

John B. Hayter, "Colloidal Dispersion Structures Analyzed by Small-Angle Neutron Scattering," Phys. Today 40, S22 (1987).

John B. Hayter and H. A. Mook, "Discr te Thin-Film Multilayer Design for X-Ray and Neutron Supermirrors," J. Appl. Crystallogr. 21, 737 (1988).

J. B. Hayter, "SANS from Polymers and Colloids," Mater. Sci. Forum 27/28, 345 (1988).

A. W. Hewat, E. A. Hewat, J. Brynestad, H. A. Mook, and E. D. Specht, "Structure and Superstructure of the Superconductor $\mathrm{T}_{2} \mathrm{Ca}_{1} \mathrm{Ba}_{2} \mathrm{Cu}_{2} \mathrm{O}_{8}$ by Neutron and Electron Diffraction," Physica $\mathrm{C} 152,438$ (1988).

Wolfgang O. Hofer, "Plasma-Surface Interactions: The Role of Charged Particle Emission," J. Vac. Sci. and Technol. A 5, 2213 (1987).

O. W. Holland, D. Fathy, S. P. Withrow, and T. P. Sjoreen, "Oxidation of TiSi2: The Role of Implanted As and Its Behavior During Oxidation," I. Vac. Sci. and Technol. B 6, 569 (1988).

O. W. Holland and D. Fathy, "Annealing of Sb+-lon-Implanted Si," I. Appl. Phys. 63, 5326 (1988).

O. W. Holland, "Low-Temperature Oxidation of Implanted TiSi, Films," Appl. Phys. Lett. 52, 789 (1988).

O. W. Holland, C. W. White, and S. J. Pennycook, "Oxidation of Si Implanted with Nondopant, Metallic lons," J. Mater. Res. 3, 898 (1988).

O. W. Holland, M. K. El-Ghor, and C. W. White, "Damage Nucleation and Annealing in MeV-lonImplanted Si," Appl. Phys. Lett. 53, 1281 (1988).

O. W. Holland, "Interaction of MeV lons with Pre-existing Damage in Si: A New Ion Beam Annealing Mechanism," Appl. Phys. Lett. 54, 320 (1989).

O. W. Holland, "New Mechanism for Diffusion of lon-Implanted Boron in Si at High Concentratioi،," Appl. Phys. Lett. 54, 798 (1989).

L. S. Hung, W. Xia, D. B. Poker, M. Fernandes, K. Tao, S. S. Lau, and J. W. Mayer, "Temperature Dependence of Atomic Transport in lon Mixing" I. Appl. Phys. 64, 2354 (1988).

S. lida, B. C. Larson, and J. Z. Tischler, "Calculation of Diffuse Scallering near Bragg Reflections from Coherent Precipitates," J. Mater. Res. 3, 267 (1988).

H. Ito, T. P. Russell, and C. D. Wignall, "Interactions in Mixtures of Poly(Ethylene Oxide) and Poly(Methyl Methacrylate)," Macromol. 20, 2213 (1987). 
G. E. Jellison, Ir., "Four-Channel Polarimeter for Time-Resolved Ellipsometry," Opt. Lett. 12, 766 (1987).

G. E. Jellison, Jr., D. H. Lowndes, and J. W. Sharp, Time-Resolved Opticai Studies of OxideEncapsulated Silicon During Pulsed-Laser Melting," J. Mater. Res. 3, 498 (1988).

R. G. Jonasson, G. M. Bancroft, and L. A. Boatner, "Surface Reactions of Synthetic, End-Member Analogues of Monazite, Xenotime, and Rhabdophane and Evolution of Natural Waters," Geochim. Cosmochim. Acte 52, 767 (1987).

Susumu Katanw, Yukio Morii, Masashi lizumi, H. R. Child, and R. M. Nicklow, "Real-Time Neutron Diffraction Study of Crystallization Kinetics in Amorphous Feg8 ${ }_{13} \mathrm{Si}_{9}$ Alloy," J. Phys. Soc. Jpm. 56, 3196 (1987).

S. Katano, M. lizumi, R. M. Nicklow, and H. R. Child, Scaling in the Kinetics of the Order-Disorder Transition in NigMn," Phys. Reo. B 38, 2659 (1988).

S. Kawarazaki, N. Kunitomi, J. R. Arthur, R. M. Moon, W. G. Stirling, and K. A. McEwen, "Evidence for Nuclear Spin Order in Double HCP-Praseodymium by Neutron Diffraction," Phys. Reo. B 37, 5336 (1988).

Shuzo Kawarazaki and John Arthur, "Structure of the Magnetic Modulation in the Coupled ElectronNuclear Magnet PrCu, "J. Phys. Soc. Ipn. 57, 1077 (1988).

Theodore Kaplan, M. J. Aziz, and L. J. Gray, "Application of Onsager's Reciprocity Relations to Interface Motion During Phase Transformation," J. Chem. Phys. 90, 1133 (1989).

Eugene J. Kelly, D. E. Heatherly, C. E. Vallet, and C. W. White, "Application of Ion Implantation to the Study of Electrocatalysis. I. Chlorine Evolution at Ru-Implanted Titanism Electrodes," J. Electrochem. Soc. 134, 1667 (1987).

H. R. Kerchner, "Geometrically Large Flux-Line Pins," Cryogenics 29, 251 (1989).

W. F. Krupke, M. D. Shinn, T. A. Kirchoff, C. B. Finch, and L. A. Boatner, "Promethium-Doped Phosphate Glass Laser at 933 and 1098 nm," Appl. Phys. Lett. 51, 2186 (1987).

P. D. Labarbe, J. S. Lin, R. A. Weeks, and A. F. Wright, "Small-Angle X-Ray Scattering and Electron Magnetic Resonance Studies of the Nucleation-Crystallization Phenomena in Basalt Classes Prepared in Various Oxydo-Reduction Conditions," Phys. Chem. Glasses 29, 77 (1988).

P. D. Labarbe, J. S. Lin, and A. F. Wright, "Small-Angle Neutron Scattering Study of Phase Separation in the Binary Glass System $\mathrm{TiO}_{2} \mathrm{SiO}_{2}$," Phys. Chem. Glasses 29, 91 (1988).

Jalil Lachter, Leo G. Henry, Robert H. Bragg, and Stephen Spooner, "Pore Growth Kinetics in HeatTreated Glass-Like Carbons," I. Mater. Ras. 1, 832 (1986).

R. A. Langley, M. Lewis, and R. A. Zuhr, "Elastic Scattering of Protons by Beryllium," Nucl. Instrum. and Methods Phys. Res. Sect. B 29, 599 (1988).

C. W. Lantman, W. J. MacKnight, S. K. Sinha, D. C. Peiffer, R. D. Lundberg, and G. D. Wignall, "SANS from Sulfonate lonomer Solutions. 2. Polyelectrolyte Effects in Polar Solvents," Macromol. 21, 1344 (1988). 
B. C. Larson and F. W. Young, Jr., "X-Ray Diffuse Scattering Study of Irradiation-Induced Dislocation Loops in Copper," Phys. Status Solidi a 104, 273 (1987).

W.-K. Lee, A. S. Nowick, and L. A. Boatner, "Protonic Conduction and Nonstoichiometry in $\mathrm{KTaO}_{3}$ Crystals," Ado. Ceram. 23, 387 (1987).

W.-H. Li, J. W. Lynn, H. A. Mook, B. C. Sales, and Z. Fisk, "Long-Range Antiferromagnetic Order of the $\mathrm{Cu}$ in Oxygen-Deficient $\mathrm{RBa}_{2} \mathrm{Cu}_{3} \mathrm{O}_{6+x}, "$ Phys. Rev. B 37, 9844 (1988).

M. A. Linne, A. Klein, L H. Sperling, and G. D. Wignall, "On the Structure and Conformation of Polymer Chains in Latex Particles. I. Small-Angle Neutron Scattering Characterization of Polystyrene Latexes of Small Diameter," I. Macromol. Sci. B 27, 181 (1988).

M. A. Linne, A. Klein, G. A. Miller, L. H. Sperling, and G. D. Wignall, "Film Formation from Latex: Hindered Initial Interdiffusion of Constrained Polystyrene Chains Characterized by Small-Angle Neutron Scattering," I. Macromol. Sci. B 27, 217 (1988).

S. H. Liu, "Electron Polaron Effects in Heavy Electron Systems," Phys. Reo. B 37, 3542 (1988).

S. H. Liu, A. J. Liu, and J. F. Cooke, "Theoretical Paramagnetic Form Factors for HCP Transition Metals," Phys. Reo. B 36, 9521 (1987).

S. H. Liu, J. F. Cooke, and A. J. Liu, "A Fast and Accurate Method for the Calculation of Orbital Susceptibility and Form Factor of Paramagnetic Transition Metals," Physica B 149, 134 (1988).

S. H. Liu, "The Itinerant Resonating-Valence-Bond Model for Superconductivity," Chinese /. Phys. 26, S17 (1988).

S. H. Liu, "Magnetic Interaction Between Rare-Earth Moments in High-Temperature Superconductors $\mathrm{RBa}_{2} \mathrm{Cu}_{3} \mathrm{O}_{7-x}$," Phys. Rev. B 37, 7470 (1988).

S. H. Liu, "Pseudo-Excitons in Mixed-Valence Metals," Phys. Rev. B 39, 1403 (1989).

C.-K. Loong, J. Zarestky, C. Stassis, O. D. Masters, and R. M. Nicklow, "Lattice Dynamics of CePd3," Phys. Reo. B 38, 7365 (1988).

D. H. Lowndes, S. J. Pennycook, G. E. Jellison, Jr., S. P. Withrow, and D. N. Mashburn, "Solidification of Highly Undercooled Liquid Silicon Produced by Pulsed-Laser Melting of lon-Implanted Amorphous Silicon: Time-Resolved and Microstructural Studies," I. Mater. Res. 2, 648 (1987).

D. H. Lowndes, D. B. Geohegan, D. Eres, S. J. Pennycook, D. N. Mashburn, and G.E. Jellison, Jr., "PhotonControlled Fabrication of Amorphous Supurlattice Structures Using ArF (193-nm) Excimer Laser Photolysis," Appl. Phys. Lett. 51, 1868 (1988).

D. H. Lowndes, D. Eres, D. B. Geohegan, D. N. Mashburn, and S. J. Pennycook, "Low-Temperature Photon-Controlled Growth of Thin Films and Multilayered Structures," Appl. Surf. Sci. 36, 59 (1988).

A. Lusnikov, I. Ohana, M. S. Dresselhaus, and S. P. Withrow, "High-Dose Fe Implantation of HOPC at Elevated Temperature," J. Appl. Phys. 63, 195 (1988).

J. W. Lynn, W.-H. Li, H. A. Mook, B. C. Sales, and Z. Fisk, "Antiferromagnetic Order of the Cu in $\mathrm{RBa}_{2} \mathrm{Cu}_{3} \mathrm{O}_{6+x}, "$ I. Phys. (France) 49, C8-2153 (1988). 
J. W. Lynn, W.-H. Li, H. A. Mook, B. C. Sales, and Z. Fisk, Nature of the Magnetic Order of Cu in Oxygen-Deficient NdBa ${ }_{2} \mathrm{Cu}_{3} \mathrm{O}_{6+x}, "$ Phys. Reo. Lett. 60, 2781 (1988).

G. D. Mahan, H. S. Cole, Y. S. Liu, and H. L. Philipp, "Theory of Polymer Ablation," Appl. Phys. Lett. 53,2377 (1988).

G. D. Mahan, T ong-Wavelength Absorption of Cermets," Phys. Rev. B 36, 9500 (1988).

C. D. Mahan and Francisco Claro, "Nonlocal Theory of Thermal Conductivity," Phys. Reo. B 38, 1963 (1988).

G. D. Mahan and Ji-Wei Wu, "Plasmons and High-Temperature Superconductivity," Phys. Reo. B 39, 265 (1989).

G. D. Mahan, "Model for Minority-Carrier Band-Gap States in Semiconductors," Phys. Reo. B 38, 12436 (1988).

G. D. Mahan, "Figure of Merit for Thermoelectronics," I. Appl. Phys. 65, 1578 (1989).

C. F. Majkrzak, Doon Gibbs, P. Boni, Alan I. Goldman, J. Kwo, M. Hong, T. C. Hsieh, R. M. Fleming, D. B. McWhan, Y. Yafet, J. W. Cable, J. Bohr, H. Grimm, and C. L. Chien, "Magnetic Rare-Earth Superlattices," I. Appl. Phys. 63, 3447 (1988).

T. E. Mason, M. F. Collins, and B. D. Gaulin, "Experimental Confirmation of the Existence of a N?w Universality Class for Stacked Triangular Lattices," I. Phys. C 20, 1945 (1987).

J. C. McCallum, C. W. White, and L. A. Boatner, "Ion Implantation and Thermal Annealing of Single Crystals of the Type $\mathrm{YBa}_{2} \mathrm{Cu}_{3} \mathrm{O}_{2}$," Mater. Lett. 6, 374 (1988).

David E. Meltzer, F. J. Pinski, and G. M. Stocks, "Rare Gas Impurities in Alkali Metals: Relation to Optical Absnrption," Phys. Rev. B 37, 6011 (1988).

F. W. Meyer, C. C. Havener, S. H. Overbury, K. J. Reed, K. J. Snowdon, and D. M. Zehner, "Inner-Shell Processes as Probes of Multicharged Ion Neutralization at Surfaces," I. Phys. (Paris), 50, Colloq. C1, 263 (1989).

F. A. Modine and H. M. Hyatt, "New Varistor Material," J. Appl. Phys. 64, 4229 (1988).

H. A. Mook, "Reanalysis of the Condersate Fraction for "He from Neutron Scattering Data," Phys. Rev. B 37, 5806 (1988).

H. A. Mook, D. McK. Paul, B. C. Sales, L. A. Boatner, and L. Cussen, "Magnetic Ordering in $\mathrm{CdBa}_{2} \mathrm{Cu}_{3} \mathrm{O}_{6.14}$," Phys. Rev. B 38, 12008 (1988).

H. A. Mook, D. McK. Paul, and Stephen Hayden, "Temperature Dependence of the High-Energy Magnetic Excitations for Ni," Phys. Rev. B 38, 12058 (1988).

H. A. Mook and John B. Hayter, "Transmission Optical Device to Produce Intense Polarized Neutron Beams," Appl. Phys. Lelt. 53, 648 (1988).

R. M. Moon, "Magnetic Structures and Form Factors from Neutron Diffraction," Trans, ACA 23, 19 (1987). 
R. M. Moon and C. D. West, "The Advanced Neutron Source," Physica B 156/157, 524 (1989).

O. Moze and J. W. Cable, "Lattice Distortions and Atomic Short-Range Order in NiMo Alloys," Physica B 156/157, 724 (1989).

J. M. Mundenar, E. W. Plummer, L. G. Sneddon, A. P. Baddorf, D. M. Zehner, and G. R. Giuzalski, "Reply to 'Comment on Oxygen Chemisorption on Cu(110),"' Surf. Sci. 198, L309 (1988).

S. E. Nagler, R. F. Shannon, Jr., C. R. HarkJess, M. A. Singh, and R. M. Nicklow, "Time-Resolved X-Ray Scattering Study of Ordering and Coarsening in Cu, Au," Phys. Rev. Lett. 61,718 (1988).

Y. Nakai, A. Onodera, N. Kunitomi, M. Hagen, and R. M. Nicklow, "A Study of the Isochoric Frequency Shift of the Phonons in Al and Cu," Solid State Commun. 64, 783 (1987).

J. Narayan, V. N. Shukla, S. J. Lukasiewicg, N. Biunno, R. Singh, A. F. Schreiner, and S. J. Pennycooi, "Microstructure and Properties of $\mathrm{YBa}_{2} \mathrm{Cu}_{3} \mathrm{O}_{9.8}$ Superconductors with Transitions at 90 and Near 290 K," Appl. Phys. Lett. 51,940 (1988).

J. Narayan, N. Biunno, R. Singh, O. W. Holland, and O. Auciello, "Formation of Thin Superconducting Films by the Laser Processing Method," Appl. Phys. Lett. 51, 1845 (1987).

D. M. Newns, M. Rasolt, and P. C. Pattnaik "Theory of High- $T_{c}$ Superconductors Within an Anderson Lattice Model," Phys. Rev. B 38, 6513 (1988).

D. M. Newns, P. C. Pattnaik, M. Rasolt, and D. A. Papaconstantopoulos, "Theory of High- $T_{c}$ Superconductors Within a Realistic Anderson Lattice Model," Phys. Reo. B 38, 7033 (1988).

J. R. Noonan and H. L. Davis, "Mixture of Ordered Domains in the NiAl(111) Surface," Phys. Rev. Lett. 59,1714 (1987).

J. R. Noonan and H. L. Davis, "Domain Mixtures in the NiAl(111) Surface," I. Vac. Sci. and Technol. A 6, 722 (1988).

Ordean S. Oen, "Cross Sections for Atomic Displacements in Solids by Fast Positrons," Nucl. Instrum. and Methods Phys. Res. Sect. B 33, 744 (1988).

V. M. Orera and Y. Chen, "Optical Detection of a Hydrogen Complex Responsible for the F Phosphorescence in Thermochemically Reduced MgO Crystals," Phys. Rev. B 36, 6120 (1987).

V. M. Orera and Y. Chen, "Superhyperfine Structure of $\mathrm{H}^{2-}$ Ion in MgO," Phys. Rev. B 36, 5576 (1987).

V. M. Orera, M. L. Sanjuan, and M. M. Abraham, "Static and Dynamical Properties of $D^{\circ}$ j (Li) in MgO:Li Studied by EPR: Isotope Effects," J. Chem. Phys. 88, 2976 (1988).

Richard A. Page, Stephen Spooner, D. Lynn Johnson, and Wayne B. Sanderson, "Pore Evolution During Plasma Sintering of Alumina," I. Am. Ceram. Soc. 71, 1125 (1988).

J. L. Park and R. Conzalez, "Infrared Absorption Spectroscopy of Hydrogen and Deuterium in $\mathrm{CaO}$ and SrO Crystals," I. Mater. Res. 4, 224 (1989). 
D. Mck. Paul, H. A. Mook, A. W. Hewat, B. C. Sales, L. A. Boatner, J. R. Thompson, and Mark Mostoller, "Magnetic Ordering in the High-Temperature Superconductor $\mathrm{GdBa}_{2} \mathrm{Cu}_{3} \mathrm{O}$," Phys. Rev. B 37, 2341 (1988).

D. Mck. Paul, P. W. Mitchell, H. A. Mook, and H. Steigenberger, "Observation of Itinerant-Electron Effects on the Magnetic Excitations of Iron," Phys. Reo. B 38, 580 (1988).

D. McK. Paul, H. A. Mook, L. A. Boatner, B. C. Sales, J. O. Romey, and L. Cussen, "Magnetic Ordering in

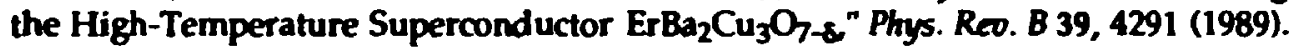

S. A. Payne, I. L. Chase, W. F. Krupke, and L. A. Boatner, "Excited State Absorption of $\mathrm{Sm}^{2+}$ in SrF and $\mathrm{SrCl}_{2}, "$ I. Chem. Phys. 88, 6751 (1988).

A. J. Pedraza, M. J. Godbole, E. A. Kenik, D. H. Lowndes, and J. R. Thompson, "Laser-Enhanced Adhesion of Copper Films to Sapphire Substrates," J. Vac. Sci. and Technol. A 6, 1763 (1988).

Antonio J. Pedraza, Mukund J. Godbole, Douglas H. Lowndes, and James R. Thompson, Jr., "Enhanced Metal-Ceramic Adhesion by Sequential Sputter Deposition and Pulsed-Laser Melting of Copper Films on Sapphire Substrates," I. Mater. Sci. 24, 115 (1989).

M. A. Pedrosa, R. Pareja, R. Gonzalez, and M. M. Abraham, "Analytical Electron Microscopic Studies and Positron Lifetime Measurements in a Doped MgO Crystal," J. Appl. Phys. 62, 429 (1987).

S. J. Pennycook, "Impurity Lattice and Sublattice Location by Electron Channeling," Scanning Microsc. 2, 21 (1988).

S. J. Pennycook, "Delocalization Corrections for Electron Channeling Analysis," Ultramicroscopy 26, 239 (1988).

S. J. Pennycook and L. A. Boatner, "Chemically Sensitive Structure Imaging with a Scanning Transmission Electron Mirroscope," Nalure 336, 565 (1988)

F. Perrot and Mark Rasolt, "Energetics and Lattice Relaxation of Light Impurities in Alumiluum," Phys. Rev. B 38, 2147 (1988).

G. J. Pogatshnik, Y. Chen, and B. D. Evans, "A Model of Lattice Defects in Sapphire," IEEE Trans. Nucl. Sci. NS-34, 1709 (1987).

G. J. Pogatshnik, Y. Cher, and B. D. Evans, "Enhancement of Color Center Luminescence in NeutronImadiated $\mathrm{Al}_{2} \mathrm{O}_{3}$," J. Lumın. 40/41, 315 (1988).

I. S. Poirot, W. K. Kot, N. M. Edelstein, M. M. Abraham, C. B. Finch, and L. A. Boatner, "Optical Study and Analysis of Put+ in Single Crystals of $\mathrm{ZrSiO}_{4}$," Phys. Rev. B 39, 6388 (1989).

I. Poirot, W. Kot, G. Shalimoff, N. Edelstein, M. M. Abraham, C. B. Finch, and L. A. Boatner, "Optical and EPR Investigations of $\mathrm{Np}^{4+}$ in Single Crystals of Doped ZrSiO, ," Phys. Rev. B 37, 3255 (1988).

D. B. Poker and D. K. Thoma:, "Effects of Different Ion-Implanted Dopant Species on the Solid-Phase Epitaxy of $\mathrm{LiNbO}_{3}$ Optical Waveguides," Nucl. Instrum. and Methods Phys. Res. Sect. B 39, 716 (1989). 
D. B. Poker and D. K. Thomas, "Solid-Phase Epitaxy of Ti-Implanted LiNbO3," I. Mater. Res. 4, 412 (1989).

O. A. Pringle, H. A. Mook, S. E. Lambert, and M. B. Maple, "Magnetism and Superconductivity in $\left(\mathrm{Er}_{0.16} \mathrm{Ho}_{0.84}\right) \mathrm{Rh}_{4} \mathrm{~B}_{4}$ " Phys. Reo. B 38, 8724 (1988).

J. Rankin, L. W. Hobbs, L. A. Boatner, and C. W. White, "An In Situ Annealing Study of LeadImplanted, Single-Crystal Calcium Titanate," Nucl. Instrum. and Methads Phys. Res. Sect. B 32, 28 (1988).

Mark Rasolt, "Functionals of the Ground-State Energy and the Band Gaps of Semiconductors and Insulators," Phys. Reo. B 36, 5041 (1987).

Mark Rasolt, Andrea Marco Malvezzi, and Heinz Kurz, "Plasmon-Phonon-Assisted Electron Hole Recombination in Silicon at High Laser Fluence," Appl. Phys. Lett. 51, 2208 (1987).

Mark Rasolt and F. Perrot, "Plasmon Broadening and Temperature Effects on the Plasmon-PhononAssisted Recombination in Silicon," Phys. Rev. B 37, 2630 (1988).

Mark Rasolt and F. Perrot, "Plasmon-Phonon-Assisted Recombinations in Germanium," Phys. Rev. B 38, 4130 (1988).

Mark Rasolt and D. J. W. Geldart, "Comment on 'Exact Electron Gas Response Functions at High Density,"' Phys. Reo. Lett. 60, 1983 (1988).

J. J. Rhyne, R. W. Erwin, J. A. Fernandez-Baca, and G. E. Fish, "Magnetic Correlations in Amorphous FeZr Alloys," I. Appl. Phys. 63, 4080 (1988).

R. Rojas, F. Claro, and R. Fuchs, "Nonlocal Response of a Small Coated Sphere," Phys. Rev. B 37, 6799 (1988).

H. E. Rorschach and J. B. Hayter, "Additional User Needs," Nucl. Instrum. and Methods Phys. Res. Sect. A 249, 123 (1986).

J. Roth, R. A. Zuhr, S. P. Withrow, and W. P. Eatherly, "Radiation Damage and Annealing in Graphite Implanted with $\mathrm{H}^{2+}$ and Mo+," I. Appl. Phys. 63, 2603 (1988).

T. P. Russell, J. S. Lin, S. Spooner, and G. D. Wignall, "Intercalibration of Small-Angle X-Ray and Neutron Scattering Data," I. Appl. Crystallogr. 21, 629 (1988).

T. P. Russell, H. Ito, and G. D. Wignall, "Neutron and X-Ray Scattering Studies of Semicrystalline Polymer Blends," Macromolecules 21, 1703 (1988).

B. Salce and L. A. Boatner, 'Thermal Conductivity of 'Pure' $\mathrm{KTaO}_{3}$," Ferroelectrics 70, 249 (1988).

B. C. Sales and L. A. Boatner, "Optical, Structural, and Chemical Characteristics of Lead-Indium and Lead-Scandium Phosphate Glasses," I. Am. Ceram. Soc, 70, 615 (1987).

B. C. Sales, J. O. Ramey, and L. A. Boatner, "Structural Alterations in the Amorphous-to-Crystalline Transformation of Lead Phosphate," Phys. Rev. Let1. 59, 1718 (1987). 
B. C. Sales, J. O. Ramey, L. A. Boatner, and J. C. McCallum, "Structural Inequivalence of the IonDamage-Produced Amorphous State and the Glass State in I.and Pyrophosphate," Phys. Reo. Lett. 62, 1138 (1989).

S. T. Sekula, J. Brynestad, D. K. Christen, J. R. Thompson, and Y. C. Kim, "Superconductivity, Critical Current Density, and Magnetic Properties of Fe-Substituted $\mathrm{Y}_{1} \mathrm{Ba}_{2}\left(\mathrm{Cu}_{1-x} \mathrm{Fe}_{2}\right)_{3} \mathrm{O}_{2}$ Materials," IEEE Trans. Magn. 25, 2266 (1989).

B. E. Sernelius, K.-F. Berggren, Z-C. Jin, I. Hamberg, and C. C. Granqvist, "Band-Gap Tailoring of ZnO by Means of Heavy Al Doping," Phys. Reo. B 37, 1024 (1988).

B. E. Sernelius, "Self-Energy Shifts in Heavily Doped, Polar Serniconductors," Phys. Reo. B 36, 4878 (1987).

B. E. Sernelius, "Polaron in n Dimensions," Phys. Reo. B 36, 9059 (1987).

B. E. Sernelius, "Dimensional Crossover for a Quasi-1-Dimensional Polaron," Phys. Reo. B 37, 7079 (1988).

K. W.-K. Shung and G. D. Mahan, "Photoemission Spectra and Band Structures of Simple Metals," Phys. Reo. B 38, 3856 (1988).

Kenneth W.-K. Shung, B. E. Sernelius, and G. D. Mahan, "Self-Energy Corrections in Photoemission of Na," Phys. Reo. B 36, 4499 (1987).

P. Sigmund, M. T. Robinson, M. I. Baskes, M. Hautala, F. Z. Cui, W. Eckstein, Y. Yamamura, S. Hosaka, T. Ishitani, V. I. Shulga, D. E. Harrison, Jr., I. R. Chakarov, D. S. Karpusov, E. Kawatoh, R. Shimizu, S. Valkealahti, R. Nieminen, G. Betz, W. Husinsky, M. H. Shapiro, M. Vicanek, and H. M. Urbassek, "Round Robin Computer Simulation of Ejection Probability in Sputtering," Nucl. Instrum. and Methods Phys. Res. Sect. B 36, 110 (1998).

A. Slaoui, E. Fogarassy, C. W. White, and P. Siffert, "Infrared Characterization of UV-Laser-Induced Silicon Oxide Films," Appl. Phys. Lett. 53, 1832 (1988).

H. G. Smith, R. Berliner, and J. D. Jorgensen, "Martensitic Transformation from BCC to 9R and FCC in Metallic Lithium," Physice B 156-157, 53 (1989).

K. J. Snowdon, C. C. Havener, F. W. Meyer, S. H. Overbury, D. M. Zehner, and W. Heiland, "Charge Exchange at Molecular-Orbital Pseudocrossings as an Important Mechanism for Nonkinetic-Electron Emission in Slow-Multicharged-Ion ( $\left.v<v_{0}\right)$ Metal-Surface Scattering," Phys. Reo. A 38, 2294 (1988).

K. J. Snowdon, C. C. Havener, F. W. Meyer, and D. M. Zehner, Technique for Determination of the Metastable Fraction of Multicharged lon Beams," Reo. Sci. Instrum. 59,902 (1988).

E. Sonder, J. R. Martinelli, R. A. Zuhr, and R. A. Weeks, "The Use of Ion Beam Analysis for Measuring lon Transport in Oxides," Cryst. Lattice Defects and Amorphous Mater. 15, 277 (1987).

E. Sonder, R. A. Zuhr, and R. E. Valiga, "Annealing of Damage and Stability of Implanted Ions in ZnO Crystals," I. Appl. Phys. 64, 1140 (1988). 
L. H. Sperling, A. Klein, Se-In Yang, and G. D. Wignall, "Development of Supermolecular Structure in Polystyrene Latexes," Polym. Mater. Sci. Eng. 58, 1109 (1988).

J. D. Stark, R. A. Weeks, G. Whichard, D. L. Kinser, and R. A. Zuhr, The Effects of lon Implantation on Optical Spectra of $\mathrm{SiO}_{2}$ Glass," I. Non-Cryst. Solids 95/96, 685 (1987).

James K. Stoops, Salih J. Wakil, Edward C. Uberbacher, and Gerard J. Bunick, "Small-Angle Neutron Scattering and Electron Microscope Studies of the Chicken Liver Fatty Acid Synthase," J. Biol. Chem. 262, 10246 (1987).

Shoji Suehiro, R. W. Hendricks, J. S. Lin, T. Kyu, P. Young, and R. S. Stein, "A Dynamic Small-Angle Scattering System for the Study of Materials Deformation and Relaxation," I. Polym. Sci. Polym. Phys. Ed. 26, 745 (1988).

Zlatko Tesanovic and Mark Rasolt, "New Type of Superconductivity in Very High Magnetic Fields," Phys. Reo. B 39, 2718 (1989).

J. R. Thompson, D. K. Christtn, S. T. Sekula, J. Brymestad, and Y. C. Kim, Magnetization Studies of the High-T ${ }_{c}$ Compound $\mathrm{Y}_{1} \mathrm{Ba}_{2} \mathrm{Cu}_{3} \mathrm{O}_{2} "$ J. Mater. Res. 2, 779 (1987).

J. R. Thompson, B. C. Sales, Y. C. Kim, S. T. Sekula, L. A. Boatner, J. Brynestad, and D. K. Christen, "Magnetic Properties of Oxygen-Reduced, Rare-Earth-Based $\mathrm{R}_{1} \mathrm{Ba}_{2} \mathrm{Cu}_{3} \mathrm{O}_{7-x}$ Compounds with $\mathrm{R}=\mathrm{Gd}$, Dy, and Ho," Phys. Reo. B 37, 9395 (1988).

J. Z. Tischler, J. D. Budai, G. E. Ice, and A. Habenschuss, "Multiple Scattering and the 200 Reflection in Silicon and Germanium," Acte Crystallogr. Sect. A 44, 22 (1988).

J. Z. Tischler, B. C. Larson, and D. M. Mills, Time-Resolved X-Ray Study of Ge During Pulsed-Laser Melting," Appl. Phys. Lett. 52, 1785 (1988).

J. Toulouse, X. M. Wang, and L. A. Buatner, "Elastic Anomalies at the Cubic-Tetragonal Transition in KTN'," Solid State Commun. 68, 353 (1988).

Roberto Triolo, Eugenio Caponetti, and Stephen Spooner, "Analysis of Small-Angle Scattering Patterns from Precipitating Alloys," Phys. Rev. B 39, 4588 (1989).

K. N. Trohidou, J. A. Blackman, and J. F. Cooke, "Spin Orbital Contributions to the Dynamical Structure Factors of Paramagnetic Transition Metals," Phys. Reo. B 37, 8154 (1988).

Y. Tsunoda, N. Kunitomi, and R. M. Nicklow, "Magnetic Structure of ץFe Precipitates in a Cu Matrix," J. Phys. F 17, 2447 (1987).

Edward C. Uberbacher and Cerard J. Bunick, "Resolving the Nucleosome Structure Controversy. A Basis for Comparison of Nucleosome Structures," Comments on Mol. Cell. Biophys. 4, 339 (1988).

E. C. Uberbacher, J. M. Harp, and G. J. Bunick, "DNA Sequence Patterns in Precisely Positioned Nucleosome," J. Biomolec. Struct. Dynamics 6, 105 (1988).

C. E. Vallet, S. E. Borns, J. S. Hendrickson, and C. W. White, "DSA RuO $/ \mathrm{TiO}_{2}$ Electroje Modeled by Ion Implantation: An In Situ Characterization by Photoacoustic and Photocurrent Spectroscopy," I. Electrochem. Soc. 135, 387 (1988). 
G. Vignale, "Quantum Electron Glass," Phys. Rao. B 36, 8192 (1987).

G. Vignale and W. Hanke, "Altshuler-Aronov Anomalies in the Density of States of Substitutional Alloys," Phys. Reo. B 36, 2924 (1987).

G. Vignale, Mark Rasolt, and D. J. W. Geldart, "Diamagnetic Susceptibility of a Dense Electron Gas," Phys. Rev. B 37, 2502 (1988).

G. Vignale and Mark Rasolt, "Density Functional Theory in Strong Magnetic Fields," Phys. Reo. Lett. 59,2360 (1987).

G. Vignale, "Acoustic Plasmons in a Two-Dimensional, Two-Component Electron Liquid," Phys. Reo. B 38,811 (1988).

G. Vignale and Mark Rasolt, "Current- and Spin-Density-Functional Theory for Inhomogeneous Electric Systems in Strong Magnetic Fields," Phys. Reo. B 37, 10685 (1988).

J. C. Wang, "Comparison of Fractal and Pore Models for Electrolyte/Electrode Interfaces," Solid State lonics 28-30, 1436 (1988).

J. C. Wang, "Impedance of a Fractal Electrolyte/Electrode Interface," Eixtrachim. Acta 33, 707 (1988).

Ping Wang, T. P. Sjoreen, Li Chang, and L. J. Demer, "A Study of Postannealing and In Situ Annealing of Silicon Wafers Implanted with a High Dose of Oxygen," J. Electrochem. Soc. 135, 186 (1988).

J. F. Wendelken, "Atomic and Molecular States of Oxygen Chemisorbed on Tungsten(112)," I. Vac. Sci. and Technol. A 6, 662 (1988).

C. W. White, L. A. Boatner, P. S. Sklad, C. J. McHargue, J. Rankin, G. C. Farlow, and M. J. Aziz, "Ion Implantation and Annealing of Crystalline Oxides and Ceramic Materials," Nucl. Instrum. and Methods Phys. Res. Sect. B 32, 11 (1988).

G. D. Wignall and F. S. Bates, "Anplications and Limitations of Deuterium Labeling Methods to Neutron Scattering Studies of Polymers," I. Die Makrom. Chemi 15, 105 (1988).

G. D. Wignall, D. K. Christen, and V. R. Ramakrishnan, "Instrumental Resolution Effects in SmallAngle Neutron Scattering," J. Appl. Crystallogr. 21, 438 (1988).

G. P. Williams, Ir., G. H. Rosenblatt, A. E. Tuttle, R. T. Williams, and Y. Chen, Time-Resolved Luminescence of $F$ and $F^{+}$Centers in Magnesium Oxide," Cryst. Lattice Defects and Amorphous Mater. 16, 209 (1987).

G. P. Williams, Jr., G. H. Rosenblatt, M. J. Ferry, R. T. Williams, and Y. Chen, "Time-Resolved Luminescence and Absorption Spectroscopy of Defects in $\mathrm{MgO}_{\mathrm{O}} \mathrm{O}$ a $\mathrm{A}_{2} \mathrm{O}_{3}$," I. Lumin. L0/41, 339 (1988).

James M. Williams, Raymond A. Buchanan, and In-Seop Lee, "Ion Beam Processing of Surgical Materials," Nucl. Instrum. and Methods Phys. Res. Sect. R 37/38, 676 (1989).

G. Wiltzius, F. S. Bates, S. B. Dierker, and G. D. Wignall, "The Structure of Porous VYCOR Class," Phys. Reo. A 36, 2991 (1987). 
Po-zen Wong and Jar-shyong Lin, Siudying Fractal Geometry on Submicron Length Scales by SmallAngle Scattering," Math. Geol. 20, 655 (1988).

C. F. Wu, S. H. Chen, L. B. Shih, and I. S. Lin, "A Direct Observation of Counterion Condensation Around Cylindrical Micelles," I. Appl. Crystallogr. 21, 853 (1988).

C. F. Wu, S. H. Chen, L. B. Shih, and J. S. Lin, "Direct Measurement of Counterion Distribution Around Cylindrical Micelles by Small-Angle X-Ray Scattering," Phys. Reo. Letl. 61, 645 (1988).

W. Xia, M. Fernandes, C. A. Hewett, S. S. Lau, D. B. Poker, and J. P. Biersack, "On the Temperature Dependence and the Moving Species During Ion Mixing," Nucl. Instrum. and Methods Phys. Res. Sect. B 37/38, 408 (1989).

W. Xia, C. A. Hewett, M. Fernandes, S. S. Lau, and D. B. Poker, "On the Thermodynamical Driving Force During Ion Mixing of the Co-Si System," J. Appl. Phys. 65, 2300 (1989).

Dan S. Zimmerman, C. Kallin, and A. J. Berlinsky, "Low-Temperature Expansions for an Ising Model on an HCP Lattice with Competing Interactions," Phys. Reo. B 37, 7766 (1988).

D. S. Zimmerman and M. R. A. Shegelski, Three-Dimensional Numerical Simulations for Activated Hopping Conduction," Phys. Reo. B 38, 2068 (1988).

R. A. Zuhr, S. J. Pennycook, T. S. Noggle, N. Herbots, T. E. Haynes, and B. R. Appleton, "lon Beam Deposition in Materials Research," Nucl. Instrum. and Methods Phys. Res. Sect. B 37/38, 16 (1989).

\section{BOOKS AND PROCEEDINGS}

J. Arthur, "An Effective Vibration Isolation System for Perfect-Crystal Neutron interferometry," p. 711 in The Investigation of Fundamental Interactions with Cold Neutrons, NBS Specia; Publication No. 124, ed. by G. L. Greene, National Bureau of Standards, Washington, D.C., 1986.

J. B. Bates and J. C. Wang, "Dielectric Response of lonic Conductors," p. 115 in Solid State Ionics-87, ed. by W. Wappner and H. Schulz, Elsevier North-Holland, Amsterdam, 1988.

J. B. Bates and Y. T. Chu, "Surface Topogruphy and Electrical Response of Metal-Electrolyte Interfaces," p. 1388 in Solid State Ionics-87, ed. by W. Weppner and H. Schulz, Elsevier North-Holland, Amsterdam, 1988.

J. B. Bates and Y. T. Chu, "Electrical Response and Electrode Topography of Metal-Electrolyte Interfaces," p. 72 in Fractal Aspects of Materials: Disordered Systems, ed. by Alan J. Hurd, David A. Weitz, and Benoit B. Mandelbrot, Materials Research Society, Pittsburgh, Pennsylvania, 1987.

S. D. Berger and S. J. Pennycook, "Qualitative and Quantitative Analysis Using High-Angle Scattering ir, the STEM," p. 157 in EM.4C '87 and Workshop on Analytical Electron Mirroscopy Proceedings, ed. by G. Lorimer, Institute of Metals, London, 1988.

L. A. Boatner and B. C. Sales, "Monazite," p. 495 in Radioactive Waste Forms for the Future, Chapter 8, ed. by Werner Lutze and Rodncy C. Ewing, Elsevier North-Holland, Amsterdam, 1988. 
G. H. Braunstein, D. Heiman, S. P. Withrow, and G. Dresselhaus, Synthesis of the Dilute Magnetic Semiconductor CdMnTe by Ion Implantation of Mn into CdTe," p. 237 in Diluted Magnetic (Semimagnetic) Semiconductors, ed. by R. L. Aggarwal, J. K. Furdyna, and S. von Molnar, Materials Research Society, Pittsburgh, Pennsylvania, 1987.

Ch. Buchal, S. Mantl, and D. K. Thomas, "Channeling Investigation of the Lattice Location of Ti in TiImplanted Optical Waveguides in LiNbO3," p. 317 in Fundamentals of Beem-Solid Interactions and Transient Thermal Proiessing, ed. by Michael J. Aziz, Lynn E. Rehn, and Bernd Stritzker, Materials Research society, Pittsburgh, Pennsylvania, 1988.

D. K. Christen, J. T. Ellis, S. T. Sekula, J. R. Thompson, J. D. Lewis, W. R. Anderson, and S. J. Pennycook, The Influence of lon-Irradiation-Induced Disonder and Post-Annealing on Thin-Film Superconductive Compounds MoN, VN, and TiN," p. 800 in Adonnces in Cryogenic Enginzering: Materials, Vol. 34, ed. by A. F. Clark and R. P. Reed, Plenum Publisiding Company, New York, 1988.

Y. T. Chu and J. B. Bates, "Model for the Transient Response of Rough Metal-Electrolyte Interface," p. 75 in Fractal Aspects of Materials: Disordered Systems, ed. by A. J. Hurd, D. A. Weitz, and B. B. Mandelbrot, Materials Research Society, Pittsburgh, Pennsylvania, 1987.

H. L. Davis and J. R. Noonan, "Atomic Structure of Binary Alloy Surfaces," p. 3 in Physical and Chemical Properties of Thin Metal Overlayers and Alloy Surfaces, ed. by D. M. Zehner and G. W. Goodman, Materials Research Society, Pittsburgh, Pennsylvania, 1987.

H. L. Davis and J. R. Noonan, "Atomic Structure of Three Low-Index Surfaces of the Ordered Binary Alloy NiAl," p. 152 in Springer Series in Surface Sciences, Vol. 11, ed. by J. F. van der Veen and M. A. van Hove, Springer Verlag, Berlin, 1988.

N. J. Dudney, "Enhanced Ionic Conductivity in Composite Electrolytes," p. 1065 in Solid State lonics-87, ed. by W. Weppner and H. Schulz. Elsevier North-Holland, Amsterdam, 1988.

Nancy J. Dudney, "Composite Electrolytes," p. 103 in Anrual Reoiew of Material Science, Vol. 19, ed. by Robert A. Huggins, Annual Reviews, Inc., Palo Alto, California, 1989.

J. A. Edmond, R. F. Davis, and S. P. Withrow, 'Structural Characterization of Ion-Implanted $\beta$-SiC Thin Films," p. 479 in Silicon Carbide '87, Ceramics Transactions, Vol. 2, ed. by J. D. Cowley and C. E. Semler, American Ceramic Society, Columbus, Ohio, 1989.

M. K. El-Ghor, S. J. Pennycook, T. P. Sjoreen, C. W. White, and J. Narayan, "Cavity Formation in SIMOX Structures," p. 79 in Silicon-on-Insulator and Buried Metals in Semiconductors, ed. by James C. Sturm, Chetuson K. Chen, Loren Pfeiffer, and Peter L. F. Hemment, Materials Research Society, Pittsburgh, Pennsylvania, 1988.

J. E. Epperson, J. S. Lin, and S. Spooner, "The Fine Scale Microstrurture in Cast and Aged Duplex Stainless Steels Investigated by Small-Angle Scattering," p. 595 in Rediation Induced Changes in Microstruciure: 13th International Symposium, ASTM Special Technical Publication No. 955, ed. by F. A. Garner, N. H. Packan, and A. S. Kumar, American Sociely for Testing and Materials, Philadelphia, Pennsylvania, 1987. 
D. Eres, D. H. Loundes, D. B. Geohegan, and D. N. Mashburn, "Laser hotochemical Growth of Amorphous Silicon at Low Temperatures and Comparison with Thermal Chemical Vapor Deposition," p. 355 in Laser and Particle Beam Clomical Processing for iaticroelectronics, ed. by D. J. Ehrlich, G. S. Higashi, and M. M. Oprysko, Malerials Pesearch Soriety. Pittsburgh, Pennsylvania, 1988.

D. Fathy, C. W. White, and O. W. holland, "Enhaneed Rate of $\mathrm{SiO}_{2}$ Growth in Ge-Implanted Si," p. 27 in Materials Madification and Growth Using lon $2.2 m s$, ed. by Ursula J. Gibson, Alice E. White, and Peter P. Pronko, Materials Research Society, Piksurgh, Pennsylvarja, 1987.

B. D. Gaulin, S. Spooner, and Y. Morii, "A Neutron Scattering Study ef the Kinetics of Phase Separation in $\mathrm{Mn}_{1-x} \mathrm{Cu}_{\mathrm{x}}$, p. 413 in Time Dependent Effects in Disordered Materis,;, ed. by R. Pyon and T. Riste, Plenum Publishing Company, New York, 1988.

D. J. W. Getdart and Mark Rasolt, "Exchange-Cot elation Energy functionals for Extended Systems," p. 151 in The Single Particle Density in Physics and Chemistry, ed. by N. H. March and B. M. Deb, Academic Press, New York, 1987.

D. E. Grider and J. F. Wendelken, "Adsorption of Oxygen on Sodium-Saturated Cu(110)," p. 101 in Physical and Chemical Properties of Thin Metal Overlayers and Alloy Surfaces, ed. by D. M. Zehner and G. W. Goodman, Materials Research Society, Pittsburgh, Pennsylvania, 1987.

K. A. Hardman-Rhyne, T. D. Coyle, E. P. Lewis, and S. Spooner, "Kinetic Investigations of Alkoxysilane Sol-Cel Processing," p. 173 in Better Ceramics Through Chemistry II, ed. by C. J. Brinker, D. J. Clark, and D. R. Ulrich, Materials Research Society, Pittsburgh, Pennsylvania, 1986.

J. B. Hayter, "Adrances in Calculating Macroionic Solution Structure," p. 500 in Procecdings of the 19th Yamada Conference: Ordering and Organization in Ionic Solutions, ed. by N. Ise, World Scientific Publishing, Singapore, 1988.

O. W. Holland, C. W. White, and D. Fathy, "Oxidation Kinetics of Ge+-Ion-Implanted Si," p. 271 in $\mathrm{SiO}_{2}$ and Its Interfaces, ed. by G. Lucovsky and S. T. Pantelides, Materials Research Society, Pittsburgh, Pennsylvania, 1988.

O. W. Holland and D. Fathy, "Enhanced Oxidation of TiSiz Films by As+ Implantation," p. 267 ir. Fundamentals of Beam-Solid Interactions and Transient Thermal Processing, ed. vy Michacl J. Aziz, Lynn E. Rehn, and Bernd Stritzker, Materials Research Society, Pittsburgh, Pennsylvania, 1988.

J. Jupile, K. G. Purcell, G. Derby, J. Wendelken, and D. A. King, "Surface Core Level Shifts for the CleanSurface and Hydrogen-Induced Phase Transitions on W(110)," p. 463 in The Structure of Surfaces II, Springer Series in Surface Sciences, Vol. 11, ed. by J. F. van der Veen and M. A. Van Hove, SpringerVerlag, Berlin, 1988.

Theodore Kaplan, M. J. Aziz, and L. J. Gray, "Application of Onsager's Reciprocity Relations to Alloy Solidification," p. 603 in Fundamentals of Beam-Solid Interactions and Transient Thermal Processing. ed. by Michael J. Aziz, Lynn E. Rehn, and Bernd Stritzker, Materials Research Socicty, Pittsburgh, Pennsylvania, 1988. 
H. R. Kerchner, R. R. Coltman, Jr., C. E. Kabunde, and F. W. Young, Jr., The Low-Temperature Neutron Irradiation Facility at ORNL," p. 701 in Influence of Rediation on Materials Properties, Thirteenth Intermational Symposium (Pert Il), Special Technical Publication 956, ed. by F. A. Garner, C. H. Henaget, Jr., and N. Igata, American Society for Testing and Materials, Philadelphia, Pennsylvania, 1987.

B. C. Larson, J. Z. Tischler, and D. Mills, "Interface Temperatures and Temperature Gradients in Silicon During Pulsed-Laser Irradiation," p. 513 in Fundementals of Beam-Solid Interactions and Transient Thermal Processing, ed. by Michaet J. Aziz, Lynn E. Rehn, and Bernd Stritzker, Materials Research Society. Pittsburgh, Pennsylvania, 1988.

In-Seop Lee, R. A. Buchanan, and J. M. Williarns, Biocorrosion Studies of Gold and Rhodium IonImplanted Titanium and Ti-6Al-4V Alloy," p. 687 in Biomedical Materials and Devices, ed. by J. S. Hanker and B. L. Giammara, Materials Research Society, Pittsburgh, Pennsylvania, 1988.

S. H. Liu, T. Kaplan, and L J. Gray, "Theory of the AC Response of Fractal Interfaces," p. 63 in Fractal Aspects of Materials: Disordered Sysiems, ed. by Alan J. Hurd, David A. Weitz, and Benoit B. Mandelbrot, Materials Research Society, Pittsburgh, Pennsylvania, 1987.

S. H. Lu, "Resonating Valence Bonds, Magnetism, and Superconductivity in the High-T, Copper Oxides," p. 11 in High-Temperaiure Superconductors II, ed. by D. W. Capone II, W. H. Butler, B. Batlogg, and C. W. Chu, Materials Research Society, Pittsburgh, Pennsylvania, 1988.

S. H. Liu, T. Kaplan, and L. J. Gray, Theory of the AC Response of Fractal Interfaces," p. 942 in Proceedings of the IEEE International Symposium on Circuits and Systems, IEEE, Philadelphia, Pennsylvania, 1987.

D. H. Lowndes, S. J. Pennycook, R. F. Wood, G. E. Jellison, Jr., and S. P. Withrow, Time-Resolved and Microstnuctural Studies of Solidification in Undercooled Liquid Silicon," p. 489 in Fundamentals of Beam-Solid Interactions and Transient Thermal Processing, ed. by Michael J. Aziz, Lynn E. Rehn, and Bernd Stritzker, Materials Research Society, Pittsburgh, Pennsylvania, 1988.

D. H. Lowndes, D. B. Geohegan, D. Eres, D. N. Mashburn, and S. J. Pennycook, "Photon-Controlled Growth of Multilayered Structures," p. 23 in Multilayers: Synthesis, Properities, and Nonelectronic Applications, ed. by T. W. Barbee, Jr., F. Spaepen, and L. Greer, Materials Research Society, Pittsburgh, Pennsylvania, 1988.

C. Q. Lu, E. Nygren, M. J. Aziz, D. Turnbull, and C. W. White, "Time-Resolved Reflectivity Measurement of the Pressure-Enhanced Crystallization Rate of Amorphous Si in a Diamond Anvil Cell," p. 435 in Fundamentals of Beam-Solid Interactions and Transient Thermal Processing, ed. by Michael J. Aziz, Lynn E. Rehn, and Bernd Stritzker, Materials Research Society, Pittsburgh, Pennsylvania, 1988.

D. Lubben, T. Motooka, J. E. Greene, J. F. Wendelken, J. E. Sundgren, and W. R. Selaneck, "XPS, UPS, and HREELS Studies of Excimer-Laser-Induced Dissociation of $\mathrm{Al}_{2}\left(\mathrm{CH}_{3}\right)_{6}$ Adsorbed on Si(100) Surface," p. 151 in Laser and Parlicle-Beam Chemical Processing for Particle Electronics, ed. by D. J. Ehrlich, G. H. Higashi, and M. M. Oprysko, Materials Research Society, Pittsburgh, Pennsylvania, 1988.

S.-C. Lui, J. Mundenar, E. W. Mummer, M. E. Mostoller, R. M. Nicklow, D. M. Zehner, and W. K. Ford, "Electronic and Vibrational Properties of Single-Crystal Surfaces of NiAl," p. 47 in Physical and Chemical Properties of Thin Metal Ooerlayers and Alluy Surfaces, ed. by D. M. Zehner and G. W. Coodman, Materials Research Society, Pitisburgh, Pennsylvania, 1987. 
R. H. Magruder III, S. H. Morgan, R. A. Weeks, and R. A. Zuhr, "Infrared Reflectance Measurement of Ion-Implanted Silica," p. 10 in Properties and Characteristics of Optical Glass-SPIE Proceedings, Vol. 970, SPIE, Bellingham, Washington, 1988.

G. D. Mahan, "Singularities in X-Ray Spectra of Metals," p. 41 in Fermi Surface Effects, ed. by J. Kondo and A. Yoshimori, Springet-Verlag, New York, 1987.

A. D. Marwick, G. S. Oehrlein, J. H. Barrett, and N. M. Johnson, "The Structure of the Boron-Hydrogen Complex in Silicon," p. 259 in Defects in Electronic Materials, ed. by Michael Stavola, Stephen I. Pearton, and Gordon Davies, Materials Research Society, Pittsburgh, Pennsylvania, 1988.

D. N. Mashbum, D. B. Geohegan, D. Eres, D. H. Lowndes, L. A. Boatner, B. C. Sales, S. J. Pennycook, R. J. Culbertson, E. Sonder, and D. K. Christen, "Deposition of High-T, Superconducto- Thin Films by Pulsed-Laser Ablation," p. 699 in High-Temperature Superconductors, ed. by Merwyn B. Brodsky. Robert C. Dynes, Koichi Kitazawa, and Harry L. Tuller, Materials Research Society, Pittsburgh, Pennsylvania, 1988.

C. J. McHargue, P. S. Sklad, C. W. White, G. C. Farlow, A. Perez, N. Kornilios, and G. Marest, The Charge State of Iron Implanted into Sapphire," p. 245 in Materials Modification by High-Fluence Ion Beams, ed. by R. Kelly and M. Fernanda da Silva, Kluwer Academic Publishers, New York, 1989.

H. A. Mook, "Neutron Scattering Studies of Magnetic Excitations in Itinerant Magnets," p. 425 in Spin Waves and Magnetic Excitations, ed. by A. S. Borovik-Romanov and S. K. Sinha, Elsevier Science Publishers B.V., Amsterdam, 1988.

J. M. Mundenar, R. H. Gaylord, S.-C. Lui, E. W. Plummer, D. M. Zehner, W. K. Ford, and L. G. Sneddon, "Carbon Monoxide Adsorption on (111) and (110) Single-Crystal Nickel-Aluminum Alloy," p. 59 in Physical and Chemical Properties of Thin Metal Ooerlayers and Alloy Surfaces, ed. by D. M. Zehner and G. W. Goodman, Materials Research Society, Pitrsburgh, Pennsylvania, 1987.

J. J. Neumeier, W. J. Nellis, M. B. Maple, M. S. Torikachvili, and B. C. Sales, "Superconductivity of A15Phase Nb3Si Synthesized by Mbar Shock Pressure," p. 477 in Proceedings of the 1987 Topical Conference on Shock Waves in Condensed Matter, ed. by S. C. Schmidt and N. C. Holmes, American Physical Society, New York, 1988.

C. G. Park and S. M. Ohr, "In Situ TEM Studies of Crack Tip Deformation in Molybdenum," p. 437 in Chemistry and Physics of Fracture, NATO Advanced Study Institute Series E, Vol. 130, ed. by R. M. Latanision and R. H. Jones, Martinus Nijhoff, Dordrecht, the Netherlands, 1987.

S. J. Pennycook, S. D. Berger, and R. J. Culbertson, "Z-Contrast Imaging of Dopant Precipitation and Redistribution During Solid-Phase and Liquid-Phase Epitaxial Grnwth of lon-Implanted Si," p. 503 in Proceedings of the 5th Oxford Conference on Microscopy of Semiconducting Materials, Institute of Physics Conference Series No. 87, Institute of Physics Publishing Lid., London, 1987.

S. J. Pennycook, "Studies of Segregation by Z-Ccntrast STEM," p. 614 in Proceedings of the 46th Annual Meeting of the Electron Microscomy Society of America, ed. by C. W. Bailey, San Francisco Press, San Francisco, Califomia, 1988.

S. J. Pennycook, R. J. Culbertson, and S. D. Berget, "Precipitation, Phase Transformation, and Enhanced Diffusion of Jon-Implanted Silicon," p. 411 in Fundamentals of Beam-Solid Interactions and Transient Thermal Processing, ed. by Michael J. Aziz, Lynn E. Rehn, and Bernd Stritzker, Materials Research Society, Pittsburgh, Pennsylvania, 1988. 
D. B. Poker, "Solid-Phase Epitaxy of Ti-Implanted LiNbO3," p. 293 in Materials Modification and Growth Using lon Beams, ed. by Ursu'a J. Gibson, Alice E. White, and Peter P. Pronko, Materials Research Society, Pittsburgh, Pennsylvania, 1987.

D. B. Poker and D. K. Thomas, Optical Waveguide Formation by lon Implantation of Ti or Ag." p. 311 in Fundomentals of Beam-Solid Interactions and Transient Thermal Processing, ed. by Michael J. Aziz, Lynn E. Rehn, and Bernd Stritzker, Materials Reseanch Society, Pittsbungh, Pennsylvania, 1988.

J. Rankin, L A. Boatner, C. W. White, and L. W. Hobbs, "A Cross-Sectional TEM Study of the Effects of Annealing Conditions on the Regrowth of Lead-Implanted Single-Crystal Calcium Titanate," p. 453 in Fundamentals of Beam-Solid Interactions and Transient Thermal Processing, ed. by Michael J. Aziz, Lynn E. Rehn, and Bernd Stritzker, Materials Research Society, Pittsburgh, Pennsylvania, 1988.

J. Rankin, J. C. McCallum, L. A. Boatner, and C. W. White, The Effects of Water Vapor on Epitaxial Regrowth in Lead-Implanted $\mathrm{CaTiO}_{3}{ }^{-}$p. 207 in Selected Topies in Electronic Materials, ed. by B. R. Appleton, W. L. Brown, D. K. Biegelsen, and J. A. Knapp, Materials Research Society, Pittsburgh, Pennsylvania, 1988.

Mark Rasolt, "Continuous Symmetries and Broken Symmetries in Multivalley Semiconductors and Semimetals," p. 43 in Solid State Physics, ed. by F. Seitz, D. Turnbull, and H. Ehrenreich, Academic Press, New York, 1989.

N. M. Ravindra, D. Fathy, O. W. Holland, and J. Narayan, "Si-SiOz Interfaces. An HTREM Study," p. 279 in Physics and Technology of Amorphous $\mathrm{SiO}_{2}$, ed. by R. A. B. Devine, Plenum Publishing Company, New York, 1988.

B. C. Sales and L. A. Boatner, "Lead-Iron Phosphate Glass," p. 195 in Redioactive Waste Forms for the Future, Chapter 3, ed. by Werner Lutze and Rodney C. Ewing, Elsevier North-Holland, Amsterdam, 1988.

B. C. Sales, J. O. Ramey, and L. A. Boatner, "Structural Nature of the Amorphous-10-Crystalline Transition in Phosphate Glasses as Studied by Liquid Chromatography," p. 315 in Effects of Modas of Fommation on the Structure of Classes, ed. by R. A. Weeks and D. L. Kinser, Trans Tech Publishers, Aedermannsdorf, Switzerland, 1988.

B. C. Sales, Y. C. Kim, J. R. Thompson, D. K. Christen, L. A. Boatner, and S. T. Sekula, "Oxygen Stoichiometry and Superconductivity in $\mathrm{Ho}_{1} \mathrm{Ba}_{2} \mathrm{Cu}_{3} \mathrm{O}_{7-x},{ }^{n}$ p. 591 in High-Temperature Superconductors, ed. by Merwyn B. Brodsky, Robert C. Dynes, Koichi Kitazawa, and Harry L. Tuller, Materials Research Society, Pittsburgh, Pennsylvania, 1988.

D. W. Schaefer, A. J. Hurd, D. K. Christen, S. Spooner, and J. S. Lin, "Growth and Structure of Pyrogenic Silicon," p. 305 in Better Ceramics Through Chemistry III, ed. by C. J. Brinker, D. E. Clark, and D. R. Ulrich, Materials Research Society, Pittsburgh, Pennsylvania, 1988.

B. E. Semelius, "Extraction of the 'Real' Band-Gap Shifts in Heavily p-Type Doped GaAs from the Comparison Between Experimental and Theoretical Photoluminescence Peaks," p. 153 in Proceedings of the Fourteenth International Symposium on Cellium Arsenide and Related Compunds, Institute of Physics Conference Series No. 91, Institute of Physics, London, 1988. 
T. P. Sjoreen, M. K. El-Ghor, D. Fathy, and N. M. Ravindra, Oxygen Ion Implantation into Germanium," p. 137 in Silicon-on-Insulator and Buried Metals in Semiconductors, ed. by James C. Sturm, Chenson K. Chen, Loren Pfeiffer, and Peter L. F. Hemment, Materials Research Society, Pittsburgh, Pennsylvania, 1988.

J. R. Thompson, S. T. Sekula, B. C. Sales, Y. C. Kim, D. K. Christen, J. Brynestad, and L. A. Boatner, Perspectives on High-T $C_{c}$ Superconductors from Magnetic Studies," p. 225 in High-Temperature Superconductors II, ed. by D. W. Capone II, W. H. Butler, B. Batlogg, and C. W. Chu, Materials Research Society, Pittsburgh, Pennsylvania, 1908.

J. R. Thompson, L A. Boatner, J. Brynestad, D. K. Christen, Y. C. Kim, B. C. Sales, and S. T. Sekula, "Magnetic Investigations of Superconductive, Polycrystalline (RE) $\mathrm{Ba}_{2} \mathrm{Cu}_{3} \mathrm{O}_{-x} \mathrm{Compounds}(\mathrm{RE}=\mathrm{Y}$, Gd, Ho, Dy)," p. 361 in High-Temperature Superconductors, ed. by Merwyn B. Brocisky. Robert C. Dynes, Koichi Kitazawa, and Harry L. Tuller, Materials Research Society. Pittsburgh, Pennsylvania, 1988.

M. Verrili, J. R. Weertman, and S. Spooner, Influence of Void Nucleation and Growth Rates in HighTemperature Fatigue," p. 89 in Basic Questions in Fatigue, Vol. II, ASTM-STP-924, ed. by R. P. Wei and R. P. Gangloff, American Society for Testing and Materials. Philadelphia, Pennsylvania, 1988.

Edward C. Uoerbacher, Elizabeth Wilkinson-Singley, Joel M. Harp, and Cerard J. Bunick, "DNA Sequence on Nucleosome Positioning and DNA Bending" p. 139 in Structure and Expression, Vol. 3, DNA Bending and Curouture, ed. by W. K. Olson, M. H. Sarma, R. H. Sarma, and M. Sundaralingam, Adenine Press, Guilderland, New York, 1988.

G.C. Wang, D. M. Zehner, and H. C. Eaton, Studies of Surface Composition and Phase Transition of Mo-5\%Re(100)," p. 29 in Physical and Chemical Properties of Thin Metal Ooerlayers and Alloy Surfaces, ed. by D. M. Zehner and G. W. Coodman, Materials Research Society, Pittsburgh, Pennsylvania, 1987.

J. C. Wang, Fractal Dimension and Impedance Frequency-Exponent of a Blocking Electrode/Electrolyte Interface," p. 69 in Fractal Aspects of Materials: Disordered Systems, ed. by Alan J. Hurd, David A. Weitz, and Benoit B. Mandelbrot, Materials Research Society, Pittsburgh, Pennsylvania, 1987.

J. C. Wang, "Comparison of Fractal and Pore Models for Electrolyte/Electrode Interfaces," p. 1436 in Solid State lonics-87, ed. by W. Weppner and H. Schulz, North Holland, Amsterdam, 1988.

G. Whichard, H. C. Mogul, R. A. Weeks, J. D. Stark, and R. A. Zuhr, "Modification of Optical Properties in Transition-Metal lon-Implanted Silica," P. 105 in Advanced Surface Processes for Optoelectronics, ed. by S. L. Bernasek, T. Venkatesan, and H. Temkin, Materials Research Society, Pittsburgh, Pennsylvania, 1988.

C. W. White, "Laser Annealing," p. 2501 in Encyclopedia of Materials Science and Engineering, ed. by Michael B. Bever, Pergamon Press Lid., London, 1986.

C. W. White, D. Fathy, and O. W. Holland, "Oxidation of Ce-Implanted Silicon," p. 485 in Photon-, Beam-, and Plasma-Enhanced Processing, ed. by E. F. Krimmel and V. T. Nguyen, Les Editions de Physique, Les Ulis Cedex, France, 1987.

C. W. White, "Pulsed-Laser Annealing of Si (2-60-ns Pulses)," p. 892 in Properties of Silicon, EMIS Datareviews Series No. 4, INSPEC, The Institution of Electrical Engineers, London, 1988. 
C. W. White and L. A. Boatner, "Materials Science," p. 275 in 1989 Science Year, World Book Encyclopedia Annual Science Supplement, World Book, Inc., Chicago, 1988.

M. K. Wilkinson, M. Bhume, D. K. Stevens, M. lizumi, and Y. Yamada, The U. S.-Japan Cooperative Program on Neutron Scattering," p. 13 in Multipurpose Research Reactors, Procedings of the LAEA International Symposium on the Utilization of Multipurpose Research Reactors and Related International Cooperation, IAEA-SM-300/50, International Atomic Energy Agency, Vienna, 1988.

S. P. Withrow, A. Lusnikov, H. J. Jimenez-Gonzalez, and G. Dresselhaus, High-Energy Ion Beam Annealing in Implanted CdTe," p. 306 in Fundamentals of Beam-Solid Interactions aud Transient Thermal Processing, ed. by Michael J. Aziz, Lynn E. Rehn, and Bernd Stritaker. Materials Research Society, Pittsburgh, Pennsylvania, 1988.

R. F. Wood, R. D. Westbrook, and G. E. Jellison, Jr., "High-Efficiency Intrinsically Pascivated LaserProcessed Silicon Solar Cells," p. 519 in Proceedings of the Nineteenth IEEE Photoooltaic Specialists Conference-1987, The Institute of Electrical and Electronic Engineers, Inc., New York, 1987.

W. Xia, M. Fernandes, C. A. Hewett, S. S. Lau, D. B. Poker, and J. P. Biersack, "On the Temperature Dependence and the Moving Species During Ion Mixing," p. 408 in Ion Implantation Technology, ed. by T. Takagi, Elsevier North-Holland, Amsterdam, 1989.

W. Xia, M. Fernandes, C. A. Hewett, S. S. Lau, D. 3. Poker, and J. P. Biersack, "On the Temperature Dependence and the Moving Species During lon Mixing," p. 193 in Proceedings of the Shanghai Workshop on Ion Implantation, ed. by Zou Shichang, Shanghai Institute of Metallurgy. Shanghai, China, 1989.

D. M. Zehner and G. R. Gruzalski, "Oxygen Adsorption on and Oxidation of NiAl Surfaces," p. 199 in Physical and Chemical Properties of Thin Metal Overlayers and Alloy Surfaces, ed. by D. M. Zehner and G. W. Coodman, Materials Research Society, Pittsburgh, Pennsylvania, 1987.

R. A. Zuhr, G. D. Alton, B. R. Appleton, N. Herbots, T. S. Nogzle, and S. J. Pennycook, "Direct Formation of Thin Films and Epitaxial Overlayers at Low Temperatures Using a Low-Energy (10-500 eV) Ion Beam Deposition System," p. 95 in Photon-, Beam-, end Plosma-Enhanced Processing, ed. by E. F. Krimmel and V. T. Nguyen, Les Editions de Physique, Les Ulis Cedex, France, 1988.

R. A. Zuhr, D. Fathy, and O. W. Holland, "Formation of Silicides by Direct Ion Beam Deposition," p. 119 in Proceedings of the Topical Symposium on Defects and Interfaces in Serniconductors, ed. by $K$. Rajan, J. Narayan, and D. G. Ast, The Metallurgical Society, Warrendale, Pennsylvania, 1988.

\section{REPORTS ISSUED}

J. D. Budai, "Defects in Quasicrystals," p. 24, in X-Ray Synchrotrons and the Deoelopment of New Materials: Workshop Report, ANL/APS-TM-1, Argonne National Laboratory, Argonne, Illinois (February 1988).

J. D. Budai, "Phason Strain in Oriented Quasicrystals." p. 3-101 in National Synchrotron Light Source Annual Report 1987, BNL-52131, Brookhaven National Laboratory, Upton, New York (October 1987). 
C. B. Finch, Y. K. Chang and M. M. Abraham, Single-Crystal Growth of Group IVB and VB Carbides by the Floating-Zone Method, ORNL/TM 1 i 103 (February 1989).

H. R. Kerchner, The Low-Temperature Neutron Irradiation Facility-Final Report, Oak Ridge National Laboratory (Octuber 1988).

B. C. Larson, Nanosecond Resolution Time-Resolved X-Ray Studies of Overheating and Undercooling During Pulsed-Laser Melting," p. 30 in Time-Resolved Studies and Ultrafast Detectors: Workshop Report, ANL/APS-TM-2, Argonne National Laboratory, Argonne, Illinois (February 1988).

F. A. Modine, Emaluation of New Metal-Insulator-Semiconductor Varistor-Final Report, ORNL/TM10386 (August 1988).

J. B. Roberto, Compiler, Workshop Report and Presentations from the Semiconductor Research Corporation-DOE Semiconductor Task Force Workshop, Oak Ridge National Laboratory, Oak Ridge, Tennessee, CONF-8711158 (February 1988).

\section{THESES}

Mohamed Kamel El-Ghor, "Characterization and Optimization of Structural Defects in Buried Oxide Materials Formed by High Dose Oxygen Implantation in Silicon," Ph.D. Thesis, North Carolina State University, Raleigh, North Carolina, 1987.

J. L. Park, "Optical Properties of Anion Vacancies and Hydride lons in CaO Crystals," Ph.D. Thesis, North Carolina State University, Raleigh, North Carolina, 1988.

\section{PATENTS GRANTED}

B. C. Sales and L. A. Boatner, Lead Phosphate Glass Compositions for Optical Components, U. S. Patent No. 4,699,889 (October 13, 1987)

\section{PAPERS PRESENTED AT TECHNICAL MEETINGS}

Theoretical (Computational) Symposium on Atomic Collisions in Solids, Tokyo, Japan, October 7-9, 1987:

Ordean S. Oen, "Extensions to the Two Atom Blocking Model" (invited paper)

Tenth Annual Cryogenic Structural Materials Workshop, Pleasanton, California, October 8 -9, 1987:

H. R. Kerchner, "The Low-Temperature Neutron Irradiation Facility" 
The Metallurgical Society Fall Meeting, Cincinnati, Ohio, October 12-15, 1987:

D. K. Christen, J. R. Thompson, S. T. Sekula, Y. C. Kim, B. C. Sales, L A. Boatner, and J. Brynestad, "Independent Magnetism and Superconductivity in (RE)Baz $\mathrm{Cu}_{3} \mathrm{O}_{7-x}{ }^{-}$(invited paper)

R. M. Moon, "Steady-State Neutron Beams: Their Role in Metallurgical Research" (invited paper)

D. M. Zehner, "Geometric, Electronic, and Vibronic Structure of Clean NiAl Surfaces" (invited paper)

Twelfth International Conference on Atomic Collisions in Solids, Okayama, Japan, October 12-15, 1967:

Ordean S. Oen, "Atomic Displacement Cross Section Calculations for Relativistic Positrons,"

P. Sigmund, M. T. Robinson, M. I. Baskes, M. Hautala, F. Z. Cui, W. Eckstein, Y. Yamamura, S. Hosaka, T. Ishitani, V. I. Shulga, D. E. Harrison, Ir., I. R. Chakarov, D. S. Karpusov, E. Kawatoh, R. Shimizu, S. Valkealahti, R. Nieminen, G. Betz, W. Husinsky, M. H. Shapiro, M. Vicanek, and H. M. Urbassek, Round Robin Computer Simulation of Ejection Probability in Sputtering"

K. J. Snowdon, C. C. Havener, W. Heiland, F. W. Meyer, S. H. Overbury, and D. M. Zehner, "Direct Evidence for the Dominant Role of Target Core Levels in the Neutralization of Slow Multicharged lons"

International Conference on the Applications and Techniques of Small-Angle Scattering October 16-19, 1987:

T. P. Russell, J. S. Lin, S. Spooner, and G. D. Wignall, "Intercalibration of Small-Angle X-Ray and Neutron Scattering Data" (invited paper)

Forty-Third Meeting of the Oak Ridge Associated Universities Council, Oak Ridge, Tennessee, October 19, 1987:

J. B. Roberto, "ORNL as a National Materials Laboratory" (invited paper)

Thirty-Fifth Midweat Solid State Conference, Notre Dame, Indiana, October 19-20, 1987:

G. Vignale and Mark Rasolt, "Density-Functional Theory in Strong Magnetic Fields"

IAEA Intermational Symposium on the Utilization of Multipurpose Research Reactors and Related International Cooperation, Grenoble, France, October 19-23, 1987:

M. K. Wilkinson, M. Blume, D. K. Stevens, M. lizumi, and Y. Yamada, "U.S.-Japan Cooperative Program on Neutron Scattering" 
Tunable Laser Conference, Williamsburg, Virginia, October 26-28, 1987:

Guangzhao Wu, R. T. Williams, G. P. Williams, Jr., C. Y. Chen, and Y. Chen, "400-nm Photoluminescence of a Color Center in Undoped YAG Grown Under Reducing Atmosphere"

G. J. Pogatshnik, M. M. Abraham, and Y. Chen, "Spectroscopy of Color Centers in CaO.Ce"

Intermational Conference in Applications aud Techniques of Small-Angle Scattering, Chicago, Illinois, October 26-29, 1987:

J. E. Epperson and J. S. Lin, "Phase Separation in a Ni-Al Alloy"

J. E. Epperson, T. E. Klippert, C. G. Windsor, V. Rainey, R. Hill, J. S. Lin, and S. Spooner, "A SmallAngle Neutron Scattering Investigation of an Fe-40 at.\% Alby"

C. F. Wu, S. H. Chen, L B. Shih, and J. S. Lin, "A Direct Measurement of Counterion Condensation Around Cylinurical Micelles"

Annual Meeting of the Ceological Society of America, Phoeniz, Arizona, October 26-29, 1987:

J. H. Fang, J. S. Lin, and P. Z. Wong, "Fractal Pore-Space Geometry of Sedimentary Rocks"

Workshop on Lightning Protection of Underground Cables, Stors, Connecticut, October 28-29, 1987:

Frank A. Modine, "Metal Oxide Varistors" (invited paper)

American Vacuum Society Thirty-Fourth National Symposium and Topical Conference, Anaheim, California, November 2-5, 1987:

H. L. Davis and J. R. Noonan, "Suriace Structure Determination by Use of LEED and SPLEED" (invited paper)

N. Herbots, T. S. Noggle, B. P. Appleton, and R. A. Zuhr, "Nonequilibrium Growth of Semiconductor Heterostructures by Ion Beam Deposition (IBD) and Also Combined Ion-Molecular Deposition (CMID)"

J. R. Noonan and H. L. Davis, 'Domain Mixtures in the NiAl(111) Surface"

J. F. Wendelken, "Atomic and Molecular States of Oxygen Chemisorbed on Tungsten (112)"

Workshop on Neutron Scattering Studies of Polymers, Los Alamos, New Mexico, November 3-5, 1987:

C. D. Wignall, V. Ramakrishnan, M. Linne, A. Klein, L. Sperling, M. Wai, R. Celman, M. Fattica, R. Hoerl, L. Fisher, S. Melpolder, and J. O'Reilly, "The Morphology of Emulsion Polymerized Latex Particles" (invited paper) 
IRUDOE Conference on Research Facilities for Materials Characterization, Argonne, Illinois, November 3-5, 1967:

J. B. Roberto, "Surface Modification Research Using Ion Beams at ORNL" (invited paper)

Workshop on X-Ray and Neutron Scattering from Magnetic Materials, Argonne, Illinois, November 6-7, 1987:

H. A. Mook, "Observation of Itinerant Electron Effects on the Magnetic Excitations of Ni and Fe" (invited paper)

Thirty-Second Annuai Conference on Magnetism and Magnetic Materials, Chicago, Illinois, November 9-12, 1987:

J. A. Fernandez-Baca, J. W. Lynn, J. J. Rhyne, and G. E. Fish, "Low-Temperature Spin Waves in Amorphous Fega- $\mathrm{Ni}_{x} \mathrm{Zr}_{10}{ }^{n}$

Fifty-Fourth Meeting of the Southeastem Section of the American Physical Society, Nashville, Tennescee, November 23-25, 1987:

R. M. Moon, "The Advanced Neutron Source: An Impurtant National User Facility" [Bull. Am. Phys. Soc. 32, 2144 (1987)]

R. T. Richard, R. L. Chaplin, R. R. Coltman, C. E. Klabunde, and H. R. Kerchner, "Stored Energy in Neutron-Irradiated Cooper" [Bull. Am. Phys. Soc. 32, 2137 (1987)]

Materials Research Society Fall Meeting, Boston, Massachusetts, November 30-December 5, 1987:

J. B. Bates and Y. T. Chu, "Electrical Response and Electrode Topography of Metal-Electrolyte Interfaces"

R. A. Buchanan and J. M. Williams, 'Noble Metal Ion Implantation of Surgical Alloys"

Y. T. C $\urcorner$ u and J. B. Bates, "Model for the Transient Response of Rough Metal-Electrolyte Interface"

R. J. Culbertson and S. J. Pennycook, "Apparent Enhanced Solubility in Dual-Implanted Silicon"

M. K. El-Ghor, S. J. Pennycook, T. P. Sjoreen, C. W. White, N. Biuono, and J. Narayan, "Cavity Formation and Pulsed-Laser Annealing of (100) and (111) SIMOX Structures"

D. Fathy, T. P. Sjoreen, and N. M. Ravindra, "Oxygen Ion Implantation into Cermanium"

D. Fathy, O. W. Holland, C. W. White, and N. M. Ravindra, "Interfacial Structure of $\mathrm{SiO}_{2} / \mathrm{Ge}$ and Cxidation Kinetics of $\mathrm{Ge}^{+}$.Implanted $\mathrm{Si}^{\prime \prime}$

O. W. Holland and D. Fathy, "Enhanced Oxidation of $\mathrm{TiSi}_{2}$ by $\mathrm{As}^{+}$Implantation" 
Theodore Kaplan, M. J. Aziz, and L. J. Gray, "Application of Onsager's Reciprocity Relations to Interface Motion"

B. C. Larson, J. Z. Tischler, and D. M. Mills, "Interface Temperatures and Thermal Transport in Si and Ge During Pulsed-Laser-Induced Melting and Solidification"

S. H. Liu, "Theory of the AC Response of Rough Elect-ode-Electrolyte Interfaces" (invited paper)

Douglas H. Lowndes. D. Eres, D. B. Geohegan, D. N. Mashburn, and S. J. Pennycook, "Laser Photochemical Growth of Amorphous and Crystalline Silicon Thin Films at Low Temperatures"

Douglas H. Lowndes, Time-Resolved and Microstructural Studies of Solidification in Pulsed-LaserMelted Undercooled Liquid Silicon" (invited paper)

Douglas H. Lowndes, D. B. Geohegan, D. Eres, D. N. Mashburn, and S. J. Pennycook, "PhotonControlled Growth of Multilayer Structures"

D. Lubben, T. Motooka, J. E. Green, J.-E. Sundgren, W. R. Salenak, and J. F. Wendelken, "XPS, UTS, and EELS Studies of Excimer-Laser-Induced Dissociation of $\mathrm{Al}_{2}\left(\mathrm{CH}_{3}\right)_{6}$ Adsorbed on $\left.\mathrm{Si}(100) 2 \times 1\right)^{n}$

A. Lusnikov, I. Ohana, C. Doll, P. J. Picone, M. S. Dresselhaus, G. Dresselhaus, and S. P. Withrow, "Lattice Mode Study of Sr-Implanted $\mathrm{La}_{2} \mathrm{CuO}_{4}$ "

A. D. Marwick, G. S. Oehrlein, J. H. Bärtett, and N. M. Johnson, "The Structure of the BorunHydroges, Complex in Silicon"

D. N. Mashbum, D. B. Geohegan, D. Eres, D. H. Lowndes, L. A. Boatner, B. C. Sales, S. J. Pennycook, and R. J. Culbertson, "Deposition of Thin Superconductor Films By Pulsed-Laser Ablation"

S. J. Pennycook, R. J. Culbertson, and S. D. Berger, "Precipitation and Redistribution of In and Sb During Recrystallization of Amorphous Si"

D. B. Poker, "Optical Waveguide Formation by Ion Implantation of Ti or Ag"

J. Rankin, L. A. Boatner, C. W. White, and L. W. Hobbs, "A Cross-Sectional TEM Study of the Effects of Annealing Conditions on the Regrowth of Lead-Implanted Single-Crystal Titanate"

N. M. Ravindra, D. Fathy, J. Narayan, A. R. Heyd, and K. Vedam, "Electrical, Optical, and Structural Properties of $\mathrm{SiO}_{2}$ Films on $\mathrm{Si}^{\prime \prime}$

B. C. Sales, Y. C. Kim, J. R. Thompson, D. K. Christen, L. A. Boatner, and S. T. Sekula, "Oxygen Stoichiometry and Superconductivity in $\mathrm{HoBa}_{2} \mathrm{Cu}_{3} \mathrm{O}_{7-x}$

J. R. Thompson, L. A. Boatner, J. Brynestad, D. K. Christen, Y. C. Kim, B. C. Sales, and S. T. Sekula, "Magnetic Investigations of Polycrystalline $\mathrm{RE}_{1} \mathrm{Ba}_{2} \mathrm{Cu}_{3} \mathrm{O}_{2}(\mathrm{RE}=\mathrm{Y}, \mathrm{Dy}, \mathrm{Cd}, \mathrm{Ho})$ "

J. C. Wang, "Fractal Dimension and Impedance Frequency-Exponent of a Blocking Electrode/Electrolyte Interface"

J. M. Williams, "Fundamental Aspects of Ion Implantation into Materials" 
S. P. Withrow, D. Fathy, A. Lusnikov, H. Jimenez-Gonzalez, and G. Dresselhaus, "Higt,-Energy Ion Beam Annealing in CdTe"

Conference on Applications of Cold Nentrona, Gaithersburg, Maryland, December 8-9, 1987:

B. D. Gaulin, "Time-Resolved Neutron Scattering Studies of the Kinetics of Phase Separation" (invited paper)

John B. Hayter, "Applications of Cold Neutron Scattering to the Study of Submicron Chemical Structures" (invited paper)

Workshop on X-Ray Synchrotrons and the Development of New Materials, Argonne, Illinois, December 10-11, 1987:

J. D. Budai, "Defects in Quasicrystals"

Workshop or. High- $T_{c}$ Superconductors, Berkeley, Culifornia, December 14-15, 1987:

F. W. Young, Jr. "High- $T_{c}$ Superconductors at Oak Ridge National Laboratory" (invited paper)

Workshop on Electron Diffraction and Channeling at Kilovolt Energies, Wickenburg, Arizona, January 10-14, 1988:

S. J. Pennycook, "Lattice and Sublattice Location Analysis of Impurities in Semiconductors" (invited paper)

Advanced Photon Source Workshop on Time-Resolved Studies and Ultrafast Detectors, Argonne, Illinois, January 25-26, 1988:

B. C. Larson, "Nanosecond Resolution Time-Resolved X-Ray Studies of Overheating and Undercooling in Semiconductors During Pulsed-Laser Melting" (invited paper)

AlME Annual Meeting, Phoenix, Arizona, January 25-28, 1988:

R. A. Zuhs, D. Fathy, and O. W. Holland, "Formation of Silicides by Direct Ion Beam Deposition"

Seventeenth Annual Symposium on Applied Vacuum Science and Technology, Clearwater Beach, Florida, February 1-3, 1988:

J. R. Noonan and H. L. Davis, "Studies of Intermetallic Compound Surfaces" (invited paper)

John F. Wendelken, "Oxygen Adsorption on Tungsten (112): Atomic and Molecular States" (invited paper) 
American Association for the Advancement of Science Annual Meeting, Boston, Massachusetts, February 11-15, 1968:

R. M. Moon, "Advanced Neutron Facilities"

Annual Symposium of the Center for Materials Processing, Knoxville, Tennessee, March 16-17, 1988:

D. H. Lowndes, D. B. Geohegan, and D. N. Mashburn, "High-T, Oxice Superconductor Thin Films Produced by Pulsed-Laser Ablation"

Twenty-Third Annual Tennessee Junior Sciences and Humanities Symposium, Oak Ridge, Tennessee, March 21, 1988:

L. A. Boatner, The Growth of Single Crystals" (invited paper)

American Physical Society Meeting, New Orleans, Louisiana, March 21-25, 1988:

C. Y. Allison and F. A. Modine, "Electrical Properties of Transition-Metal Carbides" (Bull. Am. Phys. Soc. 33, 325 (1988)]

A. P. Baddorf and E. W. Plummer, "Occupation of Subsurface Sites by Hydrogen on Cu(110)" [Bull. Am. Phys. Soc. 33, 654 (1988)].

J. B. Bates and J. C. Wang, "Dielectric Response of Stabilized Zirconia" [Bull. Am. Phys. Soc. 33, 227 (1988)]

L. A. Boatner, J. L. Boldú, and M. M. Abraham, "The Application of EPR Spectroscopy to the Characterization of Textured Ceramics" [Bull. Am. Phys. Soc. 33, 35 I (1988)]

L. S. Cain, G. J. Pogatshnik, and Y. Chen, "Absorption and Luminescence Spectroscopy of Defects in Gadolinium Gallium Garnet Crystals" [Bull. Am. Phys. Soc. 33, 564 (1988)]

G. S. Canright and G. Vignale, "Superconductivity from the Coulorr b Interaction in Two Dimensions" [Bull. Am. Phys. Soc. 33, 260 (1988)]

C. Y. Chen, G. J. Pogatshnix, and Y. Chen, "Optical and Electron Paramagnetic Resonance Studies of Fe Impurities in YAG Crystals" [Bull. Am. Phys. Soc. 33, 693 (1988)]

Y. Chen, L. S. Cain, C. Y. Chen, G. J. Pogatshnik, and M. R. Kotka, "Color Centers in Lanthanum Magnesium Aluminate" [Bull. Am. Phys. Soc. 33, 565 (1988)]

D. K. Christen, Y. C. Kim, S. T. Sekula, J. R. Thompson, J. Brynestad, B. C. Sales, L. A. Boatner, J. D. Budai, and J. Z. Tischler, "The Formation and Characterization of Magnetically Aligned Powder Samples of (RE)Ba $\mathrm{Cu}_{3} \mathrm{O}_{7-x}(\mathrm{RE}=\mathrm{Y}, \mathrm{Ho}, \mathrm{Cd}) "$ (Bull. Am. Phys. Soc. 33, 516 (1988)]

Y. T. Chu and J. B. Bates, "The Constant Phase Angle Impedance of Rough Electrodes" [Bull. Am. Phys. Soc. 33, 226 (1988)] 
Y. T. Chu and J. B. Bates, "The Constant Phase Angle Response of the Finite Sierpinskj Electrode" [Bull. An. Phys. Soc. 33, 227 (1988)]

J. F. Cooke and J. A. Blackman, Theoretical Investigation of the Inelastic Magnetic Neutron Cross Section for Itinerant Electron Sysiems at High Energies" [Bull. Am. Phys. Soc. 33, 660 (1988)]

H. L. Davis, J. R. Noonan, and K. Müllet, "Rippled Reconstruction of the HfC(100)-(1x1) Surface" [Bull. Am. Phys. Soc. 33, 232 (1988)]

N. J. Dudney, "High Conductivity Paths in AgCl-Alumina Composite Electrolytes" [Bull. Am. Phys. Soc. 33, 227 (1988)]

M. E-Ghor and S. I. Pennycook, "Formation of Cavities During High-Dose Oxygen Implantation of Si" [Bull. Am. Phys. Soc. 33, 739 (1988)]

B. D. Evans, G. J. Pogatshnik, and Y. Chen, "Optical Properties of Anion Vacancies in NeutronIrradiated Sapphire" [Bull. Am. Phys. Sac. 33, 352 (1988)]

T. R. Finlayson, D. Donovan, J. Z. Larese, and H. G. Smith, Studies of Transverse Phonon Modes in Premartensitic In-TI Alloys" [Bull. An. Phys. Soc. 33, 614 (1988)]

R. S. Fishman and D. Stroud, "Long-Range Coulomb Interactions in Josephson Junction Arrays" [Bull. Am. Fhys. Soc. 33, 341 (1988)]

B. D. Gaulin, "A Monte Carlo Study of the Kinetics of Spinodal Decomposition in One Dimension" [Bull. Am. Phys. Soc. 33, 304 (1988)]

B. D. Gaulin, "A Critical Scattering Study of the Helical Antiferromagnets Ho and Dy" [Bull. Am. Phys. Soc. 33, 539 (1988)]

B. D. Gaulin, T. E. Mason, and M. F. Collins, "A Critical Scattering Study of the Triangular Antifertomagnet CsMnBr3" [Bull. Am. Phys. Soc. 33, 538 (1988)]

B. D. Gaulin, "Experimental Observation of Kinetics of Ordering in CuMn Alloys" (Bull. Am. Phys. Soc. 33, 764 (1988)] (invited paper)

R. Conzalez, Y. Chen, and M. M. Abraham, Transmutation-Induced Tritium in $\mathrm{LiNbO}_{3}$ Single Crystals" [Bull. Am. Phys. Soc. 33, 811 (1988)]

B. Hantehzadeh, L. E. Halliburton, C. Y. Chen, R. Gonzalez, and Y. Chen, "Magnetic Resonance Investigations in Deuterated LiTaO 3 Crystals" [Bull. Am. Phys. Soc. 33, 811 (1988)]

G. E. Jellison, Jr., D. H. Lowndes, and J. W. Sharp, "Time-Resolved Optical Studies of OxideEncapsulated Silicon During Pulsed-Laser Melting" [Bull. Am. Phys. Soc. 33, 808 (1988)]

B. C. Larson, S. lida, J. Z. Tischler, G. E. Ice, and A. Habenschuss, 'X-Ray Diffuse Scattering near Bragg Reflections for the Study of Solute Precipitation in Dilute Alloys" [Bull. Am. Phys. Soc. 33, 356 (1988)!

Y. D. Lee, P. J. Phillips, and J. S. Lin, "In Situ Small-Angle X-Ray Scattering Investigations of CroseLinked Polyethylene" [Bull. Am. Phys. Soc. 33, 446 (1988)]. 
W.-H. Li, J. W. Lynn, H. A. Mook, and B. C. Sales, "Long-Range Antiferromagnetic Order in OxygenDefivient $\mathrm{YBa}_{2} \mathrm{Cu}_{3} \mathrm{O}_{5.13}{ }^{\text {" }}$ [Bull. Am. Phys. Soc. 33, 721 (1988)]

S. H. Liu, "Theory of Itinerant Resonating-Valence-Bond Superconductivity" [Bull. Am. Phys. Soc. 33, 220 (1988)]

S.-C. Lui, E. W. Plummer, D. M. Zehner, and J. Davenport, "Electronic Properties of NiAl" (Bull. Am. Phys. Soc. 33, 575 (1988)]

J. W. Lynn, W.-H. Li, S. C. Yu, R. E. Glover III, A. Santoro, B. C. Larson, and J. Z. Tischler, "Structure Study of the '240-K Superconductor' $\mathrm{Y}_{2} \mathrm{Ba}_{0.2} \mathrm{Sr}_{0.8} \mathrm{Cu}_{3} \mathrm{O}_{7-x}$ " [Bull. Ain. Phys. Soc. 33, 690 (1988)]

J. H. Magill, D. C. Sun, and J. S. Lin, "Small-Angle X-Ray Scattering from Rolltruded Polymers" [Bull. Am. Phys. Soc. 33, 717 (1988)]

D. N. Mashbum, "The Simulation and Control of a Laser Photochemical Vapor Deposition System" (Bull. Am. Phys. Soc. 33, 462 (1988)] (invited paper)

D. N. Mashbum, D. B. Geohegan, D. H. Lowndes, D. Eres, L. A. Boatner, B. C Sales, S. J. Pennycook, R. J. Culbertson, E. Sonder, and D. K. Christen, "Preparation and Cnaracterization of High- $T_{c}$ Superconducting Thin Films Using Pulsed-Laser Ablation" (Bull. Am. Phys. Soc. 33, 261 (1988)]

B. M. Ocko, Doon Gibbs, S. G. J. Mochrie, and D. M. Zehner, "Absolute X-Ray Reflectivity Study of the Au(100) Surface" [Bull. Am. Phys. Sac. 33, 395 (1988)]

J. L. Park, G. J. Pogatshnik, Y. Chen, G. P. Williams, Jr., and R. T. Williams, 'Time-Resolved Spectroscopy of $\mathrm{F}^{+}$Centers in CaO" [Bull. Am. Phys. Soc. 33, 352 (1988)]

S. J. Pennycook, R. J. Culbertson, and D. H. Lowndes, "Transient-Enhanced Diffusion in Crystalline and Amorphous Silicon" [Bull. Am. Phys. Soc. 33, 306 (1988)]

G. J. Pogatshnik, M. M. Abraham, and Y. Chen, "Spectroscopic Properties of CaO.Ce Single Crystals" [Bull. Am. Phys. Soc. 33, 564 (1988)]

M. Prakash and D. M. Zehner, 'Surface Segregation and Ordering on Clean and Oxygen-Covered MoRe Alloys" [Bull. Am. Phys. Soc. 33, 396 (1988)]

B. C. Sales, J. O. Ramey, and L. A. Boatner, "The Structure of Phosphate Glasses as Determined by High-Performance Liquid Chromatography" [Bull. Am. Phys. Soc. 33, 316 (1988)]

R. P. Shafer, R. T. Williams, G. P. Williams, Jr., C. Y. Chen, and Y. Chen, "Optical Absorption and Emission Bands in Neutron-Imadiated YAC Crystals" [Bull. Am. Phys. Soc. 33, 564 (1988)]

E. Sonder, R. A. Zuhr, and R. E. Valiga, "Annealing of Damage and Stability of Implanted lons in ZnO Crystals" |Bull. Am. Phys. Soc. 33, 780 (1988)|

J. R. Thompson, B. C. Sales, J. Brynestad, S. T. Sekula, L. A. Boatner, Y. C. Kim, and D. K. Christen, "Magnetic Susceptibility and Magnetization of Oxygen-Depleted (RE) ${ }_{1} \mathrm{Ba}_{2} \mathrm{Cu}_{3} \mathrm{O}_{(7-x)} \mid$ |Bull. Am. Phys. Soc. 33, $722(1988)]$

J. Z. Tischler, B. C. Larson, and D. M. Mills, "Ge During Pulsed-Laser Irradiation Studied by X-Ray Diffraction at Nanosecond Resolution" (Bull. Am. Phys. Soc. 33, 809 (1988)) 
G. Vignale and Mark Rasolt, Density-Functional Theory in Strong Magnetic Fields" [Bull. Am. Phys. Soc. 33, 237 (1988)]

J. C. Wang and J. B. Bates, Model for the Non-Debye Dielectric Response in lonic Cystals" [Bull. Am. Phys. Soc. 33, 226 (1988)]

J. F. Wendelken, The Termination of a Highly Stepped NiAJ111) Surface" [Bull. Am. Phys. Soc. 33, 232 (1988)]

R. F. Wood, Mark Mostoller, and J. F. Cooke, "A Spin-Polaron Approach to Pairing in High $T_{c}$ " [Bull. An. Phys. Soc. 33, 260 (1988)]

R. F. Wood, G. A. Geist, D. H. Lowndes, and C. L. Cleveland, 'Studies of Complex Morphologies in Pulsed-Laser-Irradiated a-Si" [Bull. Am. Phys. Soc. 33, 808 (1988)]

A. Wu, G. Wu, R. T. Williams, G. P. Williams, Jr., C. Y. Chen, and Y. Chen, "Color Centers in Reduced Yttrium Aluminum Garnet Crystals" [Bull. Am. Phys. Soc. 33, 564 (1988)]

D. M. Zehner, G. R. Gruzalski, and G. W. Ownby, "Formation of Surface Oxides in NiA!(110)" [Bull. Am. Phys. Soc. 33, 396 (1988)]

D. S. Zimmerman and M. R. A. Shegelski, "Three-Dimensional Numerical Simulations for Activated Hopping Conduction" [Bull. Am. Phys. Soc. 33, 816 (1988)]

Materials Research Society Meeting, Reno, Nevada, April 5-9, 1988:

David C. Eng, Nicole Herbots, Olof C. Hellman, Shahrnaz Motakef, and Robert J. Culbertson, "The Role of Interfacial Segregation and Microstructures in Interdiffusion Between Aluminum and Silicon"

Nicole Herbots, B. R. Appleton, S. J. Pennycook, T. S. Noggle, and R. A. Zuhr, "Silicon and Cermanium Oxide Formation by Ion Beam Deposition"

S. H. Liu, "Resonating Valence Bonds, Magnetism, and Superconductivity in the High-T, Copper Oxides" (invited paper)

D. B. Poker and D. K. Thomas, "Comparison of Solid-Phase Epitaxy of Ti-, Ag-, and Pb-Implanted $\mathrm{LiNbO}_{3}$ for the Production of Optical Waveguides"

J. R. Thompson, S. T. Sekula, B. C. Sales, Y. C. Kim, D. K. Christen, J. Brynestad, and L. A. Boatner, "Perspectives on High- $T_{c}$ Superconductors from Magnetic Studies" (invited paper)

Eleventh Wemer Brandt Workshop on the Penetration of Charged Particles in Matter, Oak Ridge, Tennescee, Aprit 14-15, 1988:

S. J. Pennycook, "Inner-Shell Excitation by Channeled Electrons" 
Conference on Superconductivity and Applications, Buffalo, New York, April 18-20, 1988:

D. N. Mashburt. D. B. Geohegan, D. H. Lowndes, L. A. Boatner, B. C. Sales, J. D. Budai, S. J. Pennycook, R. J. Culbertson, and D. K. Christen, The Laser Ablation Process for Producing High-Tc Thin Films"

American Physical Society Meeting, Baltimore, Maryland, April 18-21, 1988:

F. W. Meyer, C. C. Havener, W. Heiland, K. J. Snowdon, and D. M. Zehner, "Electron Emission in Collisions of Highly Charged lons with Au and Cu Surfaces"

Soth Annual Mecting of the American Ceramic Society, Cincinnati, Ohio, May 1-5, 1988:

L. A. Boatner, J. L. Boldú, and M. M. Abraham, Texturing in Cold-Pressed, Sintered MgO Ceramics Using Faceted Precursors and a New Application of EPR Spectroscopy to the Characterization of Texturing in Ceramic Materials"

N. J. Dudney, "lonic Conductivity and Microstructure of AgCl-Alumina Composites"

F. A. Modine, "New Varistor Material"

Eighth Annual Tennessee Valley Chapter-American Vacuum Society Symposium and Equipment Exhibit, Oak Ridge, Ten:tessee, May 3-5, 1988:

D. B. Geohegan, D. N. Mashburn, D. H. Lowndes, B. C. Sales, and L. A. Boatnet, "Deposition of Thin Superconducting Films of $\mathrm{Ho}_{1} \mathrm{Ba}_{2} \mathrm{Cu}_{3} \mathrm{O}_{7}$ by Pulsed-Laset Ablation" (invited paper)

Steven M. Gorbatkin, Deposition and Etching Using Electron Cyclotron Resonance (ECR) Microwave Plasmas" (invited paper)

D. H. Lowndes, D. B. Geohegan, D. Eres, D. N. Mashbum, and S. J. Pennycook, "Photon-Controlled Deposition of Thin Films and Multilayered Structures" (invited paper)

J. M. Williams, C. M. Egert, and J. G. Cooch, "Corrosion and Surface Modification Studies in Bulk Uranium and Uranium Thin Films" (invited paper)

R. A. Zuhr, B. R. Appleton, T. E. Haynes, and S. J. Pennycook, "Direct Deposition of Thin Films Using Low-Energy, Mass-Analyzed Ion Beams" (invited paper)

Intemational Polymer Processing Society Meeting, Orlando, Florida, May 8-11, 1988:

J. S. Lin, "Studies of Structural Development in Processed Polymers by Small-Angle Scattering Techniques" (invited paper) 
Fourth Annual Northeast Regional Meeting on Processing and Applications of High-T ductors: Status and Prospects, New Brunsw ick, New Jersey, May 9-11, 19a0.

D. N. Mashbaim, D. B. Geohegan, D. H. Lowndes, L. A. Boatner, B. C. Sales, J. D. Budai, S. J. Pennycook, R. J. Culbertson, and D. K. Christen, Thin Superconducting Oxide Films by Laser Ablation"

Symposium on Superconductivity, University of Alabama, Fiuntsville, Alabama, May 11, 1988

S. H. Liu, Resonating Valence Bonds, Magnetism, and Superconductivity in High- $T_{c}$ Copper Oxides"

American Geophysical Union Annual Spring Meeting. Baltimore, Maryland, May 16-20, 1988

B. C. Chakoumakos, W. C. Oliver, and R. C. Ewing, "Mechanical Properties Measurements on Alpha-Decay Damaged Zircon*

Conference on Ion Implantation and Masma-Assisted Processes for Industrial Applications, Altanta, Georgia, May 22-25, 1980:

R. A. Buchanan, In-Seop Lee, and J. M. Williams, Effects of Noble Metal Ion Implantation on Corrosion Inhibition and Charge Injection Capability of Surgical Titanium Alloy"

J. M. Williams, C. M. Egert, and T. G. Kollie, "Ion Implantation for Corrosion Inhibition of Uranium"

European Materials Research Society Meetine, Strabourg, Franc", May 30-June 2, 1980.

D. Eres, D. B. Geohegan, D. H. Lowndes, and D. N. Mashburn, "ArF (192 nm) Laser Photochemical Deposition of Amorphous Silicon from Disilane: Spectroscopic Studies and Comparison with Thermal CVD"

D. H. Lowndes, D. Eres, D. B. Ceohegan, D. N. Mashburn, and S. J. Pennycook, "Low-Temperature Photon-Controlled Growth of Thin Films and Multilayered Structures" (invited paper)

C. W. White, J. C. McCallum, and L. A. Boatner, Ion Implantation and Annealin? of High-T, Superconducting Single Crystals"

Materials Research Society International Meeting on Advanced Materials, Tokyo, Japan, May 30June 3, 198:

Y. Chen, "Hydride lons and Turable Lasers Based on Anion Vacancies in Refractory Oxide Crystals"

Shanghaj Workshop on Characterization of Ion Implantation in Silicon, Shanghad, People's Republic of China, June 2-3, 1988:

B. R. Appleton, "Damage in Ion-Implanted Semiconductors" 
W. Xia, M. Fernandes, C. A. Hewett, S. S. Lau, D. B. Poker, and J. P. Biersack, "On the Temperature Dependence and the Moving Species During Ion Mixing"

Forty-Eighth Arunual Conference on Physical Electronics, Upton, New York, June 6-8, 1988:

A. P. Baddorf, H. L Davis, and E. W. Plummer, Surface Anharmonicity: Temperature Dependence of Phonon Energies and Surface Structure"

R. A. Bartynski, D. Heskett, K. Garrison, G. Watson, D. M. Zehner, W. N. Mei, S. Y. Tong, and X. Pan, Intensity Oscillations of the Surface and Bulk 4 Core Levels of $\operatorname{Ta}(100)$ -

D. M. Zehner, Doon Gibbs, B. M. Ocko, and S. I. Mochrie, X-Ray Reflectance Studies of the Au(001) Surface Structuren

Seventh Intermational Conference on Ion Implantation Technology, Kyoto, Japan, June 7-10, 1980:

B. R. Appleton, "Ion Beam Deposition in Materials Research"

J. M. Williams, "Ion Implantation and Ion Beam Processing of Surgical Materials" (invited papter)

Sixth International Conference on Ion Beam Modification of Materials, Tokyo, Japan, June 12-17, 1989

D. B. Poker and D. K. Thomas, "Effects of Different lon-Implanted Dopant Species on the SolidPhase Epitaxy of LiNbO 3 Optical Waveguides"

J. M. Williams, C. M. Egert, and T. G. Kollie, "Ion Implantation for Corrosion Inhibition of Uranium"

Eleventh International Symposium on the Reactivity of Solids, Princeton, New Jersey, June 19-24, 1908

S. Nowick, S. O. Fu, W.-K. Lee, and L. A. Boatner, Reactions of Acceptor-Doped Oxides with Water Vapor"

Canadian Association of Physicists Annual Congress, Montreal, Canada, June 20-25, 1988:

B. D. Gaulin, E. D. Hallman, and E. C. Svensson, Thermal Evolution of Phonons in Cu 3 Au Caused by Spinodal Ordering"

NATO Workshop on Mechanisms of Reactions of Organometallic Compounds with Surfaces, St. Andrews, Scotland, June 22-24, 1988:

D. Lubben, T. Motooka, J. F. Wendelken, and J. E. Greene, "Laser-Induced Photodissociation of $\mathrm{Al}_{2}\left(\mathrm{CH}_{3}\right)_{6}$ : Cas-Phase and Adsorbed Layer Dissociation Mechanisms for Al Film Crowth" 
Eectronic Materials Conference, Boulder, Colorado, June 22-24, 190

David C. Eng Nicole Herbots, Olof C. Heliman, Shahmaz Motakef, and R. J. Culbertson, The Role of Interfacial Segregation and Microstructure in Interdiffusion Between Aluminum and Silicon"

Nicole Herbots, Olof C. Hellman, Patricia A. Cullen, Bill R. Appleton, S. J. Pennycook, Tom S. Noggje, and R. A. Zuhr, Low-Energy Ion Beam Oxidation of Silicon and Germanium"

American Cystallogaphic Ascociation Annual Meeting, Thiladelphia, Pennoylvania, June 26-July 1 , 1908

B. C. Larson and M. D. Galloway, Comparison of Bragg Diffuse Scattering with Small-Angle Scattering for the Investigation of Defects in Solids"

H. G. Smith, R. Bertines, and J. D. Jorgensen, Martensitic Transformation from BCC to 9R and FCC in Metallic Lithium"

International Conference on Neutron Scattering, Grenoble, France, July 12-15, 1908:

J. W. Cable and Y. Nakai, "Atom-Pair Correlations in Fe-13.5\% V"

B. D. Gaulin, M. F. Collins, and T. E. Mason, "Critical Scattering from Paramagnetic CsMnBr, and XY Antiferromagnet with Chiral Degeneracy"

R. M. Moon and C. D. West, The Advanced Neutron Source"

O. Moze and J. W. Cable, "Magnetic Moment Distribution in NiMo Alloys"

H. G. Smith, R. Berlines, and J. D. Jorgensen, Martensitic Transformation from BCC wo 9R and FCC to Metallic Lithium"

Thirty-Fifth International Field Emission Symposium, Oak Ridge, Tennesece, July 10-22, 1908:

S. Spooner and M. K. Miller, "A Comparison of Autocorrelograms Determined from SANS and APFIM Data"

International Conference on Magnetism 1983, Paris, France, July 25-29, 1988

J. A. Blackman and J. F. Cooke, "Inelastic Electron Scattering and Electron-Hole Excitations in Iron and Nickel"

J. W. Cable and R. M. Nicklow, "Tolarized Neutron Studies of Forbidden Magnon Scattering in Gadolinium"

J. F. Cooke and J. A. Blackman, "Magaretic Excitations in Transition-Metal Systems"

J. A. Femandez-Baca, J. J. Rhyne, and G. E. Fish, "Neutron Scattering Study of the Magnetic Correlations of Iron-Rich Fe-Ni-Zr Clasces" 

Dy"

B. D. Gaulin, M. Hagen, and H. R. Child, "A Critical Study of the Helical Antiferromagnets Ho and World Congress on Medical Physics and Biomedical Engineering San Antonio, Texas, August 6-12, 1988:

S. H. Liu, "Fractals in Physical Sciences" (invited paper)

Forty-Sixth Annual Meeting of the Electron Microscopy Society of America, Milwankee, Wisconsin, Angust 7-12, 1902

S. J. Pennycook, Studies of Segregation by Z-Contrast STEM" (inviled paper)

SPIE Thirty-Second Annual Inlemational Technical Symposium on Optical and Optoelectronic Rpplied Science and Engineerine Sin Diego, California, August 14-19, 1908

R. H. Magruder, S. H. Morgan, R. A. Weeks, and R. A. Zuhr, "Infrared Reflectance Measurements of Jon-Implanted Silica"

Workshop on Synchrotron Radiation and Semiconductor Technology, Baton Rouge, Louisiana, Auguat 15-16, 1980

D. H. Lowndes, "X-Ray/Vacuum Ultraviolet Photon-Assisted Processing" (invited paper)

Intermational Conference on Critical Currents in High-Temperature Superconductors, Snowmass, Colorado, August 16-18, 198:

H. R. Ketchner, "Ceometrically Large Flux-Line Pins" (invited paper)

1998 Applied Superconductivity Conference, San Francisco, California, August 21-25, 1988:

D. K. Christen, Y. C. Kim, S. T. Sekula, J. R. Thompson, J. Brynestad, B. C. Sakes, L. A. Boatner, and J. D. Budai, "Anisotropies in the Magnetic Penetration Depth and Critical Current Density of $\mathrm{RB}_{2} \mathrm{Cu}_{3} \mathrm{O}_{7-x}$ ( $\mathrm{R}=\mathrm{Y}$ or Ho), Measured on Magnetically Aligned Powder Composites"

S. T. Sekula, J. Brynestad, D. K. Christen, J. R. Thompson, and Y. Kim, "Superconductivity, Critical Current Density, and Magnetic Properties of Fe-Substituted $\mathrm{Y}_{1} \mathrm{Ba}_{2}\left(\mathrm{Cu}_{1-x} \mathrm{Fe}_{\mathbf{x}}\right)_{3} \mathrm{O}_{2}$ Materials"

Fifth International EXAFS Conference, Seattle, Wachington, August 22-26, 1988:

R. B. Greegor, F. W. Lytle, B. C. Chakoumakos, R. C. Ewing, R. J. Livak, F. W. Clinard, Jr., E. D. Crozier, N. Aberding, A. J. Seary, C. W. Amold, M. J. Weber, J. Wong, and W. J. Weber, "Application of Various EXAFS Techniques to the Investigation of Structurally Damaged Materials" 
International Conference on the Chemistry and Phycica of Electrified Interfaces, Bologna, Itaby, Auguet 20-September 2, 1903

S. H. Liu, "Fractal Aspects ó Electrode-Electrolyte Interfaces" (invited paper)

Intemational Conference on Defects in Inoulating Crytal, Pama, Ithy, Anguat 29-September 2, 1500

L S. Cain, G. J. Pogatshnik, and Y. Chen, "Optical Properties of Color Centers in Gadolinium Gallium Garnet Crystals"

Y. Chen, M. M. Abraham, M. R. Kikta, G. J. Pogatshnik, L S. Cain, J. L. Park, and C Y Chen, Thermochemical Reduction and Radiation Effects in Lanthanum Magnesium Aluminate Crystals"

R. Gonzalez, C. Ballesteros, Y. Chen, and M. M. Abraham, "Infrared Characterization of Tritium in $\mathrm{LiNbO}_{3}$ Single Crystals"

V. M. Orera and Y. Chen, "Electron Trapping in $\mathrm{H}^{2-}$ Defects During Photoconversion of Anion Vacancies in Thermochemically Reduced CaO Crystals"

G. P. Williams, Jr., G. H. Rosenblatt, R. T. Williams, and Y. Chen, Luminescence of $F$ and $F^{+}$Centers in Magnesium Oxide"

G. Wu, A. Wu, R. P. Shafer, G. P. Williams, Jr., R. T. Williams, C. Y. Chen, and Y. Chen, "Defect Luminescence in Undoped $Y^{\prime} G^{\circ}$

The American Society of Mechanical Engineers Holston Section Meeting, Kingsport, Tennessee, September 8, 1900

D. K. Christen, Perspectives on High-Temperature Superconductivity" (invited paper)

European Conference on Surface Cystallography, Cambridge, England, September 11-14, 1938

H. L Davis and J. R. Noonan, "LEED Techniques and Crystallographic Results for Ordered Binary Alloy Surfaces" (invited paper)

Eighteenth Rare-Earth Research Conference, Lake Geneva, Wisconsin, September 12-16, 1988;

W. Kot, G. Shalimoff, N. Edelstein, M. M. Abraham, L. A. Boatner, and C. B. Finch, Electron Paramagnetic Resonance Study and the Zero-field Splitting of $\mathrm{Cm}^{3+}$ in Single Crystals of LaPO,"

G. M. Williams, N. Edelstein, L. A. Boatner, and M. M. Abraham, "Anomalously Small 4f $\rightarrow$ 5d Oscillator Strengths and if $\rightarrow$ ff Electronic Raman Scattering Cross Sections for $\mathrm{Ce}^{3+}$ in $\mathrm{LuPO}_{4} \mathrm{Crystals"}^{n}$ 
International Conference on the Physics of Multiply Chaged Ions and International Workshop on E. C. R. Ion Sources, Grenoble, France, September 12-16, 198

F. W. Meyer, C. C. Havener, S. H. Overbury, K. J. Reed, K. J. Snowdon, and D. M. Zehner, "InnerShell Processes as Probes of Multicharged Ion Neutralization at Surfaces"

Seventh International Workshop on Inelastic Ion Surface Collisions, Krakow, Poland, September 19-23, 1989:

C. C. Havener, K. J. Reed, K. J. Snowdon, D. M. Zehner, and F. W. Meyer, Interaction of Multiply Charged Ions with Solid Surfaces"

Intermational School on Condensed Matter Physics, Vama, Bulgaria, September 19-26, 1988:

Y. Chen, R. Gonzalez, V. M. Orera, and G. J. Pogatshnik, "Optical Propcrties of Oxygen Vacancies and Hydride lons in MgO and $\mathrm{CaO}$ Crystals"

Fourth Intermational Laser Science Conference, Atlanta, Georgia, October 2-6, 1988:

D. H. Lowndes, D. Eres, D. B. Geohegan, J. Z. Tischler, D. N. Mashburn, and S. J. Perunycook, "Excimer Laser-Controlled Photochemical Deposition of Thin Films and Artificially Structured Materials"

American Va-uum Society Thirty-Fifth National Symposium and Topical Conference, Atlanta, Georgin, October 3-7, 1988:

R. A. Bartynski, D. Heskett, K. Garrison, G. Watson, D. M. Zehner, W. N. Mei, S. Y. Tong, and X. Pan, "First Interlayer Spacing of Ta(100) Determined by Photoelectron Diffraction"

H. L. Davis and J. R. Noonan, "Surface Structure and LEED 'Bric-A-Brac': A Case Study of Ag(110)"

G. R. Gruzalskj, D. M. Zehner, J. R. Noonan, H. L. Davis, and R. A. DiDio, "Mechanisms for the (1 1 1) Rippled Reconstruction of Transition-Metal Carbide Surfaces"

T. E. Haynes, R. A. Zuhr, S. J. Pennycook, and B. C. Larson, "Heteroepitaxy of ${ }^{76}$ Ge Thin Films on GaAs at Low Temperatures by Direct Deposition from Low-Energy Ion Beams"

Materials Research Society and the Electronics Division of the American Ceramic Society Symposium, San Francisco, California, October 24-26, 1988:

L. A. Boatner and C. W. White, "The Application of Rutherford Backscattering and Channeling Techniques to the Characterization of Ceramics" (invited paper)

Workhop on Momeritum Distributions, Argonne, Illir.ois, October 24-26, 1988:

H. A. Mook, "Measurements of $n(p)$ for ${ }^{3} \mathrm{He}$ and "He Using Reactor Sources" 
Twentieth Southeastern Magnetic Resonance Conference, Columbia, South Carolina, October 26-29, 1988:

M. M. Abraham, "EPR and ENDOR of Trapped-Hole Centers in Alkaline-Earth Oxides"

Annual Meeting of the Optical Society of America, Santa Barbara, Califomia, October 30-November 4, 1988:

G. E. Jellison, Jr., "A New Four-Channel Polarimeter for T'ime-Resolved Ellipsometry"

G. E. Jellison, Jr., "A Two-Channel Polarization Modulation Ellipsometer"

Thirteenth DOE Surface Studies Conference, Clearwater, Florida, November 1-3, 1988:

J. R. Noonan and H. L. Davis, "Surface Structure of Refractory Metal Oxides"

Tenth Conference on the Application of Accelerators in Research and Industry, Denton, Texas, November 7-9, 1988:

O. W. Holland, "Model for Boron Diffusion in Si at High Concentrations"

J. C. McCallum, C. W. White, and L. A. Boatner, "The Annealing Behavior of Ion-Implanted Single Crystals of the Type $\mathrm{YBa}_{2} \mathrm{Cu}_{3} \mathrm{O}_{x}{ }^{\prime \prime}$

J. B. Roberto, "Ion Implantation Technology in the National Laboratories: Four Decades of Technology Transfer" (invited paper)

R. A. Zuhr, T. E. Haynes, S. J. Pennycook, and B. R. Appleton, "Fombation of Thin Films by Direct lon Beam Deposition"

Conference on the Science and Technology of Thin-Film Superconductors, Colorado Springs, Colorado, November 14-18, 1988:

R. Feenstra, D. E. Gøshegan, D. N. Mashburn, j. D. Budai, S. J. Pennycook, L. A. Boatner, D. H. Loirndes, and D. K. Christen, "Pulsed-L'er Ablation and Thermal Evaporation of '123' Superconductor Thin Films on $\mathrm{SrTiO}_{3}$ and $\mathrm{KTaO}_{3}$

Materials Research Society Fall Meeting, Boston, Massachusetts, November 28-December 3, 1988:

J. B. Bates, N. J. Dudncy, Y. T. Chu, and P. Mazumdar, "Properties of Electrolyte and Electrode Films Prepared by RF and DC Magnetron Sputteril 'g" (iavited paper)

B. C. Chakoumakos, B. C. Sales, and E. Sonder, "Characterization of Phases in the Superconducting System $\mathrm{Bi}_{2} \mathrm{Sr}_{2} \mathrm{Ca}_{n-1} \mathrm{Cu}_{n} \mathrm{O}_{x}$

Nancy J. Dudney, "Enhanced Ionic Conductivity in Composite Electrolytes" (invited paper) 
D. Eres, D. B. Geohegan, D. H. Lowndes, D. N. Mashbum, and S. J. Pennycook, "Pyrolytic and Laser Photolytic Growth of Crystalline and Amorphous Germanium Films and Digermane $\left(\mathrm{Ce}_{2} \mathrm{H}_{6}\right)$ "

R. Feenstra, C. W. White, D. B. Poker, D. K. Christen, J. D. Budai, and S. J. Pennycook, "High-Dose Oxygen Implantation and Regrowth of $\mathrm{Y}$-Ba-Cu-O Thin Films on $\mathrm{SrTiO}_{3}$

T. E. Haynes, R. A. Zuhr, S. J. Pennycook, B. C. Larson, and B. R. Appleton, "Heteroepitaxy of GaAs on Silicon and Germanium by Low-Energy Ion Beam Deposition Using Altemating Beams"

O. W. Holland. T. P. Sjoreen, C. W. White, and M. K. El-Ghor, "Darage Formation in Semiconductors During MeV Ion Implantation"

Hiroshi Maeta, B. C. Larson, T. P. Sjoreen, and J. D. Lewis, "Interstitial and Vacancy Loops in IonIrradiated Copper"

J. C. McCallum, C. W. White, and L. A. Boatner, "The Annealing Behavior or Ion-Implanted Single Crystals of the Type $\mathrm{YBa}_{2} \mathrm{Cu}_{3} \mathrm{O}_{x} "$

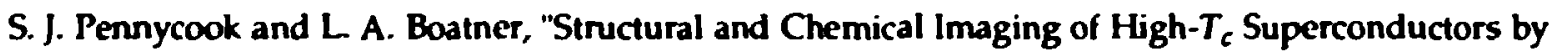
I. .gh-Resolution STEM"

J. Rankin, J. C. McCallum, L. A. Boatner, and C. W. White, "The E'fects of Water Vapor on Epitaxial Regrowth in Lead-Implanted $\mathrm{CaTiO}_{3} "$

R. K. Sirgh, N. Biunno, C. B. Lee, J. Narayan, O. W. Holland, V. N. Shukla, and S. J. Lukasiewicz, "Superconducting Y-Ba-Cu-O Thin Films"

E. Sonder, B. C. Sales, and i. A. Boatner, "Investigatior. of the Effect of Gamma Radiation on the Superconducting Properties of $\mathrm{CdBa}_{2} \mathrm{Cu}_{3} \mathrm{O}_{7-x "}$

J. R. Thompson, S. T. Sekula, D. K. Christen, J. Brynestad, Y. C. Kim, L. A. Boatner, and H. A. Mook, "Electrical Resistivity and Magnetic Susceptibility of Iron-Substituted $\mathrm{Y}_{1} \mathrm{Ba}_{2}\left(\mathrm{Cu}_{1-x} \mathrm{Fe}_{2}\right)_{3} \mathrm{O}_{2}$ Materials"

J. C. Wang and J. B. Bates, "Hopping Model for the Non-Debye Dielectric Response in lenic Crystals"

S. P. Withrow, "Implantation Damage in CdTe"

R. A. Zuhr, O. W. Holland, S. J. Pennycook, and T. E. Haynes, "Silicides Formed by Direct lon Beam Deposition"

Research and Training Opportunities, Minority Education Institutes Conference, Oak Ridge, Tennessee, November 29-December 1, 1988:

J. F. Cooke, "The ORNL Solid State Division" (invited paper)

Second International Varistor Conference, Schenectady, New York, December 5-7, 1988:

F. A. Modine, R. W. Major, S.-1. Choi, Leah B. Kerszberg, and M. N. Silver, "Zinc Oxide Varistor Time Response" 
American Society of Mechanical Engineers Meeting, Knoxville, Tennesssee, January 12, 1989.

J. R. Thompson, "Superconductivity-Current Developments and Future Applications" (invited paper)

Workshop on Atomic Steps at Interfaces, Rottach-Egern, Federal Republic of Germany, January 15-18, 1989:

J. F. Wendelken, "Atomic Step Structure of NiAl(111) and NiAl(110) Surfaces"

SRL Topical Research Conference on Plasma Etch, Cambridge, Massachusetts, February 1-2, 1989:

L. A. Berry, S. M. Gorbatkin, J. B. Roberto, C. C. Tsai, W. Holber, and J. Yeh, "Characteristics of the ORNL ECR 'Aultiple Plasma Source"

Eighteenth Annual Symposium on Applied Vacuum Science and Technology, Clearwater, Florida, February 6-9, 1989:

J. R. Noonan and H. L. Davis, "Atomic Structure of the $\mathrm{TaC}(100)$ and $\mathrm{HfC}(100)$ Surface" (invited paper)

J. F. Wendelken, "Atomic Step Structure of NiAl Surfaces" (invited paper)

Topical Meeting on Integrated and Guided Wave Optics, Houston, Texas, February 6-8, 1989:

Paul R. Ashley and Darrell K. Thomas, "Low-Loss lon-Implanted Ag Waveguides in Glass"

WATTec '89 16th Annual Technical Conference and Exhibition, Knoxville, Tennessee, February 14-17, 1989:

M. F. Chisholm, "Electron Microscopy of Interfaces in High-Temperature Superconductors" (invited paper)

D. H. Lowndes, D. Eres, D. B. Geohegan, and D. N. Mashburn, "Low-Temperature Growth of Thin Films and Artificially Structured Materials" (invited paper)

J. R. Thompson, "Anisotropic Properties of High- $T_{c}$ Superconductors" (invited paper)

J. M. Williams, "Structure and Properties of Ion Beam Modified Surfaces" (invited paper)

Third Topical Meeting on Microphysics of Surfaces, Beams, and Adsorbates, Salt Lake City, Utah, February 27-March 1, 1989:

R. A. Zuhr, T. E. Haynes, S. J. Pennycook, and B. R. Appleton, "Heteroepitaxial Growth of GaAs Films by Alternating Ion Beam Deposition" 
TMS Annual Meeting, Las Vegas, Nevada, February 27-March 3, 1989:

J. M. Vitek, S. A. David, M. Rappaz, and L. A. Boatnet, "Evaluation of Electron Beam Welds of a Single-Crystal Fe-15Ni-15Cr Alloy"

Symposium on Trends and New Applications in Thin Films, Regensburg, Federal Republic of Germany, February 27-March 3, 1909:

K. Wittmaack, D. Poker, and U. Littmark, "SIMS Depth Profiling of Multilayer Samples of Isotopically Purified Silicon"

American Vacuum Society Symposium on Ion Beam Modification of Materials, Murray Hill, New Jersey, March 8, 1989:

O. W. Holland, C. W. White, S. J. Pennycook, and D. Fathy, Thermal Oxidation of Ion-Implanted $\mathrm{Si}^{\prime \prime}$ (invited paper)

International Symposium on Magnetoelasticity and Electronic Structure of Transition Metals, Alloys, and Films, Duisburg, Federal Republic of Germany, March 20-22, 1999:

J. A. Fernandez-Baca, "Neutron Scattering Studies of Amorphous Invar Alloys"

American Physical Society Spring Meeting, St. Louis, Missouri, March 20-24, 1989:

A. P. Baddorf and J. F. Wendelken, "Molecular Oxygen on Cu(110): The Search Extended to $30 \mathrm{K"}$ [Bull. Am. Phys. Soc. 34, 535 (1989)]

C. Y. Allison, F. A. Modine, M. D. Foegelle, and C. B. Finch, "Electrical Properties of the Group V Transition-Metal Carbides" (Bull. Am. Phys. Soc. 34, 1032 (1989)]

C. Bartges, E. Ryba, and J. Budai, "Anomalous Scattering Investigation of the Structure of Icosahedral $\mathrm{Al}_{6} \mathrm{CuLi}_{3}$ Using Synchrotron Radiation" [Bull. Am. Phys. Soc. 34, 543 (1989)]

R. A. Bartynski, D. Heskett, K. Garrison, G. Watson, D. M. Zehner, W. N. Mei, S. Y. Tong, and X. Pan, "Photoelectron Study of Clean and H-Covered Ta(100)" (Bull. Am. Phys. Soc. 34, 446 (1989)]

J. D. Budai, R. Feenstra, and L. A. Boatner, "X-Ray Diffraction Studies of YBaCuO Thin Films on $\mathrm{SrTiO}_{3}$ and $\mathrm{KTaO}_{3}$ " [Bull. Am. Phys. Soc. 34, 603 (1989)]

L. S. Cain, Y. Chen, ind G. J. Pogatshnik, "Color Center Production by Neutrons in CGG" |Bull. Am. Phys. Soc. 34, 953 (1989)]

Y. Chen, V. M. Orera, R. Conzalez, R. T. Williams, C. P. Williams, Jr., and C. H. Rosenblatt, "Hydride lons and the Charge States of Oxygen Vacancies in Thermochemically Reduced $\mathrm{CaO}$ and MgO" [Bull. Am. Phys. Soc. 34, 1005 (1989)] 
D. K. Christen, R. Feenstra, L. A. Boatnet, C. E. Klabunde, J. D. Budai, M. F. Chisholm, and S. T. Sekula, Effects of Processing Parameters on the Critical Current Density of $\mathrm{Y}_{1} \mathrm{Ba}_{2} \mathrm{Cu}_{3} \mathrm{O}_{7}$ Thin Fims" [Bull. Am. Phys. Soc. 34, 1015 (1989)]

J. F. Cooke and S. H. Liu, Theory of Spin-Modulated Structures in Rare-Earth Materials: Application to Thulium" [Bull. Am. Phys. Soc. 34, 565 (1989)]

J. F. Cordaro and F. A. Modine, "Pulsed Charge Transport in Polycrystalline Materials" (Bull. Am. Phys. Soc. 34, 999 (1989)l

R. Feenstra, L. A. Boatner, J. D. Budai, D. K. Christen, M. F. Chisholm, and M. D. Galloway, "KTaO $-\mathrm{A}$ New Perovskite Substrate for Epitaxially Oriented $\mathrm{YBa}_{2} \mathrm{Cu}_{3} \mathrm{O}_{7-x}$ Thin Films" (Bull. Am. Phys. Sac. 34, 603 (1989)]

J. A. Fernandez-Baca, R. M. NickJow, and J. J. Rhyne, Neutron Scattering Study of the Magnetic Excitations of Thulium" (Bull. Am. Phys. Sac. 34, 607 (1989)

R. S. Fishman and G. D. Mahan, "Divergences in the Force-Balance Theory of Resistivity [Bull. Am. Phys. Soc. 34, 1032 (1989)l

R. S. Fishman and J. A. Sauls, "The Doublet Splitting of the Real Squashing Modes in Superfluid ${ }^{3}$ He-B" [Bull. Am. Phys. Soc. 34, 499 (1989)]

G. R. Gruzalski and D. M. Zehner, "Carbon-Vacancy-Induced Changes in the Charge Distribution in $\mathrm{TaC}_{x}$ and $\mathrm{HFC}_{x}$ " [Bull. Am. Phys. Soc. 34, 921 (1989)]

Theodore Kaplan, M. J. Aziz, and L. J. Gray, "Application of Onsager's Reciprocity Relations to Interface Motion During Phase Transformations" [Bull. Am. Phys. Soc. 34, 404 (1989)]

Y. C. Kim, D. K. Christen, J. R. Thompson, S. T. Sekula, and J. Brynestad, "Anisotropies in the Magnetic Penetration Depth, Lower Critical Field, and Critical Current Density of Aligned $\mathrm{Y}_{1} \mathrm{Ba}_{2}\left(\mathrm{Cu}_{1-\mathrm{x}} \mathrm{Fe}_{2}\right)_{3} \mathrm{O}_{2}$ Powder Composites" [Bull. Am. Phys. Soc. 34, 1014 (1989)]

J. T. Koberstein, S. H. Lee, X. Quan, I. Gancarz, F. Wilson, and G. D. Wignall, "Homopolymer Spatial Distribution in Blends with a Triblock Copolymer" [Bull. Am. Phys. Soc. 34, 711 (1989)]

B. C. Larson, M. D. Galloway, and S. lida, "Asymptotic Diffuse X-Ray Scattering from Coherent Cobalt Precipitates in Cu 1\% Co Single Crystals" [Bull. Am. Phys. Soc. 34, 607 (1989)]

Keh-Gong Lee, J. M. Schultz, and J. S. Lin, "Microstructurzl Development of Poly(Ethylene Terephthalate) Fibers Annealed Under High Threadline Force" [Bull. Am. Phys. Soc. 34, 135 (1989)]

S. H. Liu, "Mixed-Valence and Heavy Electron Materials" [Bull. Am. Phys. Soc. 34, 942 (1989)] (invited paper)

D. H. Lowndes, D. Eres, J. Z. Tischler, and D. B. Geohegan, "Pyrolytic Growth of Epitaxial Cermanium Films from Digermane ( $\mathrm{Ce}_{2} \mathrm{H}_{6}$ )" [Bull. Am. Phys. Soc. 34, 522 (1989)]

J. C. MCCallum, J. Rankin, L. A. Boatner, a ^ C. W. White, "TEM, RBS, and TRR Studies of the Effect of Implanted Species and Ambient Water Vapor on Epitaxial Regrowth of Single-Crystal Perovskites" [Bull. Am. Phys. Soc. 34, 581 (1989)] 
J. W. McCamy, G. E. Jellison, Jr., and D. H. Lowndes, "Fabrication of Ge Themrophotovoltaic (TPV) Cells with Broad Spectral Response by Pulsed-Laser Annealing" [Bull. Am. Phys. Soc. 34, 800 (1989)]

F. A. Modine, M. D. Foegelle, C. B. Finch, and C. Y. Allison, "Electrical Properties of the Group IV Transition-Metal Carbides" [Bull. Am. Phys. Soc. 34, 1032 (1989)]

J. R. Noonan and H. L. Davis, "An Expansion of the Al(110) Surface?" [Bull. Am. Phys. Soc. 34, 447 (1989)]

S. J. Pennycook, Recrystallization of High-Dose Ion-Implanted Silicon" [Bull. Am. Phys. Sac. 34, $581(1989)]$

Sune Pettersson, Thermal Conductivity of Alkali Halide Crystals" (Bull. Am. Phys. Soc. 34, 545 (1989)\}

Mark Rasolt, "Novel Superconductivity in a High Magnetic Field" (Bull. Am. Phys. Soc. 34, 474 (1989)] (invited paper)

B. C. Sales, J. O. Ramey, L. A. Boatnet, and J. C. McCallum, "Structural Inequivalence of the IonDamage-Produced Amorphous State and the Glass State in Lead Pyrophosphate" [Bull. Am. Phys. Soc. 34, 920 (1989)]

S. T. Sekula, B. C. Sales, J. R. Thompson, L. A. Boatner, D. K. Christen, and H. A. Deeds, "Magnetic Studies of Single-Crystal Superconducting $\mathrm{Er}_{1} \mathrm{Ba}_{2} \mathrm{Cu}_{3} \mathrm{O}_{7}$ [ [Bull. Am. Phys. Soc. 34, 928 (1989)]

J. W. Sharp, D. Eres, and D. H. Lowndes, "Optical Monitoring of Heteroepitaxial Thin-Film Growth: Model Calculations and Comparison with Experiment" [Bull. Am. Phys. Soc. 34, 522 (1989)]

H. G. Smith, R. Berliner, J. D. Jorgensen, and M. Nielsen, "Effects of Pressure on the Martensitic Transformation from BCC to 9R to FCC in Lithium" [Bull. Am. Phys. Soc. 34, 769 (1989)]

J. R. Thompson, J. Brynestad, D. M. Kroeger, Y. C. Kim, S. T. Sekula, D. K. Christen, and E. D. Specht, Inter- and Intra-Crain Critical Current Density of $\tau_{2} \mathrm{Ca}_{2} \mathrm{Ba}_{2} \mathrm{Cu}_{3} \mathrm{O}_{10+8}$ and $\mathrm{Tl}_{2} \mathrm{Ca}_{1} \mathrm{Ba}_{2} \mathrm{Cu}_{2} \mathrm{O}_{8+\delta}$ Materials" [Bull. Am. Phys. Soc. 34, 929 (1989)]

J. Z. Tischler, "Nuclear Bragg Scattering from Epitaxial Crystals of ${ }^{57} \mathrm{Fe}_{2} \mathrm{O}_{3}$ " [Bull. Am. Phys. Soc. 34, 892 (1989)]

J. Toulouse, X. M. Wang and L. A. Boatner, "Effect of DC Bias Field on the Ferroelectric Phase Transition of $\mathrm{KTa}_{2-x} \mathrm{Nb}_{2} \mathrm{O}_{3}$ with $x=15.8 \% "$ [Bull. Am. Phys. Soc. 34, 867 (1989)]

G. Vignale and M. Rasolt, "Current- and Spin-Density Functional Theory for Inhomogeneous Electronic Systems in Strong Magnetic Fields" [Bull. Am. Phys. Soc. 34, 998 (1989)]

J. C. Wang "Model for Lithium Intercalation into TiSz" (Bull. Am. Phys. Soc. 34, 870 (1989))

G. P. Williams, Jr., R. M. Sebek, R. T. Williams, R. Conzalez, Y. Chen, and W. Gellerman, "On the 800-nm Luminescence in Neutron-Irradiated MgO Crystals" [Bull. Am. Phys. Soc. 34, 1005 (1989)]

R. F. Wood, Mark Mostoller, and J. F. Cooke, "A Spin Polaron Model of High-T, Superconductivity" [Bull. Am. Phys. Soc. 34, 642 (1989)]

D. M. Zehner and C. R. Gruzalski, "Evidence for Metallic Layer Termination at the HiC(111) Surface from Surface Core-Level Spectroscopy" (Bull. Am. Fhys. Soc. 34, 446 (1989)) 


\section{Seminars}

\section{SOLID STATE DIVISION SEMINARS AT ORNL}

During this period H. A. Mook served as Seminar Chairman October 1987-January 1989, and H. L. Davis, February 1989-continuing. The following seininars were held:

"Why 7 GeV? An Overview of the Advanced Photon Source and Its Applications," David Moncton, Exocon Engineering and Research Laboratory, Annandale, N. J., and Argonne National Laboratory, Argonne, 111 .

"Percolation and Phase Transitions of Interacting Systems," C.-K. Hu, Institute of Physics, Academia Sinica, Taipei, Taiwan

"Applications and Limitations of Deuterium-Labeling Methods in Neutron Scattering Studies of Polymers," G. D. Wignall, Solid State Division, ORNL

"Electron Microdiffraction Applied to Oxygen Precipitates in Silicon," Y. O. Kim, Arizona State University, Tempe, Ariz.

"Devitrification of NiZra: A Time-Resolved X-Ray Scattering Study," Mark Sutton, McGill University, Montreal, Canada

"Zero Sound Mode in Normal Liquid ${ }^{3} \mathrm{He}$, " K. S. Singwi, Northwestern University, Evanston, Ill.

"Decomposition of Gallium Arsenic During Rapid Thermal Processing," Tony Haynes, University of North Carolina, Chapel Hill, N. C.

"Theory of the Fractional Hall Effect," A. H. MacDonald, Indiana University, Bloomington, Ind.

"Density Functional Theory in Strong Magnetic Fields," Giovanni Vignale, The University of Tennessee, Knoxville, Tenn.

"Ion Beam Studies of Amorphous Si, or Laser Annealing Revisited," J. M. Poate, AT\&T Bell Laboratories, Murray Hill, N. J.

"High-Pressure Synthesis of Cubic Boron Nitride," Toshio Kobayashi, Hitachi, Ltd., Tokyo, Japan

"Josephson-Coupled Hign-Temperature Superconducting Oxides," J. T. Chen, Wayne State University, Detroit, Mich.

"Single-Atom Imaging in Scanning Tunneling Electron Microscopy," Morton Lang, IBM T. J. Watson Research Center, Yorktown Heights, N. Y. 
"Structure of Phosphate Cilasses," B. C. Sales, Solid State Division, ORNL

"Magnetism in $\mathrm{La}_{2} \mathrm{CuO}_{4}$ " C. J. Peters, Massachusetts Institute of Technology, Cambridge, Mass.

"High-Temperature Superconductivity Induced by Strong Electron Correlations," Mark Rasolt, Solid State Division, ORNL

"Current Status of the Theoretical Debate on the Mechanism of High-Temperature Superconductivity." C. M. Varma, AT\&T Bell Laboratories, Murray Hill, N. J.

"The Effect of Ion Implantation on the Thermal Oxidation of Silicon," O. W. Holland, Solid State Division, ORNL

"Radiation-Induced Crystal-Amorphous Phase Transition," W. L. Johnson, California Institute of Technology, Pasadena, Calif.

"Resonating Valence Bond on a Square Lattice," Michael Johnson, University of Kentucky, Lexington, Ky.

"Collective Relaxation, Single-Particle Motion, and Short-Range Order in $\alpha^{\prime}-\mathrm{NbD}_{\mathbf{x}}$ : A Quasielastic Neutron Scattering Study," Roth Hempelmann, Kernforschungsanlage, Jülich, FRG

"Back to the Start of Time in the Laboratory-A Case for the Supercollider," Frank Close, The University of Tennessee, Knoxville, Tenn.

"Ion Beam Mixing in the Low-Temperature Regime," M.-A. Nicolet, California Institute of Technology, Pasadena, Calif.

"Channeled Helium Microbeam Analysis of Serniconductors," Jeff McCallum, University of Melboume, Melbourne, Australia

"Superconductivity and Magnetism in High-Temperature Copper Oxides," D. C. Johnston, lowa State University, Ames, lowa

"Scanning REM Study on $\mathrm{MgO}$ Surface Reactions and Scanning TEM Study on $\mathrm{Y}_{1} \mathrm{Ba}_{2} \mathrm{Cu}_{3} \mathrm{O}_{6.9}$ Superconductor," H.-J. Ou, Arizona State University, Tempe, Ariz.

"Critical Exponents for Transport in Two-Dimensional Metallic Films," Miguel Octavio, Venezuelan Institute of Scientific Investigation, Caracas, Venezuela

"Amorphization and Decrystallization by Rapid Thermal Annealing and Laser Annealing of HighEnergy lon-Implanted Si," Rudy Herring, Martin Marietta Laboratories, Baltimore, Md.

"Neutron Spectroscopy at the ISIS Spallation Source," Mark Hagan, Rutherford Appleton Laboratory, Chilton, Didcot, United Kingdom

"Microstructure in Aluminum Alloys Designed for Elevated Temperatures," J. A. Hawk, University of Virginia, Charlottesville, Va.

"The Internal Interfaces in High-Temperature Superconductors," M. F. Chisholm, IBM T. J. Watsion Research Center, Yorktown Heights, N. Y. 
"Atomic Resolution Analytical Microscopy," S. J. Pennycook, Solid State Division, ORNL

Reentrant Phenomenon in Granular Superconductors," Randy Fishman, The University of Tennessee, Knoxville, Tenn.

Diffuse Scattering in Electron Diffraction Studies of Quasicrystals," Kenneth Kelton, Washington University, St. Louis, Mo.

Pattern Formation in Iiquid Crystals," Peter Palffy-Muhoray, Kent State University, Kent, Ohio

"An Electrical Explanation of High-Temperature Superconductivity;" Martin Long, Rutherford Appleton Laboratory, Chilton, Didcot, United Kingdom

"Structure Determination of Silicon Surface by Medium-Energy Ion Scattering," Matthew Copel, IBM T. J. Watson Research Center, Yorktown Heights, N. Y.

"Superconductivity in Composites of High-Temperature Oxides with Metals," H. R. Khan, Forschungsinstitut für Edelmetalle und Metallchemie, Schwäbisch Gmünd, FRG

"Microstructure and Atomic Structure Correlations with Superconducting Properties in Novel HighTemperature Bulk Materials and Thin Films," J. Narayan, North Carolina State University, Raleigh, N.C.

The Effects of Temperature and Orientation on Microstructural Changes During Fatigue of Copper Single Crystals," Laura Lisiecki, Riso National Laboratory, Roskilde, Denmark

"Recent Advances in Atom-Probe Studies of Electronic Materials," A. Cerezo, University of Oxford, Oxford, United Kingdom1

"Wetting Behavior of Binary Liquid Mixtures on Chemically Altered Substrates," Douglas Durian, Cornell University, Ithaca, N. Y.

"What Can Critical Adsorption Experiments Tell Us?" Andrea Liu, University of Maryland, College Park, Md.

"Tunneling Spect:oscopy and the Electron-Phonon Interactions in Vanadium Nitride," John Zasadzinskj, Illinois Institute of Technology, Chicago, Ill.

"Solid State Research at KFA Jülich: GaAs Crystal Growth," Helmut Wenzl, Kernforschungsanlage, Jülich, FRG

"Reflection-Adsorption Infrared Spectroscopy of Adsorbates on a Ni(111) Single-Crystal Surface," Wulf Erley, Kernforschungsanlage, Jülich, FRG

"Composition Distribution and Magnetic Characteristics of Sputtered Permalloy Films" and "BucketType Ion Source for Ion Milling" Y. Nakagawa, Hitachi. Tokyo, Japan

"Experimental Investigations of Surface Melting," Joost Frenken, FOM Institut, Amsterdam, the Netherlands

"Resonant Tunneling in Heterostructures," Mats Jonson, Chalmers Institute of Technology, Cothenberg, Sweden 
"Correlation of Superconducting Temperature with Hole Concentration," Tom Penney, IBM T. J. Watson Research Center, Yorktown Heights, N.Y.

"Matter in Ultrathin Layers: Characterization of Multilayers Useful in X-Ray Optics by Raman Spectroscopy and Low-Angle X-Ray Diffraction," David Allred and Larry Knight, Brigham Young University, Provo, Utah

"Charge and Phonon Transport in Layered Crystals," F. M. Russell, Rutherford Appleton Laboratory. Chilton, Didcot, United Kingdom

New Approach to Highly Correlated Electrons," L. M. Falicov, University of California at Berkeley, Berkeley, Calif.

"Backscattering of Low-Energy Hydrogen from Carbon and Nickel," Wolfgang Eckstein, Max-PlanckInstitut für Plasmaphysik, Garching FRG

"What Does Electronic Structure Fermi-Liquid Theory Tell Us About High-Temperature Superconductivity?" A. J. Freeman, Northwestern University, Evanston, III.

"Surface Modification and Film Formation by the Dynamic Mixing Method IVD and Crystallization of Films," Fuminori Fujinoto, Osaka University, Osaka, Japan

"Crystal Fields in Low Symmetry Systems," Mike Loewenilaupt, Kernforschungsanlage, Jülich, FRG

"Relaxation in Disordered Systems," Derek Walton, McMaster University, Hamilton, Ontario, Canada

"Growth and Physical Properties of Thin-Film Metastable $\mathrm{Cu}_{1-x} \mathrm{Cr}_{x}$ and $\mathrm{Ti}_{0.5} \mathrm{Al}_{0.5} \mathrm{~N}$ Alloys," D. C. McIntyre, University of Illinois, Urbana, Ill.

"Optical Properties and Characterization of Copper Oxide Superconductors," Gary Doll, Massachusetts Institute of Technology, Cambridge, Mass.

"Trapping of Reflected Neutron Rare Gas Species During lon Beam Sputter Deposition of Germanium," Mark Ray, University of Illinois, Urbana, III.

"Self-Organized Criticality," Per Bak, Brookhaven National Laboratory, Upton, N. Y.

"Dynamic Martensites," R. J. Gooding, Comell University, lthaca, N. Y.

"Ultraviolet-Light-Driven Chemical Vapor Deposicion of Callium Arsenide," David Norton, Louisiana State University, Baton Rouge, La.

"Low-Temperature Deposition of $\mathrm{SiO}_{2}$ Films from ECR Microwave Plasmas," T. V. Herak, Materials and Devices Research :aboratory, University of Manitoba, Winnipeg, Canada

"Effects of Cranularity in High-Temperature Superconductors," J. R. Clem, Jowa State University, Ames, lowa

"Phase Transitions and Critical Phenomena in Polymer Blends," Dietmar Schwahn, Kernforschungsanlage, Jülich, FRC 
"Parastatistics and the Highly Correlated Two-Dimensional Hubbard Model," Eugene Mele, University of Pennsylvania, Philadelphia, Pa.

Problems and Opportunities in Surface Modification of Materials," Keith Legz, lonic Atlanta, Inc., Atlanta, Ca.

1Metals and Ceramics Division and Solid State Division Joint Seminar.

\section{LECTURES AND SEMINARS}

B. R. Appleton-Massachusetts Institute of Technology, Cambridge, Massachusetts, "Ion Beam and Laser Processing for Materials Research"

J. B. Bates-The University of Tennessee, Knoxville, Tennessee, Surface Topography and Electrical Response of Metal-Electrolyte Interfaces"

L. A. Boatner-Vanderbilt University, Nashville, Tennessee, Texturing Effects in Oxide Ceramics"; Florida State University, Tallahassee, Florida, The Formation and Characterization of Textured Ceramics"; Industrial Research Institute, Management Development Seminar, St. Petersburg, Florida, "Employee Recourse in the Performance Appraisal Process"

I. D. Budai-Harvard University, Cambridge, Massachusetts, "X-Ray Diffraction Study of Phason Defects in Al-Mn Quasicrystals"

G. J. Bunick-Baylor College of Medicine, Houston, Texas, "X-Ray Crystal Structure of the Nucleosome at 8-A Resolution"; University of Pennsylvania, "X-Ray Crystallography and Neutron Scattering Studies of the Nucleosome"

H. L. Davıs-Institut für Angewandte Physik, Universität Erlangen, FRG, "LEED Crystallographic Studies of Metallic Surfaces at Oak Ridge-From Cu(110) to NiAl(111) and HfC(100)"; Max-PlanckInstitut für Strömungforschung, Cöttingen, FRG, "Atomic Structure of Binary Alloy Surfaces"; University of Aarhus, Aarhus, Denmark, "Oak Ridge LEED Studies of Metallic Surface Relaxation-From Cu(100)to NiAl(111) and Beyond"; Institut für Grenzlachenförschung and Vakuumphysik, Kernforschungsanlage, Jülich, FRC, "LEED Studies of the Relaxation of Metallic Surfaces"; University of Califomia, Berkeley, California, "Atomic Structure of Metallic Surfaces via LEED Analysis"; Sandia National Laboratories, Livermore, California, "LEED Studies of Metallic Surface Relaxation"

N. I. Dudney-Clemson University, Clemson, South Carolina, and University of North Carolina, Chapel Hill, North Carolina, "Enhanced Ionic Conductivity in Composite Electrolytes"; Kodak Corporate Research Laboratories, Rochester, New York, "Enhanced Ionic Conduction in Silver Halide Composites"

R. S. Fishman-Southem Illinois University, Carbondale, Illinois, Illinois Institute of Technology, Chicago, Illinois, University or Central Florida, Orlando, Florida, University of Rhode Island, Kingston, Rhode Island, and State University of North Dakota, Fargo, North Dakota, "Re-entrant Behavior in Granular Superconductors" 
J. B. Hayter-Chlorox Technical Center, Pleasanton, California, 'Scattering Measurements of Micellar Structures"

H. R. Kerchner-Clemson University, Clemson, South Carolina, "Current-Carrying Capacity of HighField Superconductors"

J. S. Lin-The University of Tennessee, Knoxville, Tennessee, Recent Applications of Small-Angle Scattering of X-Ray Neutrons"

S. H. Liu-Wayne State University, Detroit, Michigan, University of Louisville, Louisville, Kentucky, University of California, Davis, California, and The University of Tennessee, Knoxville, Tennessee,

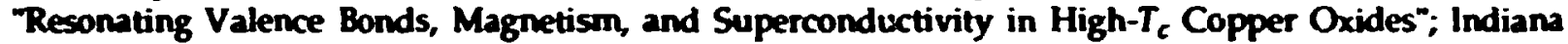
University, Bloomington, Indiana, Fractals in Condensed Matter Physics"; University of Houston, Houston, Texas, "Magnetism and Superconductivity in High- $T_{c}$ Copper Oxides"; Indiana University, Bloomington, Indiana, "New Mechanism for Mass Enhancement in Mixed-Valence and Heavy Electron Materials"

D. H. Lowndes-Martin Marietta Research Laboratories, Baltimore, Maryland, "Photon-Controlled Deposition of Thin Films and Multilayered S! :uctures"; The University of Tennessee, Knoxville, Tennessee, "Low-Temperature Vapor-Phase Growth of Thin Films and Multilayered Structures Using Photon-Controlled Reactions"

G. D. Mahan-European Conference on Thermal Physics, Umea, Sweden, "Nonlocal Theory of Thermal Conductivity"; Enrico Fermi School, Varenna, Italy, "Theory of Photoemission" (3 lectures); General Electric Research Center, Schenectady, New York, Thermoelectrics for Refrigeration"; Catholic University, Santiago, Chile, Ohio State University, Columbus, Ohio, and Canadian National Research Council, Ottawa, Canada, "Hot Electrons"; Catholic University, Santiago, Chile, "High-Temperature Superconductivity"; Centro Instituto Fisica Material Condensada, Brazilia, "Transport in Semiconductors" (4 lectures)

D. N. Mashburn-AT\&T Bell Comrinnications Research Laboratory, Red Bank, New Jersey, "Laser Ablation of Thin Superconducting Oxide Films"

5. J. Pennycook-Cornell University, lthaca, New York, AT\&T Bell Laboratories, Murray Hill, New Jersey, and The University of Tennessee, Knoxville, Tennessee, "Chemically Sensitive High-Resolution، Imaging by STEM: Semiconductors and Superconductors"

D. B. Poker-University of South Florida, Tampa, Florida, "Formation of Optical Waveguides by Ion Implantation"; The University of Tennessee, Knoxville, Tennessee, "Effects of Different Ion-Implanted Dopants on the Solid-Phase Epitaxy of $\mathrm{LiNbO}_{3}$ "; Lawrence Livermore National Laboratory, Livermore, California, "lon-Solid Interactions at ORNL"

Mark Rasolt-Centre Européen de Calcul Atomique et Moléculair, University uf Paris, Otsay, France, "Functional Density Theory in High Magnetic Fields," "Effect of the Plasma Environment on the Optical Absorption," and "Superconductivity in High Magnetic Fields"; Max-Planck-Institut, Stuttgart, FRC, Kemforschungsanlage, Jülich, FRG, IBM, Yorktown Heights, New York, Harvard University, Cambridge, Massachusetts, Massachusetts Institute of Technology, Cambridge, Massachusetts, "Functional Density Theory in High Magnetic Fields"; Institute of Theoretical Physics, Trieste, Italy, University of Pavia, Pavia, Italy, and Ecole Normal Superior, Paris, France, "Superconductivity in High Magnetic Fields"; Cornell University, University of Missouri, Columbia, Missouri, and Johns Hopkins University, Baltimore, Maryland, "New Type of Superconductivity in Strong Magnetic Fields"; 
Ohio State University, Columbus, Ohio, Indiana University, Bloomington, Indiana, and University of Missouri, Columbia, Missouri, "High- $T_{c}$ Superconductivity with the Anderson Lattice Hamiltonian"

M. T. Robinson-Simon Fraser University, Burnaby, British Columbia, The Computer Simulation of Atomic Collisions in Crystalline Solids in the Binary Collision Approximations"

J. R. Thompson-The University of Tennessee, New High-Temperature Superconductors: An Increasingty Hot Topic"; Naval Research Laboratory, Washington, D. C., "Experimental Perspectives on High$T_{c}$ Materials via Magnetic Investigations"

J. Z. Tischler-Bucknell University, Lewisburg, Pennsylvania, Time-Resolved X-Ray Study of Cermanium During Pulsed-Laser Melting"

John F. Wendelken-Universität Munich,Munich, FRG, Max-Planck Institut für Stromungsforschung, Cottingen, FRG, and Universität Erlangen, Nürnberg, FRG, "Atomic Step Studies on Metal Surfaces via Electron Scattering"

D. M. Zehner-Vanderbilt University, Nashville, Tennessee, "Ceometric Structure and Oxidation of NiAl Surfaces" 


\section{Scientific Activities, Awards, and Honors}

M. M Abraham

J. B. Bates

L. A. Boatner

J. D. Budai
Adjunct Profeseor, Universidad Nacional Autonoma de Mexico

Member, Review Panel, U.S. DOE Review of Small Business Innovation Research, Bethesda, Maryland, 1987.

Chairman, Gordon Research Conference on Solid State lonics (to be held in 1989)

Member, International Scientific Committee, 7th International Conference on Solid State lonics (to be held in 1989)

Organizer, Symposium on Electronic Ceramic Thin Films, Materials Physics Topical Group, American Physical Society, St. Louis, Missouri, 1989

Adjunct Professor, The University of Tennessee, Knoxville, Tennessee

Session Chairman, 41st Pacific Coast Regional Mceting, Northern California Section of the American Ceramic Society, San Francisco, California, 1988

Recipient, American Association for the Advancement of Science Fellowship, 1988

Recipient, The Francis F. Lucas Award of the American Society for Metals International, 1988

Recipient, The Pierre Jacquet Gold Medal Award of the International Metallographic Society, 1988

Recipient, Martin Marietta Energy Systems Technical Achievement Award, 1988

Recipient, Martin Marietta Energy Systems Significant Fvent Award, 1988

Recipient, Texas Tech University, Distinguished Alumnus Award in Physics, 1989

Member, Executive Committee, American Association for Crystal Growth

Chaiman, Membership Committee, Materials Research Society

Associate Editor, Materials Letters

Session Chairman, American Physical Society Meeting, St. Louis, Missouri, 1989

Co-Organizer and Session Chaiman, Focused Session on Quasicrystals, American Physical Society, New Orleans, Louisiana, 1988

Lecturer, Society of Physics Students Speakers Program

Recipient, Martin Marietta Energy Systems Publication Award, 1988 
G. J. Buruick

J. W. Cabie

y. Chen

D. K. Christen

J. F. Cooke

H. L. Davis
Adjunct Associate Professor, The University of Tennessee-Oak Ridge Graduate School of Biomedical Sciences

Member, Thesis Committee, Department of Biophysics, John Hopkins University, Baltimore, Maryland

Member, OHER/DOE Structural Biology Initiative Planning Workshop, Washington, D. C., 1988

Recipient, Martin Marietta Energy Systems Technical Achievement Award, 1989

Adjunct Professor, North Carolina State University, Raleigh, North Ca! olina

Member, Publications Committee, Materials Research Society

Member, Ph.D. Examination Committee, North Carolina State University, Raleigh. North Carolina

Member, M.Sc. Examination Committee, Wake Forest University, Winston Salem, North Carolina

Member, Review Committee for the Australian Government Grant Scheme, 1989

Recipient, Martin Marietta Energy Systems Significant Event Award, 1987

Adjunct Professcr, Department of Physics, The University of Tennessee, Knoxville, Tenr.essee

Session Chairman, Applied Superconductivity Conference, San Francisco, California, 1988

Recipient, Martin Marietta Eriergy Systems Technical Achievement Award, 1958

Member, Organizing Committee, Symposium on High-Temperature Superconductivity, Materials Research Scciety (to be he.d in 1989)

Recipient, American Physical Society Fellowship, 1987

Lecturer, ORAU Traveling Lecture Program, 1987-1988

President, Tennessee Valley Chapter, American Vacusum Society, 19881989

Recipient, Martin Marietta Energy Gystems Publication Award, 1987

Treasurer, Seventh Annual Symposium and Equipment Exhibit of the Tennessee Valley Chapter, American Vacuum Society. Oak Ridge, Tennessee, 1987

Vice-President, Tennessee Vailey Chapter, American Vacuum Socieiy, 1987-1988

Treasurer, Local Arrangements Committee, 35th National Symposium, American Vacuum Society, 1987-1988

Chairman, Solid State Division Cornmittee to Provide a Response to 1926 Martin Marietta Energy Systems Employee Communications Survey 
N. J. Dudney

D. J. Eres

C. B. Finch

R. S. Fishman

P. H. Green

G. R. Gruzalski

H. E. Harr:on

J. B. Hayter

G. E. Jellison, Jr.

T. Kaplan
Associate Editor, loumal of the American Ceramic Society Abstracter, American Ceramic Jociety

Judge, Southem Appalachia Science and Engiveenng Fair, 1988

Recipient, Martin Marietta Energy Systems Technical Achievement Award 1989

Recipient, Martin Marietta Energy Systems Significant Event Award, 1987

Recipient, Martin Marietta Energy Systems Technical Achievement Award, 1988

Session Chairman, American Physical Society Meeting, St. Louis, Missouri, 1989

Conference Coordinator, Conference on Ion Beam Modification of Materials (to be held in 1990)

Member, ORNL Committee on Improvement of Work Areas, 1987-1988

Member, Exploratory Studies Committee, ORNL, 1988-1989

Recipient, American Association for Crystal Growth Competition, Second Place Overall Award

Recipient, Martin Marietta Energy Systems Administrative Support/Technical Support Award, 1989

Recipient, Martin Marietta Energy Systems Inventor Award, 1988

Executive Member, National Steering Committee for the Advanced Neutron Source, 1987-1989

Member, LANSCE Advisory Committee, Los Alamos National Laboratory, 1987-1989

Session Chairman, 19th Yamada Conference on Ordering and Organization in Ionic Solutions, Kyoto, Japan, 1987

Manager, Scientific Applications, Advanced Neutron Source Project, 1989

Member, Editorial Board, Journal of Physics: Condensed Matter (Liquids), 1988-1989

Member, DOE Review Committee on HFBR Neutron Beam Lines and Facilities Upgrade, Brookhaven National Laboratory, Upton, New York, June 1988

Lecturer, ORAU Traveling Lecturer Program, 1988-1989

Recipient, Martin Marietta Energy Systems Publication Award, 1988

Recipient, Martin Marietta Energy Systems Technical Achievement A ward, 1989

Session Chairman, American Physical Society Meeting, St. Louis, Missouri, 1989 
B. C. Larson

\section{J.S. Lin}

S. H. Liu

D. H. Lowndes

G. D. Mahan

D. N. Mashburn

H. A. Mook
Member, Program Advisory Committee, Cornell High Energy Synchrotron Source

Member, Selection Committee, 1988 Bertram Eugene Warren Diffraction Physics Award

Co-Organizer, Symposium on Characterization of the Structure and Chemistry of Defects in Materials, Materials Research Society, Boston, Massachusetts, 1988

Co-Editor, Characterization of the Structure and Chemistry of Defects in Materials, Materials Research Society, Boston, Massachusetts, 1989

Session Chairman, Materials Research Society Meeting, Boston, Massachusetts, 1988

Adjunct Professor, Department of Chemical, Metallurgical, and Polymer Engineering, The University of Tennessee, Knoxville, Tennessee

Chairman, American Crystallographic Association, Small-Angle Scattering Special Interest Group Executive Committee, 1989-1990

Chairman, Panel Discussion, DOE University Instrumentation Program, $X$-Ray Diffraction and Scattering (to be held in 1989)

Recipient, Martin Marietta Energy Systems Publication Award, 1987

Member, Executive Committee, Division of Condensed Matter Physics, American Physical Society, 1985-1987

Recipient, Corporate Fellowship, Martin Marietta Energy Systems, Inc., 1989

Adjunct Professor of Materials Science, The University of Tennessee, Knoxville, Tennessee

Member, Review Panel, National Science Foundation Science and Technology Centers Program, 1988

Recipient, Martin Marietta Energy Systems Publication Award, 1988

Recipient, Martin Marietta Energy Systems Significant Event Award, October 1987

Member-at-Large, Executive Committee, Division of Condensed Matter Physics, American Physical Society, 19d9-1991

Recipient, Centennial Medal, Catholic University of Chile, January 1989

Member, National Science Foundation Review Panel of Science and Technology Centers, 1988

Chairman, Swedish Committee to Select Professor in Nonlinear Optics for Linköping University

Chairman, Scientific Committee, International Centre of Condensed Matter Physics, Brazil, 1989-1992

Director of Workshop, International Centre of Condensed Matter Physics, Brazil, January 1989

Recipient, Martin Marietta Energy Systems Publication Award, 1988

Recipient, Martin Marietta Energy Systems Significant Event Award, October 1987

Recipient, Martin Marietta Energy Systems Inventor Award, 1988 
J. R. Noonan

O.S. Oen

S. J. Pennycook

D. B. Poker

M. Rasolt

J. B. Roberto
Chairman, Scholarship Committee, Tennessee Valley Chapter, American Vacuum Society

Member, Executive Board, Tennessee Valley Chapter, American Vacuum Society, 1985-1988

Chairman, Publications Committee, American Vacuum Society, 19851987

Secretary-Treasurer, Surface Science Division, American Vacuum Society

Member, Subcommittee on Joumals, American Institute of Physics

Chairman, Editorial Board, American Vacuum Society, 1987

Chairman, Program Committee, Thirty-Fifth National Symposium of the American Vacuum Society, Atlanta, Georgia, 1988

Member, Board of Directors, American Vacuum Society, 1989-1990

Session Chairman, Twelfth Intermational Conference on Atomic Collisions in Solids, Okayama, Japan, 1987

Session Chairman, Theoretical (Computational) Symposium on Atomic Collisions in Solids, Tokyo, Japan, 1987

Recipient, Martin Marietta Energy Systems Publication Award, 1988 Recipient, Martin Marietta Energy Systems Publication Award, 1989

Recipient, Martin Marietta Energy Systems Significant Event Award, 1988

Member, Program Committee, Ion Beam Modification of Materials Conference (to be held in 1990)

Co-Chairman, Symposium on Optical Materials: Processing and Science, Materials Research Society, San Diego, California, 1989

Lecturer, ORAU Traveling Lecture Program, 1987-1989

Recipient, Martin Marietta Energy Systems Publications Award, 1988

Recipient, Martin Marietta Energy Systems Author of the Year Award, 1988

Guest Scientist, Centre Europeen de Calcul Atomique et Moleculaire, Orsay, France, May-September 1988

Visiting Scholar, Harvard University, October 1988-February 1989

Second Vice President, Materials Research Society, 1989

Co-Chairman, Symposium oul Ion Beam Processing of Advanced Electronic Materials, Materials Research Society, San Diego, Califomia, 1989

Chairman, ORNL Global Competitiveness Lecture Series, 1988-1989

Chairman, Program Committee, Materials Research Society, 1988

Recipient, Boehmische Physical Society Scientific Membership, 1988

Member, Site Visiting Committee, University of Toronto Institute for Aerospace Studies, Natural Sciences and Engineering Research Council, Canada, 1988

Member, Publications Awards Committee, Martin Marietta Energy Systems, Inc.

Member, ORNL Committee on Criteria for Ph.D. Recruiting and Hiring Member, Editorial Advisory Board, Journal of Nuclear Materials 
B. C. Sales

S. T. Sekula

H. G. Smith

S. Spoonet

J. R. Thompson
J. Z. Tischler
J.C. Wang
J. F. Wendelken

C. W. White
Lecturer, ORAU Traveling Lecture Program, 1987-1988

Adjunct Professor, Department of Physics, The University of Tennessee, Knoxville, Tennessee

Member, Editorial Board, Journal of Superconductivity

Session Chairman, American Physical Society Meeting, New Orleans, Louisiana, 1988

Session Chairman, Applied Superconductivity Conference, San Francisco, California, 1988

Session Chairman, American Physical Society Meeting, St. Louis, Missouri, 1989

Chairman, Neutron Diffraction Commission, International Union of Crystallography, 1987-1990

Recipient, Martin Marietta Energy Systems Technical Achievement Award, 1988

Adjunct Professor of Materials Engineering, Ceorgia Institute of Technology, Atlanta, Georgia

Member, Proposal Review Committee, NSERC, Canada

Chairman, Chemistry and Physics of Materials Committee, TMS, AIME

Member, Structures Activity, MSD, American Society for Metals

Professor of Physics, The University of Tennessee, Knoxville, Tennessee Member, Special Review Panel for High-T $C$ Superconductivity, Applied Physics Letters

Recipient, Martin Marietta Energy Systems Publication Award, 1988

Lecturer, ORAU Traveling Lecture Program, 1989-1990

Member, Executive Board, Tennessee Valley Chapter, American Vacuum Society, 1985-1988

Chairman, Local Arrangements Committee, 35th National Symposium of the American Vacuum Society, Atlanta, Georgia, 1988

Vice-President, Tennessee Valley Chapter, American Vacuum Society, Knoxville, Tennessee, 1988-1989

Co-Chairman, Ion Beam Modification of Materials Conference (to be held in 1990)

Councillor, Materials Research Society, 1987-1989

Principal Editor, Journal of Materials Research, 1987-1989

Member, Editorial Board, Materials Letters, 1987-1989

Member, International Materials Research Committee, External Affairs Committee, and Long-Range Planning Committee, Materials Research Society, 1987-1989 
G. D. Wignall

M. K. Wilkinson

J. M. Williams

S. P. Withrow

R. F. Wood
Adjunct Professor, Department of Chemical, Metallurgical, and Polymer Engineering, The University of Tennessee, Knoxville, Tennessee

Member, Board of Directors, American Crystallographic Asscciation Special Interest Group on Small-Angle Scattering, 1985-1988

Lecturet, ORAU Traveling Lecture Program, 1985-1986

Member, Editorial Board, Macromolecules

Executive Secretary, DOE Basic Energy Sciences Laboratory Program Panel

Member, DOE Basic Energy Sciences Council on Materials Sciences

Adjunct Professor, School of Physics, Georgia Institute of Technology, Atlanta, Georgia

Consultant to Steering Committee, U.S.Japan Cooperative Program on Neutron Scattering

Member, DOE Basic Energy Sciences Neutron Scattering Facilities Review Committee

Member, ORNL Emergency Review and Advisory Committee

Member, ORNL Steering Committee, Advanced Neutron Source

Secretary, National Steering Committee for an Advanced Neutron Source (NSCANS)

Secretary, NSCANS Executive Committee

Chairman, ORNL Research Reactor Users Committee

Member, ORNL Vulnerability Assessment Committee

Chairman, ORNL Committee on Performance, Reward, and Integrity

Member, Panel for Reactor Radiation, National Institute of Siandards and Technology

Recipient, Martin Marietta Energy Systems Operational Performance Award, 1989

Member, ORNL Commitiee on Visitor Interactions

Member, Search Committee for ORNL Director, 1988

Member, ORNL Technical Audit Review Board

Member, International Organizing Committee, Surface Modification of Metals by lon Beams Conference (to be held in 1991)

Member, Program Committee, lon Beam Modification of Materials Conference (to be held in 1990)

Member, Program Committee, 7th International Conference on Ion Implantation Technology, Kyoto, Japan, June 1988

Guest Scientist, Max-Planck-Institut für Plasmaphysik, Garching, Federal Republic of Cermany, March-August 1989

Rexipient, Martin Marietta Energy Systems Publication Award, 1988

Recipient, Martin Marietta Energy Systems Scientific Achievement Award, 1987

Member, Corporate Fellows Advisory Committee on Science and Technology

Member, Wigner Fellowship Committee, ORNL

Member, ORNL-UT Distinguished Scientist Committee

Recipient, Senior Distinguished Scientist Award from the Alexander von Humbolt Foundation, Federal Republic of Cermany 
Guest Scientist, Universität Erlangen, Nümberg, Federal Republic of Germany, July 1988-July 1989

F. W. Young, Ir.

D. M. Zehnet

R. A. Zuhr
Recipient, Martin Marietta Energy Systems Operational Performance Award, 1988

Member, Executive Committee, American Association for Crystal Growth

Member, Editorial Board, Topics in Crystal Growth

Councillor, Materials Research Society, 1987

Member, External Affairs Committee, Publications Committee, Awards Committee, Long-Range Planning Committee, and Von Hippel Selection Committee, Materials Research Society

Examining Board, Joumal of Materials Research

Member, Committee for Intemational Prize for New Materials, American Physical Society

Member, Materials Science Advisory Committee, Southeastern Universities Research Association

Session Chairman, American Physical Society Meeting, New Orleans, Louisiana, 1988

Chairman, Invited Paper Session, American Physical Society Meeting, St. Louis, Missouri, 1989

Co-Editor, Physical and Chemical Properties of Thin Metal Overlayer and Alloy Surfaces, Materials Research Society, Pittsburgh, Pennsylvania, 1987.

Program Chairman, Tennessee Valley Chapter, American Vacuum Soriety, 1988

Co-Chairman, Symposium on Beam-Solid Interactions: Physical Phenomena, Materials Research Society, Boston (to be held in 1989) 


\section{Personnel Changes}

\section{New Strff Members}

A. Scientific Staff

A. P. Baddorf, University of Pennoylvania, Philadelphia, Pennsylvania

B. C. Chakoumakos, University of New Mexico, Albuquenque, New Mexico

M. F. Chisholm, IBM, Yorktown Heights, New York

R. Feenstra, Kernforschungsanlage, Jülich, Federal Republic of Germany

S. M. Corbatkin, University of Illinois, Urbana, Ilinois

T. E. Haymes, University of North Carolina, Chapel Hill, North Carolina

B. Administrative and Technical Support Staff

E. A. Hill, Secretary

C. F. Luck, Laboratory Technician

\section{Staff Trunstern and Termination}

A. Scientific Staff

B. R. Appleton (transferred to Central Management Offices)

D. Fathy (completion of temporary assignment)

B. D. Caulin (completion of temporary assignment)

M. Prakash (completion of temporary assignment)

B. Administrative and Technical Swpport Staff

M. A. Gillespie (transferred to Health and Safety Research Division)

S. A. Richardson (transferred to Chemical Technology Division)

R. B. Wheeler (voluntary resignation)

\section{Guest Assienments}

\section{A. Scientific Staff}

C. Y. Al'ison, Oklahoma State University, Stillwater, OkJahoma

A. Chatelain, Ecole Polytechnique, Lausanne, Switzerland

C. Y. Chen, North Carolina State University, Raleigh, North Carolina

F. H. Claro, Catholic University, Santiago, Chile

R. S. Fishman, The University of Tennessee, Knoxville, Tennessee 
G. D. Mahan*, The University of Tennessee, Knoxville, Tennessee

P. Mazumdar, Perkin-Elmet Corporation, Danbury, Connecticut

P. M. L. Minnehagen, The University of Tennessee, Knoxville, Tennessee

K. Müller, Universität Erlangen, Nümberg, Federal Republic of Germany

F. Perrot, University of Paris, Paris, France

S. Pettersson, The University of Tennessee, Knoxville, Tennessee

G. J. Pogatshnik, North Carolina State University, Raleigh, North Carolina

B. Sernelius, The University of Tennessee, Knoxville, Tennessee

S. Tanaka, Kyoto University, Sakyo, Japan

G. D. Vignale, The University of Tennessee, Knoxville, Tennessee

J. L. Zarestky, Ames Laboratory/lowa State University, Ames, lowa

D. L. Zimmerman, The University of Tennessee, Knoxville, Tennessee

'ORNL/UT Distinguished Scientist

B. Oak Ridge Associated Universities Postgraduate Research Participation Program

M. K. El-Ghor, North Carolina State University, Raleigh, North Carolina

D. E. Jesson, University of Bristol, Bristol, England

S. C. Lui, University of Pennsylvania, Philadelphia, Pennsylvania

J. C. McCallum, University of Melbourne, Melbourne, Australia

C. Graduate Students

G. S. Canright, The University of Tennessee, Knoxville, Tennessee

H. A. Deeds, The University of Tennessee, Knoxville, Tennessee

M. K. El Ghor, North Carolina State University, Raleigh, North Carolina

Y. C. Kim, The University of Tennessee, Knoxville, Tennessee

A. Kirtikar, State University of New York-Stony Brook, Stony Brook, New York

J. W. McCamy, The University of Tennessee, Knoxville, Tennessee

J. Ossandon, The University of Tennessee, Knoxville, Tennessee

J. L. Park, North Carolina State University, Raleigh, North Carolina

J. Rankin, Massachusetts Institute of Technology, Cambridge, Massachusetts

B. Sanborn, State University of New York-Stony Brook, Stony Brook, New York

J. Sharp. The University of Tennessee, Knoxville, Tennessee

F. Triolo, University of Palermo, Palermo, Italy

D. Undergraduate Students

S. H. Choo, Coe College, Cedar Rapids, lowa

P. S. Ebey, St. Olaf College, Northfield, Minnesota

J. C. Lee, Massachusetts Institute of Technology, Cambridge, Massachusetts

\section{Summer Assienments (1988)}

\section{A. Scientific Staff}

S. Funahashi, Japan Atomic Energy Research Institute, Tokai-mura, Japan

M. Long, Rutherford Appleton Laboratory, Chilton, Didcot, England

M. Rappaz, Ecole Polytechnique, Lousanne, Switzerland

R. Triolo, University of Palermo, I'alermo, Italy 
B. Administrative and Tecturical Support Staff

R. Etienne-Cummings, Lincoln University, Lincoln University, Pennsylvania

M. D. Foegelle, Oklahoma State University, Stillwater, Oklahoma

D. E. Hoglund, Virginia Polytechnic Institute and State University, Blacksburg, Virginia

B. E. Kelly, The University of Tennessee, Knoxville, Tennessee

C. Oak Ridge Associated Universities-Faculty Reseanch Participants

C. Y. Allison, Oklahoma State University, Stillwater, Oklahwma

M. J. Aziz, Harvand University, Cambridge, Massachusetts

W. R. Major, University of Richmond, Richmond, Virginia 


\section{SOLID STATE DIVISION}

April 1, 1989

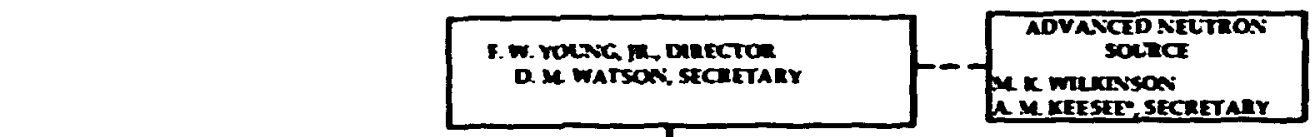

\begin{tabular}{|c|c|}
\hline 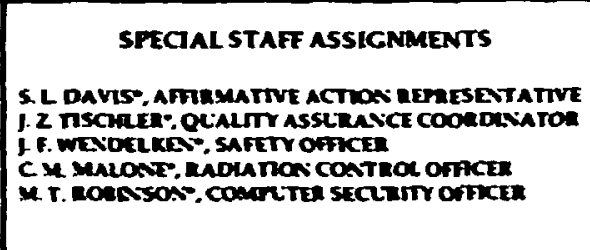 & 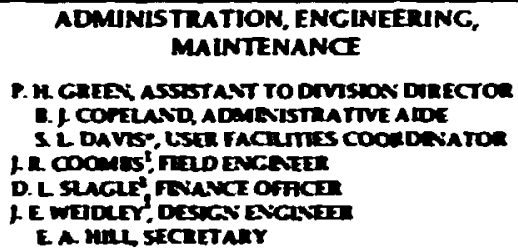 \\
\hline
\end{tabular}

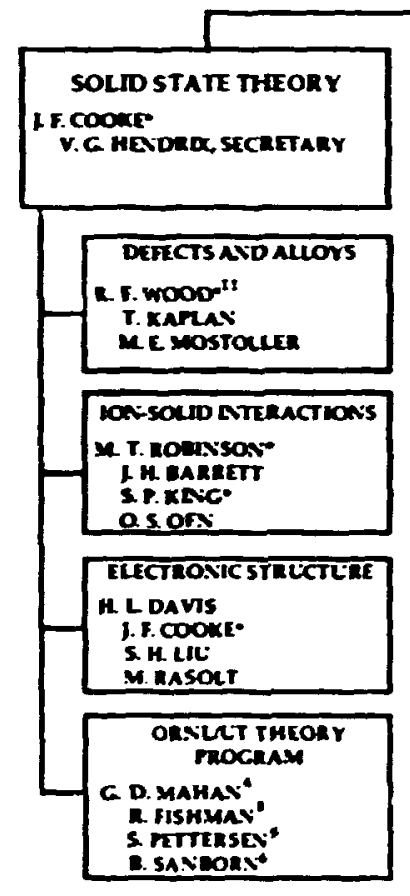

- MUlTIPLE CAPACTTY

PLANT AND EQUITMENT DIVISION

- FINANCE AND MATERIALS DIVISION

- PART-tIME EMPLOYMENT

- ORNLUT DISTINCUISMED SCIENTIST

- CUEST SCIENTIST

- GRaduate STUDENT

' COMPUTING AND TLLECOMMUNICATIONS DIVISION

ON ASSIGNMENT FROM AMES LABORATORY

- ON LOAN TO ORCDP

IORAU POS TDOCTORAL RESEARCH ELLLW

'ON FOREICN ASSICNMENT

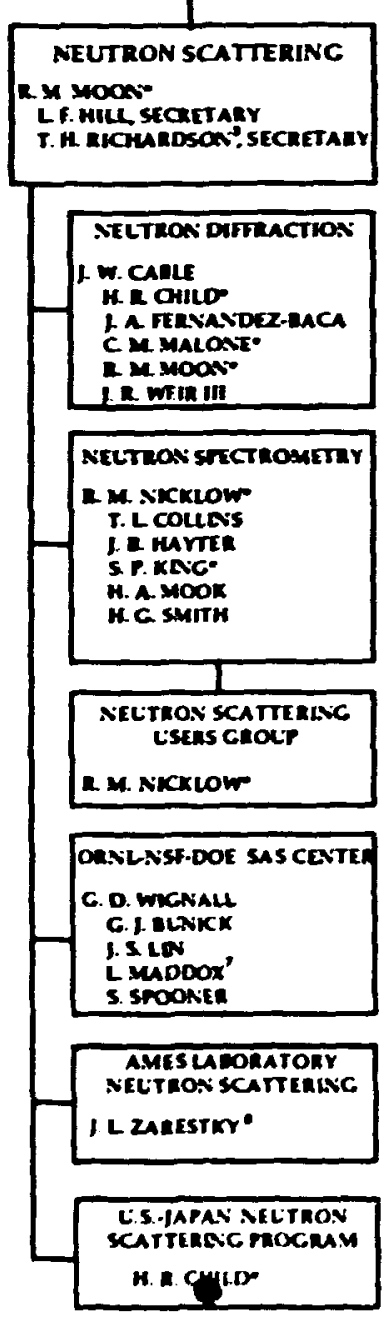

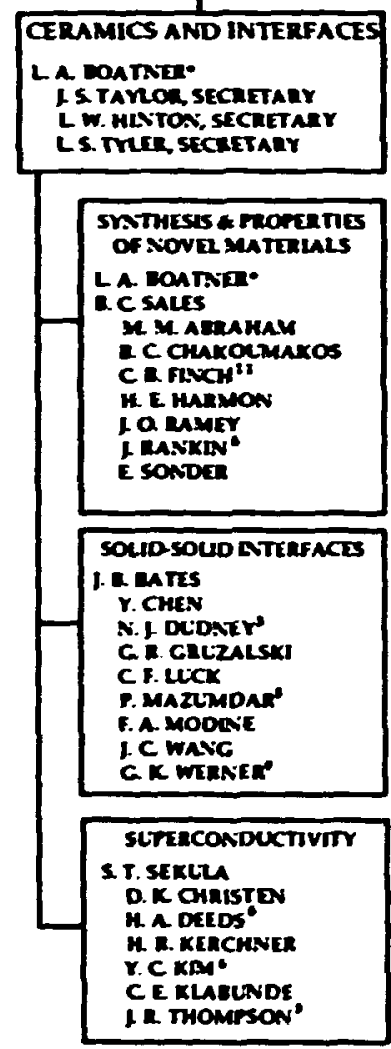

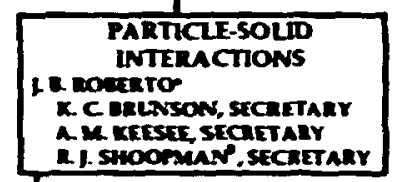

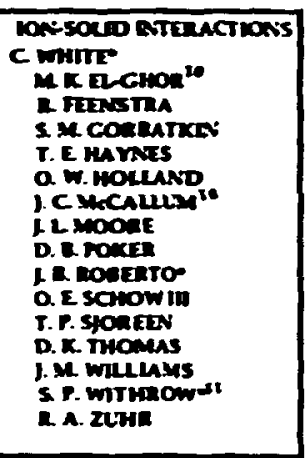

SETBACE WODTCATIOA asd ChaRaCTERIzation Faciury

c. w. mutr i. minthowal

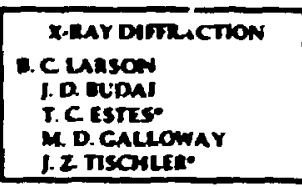

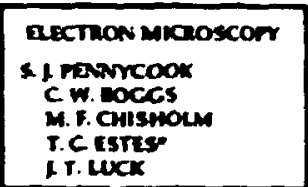

smaconoucton rimsico ANo MoTOVOLTAKC

matenials

D. H. LOWvis

a. 7. wo00'

D. I. Enes

P. H. RITManc.

D. CLONECAN

C. E Iu usow, je.

D. N. Mashduns. J. W. MCcam

\section{suAface mursics}

D. M. ZTHNE

a. padponi

3 C Luito

1. NOONAN

C W OWNar

f. WENDILKIN- 


\title{
Author Index
}

\author{
Abraham, M. M.-66, 90, 91 \\ Aeppli, G.- 14 \\ Allison, C. Y. -65 \\ Anderson, S. K-83 \\ Aziz, M. J.-21 \\ Baddorf, Arthur P.-142, 143 \\ Ballesteros, C.-94 \\ Baribeau, J. M.-150 \\ Barrett, J. H.-23, 24, 25 \\ Bartynski, R. A.-138 \\ Bates, J. B. -81, 82, 83, 86, 87 \\ Bergman, L. B-63. \\ Berliner, R. -12 \\ Berry, L. A.-109 \\ Bimbaum, H. K.-37 \\ Blackman, J. A. $-6,7$ \\ Boatnet, L A.-54, 55, 56, 67, 68, 69, 75, 78, 90, \\ $91,118,119,146,158,163,164$ \\ Boldu, J. L. 90 \\ Brown, Hugh R.-31 \\ Brynestad, J. - 55, 56, 60 \\ Buchanan, R. A. -107 \\ Budai, J. D. - 56, 58, 75, 78, 158, 160 \\ Cable, J. W.-39, 40, 46, 47, 48 \\ Cain, L. S. -95 \\ Caponetti, E.-36 \\ Chakoumakos, B. C. $-57,58,59,68,69$ \\ Chang, Y. K. -66 \\ Chaplin, R. L. -70 \\ Charles, Stuart-32 \\ Chen, S. H. -34 \\ Chen, $Y .-69,92,93,94,95$ \\ Cheong, S.-W.- 44 \\ Child, H. R. - 39, 40 \\ Chisholm, M. F.-75, 77, 148, 151, 152 \\ Choi, S.-1. -63 \\ Christen, D. K.-28, 54, 55, 56, 60, 75, 76, 77, 78 \\ Christie, W. H.-78 \\ Chu, Y. T. $-81,82,88,96$ \\ Coltman, R. R., Jr. -70 \\ Cooke, J. F. $-5,6,7,13,14$ \\ Cooper, A. S. -4
}

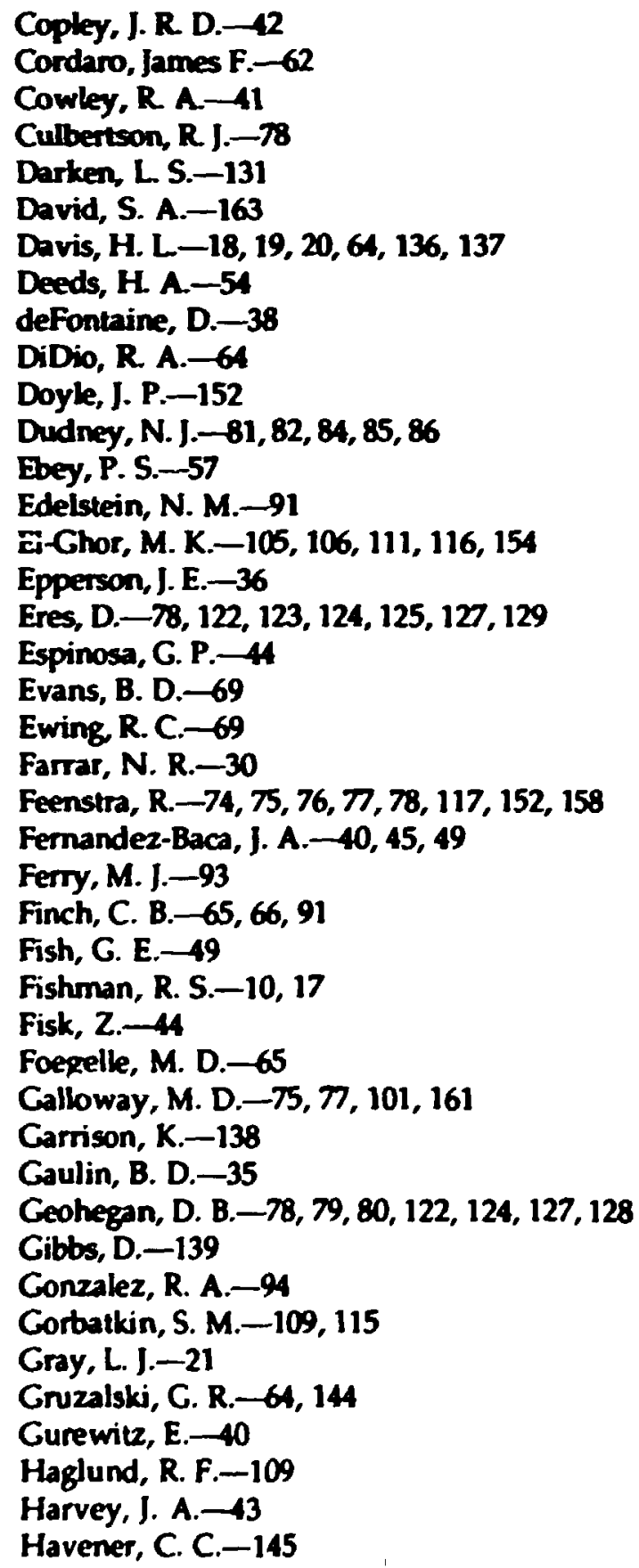


Hayden, S. M. -4

Haynes, T. E.-100, 101, 102, 104

Hayter, John B.-32, 34, 50

Hennion, B. 49

Hennion, M. -19

Heskett, D.-138, 144

Hill, N. W. -13

Holland, O. W.-101, 106, 111, 112, 113, 114, 116,148

Houghton, D. C. -150

Hoyt, J. -38

Hyatt, H. M. 63

Ito, H. -29

Jellison, G. E. Jr.-122, 130, 131, 132, 133

Johnson, N. M. -25

Kahane, S. -40

Kang, M. H.-145

Kaplan, Theodore-21

Karam, N. K.-105

Kerchner, H. R-60, 70, 76, 78

Kim, Y.C.-54, 55, 56, 60

Kabunde, C. E. -70, 76

Kot, W. K. -91

Kraitchman, M. D.-38

Kroeger, D. M. -60

Labarbe, P. D. -36

Larson, B. C-101, 157, 159, 161, 162

Lau, S. S.-122

Lee, In-Seop-10?

Lee, Youngchul-32

Lewis, J. D.-162

Lin, J. S. - 32, 34, 36, 39, 50

Littmark, U.-120

Liu, S. H. $-2,3,5,14$

Lowndes, D. H. - 78, 79, 122, 123, 124, 125, 127 , $128,129,130$

Lui, S. C. -145

Lumpkin, G. R-69

Lynn, J. E. -10

Maeta, H.-162

Mahar, G. D.-3, 4, 10, 11

Major, R. W. 63

Marwick, A. D. -25

Mashbum, L. N.-78, 79, 80, 122, 127

Mazumdar, P. $-81,82$

McCallum, J. C. $-67,69,118,119$

McCamy, J. W.-130

McHargue, C. J.-119

MCMorrow, D. F. -41

Mei, W. N. -138

Mele, E. J.-145

Meyer, F. W. -145
Mills, D. M. -159

Mitchell, P. W. -11

Mochrie, S. J. G.-139

Modine, F. A. $62,63,65,132,133$

Mogul, H. C-109

Mook, H. A.- 13, 44, 50

Moon, R. M. -10

Moore, J. L-104

More, K. L-102

Morii, Y. -35

Morris, S.-30

Mostoller, M.-13, 14, 15, 11

Mullica, D. F. -164

Müller, K.-20, 64

Nakai, Y, -39

Namavar, N. M. -105

Naramoto, H.-115

Newns, D. M.-12

Nicklow, R. M. -11, 45, 46, 47, 48

Noonan, J. R. - 18, 19, 25, 64, 136, 137

Ocko, B. M. -139

Oehrlein, G. S. -25

Oen, O.S. $-25,162$

Oliver, W. C. -69

Orera, V. M. $-92,95$

Ownby, G. W.-144

Pan, $\mathrm{X}$. -138

Pattnaik, P. C. -12

Penford, Jeff-34

Pennycook, S. J.-78, 100, 101, 105, 106, 114, 117, $122,127,146,148,149,150,151,152,154,155$, 156

Pertot, F. $-8,9$

Peterson, R. S. - 121

Plummer, E. W.-145

Pogatshnik, G. J.-69, 95

Poirot, I. S. $\rightarrow 91$

Pokex, D. B.-74, 108, 120, 122

Porter, Roger S.-32

Pynn, Roger-32

Ramakrishnan, V. R.-28

Raman, S. -10

Ramey, J. 0. 67

Rankin, J.-119

Rappaz, M. -163

Rasolt, M. $-8,9,12,16$

Reed, K. J.-145

Remeika, J. P. - 4

Rhyne, J. J. 45,49

Richard, R. T. -70

Richardson, J. W. -10

Roberto, J. B.-109 
Robinson, M. T. - 22

Rosseel, T. M.-121

Rosenblatt, G. H. -93

Roth, J.-115

Roy, R. A.-152

Russell, T. P.-29

Rytz, D. - 4

Sales, B. C.-54, 55, 56, 57, 58, 59, 67, 68, 78

Sangsinkeow, P.-131

Sappenfield, E. L.-164

Sauls, J. A. -10

Sekula, S. T. - 54, 55, 56, 60, 76, 78

Sharp, J. W.-127, 129

Sharp, K.-128

Shih, L. B. -34

Shim, Youngiae -62

Silver, M. N. -63

Sirois, E.-37

Sioreen, T. P.-116, 162

Skjeltorp, Ame T.-32

Sklad, P. S.-119

Smith, H. G. -12

Snook, lan K.-34

Snowden, K. J.-145

Sonder, E.-57, 58, 59, 68, 78

Specht, E. D. -60

Spooner, Stephen-35, 36, 37, 38, 50

Stubbs, Cerald-32

Taylor, A. D. -41

Tesanovic, Z.-16

Thomas, D. K.-108, 162

Thompson, J. R.-54, 55, 56, 60, 76, 78

Thomson, J. O. -78

Timmons, Peter-32

Tischler, J. Z.--125, 127, 157, 159

Tong S. Y. -138

Tortorelli, P. -38

Trewhella, Jill-32

Triolo, R-36

Trivisonno, J. -4

Tsai, C. C. -109

Valiga, R. E.-78

Vane, C. R.-121

Vitek, J. M.-163

Wang, J. C. $-86,87,89$

Watson, G.-138

Weeks, R. A. 109

Wendelken, J. F. -140, 141, 142, 143

Wheeler, R. B. 62

Whichard, G. W. -109

White, C. W.-106, 111, 114, 116, 117, 118, 119

Wignall, G. D.-28, 29, 30, 31, 50
Williams, G. P., Jr.-93

Williams, J. M.-107

Williams, R. T. -93

Wilson, G. A. -164

Withrow, S. P. -102

Wittmanck, K.-120

Wong Po-Zen-39

Wood, R. F.-13, 14

Wu, C. F. -34

Xia, W.-122

Yalisove, S. M. -149

Young, J. P.-121

Zarestky, J. L. 40

Zehner, D. M.-64, 138, 139, 144, 145

Zuhr, R. A.-100, 101, 102, 104, 109, 115, 121, 154 
